\title{
Classification of Genes Based on Age-Related Differential Expression in Breast Cancer
}

\author{
Gunhee Lee ${ }^{1,2}$, Minho Lee ${ }^{3 *}$ \\ ${ }^{1}$ Department of Biological Science, Sangji University, Wonju 26339, Korea, ${ }^{2}$ Department of Biomedicine \& Health Sciences, \\ Graduate School, The Catholic University of Korea, Seoul 06591, Korea, ${ }^{3}$ Catholic Precision Medicine Research Center, \\ College of Medicine, The Catholic University of Korea, Seoul 06591, Korea
}

\begin{abstract}
Transcriptome analysis has been widely used to make biomarker panels to diagnose cancers. In breast cancer, the age of the patient has been known to be associated with clinical features. As clinical transcriptome data have accumulated significantly, we classified all human genes based on age-specific differential expression between normal and breast cancer cells using public data. We retrieved the values for gene expression levels in breast cancer and matched normal cells from The Cancer Genome Atlas. We divided genes into two classes by paired t test without considering age in the first classification. We carried out a secondary classification of genes for each class into eight groups, based on the patterns of the $p$-values, which were calculated for each of the three age groups we defined. Through this two-step classification, gene expression was eventually grouped into 16 classes. We showed that this classification method could be applied to establish a more accurate prediction model to diagnose breast cancer by comparing the performance of prediction models with different combinations of genes. We expect that our scheme of classification could be used for other types of cancer data.
\end{abstract}

Keywords: biomarkers, breast cancer, differentially expressed genes, gene classification

\section{Introduction}

Breast cancer is known to one of the leading causes of cancer death among females [1]. A massive number of research studies on the genomic characterization of breast cancer, particularly the discovery of differentially expressed genes (DEGs), have revealed clinically relevant molecular subtypes [2], which has increased the accuracy of the prognosis [3-5] and has resulted in successful targeted therapy $[6,7]$. During recent decades, resources based on high-throughput sequencing technologies, such as The Cancer Genome Atlas (TCGA) [8] and International Cancer Genome Consortium (ICGC) [9], have facilitated more accurate detection of DEGs and cancer driver genes. The identification of DEGs is prominent, in that it leads to more accurate subtyping and more precise treatment for various types of cancers.

In transcriptome analysis based on microarray [10] or RNA sequencing [11] by next-generation sequencing, DEGs are usually identified by statistical tests, such as t test, nonparametric test, and Bayesian models [12]. Subsequent analysis of pathways and functional enrichment tests for DEGs are performed to increase the understanding of molecular mechanisms [13].

In the case of breast cancer, it is well known that molecular subtype and patient age are strongly associated with clinical features, such as survival rate. Fredholm et al. [14] reported that the 5-year survival rate was lowest in the of 2534-year-old age group and decreased with increasing age. Likewise, Gnerlich et al. [15] reported that younger women were more likely to die from breast cancer than older ones, based on the statistics of 243,012 breast cancer patients. Recently, Azim et al. [16] studied genomic aberrations in young and elderly breast cancer patients based on TCGA data. They found that older patients had more somatic mutations and copy number variations (CNVs) and that 11 mutations and two CNVs were independently associated with age at diagnosis.

In this work, we aimed to classify human genes based on 
age-specific differential expression between normal and breast cancer cells. DEGs were identified based on their p-values for differential gene expression between tumor and matched normal cells. DEGs and non-DEGs were then

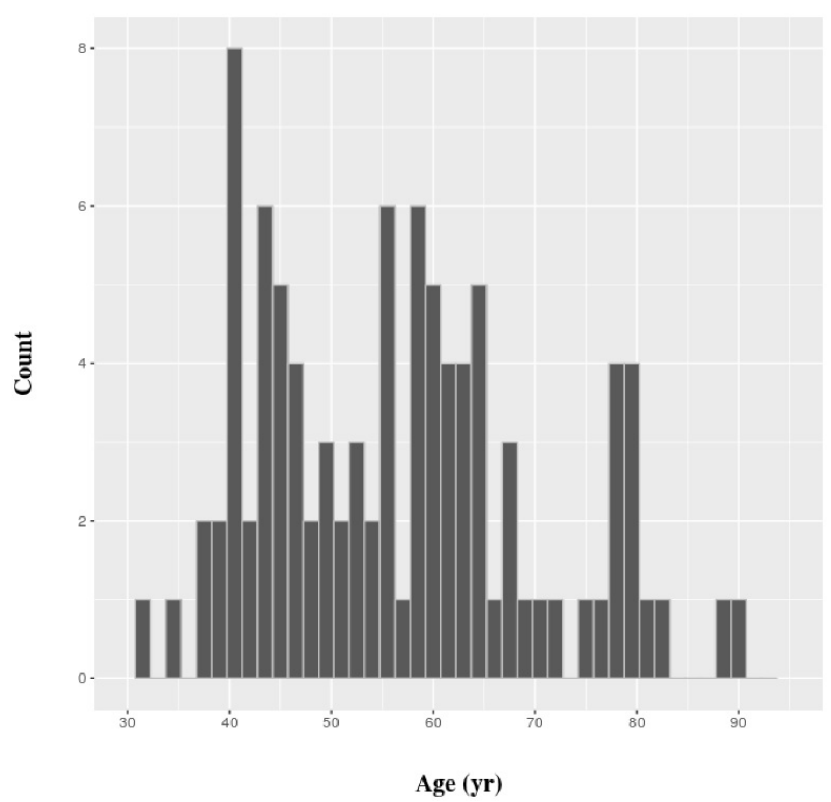

Fig. 1. Distribution of age of breast cancer patients analyzed in this work. classified based on age-specific differential expression by the three age groups we defined. To show an application of the classification, we compared the accuracy of prediction models that distinguish normal and tumor cells, constructed by support vector machine (SVM) using various combinations of genes by class. The performance of SVM was measured by the average area under the receiver operating characteristics curve value after 1,000 times bootstrap.

\section{Methods}

All gene expression values in this work were gathered from TCGA. Eligible patients had complete clinical data and a gene expression dataset of breast cancer cells and matched normal cells. Eventually, we retrieved the gene expression values of tumor and matched normal cells of 96 patients. The distribution of their ages is shown in Fig. 1. The values for gene expression level that we used were generated by the Illumina Hi-Seq platform (Illumina, Inc., San Diego, CA, USA) and normalized by root square error methods [17]. All subsequent statistical analyses were carried out using $\mathrm{R}$, version 3.2.3. SVM classifiers were constructed using the e1071 package of R, and a simple linear kernel was used. Functional enrichment analysis of gene classes was performed in ToppGene Suite [18].

Young patients were defined as $\leq 45$ years of age, and

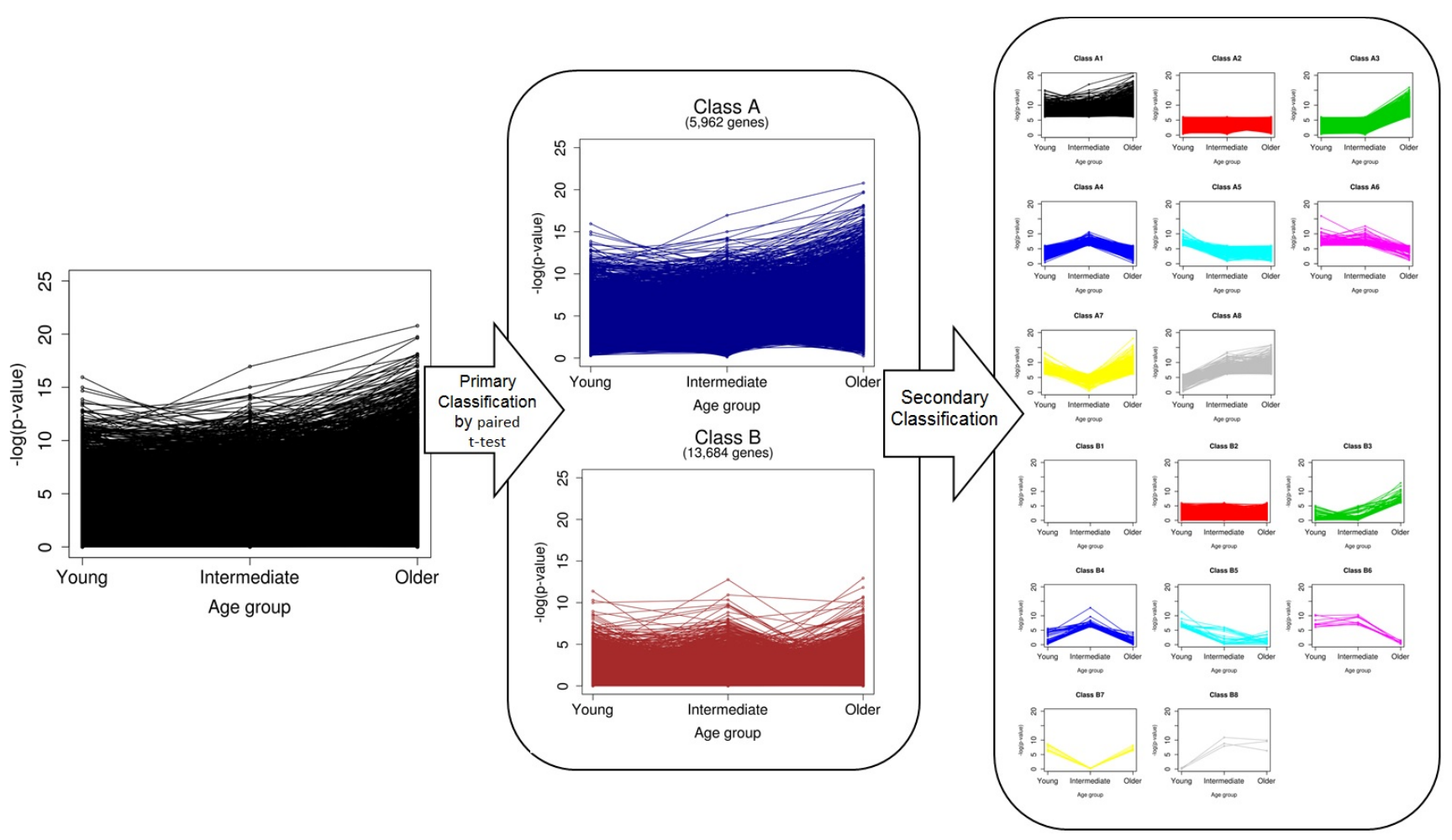

Fig. 2. Schematic view of overall procedures of the two-step classification. 
elderly patients were defined as those $\geq 60$ years of age (Fig. 1). The rest of the patients were defined as "intermediate." The statistical significance of differential expression between tumor and matched normal cells was determined by paired $t$ test, based on a p-value threshold of $8.48 \times 10^{-7}$. The threshold value was set based on the Bonferroni correction, because a test was performed for each age group, and three age groups were defined for each of the 19,646 genes.

\section{Results and Discussion}

The overall scheme of our classification is depicted in Fig. 2. We first divided genes into two classes (A and $B$ ) by paired $t$ test without considering age. A total of 5,962 genes in class A were defined as significant DEGs in breast cancer, and 13,684 in class B were nonsignificant. Ones who want to find biomarkers or driver genes are likely to investigate only genes in class A. However, we classified the genes of each class once again into eight groups, based on the pattern of p-values, which were calculated separately for every age group (secondary classification in Fig. 2). After a second round of classification, the genes were eventually divided into 16 classes (A1-B8) (Supplementary Table 1). The numbers of genes of the classes are shown in Table 1.

It was easily observed that there was no gene classified as class B1. Probably, this was because the significance of genes in class B was already tested in the primary classification step, and no genes showed significance over all age groups. The 377 genes in class A1 exhibited differential expression for every age group and all samples. These genes are the most powerful DEGs between normal and breast cancer cells. Genes of class 2, which did not have significantly different expression in any age group, accounted for the majority in both classes A and B. Indeed, classes 1 and 2 did not have any age-specific significance. Thus, we focused on the genes of classes 3-8, which showed age-specific differential expression (Fig. 3).
Functional enrichment analysis for each class was performed, but we could not find any relevant or intriguing biological implications or pathways related to breast cancer. Hence, we decided to provide the results of the analysis as raw data (Supplementary Table 2) rather than trying an unfeasible deduction.

To show an example of how the classification can be applied, we constructed prediction models that aimed to distinguish normal and breast cancer cells, using the expression values of various combinations of genes. We defined two types of combinations comprising gene lists. For type I combinations, genes were chosen evenly from each class. By contrast, genes were chosen randomly for type II combinations without considering gene class. For example, if we make a list composed of three genes from classes 3, 4, and 5, the type I combination should consist of a gene from class 3 , another from class 4 , and the other one from class 5 , but a type II combination could be composed of any genes randomly chosen from the pool of the three classes.

We compared the accuracies of the SVM of type I and II combinations for three subsets each for classes A and B (Table 2). The performance of type I was significantly better than that of type II, except in two cases (classes 6-8 in both classes A and B), the genes of which were significantly differentially expressed in the two age groups.

These results highlight the value of gene classification based on our method. Based on the first classification, 13,684 genes in class B were probably considered genes that cannot distinguish normal and breast cancer cells. However, by adapting one more classification step based on age-specific differential expression, we identified 171 age-specific DEGs in classes B3-B8. Despite the underestimated value of the classes, we showed that a balanced selection of genes from these classes could be applied as biomarkers, identifying breast cancer from normal cells. For example, genes that are known to be high-penetrance breast cancer susceptibility genes, such as TP53, STK11, and CDH11, and mode-

Table 1. Definition of classification of genes based on age-specific significance and the corresponding number of genes

\begin{tabular}{clllrr}
\hline \multirow{2}{*}{ Secondary class } & \multirow{2}{*}{ Young } & Intermediate & Older & \multicolumn{2}{c}{ Primary class } \\
\cline { 4 - 6 } & & & & A & B \\
\hline 1 & Significant & Significant & Significant & 377 & 0 \\
2 & Nonsignificant & Nonsignificant & Nonsignificant & 4,495 & 13,548 \\
3 & Nonsignificant & Nonsignificant & Significant & 781 & 69 \\
4 & Nonsignificant & Significant & Nonsignificant & 56 & 29 \\
5 & Significant & Nonsignificant & Nonsignificant & 44 & 18 \\
6 & Significant & Significant & Nonsignificant & 28 & 9 \\
7 & Significant & Nonsignificant & Nonsignificant & 71 & 8 \\
8 & Nonsignificant & Significant & Significant & 110 & 3 \\
\hline
\end{tabular}

Significances were defined based on the $\mathrm{p}$-value of paired $\mathrm{t}$ test. 

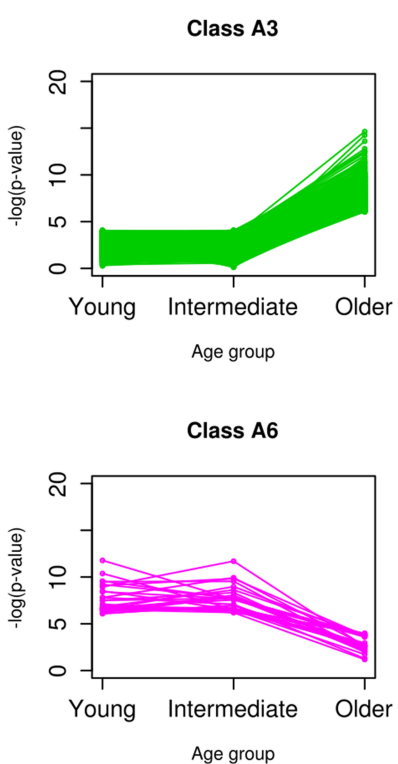

Age group

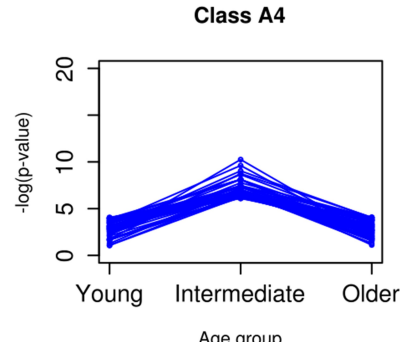

Class A7

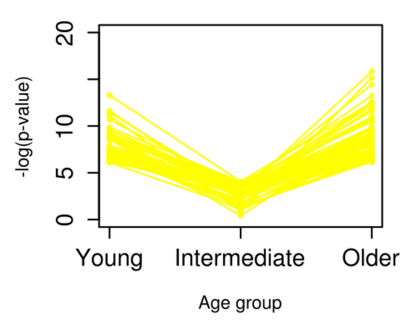

(A)

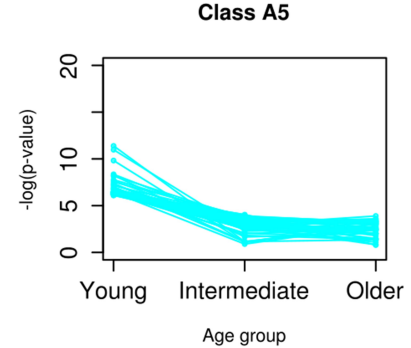

Class A8

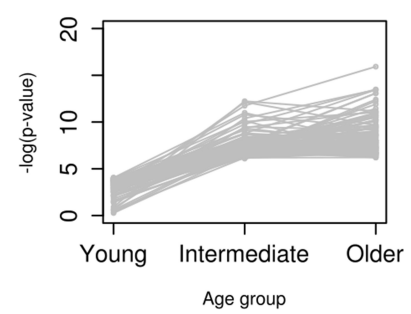

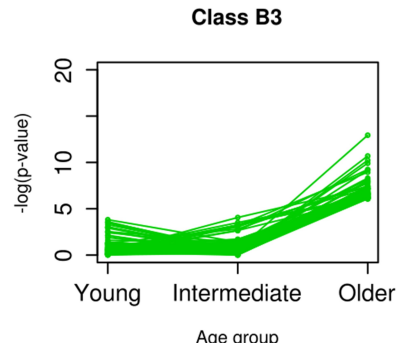

Class B6

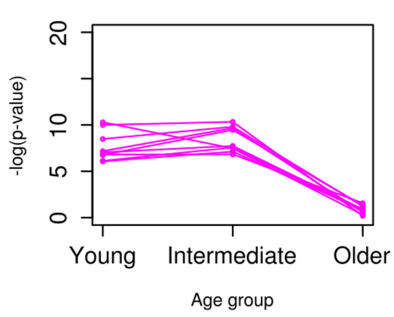

Class B4

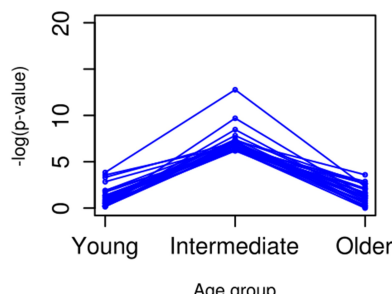

Class B7

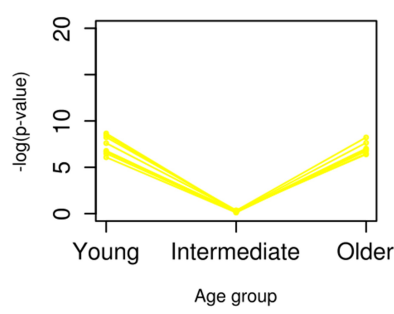

(B)

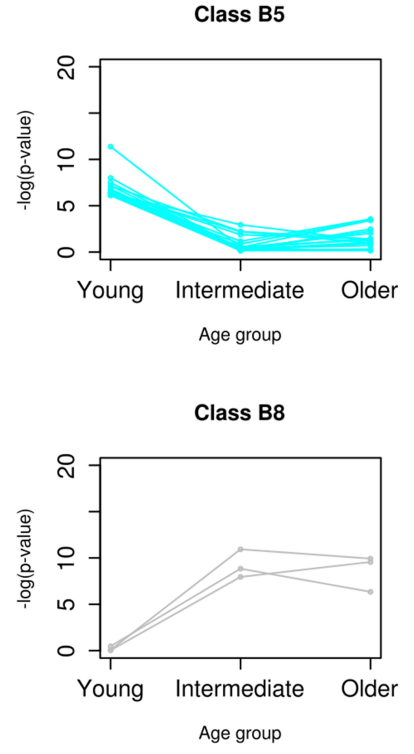

Fig. 3. Patterns of significance of agespecific differential expression of class A (A) and class B (B).

rate-penetrance genes, such as $R A D 50, R A D 51 C, R A D 51 D$, NBS1, and FANCM, were classified in class B2 [19]. In addition to proposing the possibility of genes in class $B$ as biomarkers, even for class A, we exhibited that our method of selecting biomarker genes based on secondary classification could be useful in making a combination of biomarker genes for a more accurate prediction by using different classes complementarily.

In summary, we retrieved a gene expression dataset of breast cancer and matched normal cells from TCGA and then classified the genes into 16 classes by two-step classification. This classification could be applied to generate a more accurate prediction model for identifying cancer. Furthermore, we expect that our scheme of classification could be used for other types of cancer data. 
Table 2. Comparison of performance of SVM models of different combinations of genes to distinguish breast cancer and normal cells

\begin{tabular}{cccc}
\hline Input genes $(\mathrm{N})$ & Sampling pool & Type I (one gene per class) & Type II (n random genes from the pool) \\
\hline 3 & Classes A3-A5 & 0.9351 & 0.9215 \\
3 & Classes A6-A8 & 0.9660 & 0.9662 \\
6 & Classes A3-A8 & 0.9842 & 0.9743 \\
3 & Classes B3-B5 & 0.8701 & 0.8522 \\
3 & Classes B6-B8 & 0.9269 & 0.9392 \\
6 & Classes B3-B8 & 0.9386 & 0.9227 \\
\hline
\end{tabular}

$\mathrm{N}$ input genes were sampled from each sampling pool by adopting two types of combinations (types I and II).

SVM, support vector machine.

ORCID: Gunhee Lee: http://orcid.org/0000-0001-7142-9

072; Minho Lee: http://orcid.org/0000-0002-0168-9546

\section{Authors' contribution}

\author{
Conceptualization: ML \\ Data curation: GL \\ Formal analysis: GL, ML \\ Funding acquisition: ML \\ Methodology: GL \\ Writing - original draft: GL, ML \\ Writing - review \& editing: ML
}

\section{Acknowledgments}

This work was supported by the National Research Foundation of Korea (NRF), funded by the Ministry of Science and ICT (NRF-2017R1C1B2008617, NRF-2017M3A9B6061511, and NRF-2017M3C9A604761) and KREONET (Korea Research Environment Open NETwork) which is managed and operated by KISTI (Korea Institute of Science and Technology Information). GL was supported by a Sangji University scholarship for research assistants.

\section{Supplementary materials}

Supplementary data including two tables can be found with this article online at http://www.genominfo.org/ src/sm/gni-15-156-s001.pdf.

\section{References}

1. Torre LA, Bray F, Siegel RL, Ferlay J, Lortet-Tieulent J, Jemal A. Global cancer statistics, 2012. CA Cancer J Clin 2015;65:87-108.

2. Perou CM, Sørlie T, Eisen MB, van de Rijn M, Jeffrey SS, Rees CA, et al. Molecular portraits of human breast tumours. Nature 2000;406:747-752.

3. Rouzier R, Perou CM, Symmans WF, Ibrahim N, Cristofanilli $\mathrm{M}$, Anderson $\mathrm{K}$, et al. Breast cancer molecular subtypes respond differently to preoperative chemotherapy. Clin Cancer
Res 2005;11:5678-5685.

4. Parker JS, Mullins M, Cheang MC, Leung S, Voduc D, Vickery $\mathrm{T}$, et al. Supervised risk predictor of breast cancer based on intrinsic subtypes. J Clin Oncol 2009;27:1160-1167.

5. Carey LA, Dees EC, Sawyer L, Gatti L, Moore DT, Collichio F, et al. The triple negative paradox: primary tumor chemosensitivity of breast cancer subtypes. Clin Cancer Res 2007;13: 2329-2334.

6. Nahta R, Yu D, Hung MC, Hortobagyi GN, Esteva FJ. Mechanisms of disease: understanding resistance to HER2-targeted therapy in human breast cancer. Nat Clin Pract Oncol 2006;3: 269-280.

7. Lehmann BD, Bauer JA, Chen X, Sanders ME, Chakravarthy $\mathrm{AB}$, Shyr $\mathrm{Y}$, et al. Identification of human triple-negative breast cancer subtypes and preclinical models for selection of targeted therapies. J Clin Invest 2011;121:2750-2767.

8. Cancer Genome Atlas Research Network, Weinstein JN, Collisson EA, Mills GB, Shaw KR, Ozenberger BA, et al. The Cancer Genome Atlas Pan-Cancer analysis project. Nat Genet 2013;45:1113-1120.

9. International Cancer Genome Consortium, Hudson TJ, Anderson W, Artez A, Barker AD, Bell C, et al. International network of cancer genome projects. Nature 2010;464:993-998.

10. Brown PO, Botstein D. Exploring the new world of the genome with DNA microarrays. Nat Genet 1999;21(1 Suppl): 33-37.

11. Morin R, Bainbridge M, Fejes A, Hirst M, Krzywinski M, Pugh T, et al. Profiling the HeLa S3 transcriptome using randomly primed cDNA and massively parallel short-read sequencing. Biotechniques 2008;45:81-94.

12. Efron B, Tibshirani R. Empirical bayes methods and false discovery rates for microarrays. Genet Epidemiol 2002;23:70-86.

13. Draghici S, Khatri P, Martins RP, Ostermeier GC, Krawetz SA. Global functional profiling of gene expression. Genomics 2003;81:98-104.

14. Fredholm H, Eaker S, Frisell J, Holmberg L, Fredriksson I, Lindman $\mathrm{H}$. Breast cancer in young women: poor survival despite intensive treatment. PLoS One 2009;4:e7695.

15. Gnerlich JL, Deshpande AD, Jeffe DB, Sweet A, White N, Margenthaler JA. Elevated breast cancer mortality in women younger than age 40 years compared with older women is attributed to poorer survival in early-stage disease. J Am Coll Surg 2009;208:341-347.

16. Azim HA Jr, Nguyen B, Brohée S, Zoppoli G, Sotiriou C. 
Genomic aberrations in young and elderly breast cancer patients. BMC Med 2015;13:266.

17. Cancer Genome Atlas Network. Comprehensive molecular portraits of human breast tumours. Nature 2012;490:61-70.

18. Chen J, Bardes EE, Aronow BJ, Jegga AG. ToppGene Suite for gene list enrichment analysis and candidate gene prioritization. Nucleic Acids Res 2009;37:W305-W311.

19. Economopoulou P, Dimitriadis G, Psyrri A. Beyond BRCA: new hereditary breast cancer susceptibility genes. Cancer Treat Rev 2015;41:1-8. 


\title{
SUPPLEMENTARY INFORMATION
}

\section{Classification of Genes Based on Age-Related Differential Expression in Breast Cancer}

\author{
Gunhee Lee ${ }^{1,2}$, Minho Lee ${ }^{3 *}$
}

${ }^{1}$ Department of Biological Science, Sangji University, Wonju 26339, Korea, ${ }^{2}$ Department of Biomedicine \& Health Sciences, Graduate School, The Catholic University of Korea, Seoul 06591, Korea, ${ }^{3}$ Catholic Precision Medicine Research Center, College of Medicine, The Catholic University of Korea, Seoul 06591, Korea 
Supplementary Table 1. Table of 16 gene classes based on age-specific expression

\begin{tabular}{|c|c|}
\hline \begin{tabular}{l|l} 
Gene name & Entrez ID
\end{tabular} & Gene class \\
\hline AASS|10157 & A1 \\
\hline ABCA10|10349 & $\mathrm{A} 1$ \\
\hline ABCA5|23461 & A1 \\
\hline ABCB1 15243 & $\mathrm{~A} 1$ \\
\hline ACBD3|64746 & $\mathrm{A} 1$ \\
\hline ACCS $\mid 84680$ & $\mathrm{~A} 1$ \\
\hline ACVR2A|92 & $\mathrm{A} 1$ \\
\hline ADAM33|80332 & $\mathrm{A} 1$ \\
\hline ADAMTS5|11096 & $\mathrm{A} 1$ \\
\hline ADAR|103 & $\mathrm{A} 1$ \\
\hline ADIPOR1|51094 & $\mathrm{A} 1$ \\
\hline \begin{tabular}{l|l|l|} 
ALDH1 A2 8854 \\
\end{tabular} & $\mathrm{~A} 1$ \\
\hline AMOTL1|154810 & $\mathrm{A} 1$ \\
\hline AMOTL2|51421 & $\mathrm{A} 1$ \\
\hline AMT 275 & $\mathrm{~A} 1$ \\
\hline ANAPC4|29945 & $\mathrm{A} 1$ \\
\hline ANKRD29|147463 & A1 \\
\hline ANXA1|301 & $\mathrm{A} 1$ \\
\hline AP1S1|1174 & $\mathrm{A} 1$ \\
\hline APBB1|322 & $\mathrm{A} 1$ \\
\hline APCDD1|147495 & $\mathrm{A} 1$ \\
\hline APOO|79135 & $\mathrm{A} 1$ \\
\hline ARF1|375 & $\mathrm{A} 1$ \\
\hline ARHGAP19|84986 & $\mathrm{A} 1$ \\
\hline ARHGEF10|9639 & $\mathrm{A} 1$ \\
\hline ARID5B|84159 & $\mathrm{A} 1$ \\
\hline ARRB1|408 & $\mathrm{A} 1$ \\
\hline ASB1|51665 & $\mathrm{A} 1$ \\
\hline ATP6AP1|537 & $\mathrm{A} 1$ \\
\hline ATP6V0B|533 & $\mathrm{A} 1$ \\
\hline AUH|549 & $\mathrm{A} 1$ \\
\hline BAI3|577 & $\mathrm{A} 1$ \\
\hline BBS2|583 & $\mathrm{A} 1$ \\
\hline BCL2L2|599 & $\mathrm{A} 1$ \\
\hline BCL6|604 & $\mathrm{A} 1$ \\
\hline BOLA2|552900 & $\mathrm{A} 1$ \\
\hline C16orf59|80178 & $\mathrm{A} 1$ \\
\hline C16orf86|388284 & A1 \\
\hline C17orf108|201229 & $\mathrm{A} 1$ \\
\hline C17orf48|56985 & A1 \\
\hline C1QTNF9B|387911 & $\mathrm{A} 1$ \\
\hline C1orf190|541468 & $\mathrm{A} 1$ \\
\hline C1orf31|388753 & $\mathrm{A} 1$ \\
\hline C20orf194|25943 & $\mathrm{A} 1$ \\
\hline C21orf34|388815 & $\mathrm{A} 1$ \\
\hline C2orf40|84417 & A1 \\
\hline C2orf67|151050 & $\mathrm{A} 1$ \\
\hline C4orf49|84709 & $\mathrm{A} 1$ \\
\hline
\end{tabular}




\begin{tabular}{|c|c|}
\hline C5orf4|10826 & $\mathrm{A} 1$ \\
\hline C5orf53|492311 & $\mathrm{A} 1$ \\
\hline C6orf129|154467 & $\mathrm{A} 1$ \\
\hline C8orf79|57604 & $\mathrm{A} 1$ \\
\hline CACHD1|57685 & $\mathrm{A} 1$ \\
\hline CALCOCO1|57658 & $\mathrm{A} 1$ \\
\hline CAPN11|11131 & $\mathrm{A} 1$ \\
\hline CAPZA1|829 & $\mathrm{A} 1$ \\
\hline CBX3|11335 & $\mathrm{A} 1$ \\
\hline CBX7|23492 & $\mathrm{A} 1$ \\
\hline CC2D2A|57545 & $\mathrm{A} 1$ \\
\hline CCDC46|201134 & $\mathrm{A} 1$ \\
\hline CCDC93|54520 & $\mathrm{A} 1$ \\
\hline CD200|4345 & $\mathrm{A} 1$ \\
\hline CD276|80381 & $\mathrm{A} 1$ \\
\hline CDADC1|81602 & $\mathrm{A} 1$ \\
\hline CDC14B|8555 & $\mathrm{A} 1$ \\
\hline CEP68|23177 & $\mathrm{A} 1$ \\
\hline CES2|8824 & $\mathrm{A} 1$ \\
\hline CFL1|1072 & $\mathrm{A} 1$ \\
\hline CHL1|10752 & $\mathrm{A} 1$ \\
\hline CIT|11113 & $\mathrm{A} 1$ \\
\hline CLCN6|1185 & $\mathrm{A} 1$ \\
\hline $\begin{array}{l}\text { CLDN11|5010 } \\
\end{array}$ & $\mathrm{A} 1$ \\
\hline \begin{tabular}{l|l|} 
CLDN19|149461 \\
\end{tabular} & $\mathrm{A} 1$ \\
\hline CNTNAP3|79937 & $\mathrm{A} 1$ \\
\hline COL17A1|1308 & $\mathrm{A} 1$ \\
\hline CRIM1|51232 & $\mathrm{A} 1$ \\
\hline CX3CL1|6376 & $\mathrm{A} 1$ \\
\hline CYP2U1|113612 & $\mathrm{A} 1$ \\
\hline \begin{tabular}{l|l} 
DAB2IP & 153090 \\
\end{tabular} & $\mathrm{~A} 1$ \\
\hline DCP1A|55802 & $\mathrm{A} 1$ \\
\hline DDX26B|203522 & $\mathrm{A} 1$ \\
\hline DFNB59|494513 & $\mathrm{A} 1$ \\
\hline DLGAP2|9228 & $\mathrm{A} 1$ \\
\hline DLK2|65989 & $\mathrm{A} 1$ \\
\hline DMD|1756 & $\mathrm{A} 1$ \\
\hline DNAH1|25981 & $\mathrm{A} 1$ \\
\hline DNAJC18|202052 & $\mathrm{A} 1$ \\
\hline DNAJC27|51277 & $\mathrm{A} 1$ \\
\hline DOCK1|1793 & $\mathrm{A} 1$ \\
\hline DPY19L2|283417 & $\mathrm{A} 1$ \\
\hline DPY30|84661 & $\mathrm{A} 1$ \\
\hline DPYSL2|1808 & $\mathrm{A} 1$ \\
\hline DST 667 & $\mathrm{~A} 1$ \\
\hline DTYMK|1841 & $\mathrm{A} 1$ \\
\hline DUSP6|1848 & $\mathrm{A} 1$ \\
\hline EDN3|1908 & $\mathrm{A} 1$ \\
\hline EDNRB $\mid 1910$ & $\mathrm{~A} 1$ \\
\hline EEPD1|80820 & $\mathrm{A} 1$ \\
\hline EFHA2|286097 & $\mathrm{A} 1$ \\
\hline
\end{tabular}




\begin{tabular}{|c|c|}
\hline EIF2AK1|27102 & $\mathrm{A} 1$ \\
\hline EIF3L|51386 & $\mathrm{A} 1$ \\
\hline ERCC6L|54821 & $\mathrm{A} 1$ \\
\hline ESD|2098 & $\mathrm{A} 1$ \\
\hline EVC2|132884 & $\mathrm{A} 1$ \\
\hline EZH1|2145 & $\mathrm{A} 1$ \\
\hline $\mathrm{F} 3 \mid 2152$ & $\mathrm{~A} 1$ \\
\hline FAM122A|116224 & $\mathrm{A} 1$ \\
\hline FAM126A|84668 & $\mathrm{A} 1$ \\
\hline FAM13C|220965 & $\mathrm{A} 1$ \\
\hline FAM189A2|9413 & $\mathrm{A} 1$ \\
\hline FAM82A1|151393 & $\mathrm{A} 1$ \\
\hline FBXO31|79791 & $\mathrm{A} 1$ \\
\hline FGF1|2246 & $\mathrm{A} 1$ \\
\hline FIGF|2277 & $\mathrm{A} 1$ \\
\hline FLJ10038|55056 & $\mathrm{A} 1$ \\
\hline FLJ13197|79667 & $\mathrm{A} 1$ \\
\hline FOXO4|4303 & $\mathrm{A} 1$ \\
\hline FXYD1|5348 & $\mathrm{A} 1$ \\
\hline GEMIN6|79833 & $\mathrm{A} 1$ \\
\hline GGPS1|9453 & $\mathrm{A} 1$ \\
\hline GNAL|2774 & $\mathrm{A} 1$ \\
\hline GNE|10020 & $\mathrm{A} 1$ \\
\hline GPRASP1|9737 & $\mathrm{A} 1$ \\
\hline GPRASP2|114928 & $\mathrm{A} 1$ \\
\hline GRIA4|2893 & $\mathrm{A} 1$ \\
\hline GSPT2|23708 & $\mathrm{A} 1$ \\
\hline H2AFY|9555 & $\mathrm{A} 1$ \\
\hline H2AFZ|3015 & $\mathrm{A} 1$ \\
\hline H3F3A|3020 & $\mathrm{A} 1$ \\
\hline HERC2P2|400322 & $\mathrm{A} 1$ \\
\hline HLF|3131 & $\mathrm{A} 1$ \\
\hline HNRNPAB|3182 & $\mathrm{A} 1$ \\
\hline HNRNPF|3185 & $\mathrm{A} 1$ \\
\hline HNRNPL|3191 & $\mathrm{A} 1$ \\
\hline HNRNPU|3192 & $\mathrm{A} 1$ \\
\hline HOXA2|3199 & $\mathrm{A} 1$ \\
\hline HOXA3|3200 & $\mathrm{A} 1$ \\
\hline HOXA4|3201 & $\mathrm{A} 1$ \\
\hline HOXA5|3202 & $\mathrm{A} 1$ \\
\hline HOXA6|3203 & $\mathrm{A} 1$ \\
\hline HYMAI|57061 & $\mathrm{A} 1$ \\
\hline IGSF10|285313 & $\mathrm{A} 1$ \\
\hline IL11RA|3590 & $\mathrm{A} 1$ \\
\hline IL17RD|54756 & $\mathrm{A} 1$ \\
\hline IL33|90865 & $\mathrm{A} 1$ \\
\hline ING3|54556 & $\mathrm{A} 1$ \\
\hline INPP1|3628 & $\mathrm{A} 1$ \\
\hline INTS7|25896 & $\mathrm{A} 1$ \\
\hline ISM1|140862 & $\mathrm{A} 1$ \\
\hline ITM2A|9452 & $\mathrm{A} 1$ \\
\hline
\end{tabular}




\begin{tabular}{|c|c|}
\hline KCNE1|3753 & A1 \\
\hline KCTD12|115207 & $\mathrm{A} 1$ \\
\hline KIAA1614|57710 & $\mathrm{A} 1$ \\
\hline KIRREL|55243 & $\mathrm{A} 1$ \\
\hline KLF9|687 & $\mathrm{A} 1$ \\
\hline KLHDC1|122773 & $\mathrm{A} 1$ \\
\hline KLHL21|9903 & $\mathrm{A} 1$ \\
\hline KLHL29|114818 & $\mathrm{A} 1$ \\
\hline KLHL3|26249 & $\mathrm{A} 1$ \\
\hline KL|9365 & $\mathrm{A} 1$ \\
\hline LCA5|167691 & $\mathrm{A} 1$ \\
\hline LCAT|3931 & $\mathrm{A} 1$ \\
\hline LDB2|9079 & $\mathrm{A} 1$ \\
\hline LETMD1|25875 & $\mathrm{A} 1$ \\
\hline LGR4|55366 & $\mathrm{A} 1$ \\
\hline LIFR/3977 & $\mathrm{A} 1$ \\
\hline LIMS2|55679 & $\mathrm{A} 1$ \\
\hline LMBRD1|55788 & $\mathrm{A} 1$ \\
\hline LOC100132707|100132707 & $\mathrm{A} 1$ \\
\hline LOC134466|134466 & $\mathrm{A} 1$ \\
\hline LOC285830|285830 & $\mathrm{A} 1$ \\
\hline LOC339290|339290 & $\mathrm{A} 1$ \\
\hline LOC399959|399959 & $\mathrm{A} 1$ \\
\hline LOC728264|728264 & $\mathrm{A} 1$ \\
\hline LPCAT2|54947 & $\mathrm{A} 1$ \\
\hline LRCH1|23143 & $\mathrm{A} 1$ \\
\hline LRIG3|121227 & $\mathrm{A} 1$ \\
\hline LRP4|4038 & $\mathrm{A} 1$ \\
\hline LRRC3B|116135 & $\mathrm{A} 1$ \\
\hline LRRTM2|26045 & $\mathrm{A} 1$ \\
\hline LSM4|25804 & $\mathrm{A} 1$ \\
\hline LUZP1|7798 & $\mathrm{A} 1$ \\
\hline MAB21L1|4081 & $\mathrm{A} 1$ \\
\hline MAGI1|9223 & $\mathrm{A} 1$ \\
\hline MAMDC2|256691 & $\mathrm{A} 1$ \\
\hline MAML2|84441 & $\mathrm{A} 1$ \\
\hline MANF|7873 & $\mathrm{A} 1$ \\
\hline MAPKBP1|23005 & $\mathrm{A} 1$ \\
\hline MATN2|4147 & $\mathrm{A} 1$ \\
\hline MAZ|4150 & $\mathrm{A} 1$ \\
\hline MBOAT7|79143 & $\mathrm{A} 1$ \\
\hline MEG3|55384 & $\mathrm{A} 1$ \\
\hline MEIS2|4212 & $\mathrm{A} 1$ \\
\hline METTL7A|25840 & $\mathrm{A} 1$ \\
\hline MICAL3|57553 & $\mathrm{A} 1$ \\
\hline MID1|4281 & $\mathrm{A} 1$ \\
\hline MLF1IP|79682 & $\mathrm{A} 1$ \\
\hline MME|4311 & $\mathrm{A} 1$ \\
\hline MOBKL2B|79817 & $\mathrm{A} 1$ \\
\hline MOBKL2C|148932 & $\mathrm{A} 1$ \\
\hline MPDZ|8777 & $\mathrm{A} 1$ \\
\hline
\end{tabular}




\begin{tabular}{|c|c|}
\hline MTMR10|54893 & $\mathrm{A} 1$ \\
\hline MYLK|4638 & $\mathrm{A} 1$ \\
\hline N4BP2L1|90634 & $\mathrm{A} 1$ \\
\hline NAA20|51126 & $\mathrm{A} 1$ \\
\hline NAB1|4664 & $\mathrm{A} 1$ \\
\hline NAP1L5|266812 & $\mathrm{A} 1$ \\
\hline NBLA00301|79804 & $\mathrm{A} 1$ \\
\hline NCRNA00086|399668 & $\mathrm{A} 1$ \\
\hline NCRNA00087|644596 & $\mathrm{A} 1$ \\
\hline NDEL1 $\mid 81565$ & $\mathrm{~A} 1$ \\
\hline NDRG2|57447 & $\mathrm{A} 1$ \\
\hline NEIL3|55247 & $\mathrm{A} 1$ \\
\hline NFAT5|10725 & $\mathrm{A} 1$ \\
\hline NGFR/4804 & $\mathrm{A} 1$ \\
\hline NISCH|11188 & $\mathrm{A} 1$ \\
\hline \begin{tabular}{l|l|} 
NPHP3|27031 \\
\end{tabular} & $\mathrm{A} 1$ \\
\hline NR2C1|7181 & $\mathrm{A} 1$ \\
\hline NR3C1|2908 & $\mathrm{A} 1$ \\
\hline NR3C2|4306 & $\mathrm{A} 1$ \\
\hline NRIP2|83714 & $\mathrm{A} 1$ \\
\hline NTF4|4909 & $\mathrm{A} 1$ \\
\hline OR2A1|346528 & $\mathrm{A} 1$ \\
\hline OR2A9P|441295 & $\mathrm{A} 1$ \\
\hline OXTR|5021 & $\mathrm{A} 1$ \\
\hline PAFAH1B3|5050 & $\mathrm{A} 1$ \\
\hline PAK3|5063 & $\mathrm{A} 1$ \\
\hline PAK7|57144 & $\mathrm{A} 1$ \\
\hline PAMR1|25891 & $\mathrm{A} 1$ \\
\hline PAR-SN|347746 & $\mathrm{A} 1$ \\
\hline PARK2|5071 & $\mathrm{A} 1$ \\
\hline PARP1|142 & $\mathrm{A} 1$ \\
\hline PCDHGB7|56099 & $\mathrm{A} 1$ \\
\hline PCNX|22990 & $\mathrm{A} 1$ \\
\hline PDE1C|5137 & $\mathrm{A} 1$ \\
\hline PDGFD|80310 & $\mathrm{A} 1$ \\
\hline PDLIM4|8572 & $\mathrm{A} 1$ \\
\hline PELI2|57161 & $\mathrm{A} 1$ \\
\hline PER1|5187 & $\mathrm{A} 1$ \\
\hline PHF10|55274 & $\mathrm{A} 1$ \\
\hline PHF17|79960 & $\mathrm{A} 1$ \\
\hline PIGU|128869 & $\mathrm{A} 1$ \\
\hline PIK3R1|5295 & $\mathrm{A} 1$ \\
\hline PKD2|5311 & $\mathrm{A} 1$ \\
\hline PKMYT1|9088 & $\mathrm{A} 1$ \\
\hline PLAGL1|5325 & $\mathrm{A} 1$ \\
\hline PLEKHH2|130271 & $\mathrm{A} 1$ \\
\hline PLEKHM3|389072 & $\mathrm{A} 1$ \\
\hline PLK1S1|55857 & $\mathrm{A} 1$ \\
\hline PLSCR4|57088 & $\mathrm{A} 1$ \\
\hline PMM2|5373 & $\mathrm{A} 1$ \\
\hline PNMAL2|57469 & $\mathrm{A} 1$ \\
\hline
\end{tabular}




\begin{tabular}{|c|c|}
\hline PNRC1|10957 & $\mathrm{A} 1$ \\
\hline POU6F1|5463 & $\mathrm{A} 1$ \\
\hline PPAP2B $\mid 8613$ & $\mathrm{~A} 1$ \\
\hline PPIAL4C|653598 & $\mathrm{A} 1$ \\
\hline PPL|5493 & A1 \\
\hline PPM1F|9647 & $\mathrm{A} 1$ \\
\hline PPP4C|5531 & $\mathrm{A} 1$ \\
\hline PPP4R1L|55370 & $\mathrm{A} 1$ \\
\hline PRCD|768206 & $\mathrm{A} 1$ \\
\hline PRDX1|5052 & $\mathrm{A} 1$ \\
\hline PRIMA1|145270 & $\mathrm{A} 1$ \\
\hline PRNP|5621 & $\mathrm{A} 1$ \\
\hline PROS1|5627 & $\mathrm{A} 1$ \\
\hline PRX|57716 & $\mathrm{A} 1$ \\
\hline PSENEN|55851 & $\mathrm{A} 1$ \\
\hline PSKH1|5681 & $\mathrm{A} 1$ \\
\hline PSMA5|5686 & $\mathrm{A} 1$ \\
\hline PSMC4|5704 & $\mathrm{A} 1$ \\
\hline PSMD14|10213 & $\mathrm{A} 1$ \\
\hline PTBP1|5725 & $\mathrm{A} 1$ \\
\hline PTCH1|5727 & $\mathrm{A} 1$ \\
\hline PTPN21|11099 & $\mathrm{A} 1$ \\
\hline PYCR1|5831 & $\mathrm{A} 1$ \\
\hline $\mathrm{PZP} \mid 5858$ & $\mathrm{~A} 1$ \\
\hline RAB11FIP2|22841 & $\mathrm{A} 1$ \\
\hline RAB8A|4218 & $\mathrm{A} 1$ \\
\hline RABIF|5877 & $\mathrm{A} 1$ \\
\hline RAPGEF2|9693 & $\mathrm{A} 1$ \\
\hline RAPGEF3|10411 & $\mathrm{A} 1$ \\
\hline RASSF9|9182 & $\mathrm{A} 1$ \\
\hline RBM9|23543 & $\mathrm{A} 1$ \\
\hline RBMS2|5939 & $\mathrm{A} 1$ \\
\hline RCBTB2|1102 & $\mathrm{A} 1$ \\
\hline REV1|51455 & $\mathrm{A} 1$ \\
\hline RGNEF|64283 & $\mathrm{A} 1$ \\
\hline RGN|9104 & $\mathrm{A} 1$ \\
\hline RHOJ|57381 & $\mathrm{A} 1$ \\
\hline RND3|390 & $\mathrm{A} 1$ \\
\hline RNF180|285671 & $\mathrm{A} 1$ \\
\hline RNF187|149603 & $\mathrm{A} 1$ \\
\hline RNF214|257160 & $\mathrm{A} 1$ \\
\hline RNF38|152006 & $\mathrm{A} 1$ \\
\hline ROBO3|64221 & $\mathrm{A} 1$ \\
\hline RPS6KA3|6197 & $\mathrm{A} 1$ \\
\hline RRN3P1|730092 & $\mathrm{A} 1$ \\
\hline RUNDC3B|154661 & $\mathrm{A} 1$ \\
\hline \begin{tabular}{ll|l|} 
RUSC1|23623 \\
\end{tabular} & $\mathrm{A} 1$ \\
\hline SAE1|10055 & $\mathrm{A} 1$ \\
\hline SAMD5|389432 & $\mathrm{A} 1$ \\
\hline SASH1|23328 & $\mathrm{A} 1$ \\
\hline SAV1|60485 & $\mathrm{A} 1$ \\
\hline
\end{tabular}




\begin{tabular}{|c|c|}
\hline SCN2B $\mid 6327$ & A1 \\
\hline SCN3A|6328 & $\mathrm{A} 1$ \\
\hline SCN4B $\mid 6330$ & $\mathrm{~A} 1$ \\
\hline SEC14L1|6397 & $\mathrm{A} 1$ \\
\hline SEMA3D|223117 & A1 \\
\hline SH3BGRL2|83699 & $\mathrm{A} 1$ \\
\hline SKA3|221150 & $\mathrm{A} 1$ \\
\hline SLC20A1|6574 & $\mathrm{A} 1$ \\
\hline SLC25A44|9673 & $\mathrm{A} 1$ \\
\hline SLC27A1|376497 & $\mathrm{A} 1$ \\
\hline SLC27A6|28965 & $\mathrm{A} 1$ \\
\hline SLC35A2|7355 & A1 \\
\hline SMC6|79677 & $\mathrm{A} 1$ \\
\hline SMYD4|114826 & $\mathrm{A} 1$ \\
\hline SNCA|6622 & $\mathrm{A} 1$ \\
\hline SOBP|55084 & $\mathrm{A} 1$ \\
\hline SPRY2|10253 & A1 \\
\hline SRP9|6726 & $\mathrm{A} 1$ \\
\hline SRPX|8406 & $\mathrm{A} 1$ \\
\hline SSPN|8082 & $\mathrm{A} 1$ \\
\hline SSTR1|6751 & $\mathrm{A} 1$ \\
\hline STAT5A|6776 & $\mathrm{A} 1$ \\
\hline STAT5B|6777 & $\mathrm{A} 1$ \\
\hline \begin{tabular}{l|l|} 
SYNE2|23224 &
\end{tabular} & $\mathrm{A} 1$ \\
\hline SYNM|23336 & $\mathrm{A} 1$ \\
\hline TBC1D4|9882 & $\mathrm{A} 1$ \\
\hline TBRG1|84897 & $\mathrm{A} 1$ \\
\hline TCEAL7|56849 & $\mathrm{A} 1$ \\
\hline TCP11L2|255394 & $\mathrm{A} 1$ \\
\hline TDRD10|126668 & $\mathrm{A} 1$ \\
\hline TDRD6|221400 & $\mathrm{A} 1$ \\
\hline TEF|7008 & $\mathrm{A} 1$ \\
\hline TGFBR3|7049 & $\mathrm{A} 1$ \\
\hline THRB|7068 & $\mathrm{A} 1$ \\
\hline THSD1|55901 & $\mathrm{A} 1$ \\
\hline TIMM17A|10440 & $\mathrm{A} 1$ \\
\hline TINAGL1|64129 & $\mathrm{A} 1$ \\
\hline TLE4|7091 & $\mathrm{A} 1$ \\
\hline TMEM220|388335 & $\mathrm{A} 1$ \\
\hline TMEM63B|55362 & $\mathrm{A} 1$ \\
\hline TMEM8A|58986 & $\mathrm{A} 1$ \\
\hline TMTC1|83857 & $\mathrm{A} 1$ \\
\hline TPO|7173 & $\mathrm{A} 1$ \\
\hline TRIM11|81559 & $\mathrm{A} 1$ \\
\hline TRIM59|286827 & $\mathrm{A} 1$ \\
\hline TRPC1|7220 & $\mathrm{A} 1$ \\
\hline TRPC6|7225 & $\mathrm{A} 1$ \\
\hline TSC1|7248 & $\mathrm{A} 1$ \\
\hline TSHZ2|128553 & $\mathrm{A} 1$ \\
\hline TSLP $\mid 85480$ & $\mathrm{~A} 1$ \\
\hline TSPYL2|64061 & $\mathrm{A} 1$ \\
\hline
\end{tabular}




\begin{tabular}{|c|c|}
\hline TSTA3|7264 & A1 \\
\hline TTC28|23331 & A1 \\
\hline TUBA1C $\mid 84790$ & $\mathrm{~A} 1$ \\
\hline TUBE1|51175 & $\mathrm{A} 1$ \\
\hline TUBG2|27175 & $\mathrm{A} 1$ \\
\hline USP44|84101 & $\mathrm{A} 1$ \\
\hline UTRN|7402 & $\mathrm{A} 1$ \\
\hline VAMP2|6844 & $\mathrm{A} 1$ \\
\hline WASF2|10163 & $\mathrm{A} 1$ \\
\hline WBP4|11193 & $\mathrm{A} 1$ \\
\hline WDR86|349136 & $\mathrm{A} 1$ \\
\hline WHAMML2|440253 & $\mathrm{A} 1$ \\
\hline YAP1|10413 & $\mathrm{A} 1$ \\
\hline ZBTB4|57659 & $\mathrm{A} 1$ \\
\hline ZDHHC17|23390 & $\mathrm{A} 1$ \\
\hline ZFP36L2|678 & $\mathrm{A} 1$ \\
\hline ZNF132|7691 & $\mathrm{A} 1$ \\
\hline ZNF204P|7754 & $\mathrm{A} 1$ \\
\hline ZNF280D|54816 & $\mathrm{A} 1$ \\
\hline ZNF391|346157 & $\mathrm{A} 1$ \\
\hline ZNF502|91392 & $\mathrm{A} 1$ \\
\hline ZNF660|285349 & $\mathrm{A} 1$ \\
\hline ZNF781|163115 & $\mathrm{A} 1$ \\
\hline$? \mid 10431$ & $\mathrm{~A} 2$ \\
\hline$? 653553$ & $\mathrm{~A} 2$ \\
\hline $\mathrm{A} 1 \mathrm{BG} \mid 1$ & $\mathrm{~A} 2$ \\
\hline AADAC|13 & $\mathrm{A} 2$ \\
\hline ABCA1|19 & $\mathrm{A} 2$ \\
\hline ABCA8|10351 & $\mathrm{A} 2$ \\
\hline ABCB10|23456 & $\mathrm{A} 2$ \\
\hline ABCB8|11194 & $\mathrm{A} 2$ \\
\hline ABCB9|23457 & $\mathrm{A} 2$ \\
\hline ABCC5|10057 & $\mathrm{A} 2$ \\
\hline ABCC6|368 & $\mathrm{A} 2$ \\
\hline ABCD2|225 & $\mathrm{A} 2$ \\
\hline ABCD3|5825 & $\mathrm{A} 2$ \\
\hline ABCD4|5826 & $\mathrm{A} 2$ \\
\hline $\mathrm{ABCF} 1 \mid 23$ & $\mathrm{~A} 2$ \\
\hline ABCF3|55324 & $\mathrm{A} 2$ \\
\hline ABCG1|9619 & $\mathrm{A} 2$ \\
\hline ABHD12|26090 & $\mathrm{A} 2$ \\
\hline ABHD14B|84836 & $\mathrm{A} 2$ \\
\hline ABHD1|84696 & $\mathrm{A} 2$ \\
\hline ABHD6|57406 & $\mathrm{A} 2$ \\
\hline ABL1|25 & $\mathrm{A} 2$ \\
\hline ABL2|27 & $\mathrm{A} 2$ \\
\hline ABLIM1|3983 & $\mathrm{A} 2$ \\
\hline ABLIM3|22885 & $\mathrm{A} 2$ \\
\hline ABR|29 & $\mathrm{A} 2$ \\
\hline ABTB1|80325 & $\mathrm{A} 2$ \\
\hline ACACB|32 & $\mathrm{A} 2$ \\
\hline
\end{tabular}




\begin{tabular}{|c|c|}
\hline ACAD11|84129 & A2 \\
\hline ACADS 35 & $\mathrm{~A} 2$ \\
\hline ACADVL|37 & $\mathrm{A} 2$ \\
\hline ACAT2|39 & $\mathrm{A} 2$ \\
\hline ACBD4|79777 & $\mathrm{A} 2$ \\
\hline ACOT7|11332 & $\mathrm{A} 2$ \\
\hline ACOT8|10005 & $\mathrm{A} 2$ \\
\hline ACP1|52 & $\mathrm{A} 2$ \\
\hline $\mathrm{ACP} 2 \mid 53$ & $\mathrm{~A} 2$ \\
\hline ACSBG2|81616 & $\mathrm{A} 2$ \\
\hline ACSL1|2180 & $\mathrm{A} 2$ \\
\hline ACSL5|51703 & A2 \\
\hline ACSM5|54988 & $\mathrm{A} 2$ \\
\hline ACSS2|55902 & $\mathrm{A} 2$ \\
\hline ACTA2|59 & $\mathrm{A} 2$ \\
\hline ACTB 60 & $\mathrm{~A} 2$ \\
\hline ACTG1|71 & $\mathrm{A} 2$ \\
\hline ACTL6A|86 & $\mathrm{A} 2$ \\
\hline ACTR1B|10120 & A2 \\
\hline ACTR2|10097 & $\mathrm{A} 2$ \\
\hline ACTR3|10096 & $\mathrm{A} 2$ \\
\hline ACVR1C|130399 & $\mathrm{A} 2$ \\
\hline ADAD2|161931 & $\mathrm{A} 2$ \\
\hline ADAM12|8038 & $\mathrm{A} 2$ \\
\hline ADAM15|8751 & $\mathrm{A} 2$ \\
\hline ADAM19|8728 & $\mathrm{A} 2$ \\
\hline ADAM8|101 & $\mathrm{A} 2$ \\
\hline ADAMTS12|81792 & $\mathrm{A} 2$ \\
\hline ADAMTS14|140766 & $\mathrm{A} 2$ \\
\hline ADAMTS18|170692 & $\mathrm{A} 2$ \\
\hline ADAMTS1|9510 & $\mathrm{A} 2$ \\
\hline ADAMTS3|9508 & $\mathrm{A} 2$ \\
\hline ADAMTS6|11174 & $\mathrm{A} 2$ \\
\hline ADAMTS7|11173 & $\mathrm{A} 2$ \\
\hline ADAMTS9|56999 & $\mathrm{A} 2$ \\
\hline ADAMTSL2|9719 & $\mathrm{A} 2$ \\
\hline ADAP1|11033 & $\mathrm{A} 2$ \\
\hline ADARB1|104 & $\mathrm{A} 2$ \\
\hline ADAT2|134637 & $\mathrm{A} 2$ \\
\hline ADCK2|90956 & $\mathrm{A} 2$ \\
\hline ADCK5|203054 & A2 \\
\hline ADCY4|196883 & $\mathrm{A} 2$ \\
\hline ADCY5|111 & A2 \\
\hline ADCY6|112 & $\mathrm{A} 2$ \\
\hline ADCYAP1R1|117 & $\mathrm{A} 2$ \\
\hline ADC|113451 & $\mathrm{A} 2$ \\
\hline ADD1|118 & $\mathrm{A} 2$ \\
\hline ADD3|120 & $\mathrm{A} 2$ \\
\hline ADH1A|124 & $\mathrm{A} 2$ \\
\hline ADH1B|125 & A2 \\
\hline ADH4|127 & $\mathrm{A} 2$ \\
\hline
\end{tabular}




\begin{tabular}{|c|c|}
\hline ADH5|128 & A2 \\
\hline ADHFE1|137872 & $\mathrm{A} 2$ \\
\hline ADIPOQ|9370 & $\mathrm{A} 2$ \\
\hline ADORA2A|135 & $\mathrm{A} 2$ \\
\hline ADORA3|140 & $\mathrm{A} 2$ \\
\hline ADPRHL2|54936 & $\mathrm{A} 2$ \\
\hline ADRA1A|148 & $\mathrm{A} 2$ \\
\hline ADRA2A|150 & $\mathrm{A} 2$ \\
\hline ADRB2|154 & $\mathrm{A} 2$ \\
\hline ADRBK2|157 & $\mathrm{A} 2$ \\
\hline ADRM1|11047 & $\mathrm{A} 2$ \\
\hline ADSS|159 & A2 \\
\hline AEBP1|165 & $\mathrm{A} 2$ \\
\hline AFF1|4299 & $\mathrm{A} 2$ \\
\hline AG2|387763 & $\mathrm{A} 2$ \\
\hline AGAP11|119385 & $\mathrm{A} 2$ \\
\hline AGER|177 & A2 \\
\hline AGFG2|3268 & $\mathrm{A} 2$ \\
\hline AGPAT2|10555 & A2 \\
\hline AGR3|155465 & $\mathrm{A} 2$ \\
\hline AGTRAP|57085 & $\mathrm{A} 2$ \\
\hline AGXT2L2|85007 & $\mathrm{A} 2$ \\
\hline AHCTF1|25909 & $\mathrm{A} 2$ \\
\hline AHCY|191 & $\mathrm{A} 2$ \\
\hline AHDC1|27245 & $\mathrm{A} 2$ \\
\hline AHNAK|79026 & $\mathrm{A} 2$ \\
\hline AHSA1|10598 & $\mathrm{A} 2$ \\
\hline AIF1L|83543 & $\mathrm{A} 2$ \\
\hline AIFM1|9131 & $\mathrm{A} 2$ \\
\hline AIG1|51390 & $\mathrm{A} 2$ \\
\hline AIMP2|7965 & $\mathrm{A} 2$ \\
\hline AK3|50808 & $\mathrm{A} 2$ \\
\hline AK5|26289 & $\mathrm{A} 2$ \\
\hline AKAP11|11215 & $\mathrm{A} 2$ \\
\hline AKAP13|11214 & $\mathrm{A} 2$ \\
\hline AKAP1|8165 & $\mathrm{A} 2$ \\
\hline AKAP2|11217 & $\mathrm{A} 2$ \\
\hline AKAP3|10566 & $\mathrm{A} 2$ \\
\hline AKAP5|9495 & A2 \\
\hline AKAP7|9465 & $\mathrm{A} 2$ \\
\hline AKR1C1|1645 & $\mathrm{A} 2$ \\
\hline AKR1C2|1646 & $\mathrm{A} 2$ \\
\hline AKR1C3|8644 & $\mathrm{A} 2$ \\
\hline AKT1|207 & $\mathrm{A} 2$ \\
\hline ALAD|210 & $\mathrm{A} 2$ \\
\hline ALDH18A1|5832 & $\mathrm{A} 2$ \\
\hline ALDH1A1|216 & $\mathrm{A} 2$ \\
\hline ALDH1A3|220 & $\mathrm{A} 2$ \\
\hline ALDH1B1|219 & $\mathrm{A} 2$ \\
\hline ALDH1L1|10840 & A2 \\
\hline ALDH2|217 & $\mathrm{A} 2$ \\
\hline
\end{tabular}




\begin{tabular}{|c|c|}
\hline ALDH3A2|224 & A2 \\
\hline ALDH7A1 $\mid 501$ & $\mathrm{~A} 2$ \\
\hline ALDOC|230 & $\mathrm{A} 2$ \\
\hline ALG11|440138 & $\mathrm{A} 2$ \\
\hline ALG1|56052 & $\mathrm{A} 2$ \\
\hline ALG3|10195 & $\mathrm{A} 2$ \\
\hline ALG6|29929 & $\mathrm{A} 2$ \\
\hline ALKBH3|221120 & A2 \\
\hline ALKBH6|84964 & $\mathrm{A} 2$ \\
\hline ALS2CR4|65062 & A2 \\
\hline ALS2CR8|79800 & A2 \\
\hline ALX4|60529 & $\mathrm{A} 2$ \\
\hline AMIGO1|57463 & A2 \\
\hline AMIGO3|386724 & $\overline{\mathrm{A} 2}$ \\
\hline AMMECR1|9949 & $\mathrm{A} 2$ \\
\hline AMZ2|51321 & $\mathrm{A} 2$ \\
\hline ANAPC11|51529 & $\mathrm{A} 2$ \\
\hline ANGEL2|90806 & $\mathrm{A} 2$ \\
\hline ANGPTL1|9068 & $\mathrm{A} 2$ \\
\hline ANGPTL6|83854 & $\mathrm{A} 2$ \\
\hline ANGPTL7|10218 & A2 \\
\hline ANG|283 & $\mathrm{A} 2$ \\
\hline ANK2|287 & A2 \\
\hline ANKDD1A|348094 & $\mathrm{A} 2$ \\
\hline ANKFY1|51479 & A2 \\
\hline ANKK1|255239 & A2 \\
\hline ANKRD11|29123 & $\mathrm{A} 2$ \\
\hline ANKRD22|118932 & $\mathrm{A} 2$ \\
\hline ANKRD36|375248 & A2 \\
\hline ANKRD40|91369 & A2 \\
\hline ANKRD53|79998 & $\mathrm{A} 2$ \\
\hline ANKRD58|347454 & $\mathrm{A} 2$ \\
\hline ANKRD5|63926 & $\mathrm{A} 2$ \\
\hline ANKRD6|22881 & $\mathrm{A} 2$ \\
\hline ANKS1A|23294 & A2 \\
\hline ANLN|54443 & $\overline{\mathrm{A} 2}$ \\
\hline ANO2|57101 & A2 \\
\hline ANO9|338440 & $\mathrm{A} 2$ \\
\hline ANP32E|81611 & $\mathrm{A} 2$ \\
\hline ANXA11|311 & $\mathrm{A} 2$ \\
\hline ANXA3|306 & $\mathrm{A} 2$ \\
\hline ANXA6|309 & $\mathrm{A} 2$ \\
\hline AP1G2|8906 & A2 \\
\hline AP1M2|10053 & $\mathrm{A} 2$ \\
\hline AP1S3|130340 & A2 \\
\hline AP2A2|161 & A2 \\
\hline AP2M1|1173 & $\mathrm{A} 2$ \\
\hline AP2S1|1175 & $\mathrm{A} 2$ \\
\hline AP3B1|8546 & $\mathrm{A} 2$ \\
\hline AP3D1|8943 & A2 \\
\hline AP4M1|9179 & $\mathrm{A} 2$ \\
\hline
\end{tabular}




\begin{tabular}{|c|c|}
\hline APBA1|320 & A2 \\
\hline APCDD1L $\mid 164284$ & $\mathrm{~A} 2$ \\
\hline APEH|327 & $\mathrm{A} 2$ \\
\hline APEX2|27301 & $\mathrm{A} 2$ \\
\hline APH1A|51107 & $\mathrm{A} 2$ \\
\hline APITD1|378708 & $\mathrm{A} 2$ \\
\hline APLF|200558 & $\mathrm{A} 2$ \\
\hline APOA1BP|128240 & $\mathrm{A} 2$ \\
\hline APOB48R|55911 & $\mathrm{A} 2$ \\
\hline APOBEC3C|27350 & $\mathrm{A} 2$ \\
\hline APOB|338 & $\mathrm{A} 2$ \\
\hline APOC2|344 & A2 \\
\hline APPL2|55198 & $\mathrm{A} 2$ \\
\hline APTX|54840 & $\mathrm{A} 2$ \\
\hline AQP1|358 & $\mathrm{A} 2$ \\
\hline AQP7P1|375719 & $\mathrm{A} 2$ \\
\hline AQP7P3|441432 & A2 \\
\hline AQPEP|206338 & $\mathrm{A} 2$ \\
\hline ARAP1|116985 & A2 \\
\hline ARAP3|64411 & $\mathrm{A} 2$ \\
\hline ARC|23237 & $\mathrm{A} 2$ \\
\hline ARF3|377 & $\mathrm{A} 2$ \\
\hline ARF4|378 & $\mathrm{A} 2$ \\
\hline ARF5|381 & $\mathrm{A} 2$ \\
\hline ARFGAP1|55738 & $\mathrm{A} 2$ \\
\hline ARFIP2|23647 & $\mathrm{A} 2$ \\
\hline ARG2|384 & $\mathrm{A} 2$ \\
\hline ARGLU1|55082 & $\mathrm{A} 2$ \\
\hline ARHGAP11A|9824 & $\mathrm{A} 2$ \\
\hline ARHGAP20|57569 & $\mathrm{A} 2$ \\
\hline ARHGAP21|57584 & $\mathrm{A} 2$ \\
\hline $\begin{array}{l}\text { ARHGAP23|57636 } \\
\end{array}$ & $\mathrm{A} 2$ \\
\hline ARHGAP24|83478 & $\mathrm{A} 2$ \\
\hline ARHGAP26|23092 & A2 \\
\hline ARHGAP29|9411 & $\mathrm{A} 2$ \\
\hline ARHGAP32|9743 & $\mathrm{A} 2$ \\
\hline \begin{tabular}{l|l|} 
ARHGAP39|80728 & \\
\end{tabular} & $\mathrm{A} 2$ \\
\hline ARHGAP42|143872 & $\mathrm{A} 2$ \\
\hline ARHGAP5|394 & $\mathrm{A} 2$ \\
\hline ARHGAP6|395 & $\mathrm{A} 2$ \\
\hline ARHGDIB|397 & A2 \\
\hline ARHGEF11|9826 & $\mathrm{A} 2$ \\
\hline ARHGEF17|9828 & A2 \\
\hline ARHGEF37|389337 & $\mathrm{A} 2$ \\
\hline ARHGEF6|9459 & $\mathrm{A} 2$ \\
\hline ARHGEF7|8874 & $\mathrm{A} 2$ \\
\hline ARID1B|57492 & $\mathrm{A} 2$ \\
\hline ARID3A|1820 & $\mathrm{A} 2$ \\
\hline ARID4A|5926 & $\mathrm{A} 2$ \\
\hline ARID5A|10865 & A2 \\
\hline ARL10|285598 & $\mathrm{A} 2$ \\
\hline
\end{tabular}




\begin{tabular}{|c|c|}
\hline ARL11|115761 & A2 \\
\hline ARL1|400 & $\mathrm{A} 2$ \\
\hline ARL2|402 & $\mathrm{A} 2$ \\
\hline ARL3|403 & $\mathrm{A} 2$ \\
\hline ARL4A|10124 & $\mathrm{A} 2$ \\
\hline ARL4C|10123 & $\mathrm{A} 2$ \\
\hline ARL6IP1|23204 & $\mathrm{A} 2$ \\
\hline ARL8A|127829 & $\mathrm{A} 2$ \\
\hline ARL8B|55207 & A2 \\
\hline ARMC10|83787 & $\mathrm{A} 2$ \\
\hline ARMC1|55156 & $\mathrm{A} 2$ \\
\hline ARMC6|93436 & A2 \\
\hline ARMC7|79637 & $\mathrm{A} 2$ \\
\hline ARMCX1|51309 & $\mathrm{A} 2$ \\
\hline ARNT2|9915 & $\mathrm{A} 2$ \\
\hline ARPC1B|10095 & $\mathrm{A} 2$ \\
\hline ARPC2|10109 & $\mathrm{A} 2$ \\
\hline ARPC3|10094 & $\mathrm{A} 2$ \\
\hline ARPC4|10093 & A2 \\
\hline ARPC5L|81873 & $\mathrm{A} 2$ \\
\hline ARPC5|10092 & $\mathrm{A} 2$ \\
\hline ARRDC2|27106 & $\mathrm{A} 2$ \\
\hline ARRDC3|57561 & $\mathrm{A} 2$ \\
\hline ARSB|411 & $\mathrm{A} 2$ \\
\hline ARSI|340075 & $\mathrm{A} 2$ \\
\hline ART4|420 & $\mathrm{A} 2$ \\
\hline ARV1|64801 & $\mathrm{A} 2$ \\
\hline ASB13|79754 & $\mathrm{A} 2$ \\
\hline ASF1B|55723 & $\mathrm{A} 2$ \\
\hline ASNA1|439 & $\mathrm{A} 2$ \\
\hline ASPH|444 & $\mathrm{A} 2$ \\
\hline ASPM|259266 & $\mathrm{A} 2$ \\
\hline ASPN|54829 & $\mathrm{A} 2$ \\
\hline ASPRV1|151516 & A2 \\
\hline ASPSCR1|79058 & $\mathrm{A} 2$ \\
\hline ASTN1|460 & $\mathrm{A} 2$ \\
\hline ASXL1|171023 & $\mathrm{A} 2$ \\
\hline ATAD2|29028 & $\mathrm{A} 2$ \\
\hline ATF3|467 & $\mathrm{A} 2$ \\
\hline ATF5|22809 & $\mathrm{A} 2$ \\
\hline ATF6B|1388 & $\mathrm{A} 2$ \\
\hline ATF7|11016 & $\mathrm{A} 2$ \\
\hline ATG16L1|55054 & $\mathrm{A} 2$ \\
\hline ATG2B|55102 & $\mathrm{A} 2$ \\
\hline ATG4C|84938 & $\mathrm{A} 2$ \\
\hline \begin{tabular}{l|l|} 
ATG4D|84971 &
\end{tabular} & $\mathrm{A} 2$ \\
\hline ATG9B|285973 & $\mathrm{A} 2$ \\
\hline ATIC|471 & $\mathrm{A} 2$ \\
\hline ATL1|51062 & $\mathrm{A} 2$ \\
\hline ATM|472 & A2 \\
\hline ATOH8|84913 & $\mathrm{A} 2$ \\
\hline
\end{tabular}




\begin{tabular}{|c|c|}
\hline ATOX1|475 & A2 \\
\hline ATP13A1|57130 & $\mathrm{A} 2$ \\
\hline ATP13A2|23400 & $\mathrm{A} 2$ \\
\hline ATP1B2|482 & $\mathrm{A} 2$ \\
\hline ATP2B4|493 & A2 \\
\hline ATP2C2|9914 & $\mathrm{A} 2$ \\
\hline ATP5C1|509 & $\mathrm{A} 2$ \\
\hline ATP6AP2|10159 & $\mathrm{A} 2$ \\
\hline ATP6V1D|51382 & $\mathrm{A} 2$ \\
\hline ATP6V1F|9296 & $\mathrm{A} 2$ \\
\hline ATP7B|540 & $\mathrm{A} 2$ \\
\hline ATP8B1|5205 & A2 \\
\hline ATPIF1|93974 & $\mathrm{A} 2$ \\
\hline ATRIP|84126 & $\mathrm{A} 2$ \\
\hline ATXN1L|342371 & $\mathrm{A} 2$ \\
\hline ATXN3|4287 & $\mathrm{A} 2$ \\
\hline ATXN7L3|56970 & $\mathrm{A} 2$ \\
\hline AUP1|550 & $\mathrm{A} 2$ \\
\hline AURKAPS1|6791 & $\mathrm{A} 2$ \\
\hline AURKA|6790 & $\mathrm{A} 2$ \\
\hline AURKB|9212 & $\mathrm{A} 2$ \\
\hline AVPI1|60370 & $\mathrm{A} 2$ \\
\hline AVPR1A|552 & $\mathrm{A} 2$ \\
\hline AXIN1|8312 & $\mathrm{A} 2$ \\
\hline AXIN2|8313 & $\mathrm{A} 2$ \\
\hline AZI2|64343 & $\mathrm{A} 2$ \\
\hline B3GALNT1|8706 & $\mathrm{A} 2$ \\
\hline B3GALNT2|148789 & $\mathrm{A} 2$ \\
\hline B3GALT1|8708 & $\mathrm{A} 2$ \\
\hline B3GALT2|8707 & $\mathrm{A} 2$ \\
\hline B3GAT3|26229 & $\mathrm{A} 2$ \\
\hline B3GNT1|11041 & $\mathrm{A} 2$ \\
\hline B3GNT4|79369 & $\mathrm{A} 2$ \\
\hline B4GALNT3|283358 & $\mathrm{A} 2$ \\
\hline B4GALT1|2683 & $\mathrm{A} 2$ \\
\hline B4GALT3|8703 & $\mathrm{A} 2$ \\
\hline B4GALT4|8702 & $\mathrm{A} 2$ \\
\hline B4GALT6|9331 & $\mathrm{A} 2$ \\
\hline BACH2|60468 & $\mathrm{A} 2$ \\
\hline BAI2|576 & $\mathrm{A} 2$ \\
\hline BAIAP2L1|55971 & $\mathrm{A} 2$ \\
\hline BAIAP2|10458 & $\mathrm{A} 2$ \\
\hline BAK1|578 & $\mathrm{A} 2$ \\
\hline BANF1|8815 & $\mathrm{A} 2$ \\
\hline BASP1|10409 & $\mathrm{A} 2$ \\
\hline BAT5|7920 & $\mathrm{A} 2$ \\
\hline BAX|581 & $\mathrm{A} 2$ \\
\hline \begin{tabular}{ll|l|} 
BAZ2B 29994 \\
\end{tabular} & $\mathrm{~A} 2$ \\
\hline BBC3|27113 & $\mathrm{A} 2$ \\
\hline BBOX1|8424 & $\mathrm{A} 2$ \\
\hline BBS10|79738 & $\mathrm{A} 2$ \\
\hline
\end{tabular}




\begin{tabular}{|c|c|}
\hline BBS12|166379 & A2 \\
\hline BBS7|55212 & A2 \\
\hline BCKDK|10295 & $\mathrm{A} 2$ \\
\hline BCL2L12|83596 & $\mathrm{A} 2$ \\
\hline BCL2L1|598 & $\mathrm{A} 2$ \\
\hline BCL3|602 & $\mathrm{A} 2$ \\
\hline BCL9|607 & $\mathrm{A} 2$ \\
\hline BCOR|54880 & $\mathrm{A} 2$ \\
\hline BDH2|56898 & $\mathrm{A} 2$ \\
\hline BDNFOS|497258 & $\mathrm{A} 2$ \\
\hline BEGAIN|57596 & $\mathrm{A} 2$ \\
\hline BEND3|57673 & $\mathrm{A} 2$ \\
\hline \begin{tabular}{l|l|} 
BEND5|79656 \\
\end{tabular} & $\mathrm{A} 2$ \\
\hline BEND7|222389 & A2 \\
\hline BFAR|51283 & $\mathrm{A} 2$ \\
\hline BGN|633 & $\mathrm{A} 2$ \\
\hline BICD2|23299 & $\mathrm{A} 2$ \\
\hline BIK|638 & $\mathrm{A} 2$ \\
\hline BIRC5|332 & $\mathrm{A} 2$ \\
\hline BIVM|54841 & $\mathrm{A} 2$ \\
\hline BLCAP|10904 & $\mathrm{A} 2$ \\
\hline BLNK|29760 & $\mathrm{A} 2$ \\
\hline BLOC1S2|282991 & $\mathrm{A} 2$ \\
\hline BLOC1S3|388552 & $\mathrm{A} 2$ \\
\hline BLZF1|8548 & $\mathrm{A} 2$ \\
\hline BMI1|648 & $\mathrm{A} 2$ \\
\hline BMP8A|353500 & $\mathrm{A} 2$ \\
\hline BMPER|168667 & $\mathrm{A} 2$ \\
\hline BMS1P5|399761 & $\mathrm{A} 2$ \\
\hline BMX|660 & $\mathrm{A} 2$ \\
\hline BNIP1|662 & $\mathrm{A} 2$ \\
\hline BNIP2|663 & $\mathrm{A} 2$ \\
\hline BNIP3L|665 & $\mathrm{A} 2$ \\
\hline BOC $\mid 91653$ & $\mathrm{~A} 2$ \\
\hline BOD1L|259282 & $\mathrm{A} 2$ \\
\hline BOK|666 & $\mathrm{A} 2$ \\
\hline BOLA1|51027 & $\mathrm{A} 2$ \\
\hline BOLA3|388962 & $\mathrm{A} 2$ \\
\hline BOP1|23246 & $\mathrm{A} 2$ \\
\hline BPNT1|10380 & $\mathrm{A} 2$ \\
\hline BRCA1|672 & $\mathrm{A} 2$ \\
\hline \begin{tabular}{l|l|} 
BRCC3|79184 \\
\end{tabular} & $\mathrm{A} 2$ \\
\hline BRI3BP|140707 & $\mathrm{A} 2$ \\
\hline BRIX1|55299 & $\mathrm{A} 2$ \\
\hline BRMS1|25855 & $\mathrm{A} 2$ \\
\hline BSCL2|26580 & $\mathrm{A} 2$ \\
\hline BST2|684 & $\mathrm{A} 2$ \\
\hline BTAF1|9044 & $\mathrm{A} 2$ \\
\hline BTBD10|84280 & $\mathrm{A} 2$ \\
\hline BTBD11|121551 & $\mathrm{A} 2$ \\
\hline BTBD7|55727 & $\mathrm{A} 2$ \\
\hline
\end{tabular}




\begin{tabular}{|c|c|}
\hline BTBD8|284697 & A2 \\
\hline BTF3L4|91408 & A2 \\
\hline BTN2A1|11120 & $\mathrm{A} 2$ \\
\hline BTN2A3|54718 & $\mathrm{A} 2$ \\
\hline BTNL9|153579 & $\mathrm{A} 2$ \\
\hline BUB1B|701 & $\mathrm{A} 2$ \\
\hline BUB1|699 & $\mathrm{A} 2$ \\
\hline BUB3|9184 & $\mathrm{A} 2$ \\
\hline BUD13|84811 & $\mathrm{A} 2$ \\
\hline BUD31|8896 & $\mathrm{A} 2$ \\
\hline BZRAP1|9256 & $\mathrm{A} 2$ \\
\hline BZW2|28969 & $\mathrm{A} 2$ \\
\hline C10orf10|11067 & $\mathrm{A} 2$ \\
\hline C10orf116|10974 & $\mathrm{A} 2$ \\
\hline C10orf11|83938 & $\mathrm{A} 2$ \\
\hline C10orf128|170371 & $\mathrm{A} 2$ \\
\hline C10orf18|54906 & $\mathrm{A} 2$ \\
\hline C10orf35|219738 & $\mathrm{A} 2$ \\
\hline C10orf46|143384 & $\mathrm{A} 2$ \\
\hline C10orf4|118924 & $\mathrm{A} 2$ \\
\hline C10orf50|645528 & $\mathrm{A} 2$ \\
\hline C10orf54|64115 & $\mathrm{A} 2$ \\
\hline C10orf57|80195 & $\mathrm{A} 2$ \\
\hline C10orf58|84293 & $\mathrm{A} 2$ \\
\hline C10orf95|79946 & $\mathrm{A} 2$ \\
\hline C11orf10|746 & $\mathrm{A} 2$ \\
\hline C11orf24|53838 & $\mathrm{A} 2$ \\
\hline C11 orf2|738 & $\mathrm{A} 2$ \\
\hline C11orf48|79081 & $\mathrm{A} 2$ \\
\hline C11orf49|79096 & $\mathrm{A} 2$ \\
\hline C11orf61|79684 & $\mathrm{A} 2$ \\
\hline C11 orf63|79864 & $\mathrm{A} 2$ \\
\hline C11orf66|220004 & $\mathrm{A} 2$ \\
\hline C11orf82|220042 & $\mathrm{A} 2$ \\
\hline C11orf83|790955 & $\mathrm{A} 2$ \\
\hline C11orf84|144097 & $\mathrm{A} 2$ \\
\hline C11orf95|65998 & $\mathrm{A} 2$ \\
\hline C12orf11|55726 & $\mathrm{A} 2$ \\
\hline C12orf23|90488 & $\mathrm{A} 2$ \\
\hline C12orf26|84190 & $\mathrm{A} 2$ \\
\hline C12orf32|83695 & $\mathrm{A} 2$ \\
\hline C12orf34|84915 & $\mathrm{A} 2$ \\
\hline C12orf43|64897 & $\mathrm{A} 2$ \\
\hline C12orf44|60673 & $\mathrm{A} 2$ \\
\hline $\begin{array}{l}\text { C12orf52|84934 } \\
\end{array}$ & $\mathrm{A} 2$ \\
\hline C12orf68|387856 & $\mathrm{A} 2$ \\
\hline C12orf70|341346 & $\mathrm{A} 2$ \\
\hline C13orf34|79866 & $\mathrm{A} 2$ \\
\hline C13orf36|400120 & $\mathrm{A} 2$ \\
\hline C13orf37|440145 & $\mathrm{A} 2$ \\
\hline C14orf118|55668 & $\mathrm{A} 2$ \\
\hline
\end{tabular}




\begin{tabular}{|c|c|}
\hline C14orf119|55017 & $\mathrm{A} 2$ \\
\hline C14orf128|84837 & A2 \\
\hline C14orf129|51527 & $\mathrm{A} 2$ \\
\hline C14orf132|56967 & $\mathrm{A} 2$ \\
\hline C14orf142|84520 & $\mathrm{A} 2$ \\
\hline C14orf149|112849 & $\mathrm{A} 2$ \\
\hline C14orf156|81892 & $\mathrm{A} 2$ \\
\hline C14orf159|80017 & $\mathrm{A} 2$ \\
\hline C14orf169|79697 & $\mathrm{A} 2$ \\
\hline C14orf174|161394 & $\mathrm{A} 2$ \\
\hline C14orf180|400258 & $\mathrm{A} 2$ \\
\hline C14orf1|11161 & $\mathrm{A} 2$ \\
\hline C14orf64|388011 & $\mathrm{A} 2$ \\
\hline C14orf80|283643 & $\mathrm{A} 2$ \\
\hline C15orf17|57184 & A2 \\
\hline C15orf21|283651 & $\mathrm{A} 2$ \\
\hline C15orf23|90417 & $\mathrm{A} 2$ \\
\hline C15orf29|79768 & $\mathrm{A} 2$ \\
\hline C15orf32|145858 & $\mathrm{A} 2$ \\
\hline C15orf40|123207 & $\mathrm{A} 2$ \\
\hline C15orf42|90381 & $\mathrm{A} 2$ \\
\hline C15orf51|196968 & $\mathrm{A} 2$ \\
\hline C15orf52|388115 & $\mathrm{A} 2$ \\
\hline C15orf54|400360 & $\mathrm{A} 2$ \\
\hline C15orf63|25764 & $\mathrm{A} 2$ \\
\hline C16orf13|84326 & $\mathrm{A} 2$ \\
\hline C16orf53|79447 & $\mathrm{A} 2$ \\
\hline C16orf55|124045 & $\mathrm{A} 2$ \\
\hline C16orf58|64755 & $\mathrm{A} 2$ \\
\hline C16orf5|29965 & $\mathrm{A} 2$ \\
\hline C16orf61|56942 & $\mathrm{A} 2$ \\
\hline C16orf70|80262 & $\mathrm{A} 2$ \\
\hline C16orf71|146562 & $\mathrm{A} 2$ \\
\hline C16orf72|29035 & $\mathrm{A} 2$ \\
\hline C16orf75|116028 & $\mathrm{A} 2$ \\
\hline C16orf88|400506 & $\mathrm{A} 2$ \\
\hline C16orf91|283951 & $\mathrm{A} 2$ \\
\hline C17orf100|388327 & $\mathrm{A} 2$ \\
\hline C17orf103|256302 & $\mathrm{A} 2$ \\
\hline C17orf107|100130311 & $\mathrm{A} 2$ \\
\hline C17orf28|283987 & $\mathrm{A} 2$ \\
\hline C17orf39|79018 & $\mathrm{A} 2$ \\
\hline C17orf51|339263 & $\mathrm{A} 2$ \\
\hline C17orf57|124989 & $\mathrm{A} 2$ \\
\hline C17orf62|79415 & $\mathrm{A} 2$ \\
\hline C17orf65|339201 & $\mathrm{A} 2$ \\
\hline C17orf69|147081 & $\mathrm{A} 2$ \\
\hline C17orf70|80233 & $\mathrm{A} 2$ \\
\hline C17orf81|23587 & $\mathrm{A} 2$ \\
\hline C17orf85|55421 & $\mathrm{A} 2$ \\
\hline C17orf90|339229 & $\mathrm{A} 2$ \\
\hline
\end{tabular}




\begin{tabular}{|c|c|}
\hline C17orf91|84981 & A2 \\
\hline C18orf18|147525 & A2 \\
\hline C18orf32|497661 & $\mathrm{A} 2$ \\
\hline C18orf34|374864 & $\mathrm{A} 2$ \\
\hline C18orf56|494514 & $\mathrm{A} 2$ \\
\hline C19orf10|56005 & $\mathrm{A} 2$ \\
\hline C19orf21|126353 & $\mathrm{A} 2$ \\
\hline C19orf22|91300 & $\mathrm{A} 2$ \\
\hline C19orf24|55009 & $\mathrm{A} 2$ \\
\hline C19orf28|126321 & $\mathrm{A} 2$ \\
\hline C19orf39|126074 & $\mathrm{A} 2$ \\
\hline C19orf40|91442 & $\mathrm{A} 2$ \\
\hline C19orf45|374877 & $\mathrm{A} 2$ \\
\hline C19orf46|163183 & $\mathrm{A} 2$ \\
\hline C19orf48|84798 & A2 \\
\hline C19orf51|352909 & $\mathrm{A} 2$ \\
\hline C19orf52|90580 & $\mathrm{A} 2$ \\
\hline C19orf53|28974 & $\mathrm{A} 2$ \\
\hline C19orf61|56006 & $\mathrm{A} 2$ \\
\hline C19orf63|284361 & $\mathrm{A} 2$ \\
\hline C19orf69|100170765 & $\mathrm{A} 2$ \\
\hline C1GALT1C1|29071 & $\mathrm{A} 2$ \\
\hline C1QL2|165257 & $\mathrm{A} 2$ \\
\hline C1QTNF6|114904 & $\mathrm{A} 2$ \\
\hline C1QTNF9|338872 & $\mathrm{A} 2$ \\
\hline C1RL|51279 & $\mathrm{A} 2$ \\
\hline C1orf103|55791 & $\mathrm{A} 2$ \\
\hline C1orf112|55732 & $\mathrm{A} 2$ \\
\hline C1orf115|79762 & $\mathrm{A} 2$ \\
\hline C1orf122|127687 & $\mathrm{A} 2$ \\
\hline C1orf124|83932 & $\mathrm{A} 2$ \\
\hline C1orf130|400746 & $\mathrm{A} 2$ \\
\hline C1orf131|128061 & $\mathrm{A} 2$ \\
\hline C1orf135|79000 & $\mathrm{A} 2$ \\
\hline C1orf156|92342 & $\mathrm{A} 2$ \\
\hline C1orf175|374977 & $\mathrm{A} 2$ \\
\hline C1orf182|128229 & $\mathrm{A} 2$ \\
\hline C1orf183|55924 & $\mathrm{A} 2$ \\
\hline C1orf220|400798 & $\mathrm{A} 2$ \\
\hline C1orf27|54953 & $\mathrm{A} 2$ \\
\hline C1orf35|79169 & $\mathrm{A} 2$ \\
\hline C1orf43|25912 & $\mathrm{A} 2$ \\
\hline C1orf52|148423 & $\mathrm{A} 2$ \\
\hline C1orf53|388722 & $\mathrm{A} 2$ \\
\hline C1orf56|54964 & $\mathrm{A} 2$ \\
\hline C1orf57|84284 & $\mathrm{A} 2$ \\
\hline C1orf58|148362 & $\mathrm{A} 2$ \\
\hline C1orf77|26097 & $\mathrm{A} 2$ \\
\hline C1orf85|112770 & $\mathrm{A} 2$ \\
\hline C1orf93|127281 & $\mathrm{A} 2$ \\
\hline C1orf97|84791 & $\mathrm{A} 2$ \\
\hline
\end{tabular}




\begin{tabular}{|c|c|}
\hline C1orf9|51430 & A2 \\
\hline C20orf103|24141 & A2 \\
\hline C20orf112|140688 & $\mathrm{A} 2$ \\
\hline C20orf132|140699 & $\mathrm{A} 2$ \\
\hline C20orf151|140893 & $\mathrm{A} 2$ \\
\hline C20orf160|140706 & $\mathrm{A} 2$ \\
\hline C20orf165|128497 & $\mathrm{A} 2$ \\
\hline C20orf203|284805 & $\mathrm{A} 2$ \\
\hline C20orf20|55257 & $\mathrm{A} 2$ \\
\hline C20orf24|55969 & $\mathrm{A} 2$ \\
\hline C20orf54|113278 & $\mathrm{A} 2$ \\
\hline C21orf2|755 & $\mathrm{A} 2$ \\
\hline $\begin{array}{l}\text { C21 orf45|54069 } \\
\end{array}$ & $\mathrm{A} 2$ \\
\hline C21orf49|54067 & $\mathrm{A} 2$ \\
\hline C21orf59|56683 & $\mathrm{A} 2$ \\
\hline $\begin{array}{l}\text { C21orf63|59271 } \\
\end{array}$ & $\mathrm{A} 2$ \\
\hline C22orf46|79640 & $\mathrm{A} 2$ \\
\hline C2CD2L|9854 & $\mathrm{A} 2$ \\
\hline C2orf18|54978 & $\mathrm{A} 2$ \\
\hline \begin{tabular}{l|l|} 
C2orf29|55571 \\
\end{tabular} & $\mathrm{A} 2$ \\
\hline C2orf48|348738 & $\mathrm{A} 2$ \\
\hline C2orf58|285154 & $\mathrm{A} 2$ \\
\hline C2orf74|339804 & $\mathrm{A} 2$ \\
\hline C2orf86|51057 & $\mathrm{A} 2$ \\
\hline C2orf88|84281 & $\mathrm{A} 2$ \\
\hline C3orf14|57415 & $\mathrm{A} 2$ \\
\hline C3orf18|51161 & $\mathrm{A} 2$ \\
\hline C3orf21|152002 & $\mathrm{A} 2$ \\
\hline C3orf36|80111 & $\mathrm{A} 2$ \\
\hline C3orf38|285237 & $\mathrm{A} 2$ \\
\hline C3orf42|84657 & $\mathrm{A} 2$ \\
\hline C3orf47|339942 & $\mathrm{A} 2$ \\
\hline C3orf50|93556 & $\mathrm{A} 2$ \\
\hline C3orf52|79669 & $\mathrm{A} 2$ \\
\hline C3orf54|389119 & $\mathrm{A} 2$ \\
\hline C3orf67|200844 & $\mathrm{A} 2$ \\
\hline C4A|720 & $\mathrm{A} 2$ \\
\hline C4orf12|404201 & $\mathrm{A} 2$ \\
\hline C4orf23|152992 & $\mathrm{A} 2$ \\
\hline C4orf34|201895 & $\mathrm{A} 2$ \\
\hline C4orf38|152641 & $\mathrm{A} 2$ \\
\hline C4orf42|92070 & $\mathrm{A} 2$ \\
\hline C4orf46|201725 & $\mathrm{A} 2$ \\
\hline C4orf48|401115 & $\mathrm{A} 2$ \\
\hline C5orf13|9315 & $\mathrm{A} 2$ \\
\hline C5orf15|56951 & $\mathrm{A} 2$ \\
\hline \begin{tabular}{l|l|} 
C5orf22|55322 \\
\end{tabular} & $\mathrm{A} 2$ \\
\hline C5orf30|90355 & $\mathrm{A} 2$ \\
\hline C5orf41|153222 & $\mathrm{A} 2$ \\
\hline C5orf43|643155 & $\mathrm{A} 2$ \\
\hline C5orf45|51149 & $\mathrm{A} 2$ \\
\hline
\end{tabular}




\begin{tabular}{|c|c|}
\hline C5orf49|134121 & A2 \\
\hline C5orf56|441108 & $\mathrm{A} 2$ \\
\hline C5orf62|85027 & $\mathrm{A} 2$ \\
\hline C6orf115|58527 & $\mathrm{A} 2$ \\
\hline C6orf120|387263 & $\mathrm{A} 2$ \\
\hline C6orf122|401288 & $\mathrm{A} 2$ \\
\hline C6orf123|26238 & $\mathrm{A} 2$ \\
\hline C6orf125|84300 & $\mathrm{A} 2$ \\
\hline C6orf126|389383 & $\mathrm{A} 2$ \\
\hline C6orf127|340204 & $\mathrm{A} 2$ \\
\hline C6orf145|221749 & $\mathrm{A} 2$ \\
\hline C6orf150|115004 & $\mathrm{A} 2$ \\
\hline C6orf153|88745 & $\mathrm{A} 2$ \\
\hline C6orf154|221424 & $\mathrm{A} 2$ \\
\hline C6orf163|206412 & $\mathrm{A} 2$ \\
\hline C6orf164|63914 & $\mathrm{A} 2$ \\
\hline C6orf191|253582 & $\mathrm{A} 2$ \\
\hline C6orf1|221491 & $\mathrm{A} 2$ \\
\hline C6orf204|387119 & $\mathrm{A} 2$ \\
\hline C6orf35|729515 & $\mathrm{A} 2$ \\
\hline C6orf57|135154 & $\mathrm{A} 2$ \\
\hline $\begin{array}{l}\text { C6orf59|79992 } \\
\end{array}$ & $\mathrm{A} 2$ \\
\hline \begin{tabular}{l|l|} 
C6orf62|81688 \\
\end{tabular} & $\mathrm{A} 2$ \\
\hline C6orf64|55776 & $\mathrm{A} 2$ \\
\hline C6orf89|221477 & $\mathrm{A} 2$ \\
\hline C7orf11|136647 & $\mathrm{A} 2$ \\
\hline C7orf28A|51622 & $\mathrm{A} 2$ \\
\hline C7orf47|221908 & $\mathrm{A} 2$ \\
\hline C7orf58|79974 & $\mathrm{A} 2$ \\
\hline \begin{tabular}{l|l|} 
C7orf70|84792 \\
\end{tabular} & $\mathrm{A} 2$ \\
\hline C8orf30A|51236 & $\mathrm{A} 2$ \\
\hline C8orf38|137682 & $\mathrm{A} 2$ \\
\hline C8orf51|78998 & $\mathrm{A} 2$ \\
\hline C8orf58|541565 & $\mathrm{A} 2$ \\
\hline C8orf76|84933 & $\mathrm{A} 2$ \\
\hline C8orf83|286144 & $\mathrm{A} 2$ \\
\hline C9orf100|84904 & $\mathrm{A} 2$ \\
\hline C9orf102|375748 & $\mathrm{A} 2$ \\
\hline C9orf116|138162 & $\mathrm{A} 2$ \\
\hline C9orf119|375757 & $\mathrm{A} 2$ \\
\hline C9orf11|54586 & $\mathrm{A} 2$ \\
\hline C9orf125|84302 & $\mathrm{A} 2$ \\
\hline C9orf130|100128782 & $\mathrm{A} 2$ \\
\hline C9orf131|138724 & $\mathrm{A} 2$ \\
\hline C9orf140|89958 & $\mathrm{A} 2$ \\
\hline C9orf142|286257 & $\mathrm{A} 2$ \\
\hline \begin{tabular}{l|l|} 
C9orf144B 259308 \\
\end{tabular} & $\mathrm{~A} 2$ \\
\hline C9orf163|158055 & $\mathrm{A} 2$ \\
\hline C9orf172|389813 & $\mathrm{A} 2$ \\
\hline C9orf21|195827 & $\mathrm{A} 2$ \\
\hline C9orf30|91283 & $\mathrm{A} 2$ \\
\hline
\end{tabular}




\begin{tabular}{|c|c|}
\hline C9orf46|55848 & A2 \\
\hline C9orf5|23731 & A2 \\
\hline C9orf64|84267 & $\mathrm{A} 2$ \\
\hline C9orf72|203228 & $\mathrm{A} 2$ \\
\hline C9orf7|11094 & $\mathrm{A} 2$ \\
\hline C9orf85|138241 & $\mathrm{A} 2$ \\
\hline C9orf89|84270 & $\mathrm{A} 2$ \\
\hline C9orf95|54981 & $\mathrm{A} 2$ \\
\hline C9orf98|158067 & $\mathrm{A} 2$ \\
\hline CA4|762 & $\mathrm{A} 2$ \\
\hline CA5B|11238 & $\mathrm{A} 2$ \\
\hline CAB39L|81617 & $\mathrm{A} 2$ \\
\hline $\begin{array}{l}\text { CABLES2|81928 } \\
\end{array}$ & $\mathrm{A} 2$ \\
\hline CABP1|9478 & $\mathrm{A} 2$ \\
\hline CABYR|26256 & $\mathrm{A} 2$ \\
\hline CACNA1C|775 & $\mathrm{A} 2$ \\
\hline CACNA1D|776 & $\mathrm{A} 2$ \\
\hline $\begin{array}{l}\text { CACNB3|784 } \\
\end{array}$ & $\mathrm{A} 2$ \\
\hline $\begin{array}{l}\text { CACNG4|27092 } \\
\end{array}$ & $\mathrm{A} 2$ \\
\hline CADM3|57863 & $\mathrm{A} 2$ \\
\hline CADPS|8618 & $\mathrm{A} 2$ \\
\hline CALM2|805 & $\mathrm{A} 2$ \\
\hline \begin{tabular}{l|l|} 
CALN1|83698 \\
\end{tabular} & $\mathrm{A} 2$ \\
\hline CALR $\mid 811$ & $\mathrm{~A} 2$ \\
\hline CALU|813 & $\mathrm{A} 2$ \\
\hline CAMK2N2|94032 & $\mathrm{A} 2$ \\
\hline CAMKK2|10645 & $\mathrm{A} 2$ \\
\hline CAMTA2|23125 & $\mathrm{A} 2$ \\
\hline CAND1|55832 & $\mathrm{A} 2$ \\
\hline CANT1|124583 & $\mathrm{A} 2$ \\
\hline CAP1|10487 & $\mathrm{A} 2$ \\
\hline CAPG $\mid 822$ & $\mathrm{~A} 2$ \\
\hline CAPN1|823 & $\mathrm{A} 2$ \\
\hline CAPN6|827 & $\mathrm{A} 2$ \\
\hline CAPRIN1|4076 & $\mathrm{A} 2$ \\
\hline CAPRIN2|65981 & $\mathrm{A} 2$ \\
\hline $\begin{array}{l}\text { CAPS2|84698 } \\
\end{array}$ & $\mathrm{A} 2$ \\
\hline CAPS $\mid 828$ & $\mathrm{~A} 2$ \\
\hline CARD10|29775 & $\mathrm{A} 2$ \\
\hline CARD6|84674 & $\mathrm{A} 2$ \\
\hline $\begin{array}{l}\text { CARD8|22900 } \\
\end{array}$ & $\mathrm{A} 2$ \\
\hline CARM1|10498 & $\mathrm{A} 2$ \\
\hline \begin{tabular}{ll|l|} 
CASC57082 \\
\end{tabular} & $\mathrm{A} 2$ \\
\hline CASP2|835 & $\mathrm{A} 2$ \\
\hline CASP3|836 & $\mathrm{A} 2$ \\
\hline \begin{tabular}{l|l} 
CASP6|839 \\
\end{tabular} & $\mathrm{A} 2$ \\
\hline CATSPER1|117144 & $\mathrm{A} 2$ \\
\hline CATSPER2|117155 & $\mathrm{A} 2$ \\
\hline CAV1|857 & $\mathrm{A} 2$ \\
\hline CAV2|858 & $\mathrm{A} 2$ \\
\hline CBFB|865 & $\mathrm{A} 2$ \\
\hline
\end{tabular}




\begin{tabular}{|c|c|}
\hline CBLC|23624 & $\mathrm{A} 2$ \\
\hline CBL $\mid 867$ & $\mathrm{~A} 2$ \\
\hline CBX1|10951 & $\mathrm{A} 2$ \\
\hline CBX2|84733 & $\mathrm{A} 2$ \\
\hline CBX4|8535 & $\mathrm{A} 2$ \\
\hline CBX6|23466 & A2 \\
\hline CC2D2B|387707 & $\mathrm{A} 2$ \\
\hline CCAR1|55749 & $\mathrm{A} 2$ \\
\hline CCBE1|147372 & $\mathrm{A} 2$ \\
\hline CCDC102A|92922 & $\mathrm{A} 2$ \\
\hline CCDC109B|55013 & $\mathrm{A} 2$ \\
\hline CCDC111|201973 & $\mathrm{A} 2$ \\
\hline CCDC112|153733 & $\mathrm{A} 2$ \\
\hline CCDC116|164592 & $\mathrm{A} 2$ \\
\hline CCDC121|79635 & $\mathrm{A} 2$ \\
\hline 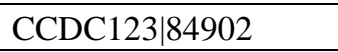 & $\mathrm{A} 2$ \\
\hline CCDC125|202243 & $\mathrm{A} 2$ \\
\hline CCDC136|64753 & $\mathrm{A} 2$ \\
\hline CCDC137|339230 & $\mathrm{A} 2$ \\
\hline CCDC138|165055 & $\mathrm{A} 2$ \\
\hline CCDC150|284992 & $\mathrm{A} 2$ \\
\hline CCDC152|100129792 & $\mathrm{A} 2$ \\
\hline CCDC158|339965 & $\mathrm{A} 2$ \\
\hline CCDC19|25790 & $\mathrm{A} 2$ \\
\hline CCDC22|28952 & $\mathrm{A} 2$ \\
\hline CCDC24|149473 & $\mathrm{A} 2$ \\
\hline CCDC25|55246 & $\mathrm{A} 2$ \\
\hline CCDC28A|25901 & $\mathrm{A} 2$ \\
\hline \begin{tabular}{ll|l|} 
CCDC43|124808 \\
\end{tabular} & $\mathrm{A} 2$ \\
\hline CCDC50|152137 & $\mathrm{A} 2$ \\
\hline CCDC51|79714 & $\mathrm{A} 2$ \\
\hline CCDC52|152185 & $\mathrm{A} 2$ \\
\hline CCDC54|84692 & $\mathrm{A} 2$ \\
\hline CCDC58|131076 & $\mathrm{A} 2$ \\
\hline CCDC64|92558 & $\mathrm{A} 2$ \\
\hline CCDC75|253635 & $\mathrm{A} 2$ \\
\hline CCDC76|54482 & $\mathrm{A} 2$ \\
\hline CCDC78|124093 & $\mathrm{A} 2$ \\
\hline CCDC82|79780 & $\mathrm{A} 2$ \\
\hline CCDC87|55231 & $\mathrm{A} 2$ \\
\hline CCDC $88 C \mid 440193$ & $\mathrm{~A} 2$ \\
\hline CCDC8|83987 & $\mathrm{A} 2$ \\
\hline CCDC96|257236 & $\mathrm{A} 2$ \\
\hline CCDC99|54908 & $\mathrm{A} 2$ \\
\hline CCL16|6360 & $\mathrm{A} 2$ \\
\hline CCNA2|890 & $\mathrm{A} 2$ \\
\hline CCNB1IP1|57820 & $\mathrm{A} 2$ \\
\hline CCNB1|891 & $\mathrm{A} 2$ \\
\hline CCNB2|9133 & $\mathrm{A} 2$ \\
\hline \begin{tabular}{ll|} 
CCND2|894 &
\end{tabular} & $\mathrm{A} 2$ \\
\hline CCNE1|898 & $\mathrm{A} 2$ \\
\hline
\end{tabular}




\begin{tabular}{|c|c|}
\hline CCNE2|9134 & $\mathrm{A} 2$ \\
\hline CCNF|899 & $\mathrm{A} 2$ \\
\hline CCNG1|900 & $\mathrm{A} 2$ \\
\hline CCNK|8812 & $\mathrm{A} 2$ \\
\hline CCNL1|57018 & $\mathrm{A} 2$ \\
\hline $\mathrm{CCNO} \mid 10309$ & A2 \\
\hline CCR10|2826 & $\mathrm{A} 2$ \\
\hline CCR8|1237 & $\mathrm{A} 2$ \\
\hline СCT3|7203 & $\mathrm{A} 2$ \\
\hline CCT5|22948 & $\mathrm{A} 2$ \\
\hline CCT7|10574 & $\mathrm{A} 2$ \\
\hline ССТ8|10694 & $\mathrm{A} 2$ \\
\hline CD160|11126 & $\mathrm{A} 2$ \\
\hline CD164L2|388611 & $\mathrm{A} 2$ \\
\hline CD209|30835 & $\mathrm{A} 2$ \\
\hline CD300LG|146894 & $\mathrm{A} 2$ \\
\hline CD302|9936 & $\mathrm{A} 2$ \\
\hline CD34|947 & $\mathrm{A} 2$ \\
\hline \begin{tabular}{l|l|l|} 
CD46179 &
\end{tabular} & $\mathrm{A} 2$ \\
\hline $\begin{array}{l}\text { CD86|942 } \\
\end{array}$ & $\mathrm{A} 2$ \\
\hline CD9|928 & $\mathrm{A} 2$ \\
\hline CDC123|8872 & $\mathrm{A} 2$ \\
\hline CDC14C|168448 & $\mathrm{A} 2$ \\
\hline $\begin{array}{l}\text { CDC20|991 } \\
\end{array}$ & $\mathrm{A} 2$ \\
\hline CDC25A|993 & $\mathrm{A} 2$ \\
\hline CDC25C $\mid 995$ & $\mathrm{~A} 2$ \\
\hline CDC42EP4|23580 & $\mathrm{A} 2$ \\
\hline CDC42SE1|56882 & $\mathrm{A} 2$ \\
\hline CDC42|998 & $\mathrm{A} 2$ \\
\hline CDC45|8318 & $\mathrm{A} 2$ \\
\hline \begin{tabular}{l|l|l|} 
CDC73 & \\
\end{tabular} & $\mathrm{A} 2$ \\
\hline CDC7|8317 & $\mathrm{A} 2$ \\
\hline CDCA2|157313 & $\mathrm{A} 2$ \\
\hline CDCA3|83461 & $\mathrm{A} 2$ \\
\hline CDCA4|55038 & $\mathrm{A} 2$ \\
\hline CDCA5|113130 & $\mathrm{A} 2$ \\
\hline CDCA8|55143 & $\mathrm{A} 2$ \\
\hline CDCP2|200008 & $\mathrm{A} 2$ \\
\hline CDH11|1009 & $\mathrm{A} 2$ \\
\hline CDH12|1010 & $\mathrm{A} 2$ \\
\hline CDH20|28316 & $\mathrm{A} 2$ \\
\hline \begin{tabular}{ll|l} 
CDH23 64072 \\
\end{tabular} & $\mathrm{~A} 2$ \\
\hline CDH4|1002 & $\mathrm{A} 2$ \\
\hline CDK10|8558 & $\mathrm{A} 2$ \\
\hline CDK16|5127 & $\mathrm{A} 2$ \\
\hline CDK1|983 & $\mathrm{A} 2$ \\
\hline CDK2AP2|10263 & $\mathrm{A} 2$ \\
\hline CDK2|1017 & $\mathrm{A} 2$ \\
\hline CDK3|1018 & $\mathrm{A} 2$ \\
\hline CDK4|1019 & $\mathrm{A} 2$ \\
\hline CDK5|1020 & $\mathrm{A} 2$ \\
\hline
\end{tabular}




\begin{tabular}{|c|c|}
\hline CDK7|1022 & A2 \\
\hline CDKL2|8999 & A2 \\
\hline CDKN1C|1028 & $\mathrm{A} 2$ \\
\hline CDKN2AIP|55602 & $\mathrm{A} 2$ \\
\hline CDKN2BAS|100048912 & $\mathrm{A} 2$ \\
\hline CDKN2C|1031 & $\mathrm{A} 2$ \\
\hline CDKN2D|1032 & $\mathrm{A} 2$ \\
\hline CDKN3|1033 & $\mathrm{A} 2$ \\
\hline CDR2L/30850 & $\mathrm{A} 2$ \\
\hline CDR2|1039 & $\mathrm{A} 2$ \\
\hline CDT1|81620 & $\mathrm{A} 2$ \\
\hline CEBPD|1052 & $\mathrm{A} 2$ \\
\hline CECR7|100130418 & $\mathrm{A} 2$ \\
\hline CELF2|10659 & $\mathrm{A} 2$ \\
\hline CELSR1|9620 & A2 \\
\hline $\begin{array}{l}\text { CELSR3|1951 } \\
\end{array}$ & $\mathrm{A} 2$ \\
\hline CENPA|1058 & $\mathrm{A} 2$ \\
\hline CENPC1|1060 & $\mathrm{A} 2$ \\
\hline CENPE|1062 & $\mathrm{A} 2$ \\
\hline CENPF|1063 & $\mathrm{A} 2$ \\
\hline CENPH|64946 & $\mathrm{A} 2$ \\
\hline CENPI|2491 & $\mathrm{A} 2$ \\
\hline CENPK|64105 & $\mathrm{A} 2$ \\
\hline CENPL|91687 & $\mathrm{A} 2$ \\
\hline CENPN|55839 & $\mathrm{A} 2$ \\
\hline CENPO|79172 & $\mathrm{A} 2$ \\
\hline CENPP|401541 & $\mathrm{A} 2$ \\
\hline CEP120|153241 & $\mathrm{A} 2$ \\
\hline CEP350|9857 & $\mathrm{A} 2$ \\
\hline CEP55|55165 & $\mathrm{A} 2$ \\
\hline CEP57|9702 & $\mathrm{A} 2$ \\
\hline CEP78|84131 & $\mathrm{A} 2$ \\
\hline CEP97|79598 & $\mathrm{A} 2$ \\
\hline CERKL|375298 & $\mathrm{A} 2$ \\
\hline CES4|51716 & $\mathrm{A} 2$ \\
\hline CETN2|1069 & $\mathrm{A} 2$ \\
\hline \begin{tabular}{ll|l|} 
CETN4P|729338 \\
\end{tabular} & $\mathrm{A} 2$ \\
\hline CFB|629 & $\mathrm{A} 2$ \\
\hline CFD|1675 & $\mathrm{A} 2$ \\
\hline $\mathrm{CFI} \mid 3426$ & $\mathrm{~A} 2$ \\
\hline CFLAR|8837 & $\mathrm{A} 2$ \\
\hline CFLP1|142913 & $\mathrm{A} 2$ \\
\hline CG030|116828 & $\mathrm{A} 2$ \\
\hline CGB7|94027 & $\mathrm{A} 2$ \\
\hline \begin{tabular}{ll|} 
CGNL1|84952 \\
\end{tabular} & $\mathrm{A} 2$ \\
\hline CGN|57530 & $\mathrm{A} 2$ \\
\hline CGRRF1|10668 & $\mathrm{A} 2$ \\
\hline CH25H|9023 & $\mathrm{A} 2$ \\
\hline CHAC2|494143 & $\mathrm{A} 2$ \\
\hline CHAF1A|10036 & $\mathrm{A} 2$ \\
\hline CHAF1B|8208 & $\mathrm{A} 2$ \\
\hline
\end{tabular}




\begin{tabular}{|c|c|}
\hline CHCHD1|118487 & A2 \\
\hline CHCHD5|84269 & A2 \\
\hline CHCHD8|51287 & $\mathrm{A} 2$ \\
\hline CHD4|1108 & $\mathrm{A} 2$ \\
\hline CHD9 $\mid 80205$ & $\mathrm{~A} 2$ \\
\hline CHEK1|1111 & $\mathrm{A} 2$ \\
\hline CHEK2|11200 & $\mathrm{A} 2$ \\
\hline CHFR|55743 & $\mathrm{A} 2$ \\
\hline CHIC2|26511 & $\mathrm{A} 2$ \\
\hline CHKB-CPT1B|386593 & $\mathrm{A} 2$ \\
\hline CHMP4B|128866 & $\mathrm{A} 2$ \\
\hline CHMP5|51510 & $\mathrm{A} 2$ \\
\hline CHORDC1|26973 & $\mathrm{A} 2$ \\
\hline CHPF2|54480 & $\mathrm{A} 2$ \\
\hline CHPF|79586 & A2 \\
\hline CHRAC1|54108 & $\mathrm{A} 2$ \\
\hline CHRFAM7A|89832 & $\mathrm{A} 2$ \\
\hline CHRNA6|8973 & $\mathrm{A} 2$ \\
\hline CHRNA7|1139 & $\mathrm{A} 2$ \\
\hline CHST11|50515 & $\mathrm{A} 2$ \\
\hline CHST15|51363 & $\mathrm{A} 2$ \\
\hline CHST6|4166 & $\mathrm{A} 2$ \\
\hline CHST7|56548 & $\mathrm{A} 2$ \\
\hline CHSY3|337876 & $\mathrm{A} 2$ \\
\hline CIB3|117286 & $\mathrm{A} 2$ \\
\hline CIDEA|1149 & $\mathrm{A} 2$ \\
\hline CIRBP|1153 & $\mathrm{A} 2$ \\
\hline CISD2|493856 & $\mathrm{A} 2$ \\
\hline CITED2|10370 & $\mathrm{A} 2$ \\
\hline CKAP2L|150468 & $\mathrm{A} 2$ \\
\hline CKAP2|26586 & $\mathrm{A} 2$ \\
\hline CKAP4|10970 & $\mathrm{A} 2$ \\
\hline CKAP5|9793 & $\mathrm{A} 2$ \\
\hline CKLF|51192 & $\mathrm{A} 2$ \\
\hline CKS1B|1163 & $\mathrm{A} 2$ \\
\hline CKS2|1164 & $\mathrm{A} 2$ \\
\hline CLASP1|23332 & $\mathrm{A} 2$ \\
\hline CLCN2|1181 & $\mathrm{A} 2$ \\
\hline CLCN7|1186 & $\mathrm{A} 2$ \\
\hline CLDN14|23562 & $\mathrm{A} 2$ \\
\hline CLDN15|24146 & $\mathrm{A} 2$ \\
\hline CLDN18|51208 & $\mathrm{A} 2$ \\
\hline CLDN5|7122 & $\mathrm{A} 2$ \\
\hline CLDN7|1366 & $\mathrm{A} 2$ \\
\hline CLEC11A|6320 & $\mathrm{A} 2$ \\
\hline CLEC1A|51267 & $\mathrm{A} 2$ \\
\hline CLEC4GP1|440508 & $\mathrm{A} 2$ \\
\hline CLEC5A|23601 & $\mathrm{A} 2$ \\
\hline CLGN|1047 & $\mathrm{A} 2$ \\
\hline CLIC1|1192 & $\mathrm{A} 2$ \\
\hline CLIP3|25999 & $\mathrm{A} 2$ \\
\hline
\end{tabular}




\begin{tabular}{|c|c|}
\hline CLIP4|79745 & A2 \\
\hline CLK1|1195 & $\mathrm{A} 2$ \\
\hline CLK3|1198 & $\mathrm{A} 2$ \\
\hline CLK4|57396 & $\mathrm{A} 2$ \\
\hline CLN6|54982 & $\mathrm{A} 2$ \\
\hline CLN8|2055 & $\mathrm{A} 2$ \\
\hline CLPB $\mid 81570$ & $\mathrm{~A} 2$ \\
\hline CLPP|8192 & $\mathrm{A} 2$ \\
\hline CLPTM1L|81037 & $\mathrm{A} 2$ \\
\hline CLPTM1|1209 & $\mathrm{A} 2$ \\
\hline CLSPN|63967 & $\mathrm{A} 2$ \\
\hline CLVS1|157807 & $\mathrm{A} 2$ \\
\hline CLYBL|171425 & $\mathrm{A} 2$ \\
\hline CMA1|1215 & $\mathrm{A} 2$ \\
\hline CMPK1|51727 & $\mathrm{A} 2$ \\
\hline CNIH2|254263 & $\mathrm{A} 2$ \\
\hline CNIH4|29097 & $\mathrm{A} 2$ \\
\hline $\mathrm{CNIH} \mid 10175$ & $\mathrm{~A} 2$ \\
\hline CNN1|1264 & $\mathrm{A} 2$ \\
\hline CNNM4|26504 & $\mathrm{A} 2$ \\
\hline CNOT3|4849 & $\mathrm{A} 2$ \\
\hline CNOT6L|246175 & $\mathrm{A} 2$ \\
\hline $\mathrm{CNO} 55330$ & $\mathrm{~A} 2$ \\
\hline CNPY2|10330 & $\mathrm{A} 2$ \\
\hline CNPY3|10695 & $\mathrm{A} 2$ \\
\hline COBLL1|22837 & $\mathrm{A} 2$ \\
\hline COG2|22796 & $\mathrm{A} 2$ \\
\hline COG7|91949 & $\mathrm{A} 2$ \\
\hline COIL|8161 & $\mathrm{A} 2$ \\
\hline COL10A1|1300 & $\mathrm{A} 2$ \\
\hline COL11A1|1301 & $\mathrm{A} 2$ \\
\hline COL12A1|1303 & $\mathrm{A} 2$ \\
\hline COL14A1|7373 & $\mathrm{A} 2$ \\
\hline COL1A1|1277 & $\mathrm{A} 2$ \\
\hline COL23A1|91522 & $\mathrm{A} 2$ \\
\hline COL24A1|255631 & $\mathrm{A} 2$ \\
\hline COL25A1|84570 & $\mathrm{A} 2$ \\
\hline COL4A3BP|10087 & $\mathrm{A} 2$ \\
\hline COL5A1|1289 & $\mathrm{A} 2$ \\
\hline COL5A2|1290 & $\mathrm{A} 2$ \\
\hline COL6A3|1293 & $\mathrm{A} 2$ \\
\hline COL6A6|131873 & $\mathrm{A} 2$ \\
\hline COL8A1|1295 & $\mathrm{A} 2$ \\
\hline COLEC11|78989 & $\mathrm{A} 2$ \\
\hline COMMD10|51397 & $\mathrm{A} 2$ \\
\hline COMMD3|23412 & $\mathrm{A} 2$ \\
\hline COMMD4|54939 & $\mathrm{A} 2$ \\
\hline COMMD5|28991 & $\mathrm{A} 2$ \\
\hline COMMD7|149951 & $\mathrm{A} 2$ \\
\hline COMP|1311 & $\mathrm{A} 2$ \\
\hline COMTD1|118881 & $\mathrm{A} 2$ \\
\hline
\end{tabular}




\begin{tabular}{|c|c|}
\hline COPB1|1315 & A2 \\
\hline COPB2|9276 & A2 \\
\hline COPE|11316 & A2 \\
\hline COPG $\mid 22820$ & $\mathrm{~A} 2$ \\
\hline COPS2|9318 & $\mathrm{A} 2$ \\
\hline COPS5|10987 & A2 \\
\hline COPS8|10920 & $\mathrm{A} 2$ \\
\hline COPZ1|22818 & $\mathrm{A} 2$ \\
\hline CORIN|10699 & A2 \\
\hline CORO1B|57175 & $\mathrm{A} 2$ \\
\hline CORO1C|23603 & A2 \\
\hline CORO2A|7464 & $\mathrm{A} 2$ \\
\hline CORO2B|10391 & $\mathrm{A} 2$ \\
\hline CORO7|79585 & A2 \\
\hline COX16|51241 & $\mathrm{A} 2$ \\
\hline COX17|10063 & $\mathrm{A} 2$ \\
\hline COX4I2|84701 & $\mathrm{A} 2$ \\
\hline COX6C|1345 & $\mathrm{A} 2$ \\
\hline COX7A2|1347 & $\mathrm{A} 2$ \\
\hline COX8A|1351 & $\mathrm{A} 2$ \\
\hline CPA1|1357 & $\mathrm{A} 2$ \\
\hline CPM|1368 & $\mathrm{A} 2$ \\
\hline CPNE3|8895 & $\mathrm{A} 2$ \\
\hline CPNE8|144402 & $\mathrm{A} 2$ \\
\hline CPSF2|53981 & $\mathrm{A} 2$ \\
\hline CPVL|54504 & $\mathrm{A} 2$ \\
\hline CPXM2|119587 & $\mathrm{A} 2$ \\
\hline CR1|1378 & $\mathrm{A} 2$ \\
\hline CRABP2|1382 & $\mathrm{A} 2$ \\
\hline CRADD|8738 & $\mathrm{A} 2$ \\
\hline CRB2|286204 & $\mathrm{A} 2$ \\
\hline CRB3|92359 & $\mathrm{A} 2$ \\
\hline CRBN|51185 & $\mathrm{A} 2$ \\
\hline CRCP|27297 & $\mathrm{A} 2$ \\
\hline CREB3L1|90993 & $\mathrm{A} 2$ \\
\hline CREB3L4|148327 & $\mathrm{A} 2$ \\
\hline CREB5|9586 & $\mathrm{A} 2$ \\
\hline CRELD1|78987 & $\mathrm{A} 2$ \\
\hline CRELD2|79174 & $\mathrm{A} 2$ \\
\hline CRHBP|1393 & $\mathrm{A} 2$ \\
\hline CRIP1|1396 & $\mathrm{A} 2$ \\
\hline CRK|1398 & $\mathrm{A} 2$ \\
\hline CRTC2|200186 & $\mathrm{A} 2$ \\
\hline CRY2|1408 & $\mathrm{A} 2$ \\
\hline CRYAB|1410 & $\mathrm{A} 2$ \\
\hline $\begin{array}{l}\text { CRYBG3|131544 } \\
\end{array}$ & $\mathrm{A} 2$ \\
\hline CRYGS|1427 & $\mathrm{A} 2$ \\
\hline CRYL1|51084 & $\mathrm{A} 2$ \\
\hline CRYZL1|9946 & $\mathrm{A} 2$ \\
\hline CSAD|51380 & A2 \\
\hline CSDE1|7812 & A2 \\
\hline
\end{tabular}




\begin{tabular}{|c|c|}
\hline CSF1|1435 & $\mathrm{A} 2$ \\
\hline CSGALNACT1|55790 & $\mathrm{A} 2$ \\
\hline CSK|1445 & $\mathrm{A} 2$ \\
\hline CSMD2|114784 & $\mathrm{A} 2$ \\
\hline CSNK2B $\mid 1460$ & $\mathrm{~A} 2$ \\
\hline CSRNP1|64651 & $\mathrm{A} 2$ \\
\hline CSRNP2|81566 & $\mathrm{A} 2$ \\
\hline CSRNP3|80034 & $\mathrm{A} 2$ \\
\hline CSRP1|1465 & $\mathrm{A} 2$ \\
\hline CST2|1470 & $\mathrm{A} 2$ \\
\hline CSTF2|1478 & $\mathrm{A} 2$ \\
\hline CTAGE5|4253 & $\mathrm{A} 2$ \\
\hline CTDSP2|10106 & $\mathrm{A} 2$ \\
\hline CTDSPL|10217 & $\mathrm{A} 2$ \\
\hline CTHRC1|115908 & $\mathrm{A} 2$ \\
\hline CTNNAL1|8727 & $\mathrm{A} 2$ \\
\hline CTPS2|56474 & $\mathrm{A} 2$ \\
\hline CTPS|1503 & $\mathrm{A} 2$ \\
\hline CTSG|1511 & $\mathrm{A} 2$ \\
\hline CTXN1|404217 & $\mathrm{A} 2$ \\
\hline CUBN|8029 & $\mathrm{A} 2$ \\
\hline CUTA|51596 & $\mathrm{A} 2$ \\
\hline CWC22|57703 & $\mathrm{A} 2$ \\
\hline CWF19L2|143884 & $\mathrm{A} 2$ \\
\hline CX3CR1|1524 & $\mathrm{A} 2$ \\
\hline CXCL12|6387 & $\mathrm{A} 2$ \\
\hline CXCL2|2920 & $\mathrm{A} 2$ \\
\hline CXCL3|2921 & $\mathrm{A} 2$ \\
\hline \begin{tabular}{l|l|} 
CXXC5|51523 \\
\end{tabular} & $\mathrm{A} 2$ \\
\hline CXorf36|79742 & $\mathrm{A} 2$ \\
\hline CXorf40A|91966 & $\mathrm{A} 2$ \\
\hline CXorf40B|541578 & $\mathrm{A} 2$ \\
\hline CYB561D1|284613 & $\mathrm{A} 2$ \\
\hline CYB561D2|11068 & $\mathrm{A} 2$ \\
\hline CYB561|1534 & $\mathrm{A} 2$ \\
\hline CYB5D2|124936 & $\mathrm{A} 2$ \\
\hline CYBRD1|79901 & $\mathrm{A} 2$ \\
\hline CYCS|54205 & $\mathrm{A} 2$ \\
\hline CYFIP2|26999 & $\mathrm{A} 2$ \\
\hline CYMP|643160 & $\mathrm{A} 2$ \\
\hline CYP2B7P1|1556 & $\mathrm{A} 2$ \\
\hline CYP46A1|10858 & $\mathrm{A} 2$ \\
\hline CYP51A1|1595 & $\mathrm{A} 2$ \\
\hline CYP7A1|1581 & $\mathrm{A} 2$ \\
\hline CYR61|3491 & $\mathrm{A} 2$ \\
\hline CYTH3|9265 & $\mathrm{A} 2$ \\
\hline DACT1|51339 & $\mathrm{A} 2$ \\
\hline DACT2|168002 & $\mathrm{A} 2$ \\
\hline DAD1|1603 & $\mathrm{A} 2$ \\
\hline DAGLB|221955 & $\mathrm{A} 2$ \\
\hline DAPK2|23604 & $\mathrm{A} 2$ \\
\hline
\end{tabular}




\begin{tabular}{|c|c|}
\hline DAP|1611 & A2 \\
\hline DARC 2532 & $\mathrm{~A} 2$ \\
\hline DARS2|55157 & A2 \\
\hline DAXX|1616 & A2 \\
\hline DAZAP1|26528 & A2 \\
\hline DBC1 $\mid 1620$ & $\mathrm{~A} 2$ \\
\hline DBNDD1|79007 & A2 \\
\hline DBX2|440097 & A2 \\
\hline DCAF10|79269 & A2 \\
\hline DCAF12|25853 & A2 \\
\hline DCAF13|25879 & A2 \\
\hline DCAF5|8816 & A2 \\
\hline DCAKD|79877 & $\mathrm{A} 2$ \\
\hline DCBLD1|285761 & A2 \\
\hline DCBLD2|131566 & A2 \\
\hline DCK|1633 & $\mathrm{A} 2$ \\
\hline DCTN5|84516 & $\mathrm{A} 2$ \\
\hline DCTPP1|79077 & A2 \\
\hline DCUN1D3|123879 & $\mathrm{A} 2$ \\
\hline DCUN1D4|23142 & $\mathrm{A} 2$ \\
\hline DCUN1D5|84259 & A2 \\
\hline DDAH1|23576 & A2 \\
\hline DDB1|1642 & $\mathrm{A} 2$ \\
\hline DDB2|1643 & $\mathrm{A} 2$ \\
\hline DDOST|1650 & $\mathrm{A} 2$ \\
\hline DDO|8528 & A2 \\
\hline DDR1|780 & $\mathrm{A} 2$ \\
\hline DDR2|4921 & $\mathrm{A} 2$ \\
\hline DDX17|10521 & A2 \\
\hline DDX19A|55308 & $\mathrm{A} 2$ \\
\hline DDX19B|11269 & $\mathrm{A} 2$ \\
\hline DDX23|9416 & $\mathrm{A} 2$ \\
\hline DDX24|57062 & $\mathrm{A} 2$ \\
\hline \begin{tabular}{l|l|} 
DDX25|29118 \\
\end{tabular} & $\mathrm{A} 2$ \\
\hline DDX39|10212 & $\mathrm{A} 2$ \\
\hline DDX3X|1654 & $\mathrm{A} 2$ \\
\hline DDX41|51428 & $\mathrm{A} 2$ \\
\hline DDX49|54555 & $\mathrm{A} 2$ \\
\hline DDX56|54606 & $\mathrm{A} 2$ \\
\hline DDX58|23586 & $\mathrm{A} 2$ \\
\hline DEDD2|162989 & $\mathrm{A} 2$ \\
\hline DEFB1|1672 & $\mathrm{A} 2$ \\
\hline DEGS2|123099 & $\mathrm{A} 2$ \\
\hline DEM1|64789 & $\mathrm{A} 2$ \\
\hline DENND2A|27147 & $\mathrm{A} 2$ \\
\hline DENND3|22898 & $\mathrm{A} 2$ \\
\hline DENND4C|55667 & $\mathrm{A} 2$ \\
\hline DENND5A|23258 & $\mathrm{A} 2$ \\
\hline DENND5B|160518 & $\mathrm{A} 2$ \\
\hline DEPDC1B|55789 & $\mathrm{A} 2$ \\
\hline DEPDC1 155635 & A2 \\
\hline
\end{tabular}




\begin{tabular}{|c|c|}
\hline DEPDC7|91614 & $\mathrm{A} 2$ \\
\hline DERL1|79139 & $\mathrm{A} 2$ \\
\hline DET1|55070 & $\mathrm{A} 2$ \\
\hline DGUOK|1716 & $\mathrm{A} 2$ \\
\hline DHCR24|1718 & A2 \\
\hline DHDPSL|112817 & $\mathrm{A} 2$ \\
\hline DHRS12|79758 & $\mathrm{A} 2$ \\
\hline DHTKD1|55526 & $\mathrm{A} 2$ \\
\hline DHX15|1665 & $\mathrm{A} 2$ \\
\hline DHX29|54505 & $\mathrm{A} 2$ \\
\hline DHX38|9785 & $\mathrm{A} 2$ \\
\hline DIABLO|56616 & $\mathrm{A} 2$ \\
\hline DIAPH2|1730 & $\mathrm{A} 2$ \\
\hline DIO3OS|64150 & $\mathrm{A} 2$ \\
\hline DIP2C|22982 & $\mathrm{A} 2$ \\
\hline DIRC1|116093 & $\mathrm{A} 2$ \\
\hline DIXDC1|85458 & $\mathrm{A} 2$ \\
\hline DKC1|1736 & $\mathrm{A} 2$ \\
\hline DKFZP434L187|26082 & $\mathrm{A} 2$ \\
\hline DKFZp779M0652|374387 & $\mathrm{A} 2$ \\
\hline DKK3|27122 & $\mathrm{A} 2$ \\
\hline DLEC1 19940 & $\mathrm{~A} 2$ \\
\hline DLEU1|10301 & $\mathrm{A} 2$ \\
\hline DLG3|1741 & $\mathrm{A} 2$ \\
\hline DLGAP5|9787 & $\mathrm{A} 2$ \\
\hline DLL1|28514 & $\mathrm{A} 2$ \\
\hline DLL4|54567 & $\mathrm{A} 2$ \\
\hline DLST|1743 & $\mathrm{A} 2$ \\
\hline DMRT2|10655 & $\mathrm{A} 2$ \\
\hline DMXL1|1657 & $\mathrm{A} 2$ \\
\hline DNA2|1763 & $\mathrm{A} 2$ \\
\hline DNAH14|127602 & $\mathrm{A} 2$ \\
\hline DNAH6|1768 & $\mathrm{A} 2$ \\
\hline DNAH9|1770 & $\mathrm{A} 2$ \\
\hline DNAJA1|3301 & $\mathrm{A} 2$ \\
\hline DNAJA3|9093 & $\mathrm{A} 2$ \\
\hline DNAJA4|55466 & $\mathrm{A} 2$ \\
\hline DNAJB4|11080 & $\mathrm{A} 2$ \\
\hline DNAJC10|54431 & $\mathrm{A} 2$ \\
\hline DNAJC14|85406 & $\mathrm{A} 2$ \\
\hline DNAJC1|64215 & $\mathrm{A} 2$ \\
\hline DNAJC22|79962 & $\mathrm{A} 2$ \\
\hline DNAJC2|27000 & $\mathrm{A} 2$ \\
\hline DNAJC7|7266 & $\mathrm{A} 2$ \\
\hline DNAJC9|23234 & $\mathrm{A} 2$ \\
\hline DNASE1L2|1775 & $\mathrm{A} 2$ \\
\hline DNASE1|1773 & $\mathrm{A} 2$ \\
\hline DNASE2|1777 & $\mathrm{A} 2$ \\
\hline DNM3|26052 & $\mathrm{A} 2$ \\
\hline DNMT1|1786 & $\mathrm{A} 2$ \\
\hline DNMT3A|1788 & $\mathrm{A} 2$ \\
\hline
\end{tabular}




\begin{tabular}{|c|c|}
\hline DNMT3B|1789 & A2 \\
\hline DNPEP|23549 & $\mathrm{A} 2$ \\
\hline DOCK11|139818 & A2 \\
\hline DOCK4|9732 & $\mathrm{A} 2$ \\
\hline DOCK8|81704 & $\mathrm{A} 2$ \\
\hline DOCK9|23348 & A2 \\
\hline DOK3|79930 & $\mathrm{A} 2$ \\
\hline DOLK|22845 & $\mathrm{A} 2$ \\
\hline DOLPP1|57171 & A2 \\
\hline DONSON|29980 & $\mathrm{A} 2$ \\
\hline DOPEY1|23033 & $\mathrm{A} 2$ \\
\hline DOPEY2|9980 & $\mathrm{A} 2$ \\
\hline DPAGT1|1798 & $\mathrm{A} 2$ \\
\hline DPCD|25911 & $\mathrm{A} 2$ \\
\hline DPEP1|1800 & $\mathrm{A} 2$ \\
\hline DPH2|1802 & $\mathrm{A} 2$ \\
\hline DPM1|8813 & $\mathrm{A} 2$ \\
\hline DPP3|10072 & $\mathrm{A} 2$ \\
\hline DPP6|1804 & A2 \\
\hline DPP9|91039 & $\mathrm{A} 2$ \\
\hline DPRX|503834 & $\mathrm{A} 2$ \\
\hline DPYD|1806 & $\mathrm{A} 2$ \\
\hline DRAP1|10589 & $\mathrm{A} 2$ \\
\hline DRD1|1812 & $\mathrm{A} 2$ \\
\hline DRD4|1815 & $\mathrm{A} 2$ \\
\hline DSC1|1823 & $\mathrm{A} 2$ \\
\hline DSCC1|79075 & A2 \\
\hline DSCR6|53820 & A2 \\
\hline DSE|29940 & $\mathrm{A} 2$ \\
\hline DTL|51514 & $\mathrm{A} 2$ \\
\hline DTWD1|56986 & $\mathrm{A} 2$ \\
\hline DTX2|113878 & $\mathrm{A} 2$ \\
\hline DTX3L|151636 & $\mathrm{A} 2$ \\
\hline DUSP12|11266 & A2 \\
\hline DUSP14|11072 & $\mathrm{A} 2$ \\
\hline \begin{tabular}{l|l|} 
DUSP19|142679 \\
\end{tabular} & $\mathrm{A} 2$ \\
\hline DUSP1|1843 & $\mathrm{A} 2$ \\
\hline DUSP7|1849 & $\mathrm{A} 2$ \\
\hline DVL3|1857 & $\mathrm{A} 2$ \\
\hline DYNC1LI1|51143 & $\mathrm{A} 2$ \\
\hline DYNC1LI2|1783 & A2 \\
\hline DYNLL1|8655 & $\mathrm{A} 2$ \\
\hline DYNLT1|6993 & A2 \\
\hline DYX1C1|161582 & $\mathrm{A} 2$ \\
\hline DZIP1L|199221 & $\mathrm{A} 2$ \\
\hline E2F2|1870 & $\mathrm{A} 2$ \\
\hline E2F3|1871 & $\mathrm{A} 2$ \\
\hline E2F5|1875 & $\mathrm{A} 2$ \\
\hline E2F7|144455 & A2 \\
\hline E2F8|79733 & A2 \\
\hline E4F1|1877 & $\mathrm{A} 2$ \\
\hline
\end{tabular}




\begin{tabular}{|c|c|}
\hline EAF1|85403 & $\mathrm{A} 2$ \\
\hline EBNA1BP2|10969 & $\mathrm{A} 2$ \\
\hline EBP|10682 & $\mathrm{A} 2$ \\
\hline ECE2|9718 & $\mathrm{A} 2$ \\
\hline ECHDC1|55862 & $\mathrm{A} 2$ \\
\hline ECHDC3|79746 & A2 \\
\hline ECT2|1894 & $\mathrm{A} 2$ \\
\hline EDA2R|60401 & $\mathrm{A} 2$ \\
\hline EDA|1896 & $\mathrm{A} 2$ \\
\hline EDEM2|55741 & $\mathrm{A} 2$ \\
\hline EDEM3|80267 & $\mathrm{A} 2$ \\
\hline EDN1|1906 & $\mathrm{A} 2$ \\
\hline EDN2|1907 & $\mathrm{A} 2$ \\
\hline EEA1|8411 & $\mathrm{A} 2$ \\
\hline EEF1A1P9|441032 & $\mathrm{A} 2$ \\
\hline EEF1A1|1915 & $\mathrm{A} 2$ \\
\hline EEF1B2|1933 & $\mathrm{A} 2$ \\
\hline EEF1E1|9521 & $\mathrm{A} 2$ \\
\hline EEF2K|29904 & $\mathrm{A} 2$ \\
\hline EEF2|1938 & $\mathrm{A} 2$ \\
\hline EFCAB1|79645 & $\mathrm{A} 2$ \\
\hline EFEMP1|2202 & $\mathrm{A} 2$ \\
\hline EFNA1|1942 & $\mathrm{A} 2$ \\
\hline EFNA3|1944 & $\mathrm{A} 2$ \\
\hline EFNA4|1945 & $\mathrm{A} 2$ \\
\hline EFNB1|1947 & $\mathrm{A} 2$ \\
\hline EFNB3|1949 & $\mathrm{A} 2$ \\
\hline EFR3A|23167 & $\mathrm{A} 2$ \\
\hline EFTUD2|9343 & $\mathrm{A} 2$ \\
\hline EGFL7|51162 & $\mathrm{A} 2$ \\
\hline $\begin{array}{l}\text { EGFL8|80864 } \\
\end{array}$ & $\mathrm{A} 2$ \\
\hline EGFLAM|133584 & $\mathrm{A} 2$ \\
\hline EGR1|1958 & $\mathrm{A} 2$ \\
\hline EGR2|1959 & $\mathrm{A} 2$ \\
\hline EGR3|1960 & $\mathrm{A} 2$ \\
\hline EHD3|30845 & $\mathrm{A} 2$ \\
\hline EHHADH|1962 & $\mathrm{A} 2$ \\
\hline EHMT2|10919 & $\mathrm{A} 2$ \\
\hline EID1|23741 & $\mathrm{A} 2$ \\
\hline EID2B|126272 & $\mathrm{A} 2$ \\
\hline EIF1AD|84285 & $\mathrm{A} 2$ \\
\hline EIF1B|10289 & $\mathrm{A} 2$ \\
\hline EIF2AK2|5610 & $\mathrm{A} 2$ \\
\hline EIF2AK3|9451 & $\mathrm{A} 2$ \\
\hline EIF2B3|8891 & $\mathrm{A} 2$ \\
\hline EIF2C4|192670 & $\mathrm{A} 2$ \\
\hline EIF2S2|8894 & $\mathrm{A} 2$ \\
\hline \begin{tabular}{l|l} 
EIF3D|8664 &
\end{tabular} & $\mathrm{A} 2$ \\
\hline EIF3H|8667 & $\mathrm{A} 2$ \\
\hline EIF4A2|1974 & $\mathrm{A} 2$ \\
\hline EIF4A3|9775 & $\mathrm{A} 2$ \\
\hline
\end{tabular}




\begin{tabular}{|c|c|}
\hline EIF4B|1975 & A2 \\
\hline EIF4E|1977 & $\mathrm{A} 2$ \\
\hline EIF4G1|1981 & $\mathrm{A} 2$ \\
\hline EIF5AL1|143244 & $\mathrm{A} 2$ \\
\hline EIF5A|1984 & A2 \\
\hline EIF5B $\mid 9669$ & $\mathrm{~A} 2$ \\
\hline EIF6|3692 & $\mathrm{A} 2$ \\
\hline ELANE|1991 & $\mathrm{A} 2$ \\
\hline ELAVL1|1994 & $\mathrm{A} 2$ \\
\hline ELF4|2000 & $\mathrm{A} 2$ \\
\hline ELF5|2001 & $\mathrm{A} 2$ \\
\hline ELK1|2002 & $\mathrm{A} 2$ \\
\hline \begin{tabular}{l|l} 
ELL3|80237 &
\end{tabular} & $\mathrm{A} 2$ \\
\hline ELMO2|63916 & $\mathrm{A} 2$ \\
\hline ELMOD2|255520 & $\mathrm{A} 2$ \\
\hline ELMOD3|84173 & $\mathrm{A} 2$ \\
\hline EMCN|51705 & $\mathrm{A} 2$ \\
\hline EME1|146956 & $\mathrm{A} 2$ \\
\hline EMILIN2|84034 & $\mathrm{A} 2$ \\
\hline EMILIN3|90187 & $\mathrm{A} 2$ \\
\hline EML3|256364 & $\mathrm{A} 2$ \\
\hline EML5|161436 & $\mathrm{A} 2$ \\
\hline EMP1|2012 & $\mathrm{A} 2$ \\
\hline EMP2|2013 & $\mathrm{A} 2$ \\
\hline ENC1|8507 & $\mathrm{A} 2$ \\
\hline ENDOU|8909 & $\mathrm{A} 2$ \\
\hline ENGASE|64772 & $\mathrm{A} 2$ \\
\hline ENG|2022 & $\mathrm{A} 2$ \\
\hline ENO2|2026 & $\mathrm{A} 2$ \\
\hline ENOPH1|58478 & $\mathrm{A} 2$ \\
\hline ENPP2|5168 & $\mathrm{A} 2$ \\
\hline ENPP6|133121 & $\mathrm{A} 2$ \\
\hline ENSA|2029 & $\mathrm{A} 2$ \\
\hline ENTPD7|57089 & $\mathrm{A} 2$ \\
\hline ENY2|56943 & $\mathrm{A} 2$ \\
\hline EPAS1|2034 & $\mathrm{A} 2$ \\
\hline EPB41L2|2037 & $\mathrm{A} 2$ \\
\hline EPB41L4B|54566 & $\mathrm{A} 2$ \\
\hline EPB49|2039 & $\mathrm{A} 2$ \\
\hline EPC1|80314 & $\mathrm{A} 2$ \\
\hline EPCAM|4072 & $\mathrm{A} 2$ \\
\hline EPHA2|1969 & $\mathrm{A} 2$ \\
\hline EPHB6|2051 & $\mathrm{A} 2$ \\
\hline EPHX2|2053 & $\mathrm{A} 2$ \\
\hline EPN3|55040 & $\mathrm{A} 2$ \\
\hline EPR1|8475 & $\mathrm{A} 2$ \\
\hline EPS15L1|58513 & $\mathrm{A} 2$ \\
\hline EPS15|2060 & $\mathrm{A} 2$ \\
\hline EPS8L1|54869 & $\mathrm{A} 2$ \\
\hline EPS8|2059 & $\mathrm{A} 2$ \\
\hline EPSTI1|94240 & $\mathrm{A} 2$ \\
\hline
\end{tabular}




\begin{tabular}{|c|c|}
\hline ERAP1|51752 & $\mathrm{A} 2$ \\
\hline ERBB2IP|55914 & $\mathrm{A} 2$ \\
\hline ERCC5|2073 & $\mathrm{A} 2$ \\
\hline ERGIC1|57222 & $\mathrm{A} 2$ \\
\hline ERGIC2|51290 & $\mathrm{A} 2$ \\
\hline ERG|2078 & A2 \\
\hline ERH|2079 & $\mathrm{A} 2$ \\
\hline ERI2|112479 & $\mathrm{A} 2$ \\
\hline ERI3|79033 & $\mathrm{A} 2$ \\
\hline ERICH1|157697 & $\mathrm{A} 2$ \\
\hline ERLEC1|27248 & $\mathrm{A} 2$ \\
\hline ERMAP|114625 & $\mathrm{A} 2$ \\
\hline ERMN|57471 & $\mathrm{A} 2$ \\
\hline ERMP1|79956 & $\mathrm{A} 2$ \\
\hline ERO1L|30001 & $\mathrm{A} 2$ \\
\hline ERP27|121506 & $\mathrm{A} 2$ \\
\hline ERRFI1|54206 & $\mathrm{A} 2$ \\
\hline \begin{tabular}{l|l|l|} 
ESCO2|1570 \\
\end{tabular} & $\mathrm{A} 2$ \\
\hline ESM1|11082 & $\mathrm{A} 2$ \\
\hline ESPL1|9700 & $\mathrm{A} 2$ \\
\hline ESPN|83715 & $\mathrm{A} 2$ \\
\hline ESR1|2099 & $\mathrm{A} 2$ \\
\hline ESR2|2100 & $\mathrm{A} 2$ \\
\hline ESRP1|54845 & $\mathrm{A} 2$ \\
\hline ESRP2|80004 & $\mathrm{A} 2$ \\
\hline ESYT1|23344 & $\mathrm{A} 2$ \\
\hline ESYT2|57488 & $\mathrm{A} 2$ \\
\hline \begin{tabular}{ll|l|} 
ETFB & 2109 \\
\end{tabular} & $\mathrm{~A} 2$ \\
\hline ETFDH|2110 & $\mathrm{A} 2$ \\
\hline $\begin{array}{l}\text { ETNK2|55224 } \\
\end{array}$ & $\mathrm{A} 2$ \\
\hline \begin{tabular}{ll|l|} 
ETS2|114 \\
\end{tabular} & $\mathrm{A} 2$ \\
\hline ETV1|2115 & $\mathrm{A} 2$ \\
\hline ETV3L|440695 & $\mathrm{A} 2$ \\
\hline ETV5|2119 & $\mathrm{A} 2$ \\
\hline ETV7|51513 & $\mathrm{A} 2$ \\
\hline EVI5|7813 & $\mathrm{A} 2$ \\
\hline EVL|51466 & $\mathrm{A} 2$ \\
\hline EVPL|2125 & $\mathrm{A} 2$ \\
\hline EXO1|9156 & $\mathrm{A} 2$ \\
\hline EXOC3|11336 & $\mathrm{A} 2$ \\
\hline EXOC6B|23233 & $\mathrm{A} 2$ \\
\hline EXOC6|54536 & $\mathrm{A} 2$ \\
\hline EXOSC1|51013 & $\mathrm{A} 2$ \\
\hline EXOSC3|51010 & $\mathrm{A} 2$ \\
\hline EXOSC4|54512 & $\mathrm{A} 2$ \\
\hline EXTL2|2135 & $\mathrm{A} 2$ \\
\hline EZH2|2146 & $\mathrm{A} 2$ \\
\hline EZR|7430 & $\mathrm{A} 2$ \\
\hline F10|2159 & $\mathrm{A} 2$ \\
\hline F11R|50848 & $\mathrm{A} 2$ \\
\hline F12|2161 & $\mathrm{A} 2$ \\
\hline
\end{tabular}




\begin{tabular}{|c|c|}
\hline F2RL2|2151 & A2 \\
\hline F2R/2149 & A2 \\
\hline F7|2155 & $\mathrm{A} 2$ \\
\hline F8|2157 & $\mathrm{A} 2$ \\
\hline FAAH|2166 & $\mathrm{A} 2$ \\
\hline FADD $\mid 8772$ & $\mathrm{~A} 2$ \\
\hline FAF2|23197 & $\mathrm{A} 2$ \\
\hline FAHD1|81889 & $\mathrm{A} 2$ \\
\hline FAM103A1|83640 & A2 \\
\hline FAM104A|84923 & $\mathrm{A} 2$ \\
\hline FAM107A|11170 & $\mathrm{A} 2$ \\
\hline FAM108C1|58489 & A2 \\
\hline FAM10A4|145165 & $\mathrm{A} 2$ \\
\hline FAM110A|83541 & $\mathrm{A} 2$ \\
\hline FAM110B|90362 & $\mathrm{A} 2$ \\
\hline FAM111B|374393 & $\mathrm{A} 2$ \\
\hline FAM114A1|92689 & $\mathrm{A} 2$ \\
\hline FAM117A|81558 & $\mathrm{A} 2$ \\
\hline FAM122B|159090 & A2 \\
\hline FAM124A|220108 & $\mathrm{A} 2$ \\
\hline FAM125B|89853 & $\mathrm{A} 2$ \\
\hline FAM128A|653784 & $\mathrm{A} 2$ \\
\hline FAM131A|131408 & $\mathrm{A} 2$ \\
\hline FAM135A|57579 & $\mathrm{A} 2$ \\
\hline FAM136A|84908 & $\mathrm{A} 2$ \\
\hline FAM13AOS|285512 & $\mathrm{A} 2$ \\
\hline FAM13A|10144 & $\mathrm{A} 2$ \\
\hline FAM149A|25854 & $\mathrm{A} 2$ \\
\hline FAM151B|167555 & $\mathrm{A} 2$ \\
\hline FAM153A|285596 & $\mathrm{A} 2$ \\
\hline FAM156A|29057 & $\mathrm{A} 2$ \\
\hline FAM158A|51016 & $\mathrm{A} 2$ \\
\hline FAM162A|26355 & $\mathrm{A} 2$ \\
\hline FAM162B 221303 & $\mathrm{~A} 2$ \\
\hline FAM168B|130074 & $\mathrm{A} 2$ \\
\hline FAM170B|170370 & $\mathrm{A} 2$ \\
\hline FAM171A1|221061 & $\mathrm{A} 2$ \\
\hline FAM172A|83989 & $\mathrm{A} 2$ \\
\hline FAM173A|65990 & A2 \\
\hline FAM174B|400451 & $\mathrm{A} 2$ \\
\hline FAM175A|84142 & A2 \\
\hline FAM180A|389558 & $\mathrm{A} 2$ \\
\hline FAM181A|90050 & $\mathrm{A} 2$ \\
\hline $\begin{array}{l}\text { FAM186B } \mid 84070 \\
\end{array}$ & $\mathrm{~A} 2$ \\
\hline FAM189B|10712 & $\mathrm{A} 2$ \\
\hline FAM18A|780776 & $\mathrm{A} 2$ \\
\hline FAM190B|54462 & $\mathrm{A} 2$ \\
\hline \begin{tabular}{l|l|l|} 
FAM193B 54540 \\
\end{tabular} & $\mathrm{~A} 2$ \\
\hline FAM195A|84331 & $\mathrm{A} 2$ \\
\hline FAM196B|100131897 & A2 \\
\hline FAM19A1|407738 & $\mathrm{A} 2$ \\
\hline
\end{tabular}




\begin{tabular}{|c|c|}
\hline FAM200B 285550 & $\mathrm{~A} 2$ \\
\hline FAM23A|653567 & $\mathrm{A} 2$ \\
\hline FAM26D|221301 & $\mathrm{A} 2$ \\
\hline FAM36A|116228 & $\mathrm{A} 2$ \\
\hline FAM43A|131583 & $\mathrm{A} 2$ \\
\hline FAM47E|100129583 & $\mathrm{A} 2$ \\
\hline FAM49B|51571 & $\mathrm{A} 2$ \\
\hline FAM53C|51307 & $\mathrm{A} 2$ \\
\hline FAM54A|113115 & $\mathrm{A} 2$ \\
\hline FAM54B|56181 & $\mathrm{A} 2$ \\
\hline FAM55D|54827 & $\mathrm{A} 2$ \\
\hline FAM64A|54478 & $\mathrm{A} 2$ \\
\hline FAM65A|79567 & $\mathrm{A} 2$ \\
\hline FAM66A|100133172 & $\mathrm{A} 2$ \\
\hline FAM69A|388650 & $\mathrm{A} 2$ \\
\hline FAM70A|55026 & $\mathrm{A} 2$ \\
\hline FAM72B $\mid 653820$ & $\mathrm{~A} 2$ \\
\hline FAM72D|728833 & $\mathrm{A} 2$ \\
\hline FAM76A|199870 & $\mathrm{A} 2$ \\
\hline FAM7A2|100288380 & $\mathrm{A} 2$ \\
\hline FAM83D|81610 & $\mathrm{A} 2$ \\
\hline FAM95B1|100133036 & $\mathrm{A} 2$ \\
\hline FAM96A|84191 & $\mathrm{A} 2$ \\
\hline FANCA|2175 & $\mathrm{A} 2$ \\
\hline FANCD2|2177 & $\mathrm{A} 2$ \\
\hline FANCF|2188 & $\mathrm{A} 2$ \\
\hline FANCG|2189 & $\mathrm{A} 2$ \\
\hline FANCI $\mid 55215$ & $\mathrm{~A} 2$ \\
\hline FARP1|10160 & $\mathrm{A} 2$ \\
\hline FARSA|2193 & $\mathrm{A} 2$ \\
\hline FARSB|10056 & $\mathrm{A} 2$ \\
\hline FASTKD3|79072 & $\mathrm{A} 2$ \\
\hline FAT1|2195 & $\mathrm{A} 2$ \\
\hline FAT4|79633 & $\mathrm{A} 2$ \\
\hline \begin{tabular}{l|l|l|} 
FBLN5 & 10516 \\
\end{tabular} & $\mathrm{~A} 2$ \\
\hline FBP1|2203 & $\mathrm{A} 2$ \\
\hline FBRS|64319 & $\mathrm{A} 2$ \\
\hline FBXL14|144699 & $\mathrm{A} 2$ \\
\hline FBXL16|146330 & $\mathrm{A} 2$ \\
\hline FBXL19|54620 & $\mathrm{A} 2$ \\
\hline FBXL22|283807 & $\mathrm{A} 2$ \\
\hline FBXL5|26234 & $\mathrm{A} 2$ \\
\hline FBXL6|26233 & $\mathrm{A} 2$ \\
\hline FBXO16|157574 & $\mathrm{A} 2$ \\
\hline FBXO17|115290 & $\mathrm{A} 2$ \\
\hline FBXO22OS|692224 & $\mathrm{A} 2$ \\
\hline FBXO28|23219 & $\mathrm{A} 2$ \\
\hline FBXO33|254170 & $\mathrm{A} 2$ \\
\hline FBXO41|150726 & $\mathrm{A} 2$ \\
\hline FBXO42|54455 & $\mathrm{A} 2$ \\
\hline FBXO43|286151 & $\mathrm{A} 2$ \\
\hline
\end{tabular}




\begin{tabular}{|c|c|}
\hline FBXO45|200933 & $\mathrm{A} 2$ \\
\hline FBXO48|554251 & $\mathrm{A} 2$ \\
\hline FBXO5|26271 & $\mathrm{A} 2$ \\
\hline FBXO6|26270 & $\mathrm{A} 2$ \\
\hline FBXO8|26269 & $\mathrm{A} 2$ \\
\hline FCGR1A|2209 & $\mathrm{A} 2$ \\
\hline FCGR1B|2210 & $\mathrm{A} 2$ \\
\hline FCGR1C|100132417 & $\mathrm{A} 2$ \\
\hline FCGR3A|2214 & $\mathrm{A} 2$ \\
\hline FCGRT|2217 & $\mathrm{A} 2$ \\
\hline FDPS|2224 & $\mathrm{A} 2$ \\
\hline FDXR 2232 & $\mathrm{~A} 2$ \\
\hline FEN1|2237 & $\mathrm{A} 2$ \\
\hline FER|2241 & $\mathrm{A} 2$ \\
\hline FEZ2|9637 & $\mathrm{A} 2$ \\
\hline FGD1|2245 & $\mathrm{A} 2$ \\
\hline FGD3|89846 & $\mathrm{A} 2$ \\
\hline FGD4|121512 & $\mathrm{A} 2$ \\
\hline FGF17|8822 & $\mathrm{A} 2$ \\
\hline FGF2|2247 & $\mathrm{A} 2$ \\
\hline FGF7|2252 & $\mathrm{A} 2$ \\
\hline FGF8|2253 & $\mathrm{A} 2$ \\
\hline FGF9|2254 & $\mathrm{A} 2$ \\
\hline FGFBP3|143282 & $\mathrm{A} 2$ \\
\hline FGFR1OP2|26127 & $\mathrm{A} 2$ \\
\hline FGFR1|2260 & $\mathrm{A} 2$ \\
\hline FGL2|10875 & $\mathrm{A} 2$ \\
\hline FHAD1|114827 & $\mathrm{A} 2$ \\
\hline FHL5|9457 & $\mathrm{A} 2$ \\
\hline FHOD3|80206 & $\mathrm{A} 2$ \\
\hline $\mathrm{FH} \mid 2271$ & $\mathrm{~A} 2$ \\
\hline FIBP $\mid 9158$ & $\mathrm{~A} 2$ \\
\hline FIGNL2|401720 & $\mathrm{A} 2$ \\
\hline FIGN|55137 & $\mathrm{A} 2$ \\
\hline FKBP10|60681 & $\mathrm{A} 2$ \\
\hline FKBP3|2287 & $\mathrm{A} 2$ \\
\hline FKBP4|2288 & $\mathrm{A} 2$ \\
\hline FKBPL|63943 & $\mathrm{A} 2$ \\
\hline FKTN|2218 & $\mathrm{A} 2$ \\
\hline FLJ10357|55701 & $\mathrm{A} 2$ \\
\hline FLJ26850|400710 & $\mathrm{A} 2$ \\
\hline FLJ33360|401172 & $\mathrm{A} 2$ \\
\hline FLJ35024|401491 & $\mathrm{A} 2$ \\
\hline FLJ36777|730971 & $\mathrm{A} 2$ \\
\hline FLJ37543|285668 & $\mathrm{A} 2$ \\
\hline FLJ39653|202020 & $\mathrm{A} 2$ \\
\hline FLJ42875|440556 & $\mathrm{A} 2$ \\
\hline FLJ43390|646113 & $\mathrm{A} 2$ \\
\hline FLJ43663|378805 & $\mathrm{A} 2$ \\
\hline FLJ44635|392490 & $\mathrm{A} 2$ \\
\hline FLJ45340|402483 & $\mathrm{A} 2$ \\
\hline
\end{tabular}




\begin{tabular}{|c|c|}
\hline FLJ45983|399717 & $\mathrm{A} 2$ \\
\hline \begin{tabular}{ll|l|} 
FLRT23768 \\
\end{tabular} & $\mathrm{A} 2$ \\
\hline FLT4|2324 & $\mathrm{A} 2$ \\
\hline FLVCR1|28982 & $\mathrm{A} 2$ \\
\hline FLYWCH2|114984 & $\mathrm{A} 2$ \\
\hline FMNL2|114793 & $\mathrm{A} 2$ \\
\hline FMO2|2327 & $\mathrm{A} 2$ \\
\hline FMO4|2329 & $\mathrm{A} 2$ \\
\hline FMOD|2331 & $\mathrm{A} 2$ \\
\hline FN1|2335 & $\mathrm{A} 2$ \\
\hline FNDC1|84624 & $\mathrm{A} 2$ \\
\hline FNDC3A|22862 & $\mathrm{A} 2$ \\
\hline FNIP2|57600 & $\mathrm{A} 2$ \\
\hline FOLR2|2350 & $\mathrm{A} 2$ \\
\hline FOSB|2354 & $\mathrm{A} 2$ \\
\hline FOS $\mid 2353$ & $\mathrm{~A} 2$ \\
\hline FOXA1|3169 & $\mathrm{A} 2$ \\
\hline FOXD4L1|200350 & $\mathrm{A} 2$ \\
\hline FOXH1|8928 & $\mathrm{A} 2$ \\
\hline FOXK2|3607 & $\mathrm{A} 2$ \\
\hline FOXM1|2305 & $\mathrm{A} 2$ \\
\hline FOXN2|3344 & $\mathrm{A} 2$ \\
\hline FOXN3|1112 & $\mathrm{A} 2$ \\
\hline FOXO1|2308 & $\mathrm{A} 2$ \\
\hline FOXP2|93986 & $\mathrm{A} 2$ \\
\hline FOXP3|50943 & $\mathrm{A} 2$ \\
\hline FOXRED2|80020 & $\mathrm{A} 2$ \\
\hline FOXS1|2307 & $\mathrm{A} 2$ \\
\hline FRAT2|23401 & $\mathrm{A} 2$ \\
\hline FREM1|158326 & $\mathrm{A} 2$ \\
\hline FRG1B|284802 & $\mathrm{A} 2$ \\
\hline FRG1|2483 & $\mathrm{A} 2$ \\
\hline FRK|2444 & $\mathrm{A} 2$ \\
\hline FRMD4A|55691 & $\mathrm{A} 2$ \\
\hline FRMD4B|23150 & $\mathrm{A} 2$ \\
\hline FRMPD1|22844 & $\mathrm{A} 2$ \\
\hline FRMPD4|9758 & $\mathrm{A} 2$ \\
\hline FRY|10129 & $\mathrm{A} 2$ \\
\hline FSIP1|161835 & $\mathrm{A} 2$ \\
\hline FTO|79068 & $\mathrm{A} 2$ \\
\hline FTSJ1|24140 & $\mathrm{A} 2$ \\
\hline FTSJ2|29960 & $\mathrm{A} 2$ \\
\hline FUNDC1|139341 & $\mathrm{A} 2$ \\
\hline $\begin{array}{l}\text { FUT10|84750 } \\
\end{array}$ & $\mathrm{A} 2$ \\
\hline FUT2|2524 & $\mathrm{A} 2$ \\
\hline FUT8|2530 & $\mathrm{A} 2$ \\
\hline FXYD3|5349 & $\mathrm{A} 2$ \\
\hline FZD5|7855 & $\mathrm{A} 2$ \\
\hline FZD7|8324 & $\mathrm{A} 2$ \\
\hline G3BP1|10146 & $\mathrm{A} 2$ \\
\hline G6PC3|92579 & $\mathrm{A} 2$ \\
\hline
\end{tabular}




\begin{tabular}{|c|c|}
\hline GAB3|139716 & A2 \\
\hline GABARAPL1|23710 & $\mathrm{A} 2$ \\
\hline GABARAPL3|23766 & $\mathrm{A} 2$ \\
\hline GABBR1|2550 & $\mathrm{A} 2$ \\
\hline GABPA|2551 & $\mathrm{A} 2$ \\
\hline GABRA4|2557 & $\mathrm{A} 2$ \\
\hline GABRD|2563 & $\mathrm{A} 2$ \\
\hline GABRE|2564 & $\mathrm{A} 2$ \\
\hline GABRG1|2565 & $\mathrm{A} 2$ \\
\hline GAK|2580 & $\mathrm{A} 2$ \\
\hline GAL3ST3|89792 & $\mathrm{A} 2$ \\
\hline GAL3ST4|79690 & A2 \\
\hline GALE|2582 & $\mathrm{A} 2$ \\
\hline GALNS|2588 & $\mathrm{A} 2$ \\
\hline GALNT10|55568 & $\mathrm{A} 2$ \\
\hline GALNT11|63917 & $\mathrm{A} 2$ \\
\hline GALNT1|2589 & $\mathrm{A} 2$ \\
\hline GALNT6|11226 & $\mathrm{A} 2$ \\
\hline GALNT7|51809 & A2 \\
\hline GALNTL1|57452 & $\mathrm{A} 2$ \\
\hline GALR1|2587 & $\mathrm{A} 2$ \\
\hline GALT|2592 & $\mathrm{A} 2$ \\
\hline GANAB|23193 & $\mathrm{A} 2$ \\
\hline GAPT|202309 & $\mathrm{A} 2$ \\
\hline GARS|2617 & $\mathrm{A} 2$ \\
\hline GART|2618 & $\mathrm{A} 2$ \\
\hline GAS2L2|246176 & $\mathrm{A} 2$ \\
\hline GAS2L3|283431 & $\mathrm{A} 2$ \\
\hline GAS2|2620 & $\mathrm{A} 2$ \\
\hline GAS7|8522 & $\mathrm{A} 2$ \\
\hline GATA3|2625 & $\mathrm{A} 2$ \\
\hline GATA6|2627 & $\mathrm{A} 2$ \\
\hline GATAD2A|54815 & $\mathrm{A} 2$ \\
\hline GBA|2629 & A2 \\
\hline GBE1|2632 & $\mathrm{A} 2$ \\
\hline GBF1|8729 & $\mathrm{A} 2$ \\
\hline GCAT|23464 & $\mathrm{A} 2$ \\
\hline GCH1|2643 & $\mathrm{A} 2$ \\
\hline GCNT4|51301 & A2 \\
\hline GDE1|51573 & $\mathrm{A} 2$ \\
\hline GDF10|2662 & A2 \\
\hline GDI1|2664 & $\mathrm{A} 2$ \\
\hline GDI2|2665 & $\mathrm{A} 2$ \\
\hline GEFT|115557 & $\mathrm{A} 2$ \\
\hline GEMIN4|50628 & $\mathrm{A} 2$ \\
\hline GEMIN7|79760 & $\mathrm{A} 2$ \\
\hline $\begin{array}{ll}\text { GFAP } & 2670 \\
\end{array}$ & $\mathrm{~A} 2$ \\
\hline GFER|2671 & $\mathrm{A} 2$ \\
\hline GFM1|85476 & $\mathrm{A} 2$ \\
\hline GFOD1|54438 & A2 \\
\hline GFRA2|2675 & $\mathrm{A} 2$ \\
\hline
\end{tabular}




\begin{tabular}{|c|c|}
\hline GGCT|79017 & A2 \\
\hline GGT7|2686 & $\mathrm{A} 2$ \\
\hline GGTA1|2681 & $\mathrm{A} 2$ \\
\hline GHR|2690 & $\mathrm{A} 2$ \\
\hline GIGYF1|64599 & $\mathrm{A} 2$ \\
\hline GIMAP4|55303 & $\mathrm{A} 2$ \\
\hline GIMAP5|55340 & $\mathrm{A} 2$ \\
\hline GIMAP6|474344 & $\mathrm{A} 2$ \\
\hline GIMAP8|155038 & $\mathrm{A} 2$ \\
\hline GINS1|9837 & $\mathrm{A} 2$ \\
\hline GINS2|51659 & $\mathrm{A} 2$ \\
\hline GINS3|64785 & A2 \\
\hline GIPC1|10755 & $\mathrm{A} 2$ \\
\hline GIPC2|54810 & $\mathrm{A} 2$ \\
\hline GIT2|9815 & $\mathrm{A} 2$ \\
\hline GIYD2|79008 & $\mathrm{A} 2$ \\
\hline GJA3|2700 & A2 \\
\hline GJB2|2706 & $\mathrm{A} 2$ \\
\hline GJD3|125111 & A2 \\
\hline GK|2710 & $\mathrm{A} 2$ \\
\hline GLB1|2720 & $\mathrm{A} 2$ \\
\hline GLDN|342035 & $\mathrm{A} 2$ \\
\hline GLE1|2733 & $\mathrm{A} 2$ \\
\hline GLG1|2734 & $\mathrm{A} 2$ \\
\hline GLIS2|84662 & $\mathrm{A} 2$ \\
\hline GLIS3|169792 & $\mathrm{A} 2$ \\
\hline GLO1|2739 & $\mathrm{A} 2$ \\
\hline GLP2R|9340 & $\mathrm{A} 2$ \\
\hline GLRA4|441509 & $\mathrm{A} 2$ \\
\hline GLRX2|51022 & $\mathrm{A} 2$ \\
\hline \begin{tabular}{l|l|} 
GLRX3|10539 \\
\end{tabular} & $\mathrm{A} 2$ \\
\hline GLT25D1|79709 & A2 \\
\hline GLTSCR2|29997 & $\mathrm{A} 2$ \\
\hline GLYR1|84656 & A2 \\
\hline GMDS|2762 & $\mathrm{A} 2$ \\
\hline GMIP|51291 & $\mathrm{A} 2$ \\
\hline GMPPA|29926 & $\mathrm{A} 2$ \\
\hline GMPS|8833 & $\mathrm{A} 2$ \\
\hline GNA11|2767 & A2 \\
\hline GNAI3|2773 & $\mathrm{A} 2$ \\
\hline GNAZ|2781 & $\mathrm{A} 2$ \\
\hline GNB2|2783 & $\mathrm{A} 2$ \\
\hline GNG11|2791 & $\mathrm{A} 2$ \\
\hline \begin{tabular}{l|l|l|} 
GNG125970 \\
\end{tabular} & $\mathrm{A} 2$ \\
\hline $\begin{array}{l}\text { GNG5|2787 } \\
\end{array}$ & $\mathrm{A} 2$ \\
\hline GNG7|2788 & $\mathrm{A} 2$ \\
\hline \begin{tabular}{l|l|} 
GNL1|2794 \\
\end{tabular} & $\mathrm{A} 2$ \\
\hline \begin{tabular}{l|l|} 
GNL2|2989 \\
\end{tabular} & $\mathrm{A} 2$ \\
\hline GNPDA1|10007 & $\mathrm{A} 2$ \\
\hline GNPNAT1|64841 & A2 \\
\hline GOLGA2|2801 & $\overline{\mathrm{A} 2}$ \\
\hline
\end{tabular}




\begin{tabular}{|c|c|}
\hline GOLGA5|9950 & A2 \\
\hline GOLGA6L5|374650 & $\mathrm{A} 2$ \\
\hline GOLGA8A|23015 & $\mathrm{A} 2$ \\
\hline GOLGA8B|440270 & $\mathrm{A} 2$ \\
\hline GOLGA8C|729786 & $\mathrm{A} 2$ \\
\hline GOLGA9P|283796 & $\mathrm{A} 2$ \\
\hline GOLM1|51280 & $\mathrm{A} 2$ \\
\hline GOLPH3L|55204 & $\mathrm{A} 2$ \\
\hline GOLPH3|64083 & A2 \\
\hline GOLT1A|127845 & $\mathrm{A} 2$ \\
\hline GOLT1B|51026 & $\mathrm{A} 2$ \\
\hline GON4L|54856 & $\mathrm{A} 2$ \\
\hline GORASP1|64689 & $\mathrm{A} 2$ \\
\hline GORASP2|26003 & $\mathrm{A} 2$ \\
\hline GPAA1 $\mid 8733$ & $\mathrm{~A} 2$ \\
\hline GPAM|57678 & $\mathrm{A} 2$ \\
\hline GPATCH4|54865 & $\mathrm{A} 2$ \\
\hline GPC3|2719 & $\mathrm{A} 2$ \\
\hline GPC4|2239 & A2 \\
\hline GPIHBP1|338328 & $\mathrm{A} 2$ \\
\hline GPLD1|2822 & $\mathrm{A} 2$ \\
\hline GPM6B|2824 & $\mathrm{A} 2$ \\
\hline GPN1|11321 & $\mathrm{A} 2$ \\
\hline GPR109B|8843 & $\mathrm{A} 2$ \\
\hline GPR112|139378 & $\mathrm{A} 2$ \\
\hline GPR120|338557 & $\mathrm{A} 2$ \\
\hline GPR124|25960 & $\mathrm{A} 2$ \\
\hline GPR135|64582 & $\mathrm{A} 2$ \\
\hline GPR137B|7107 & $\mathrm{A} 2$ \\
\hline GPR137C|283554 & $\mathrm{A} 2$ \\
\hline GPR137|56834 & $\mathrm{A} 2$ \\
\hline GPR142|350383 & $\mathrm{A} 2$ \\
\hline GPR143|4935 & $\mathrm{A} 2$ \\
\hline GPR144|347088 & $\mathrm{A} 2$ \\
\hline GPR146|115330 & $\mathrm{A} 2$ \\
\hline GPR149|344758 & $\mathrm{A} 2$ \\
\hline GPR160|26996 & $\mathrm{A} 2$ \\
\hline GPR172A|79581 & $\mathrm{A} 2$ \\
\hline GPR17|2840 & A2 \\
\hline GPR182|11318 & $\mathrm{A} 2$ \\
\hline GPR19|2842 & A2 \\
\hline GPR45|11250 & $\mathrm{A} 2$ \\
\hline GPR68|8111 & $\mathrm{A} 2$ \\
\hline GPR75|10936 & $\mathrm{A} 2$ \\
\hline GPR77|27202 & A2 \\
\hline GPR81|27198 & $\mathrm{A} 2$ \\
\hline GPR84|53831 & $\mathrm{A} 2$ \\
\hline GPR88|54112 & $\mathrm{A} 2$ \\
\hline GPR89A|653519 & $\mathrm{A} 2$ \\
\hline GPR97|222487 & A2 \\
\hline GPRC5A|9052 & $\mathrm{A} 2$ \\
\hline
\end{tabular}




\begin{tabular}{|c|c|}
\hline GPRC5B|51704 & A2 \\
\hline GPSM2|29899 & $\mathrm{A} 2$ \\
\hline GPX8|493869 & $\mathrm{A} 2$ \\
\hline GRAMD1A|57655 & $\mathrm{A} 2$ \\
\hline GRAMD3|65983 & $\mathrm{A} 2$ \\
\hline GRASP|160622 & $\mathrm{A} 2$ \\
\hline GRB2|2885 & $\mathrm{A} 2$ \\
\hline GREM2|64388 & $\mathrm{A} 2$ \\
\hline GRHL2|79977 & $\mathrm{A} 2$ \\
\hline GRIK5|2901 & $\mathrm{A} 2$ \\
\hline GRIN2B|2904 & $\mathrm{A} 2$ \\
\hline GRINL1A|81488 & $\mathrm{A} 2$ \\
\hline GRM7|2917 & $\mathrm{A} 2$ \\
\hline GRM8|2918 & $\mathrm{A} 2$ \\
\hline GRN|2896 & $\mathrm{A} 2$ \\
\hline GRPEL2|134266 & $\mathrm{A} 2$ \\
\hline GRRP1|79927 & A2 \\
\hline GRTP1|79774 & $\mathrm{A} 2$ \\
\hline GSG2|83903 & A2 \\
\hline GSK3A|2931 & $\mathrm{A} 2$ \\
\hline GSK3B|2932 & $\mathrm{A} 2$ \\
\hline GSN|2934 & $\mathrm{A} 2$ \\
\hline GTF2E1|2960 & $\mathrm{A} 2$ \\
\hline GTF2H1|2965 & $\mathrm{A} 2$ \\
\hline GTF2IRD1|9569 & $\mathrm{A} 2$ \\
\hline GTF2IRD2B|389524 & $\mathrm{A} 2$ \\
\hline GTF2IRD2P1|401375 & $\mathrm{A} 2$ \\
\hline GTF2IRD2|84163 & $\mathrm{A} 2$ \\
\hline GTF3C1|2975 & $\mathrm{A} 2$ \\
\hline GTF3C2|2976 & $\mathrm{A} 2$ \\
\hline GTPBP3|84705 & $\mathrm{A} 2$ \\
\hline GTPBP4|23560 & $\mathrm{A} 2$ \\
\hline GTSE1|51512 & $\mathrm{A} 2$ \\
\hline GUCA1A|2978 & $\mathrm{A} 2$ \\
\hline GUK1|2987 & $\mathrm{A} 2$ \\
\hline GYPC|2995 & $\mathrm{A} 2$ \\
\hline GYPE|2996 & $\mathrm{A} 2$ \\
\hline H1F0|3005 & $\mathrm{A} 2$ \\
\hline H2AFV|94239 & A2 \\
\hline H2AFX|3014 & $\mathrm{A} 2$ \\
\hline $\begin{array}{l}\text { H6PD|9563 } \\
\end{array}$ & A2 \\
\hline HABP4|22927 & $\mathrm{A} 2$ \\
\hline HACL1|26061 & A2 \\
\hline HADHA|3030 & $\mathrm{A} 2$ \\
\hline HADH|3033 & A2 \\
\hline HAGHL|84264 & $\mathrm{A} 2$ \\
\hline HAND2|9464 & $\mathrm{A} 2$ \\
\hline HAP1|9001 & $\mathrm{A} 2$ \\
\hline HAPLN4|404037 & $\mathrm{A} 2$ \\
\hline HARBI1|283254 & A2 \\
\hline HARS2|23438 & $\mathrm{A} 2$ \\
\hline
\end{tabular}




\begin{tabular}{|c|c|}
\hline HAS3|3038 & A2 \\
\hline HAUS1|115106 & A2 \\
\hline HAUS4|54930 & $\mathrm{A} 2$ \\
\hline HAUS8|93323 & $\mathrm{A} 2$ \\
\hline HBEGF|1839 & $\mathrm{A} 2$ \\
\hline HBP1|26959 & $\mathrm{A} 2$ \\
\hline HCFC2|29915 & $\mathrm{A} 2$ \\
\hline HCG11|493812 & $\mathrm{A} 2$ \\
\hline HCG22|285834 & $\mathrm{A} 2$ \\
\hline HCN3|57657 & $\mathrm{A} 2$ \\
\hline HCRTR1|3061 & $\mathrm{A} 2$ \\
\hline HDAC1|3065 & $\mathrm{A} 2$ \\
\hline \begin{tabular}{l|l|} 
HDAC5|10014 \\
\end{tabular} & $\mathrm{A} 2$ \\
\hline HDAC6 10013 & $\mathrm{~A} 2$ \\
\hline HDAC7|51564 & $\mathrm{A} 2$ \\
\hline HDAC8|55869 & $\mathrm{A} 2$ \\
\hline HDGF|3068 & $\mathrm{A} 2$ \\
\hline HEATR2|54919 & $\mathrm{A} 2$ \\
\hline HEATR3|55027 & $\mathrm{A} 2$ \\
\hline HELLS|3070 & $\mathrm{A} 2$ \\
\hline HELQ|113510 & $\mathrm{A} 2$ \\
\hline HERC2|8924 & $\mathrm{A} 2$ \\
\hline HERPUD2|64224 & $\mathrm{A} 2$ \\
\hline HES6|55502 & $\mathrm{A} 2$ \\
\hline HEYL|26508 & $\mathrm{A} 2$ \\
\hline HFM1|164045 & $\mathrm{A} 2$ \\
\hline HGF|3082 & $\mathrm{A} 2$ \\
\hline HHAT|55733 & $\mathrm{A} 2$ \\
\hline $\begin{array}{l}\text { HIATL1|84641 } \\
\end{array}$ & $\mathrm{A} 2$ \\
\hline HIF3A|64344 & $\mathrm{A} 2$ \\
\hline HIPK2|28996 & $\mathrm{A} 2$ \\
\hline HIPK3|10114 & $\mathrm{A} 2$ \\
\hline HIST1H1C|3006 & $\mathrm{A} 2$ \\
\hline HIST1H1T|3010 & $\mathrm{A} 2$ \\
\hline HIST1H2AC|8334 & $\mathrm{A} 2$ \\
\hline HIST1H2AD|3013 & $\mathrm{A} 2$ \\
\hline HIST1H2AJ|8331 & $\mathrm{A} 2$ \\
\hline HIST1H2AM|8336 & $\mathrm{A} 2$ \\
\hline HIST1H2BC|8347 & $\mathrm{A} 2$ \\
\hline HIST1H2BD|3017 & $\mathrm{A} 2$ \\
\hline HIST1H2BE|8344 & $\mathrm{A} 2$ \\
\hline HIST1H2BG|8339 & $\mathrm{A} 2$ \\
\hline HIST1H2BH| 8345 & $\mathrm{~A} 2$ \\
\hline HIST1H2BJ|8970 & $\mathrm{A} 2$ \\
\hline HIST1H2BK|85236 & $\mathrm{A} 2$ \\
\hline HIST1H2BN|8341 & $\mathrm{A} 2$ \\
\hline HIST1H2BO|8348 & $\mathrm{A} 2$ \\
\hline HIST1H3D|8351 & $\mathrm{A} 2$ \\
\hline HIST1H3E $\mid 8353$ & $\mathrm{~A} 2$ \\
\hline HIST1H3F|8968 & $\mathrm{A} 2$ \\
\hline HIST1H3H|8357 & $\mathrm{A} 2$ \\
\hline
\end{tabular}




\begin{tabular}{|c|c|}
\hline HIST1H3J|8356 & $\mathrm{A} 2$ \\
\hline HIST1H4A|8359 & $\mathrm{A} 2$ \\
\hline HIST1H4D|8360 & $\mathrm{A} 2$ \\
\hline HIST1H4I|8294 & $\mathrm{A} 2$ \\
\hline HIST1H4J|8363 & A2 \\
\hline HIST2H2AA3|8337 & $\mathrm{A} 2$ \\
\hline HIST2H2AC|8338 & A2 \\
\hline HIST2H2BE|8349 & $\mathrm{A} 2$ \\
\hline HIST2H2BF|440689 & $\mathrm{A} 2$ \\
\hline HIST3H2BB|128312 & A2 \\
\hline HIVEP2|3097 & $\mathrm{A} 2$ \\
\hline HJURP|55355 & $\mathrm{A} 2$ \\
\hline HK1|3098 & $\mathrm{A} 2$ \\
\hline HLA-C|3107 & $\mathrm{A} 2$ \\
\hline HLA-E|3133 & $\mathrm{A} 2$ \\
\hline HLCS|3141 & $\mathrm{A} 2$ \\
\hline HLTF $\mid 6596$ & $\mathrm{~A} 2$ \\
\hline HM13|81502 & $\mathrm{A} 2$ \\
\hline HMBS|3145 & $\mathrm{A} 2$ \\
\hline HMG20B|10362 & $\mathrm{A} 2$ \\
\hline HMGA2|8091 & $\mathrm{A} 2$ \\
\hline HMGB2|3148 & $\mathrm{A} 2$ \\
\hline HMGB3|3149 & $\mathrm{A} 2$ \\
\hline HMGCLL1|54511 & $\mathrm{A} 2$ \\
\hline HMGN1|3150 & $\mathrm{A} 2$ \\
\hline HMGN2|3151 & $\mathrm{A} 2$ \\
\hline HMGN3|9324 & $\mathrm{A} 2$ \\
\hline HMMR|3161 & $\mathrm{A} 2$ \\
\hline HMOX2|3163 & $\mathrm{A} 2$ \\
\hline HN1L|90861 & $\mathrm{A} 2$ \\
\hline HN1|51155 & $\mathrm{A} 2$ \\
\hline HNMT|3176 & $\mathrm{A} 2$ \\
\hline HNRNPA2B1|3181 & $\mathrm{A} 2$ \\
\hline HNRNPC|3183 & $\mathrm{A} 2$ \\
\hline HNRNPK 3190 & $\mathrm{~A} 2$ \\
\hline HNRNPUL1|11100 & $\mathrm{A} 2$ \\
\hline HNRPDL|9987 & $\mathrm{A} 2$ \\
\hline HOMER3|9454 & $\mathrm{A} 2$ \\
\hline HOOK2|29911 & $\mathrm{A} 2$ \\
\hline HOOK $3 \mid 84376$ & $\mathrm{~A} 2$ \\
\hline HOPX|84525 & $\mathrm{A} 2$ \\
\hline HOTAIR|100124700 & $\mathrm{A} 2$ \\
\hline HOXA10|3206 & $\mathrm{A} 2$ \\
\hline HOXA7|3204 & $\mathrm{A} 2$ \\
\hline HOXA9|3205 & $\mathrm{A} 2$ \\
\hline HOXC10|3226 & $\mathrm{A} 2$ \\
\hline HOXC11|3227 & $\mathrm{A} 2$ \\
\hline HOXC13|3229 & $\mathrm{A} 2$ \\
\hline HOXC4|3221 & $\mathrm{A} 2$ \\
\hline HOXD10|3236 & $\mathrm{A} 2$ \\
\hline HOXD4|3233 & $\mathrm{A} 2$ \\
\hline
\end{tabular}




\begin{tabular}{|c|c|}
\hline HPN|3249 & $\mathrm{A} 2$ \\
\hline HPS1|3257 & $\mathrm{A} 2$ \\
\hline HPS4|89781 & $\mathrm{A} 2$ \\
\hline HPS5|11234 & $\mathrm{A} 2$ \\
\hline HPSE2|60495 & $\mathrm{A} 2$ \\
\hline HRASLS5|117245 & $\mathrm{A} 2$ \\
\hline HRH4|59340 & $\mathrm{A} 2$ \\
\hline HRNR|388697 & $\mathrm{A} 2$ \\
\hline HS3ST3A1|9955 & $\mathrm{A} 2$ \\
\hline HS3ST4|9951 & $\mathrm{A} 2$ \\
\hline HS6ST1|9394 & $\mathrm{A} 2$ \\
\hline HS6ST3|266722 & $\mathrm{A} 2$ \\
\hline HSD11B1|3290 & $\mathrm{A} 2$ \\
\hline HSD17B10|3028 & $\mathrm{A} 2$ \\
\hline HSD17B11|51170 & $\mathrm{A} 2$ \\
\hline HSD17B6|8630 & $\mathrm{A} 2$ \\
\hline HSD17B7|51478 & $\mathrm{A} 2$ \\
\hline $\begin{array}{l}\text { HSDL2|84263 } \\
\end{array}$ & $\mathrm{A} 2$ \\
\hline HSF2BP|11077 & $\mathrm{A} 2$ \\
\hline HSF4|3299 & $\mathrm{A} 2$ \\
\hline HSH2D|84941 & $\mathrm{A} 2$ \\
\hline HSP90AA $1 \mid 3320$ & $\mathrm{~A} 2$ \\
\hline HSP90AB 13326 & $\mathrm{~A} 2$ \\
\hline HSP90B1|7184 & $\mathrm{A} 2$ \\
\hline HSPA12B|116835 & $\mathrm{A} 2$ \\
\hline HSPA13|6782 & $\mathrm{A} 2$ \\
\hline HSPA4|3308 & $\mathrm{A} 2$ \\
\hline HSPA5|3309 & $\mathrm{A} 2$ \\
\hline HSPA8|3312 & $\mathrm{A} 2$ \\
\hline HSPA9|3313 & $\mathrm{A} 2$ \\
\hline HSPB1|3315 & $\mathrm{A} 2$ \\
\hline HSPB2|3316 & $\mathrm{A} 2$ \\
\hline HSPB6|126393 & $\mathrm{A} 2$ \\
\hline HSPBP1|23640 & $\mathrm{A} 2$ \\
\hline HSPC157|29092 & $\mathrm{A} 2$ \\
\hline HSPD1|3329 & $\mathrm{A} 2$ \\
\hline HSPE1|3336 & $\mathrm{A} 2$ \\
\hline HSPH1|10808 & $\mathrm{A} 2$ \\
\hline HTATIP2|10553 & $\mathrm{A} 2$ \\
\hline HTR1D|3352 & $\mathrm{A} 2$ \\
\hline HTR2A|3356 & $\mathrm{A} 2$ \\
\hline HTR3C|170572 & $\mathrm{A} 2$ \\
\hline HTR4|3360 & $\mathrm{A} 2$ \\
\hline HUS1|3364 & $\mathrm{A} 2$ \\
\hline HYAL3|8372 & $\mathrm{A} 2$ \\
\hline HYLS1|219844 & $\mathrm{A} 2$ \\
\hline HYOU1|10525 & $\mathrm{A} 2$ \\
\hline $\begin{array}{l}\text { IARS2|55699 } \\
\end{array}$ & $\mathrm{A} 2$ \\
\hline IARS|3376 & $\mathrm{A} 2$ \\
\hline ICAM2|3384 & $\mathrm{A} 2$ \\
\hline ICT1|3396 & $\mathrm{A} 2$ \\
\hline
\end{tabular}




\begin{tabular}{|c|c|}
\hline ID1|3397 & A2 \\
\hline IDE|3416 & A2 \\
\hline IER3IP1|51124 & $\mathrm{A} 2$ \\
\hline IER5L|389792 & $\mathrm{A} 2$ \\
\hline IFFO2|126917 & A2 \\
\hline IFI30|10437 & $\mathrm{A} 2$ \\
\hline IFI35|3430 & $\mathrm{A} 2$ \\
\hline IFI6|2537 & $\mathrm{A} 2$ \\
\hline IFNE|338376 & $\mathrm{A} 2$ \\
\hline IFNGR1|3459 & $\mathrm{A} 2$ \\
\hline IGF1|3479 & $\mathrm{A} 2$ \\
\hline IGFBP6|3489 & A2 \\
\hline IGFBPL1|347252 & $\mathrm{A} 2$ \\
\hline IGJ/3512 & $\mathrm{A} 2$ \\
\hline IKBKE|9641 & $\mathrm{A} 2$ \\
\hline IKZF4|64375 & $\mathrm{A} 2$ \\
\hline IL11|3589 & A2 \\
\hline IL15RA|3601 & $\mathrm{A} 2$ \\
\hline IL17RA|23765 & $\mathrm{A} 2$ \\
\hline IL18|3606 & $\mathrm{A} 2$ \\
\hline IL1R1|3554 & $\mathrm{A} 2$ \\
\hline IL1RL2|8808 & $\mathrm{A} 2$ \\
\hline IL21R|50615 & $\mathrm{A} 2$ \\
\hline IL22RA1|58985 & $\mathrm{A} 2$ \\
\hline IL28RA|163702 & A2 \\
\hline IL4I1|259307 & $\mathrm{A} 2$ \\
\hline IL4R|3566 & $\mathrm{A} 2$ \\
\hline IL9R|3581 & $\mathrm{A} 2$ \\
\hline ILDR1|286676 & $\mathrm{A} 2$ \\
\hline ILDR2|387597 & $\mathrm{A} 2$ \\
\hline IMMP2L|83943 & $\mathrm{A} 2$ \\
\hline INCENP|3619 & $\mathrm{A} 2$ \\
\hline INE1|8552 & $\mathrm{A} 2$ \\
\hline INHBA|3624 & A2 \\
\hline INHBB|3625 & $\mathrm{A} 2$ \\
\hline INO80|54617 & $\mathrm{A} 2$ \\
\hline INPP5A|3632 & $\mathrm{A} 2$ \\
\hline INTS8|55656 & $\mathrm{A} 2$ \\
\hline INTU|27152 & $\mathrm{A} 2$ \\
\hline IP6K1|9807 & $\mathrm{A} 2$ \\
\hline IPO9|55705 & $\mathrm{A} 2$ \\
\hline IPPK|64768 & $\mathrm{A} 2$ \\
\hline IPW|3653 & $\mathrm{A} 2$ \\
\hline IQCD|115811 & $\mathrm{A} 2$ \\
\hline IQCK|124152 & $\mathrm{A} 2$ \\
\hline IQGAP3|128239 & $\mathrm{A} 2$ \\
\hline IQSEC1|9922 & $\mathrm{A} 2$ \\
\hline IRAK2|3656 & $\mathrm{A} 2$ \\
\hline IRF7|3665 & $\mathrm{A} 2$ \\
\hline IRF9|10379 & $\mathrm{A} 2$ \\
\hline IRS2|8660 & $\mathrm{A} 2$ \\
\hline
\end{tabular}




\begin{tabular}{|c|c|}
\hline IRX1|79192 & A2 \\
\hline IRX5|10265 & A2 \\
\hline IRX6|79190 & A2 \\
\hline ISG15|9636 & A2 \\
\hline ISG20L2|81875 & A2 \\
\hline ISLR2|57611 & $\mathrm{A} 2$ \\
\hline ISOC2|79763 & A2 \\
\hline ITFG2|55846 & $\mathrm{A} 2$ \\
\hline ITGA1|3672 & $\mathrm{A} 2$ \\
\hline ITGA3|3675 & A2 \\
\hline ITGA6|3655 & A2 \\
\hline ITGA9|3680 & A2 \\
\hline ITIH5|80760 & $\mathrm{A} 2$ \\
\hline ITPR1|3708 & A2 \\
\hline ITPR3|3710 & A2 \\
\hline ITPRIPL1|150771 & $\mathrm{A} 2$ \\
\hline JAG1|182 & A2 \\
\hline JAG2|3714 & A2 \\
\hline JAK1|3716 & $\mathrm{A} 2$ \\
\hline JAKMIP1|152789 & A2 \\
\hline JAM2|58494 & $\mathrm{A} 2$ \\
\hline JAM3|83700 & $\mathrm{A} 2$ \\
\hline JMJD1C|221037 & A2 \\
\hline JMJD4|65094 & A2 \\
\hline JMY|133746 & A2 \\
\hline JPH4|84502 & A2 \\
\hline JRKL|8690 & A2 \\
\hline JRK|8629 & $\mathrm{A} 2$ \\
\hline JTB|10899 & A2 \\
\hline JUND|3727 & A2 \\
\hline JUN|3725 & A2 \\
\hline KALRN|8997 & A2 \\
\hline KANK1|23189 & A2 \\
\hline KANK3|256949 & $\mathrm{A} 2$ \\
\hline KAT2B $\mid 8850$ & $\mathrm{~A} 2$ \\
\hline KATNAL1|84056 & $\mathrm{A} 2$ \\
\hline KBTBD3|143879 & A2 \\
\hline \begin{tabular}{l|l} 
KBTBD6|89890 \\
\end{tabular} & $\mathrm{A} 2$ \\
\hline KCNA1|3736 & A2 \\
\hline KCNA2|3737 & A2 \\
\hline KCNA4|3739 & A2 \\
\hline KCNAB1|7881 & A2 \\
\hline KCNH1|3756 & $\mathrm{A} 2$ \\
\hline KCNH3|23416 & $\mathrm{A} 2$ \\
\hline \begin{tabular}{l|l|} 
KCNH5|27133 & \\
\end{tabular} & A2 \\
\hline KCNH8|131096 & $\mathrm{A} 2$ \\
\hline KCNIP3|30818 & A2 \\
\hline KCNIP4|80333 & A2 \\
\hline KCNJ2|3759 & A2 \\
\hline KCNK15|60598 & $\mathrm{A} 2$ \\
\hline KCNK1|3775 & A2 \\
\hline
\end{tabular}




\begin{tabular}{|c|c|}
\hline KCNK6|9424 & A2 \\
\hline KCNMB1|3779 & $\mathrm{A} 2$ \\
\hline KCNS3|3790 & $\mathrm{A} 2$ \\
\hline KCTD10|83892 & $\mathrm{A} 2$ \\
\hline KCTD13|253980 & A2 \\
\hline KCTD15|79047 & $\mathrm{A} 2$ \\
\hline KCTD2|23510 & $\mathrm{A} 2$ \\
\hline KCTD3|51133 & $\mathrm{A} 2$ \\
\hline KCTD4|386618 & $\mathrm{A} 2$ \\
\hline KCTD6|200845 & $\mathrm{A} 2$ \\
\hline KCTD7|154881 & $\mathrm{A} 2$ \\
\hline KDELC1|79070 & $\mathrm{A} 2$ \\
\hline KDELR1|10945 & $\mathrm{A} 2$ \\
\hline KDELR2|11014 & $\mathrm{A} 2$ \\
\hline KDELR3|11015 & $\mathrm{A} 2$ \\
\hline KDM1A|23028 & $\mathrm{A} 2$ \\
\hline KDM1B|221656 & $\mathrm{A} 2$ \\
\hline KDM4B|23030 & $\mathrm{A} 2$ \\
\hline KDM5B|10765 & $\mathrm{A} 2$ \\
\hline KDM6B|23135 & $\mathrm{A} 2$ \\
\hline KHDRBS1|10657 & $\mathrm{A} 2$ \\
\hline KIAA0040|9674 & $\mathrm{A} 2$ \\
\hline KIAA0101|9768 & $\mathrm{A} 2$ \\
\hline KIAA0141|9812 & $\mathrm{A} 2$ \\
\hline KIAA0196|9897 & $\mathrm{A} 2$ \\
\hline KIAA0226|9711 & $\mathrm{A} 2$ \\
\hline KIAA0240|23506 & $\mathrm{A} 2$ \\
\hline KIAA0355|9710 & $\mathrm{A} 2$ \\
\hline KIAA0391|9692 & $\mathrm{A} 2$ \\
\hline KIAA0406|9675 & $\mathrm{A} 2$ \\
\hline KIAA0408|9729 & $\mathrm{A} 2$ \\
\hline KIAA0427|9811 & $\mathrm{A} 2$ \\
\hline KIAA0430|9665 & $\mathrm{A} 2$ \\
\hline KIAA0467|23334 & $\mathrm{A} 2$ \\
\hline KIAA0494|9813 & $\mathrm{A} 2$ \\
\hline KIAA0495|57212 & $\mathrm{A} 2$ \\
\hline KIAA0556|23247 & $\mathrm{A} 2$ \\
\hline KIAA0754|643314 & $\mathrm{A} 2$ \\
\hline KIAA0831|22863 & $\mathrm{A} 2$ \\
\hline KIAA0895|23366 & $\mathrm{A} 2$ \\
\hline KIAA1009|22832 & $\mathrm{A} 2$ \\
\hline KIAA1045|23349 & $\mathrm{A} 2$ \\
\hline KIAA1107|23285 & $\mathrm{A} 2$ \\
\hline KIAA1109|84162 & $\mathrm{A} 2$ \\
\hline KIAA1143|57456 & $\mathrm{A} 2$ \\
\hline KIAA1199|57214 & $\mathrm{A} 2$ \\
\hline KIAA1211|57482 & $\mathrm{A} 2$ \\
\hline KIAA1328|57536 & $\mathrm{A} 2$ \\
\hline KIAA1522|57648 & $\mathrm{A} 2$ \\
\hline KIAA1524|57650 & $\mathrm{A} 2$ \\
\hline KIAA1598|57698 & $\mathrm{A} 2$ \\
\hline
\end{tabular}




\begin{tabular}{|c|c|}
\hline KIAA1671|85379 & A2 \\
\hline KIAA1683|80726 & $\mathrm{A} 2$ \\
\hline KIAA1841|84542 & $\mathrm{A} 2$ \\
\hline KIAA1908|114796 & $\mathrm{A} 2$ \\
\hline KIF11|3832 & A2 \\
\hline KIF13A|63971 & $\mathrm{A} 2$ \\
\hline KIF14|9928 & $\mathrm{A} 2$ \\
\hline KIF15|56992 & $\mathrm{A} 2$ \\
\hline KIF18A|81930 & $\mathrm{A} 2$ \\
\hline KIF18B|146909 & $\mathrm{A} 2$ \\
\hline KIF1C|10749 & $\mathrm{A} 2$ \\
\hline KIF20A|10112 & A2 \\
\hline KIF21A|55605 & $\mathrm{A} 2$ \\
\hline KIF22|3835 & $\mathrm{A} 2$ \\
\hline KIF23|9493 & $\mathrm{A} 2$ \\
\hline KIF24|347240 & $\mathrm{A} 2$ \\
\hline KIF25|3834 & A2 \\
\hline KIF26A|26153 & $\mathrm{A} 2$ \\
\hline \begin{tabular}{l|l|l|} 
KIF26B & 55083
\end{tabular} & A2 \\
\hline KIF2C|11004 & $\mathrm{A} 2$ \\
\hline KIF3B|9371 & $\mathrm{A} 2$ \\
\hline KIF4A|24137 & $\mathrm{A} 2$ \\
\hline KIF4B|285643 & $\mathrm{A} 2$ \\
\hline KIFAP3|22920 & $\mathrm{A} 2$ \\
\hline KIFC1|3833 & $\mathrm{A} 2$ \\
\hline KIFC2|90990 & $\mathrm{A} 2$ \\
\hline KIT 3815 & $\mathrm{~A} 2$ \\
\hline KLC2|64837 & $\mathrm{A} 2$ \\
\hline KLF10|7071 & $\mathrm{A} 2$ \\
\hline KLF11|8462 & $\mathrm{A} 2$ \\
\hline KLF13|51621 & $\mathrm{A} 2$ \\
\hline KLF15|28999 & A2 \\
\hline KLF1|10661 & $\mathrm{A} 2$ \\
\hline KLF2|10365 & $\mathrm{A} 2$ \\
\hline KLF3|51274 & $\mathrm{A} 2$ \\
\hline KLF4|9314 & $\mathrm{A} 2$ \\
\hline KLF6|1316 & $\mathrm{A} 2$ \\
\hline KLF8|11279 & $\mathrm{A} 2$ \\
\hline KLHDC2|23588 & A2 \\
\hline KLHDC8B|200942 & $\mathrm{A} 2$ \\
\hline KLHL10|317719 & A2 \\
\hline KLHL12|59349 & $\mathrm{A} 2$ \\
\hline KLHL13|90293 & A2 \\
\hline KLHL15|80311 & $\mathrm{A} 2$ \\
\hline KLHL32|114792 & $\mathrm{A} 2$ \\
\hline KLK4|9622 & $\mathrm{A} 2$ \\
\hline KLKP1|606293 & $\mathrm{A} 2$ \\
\hline KLRAQ1|129285 & $\mathrm{A} 2$ \\
\hline KLRF1|51348 & $\mathrm{A} 2$ \\
\hline KNTC1|9735 & A2 \\
\hline KPNA5|3841 & A2 \\
\hline
\end{tabular}




\begin{tabular}{|c|c|}
\hline KPNB1|3837 & A2 \\
\hline KPTN|11133 & $\mathrm{A} 2$ \\
\hline KRAS|3845 & $\mathrm{A} 2$ \\
\hline KRBA2|124751 & $\mathrm{A} 2$ \\
\hline KREMEN1|83999 & $\mathrm{A} 2$ \\
\hline KRT14|3861 & $\mathrm{A} 2$ \\
\hline KRT15|3866 & $\mathrm{A} 2$ \\
\hline KRT17|3872 & $\mathrm{A} 2$ \\
\hline KRT5|3852 & $\mathrm{A} 2$ \\
\hline KRT8|3856 & $\mathrm{A} 2$ \\
\hline KRTAP13-4|284827 & $\mathrm{A} 2$ \\
\hline KRTCAP2|200185 & A2 \\
\hline KY|339855 & $\mathrm{A} 2$ \\
\hline L3MBTL|26013 & $\mathrm{A} 2$ \\
\hline LAGE3|8270 & $\mathrm{A} 2$ \\
\hline LAMA3|3909 & $\mathrm{A} 2$ \\
\hline LAMB2L|22973 & A2 \\
\hline LAMB3|3914 & $\mathrm{A} 2$ \\
\hline LAMC2|3918 & A2 \\
\hline LAMC3|10319 & $\mathrm{A} 2$ \\
\hline LANCL1|10314 & $\mathrm{A} 2$ \\
\hline LAPTM4B|55353 & $\mathrm{A} 2$ \\
\hline LARP1B|55132 & $\mathrm{A} 2$ \\
\hline LARP4B|23185 & $\mathrm{A} 2$ \\
\hline LARP4|113251 & $\mathrm{A} 2$ \\
\hline LARP6|55323 & $\mathrm{A} 2$ \\
\hline LAS1L|81887 & $\mathrm{A} 2$ \\
\hline LASS2|29956 & $\mathrm{A} 2$ \\
\hline LASS6|253782 & $\mathrm{A} 2$ \\
\hline LAT2|7462 & $\mathrm{A} 2$ \\
\hline LCA5L|150082 & $\mathrm{A} 2$ \\
\hline LCMT1|51451 & $\mathrm{A} 2$ \\
\hline LCN10|414332 & $\mathrm{A} 2$ \\
\hline LDHA|3939 & $\mathrm{A} 2$ \\
\hline LDHB|3945 & $\mathrm{A} 2$ \\
\hline LDHD|197257 & $\mathrm{A} 2$ \\
\hline LDLRAD2|401944 & $\mathrm{A} 2$ \\
\hline LEF1|51176 & $\mathrm{A} 2$ \\
\hline LEMD2|221496 & A2 \\
\hline LEPREL2|10536 & $\mathrm{A} 2$ \\
\hline LEPROT|54741 & A2 \\
\hline LETM1|3954 & $\mathrm{A} 2$ \\
\hline LGALS12|85329 & A2 \\
\hline LGALS8|3964 & $\mathrm{A} 2$ \\
\hline LGI4|163175 & $\mathrm{A} 2$ \\
\hline LGR6|59352 & $\mathrm{A} 2$ \\
\hline LHCGR|3973 & $\mathrm{A} 2$ \\
\hline LHFP|10186 & $\mathrm{A} 2$ \\
\hline LIAS|11019 & $\mathrm{A} 2$ \\
\hline LIF|3976 & A2 \\
\hline $\begin{array}{l}\text { LILRB5|10990 } \\
\end{array}$ & $\mathrm{A} 2$ \\
\hline
\end{tabular}




\begin{tabular}{|c|c|}
\hline LIMA1|51474 & A2 \\
\hline LIN9|286826 & $\mathrm{A} 2$ \\
\hline LIPC|3990 & $\mathrm{A} 2$ \\
\hline LIPT1|51601 & $\mathrm{A} 2$ \\
\hline LLGL2|3993 & $\mathrm{A} 2$ \\
\hline LLPH $\mid 84298$ & $\mathrm{~A} 2$ \\
\hline LMAN1|3998 & $\mathrm{A} 2$ \\
\hline LMAN2|10960 & $\mathrm{A} 2$ \\
\hline LMBR1|64327 & A2 \\
\hline LMNB2|84823 & $\mathrm{A} 2$ \\
\hline LMO2|4005 & $\mathrm{A} 2$ \\
\hline LMOD1|25802 & A2 \\
\hline LOC100126784|100126784 & $\mathrm{A} 2$ \\
\hline LOC100128164|100128164 & $\mathrm{A} 2$ \\
\hline LOC100128191|100128191 & $\mathrm{A} 2$ \\
\hline LOC100128288|100128288 & $\mathrm{A} 2$ \\
\hline LOC100128640|100128640 & $\mathrm{A} 2$ \\
\hline LOC100128675|100128675 & $\mathrm{A} 2$ \\
\hline LOC100129066|100129066 & $\mathrm{A} 2$ \\
\hline LOC100129387|100129387 & $\mathrm{A} 2$ \\
\hline LOC100129550|100129550 & $\mathrm{A} 2$ \\
\hline LOC100129637|100129637 & $\mathrm{A} 2$ \\
\hline LOC100129726|100129726 & $\mathrm{A} 2$ \\
\hline LOC100130015|100130015 & $\mathrm{A} 2$ \\
\hline LOC100130932|100130932 & $\mathrm{A} 2$ \\
\hline LOC100131193|100131193 & $\mathrm{A} 2$ \\
\hline LOC100131434|100131434 & $\mathrm{A} 2$ \\
\hline LOC100131496|100131496 & $\mathrm{A} 2$ \\
\hline LOC100132724|100132724 & $\mathrm{A} 2$ \\
\hline LOC100133545|100133545 & $\mathrm{A} 2$ \\
\hline LOC100134259|100134259 & $\mathrm{A} 2$ \\
\hline LOC100134713|100134713 & $\mathrm{A} 2$ \\
\hline LOC100144603|100144603 & $\mathrm{A} 2$ \\
\hline LOC100190938|100190938 & $\mathrm{A} 2$ \\
\hline LOC100190939|100190939 & $\mathrm{A} 2$ \\
\hline LOC100240726|100240726 & $\mathrm{A} 2$ \\
\hline LOC100240735|100240735 & $\mathrm{A} 2$ \\
\hline LOC100271722|100271722 & $\mathrm{A} 2$ \\
\hline LOC100302650|100302650 & $\mathrm{A} 2$ \\
\hline LOC113230|113230 & $\mathrm{A} 2$ \\
\hline LOC143188|143188 & $\mathrm{A} 2$ \\
\hline LOC144571|144571 & $\mathrm{A} 2$ \\
\hline LOC145820|145820 & $\mathrm{A} 2$ \\
\hline LOC147727|147727 & $\mathrm{A} 2$ \\
\hline LOC148145|148145 & $\mathrm{A} 2$ \\
\hline LOC148709|148709 & A2 \\
\hline LOC151009|151009 & $\mathrm{A} 2$ \\
\hline LOC201651|201651 & $\mathrm{A} 2$ \\
\hline LOC221122|221122 & $\mathrm{A} 2$ \\
\hline LOC221442|221442 & $\mathrm{A} 2$ \\
\hline LOC283392|283392 & $\mathrm{A} 2$ \\
\hline
\end{tabular}




\begin{tabular}{|c|c|}
\hline LOC283856|283856 & $\mathrm{A} 2$ \\
\hline LOC283867|283867 & $\mathrm{A} 2$ \\
\hline LOC283922|283922 & A2 \\
\hline LOC284009|284009 & $\mathrm{A} 2$ \\
\hline LOC284440|284440 & $\mathrm{A} 2$ \\
\hline LOC284661|284661 & $\mathrm{A} 2$ \\
\hline LOC285074|285074 & $\mathrm{A} 2$ \\
\hline LOC285205|285205 & $\mathrm{A} 2$ \\
\hline LOC285796|285796 & $\mathrm{A} 2$ \\
\hline LOC338588|338588 & A2 \\
\hline LOC338758|338758 & $\mathrm{A} 2$ \\
\hline LOC339674|339674 & $\mathrm{A} 2$ \\
\hline LOC341056|341056 & $\mathrm{A} 2$ \\
\hline LOC342346|342346 & $\mathrm{A} 2$ \\
\hline LOC344967|344967 & $\mathrm{A} 2$ \\
\hline LOC349196|349196 & A2 \\
\hline LOC374443|3744443 & $\mathrm{A} 2$ \\
\hline LOC375190|375190 & $\mathrm{A} 2$ \\
\hline LOC387647|387647 & $\mathrm{A} 2$ \\
\hline LOC388955|388955 & $\mathrm{A} 2$ \\
\hline LOC389493|389493 & $\mathrm{A} 2$ \\
\hline LOC390595|390595 & $\mathrm{A} 2$ \\
\hline LOC399815|399815 & $\mathrm{A} 2$ \\
\hline LOC400027|400027 & $\mathrm{A} 2$ \\
\hline LOC400931|400931 & $\mathrm{A} 2$ \\
\hline LOC401010|401010 & $\mathrm{A} 2$ \\
\hline LOC401093|401093 & $\mathrm{A} 2$ \\
\hline LOC401431|401431 & $\mathrm{A} 2$ \\
\hline LOC415056|415056 & $\mathrm{A} 2$ \\
\hline LOC493754|493754 & $\mathrm{A} 2$ \\
\hline LOC541471|541471 & $\mathrm{A} 2$ \\
\hline LOC550643|550643 & $\mathrm{A} 2$ \\
\hline LOC55908|55908 & $\mathrm{A} 2$ \\
\hline LOC572558|572558 & $\mathrm{A} 2$ \\
\hline LOC642587|642587 & $\mathrm{A} 2$ \\
\hline LOC642826|642826 & $\mathrm{A} 2$ \\
\hline LOC643387|643387 & $\mathrm{A} 2$ \\
\hline LOC644538|644538 & $\mathrm{A} 2$ \\
\hline LOC646982|646982 & $\mathrm{A} 2$ \\
\hline LOC647309|647309 & $\mathrm{A} 2$ \\
\hline LOC648740|648740 & $\mathrm{A} 2$ \\
\hline LOC650368|650368 & $\mathrm{A} 2$ \\
\hline LOC650623|650623 & $\mathrm{A} 2$ \\
\hline LOC653501|653501 & $\mathrm{A} 2$ \\
\hline LOC653566|653566 & $\mathrm{A} 2$ \\
\hline LOC654342|654342 & $\mathrm{A} 2$ \\
\hline LOC728392|728392 & $\mathrm{A} 2$ \\
\hline LOC728554|728554 & $\mathrm{A} 2$ \\
\hline LOC729467|729467 & $\mathrm{A} 2$ \\
\hline LOC729799|729799 & $\mathrm{A} 2$ \\
\hline LOC90784|90784 & $\mathrm{A} 2$ \\
\hline
\end{tabular}




\begin{tabular}{|c|c|}
\hline LOC90834|90834 & A2 \\
\hline LOC91149|91149 & $\mathrm{A} 2$ \\
\hline LOC92659|92659 & $\mathrm{A} 2$ \\
\hline LOH12CR2|503693 & $\mathrm{A} 2$ \\
\hline LOH3CR2A|29931 & $\mathrm{A} 2$ \\
\hline LONRF1|91694 & $\mathrm{A} 2$ \\
\hline LONRF3|79836 & $\mathrm{A} 2$ \\
\hline LOXL1|4016 & $\mathrm{A} 2$ \\
\hline LPAR1|1902 & A2 \\
\hline LPAR5|57121 & $\mathrm{A} 2$ \\
\hline LPAR6|10161 & $\mathrm{A} 2$ \\
\hline LPCAT1|79888 & $\mathrm{A} 2$ \\
\hline LPHN2|23266 & $\mathrm{A} 2$ \\
\hline \begin{tabular}{|l|l|} 
LPHN3 23284 \\
\end{tabular} & $\mathrm{~A} 2$ \\
\hline LPL|4023 & $\mathrm{A} 2$ \\
\hline LPPR1|54886 & $\mathrm{A} 2$ \\
\hline LPPR2|64748 & $\mathrm{A} 2$ \\
\hline LPPR4|9890 & $\mathrm{A} 2$ \\
\hline LRBA|987 & A2 \\
\hline LRFN1|57622 & $\mathrm{A} 2$ \\
\hline LRFN2|57497 & $\mathrm{A} 2$ \\
\hline LRIG2|9860 & $\mathrm{A} 2$ \\
\hline LRMP|4033 & $\mathrm{A} 2$ \\
\hline LRP8|7804 & $\mathrm{A} 2$ \\
\hline \begin{tabular}{|l|} 
LRRC29|26231 \\
\end{tabular} & $\mathrm{A} 2$ \\
\hline LRRC33|375387 & $\mathrm{A} 2$ \\
\hline LRRC34|151827 & $\mathrm{A} 2$ \\
\hline LRRC37A4|55073 & $\mathrm{A} 2$ \\
\hline LRRC3|81543 & $\mathrm{A} 2$ \\
\hline LRRC42|115353 & $\mathrm{A} 2$ \\
\hline LRRC45|201255 & $\mathrm{A} 2$ \\
\hline LRRC4B|94030 & $\mathrm{A} 2$ \\
\hline LRRC56|115399 & $\mathrm{A} 2$ \\
\hline LRRC61|65999 & $\mathrm{A} 2$ \\
\hline LRRC6|23639 & $\mathrm{A} 2$ \\
\hline LRRC70|100130733 & $\mathrm{A} 2$ \\
\hline LRRC8C|84230 & $\mathrm{A} 2$ \\
\hline LRRC8E|80131 & $\mathrm{A} 2$ \\
\hline LRRCC1|85444 & $\mathrm{A} 2$ \\
\hline LRRN2|10446 & $\mathrm{A} 2$ \\
\hline LRRN4CL|221091 & A2 \\
\hline LRRTM1|347730 & $\mathrm{A} 2$ \\
\hline LRSAM1|90678 & A2 \\
\hline LRWD1|222229 & $\mathrm{A} 2$ \\
\hline LSM12|124801 & $\mathrm{A} 2$ \\
\hline LSM14B|149986 & $\mathrm{A} 2$ \\
\hline LSM2|57819 & $\mathrm{A} 2$ \\
\hline LSM3|27258 & $\mathrm{A} 2$ \\
\hline LSM5|23658 & $\mathrm{A} 2$ \\
\hline LSR|51599 & A2 \\
\hline LTBP2|4053 & $\mathrm{A} 2$ \\
\hline
\end{tabular}




\begin{tabular}{|c|c|}
\hline LTBP4|8425 & A2 \\
\hline LTC4S|4056 & $\mathrm{A} 2$ \\
\hline LUZP2|338645 & $\mathrm{A} 2$ \\
\hline LY6G5B|58496 & $\mathrm{A} 2$ \\
\hline LY75|4065 & A2 \\
\hline LYPD1|116372 & $\mathrm{A} 2$ \\
\hline LYPLA1|10434 & $\mathrm{A} 2$ \\
\hline LYPLA2P1|653639 & $\mathrm{A} 2$ \\
\hline LYPLA2|11313 & $\mathrm{A} 2$ \\
\hline LYPLAL1|127018 & $\mathrm{A} 2$ \\
\hline LYRM1|57149 & $\mathrm{A} 2$ \\
\hline LYRM7|90624 & $\mathrm{A} 2$ \\
\hline LYSMD1|388695 & $\mathrm{A} 2$ \\
\hline LYVE1|10894 & $\mathrm{A} 2$ \\
\hline MAD2L1BP|9587 & $\mathrm{A} 2$ \\
\hline MAD2L1|4085 & $\mathrm{A} 2$ \\
\hline MAFG|4097 & $\mathrm{A} 2$ \\
\hline MAFK|7975 & $\mathrm{A} 2$ \\
\hline MAF|4094 & $\mathrm{A} 2$ \\
\hline MAGED1|9500 & $\mathrm{A} 2$ \\
\hline MAGED2|10916 & $\mathrm{A} 2$ \\
\hline MAGEF1|64110 & $\mathrm{A} 2$ \\
\hline MAGIX|79917 & $\mathrm{A} 2$ \\
\hline MAGOHB|55110 & $\mathrm{A} 2$ \\
\hline MAL2|114569 & $\mathrm{A} 2$ \\
\hline MAML3|55534 & $\mathrm{A} 2$ \\
\hline MAN2A2|4122 & $\mathrm{A} 2$ \\
\hline MAN2B1|4125 & $\mathrm{A} 2$ \\
\hline MANEAL|149175 & $\mathrm{A} 2$ \\
\hline MAOA|4128 & $\mathrm{A} 2$ \\
\hline MAP1D|254042 & $\mathrm{A} 2$ \\
\hline MAP2K6|5608 & $\mathrm{A} 2$ \\
\hline MAP3K14|9020 & $\mathrm{A} 2$ \\
\hline MAP3K3|4215 & $\mathrm{A} 2$ \\
\hline MAP3K4|4216 & $\mathrm{A} 2$ \\
\hline MAP3K5|4217 & $\mathrm{A} 2$ \\
\hline MAP3K6|9064 & $\mathrm{A} 2$ \\
\hline MAP3K8|1326 & $\mathrm{A} 2$ \\
\hline MAP4K2|5871 & $\mathrm{A} 2$ \\
\hline MAP7|9053 & $\mathrm{A} 2$ \\
\hline MAPK10|5602 & $\mathrm{A} 2$ \\
\hline MAPK11|5600 & $\mathrm{A} 2$ \\
\hline MAPK13|5603 & $\mathrm{A} 2$ \\
\hline MAPK15|225689 & $\mathrm{A} 2$ \\
\hline MAPK8IP2|23542 & $\mathrm{A} 2$ \\
\hline MAPK9|5601 & A2 \\
\hline MAPKAPK2|9261 & $\mathrm{A} 2$ \\
\hline MAPKAPK5|8550 & $\mathrm{A} 2$ \\
\hline MAPRE1|22919 & $\mathrm{A} 2$ \\
\hline MARCH9|92979 & $\mathrm{A} 2$ \\
\hline MARCKSL1|65108 & $\mathrm{A} 2$ \\
\hline
\end{tabular}




\begin{tabular}{|c|c|}
\hline MARS|4141 & A2 \\
\hline MASP1|5648 & $\mathrm{A} 2$ \\
\hline MASP2|10747 & $\mathrm{A} 2$ \\
\hline MASTL|84930 & $\mathrm{A} 2$ \\
\hline MAT2B|27430 & $\mathrm{A} 2$ \\
\hline MATN3|4148 & $\mathrm{A} 2$ \\
\hline MAVS|57506 & $\mathrm{A} 2$ \\
\hline MBD1|4152 & $\mathrm{A} 2$ \\
\hline MBD4|8930 & $\mathrm{A} 2$ \\
\hline MBL1P $\mid 8512$ & $\mathrm{~A} 2$ \\
\hline MBLAC2|153364 & $\mathrm{A} 2$ \\
\hline MBNL1|4154 & A2 \\
\hline MBNL2|10150 & $\mathrm{A} 2$ \\
\hline MBNL3|55796 & $\mathrm{A} 2$ \\
\hline MBOAT2|129642 & $\mathrm{A} 2$ \\
\hline MBP|4155 & $\mathrm{A} 2$ \\
\hline $\mathrm{MC} 1 \mathrm{R} \mid 4157$ & $\mathrm{~A} 2$ \\
\hline MCC|4163 & $\mathrm{A} 2$ \\
\hline MCFD2|90411 & A2 \\
\hline MCM10|55388 & $\mathrm{A} 2$ \\
\hline MCM2|4171 & $\mathrm{A} 2$ \\
\hline MCM4|4173 & $\mathrm{A} 2$ \\
\hline MCM6|4175 & $\mathrm{A} 2$ \\
\hline MCOLN2|255231 & $\mathrm{A} 2$ \\
\hline MCPH1|79648 & $\mathrm{A} 2$ \\
\hline MCTS1|28985 & $\mathrm{A} 2$ \\
\hline MDN1|23195 & $\mathrm{A} 2$ \\
\hline ME3|10873 & $\mathrm{A} 2$ \\
\hline MEA1|4201 & $\mathrm{A} 2$ \\
\hline MEAF6|64769 & $\mathrm{A} 2$ \\
\hline MECP2|4204 & $\mathrm{A} 2$ \\
\hline MED14|9282 & $\mathrm{A} 2$ \\
\hline MED20|9477 & $\mathrm{A} 2$ \\
\hline MED25|81857 & A2 \\
\hline MED8|112950 & $\mathrm{A} 2$ \\
\hline MED9|55090 & $\mathrm{A} 2$ \\
\hline MEGF8|1954 & $\mathrm{A} 2$ \\
\hline MEIS1|4211 & $\mathrm{A} 2$ \\
\hline MEIS3P1|4213 & $\mathrm{A} 2$ \\
\hline MEIS3|56917 & $\mathrm{A} 2$ \\
\hline MELK|9833 & A2 \\
\hline MEMO1|51072 & $\mathrm{A} 2$ \\
\hline MEN1|4221 & A2 \\
\hline MEOX2|4223 & $\mathrm{A} 2$ \\
\hline MERTK|10461 & $\mathrm{A} 2$ \\
\hline MESDC2|23184 & $\mathrm{A} 2$ \\
\hline MESTIT1|317751 & $\mathrm{A} 2$ \\
\hline METRN|79006 & $\mathrm{A} 2$ \\
\hline METT10D|79066 & $\mathrm{A} 2$ \\
\hline METTL11B|149281 & A2 \\
\hline METTL13|51603 & $\mathrm{A} 2$ \\
\hline
\end{tabular}




\begin{tabular}{|c|c|}
\hline METTL2A|339175 & $\mathrm{A} 2$ \\
\hline MEX3A|92312 & $\mathrm{A} 2$ \\
\hline MEX3D|399664 & $\mathrm{A} 2$ \\
\hline MFAP2|4237 & $\mathrm{A} 2$ \\
\hline MFSD10|10227 & $\mathrm{A} 2$ \\
\hline MFSD3|113655 & $\mathrm{A} 2$ \\
\hline MFSD5|84975 & $\mathrm{A} 2$ \\
\hline MGAT2|4247 & $\mathrm{A} 2$ \\
\hline MGAT3|4248 & $\mathrm{A} 2$ \\
\hline MGAT4A|11320 & $\mathrm{A} 2$ \\
\hline MGAT4B|11282 & $\mathrm{A} 2$ \\
\hline MGC15885|197003 & $\mathrm{A} 2$ \\
\hline MGC21881|389741 & $\mathrm{A} 2$ \\
\hline MGC23284|197187 & $\mathrm{A} 2$ \\
\hline MGC87042|256227 & $\mathrm{A} 2$ \\
\hline MGRN1|23295 & $\mathrm{A} 2$ \\
\hline MICAL2|9645 & $\mathrm{A} 2$ \\
\hline MICALL2|79778 & $\mathrm{A} 2$ \\
\hline MICB $\mid 4277$ & $\mathrm{~A} 2$ \\
\hline MIF|4282 & $\mathrm{A} 2$ \\
\hline MINK1|50488 & $\mathrm{A} 2$ \\
\hline MINPP1|9562 & $\mathrm{A} 2$ \\
\hline MIR17HG|407975 & $\mathrm{A} 2$ \\
\hline MKI67IP|84365 & $\mathrm{A} 2$ \\
\hline MKI67|4288 & $\mathrm{A} 2$ \\
\hline MKLN1|4289 & $\mathrm{A} 2$ \\
\hline MKNK1|8569 & $\mathrm{A} 2$ \\
\hline MLF2|8079 & $\mathrm{A} 2$ \\
\hline MLLT11|10962 & $\mathrm{A} 2$ \\
\hline MLL|4297 & $\mathrm{A} 2$ \\
\hline MLPH|79083 & $\mathrm{A} 2$ \\
\hline MLST8|64223 & $\mathrm{A} 2$ \\
\hline MLXIPL|51085 & $\mathrm{A} 2$ \\
\hline MLYCD|23417 & $\mathrm{A} 2$ \\
\hline MMGT1|93380 & $\mathrm{A} 2$ \\
\hline MMP11/4320 & $\mathrm{A} 2$ \\
\hline MMP14|4323 & $\mathrm{A} 2$ \\
\hline MMP19|4327 & $\mathrm{A} 2$ \\
\hline MMP3|4314 & $\mathrm{A} 2$ \\
\hline MMRN2|79812 & $\mathrm{A} 2$ \\
\hline MND1|84057 & $\mathrm{A} 2$ \\
\hline MOCS1|4337 & $\mathrm{A} 2$ \\
\hline MOCS3|27304 & $\mathrm{A} 2$ \\
\hline MOGS|7841 & $\mathrm{A} 2$ \\
\hline MORC2|22880 & $\mathrm{A} 2$ \\
\hline MORF4L2|9643 & $\mathrm{A} 2$ \\
\hline MOSPD3|64598 & $\mathrm{A} 2$ \\
\hline MOV10|4343 & $\mathrm{A} 2$ \\
\hline MOXD1|26002 & $\mathrm{A} 2$ \\
\hline MPDU1|9526 & $\mathrm{A} 2$ \\
\hline MPHOSPH8|54737 & $\mathrm{A} 2$ \\
\hline
\end{tabular}




\begin{tabular}{|c|c|}
\hline MPHOSPH9|10198 & $\mathrm{A} 2$ \\
\hline MPL|4352 & $\mathrm{A} 2$ \\
\hline MPP6|51678 & $\mathrm{A} 2$ \\
\hline MPPED2|744 & $\mathrm{A} 2$ \\
\hline MPRIP|23164 & $\mathrm{A} 2$ \\
\hline MPV17L2|84769 & $\mathrm{A} 2$ \\
\hline MPV17|4358 & $\mathrm{A} 2$ \\
\hline MR1|3140 & $\mathrm{A} 2$ \\
\hline MRAS|22808 & A2 \\
\hline MRC1|4360 & $\mathrm{A} 2$ \\
\hline MRGPRF|116535 & $\mathrm{A} 2$ \\
\hline MRGPRX2|117194 & $\mathrm{A} 2$ \\
\hline MRGPRX3|117195 & $\mathrm{A} 2$ \\
\hline MRPL12|6182 & $\mathrm{A} 2$ \\
\hline MRPL13|28998 & $\mathrm{A} 2$ \\
\hline MRPL14|64928 & $\mathrm{A} 2$ \\
\hline MRPL15|29088 & $\mathrm{A} 2$ \\
\hline MRPL17|63875 & $\mathrm{A} 2$ \\
\hline MRPL19|9801 & $\mathrm{A} 2$ \\
\hline MRPL22|29093 & $\mathrm{A} 2$ \\
\hline MRPL24|79590 & $\mathrm{A} 2$ \\
\hline MRPL28|10573 & $\mathrm{A} 2$ \\
\hline MRPL36|64979 & $\mathrm{A} 2$ \\
\hline MRPL3|11222 & $\mathrm{A} 2$ \\
\hline MRPL42|28977 & $\mathrm{A} 2$ \\
\hline MRPL47|57129 & $\mathrm{A} 2$ \\
\hline MRPL49|740 & $\mathrm{A} 2$ \\
\hline MRPL51|51258 & $\mathrm{A} 2$ \\
\hline MRPL55|128308 & $\mathrm{A} 2$ \\
\hline MRPL9|65005 & $\mathrm{A} 2$ \\
\hline MRPS10|55173 & $\mathrm{A} 2$ \\
\hline MRPS14|63931 & $\mathrm{A} 2$ \\
\hline MRPS16|51021 & $\mathrm{A} 2$ \\
\hline MRPS21|54460 & $\mathrm{A} 2$ \\
\hline MRPS23|51649 & $\mathrm{A} 2$ \\
\hline MRPS31|10240 & $\mathrm{A} 2$ \\
\hline MRPS34|65993 & $\mathrm{A} 2$ \\
\hline MRPS35|60488 & $\mathrm{A} 2$ \\
\hline MSH2|4436 & $\mathrm{A} 2$ \\
\hline MSH6|2956 & $\mathrm{A} 2$ \\
\hline MSI2|124540 & $\mathrm{A} 2$ \\
\hline MSR1|4481 & $\mathrm{A} 2$ \\
\hline MSRA|4482 & $\mathrm{A} 2$ \\
\hline MSRB3|253827 & $\mathrm{A} 2$ \\
\hline MST1P9|11223 & $\mathrm{A} 2$ \\
\hline MST1R|4486 & $\mathrm{A} 2$ \\
\hline MT1E|4493 & $\mathrm{A} 2$ \\
\hline MT1L|4500 & $\mathrm{A} 2$ \\
\hline MT1M|4499 & $\mathrm{A} 2$ \\
\hline MT1X|4501 & $\mathrm{A} 2$ \\
\hline MTBP|27085 & $\mathrm{A} 2$ \\
\hline
\end{tabular}




\begin{tabular}{|c|c|}
\hline MTCH2|23788 & $\mathrm{A} 2$ \\
\hline MTDH $\mid 92140$ & $\mathrm{~A} 2$ \\
\hline MTERFD1|51001 & $\mathrm{A} 2$ \\
\hline MTERFD2|130916 & $\mathrm{A} 2$ \\
\hline MTFR1|9650 & A2 \\
\hline MTHFD2|10797 & $\mathrm{A} 2$ \\
\hline MTMR12|54545 & $\mathrm{A} 2$ \\
\hline MTMR15|22909 & $\mathrm{A} 2$ \\
\hline MTMR3|8897 & $\mathrm{A} 2$ \\
\hline MTMR8|55613 & $\mathrm{A} 2$ \\
\hline MTMR9L|339483 & $\mathrm{A} 2$ \\
\hline MTMR9|66036 & $\mathrm{A} 2$ \\
\hline MTP18|51537 & $\mathrm{A} 2$ \\
\hline MTUS1|57509 & $\mathrm{A} 2$ \\
\hline MTX1|4580 & $\mathrm{A} 2$ \\
\hline MTX2|10651 & $\mathrm{A} 2$ \\
\hline MUC1|4582 & $\mathrm{A} 2$ \\
\hline MUM1|84939 & $\mathrm{A} 2$ \\
\hline MVP|9961 & $\mathrm{A} 2$ \\
\hline MX1|4599 & $\mathrm{A} 2$ \\
\hline MXD3|83463 & $\mathrm{A} 2$ \\
\hline MXI1|4601 & $\mathrm{A} 2$ \\
\hline MXRA5|25878 & $\mathrm{A} 2$ \\
\hline MYADM|91663 & $\mathrm{A} 2$ \\
\hline MYBL2|4605 & $\mathrm{A} 2$ \\
\hline MYB|4602 & $\mathrm{A} 2$ \\
\hline MYCBP2|23077 & $\mathrm{A} 2$ \\
\hline MYCBP|26292 & $\mathrm{A} 2$ \\
\hline MYCT1|80177 & $\mathrm{A} 2$ \\
\hline MYC|4609 & $\mathrm{A} 2$ \\
\hline MYD88|4615 & $\mathrm{A} 2$ \\
\hline MYEF2|50804 & $\mathrm{A} 2$ \\
\hline MYH10|4628 & $\mathrm{A} 2$ \\
\hline MYO19|80179 & $\mathrm{A} 2$ \\
\hline MYO1D|4642 & $\mathrm{A} 2$ \\
\hline MYO1H|283446 & $\mathrm{A} 2$ \\
\hline MYO5B|4645 & $\mathrm{A} 2$ \\
\hline MYO6|4646 & $\mathrm{A} 2$ \\
\hline MYOCD|93649 & $\mathrm{A} 2$ \\
\hline MYOC|4653 & $\mathrm{A} 2$ \\
\hline N4BP2L2|10443 & $\mathrm{A} 2$ \\
\hline N4BP3|23138 & $\mathrm{A} 2$ \\
\hline NAA15|80155 & $\mathrm{A} 2$ \\
\hline $\begin{array}{l}\text { NAA16|79612 } \\
\end{array}$ & $\mathrm{A} 2$ \\
\hline NAA38|51691 & $\mathrm{A} 2$ \\
\hline NAALAD2|10003 & $\mathrm{A} 2$ \\
\hline NAALADL1|10004 & $\mathrm{A} 2$ \\
\hline NACAD|23148 & $\mathrm{A} 2$ \\
\hline NACA|4666 & $\mathrm{A} 2$ \\
\hline NACC1|112939 & $\mathrm{A} 2$ \\
\hline NAGK|55577 & $\mathrm{A} 2$ \\
\hline
\end{tabular}




\begin{tabular}{|c|c|}
\hline NANS|54187 & A2 \\
\hline NAP1L2|4674 & $\mathrm{A} 2$ \\
\hline NAPA $\mid 8775$ & $\mathrm{~A} 2$ \\
\hline NARFL|64428 & $\mathrm{A} 2$ \\
\hline NARS $\mid 4677$ & $\mathrm{~A} 2$ \\
\hline NAT14|57106 & $\mathrm{A} 2$ \\
\hline NAT15|79903 & $\mathrm{A} 2$ \\
\hline NAT6|24142 & $\mathrm{A} 2$ \\
\hline NAT8L|339983 & $\mathrm{A} 2$ \\
\hline NAV2|89797 & $\mathrm{A} 2$ \\
\hline NBEAL1|65065 & $\mathrm{A} 2$ \\
\hline NCAM1|4684 & A2 \\
\hline NCAPD2|9918 & $\mathrm{A} 2$ \\
\hline NCAPD3|23310 & $\mathrm{A} 2$ \\
\hline NCAPG2|54892 & $\mathrm{A} 2$ \\
\hline NCAPG|64151 & $\mathrm{A} 2$ \\
\hline NCAPH|23397 & A2 \\
\hline NCBP2|22916 & $\mathrm{A} 2$ \\
\hline NCDN|23154 & A2 \\
\hline NCEH1|57552 & $\mathrm{A} 2$ \\
\hline NCKAP5L|57701 & $\mathrm{A} 2$ \\
\hline NCLN|56926 & $\mathrm{A} 2$ \\
\hline NCOA1 $\mid 8648$ & $\mathrm{~A} 2$ \\
\hline NCOA4|8031 & $\mathrm{A} 2$ \\
\hline NCOA7|135112 & $\mathrm{A} 2$ \\
\hline NCRNA00032|158035 & $\mathrm{A} 2$ \\
\hline NCRNA00092|100188953 & $\mathrm{A} 2$ \\
\hline NCRNA00120|55389 & $\mathrm{A} 2$ \\
\hline NCRNA00152|112597 & $\mathrm{A} 2$ \\
\hline NCRNA00176|284739 & $\mathrm{A} 2$ \\
\hline NCRNA00182|100302692 & $\mathrm{A} 2$ \\
\hline NCRNA00188|125144 & $\mathrm{A} 2$ \\
\hline NCRNA00204B|286967 & $\mathrm{A} 2$ \\
\hline NCRNA00219|114915 & $\mathrm{A} 2$ \\
\hline NCSTN|23385 & $\mathrm{A} 2$ \\
\hline NDC80|10403 & $\mathrm{A} 2$ \\
\hline NDE1|54820 & $\mathrm{A} 2$ \\
\hline NDN|4692 & $\mathrm{A} 2$ \\
\hline NDOR1|27158 & $\mathrm{A} 2$ \\
\hline NDRG3|57446 & $\mathrm{A} 2$ \\
\hline NDST1|3340 & A2 \\
\hline NDUFA13|51079 & $\mathrm{A} 2$ \\
\hline NDUFA7|4701 & $\mathrm{A} 2$ \\
\hline NDUFB4|4710 & $\mathrm{A} 2$ \\
\hline NDUFB9|4715 & A2 \\
\hline NDUFS2|4720 & $\mathrm{A} 2$ \\
\hline NDUFS4|4724 & $\mathrm{A} 2$ \\
\hline NDUFV2|4729 & $\mathrm{A} 2$ \\
\hline NECAB3|63941 & $\mathrm{A} 2$ \\
\hline NECAP1|25977 & A2 \\
\hline NEDD9|4739 & $\mathrm{A} 2$ \\
\hline
\end{tabular}




\begin{tabular}{|c|c|}
\hline NEIL1|79661 & $\mathrm{A} 2$ \\
\hline NEK1|4750 & $\mathrm{A} 2$ \\
\hline NEK2|4751 & $\mathrm{A} 2$ \\
\hline NEK3|4752 & $\mathrm{A} 2$ \\
\hline NEK5|341676 & $\mathrm{A} 2$ \\
\hline NEK7|140609 & $\mathrm{A} 2$ \\
\hline NEK9|91754 & $\mathrm{A} 2$ \\
\hline NES|10763 & $\mathrm{A} 2$ \\
\hline NEU1|4758 & $\mathrm{A} 2$ \\
\hline NEURL2|140825 & $\mathrm{A} 2$ \\
\hline NEURL4|84461 & $\mathrm{A} 2$ \\
\hline NFATC2|4773 & $\mathrm{A} 2$ \\
\hline NFATC3|4775 & $\mathrm{A} 2$ \\
\hline NFIB|4781 & $\mathrm{A} 2$ \\
\hline NFIL3|4783 & $\mathrm{A} 2$ \\
\hline NFKBIE|4794 & $\mathrm{A} 2$ \\
\hline NFKBIL2|4796 & $\mathrm{A} 2$ \\
\hline NFU1|27247 & $\mathrm{A} 2$ \\
\hline NFYA|4800 & $\mathrm{A} 2$ \\
\hline NGF|4803 & $\mathrm{A} 2$ \\
\hline NHEJ1|79840 & $\mathrm{A} 2$ \\
\hline NHLRC3|387921 & $\mathrm{A} 2$ \\
\hline NHP2|55651 & $\mathrm{A} 2$ \\
\hline NICN1 $\mid 84276$ & $\mathrm{~A} 2$ \\
\hline NINJ1|4814 & $\mathrm{A} 2$ \\
\hline NIPA1|123606 & $\mathrm{A} 2$ \\
\hline NIPSNAP3B|55335 & $\mathrm{A} 2$ \\
\hline NIT2|56954 & $\mathrm{A} 2$ \\
\hline NKAIN1|79570 & $\mathrm{A} 2$ \\
\hline NKAIN2|154215 & $\mathrm{A} 2$ \\
\hline NKAPL|222698 & $\mathrm{A} 2$ \\
\hline NKAP|79576 & $\mathrm{A} 2$ \\
\hline NKD2|85409 & $\mathrm{A} 2$ \\
\hline NKTR|4820 & $\mathrm{A} 2$ \\
\hline NKX3-2|579 & $\mathrm{A} 2$ \\
\hline NLGN1|22871 & $\mathrm{A} 2$ \\
\hline \begin{tabular}{l|l|} 
NLGN3|54413 \\
\end{tabular} & $\mathrm{A} 2$ \\
\hline NLGN4X|57502 & $\mathrm{A} 2$ \\
\hline NLK|51701 & $\mathrm{A} 2$ \\
\hline NLN|57486 & $\mathrm{A} 2$ \\
\hline NLRP1|22861 & $\mathrm{A} 2$ \\
\hline NLRX1|79671 & $\mathrm{A} 2$ \\
\hline NMBR $\mid 4829$ & $\mathrm{~A} 2$ \\
\hline NME1|4830 & $\mathrm{A} 2$ \\
\hline NME3|4832 & $\mathrm{A} 2$ \\
\hline NME4|4833 & $\mathrm{A} 2$ \\
\hline NME7|29922 & $\mathrm{A} 2$ \\
\hline NMNAT2|23057 & $\mathrm{A} 2$ \\
\hline NMRAL1|57407 & $\mathrm{A} 2$ \\
\hline NMT1|4836 & $\mathrm{A} 2$ \\
\hline NMT2|9397 & $\mathrm{A} 2$ \\
\hline
\end{tabular}




\begin{tabular}{|c|c|}
\hline NOB1|28987 & A2 \\
\hline NOC2L|26155 & $\mathrm{A} 2$ \\
\hline NOC4L|79050 & $\mathrm{A} 2$ \\
\hline NOD1|10392 & $\mathrm{A} 2$ \\
\hline NODAL|4838 & $\mathrm{A} 2$ \\
\hline NOL10|79954 & $\mathrm{A} 2$ \\
\hline NOMO2|283820 & $\mathrm{A} 2$ \\
\hline NOMO3|408050 & $\mathrm{A} 2$ \\
\hline NOP16|51491 & A2 \\
\hline NOP2|4839 & $\mathrm{A} 2$ \\
\hline NOP56|10528 & $\mathrm{A} 2$ \\
\hline NOP58|51602 & $\mathrm{A} 2$ \\
\hline NOTCH4|4855 & $\mathrm{A} 2$ \\
\hline NPAS4|266743 & $\mathrm{A} 2$ \\
\hline NPIP $\mid 9284$ & $\mathrm{~A} 2$ \\
\hline NPM2|10361 & $\mathrm{A} 2$ \\
\hline NPNT|255743 & $\mathrm{A} 2$ \\
\hline NPR2|4882 & $\mathrm{A} 2$ \\
\hline NPRL2|10641 & A2 \\
\hline NPY2R|4887 & $\mathrm{A} 2$ \\
\hline NR1D2|9975 & $\mathrm{A} 2$ \\
\hline NR1H3|10062 & $\mathrm{A} 2$ \\
\hline NR2C2AP|126382 & $\mathrm{A} 2$ \\
\hline NR2C2|7182 & $\mathrm{A} 2$ \\
\hline NR2F6|2063 & $\mathrm{A} 2$ \\
\hline NR4A1|3164 & $\mathrm{A} 2$ \\
\hline NR4A3|8013 & $\mathrm{A} 2$ \\
\hline NR5A2|2494 & $\mathrm{A} 2$ \\
\hline NRAS|4893 & $\mathrm{A} 2$ \\
\hline NRBP1|29959 & A2 \\
\hline NRBP2|340371 & $\mathrm{A} 2$ \\
\hline NRF1|4899 & $\mathrm{A} 2$ \\
\hline NRG1|3084 & $\mathrm{A} 2$ \\
\hline NRG2|9542 & $\mathrm{A} 2$ \\
\hline NRM|11270 & $\mathrm{A} 2$ \\
\hline NRN1|51299 & $\mathrm{A} 2$ \\
\hline NRSN2|80023 & $\mathrm{A} 2$ \\
\hline NSDHL|50814 & $\mathrm{A} 2$ \\
\hline NSL1|25936 & $\mathrm{A} 2$ \\
\hline NSMCE1|197370 & $\mathrm{A} 2$ \\
\hline NSMCE2|286053 & A2 \\
\hline NSUN5|55695 & $\mathrm{A} 2$ \\
\hline NT5C3|51251 & $\mathrm{A} 2$ \\
\hline NT5DC3|51559 & $\mathrm{A} 2$ \\
\hline NTF3|4908 & A2 \\
\hline NTHL1|4913 & $\mathrm{A} 2$ \\
\hline \begin{tabular}{l|l|} 
NTNG2|84628 \\
\end{tabular} & $\mathrm{A} 2$ \\
\hline NTRK2|4915 & $\mathrm{A} 2$ \\
\hline NUAK2|81788 & $\mathrm{A} 2$ \\
\hline NUBP1|4682 & A2 \\
\hline NUBP2|10101 & $\mathrm{A} 2$ \\
\hline
\end{tabular}




\begin{tabular}{|c|c|}
\hline NUCB2|4925 & $\mathrm{A} 2$ \\
\hline NUDT11|55190 & $\mathrm{A} 2$ \\
\hline NUDT16L1|84309 & $\mathrm{A} 2$ \\
\hline NUDT16|131870 & $\mathrm{A} 2$ \\
\hline NUDT19|390916 & A2 \\
\hline NUDT1|4521 & A2 \\
\hline NUDT22|84304 & $\mathrm{A} 2$ \\
\hline NUDT3|11165 & A2 \\
\hline NUDT5|11164 & A2 \\
\hline NUDT9P1|119369 & A2 \\
\hline NUF2|83540 & $\mathrm{A} 2$ \\
\hline NUP107|57122 & $\mathrm{A} 2$ \\
\hline NUP133|55746 & A2 \\
\hline NUP205|23165 & $\mathrm{A} 2$ \\
\hline NUP210|23225 & $\mathrm{A} 2$ \\
\hline NUP37|79023 & $\mathrm{A} 2$ \\
\hline NUP62|23636 & A2 \\
\hline NUP85|79902 & A2 \\
\hline NUPL1|9818 & A2 \\
\hline NUSAP1|51203 & $\mathrm{A} 2$ \\
\hline NUTF2|10204 & A2 \\
\hline NVL|4931 & $\mathrm{A} 2$ \\
\hline NXNL1|115861 & $\mathrm{A} 2$ \\
\hline NYNRIN|57523 & $\mathrm{A} 2$ \\
\hline OAS1|4938 & $\mathrm{A} 2$ \\
\hline OAS2|4939 & $\mathrm{A} 2$ \\
\hline OAS3|4940 & A2 \\
\hline OAZ3|51686 & A2 \\
\hline OBFC1|79991 & $\mathrm{A} 2$ \\
\hline OBFC2B|79035 & $\mathrm{A} 2$ \\
\hline OCIAD2|132299 & $\mathrm{A} 2$ \\
\hline ODF2|4957 & A2 \\
\hline ODF3L1|161753 & $\mathrm{A} 2$ \\
\hline ODZ1|10178 & $\overline{\mathrm{A} 2}$ \\
\hline ODZ2|57451 & $\mathrm{A} 2$ \\
\hline OGN|4969 & $\overline{\mathrm{A} 2}$ \\
\hline OGT|8473 & $\mathrm{A} 2$ \\
\hline OIP5|11339 & $\mathrm{A} 2$ \\
\hline OLA1|29789 & A2 \\
\hline OLFML2A|169611 & $\mathrm{A} 2$ \\
\hline OLFML2B|25903 & A2 \\
\hline OLR1|4973 & A2 \\
\hline OPTN|10133 & A2 \\
\hline OR10A3|26496 & $\mathrm{A} 2$ \\
\hline OR10A6|390093 & A2 \\
\hline OR10V1|390201 & A2 \\
\hline $\begin{array}{l}\text { OR2B6|26212 } \\
\end{array}$ & $\mathrm{A} 2$ \\
\hline \begin{tabular}{l|l|} 
OR2L13|284521 &
\end{tabular} & $\mathrm{A} 2$ \\
\hline OR2L3|391192 & A2 \\
\hline OR52N4|390072 & A2 \\
\hline OR5K1|26339 & $\mathrm{A} 2$ \\
\hline
\end{tabular}




\begin{tabular}{|c|c|}
\hline OR5K2|402135 & A2 \\
\hline $\begin{array}{l}\text { ORAI2|80228 } \\
\end{array}$ & $\mathrm{A} 2$ \\
\hline ORC1L|4998 & $\mathrm{A} 2$ \\
\hline ORC6L|23594 & A2 \\
\hline ORMDL2|29095 & $\mathrm{A} 2$ \\
\hline OSBPL1A|114876 & $\mathrm{A} 2$ \\
\hline OSBPL9|114883 & $\mathrm{A} 2$ \\
\hline OST4|100128731 & $\mathrm{A} 2$ \\
\hline OSTC 58505 & $\mathrm{~A} 2$ \\
\hline OTUB1|55611 & $\mathrm{A} 2$ \\
\hline OTUB2|78990 & $\mathrm{A} 2$ \\
\hline OTUD7A|161725 & A2 \\
\hline OTUD7B|56957 & $\mathrm{A} 2$ \\
\hline OVCA2|124641 & $\mathrm{A} 2$ \\
\hline OVCH2|341277 & $\mathrm{A} 2$ \\
\hline OVOL1|5017 & $\mathrm{A} 2$ \\
\hline OXCT1|5019 & $\mathrm{A} 2$ \\
\hline OXGR1|27199 & $\mathrm{A} 2$ \\
\hline OXSM|54995 & $\mathrm{A} 2$ \\
\hline P2RX4|5025 & $\mathrm{A} 2$ \\
\hline P2RY12|64805 & $\mathrm{A} 2$ \\
\hline P2RY13|53829 & $\mathrm{A} 2$ \\
\hline P2RY14|9934 & A2 \\
\hline P4HA2|8974 & $\mathrm{A} 2$ \\
\hline P4HA3|283208 & $\mathrm{A} 2$ \\
\hline P4HB|5034 & $\mathrm{A} 2$ \\
\hline PA2G4P4|647033 & $\mathrm{A} 2$ \\
\hline PABPC4L|132430 & $\mathrm{A} 2$ \\
\hline PACRGL|133015 & $\mathrm{A} 2$ \\
\hline PACRG|135138 & $\mathrm{A} 2$ \\
\hline PACSIN1|29993 & $\mathrm{A} 2$ \\
\hline PAFAH1B1|5048 & $\mathrm{A} 2$ \\
\hline PAFAH2|5051 & $\mathrm{A} 2$ \\
\hline PAICS|10606 & $\mathrm{A} 2$ \\
\hline PAIP1|10605 & $\mathrm{A} 2$ \\
\hline PAK2|5062 & $\mathrm{A} 2$ \\
\hline PAK4|10298 & $\mathrm{A} 2$ \\
\hline PALMD|54873 & $\mathrm{A} 2$ \\
\hline $\begin{array}{l}\text { PAN2|9924 } \\
\end{array}$ & $\mathrm{A} 2$ \\
\hline PANK3|79646 & $\mathrm{A} 2$ \\
\hline PANX1|24145 & $\mathrm{A} 2$ \\
\hline PAPLN|89932 & $\mathrm{A} 2$ \\
\hline PAPPA2|60676 & $\mathrm{A} 2$ \\
\hline PAR1|145624 & $\mathrm{A} 2$ \\
\hline PAR5|8123 & $\mathrm{A} 2$ \\
\hline PARD3B|117583 & $\mathrm{A} 2$ \\
\hline PARN|5073 & $\mathrm{A} 2$ \\
\hline PARP12|64761 & $\mathrm{A} 2$ \\
\hline PARP14|54625 & $\mathrm{A} 2$ \\
\hline PARP9|83666 & $\mathrm{A} 2$ \\
\hline PATZ1|23598 & $\mathrm{A} 2$ \\
\hline
\end{tabular}




\begin{tabular}{|c|c|}
\hline PAX2|5076 & A2 \\
\hline \begin{tabular}{l|l|l|} 
PAX9 & \\
\end{tabular} & A2 \\
\hline PAXIP1|22976 & $\mathrm{A} 2$ \\
\hline PBK|55872 & $\mathrm{A} 2$ \\
\hline PBX1|5087 & A2 \\
\hline PCBP3|54039 & $\mathrm{A} 2$ \\
\hline PCCA|5095 & A2 \\
\hline PCDH17|27253 & $\mathrm{A} 2$ \\
\hline PCDH18|54510 & $\mathrm{A} 2$ \\
\hline PCDH19|57526 & $\mathrm{A} 2$ \\
\hline PCDHB12|56124 & $\mathrm{A} 2$ \\
\hline PCDHB15|56121 & $\mathrm{A} 2$ \\
\hline PCDHB18|54660 & $\mathrm{A} 2$ \\
\hline PCDHB19P|84054 & $\mathrm{A} 2$ \\
\hline PCDHB7|56129 & $\mathrm{A} 2$ \\
\hline PCDHGA11|56105 & $\mathrm{A} 2$ \\
\hline PCDHGA2|56113 & $\mathrm{A} 2$ \\
\hline PCDHGA6|56109 & $\mathrm{A} 2$ \\
\hline PCDHGA9|56107 & $\mathrm{A} 2$ \\
\hline PCDHGB6|56100 & $\mathrm{A} 2$ \\
\hline $\begin{array}{l}\text { PCGF5|84333 } \\
\end{array}$ & $\mathrm{A} 2$ \\
\hline PCK1|5105 & $\mathrm{A} 2$ \\
\hline PCNA|5111 & $\mathrm{A} 2$ \\
\hline PCNXL3|399909 & $\mathrm{A} 2$ \\
\hline PCYOX1L|78991 & $\mathrm{A} 2$ \\
\hline PCYT1B|9468 & $\mathrm{A} 2$ \\
\hline PC|5091 & $\mathrm{A} 2$ \\
\hline PDCD10|11235 & $\mathrm{A} 2$ \\
\hline PDCD6|10016 & $\mathrm{A} 2$ \\
\hline $\begin{array}{l}\text { PDCL3|79031 } \\
\end{array}$ & $\mathrm{A} 2$ \\
\hline PDE11A|50940 & $\mathrm{A} 2$ \\
\hline PDE1B|5153 & $\mathrm{A} 2$ \\
\hline PDE2A|5138 & $\mathrm{A} 2$ \\
\hline PDE3B|5140 & $\mathrm{A} 2$ \\
\hline PDE5A|8654 & $\mathrm{A} 2$ \\
\hline PDE6D|5147 & $\mathrm{A} 2$ \\
\hline PDE8A|5151 & $\mathrm{A} 2$ \\
\hline PDF|64146 & $\mathrm{A} 2$ \\
\hline PDGFA|5154 & $\mathrm{A} 2$ \\
\hline PDGFRA|5156 & $\mathrm{A} 2$ \\
\hline PDIA3P|171423 & $\mathrm{A} 2$ \\
\hline PDIA3|2923 & $\mathrm{A} 2$ \\
\hline PDIA4|9601 & $\mathrm{A} 2$ \\
\hline PDIA5|10954 & $\mathrm{A} 2$ \\
\hline PDIA6|10130 & $\mathrm{A} 2$ \\
\hline \begin{tabular}{l|l|l|} 
PDK2|5164 & \\
\end{tabular} & $\mathrm{A} 2$ \\
\hline PDK3|5165 & $\mathrm{A} 2$ \\
\hline PDK4|5166 & $\mathrm{A} 2$ \\
\hline PDP2|57546 & $\mathrm{A} 2$ \\
\hline PDPR|55066 & $\mathrm{A} 2$ \\
\hline PDSS1|23590 & $\mathrm{A} 2$ \\
\hline
\end{tabular}




\begin{tabular}{|c|c|}
\hline PDXDC1|23042 & $\mathrm{A} 2$ \\
\hline PDXP|57026 & $\mathrm{A} 2$ \\
\hline PDZD11|51248 & $\mathrm{A} 2$ \\
\hline PDZD4|57595 & $\mathrm{A} 2$ \\
\hline PEAR1|375033 & $\mathrm{A} 2$ \\
\hline PELI1|57162 & A2 \\
\hline PEMT $\mid 10400$ & $\mathrm{~A} 2$ \\
\hline PER2|8864 & $\mathrm{A} 2$ \\
\hline PER3|8863 & $\mathrm{A} 2$ \\
\hline PEX11B|8799 & $\mathrm{A} 2$ \\
\hline PEX13|5194 & $\mathrm{A} 2$ \\
\hline PEX5|5830 & $\mathrm{A} 2$ \\
\hline PFDN2|5202 & $\mathrm{A} 2$ \\
\hline PFDN5|5204 & $\mathrm{A} 2$ \\
\hline PFKFB1|5207 & $\mathrm{A} 2$ \\
\hline PFKFB3|5209 & $\mathrm{A} 2$ \\
\hline PFKFB4|5210 & $\mathrm{A} 2$ \\
\hline PFN1|5216 & $\mathrm{A} 2$ \\
\hline \begin{tabular}{l|l|} 
PFN2|5217 &
\end{tabular} & $\mathrm{A} 2$ \\
\hline PGA3|643834 & $\mathrm{A} 2$ \\
\hline PGAM1|5223 & $\mathrm{A} 2$ \\
\hline PGAM5|192111 & $\mathrm{A} 2$ \\
\hline PGAP1|80055 & $\mathrm{A} 2$ \\
\hline PGBD4|161779 & $\mathrm{A} 2$ \\
\hline PGGT1B|5229 & $\mathrm{A} 2$ \\
\hline PGK1|5230 & $\mathrm{A} 2$ \\
\hline PGM1|5236 & $\mathrm{A} 2$ \\
\hline PGM2|55276 & $\mathrm{A} 2$ \\
\hline \begin{tabular}{l|l|} 
PGM3|5238 \\
\end{tabular} & $\mathrm{A} 2$ \\
\hline PGM5P2|595135 & $\mathrm{A} 2$ \\
\hline $\begin{array}{l}\text { PGM5|5239 } \\
\end{array}$ & $\mathrm{A} 2$ \\
\hline PGPEP1|54858 & $\mathrm{A} 2$ \\
\hline PGP|283871 & $\mathrm{A} 2$ \\
\hline PHACTR2|9749 & $\mathrm{A} 2$ \\
\hline PHF14|9678 & $\mathrm{A} 2$ \\
\hline PHF16|9767 & $\mathrm{A} 2$ \\
\hline PHF1|5252 & $\mathrm{A} 2$ \\
\hline PHF21A|51317 & $\mathrm{A} 2$ \\
\hline PHF2|5253 & $\mathrm{A} 2$ \\
\hline PHF7|51533 & $\mathrm{A} 2$ \\
\hline PHKA2|5256 & $\mathrm{A} 2$ \\
\hline PHKG2|5261 & $\mathrm{A} 2$ \\
\hline PHLDA1|22822 & $\mathrm{A} 2$ \\
\hline PHLDA3|23612 & $\mathrm{A} 2$ \\
\hline PHLDB2|90102 & $\mathrm{A} 2$ \\
\hline PHLPP1|23239 & A2 \\
\hline PHTF1|10745 & $\mathrm{A} 2$ \\
\hline PHYHD1|254295 & $\mathrm{A} 2$ \\
\hline PHYHIPL|84457 & $\mathrm{A} 2$ \\
\hline PHYHIP|9796 & $\mathrm{A} 2$ \\
\hline PI15|51050 & $\mathrm{A} 2$ \\
\hline
\end{tabular}




\begin{tabular}{|c|c|}
\hline PI4KAP1|728233 & $\mathrm{A} 2$ \\
\hline PI4KAP2|375133 & $\mathrm{A} 2$ \\
\hline PICALM|8301 & $\mathrm{A} 2$ \\
\hline PIF1|80119 & $\mathrm{A} 2$ \\
\hline PIGC|5279 & A2 \\
\hline PIGL|9487 & $\mathrm{A} 2$ \\
\hline PIGM|93183 & $\mathrm{A} 2$ \\
\hline PIGO|84720 & $\mathrm{A} 2$ \\
\hline PIGQ|9091 & $\mathrm{A} 2$ \\
\hline PIGW|284098 & $\mathrm{A} 2$ \\
\hline PIGX|54965 & $\mathrm{A} 2$ \\
\hline PIK3C2B|5287 & $\mathrm{A} 2$ \\
\hline PIK3C2G|5288 & $\mathrm{A} 2$ \\
\hline PIK3CA|5290 & $\mathrm{A} 2$ \\
\hline PIK3IP1|113791 & $\mathrm{A} 2$ \\
\hline PIK3R2|5296 & $\mathrm{A} 2$ \\
\hline PIK3R3|8503 & $\mathrm{A} 2$ \\
\hline PIKFYVE|200576 & $\mathrm{A} 2$ \\
\hline PINK1|65018 & $\mathrm{A} 2$ \\
\hline PINX1|54984 & $\mathrm{A} 2$ \\
\hline PIP4K2C|79837 & $\mathrm{A} 2$ \\
\hline PIP5K1A|8394 & $\mathrm{A} 2$ \\
\hline PIPSL|266971 & $\mathrm{A} 2$ \\
\hline PISD|23761 & $\mathrm{A} 2$ \\
\hline PITPNM2|57605 & $\mathrm{A} 2$ \\
\hline PITX1|5307 & $\mathrm{A} 2$ \\
\hline PIWIL4|143689 & $\mathrm{A} 2$ \\
\hline PJA2|9867 & $\mathrm{A} 2$ \\
\hline PKD1L2|114780 & $\mathrm{A} 2$ \\
\hline PKD1L3|342372 & $\mathrm{A} 2$ \\
\hline PKIB|5570 & $\mathrm{A} 2$ \\
\hline PKN2|5586 & $\mathrm{A} 2$ \\
\hline PKN3|29941 & $\mathrm{A} 2$ \\
\hline PL-5283|647087 & $\mathrm{A} 2$ \\
\hline PLA2G16|11145 & $\mathrm{A} 2$ \\
\hline PLA2G4F|255189 & $\mathrm{A} 2$ \\
\hline PLA2G6|8398 & $\mathrm{A} 2$ \\
\hline PLA2R1|22925 & $\mathrm{A} 2$ \\
\hline PLAA|9373 & $\mathrm{A} 2$ \\
\hline PLAC1|10761 & $\mathrm{A} 2$ \\
\hline PLAC2|257000 & $\mathrm{A} 2$ \\
\hline PLAUR|5329 & $\mathrm{A} 2$ \\
\hline PLAU|5328 & $\mathrm{A} 2$ \\
\hline PLB1|151056 & $\mathrm{A} 2$ \\
\hline PLCB3|5331 & $\mathrm{A} 2$ \\
\hline PLCD1|5333 & $\mathrm{A} 2$ \\
\hline PLCD3|113026 & $\mathrm{A} 2$ \\
\hline PLCH2|9651 & $\mathrm{A} 2$ \\
\hline PLCL2|23228 & $\mathrm{A} 2$ \\
\hline $\begin{array}{l}\text { PLCXD3|345557 } \\
\end{array}$ & $\mathrm{A} 2$ \\
\hline PLCZ1|89869 & $\mathrm{A} 2$ \\
\hline
\end{tabular}




\begin{tabular}{|c|c|}
\hline PLEK2|26499 & $\mathrm{A} 2$ \\
\hline PLEKHA4|57664 & $\mathrm{A} 2$ \\
\hline PLEKHA7|144100 & $\mathrm{A} 2$ \\
\hline PLEKHA8 $\mid 84725$ & $\mathrm{~A} 2$ \\
\hline PLEKHF2|79666 & $\mathrm{A} 2$ \\
\hline PLEKHG1|57480 & $\mathrm{A} 2$ \\
\hline PLEKHG5|57449 & $\mathrm{A} 2$ \\
\hline PLEKHM1P|440456 & $\mathrm{A} 2$ \\
\hline PLEKHM1|9842 & $\mathrm{A} 2$ \\
\hline PLEKHN1|84069 & $\mathrm{A} 2$ \\
\hline PLIN2|123 & $\mathrm{A} 2$ \\
\hline PLIN3|10226 & $\mathrm{A} 2$ \\
\hline PLK1|5347 & $\mathrm{A} 2$ \\
\hline PLK3|1263 & $\mathrm{A} 2$ \\
\hline PLK4|10733 & $\mathrm{A} 2$ \\
\hline PLLP|51090 & $\mathrm{A} 2$ \\
\hline PLS3|5358 & $\mathrm{A} 2$ \\
\hline PLTP|5360 & $\mathrm{A} 2$ \\
\hline $\begin{array}{l}\text { PLXNA3|55558 } \\
\end{array}$ & $\mathrm{A} 2$ \\
\hline PLXNC1|10154 & $\mathrm{A} 2$ \\
\hline PM20D2|135293 & $\mathrm{A} 2$ \\
\hline PMAIP1|5366 & $\mathrm{A} 2$ \\
\hline PMCH|5367 & $\mathrm{A} 2$ \\
\hline PMS2L1|5379 & $\mathrm{A} 2$ \\
\hline PMS2L4|5382 & $\mathrm{A} 2$ \\
\hline PNKD|25953 & $\mathrm{A} 2$ \\
\hline PNKP|11284 & $\mathrm{A} 2$ \\
\hline PNPLA2|57104 & $\mathrm{A} 2$ \\
\hline PNPLA7|375775 & $\mathrm{A} 2$ \\
\hline PNP|4860 & $\mathrm{A} 2$ \\
\hline POC1A|25886 & $\mathrm{A} 2$ \\
\hline PODXL2|50512 & $\mathrm{A} 2$ \\
\hline POGK|57645 & $\mathrm{A} 2$ \\
\hline POLA2|23649 & $\mathrm{A} 2$ \\
\hline POLD1|5424 & $\mathrm{A} 2$ \\
\hline POLD2|5425 & $\mathrm{A} 2$ \\
\hline POLD4|57804 & $\mathrm{A} 2$ \\
\hline POLE2|5427 & $\mathrm{A} 2$ \\
\hline POLE3|54107 & $\mathrm{A} 2$ \\
\hline POLE|5426 & $\mathrm{A} 2$ \\
\hline POLI|11201 & $\mathrm{A} 2$ \\
\hline POLL|27343 & $\mathrm{A} 2$ \\
\hline POLQ|10721 & $\mathrm{A} 2$ \\
\hline POLR1E|64425 & $\mathrm{A} 2$ \\
\hline POLR2D|5433 & $\mathrm{A} 2$ \\
\hline POLR2E|5434 & A2 \\
\hline POLR2G|5436 & $\mathrm{A} 2$ \\
\hline POLR2H|5437 & $\mathrm{A} 2$ \\
\hline POLR2J4|84820 & $\mathrm{A} 2$ \\
\hline POLR2J|5439 & $\mathrm{A} 2$ \\
\hline POLR2K|5440 & $\mathrm{A} 2$ \\
\hline
\end{tabular}




\begin{tabular}{|c|c|}
\hline POLR3K|51728 & $\mathrm{A} 2$ \\
\hline POM121L9P|29774 & $\mathrm{A} 2$ \\
\hline POMP|51371 & $\mathrm{A} 2$ \\
\hline POP1|10940 & $\mathrm{A} 2$ \\
\hline POP4|10775 & $\mathrm{A} 2$ \\
\hline POP7|10248 & A2 \\
\hline POSTN|10631 & $\mathrm{A} 2$ \\
\hline POU2F1|5451 & $\mathrm{A} 2$ \\
\hline PP14571|100130449 & $\mathrm{A} 2$ \\
\hline PPA2|27068 & $\mathrm{A} 2$ \\
\hline PPAP2A|8611 & $\mathrm{A} 2$ \\
\hline PPAPDC1A|196051 & $\mathrm{A} 2$ \\
\hline PPARA|5465 & $\mathrm{A} 2$ \\
\hline PPARGC1B|133522 & $\mathrm{A} 2$ \\
\hline PPCS|79717 & $\mathrm{A} 2$ \\
\hline PPDPF|79144 & $\mathrm{A} 2$ \\
\hline PPFIA3|8541 & $\mathrm{A} 2$ \\
\hline PPIAL4G|644591 & $\mathrm{A} 2$ \\
\hline PPIA|5478 & $\mathrm{A} 2$ \\
\hline PPIB|5479 & $\mathrm{A} 2$ \\
\hline PPIH|10465 & $\mathrm{A} 2$ \\
\hline PPIL1|51645 & $\mathrm{A} 2$ \\
\hline PPIL2|23759 & $\mathrm{A} 2$ \\
\hline PPIL5|122769 & $\mathrm{A} 2$ \\
\hline PPM1G|5496 & $\mathrm{A} 2$ \\
\hline PPM1J|333926 & $\mathrm{A} 2$ \\
\hline PPOX|5498 & $\mathrm{A} 2$ \\
\hline PPP1CA|5499 & $\mathrm{A} 2$ \\
\hline PPP1R10|5514 & $\mathrm{A} 2$ \\
\hline PPP1R11|6992 & $\mathrm{A} 2$ \\
\hline PPP1R12A|4659 & $\mathrm{A} 2$ \\
\hline \begin{tabular}{ll|l|} 
PPP1R12B 4660 \\
\end{tabular} & $\mathrm{~A} 2$ \\
\hline PPP1R14A|94274 & $\mathrm{A} 2$ \\
\hline PPP1R14B|26472 & $\mathrm{A} 2$ \\
\hline PPP1R15A|23645 & $\mathrm{A} 2$ \\
\hline PPP1R16B|26051 & $\mathrm{A} 2$ \\
\hline PPP1R3E|90673 & $\mathrm{A} 2$ \\
\hline PPP1R9B|84687 & $\mathrm{A} 2$ \\
\hline PPP2CB|5516 & $\mathrm{A} 2$ \\
\hline PPP2R1A|5518 & $\mathrm{A} 2$ \\
\hline PPP2R1B|5519 & $\mathrm{A} 2$ \\
\hline PPP2R2B|5521 & $\mathrm{A} 2$ \\
\hline PPP2R2C|5522 & $\mathrm{A} 2$ \\
\hline PPP2R4|5524 & $\mathrm{A} 2$ \\
\hline PPP2R5E|5529 & $\mathrm{A} 2$ \\
\hline PPP3CC $\mid 5533$ & $\mathrm{~A} 2$ \\
\hline PPP5C|5536 & $\mathrm{A} 2$ \\
\hline PPPDE1|51029 & $\mathrm{A} 2$ \\
\hline PPT1|5538 & $\mathrm{A} 2$ \\
\hline PPWD1|23398 & $\mathrm{A} 2$ \\
\hline PQLC1|80148 & $\mathrm{A} 2$ \\
\hline
\end{tabular}




\begin{tabular}{|c|c|}
\hline PQLC2|54896 & $\mathrm{A} 2$ \\
\hline PRAF2|11230 & $\mathrm{A} 2$ \\
\hline PRC1|9055 & $\mathrm{A} 2$ \\
\hline PRCC $\mid 5546$ & $\mathrm{~A} 2$ \\
\hline PRDM11|56981 & $\mathrm{A} 2$ \\
\hline PRDM16|63976 & A2 \\
\hline PRDM2|7799 & $\mathrm{A} 2$ \\
\hline PRDM5|11107 & $\mathrm{A} 2$ \\
\hline PRDM8|56978 & $\mathrm{A} 2$ \\
\hline PRDX2|7001 & $\mathrm{A} 2$ \\
\hline PRDX4|10549 & $\mathrm{A} 2$ \\
\hline PRELID1|27166 & $\mathrm{A} 2$ \\
\hline PREX2|80243 & $\mathrm{A} 2$ \\
\hline PRICKLE2|166336 & $\mathrm{A} 2$ \\
\hline PRIM2|5558 & $\mathrm{A} 2$ \\
\hline PRKAG2|51422 & $\mathrm{A} 2$ \\
\hline PRKAR1B|5575 & $\mathrm{A} 2$ \\
\hline PRKAR2B|5577 & $\mathrm{A} 2$ \\
\hline PRKCA|5578 & $\mathrm{A} 2$ \\
\hline PRKCH|5583 & $\mathrm{A} 2$ \\
\hline PRKD1|5587 & $\mathrm{A} 2$ \\
\hline PRKD3|23683 & $\mathrm{A} 2$ \\
\hline PRKDC|5591 & $\mathrm{A} 2$ \\
\hline PRMT10|90826 & $\mathrm{A} 2$ \\
\hline PROKR1|10887 & $\mathrm{A} 2$ \\
\hline PROM2|150696 & $\mathrm{A} 2$ \\
\hline PROX1|5629 & $\mathrm{A} 2$ \\
\hline PRPF19|27339 & $\mathrm{A} 2$ \\
\hline PRPS1|5631 & $\mathrm{A} 2$ \\
\hline $\begin{array}{l}\text { PRR13|54458 } \\
\end{array}$ & $\mathrm{A} 2$ \\
\hline PRR14|78994 & $\mathrm{A} 2$ \\
\hline \begin{tabular}{l|l|l|} 
PRR15 22171 \\
\end{tabular} & $\mathrm{~A} 2$ \\
\hline PRR19|284338 & $\mathrm{A} 2$ \\
\hline PRR7|80758 & $\mathrm{A} 2$ \\
\hline PRRC1|133619 & $\mathrm{A} 2$ \\
\hline PRRG1|5638 & $\mathrm{A} 2$ \\
\hline PRRG3|79057 & $\mathrm{A} 2$ \\
\hline PRRT3|285368 & $\mathrm{A} 2$ \\
\hline PRSS50|29122 & $\mathrm{A} 2$ \\
\hline \begin{tabular}{l|l|} 
PRSS55|203074 \\
\end{tabular} & $\mathrm{A} 2$ \\
\hline PRSS8|5652 & $\mathrm{A} 2$ \\
\hline PRUNE|58497 & $\mathrm{A} 2$ \\
\hline PSEN1|5663 & $\mathrm{A} 2$ \\
\hline PSEN2|5664 & $\mathrm{A} 2$ \\
\hline PSMA2|5683 & $\mathrm{A} 2$ \\
\hline PSMA3|5684 & $\mathrm{A} 2$ \\
\hline PSMA4|5685 & $\mathrm{A} 2$ \\
\hline PSMA6|5687 & $\mathrm{A} 2$ \\
\hline PSMA7|5688 & $\mathrm{A} 2$ \\
\hline PSMB2|5690 & $\mathrm{A} 2$ \\
\hline PSMB3|5691 & $\mathrm{A} 2$ \\
\hline
\end{tabular}




\begin{tabular}{|c|c|}
\hline PSMB4|5692 & A2 \\
\hline PSMB5|5693 & $\mathrm{A} 2$ \\
\hline PSMC2|5701 & $\mathrm{A} 2$ \\
\hline PSMC3IP|29893 & $\mathrm{A} 2$ \\
\hline PSMC6|5706 & A2 \\
\hline PSMD10|5716 & A2 \\
\hline PSMD11|5717 & $\mathrm{A} 2$ \\
\hline PSMD12|5718 & $\mathrm{A} 2$ \\
\hline PSMD1|5707 & $\mathrm{A} 2$ \\
\hline PSMD2|5708 & $\mathrm{A} 2$ \\
\hline PSMD4|5710 & $\mathrm{A} 2$ \\
\hline PSMD5|5711 & A2 \\
\hline PSME2|5721 & $\mathrm{A} 2$ \\
\hline PSME3|10197 & $\mathrm{A} 2$ \\
\hline PSME4|23198 & $\mathrm{A} 2$ \\
\hline PSMG3|84262 & $\mathrm{A} 2$ \\
\hline PSPC1|55269 & $\mathrm{A} 2$ \\
\hline PSRC1|84722 & $\mathrm{A} 2$ \\
\hline PTAR1|375743 & A2 \\
\hline PTCD1|26024 & A2 \\
\hline PTCH2|8643 & $\mathrm{A} 2$ \\
\hline PTCHD1|139411 & A2 \\
\hline PTEN|5728 & $\mathrm{A} 2$ \\
\hline PTGDS|5730 & $\mathrm{A} 2$ \\
\hline PTGER3|5733 & $\mathrm{A} 2$ \\
\hline PTGER4|5734 & $\mathrm{A} 2$ \\
\hline PTK2|5747 & A2 \\
\hline PTK7|5754 & A2 \\
\hline PTMA|5757 & $\mathrm{A} 2$ \\
\hline PTMS|5763 & $\mathrm{A} 2$ \\
\hline PTN|5764 & $\mathrm{A} 2$ \\
\hline PTPLAD1|51495 & $\mathrm{A} 2$ \\
\hline PTPLA|9200 & $\mathrm{A} 2$ \\
\hline $\begin{array}{l}\text { PTPN11|5781 } \\
\end{array}$ & $\mathrm{A} 2$ \\
\hline PTPN14|5784 & $\mathrm{A} 2$ \\
\hline PTPN4|5775 & $\mathrm{A} 2$ \\
\hline PTPN6|5777 & $\mathrm{A} 2$ \\
\hline PTPRG|5793 & A2 \\
\hline PTPRS|5802 & $\mathrm{A} 2$ \\
\hline PTRF|284119 & $\mathrm{A} 2$ \\
\hline PTRH1|138428 & A2 \\
\hline PTS|5805 & A2 \\
\hline PTTG1IP|754 & A2 \\
\hline PTTG1|9232 & $\mathrm{A} 2$ \\
\hline PTTG3P|26255 & $\mathrm{A} 2$ \\
\hline PUF60|22827 & $\mathrm{A} 2$ \\
\hline PUS7L|83448 & $\mathrm{A} 2$ \\
\hline PUS7|54517 & $\mathrm{A} 2$ \\
\hline PUSL1|126789 & A2 \\
\hline PVRL2|5819 & A2 \\
\hline PVRL4|81607 & $\mathrm{A} 2$ \\
\hline
\end{tabular}




\begin{tabular}{|c|c|}
\hline PXN|5829 & A2 \\
\hline PYCARD|29108 & $\mathrm{A} 2$ \\
\hline PYCR2|29920 & $\mathrm{A} 2$ \\
\hline PYCRL|65263 & $\mathrm{A} 2$ \\
\hline PYGO2|90780 & A2 \\
\hline ProSAPiP1|9762 & $\mathrm{A} 2$ \\
\hline QKI|9444 & $\mathrm{A} 2$ \\
\hline QTRTD1|79691 & $\mathrm{A} 2$ \\
\hline R3HDM1|23518 & $\mathrm{A} 2$ \\
\hline RAB10|10890 & $\mathrm{A} 2$ \\
\hline RAB11FIP3|9727 & $\mathrm{A} 2$ \\
\hline RAB11FIP4|84440 & $\mathrm{A} 2$ \\
\hline RAB13|5872 & $\mathrm{A} 2$ \\
\hline RAB17|64284 & $\mathrm{A} 2$ \\
\hline RAB1A|5861 & $\mathrm{A} 2$ \\
\hline RAB25|57111 & $\mathrm{A} 2$ \\
\hline RAB26|25837 & $\mathrm{A} 2$ \\
\hline RAB28|9364 & $\mathrm{A} 2$ \\
\hline RAB2A|5862 & $\mathrm{A} 2$ \\
\hline RAB31|11031 & $\mathrm{A} 2$ \\
\hline RAB33A|9363 & $\mathrm{A} 2$ \\
\hline RAB3D|9545 & $\mathrm{A} 2$ \\
\hline RAB3IP|117177 & $\mathrm{A} 2$ \\
\hline RAB40A|142684 & $\mathrm{A} 2$ \\
\hline RAB40B|10966 & $\mathrm{A} 2$ \\
\hline RAB40C|57799 & $\mathrm{A} 2$ \\
\hline RAB4B|53916 & $\mathrm{A} 2$ \\
\hline RAB6A|5870 & $\mathrm{A} 2$ \\
\hline \begin{tabular}{l|l|} 
RAB9B|51209 \\
\end{tabular} & $\mathrm{A} 2$ \\
\hline RABEPK|10244 & $\mathrm{A} 2$ \\
\hline RABL5|64792 & $\mathrm{A} 2$ \\
\hline RAC3|5881 & $\mathrm{A} 2$ \\
\hline RACGAP1P|83956 & $\mathrm{A} 2$ \\
\hline RACGAP1|29127 & $\mathrm{A} 2$ \\
\hline RAD21|5885 & $\mathrm{A} 2$ \\
\hline RAD51AP1|10635 & $\mathrm{A} 2$ \\
\hline RAD51|5888 & $\mathrm{A} 2$ \\
\hline RAD54B|25788 & $\mathrm{A} 2$ \\
\hline RAD54L2|23132 & $\mathrm{A} 2$ \\
\hline RAD54L|8438 & $\mathrm{A} 2$ \\
\hline RADIL|55698 & $\mathrm{A} 2$ \\
\hline RAE1|8480 & $\mathrm{A} 2$ \\
\hline RAET1K|646024 & $\mathrm{A} 2$ \\
\hline RAG1AP1|55974 & $\mathrm{A} 2$ \\
\hline RAI14|26064 & $\mathrm{A} 2$ \\
\hline RAI2|10742 & $\mathrm{A} 2$ \\
\hline RALA|5898 & $\mathrm{A} 2$ \\
\hline RALGDS|5900 & $\mathrm{A} 2$ \\
\hline RALGPS2|55103 & $\mathrm{A} 2$ \\
\hline RALY|22913 & $\mathrm{A} 2$ \\
\hline RANBP10|57610 & $\mathrm{A} 2$ \\
\hline
\end{tabular}




\begin{tabular}{|c|c|}
\hline RANBP17|64901 & $\mathrm{A} 2$ \\
\hline RANBP1|5902 & $\mathrm{A} 2$ \\
\hline RANBP9|10048 & $\mathrm{A} 2$ \\
\hline RANGAP1|5905 & $\mathrm{A} 2$ \\
\hline RAN|5901 & $\mathrm{A} 2$ \\
\hline RAP2C|57826 & $\mathrm{A} 2$ \\
\hline RAPGEF1|2889 & $\mathrm{A} 2$ \\
\hline RARB|5915 & $\mathrm{A} 2$ \\
\hline RARRES2|5919 & A2 \\
\hline RARRES3|5920 & $\mathrm{A} 2$ \\
\hline RARS|5917 & $\mathrm{A} 2$ \\
\hline RASA2|5922 & $\mathrm{A} 2$ \\
\hline RASA4|10156 & $\mathrm{A} 2$ \\
\hline RASGEF1B|153020 & $\mathrm{A} 2$ \\
\hline RASGRP2|10235 & $\mathrm{A} 2$ \\
\hline RASIP1|54922 & $\mathrm{A} 2$ \\
\hline RASL10A|10633 & $\mathrm{A} 2$ \\
\hline RASL11B|65997 & $\mathrm{A} 2$ \\
\hline RASSF1|11186 & $\mathrm{A} 2$ \\
\hline RASSF3|283349 & $\mathrm{A} 2$ \\
\hline RASSF6|166824 & $\mathrm{A} 2$ \\
\hline RAVER1|125950 & $\mathrm{A} 2$ \\
\hline RAVER2|55225 & $\mathrm{A} 2$ \\
\hline RAX2|84839 & $\mathrm{A} 2$ \\
\hline RBBP4|5928 & $\mathrm{A} 2$ \\
\hline RBKS|64080 & $\mathrm{A} 2$ \\
\hline RBL1|5933 & $\mathrm{A} 2$ \\
\hline RBM33|155435 & $\mathrm{A} 2$ \\
\hline RBM34|23029 & $\mathrm{A} 2$ \\
\hline RBM47|54502 & $\mathrm{A} 2$ \\
\hline RBM4|5936 & $\mathrm{A} 2$ \\
\hline RBM6|10180 & $\mathrm{A} 2$ \\
\hline RBM7|10179 & $\mathrm{A} 2$ \\
\hline RBM8A|9939 & $\mathrm{A} 2$ \\
\hline RBMS3|27303 & $\mathrm{A} 2$ \\
\hline RBMXL1|494115 & $\mathrm{A} 2$ \\
\hline RBP7|116362 & $\mathrm{A} 2$ \\
\hline RBPMS|11030 & $\mathrm{A} 2$ \\
\hline RBX1|9978 & $\mathrm{A} 2$ \\
\hline RCAN1|1827 & $\mathrm{A} 2$ \\
\hline RCC1|1104 & $\mathrm{A} 2$ \\
\hline RCC2|55920 & $\mathrm{A} 2$ \\
\hline RCSD1|92241 & $\mathrm{A} 2$ \\
\hline RCVRN|5957 & $\mathrm{A} 2$ \\
\hline RDH13|112724 & $\mathrm{A} 2$ \\
\hline RDM1|201299 & $\mathrm{A} 2$ \\
\hline RECK|8434 & $\mathrm{A} 2$ \\
\hline RECQL4|9401 & $\mathrm{A} 2$ \\
\hline REEP4|80346 & $\mathrm{A} 2$ \\
\hline RELL2|285613 & $\mathrm{A} 2$ \\
\hline RELN|5649 & $\mathrm{A} 2$ \\
\hline
\end{tabular}




\begin{tabular}{|c|c|}
\hline RELT $\mid 84957$ & $\mathrm{~A} 2$ \\
\hline REM2|161253 & $\mathrm{A} 2$ \\
\hline REPIN1|29803 & $\mathrm{A} 2$ \\
\hline REPS2|9185 & $\mathrm{A} 2$ \\
\hline RERE|473 & $\mathrm{A} 2$ \\
\hline RERGL|79785 & $\mathrm{A} 2$ \\
\hline REV3L|5980 & $\mathrm{A} 2$ \\
\hline RFC2|5982 & $\mathrm{A} 2$ \\
\hline RFC3|5983 & A2 \\
\hline RFC4|5984 & $\mathrm{A} 2$ \\
\hline RFTN2|130132 & $\mathrm{A} 2$ \\
\hline RFX2|5990 & $\mathrm{A} 2$ \\
\hline RFXANK|8625 & $\mathrm{A} 2$ \\
\hline RG9MTD2|93587 & $\mathrm{A} 2$ \\
\hline RG9MTD3|158234 & $\mathrm{A} 2$ \\
\hline RGL1|23179 & $\mathrm{A} 2$ \\
\hline RGMA|56963 & $\mathrm{A} 2$ \\
\hline RGMB|285704 & $\mathrm{A} 2$ \\
\hline RGPD3|653489 & $\mathrm{A} 2$ \\
\hline RGPD4|285190 & $\mathrm{A} 2$ \\
\hline RGPD5|84220 & $\mathrm{A} 2$ \\
\hline RGPD6|729540 & $\mathrm{A} 2$ \\
\hline RGS10|6001 & $\mathrm{A} 2$ \\
\hline RGS12|6002 & $\mathrm{A} 2$ \\
\hline RGS14|10636 & $\mathrm{A} 2$ \\
\hline RGS19|10287 & $\mathrm{A} 2$ \\
\hline RGS4|5999 & $\mathrm{A} 2$ \\
\hline RGS6|9628 & $\mathrm{A} 2$ \\
\hline RGS7BP|401190 & $\mathrm{A} 2$ \\
\hline RHBDD2|57414 & $\mathrm{A} 2$ \\
\hline RHBDD3|25807 & $\mathrm{A} 2$ \\
\hline RHBDF1|64285 & $\mathrm{A} 2$ \\
\hline RHBDF2|79651 & $\mathrm{A} 2$ \\
\hline $\begin{array}{l}\text { RHBDL1|9028 } \\
\end{array}$ & $\mathrm{A} 2$ \\
\hline RHBDL2|54933 & $\mathrm{A} 2$ \\
\hline RHCE $\mid 6006$ & $\mathrm{~A} 2$ \\
\hline RHOD|29984 & $\mathrm{A} 2$ \\
\hline RHOF|54509 & $\mathrm{A} 2$ \\
\hline RHOT2|89941 & $\mathrm{A} 2$ \\
\hline RHOXF1|158800 & $\mathrm{A} 2$ \\
\hline RHPN1|114822 & $\mathrm{A} 2$ \\
\hline RIC3|79608 & $\mathrm{A} 2$ \\
\hline RILPL1|353116 & $\mathrm{A} 2$ \\
\hline RILPL2|196383 & $\mathrm{A} 2$ \\
\hline RIMS3|9783 & $\mathrm{A} 2$ \\
\hline RIMS4|140730 & $\mathrm{A} 2$ \\
\hline RINT1|60561 & $\mathrm{A} 2$ \\
\hline RIPK4|54101 & $\mathrm{A} 2$ \\
\hline RIT1|6016 & $\mathrm{A} 2$ \\
\hline RMI1|80010 & $\mathrm{A} 2$ \\
\hline RMND5B|64777 & $\mathrm{A} 2$ \\
\hline
\end{tabular}




\begin{tabular}{|c|c|}
\hline RNASE13|440163 & $\mathrm{A} 2$ \\
\hline RNASE1|6035 & $\mathrm{A} 2$ \\
\hline RNASEH2A|10535 & $\mathrm{A} 2$ \\
\hline RND1|27289 & $\mathrm{A} 2$ \\
\hline \begin{tabular}{ll|l|} 
RNF103|7844 \\
\end{tabular} & $\mathrm{A} 2$ \\
\hline RNF112|7732 & $\mathrm{A} 2$ \\
\hline RNF115|27246 & $\mathrm{A} 2$ \\
\hline RNF11|26994 & $\mathrm{A} 2$ \\
\hline RNF125|54941 & $\mathrm{A} 2$ \\
\hline RNF138P1|379013 & $\mathrm{A} 2$ \\
\hline RNF139|11236 & $\mathrm{A} 2$ \\
\hline RNF145|153830 & $\mathrm{A} 2$ \\
\hline RNF146|81847 & $\mathrm{A} 2$ \\
\hline $\begin{array}{l}\text { RNF149|284996 } \\
\end{array}$ & $\mathrm{A} 2$ \\
\hline \begin{tabular}{l|l|} 
RNF150|57484 \\
\end{tabular} & $\mathrm{A} 2$ \\
\hline RNF157|114804 & $\mathrm{A} 2$ \\
\hline RNF181|51255 & $\mathrm{A} 2$ \\
\hline RNF185|91445 & $\mathrm{A} 2$ \\
\hline RNF186|54546 & $\mathrm{A} 2$ \\
\hline RNF208|727800 & $\mathrm{A} 2$ \\
\hline RNF213|57674 & $\mathrm{A} 2$ \\
\hline RNF217|154214 & $\mathrm{A} 2$ \\
\hline RNF34|80196 & $\mathrm{A} 2$ \\
\hline RNF40|9810 & $\mathrm{A} 2$ \\
\hline RNF41|10193 & $\mathrm{A} 2$ \\
\hline RNF4|6047 & $\mathrm{A} 2$ \\
\hline RNF7|9616 & $\mathrm{A} 2$ \\
\hline RNFT2|84900 & $\mathrm{A} 2$ \\
\hline $\begin{array}{l}\text { RNLS|55328 } \\
\end{array}$ & $\mathrm{A} 2$ \\
\hline RNPEP|6051 & $\mathrm{A} 2$ \\
\hline RNPS1|10921 & $\mathrm{A} 2$ \\
\hline ROBLD3|28956 & $\mathrm{A} 2$ \\
\hline ROCK2|9475 & $\mathrm{A} 2$ \\
\hline ROR1|4919 & $\mathrm{A} 2$ \\
\hline ROR2|4920 & $\mathrm{A} 2$ \\
\hline RPA3|6119 & $\mathrm{A} 2$ \\
\hline RPAP3|79657 & $\mathrm{A} 2$ \\
\hline RPE65|6121 & $\mathrm{A} 2$ \\
\hline RPGRIP1L|23322 & $\mathrm{A} 2$ \\
\hline RPGR|6103 & $\mathrm{A} 2$ \\
\hline RPL10A|4736 & $\mathrm{A} 2$ \\
\hline RPL11|6135 & $\mathrm{A} 2$ \\
\hline RPL15|6138 & $\mathrm{A} 2$ \\
\hline RPL21P44|402176 & $\mathrm{A} 2$ \\
\hline RPL21|6144 & $\mathrm{A} 2$ \\
\hline RPL22|6146 & $\mathrm{A} 2$ \\
\hline RPL23AP53|644128 & $\mathrm{A} 2$ \\
\hline RPL23AP64|649946 & $\mathrm{A} 2$ \\
\hline RPL26L1|51121 & $\mathrm{A} 2$ \\
\hline RPL26|6154 & $\mathrm{A} 2$ \\
\hline RPL31P11|641311 & $\mathrm{A} 2$ \\
\hline
\end{tabular}




\begin{tabular}{|c|c|}
\hline RPL32|6161 & $\mathrm{A} 2$ \\
\hline RPL34|6164 & $\mathrm{A} 2$ \\
\hline RPL39L|116832 & $\mathrm{A} 2$ \\
\hline RPL3|6122 & $\mathrm{A} 2$ \\
\hline RPL4|6124 & $\mathrm{A} 2$ \\
\hline RPL5|6125 & A2 \\
\hline RPLP0P2|113157 & $\mathrm{A} 2$ \\
\hline RPN1|6184 & $\mathrm{A} 2$ \\
\hline RPN2|6185 & $\mathrm{A} 2$ \\
\hline RPP21|79897 & $\mathrm{A} 2$ \\
\hline RPP40|10799 & $\mathrm{A} 2$ \\
\hline RPRD2|23248 & $\mathrm{A} 2$ \\
\hline RPS13|6207 & $\mathrm{A} 2$ \\
\hline RPS14|6208 & $\mathrm{A} 2$ \\
\hline RPS20|6224 & $\mathrm{A} 2$ \\
\hline RPS23|6228 & $\mathrm{A} 2$ \\
\hline RPS25|6230 & $\mathrm{A} 2$ \\
\hline RPS27A|6233 & $\mathrm{A} 2$ \\
\hline RPS3A|6189 & $\mathrm{A} 2$ \\
\hline RPS6KA1|6195 & $\mathrm{A} 2$ \\
\hline RPS6KA5|9252 & $\mathrm{A} 2$ \\
\hline RPS6KB2|6199 & $\mathrm{A} 2$ \\
\hline RPS9|6203 & $\mathrm{A} 2$ \\
\hline RPUSD1|113000 & $\mathrm{A} 2$ \\
\hline RRAGD|58528 & $\mathrm{A} 2$ \\
\hline RRAS2|22800 & $\mathrm{A} 2$ \\
\hline RRM1|6240 & $\mathrm{A} 2$ \\
\hline RRM2|6241 & $\mathrm{A} 2$ \\
\hline RRN3|54700 & $\mathrm{A} 2$ \\
\hline RRS1|23212 & $\mathrm{A} 2$ \\
\hline RSAD2|91543 & $\mathrm{A} 2$ \\
\hline RSPH1|89765 & $\mathrm{A} 2$ \\
\hline RSPO3|84870 & $\mathrm{A} 2$ \\
\hline RTCD1|8634 & $\mathrm{A} 2$ \\
\hline RTKN2|219790 & $\mathrm{A} 2$ \\
\hline RTKN|6242 & $\mathrm{A} 2$ \\
\hline RTN4RL2|349667 & $\mathrm{A} 2$ \\
\hline RTN4|57142 & $\mathrm{A} 2$ \\
\hline RUNX1T1|862 & $\mathrm{A} 2$ \\
\hline RUNX2|860 & $\mathrm{A} 2$ \\
\hline RUSC2|9853 & $\mathrm{A} 2$ \\
\hline RUVBL1|8607 & $\mathrm{A} 2$ \\
\hline RUVBL2|10856 & $\mathrm{A} 2$ \\
\hline \begin{tabular}{l|l|l|} 
RXFP3 & \\
\end{tabular} & $\mathrm{A} 2$ \\
\hline S100A11|6282 & $\mathrm{A} 2$ \\
\hline S100A14|57402 & $\mathrm{A} 2$ \\
\hline S100A16|140576 & $\mathrm{A} 2$ \\
\hline S1PR1|1901 & $\mathrm{A} 2$ \\
\hline SAA2|6289 & $\mathrm{A} 2$ \\
\hline SAA4|6291 & $\mathrm{A} 2$ \\
\hline SAAL1|113174 & $\mathrm{A} 2$ \\
\hline
\end{tabular}




\begin{tabular}{|c|c|}
\hline SAC3D1|29901 & A2 \\
\hline SACS|26278 & $\mathrm{A} 2$ \\
\hline SALL4|57167 & $\mathrm{A} 2$ \\
\hline SAMD11|148398 & $\mathrm{A} 2$ \\
\hline SAMD14|201191 & $\mathrm{A} 2$ \\
\hline SAMD1|90378 & $\mathrm{A} 2$ \\
\hline SAMD4A|23034 & $\mathrm{A} 2$ \\
\hline SAMSN1|64092 & $\mathrm{A} 2$ \\
\hline SAP130|79595 & $\mathrm{A} 2$ \\
\hline SAP30L|79685 & $\mathrm{A} 2$ \\
\hline SAP30|8819 & $\mathrm{A} 2$ \\
\hline SAPS1|22870 & $\mathrm{A} 2$ \\
\hline SAR1B|51128 & $\mathrm{A} 2$ \\
\hline \begin{tabular}{l|l|} 
SARNP & 84324 \\
\end{tabular} & $\mathrm{~A} 2$ \\
\hline SASS6|163786 & $\mathrm{A} 2$ \\
\hline SAT2|112483 & $\mathrm{A} 2$ \\
\hline SATB1|6304 & $\mathrm{A} 2$ \\
\hline SBDS|51119 & $\mathrm{A} 2$ \\
\hline SBF1|6305 & A2 \\
\hline SBF2|81846 & $\mathrm{A} 2$ \\
\hline SBK1|388228 & $\mathrm{A} 2$ \\
\hline SC65|10609 & $\mathrm{A} 2$ \\
\hline SCAMP3|10067 & $\mathrm{A} 2$ \\
\hline SCAPER|49855 & $\mathrm{A} 2$ \\
\hline SCARA5|286133 & $\mathrm{A} 2$ \\
\hline SCARNA17|677769 & $\mathrm{A} 2$ \\
\hline SCCPDH|51097 & $\mathrm{A} 2$ \\
\hline SCLY|51540 & $\mathrm{A} 2$ \\
\hline SCML1|6322 & $\mathrm{A} 2$ \\
\hline SCN11A|11280 & $\mathrm{A} 2$ \\
\hline SCN2A|6326 & $\mathrm{A} 2$ \\
\hline \begin{tabular}{l|l|l|} 
SCN3B 55800 \\
\end{tabular} & $\mathrm{~A} 2$ \\
\hline SCN5A|6331 & $\mathrm{A} 2$ \\
\hline SCNM1|79005 & $\mathrm{A} 2$ \\
\hline SCO2|9997 & $\mathrm{A} 2$ \\
\hline SCRN2|90507 & $\mathrm{A} 2$ \\
\hline SCT $\mid 6343$ & $\mathrm{~A} 2$ \\
\hline SDC1|6382 & $\mathrm{A} 2$ \\
\hline \begin{tabular}{ll|l|l|} 
SDF2L1 23753 \\
\end{tabular} & $\mathrm{~A} 2$ \\
\hline SDHAP1|255812 & $\mathrm{A} 2$ \\
\hline SDHAP2|727956 & A2 \\
\hline $\begin{array}{l}\text { SDHAP3|728609 } \\
\end{array}$ & $\mathrm{A} 2$ \\
\hline SDHD|6392 & $\mathrm{A} 2$ \\
\hline SDK1|221935 & $\mathrm{A} 2$ \\
\hline SDPR|8436 & $\mathrm{A} 2$ \\
\hline SDS|10993 & A2 \\
\hline SEC11C|90701 & $\mathrm{A} 2$ \\
\hline SEC13|6396 & $\mathrm{A} 2$ \\
\hline SEC16B|89866 & $\mathrm{A} 2$ \\
\hline SEC22C|9117 & A2 \\
\hline \begin{tabular}{l|l|l|} 
SEC24D & 9871 \\
\end{tabular} & $\mathrm{~A} 2$ \\
\hline
\end{tabular}




\begin{tabular}{|c|c|}
\hline SEC31A|22872 & $\mathrm{A} 2$ \\
\hline SEC31B|25956 & A2 \\
\hline SEC61A1|29927 & $\mathrm{A} 2$ \\
\hline SEC61B|10952 & A2 \\
\hline SECISBP2L|9728 & $\mathrm{A} 2$ \\
\hline SECTM1|6398 & $\mathrm{A} 2$ \\
\hline SELS|55829 & $\mathrm{A} 2$ \\
\hline SELT|51714 & $\mathrm{A} 2$ \\
\hline SEMA3C|10512 & $\mathrm{A} 2$ \\
\hline SEMA3F|6405 & $\mathrm{A} 2$ \\
\hline SEMA3G|56920 & $\mathrm{A} 2$ \\
\hline SEMA5A|9037 & $\mathrm{A} 2$ \\
\hline SEMA7A|8482 & $\mathrm{A} 2$ \\
\hline SENP1|29843 & $\mathrm{A} 2$ \\
\hline SEPHS2|22928 & $\mathrm{A} 2$ \\
\hline SEPT10|151011 & $\mathrm{A} 2$ \\
\hline SEPT8|23176 & $\mathrm{A} 2$ \\
\hline \begin{tabular}{l|l|} 
SEPT9 & 10801 \\
\end{tabular} & $\mathrm{~A} 2$ \\
\hline SEPX1|51734 & $\mathrm{A} 2$ \\
\hline SERINC1|57515 & $\mathrm{A} 2$ \\
\hline SERINC2|347735 & $\mathrm{A} 2$ \\
\hline SERP1|27230 & $\mathrm{A} 2$ \\
\hline SERPINH1|871 & $\mathrm{A} 2$ \\
\hline \begin{tabular}{l|l|l|} 
SESN1 & 2724 \\
\end{tabular} & $\mathrm{~A} 2$ \\
\hline SETD7|80854 & $\mathrm{A} 2$ \\
\hline SETDB1|9869 & $\mathrm{A} 2$ \\
\hline SETMAR|6419 & $\mathrm{A} 2$ \\
\hline SETX|23064 & $\mathrm{A} 2$ \\
\hline SEZ6L2|26470 & $\mathrm{A} 2$ \\
\hline SF3A1|10291 & $\mathrm{A} 2$ \\
\hline SF3B14|51639 & $\mathrm{A} 2$ \\
\hline SF3B4|10262 & $\mathrm{A} 2$ \\
\hline SFPQ|6421 & $\mathrm{A} 2$ \\
\hline SFRP2|6423 & $\mathrm{A} 2$ \\
\hline SFRS18|25957 & $\mathrm{A} 2$ \\
\hline SFRS1|6426 & $\mathrm{A} 2$ \\
\hline SFRS2B|10929 & $\mathrm{A} 2$ \\
\hline SFRS2|6427 & $\mathrm{A} 2$ \\
\hline SFRS4|6429 & $\mathrm{A} 2$ \\
\hline SFRS9|8683 & $\mathrm{A} 2$ \\
\hline \begin{tabular}{l|l|} 
SFXN1|94081 \\
\end{tabular} & $\mathrm{A} 2$ \\
\hline SFXN2|118980 & $\mathrm{A} 2$ \\
\hline \begin{tabular}{l|l|} 
SFXN5|94097 \\
\end{tabular} & $\mathrm{A} 2$ \\
\hline SGCE $\mid 8910$ & $\mathrm{~A} 2$ \\
\hline SGCZ|137868 & $\mathrm{A} 2$ \\
\hline SGK1|6446 & $\mathrm{A} 2$ \\
\hline SGK269|79834 & $\mathrm{A} 2$ \\
\hline SGOL1|151648 & $\mathrm{A} 2$ \\
\hline SGOL2|151246 & $\mathrm{A} 2$ \\
\hline SGPL1|8879 & $\mathrm{A} 2$ \\
\hline SGSM2|9905 & $\mathrm{A} 2$ \\
\hline
\end{tabular}




\begin{tabular}{|c|c|}
\hline SH2D2A|9047 & $\mathrm{A} 2$ \\
\hline SH2D4A|63898 & $\mathrm{A} 2$ \\
\hline SH3BGRL|6451 & $\mathrm{A} 2$ \\
\hline SH3BP2|6452 & $\mathrm{A} 2$ \\
\hline SH3BP5L|80851 & $\mathrm{A} 2$ \\
\hline SH3BP5|9467 & $\mathrm{A} 2$ \\
\hline SH3D19|152503 & $\mathrm{A} 2$ \\
\hline SH3GL1|6455 & $\mathrm{A} 2$ \\
\hline SH3KBP1|30011 & A2 \\
\hline SH3RF1|57630 & $\mathrm{A} 2$ \\
\hline SH3TC2|79628 & $\mathrm{A} 2$ \\
\hline SHANK3|85358 & $\mathrm{A} 2$ \\
\hline SHARPIN|81858 & $\mathrm{A} 2$ \\
\hline SHBG|6462 & $\mathrm{A} 2$ \\
\hline SHCBP1|79801 & $\mathrm{A} 2$ \\
\hline SHE|126669 & $\mathrm{A} 2$ \\
\hline SHFM1|7979 & $\mathrm{A} 2$ \\
\hline SHISA5|51246 & $\mathrm{A} 2$ \\
\hline SHISA9|729993 & $\mathrm{A} 2$ \\
\hline SHKBP1|92799 & $\mathrm{A} 2$ \\
\hline SHMT1|6470 & $\mathrm{A} 2$ \\
\hline SHMT2|6472 & $\mathrm{A} 2$ \\
\hline SHOX|6473 & $\mathrm{A} 2$ \\
\hline SHQ1|55164 & $\mathrm{A} 2$ \\
\hline SHROOM2|357 & $\mathrm{A} 2$ \\
\hline SHROOM4|57477 & $\mathrm{A} 2$ \\
\hline SIAH2|6478 & $\mathrm{A} 2$ \\
\hline SIDT1|54847 & $\mathrm{A} 2$ \\
\hline SIDT2|51092 & $\mathrm{A} 2$ \\
\hline SIGIRR|59307 & $\mathrm{A} 2$ \\
\hline SIK3|23387 & $\mathrm{A} 2$ \\
\hline SIM2|6493 & $\mathrm{A} 2$ \\
\hline $\begin{array}{l}\text { SIPA1L3|23094 } \\
\end{array}$ & $\mathrm{A} 2$ \\
\hline SIRT1|23411 & $\mathrm{A} 2$ \\
\hline SIRT6|51548 & $\mathrm{A} 2$ \\
\hline SIRT7|51547 & $\mathrm{A} 2$ \\
\hline SIX4|51804 & $\mathrm{A} 2$ \\
\hline SKA2|348235 & $\mathrm{A} 2$ \\
\hline SKAP2|8935 & $\mathrm{A} 2$ \\
\hline SKIL|6498 & $\mathrm{A} 2$ \\
\hline SKINTL|391037 & $\mathrm{A} 2$ \\
\hline SKI|6497 & $\mathrm{A} 2$ \\
\hline SLAMF8|56833 & $\mathrm{A} 2$ \\
\hline SLBP|7884 & $\mathrm{A} 2$ \\
\hline SLC10A6|345274 & $\mathrm{A} 2$ \\
\hline SLC11A1|6556 & $\mathrm{A} 2$ \\
\hline SLC12A4|6560 & $\mathrm{A} 2$ \\
\hline SLC12A6|9990 & $\mathrm{A} 2$ \\
\hline SLC12A7|10723 & $\mathrm{A} 2$ \\
\hline SLC12A8|84561 & $\mathrm{A} 2$ \\
\hline SLC16A11|162515 & $\mathrm{A} 2$ \\
\hline
\end{tabular}




\begin{tabular}{|c|c|}
\hline SLC16A12|387700 & $\mathrm{A} 2$ \\
\hline SLC16A2|6567 & $\mathrm{A} 2$ \\
\hline SLC16A3|9123 & $\mathrm{A} 2$ \\
\hline SLC16A7|9194 & $\mathrm{A} 2$ \\
\hline SLC19A1|6573 & A2 \\
\hline SLC19A2|10560 & $\mathrm{A} 2$ \\
\hline \begin{tabular}{ll|l|} 
SLC19A3|80704 \\
\end{tabular} & $\mathrm{A} 2$ \\
\hline SLC1A3|6507 & $\mathrm{A} 2$ \\
\hline SLC1A4|6509 & $\mathrm{A} 2$ \\
\hline SLC1A7|6512 & $\mathrm{A} 2$ \\
\hline SLC22A11|55867 & $\mathrm{A} 2$ \\
\hline SLC22A12|116085 & $\mathrm{A} 2$ \\
\hline SLC22A18|5002 & $\mathrm{A} 2$ \\
\hline SLC23A1|9963 & $\mathrm{A} 2$ \\
\hline SLC24A2|25769 & $\mathrm{A} 2$ \\
\hline SLC25A14|9016 & $\mathrm{A} 2$ \\
\hline SLC25A15|10166 & $\mathrm{A} 2$ \\
\hline SLC25A19|60386 & $\mathrm{A} 2$ \\
\hline SLC25A20|788 & $\mathrm{A} 2$ \\
\hline SLC25A22|79751 & $\mathrm{A} 2$ \\
\hline SLC25A24|29957 & $\mathrm{A} 2$ \\
\hline SLC25A25|114789 & $\mathrm{A} 2$ \\
\hline SLC25A26|115286 & $\mathrm{A} 2$ \\
\hline SLC25A27|9481 & $\mathrm{A} 2$ \\
\hline SLC25A28|81894 & $\mathrm{A} 2$ \\
\hline SLC25A30|253512 & $\mathrm{A} 2$ \\
\hline SLC25A36|55186 & $\mathrm{A} 2$ \\
\hline SLC25A39|51629 & $\mathrm{A} 2$ \\
\hline SLC25A42|284439 & $\mathrm{A} 2$ \\
\hline SLC25A5|292 & $\mathrm{A} 2$ \\
\hline SLC26A10|65012 & $\mathrm{A} 2$ \\
\hline SLC26A4|5172 & $\mathrm{A} 2$ \\
\hline SLC27A4|10999 & $\mathrm{A} 2$ \\
\hline SLC29A1|2030 & $\mathrm{A} 2$ \\
\hline \begin{tabular}{l|l|l|} 
SLC29A3|55315 \\
\end{tabular} & $\mathrm{A} 2$ \\
\hline SLC2A10|81031 & $\mathrm{A} 2$ \\
\hline SLC2A12|154091 & $\mathrm{A} 2$ \\
\hline SLC2A1|6513 & $\mathrm{A} 2$ \\
\hline SLC2A4|6517 & $\mathrm{A} 2$ \\
\hline SLC2A6|11182 & $\mathrm{A} 2$ \\
\hline SLC30A1|7779 & $\mathrm{A} 2$ \\
\hline SLC30A7|148867 & $\mathrm{A} 2$ \\
\hline SLC35A3|23443 & $\mathrm{A} 2$ \\
\hline SLC35B1|10237 & $\mathrm{A} 2$ \\
\hline SLC35B2|347734 & $\mathrm{A} 2$ \\
\hline SLC35B4|84912 & A2 \\
\hline SLC35D1|23169 & $\mathrm{A} 2$ \\
\hline $\begin{array}{l}\text { SLC35E3|55508 } \\
\end{array}$ & $\mathrm{A} 2$ \\
\hline SLC35F5|80255 & $\mathrm{A} 2$ \\
\hline SLC38A5|92745 & $\mathrm{A} 2$ \\
\hline SLC38A7|55238 & $\mathrm{A} 2$ \\
\hline
\end{tabular}




\begin{tabular}{|c|c|}
\hline SLC39A10|57181 & A2 \\
\hline SLC39A11|201266 & $\mathrm{A} 2$ \\
\hline SLC39A1|27173 & $\mathrm{A} 2$ \\
\hline SLC39A3|29985 & $\mathrm{A} 2$ \\
\hline SLC39A4|55630 & $\mathrm{A} 2$ \\
\hline SLC39A7|7922 & $\mathrm{A} 2$ \\
\hline SLC41A3|54946 & $\mathrm{A} 2$ \\
\hline SLC44A1|23446 & $\mathrm{A} 2$ \\
\hline SLC44A4|80736 & $\mathrm{A} 2$ \\
\hline SLC47A2|146802 & $\mathrm{A} 2$ \\
\hline SLC48A1|55652 & $\mathrm{A} 2$ \\
\hline SLC4A2|6522 & $\mathrm{A} 2$ \\
\hline SLC4A8|9498 & $\mathrm{A} 2$ \\
\hline SLC5A4|6527 & $\mathrm{A} 2$ \\
\hline SLC5A9|200010 & $\mathrm{A} 2$ \\
\hline SLC6A16|28968 & $\mathrm{A} 2$ \\
\hline SLC6A1|6529 & $\mathrm{A} 2$ \\
\hline SLC6A9|6536 & $\mathrm{A} 2$ \\
\hline SLC7A10|56301 & $\mathrm{A} 2$ \\
\hline SLC7A11|23657 & $\mathrm{A} 2$ \\
\hline SLC7A3|84889 & $\mathrm{A} 2$ \\
\hline SLC7A8|23428 & $\mathrm{A} 2$ \\
\hline SLC9A6|10479 & $\mathrm{A} 2$ \\
\hline SLCO1A2|6579 & $\mathrm{A} 2$ \\
\hline SLCO1C1|53919 & $\mathrm{A} 2$ \\
\hline SLITRK2|84631 & $\mathrm{A} 2$ \\
\hline SLMO2|51012 & $\mathrm{A} 2$ \\
\hline SLU7|10569 & $\mathrm{A} 2$ \\
\hline SMAD4|4089 & $\mathrm{A} 2$ \\
\hline SMAD9|4093 & $\mathrm{A} 2$ \\
\hline SMARCA4|6597 & $\mathrm{A} 2$ \\
\hline SMARCB1|6598 & $\mathrm{A} 2$ \\
\hline SMARCC1|6599 & $\mathrm{A} 2$ \\
\hline SMARCC2|6601 & $\mathrm{A} 2$ \\
\hline SMC1A|8243 & $\mathrm{A} 2$ \\
\hline SMC2|10592 & $\mathrm{A} 2$ \\
\hline SMC4|10051 & $\mathrm{A} 2$ \\
\hline SMG5|23381 & $\mathrm{A} 2$ \\
\hline SMG7|9887 & $\mathrm{A} 2$ \\
\hline SMPD2|6610 & $\mathrm{A} 2$ \\
\hline SMPD4|55627 & $\mathrm{A} 2$ \\
\hline SMS|6611 & $\mathrm{A} 2$ \\
\hline SMTN|6525 & $\mathrm{A} 2$ \\
\hline SMUG1|23583 & $\mathrm{A} 2$ \\
\hline \begin{tabular}{l|l|l|} 
SMYD3|64754 \\
\end{tabular} & $\mathrm{A} 2$ \\
\hline SNAI2|6591 & $\mathrm{A} 2$ \\
\hline SNAP47|116841 & $\mathrm{A} 2$ \\
\hline SNAPC2|6618 & $\mathrm{A} 2$ \\
\hline SNCG|6623 & $\mathrm{A} 2$ \\
\hline SNED1|25992 & $\mathrm{A} 2$ \\
\hline SNHG8|100093630 & $\mathrm{A} 2$ \\
\hline
\end{tabular}




\begin{tabular}{|c|c|}
\hline SNN|8303 & $\mathrm{A} 2$ \\
\hline SNORD116-20|100033431 & A2 \\
\hline SNRK|54861 & $\mathrm{A} 2$ \\
\hline SNRNP25|79622 & $\mathrm{A} 2$ \\
\hline SNRNP40|9410 & A2 \\
\hline SNRPA1|6627 & $\mathrm{A} 2$ \\
\hline SNRPB2|6629 & $\mathrm{A} 2$ \\
\hline SNRPB|6628 & $\mathrm{A} 2$ \\
\hline SNRPC|6631 & $\mathrm{A} 2$ \\
\hline SNRPD3|6634 & $\mathrm{A} 2$ \\
\hline SNRPE|6635 & $\mathrm{A} 2$ \\
\hline SNRPF|6636 & $\mathrm{A} 2$ \\
\hline SNRPG|6637 & $\mathrm{A} 2$ \\
\hline SNTB1|6641 & $\mathrm{A} 2$ \\
\hline SNTB2|6645 & $\mathrm{A} 2$ \\
\hline SNTG2|54221 & $\mathrm{A} 2$ \\
\hline SNX15|29907 & $\mathrm{A} 2$ \\
\hline SNX18|112574 & $\mathrm{A} 2$ \\
\hline SNX21|90203 & $\mathrm{A} 2$ \\
\hline SNX24|28966 & $\mathrm{A} 2$ \\
\hline SNX27|81609 & $\mathrm{A} 2$ \\
\hline \begin{tabular}{l|c|} 
SNX29|92017 \\
\end{tabular} & $\mathrm{A} 2$ \\
\hline SOCS3|9021 & $\mathrm{A} 2$ \\
\hline SOCS5|9655 & $\mathrm{A} 2$ \\
\hline SOD2|6648 & $\mathrm{A} 2$ \\
\hline SOLH|6650 & $\mathrm{A} 2$ \\
\hline SORBS2|8470 & $\mathrm{A} 2$ \\
\hline SOS1|6654 & $\mathrm{A} 2$ \\
\hline SOS2|6655 & $\mathrm{A} 2$ \\
\hline SOSTDC1|25928 & $\mathrm{A} 2$ \\
\hline SOX12|6666 & $\mathrm{A} 2$ \\
\hline SOX17|64321 & $\mathrm{A} 2$ \\
\hline SOX4|6659 & $\mathrm{A} 2$ \\
\hline SOX5|6660 & $\mathrm{A} 2$ \\
\hline SOX7|83595 & $\mathrm{A} 2$ \\
\hline SP110|3431 & $\mathrm{A} 2$ \\
\hline SPA17|53340 & $\mathrm{A} 2$ \\
\hline SPAG5|10615 & $\mathrm{A} 2$ \\
\hline SPATA17|128153 & $\mathrm{A} 2$ \\
\hline SPATA18|132671 & $\mathrm{A} 2$ \\
\hline SPATA7|55812 & $\mathrm{A} 2$ \\
\hline SPATS2|65244 & $\mathrm{A} 2$ \\
\hline SPC24|147841 & $\mathrm{A} 2$ \\
\hline SPC25|57405 & $\mathrm{A} 2$ \\
\hline SPCS1|28972 & $\mathrm{A} 2$ \\
\hline SPCS2|9789 & $\mathrm{A} 2$ \\
\hline SPDEF|25803 & $\mathrm{A} 2$ \\
\hline SPEF1|25876 & $\mathrm{A} 2$ \\
\hline SPEN|23013 & $\mathrm{A} 2$ \\
\hline SPG20|23111 & $\mathrm{A} 2$ \\
\hline SPHKAP|80309 & $\mathrm{A} 2$ \\
\hline
\end{tabular}




\begin{tabular}{|c|c|}
\hline SPIN2A|54466 & $\mathrm{A} 2$ \\
\hline SPINT1|6692 & $\mathrm{A} 2$ \\
\hline SPINT2|10653 & $\mathrm{A} 2$ \\
\hline SPIRE2|84501 & $\mathrm{A} 2$ \\
\hline SPNS1|83985 & A2 \\
\hline SPOCD1|90853 & $\mathrm{A} 2$ \\
\hline SPP1|6696 & $\mathrm{A} 2$ \\
\hline SPRY1|10252 & $\mathrm{A} 2$ \\
\hline SPR|6697 & $\mathrm{A} 2$ \\
\hline SPTBN1|6711 & $\mathrm{A} 2$ \\
\hline SQRDL|58472 & $\mathrm{A} 2$ \\
\hline SRA1|10011 & $\mathrm{A} 2$ \\
\hline SRCAP|10847 & $\mathrm{A} 2$ \\
\hline SRC|6714 & $\mathrm{A} 2$ \\
\hline SRD5A3|79644 & $\mathrm{A} 2$ \\
\hline SREBF1|6720 & $\mathrm{A} 2$ \\
\hline SRF|6722 & $\mathrm{A} 2$ \\
\hline SRGAP2|23380 & $\mathrm{A} 2$ \\
\hline SRMS|6725 & $\mathrm{A} 2$ \\
\hline SRP54|6729 & $\mathrm{A} 2$ \\
\hline SRP72|6731 & $\mathrm{A} 2$ \\
\hline SRPK2|6733 & $\mathrm{A} 2$ \\
\hline SRPRB|58477 & $\mathrm{A} 2$ \\
\hline SRXN1|140809 & $\mathrm{A} 2$ \\
\hline SSBP1|6742 & $\mathrm{A} 2$ \\
\hline SSBP2|23635 & $\mathrm{A} 2$ \\
\hline SSNA1|8636 & $\mathrm{A} 2$ \\
\hline SSR2|6746 & $\mathrm{A} 2$ \\
\hline SSR4|6748 & $\mathrm{A} 2$ \\
\hline SSX2IP|117178 & $\mathrm{A} 2$ \\
\hline $\begin{array}{l}\text { ST13|6767 } \\
\end{array}$ & $\mathrm{A} 2$ \\
\hline ST14|6768 & $\mathrm{A} 2$ \\
\hline ST20|400410 & $\mathrm{A} 2$ \\
\hline ST3GAL3|6487 & $\mathrm{A} 2$ \\
\hline ST5|6764 & $\mathrm{A} 2$ \\
\hline $\begin{array}{l}\text { ST6GAL2|84620 } \\
\end{array}$ & $\mathrm{A} 2$ \\
\hline ST8SIA2|8128 & $\mathrm{A} 2$ \\
\hline STAB1|23166 & $\mathrm{A} 2$ \\
\hline \begin{tabular}{l|l|} 
STAC2|342667 \\
\end{tabular} & $\mathrm{A} 2$ \\
\hline STAG3L3|442578 & $\mathrm{A} 2$ \\
\hline STAMBP|10617 & $\mathrm{A} 2$ \\
\hline STAM|8027 & $\mathrm{A} 2$ \\
\hline STARD10|10809 & $\mathrm{A} 2$ \\
\hline STARD13|90627 & $\mathrm{A} 2$ \\
\hline STAT1|6772 & $\mathrm{A} 2$ \\
\hline STAT6|6778 & $\mathrm{A} 2$ \\
\hline STBD1|8987 & $\mathrm{A} 2$ \\
\hline STIL|6491 & $\mathrm{A} 2$ \\
\hline STIM1|6786 & $\mathrm{A} 2$ \\
\hline STIP1|10963 & $\mathrm{A} 2$ \\
\hline STK11IP|114790 & $\mathrm{A} 2$ \\
\hline
\end{tabular}




\begin{tabular}{|c|c|}
\hline STMN1|3925 & A2 \\
\hline STON1|11037 & A2 \\
\hline STRA13|201254 & A2 \\
\hline STRBP|55342 & A2 \\
\hline STT3A|3703 & A2 \\
\hline STX12|23673 & $\mathrm{A} 2$ \\
\hline STX1A|6804 & A2 \\
\hline STX3|6809 & A2 \\
\hline STXBP1|6812 & A2 \\
\hline STXBP2|6813 & A2 \\
\hline STXBP6|29091 & A2 \\
\hline STYXL1|51657 & A2 \\
\hline SUCLA2|8803 & A2 \\
\hline SUCLG1|8802 & $\mathrm{A} 2$ \\
\hline SUGT1L1|283507 & $\mathrm{A} 2$ \\
\hline SUGT1P1|441394 & A2 \\
\hline SULF1|23213 & $\mathrm{A} 2$ \\
\hline SULF2|55959 & A2 \\
\hline SUMO1P3|474338 & $\mathrm{A} 2$ \\
\hline SUMO1|7341 & $\mathrm{A} 2$ \\
\hline SUMO2|6613 & A2 \\
\hline $\begin{array}{l}\text { SUMO3|6612 } \\
\end{array}$ & $\mathrm{A} 2$ \\
\hline SUN2|25777 & $\mathrm{A} 2$ \\
\hline SUPT16H|11198 & A2 \\
\hline SUPT3H|8464 & $\mathrm{A} 2$ \\
\hline SUSD3|203328 & $\mathrm{A} 2$ \\
\hline SUV39H1|6839 & $\mathrm{A} 2$ \\
\hline SUV39H2|79723 & $\mathrm{A} 2$ \\
\hline $\begin{array}{l}\text { SUV420H2|84787 } \\
\end{array}$ & $\mathrm{A} 2$ \\
\hline SV2B|9899 & $\mathrm{A} 2$ \\
\hline SVEP1|79987 & $\mathrm{A} 2$ \\
\hline SWAP70|23075 & $\mathrm{A} 2$ \\
\hline SYAP1|94056 & $\mathrm{A} 2$ \\
\hline SYCE2|256126 & $\mathrm{A} 2$ \\
\hline SYDE1|85360 & $\mathrm{A} 2$ \\
\hline SYF2|25949 & A2 \\
\hline \begin{tabular}{ll|l|} 
SYN2|6854 \\
\end{tabular} & $\mathrm{A} 2$ \\
\hline SYNE1|23345 & $\mathrm{A} 2$ \\
\hline SYNGR2|9144 & $\mathrm{A} 2$ \\
\hline SYNGR3|9143 & $\mathrm{A} 2$ \\
\hline SYNGR4|23546 & $\mathrm{A} 2$ \\
\hline SYNPO|11346 & $\mathrm{A} 2$ \\
\hline $\begin{array}{l}\text { SYT15|83849 } \\
\end{array}$ & $\mathrm{A} 2$ \\
\hline SYT2|127833 & $\mathrm{A} 2$ \\
\hline TAC1|6863 & $\mathrm{A} 2$ \\
\hline TACC3|10460 & $\mathrm{A} 2$ \\
\hline TACO1|51204 & $\mathrm{A} 2$ \\
\hline TACR1|6869 & $\mathrm{A} 2$ \\
\hline TAF11|6882 & $\mathrm{A} 2$ \\
\hline TAF12|6883 & $\mathrm{A} 2$ \\
\hline TAF4|6874 & A2 \\
\hline
\end{tabular}




\begin{tabular}{|c|c|}
\hline TAF5L|27097 & A2 \\
\hline TAF6|6878 & $\mathrm{A} 2$ \\
\hline TAF8|129685 & $\mathrm{A} 2$ \\
\hline TAGLN2|8407 & $\mathrm{A} 2$ \\
\hline TAL1|6886 & $\mathrm{A} 2$ \\
\hline TANC2|26115 & $\mathrm{A} 2$ \\
\hline TANK|10010 & $\mathrm{A} 2$ \\
\hline TAP1|6890 & $\mathrm{A} 2$ \\
\hline TAPBP|6892 & $\mathrm{A} 2$ \\
\hline TARBP2|6895 & $\mathrm{A} 2$ \\
\hline TARDBP|23435 & $\mathrm{A} 2$ \\
\hline TARS2|80222 & A2 \\
\hline TARS|6897 & $\mathrm{A} 2$ \\
\hline TAS2R5|54429 & $\mathrm{A} 2$ \\
\hline TAX1BP1|8887 & $\mathrm{A} 2$ \\
\hline TBC1D10B|26000 & $\mathrm{A} 2$ \\
\hline TBC1D15|64786 & A2 \\
\hline TBC1D17|79735 & $\mathrm{A} 2$ \\
\hline TBC1D25|4943 & A2 \\
\hline TBC1D5|9779 & $\mathrm{A} 2$ \\
\hline TBC1D9|23158 & $\mathrm{A} 2$ \\
\hline TBCA|6902 & $\mathrm{A} 2$ \\
\hline TBCB $\mid 1155$ & $\mathrm{~A} 2$ \\
\hline TBCE|6905 & $\mathrm{A} 2$ \\
\hline TBKBP1|9755 & $\mathrm{A} 2$ \\
\hline TBL1XR1|79718 & $\mathrm{A} 2$ \\
\hline TBL3|10607 & $\mathrm{A} 2$ \\
\hline TBRG4|9238 & $\mathrm{A} 2$ \\
\hline TBX15|6913 & $\mathrm{A} 2$ \\
\hline TBX18|9096 & $\mathrm{A} 2$ \\
\hline TCEA1|6917 & $\mathrm{A} 2$ \\
\hline TCEAL2|140597 & A2 \\
\hline TCEAL5|340543 & $\mathrm{A} 2$ \\
\hline TCEB1|6921 & A2 \\
\hline TCEB2|6923 & $\mathrm{A} 2$ \\
\hline TCF15|6939 & $\mathrm{A} 2$ \\
\hline TCF19|6941 & $\mathrm{A} 2$ \\
\hline TCF21|6943 & $\mathrm{A} 2$ \\
\hline TCF25|22980 & A2 \\
\hline TCF3|6929 & $\mathrm{A} 2$ \\
\hline TCF4|6925 & A2 \\
\hline TCF7L1|83439 & $\mathrm{A} 2$ \\
\hline TCF7L2|6934 & A2 \\
\hline TCF7|6932 & $\mathrm{A} 2$ \\
\hline TCTEX1D2|255758 & $\mathrm{A} 2$ \\
\hline TDP1|55775 & $\mathrm{A} 2$ \\
\hline TDRKH|11022 & $\mathrm{A} 2$ \\
\hline TEAD4|7004 & $\mathrm{A} 2$ \\
\hline TECPR2|9895 & $\mathrm{A} 2$ \\
\hline TENC1|23371 & A2 \\
\hline TEPP|374739 & A2 \\
\hline
\end{tabular}




\begin{tabular}{|c|c|}
\hline TFAP2A|7020 & A2 \\
\hline TFDP1|7027 & $\mathrm{A} 2$ \\
\hline TFG|10342 & $\mathrm{A} 2$ \\
\hline TFPI|7035 & $\mathrm{A} 2$ \\
\hline TFPT|29844 & $\mathrm{A} 2$ \\
\hline TFR2|7036 & $\mathrm{A} 2$ \\
\hline TFRC|7037 & $\mathrm{A} 2$ \\
\hline TF|7018 & $\mathrm{A} 2$ \\
\hline TGFB1I1|7041 & $\mathrm{A} 2$ \\
\hline TGFB3|7043 & $\mathrm{A} 2$ \\
\hline TGFBR2|7048 & $\mathrm{A} 2$ \\
\hline TGIF1|7050 & A2 \\
\hline THBD|7056 & $\mathrm{A} 2$ \\
\hline THOC3|84321 & $\mathrm{A} 2$ \\
\hline THOC4|10189 & $\mathrm{A} 2$ \\
\hline THOC6|79228 & $\mathrm{A} 2$ \\
\hline THRA|7067 & A2 \\
\hline THRSP|7069 & $\mathrm{A} 2$ \\
\hline THSD1P1|374500 & A2 \\
\hline THSD7A|221981 & $\mathrm{A} 2$ \\
\hline THUMPD3|25917 & $\mathrm{A} 2$ \\
\hline THY1|7070 & $\mathrm{A} 2$ \\
\hline TIGD3|220359 & $\mathrm{A} 2$ \\
\hline TIGD5|84948 & $\mathrm{A} 2$ \\
\hline TIGIT|201633 & $\mathrm{A} 2$ \\
\hline TIMELESS|8914 & $\mathrm{A} 2$ \\
\hline TIMM16|51025 & $\mathrm{A} 2$ \\
\hline TIMM17B|10245 & $\mathrm{A} 2$ \\
\hline TIMM8A|1678 & $\mathrm{A} 2$ \\
\hline TIMP1|7076 & $\mathrm{A} 2$ \\
\hline TIMP4|7079 & $\mathrm{A} 2$ \\
\hline TIPARP|25976 & $\mathrm{A} 2$ \\
\hline TIPIN|54962 & $\mathrm{A} 2$ \\
\hline TIPRL|261726 & $\mathrm{A} 2$ \\
\hline TJP1|7082 & $\mathrm{A} 2$ \\
\hline TJP2|9414 & $\mathrm{A} 2$ \\
\hline TJP3|27134 & $\mathrm{A} 2$ \\
\hline TK1|7083 & $\mathrm{A} 2$ \\
\hline TK2|7084 & A2 \\
\hline TKTL1|8277 & $\mathrm{A} 2$ \\
\hline TLE2|7089 & $\mathrm{A} 2$ \\
\hline TLE3|7090 & $\mathrm{A} 2$ \\
\hline TLE6|79816 & $\mathrm{A} 2$ \\
\hline TLL2|7093 & $\mathrm{A} 2$ \\
\hline TLN2|83660 & A2 \\
\hline TLR3|7098 & $\mathrm{A} 2$ \\
\hline TLR4|7099 & $\mathrm{A} 2$ \\
\hline TM4SF18|116441 & $\mathrm{A} 2$ \\
\hline TM6SF2|53345 & $\mathrm{A} 2$ \\
\hline TM9SF1|10548 & A2 \\
\hline TM9SF2|9375 & $\mathrm{A} 2$ \\
\hline
\end{tabular}




\begin{tabular}{|c|c|}
\hline TM9SF4|9777 & A2 \\
\hline TMBIM6|7009 & A2 \\
\hline TMC4|147798 & $\mathrm{A} 2$ \\
\hline TMC6|11322 & $\mathrm{A} 2$ \\
\hline TMCC $3 \mid 57458$ & $\mathrm{~A} 2$ \\
\hline TMCO1|54499 & $\mathrm{A} 2$ \\
\hline TMCO3|55002 & $\mathrm{A} 2$ \\
\hline TMCO4|255104 & $\mathrm{A} 2$ \\
\hline TMCO6|55374 & $\mathrm{A} 2$ \\
\hline TMCO7|79613 & $\mathrm{A} 2$ \\
\hline TMED10P1|286102 & $\mathrm{A} 2$ \\
\hline TMED2|10959 & A2 \\
\hline TMED3|23423 & $\mathrm{A} 2$ \\
\hline TMED4|222068 & $\mathrm{A} 2$ \\
\hline TMED7|51014 & $\mathrm{A} 2$ \\
\hline TMED9|54732 & $\mathrm{A} 2$ \\
\hline TMEM100|55273 & A2 \\
\hline TMEM104|54868 & $\mathrm{A} 2$ \\
\hline TMEM106C|79022 & A2 \\
\hline TMEM109|79073 & $\mathrm{A} 2$ \\
\hline TMEM117|84216 & $\mathrm{A} 2$ \\
\hline TMEM120B|144404 & $\mathrm{A} 2$ \\
\hline TMEM127|55654 & $\mathrm{A} 2$ \\
\hline TMEM132A|54972 & $\mathrm{A} 2$ \\
\hline TMEM133|83935 & $\mathrm{A} 2$ \\
\hline TMEM134|80194 & $\mathrm{A} 2$ \\
\hline TMEM141|85014 & $\mathrm{A} 2$ \\
\hline TMEM147|10430 & $\mathrm{A} 2$ \\
\hline TMEM14A|28978 & $\mathrm{A} 2$ \\
\hline TMEM165|55858 & $\mathrm{A} 2$ \\
\hline TMEM170A|124491 & $\mathrm{A} 2$ \\
\hline TMEM170B|100113407 & $\mathrm{A} 2$ \\
\hline TMEM178|130733 & $\mathrm{A} 2$ \\
\hline TMEM179B|374395 & $\mathrm{A} 2$ \\
\hline TMEM183A|92703 & $\mathrm{A} 2$ \\
\hline TMEM18|129787 & $\mathrm{A} 2$ \\
\hline TMEM191A|84222 & $\mathrm{A} 2$ \\
\hline TMEM194A|23306 & $\mathrm{A} 2$ \\
\hline TMEM195|392636 & A2 \\
\hline TMEM199|147007 & $\mathrm{A} 2$ \\
\hline TMEM200B|399474 & A2 \\
\hline TMEM205|374882 & $\mathrm{A} 2$ \\
\hline TMEM206|55248 & A2 \\
\hline TMEM208|29100 & $\mathrm{A} 2$ \\
\hline TMEM209|84928 & A2 \\
\hline TMEM218|219854 & $\mathrm{A} 2$ \\
\hline TMEM223|79064 & $\mathrm{A} 2$ \\
\hline TMEM33|55161 & $\mathrm{A} 2$ \\
\hline TMEM41A|90407 & $\mathrm{A} 2$ \\
\hline TMEM42|131616 & A2 \\
\hline TMEM43|79188 & $\mathrm{A} 2$ \\
\hline
\end{tabular}




\begin{tabular}{|c|c|}
\hline TMEM47|83604 & A2 \\
\hline TMEM54|113452 & $\mathrm{A} 2$ \\
\hline TMEM55A|55529 & A2 \\
\hline TMEM57|55219 & A2 \\
\hline TMEM69|51249 & A2 \\
\hline TMEM71|137835 & $\mathrm{A} 2$ \\
\hline TMEM80|283232 & A2 \\
\hline TMEM87B|84910 & A2 \\
\hline TMEM88|92162 & A2 \\
\hline TMEM90B|79953 & A2 \\
\hline TMEM9B|56674 & A2 \\
\hline TMEM9|252839 & A2 \\
\hline TMIE|259236 & $\mathrm{A} 2$ \\
\hline TMLHE|55217 & A2 \\
\hline TMSB10|9168 & A2 \\
\hline TMTC3|160418 & $\mathrm{A} 2$ \\
\hline TMTC4|84899 & $\mathrm{A} 2$ \\
\hline TMX1|81542 & A2 \\
\hline TNFAIP8L1|126282 & $\mathrm{A} 2$ \\
\hline TNFRSF10D|8793 & $\mathrm{A} 2$ \\
\hline TNFRSF18|8784 & A2 \\
\hline TNFRSF1B|7133 & $\mathrm{A} 2$ \\
\hline TNFRSF21|27242 & $\mathrm{A} 2$ \\
\hline TNFRSF4|7293 & $\mathrm{A} 2$ \\
\hline TNFRSF8|943 & $\mathrm{A} 2$ \\
\hline TNFSF12|8742 & A2 \\
\hline TNFSF4|7292 & $\mathrm{A} 2$ \\
\hline TNRC6B|23112 & $\mathrm{A} 2$ \\
\hline TNXB|7148 & A2 \\
\hline TOM1L2|146691 & $\mathrm{A} 2$ \\
\hline TOMM40L|84134 & $\mathrm{A} 2$ \\
\hline TOMM40|10452 & $\mathrm{A} 2$ \\
\hline TOMM6|100188893 & $\mathrm{A} 2$ \\
\hline TOMM7|54543 & $\mathrm{A} 2$ \\
\hline TOP1P2|7152 & $\mathrm{A} 2$ \\
\hline TOP1|7150 & A2 \\
\hline TOPBP1|11073 & $\mathrm{A} 2$ \\
\hline TOR1A|1861 & $\mathrm{A} 2$ \\
\hline TOR1B|27348 & $\mathrm{A} 2$ \\
\hline TOR2A|27433 & $\mathrm{A} 2$ \\
\hline TOR3A|64222 & $\mathrm{A} 2$ \\
\hline TOX3|27324 & $\mathrm{A} 2$ \\
\hline TP53AIP1|63970 & $\mathrm{A} 2$ \\
\hline TP53BP2|7159 & $\mathrm{A} 2$ \\
\hline TP53I11|9537 & $\mathrm{A} 2$ \\
\hline TP53INP1|94241 & $\mathrm{A} 2$ \\
\hline TP53RK|112858 & $\mathrm{A} 2$ \\
\hline TP53TG3B|729355 & $\mathrm{A} 2$ \\
\hline TP63|8626 & $\mathrm{A} 2$ \\
\hline TPD52|7163 & $\mathrm{A} 2$ \\
\hline TPI1P2|286016 & A2 \\
\hline
\end{tabular}




\begin{tabular}{|c|c|}
\hline TPI1|7167 & A2 \\
\hline TPM4|7171 & $\mathrm{A} 2$ \\
\hline TPMT|7172 & $\mathrm{A} 2$ \\
\hline TPPP2|122664 & $\mathrm{A} 2$ \\
\hline TPRKB|51002 & $\mathrm{A} 2$ \\
\hline TPRN|286262 & $\mathrm{A} 2$ \\
\hline TPT1|7178 & $\mathrm{A} 2$ \\
\hline TPX2|22974 & $\mathrm{A} 2$ \\
\hline TRAF2|7186 & $\mathrm{A} 2$ \\
\hline TRAF5|7188 & $\mathrm{A} 2$ \\
\hline TRAF6|7189 & $\mathrm{A} 2$ \\
\hline TRAF7|84231 & $\mathrm{A} 2$ \\
\hline TRAIP|10293 & $\mathrm{A} 2$ \\
\hline TRAPPC9|83696 & $\mathrm{A} 2$ \\
\hline TRDMT1|1787 & $\mathrm{A} 2$ \\
\hline TREH|11181 & $\mathrm{A} 2$ \\
\hline TREM1|54210 & $\mathrm{A} 2$ \\
\hline TRHDE|29953 & $\mathrm{A} 2$ \\
\hline TRIB3|57761 & $\mathrm{A} 2$ \\
\hline TRIM23|373 & $\mathrm{A} 2$ \\
\hline TRIM24|8805 & $\mathrm{A} 2$ \\
\hline TRIM26|7726 & $\mathrm{A} 2$ \\
\hline TRIM27|5987 & $\mathrm{A} 2$ \\
\hline TRIM28|10155 & $\mathrm{A} 2$ \\
\hline TRIM29|23650 & $\mathrm{A} 2$ \\
\hline TRIM2|23321 & $\mathrm{A} 2$ \\
\hline TRIM35|23087 & $\mathrm{A} 2$ \\
\hline TRIM46|80128 & $\mathrm{A} 2$ \\
\hline TRIM50|135892 & $\mathrm{A} 2$ \\
\hline TRIM56|81844 & $\mathrm{A} 2$ \\
\hline TRIM61|391712 & $\mathrm{A} 2$ \\
\hline TRMT11|60487 & $\mathrm{A} 2$ \\
\hline TRMT12|55039 & $\mathrm{A} 2$ \\
\hline TRMT5|57570 & $\mathrm{A} 2$ \\
\hline TRMT6|51605 & $\mathrm{A} 2$ \\
\hline TRNP1|388610 & $\mathrm{A} 2$ \\
\hline TROAP|10024 & $\mathrm{A} 2$ \\
\hline TROVE2|6738 & $\mathrm{A} 2$ \\
\hline TRO|7216 & $\mathrm{A} 2$ \\
\hline TRPM2|7226 & $\mathrm{A} 2$ \\
\hline TRPM3|80036 & $\mathrm{A} 2$ \\
\hline TRPM6|140803 & $\mathrm{A} 2$ \\
\hline TRUB1|142940 & $\mathrm{A} 2$ \\
\hline TSC22D1|8848 & $\mathrm{A} 2$ \\
\hline TSC22D3|1831 & $\mathrm{A} 2$ \\
\hline TSEN15|116461 & $\mathrm{A} 2$ \\
\hline TSEN34|79042 & $\mathrm{A} 2$ \\
\hline TSFM|10102 & $\mathrm{A} 2$ \\
\hline TSG101|7251 & $\mathrm{A} 2$ \\
\hline TSHZ1|10194 & $\mathrm{A} 2$ \\
\hline TSKS|60385 & $\mathrm{A} 2$ \\
\hline
\end{tabular}




\begin{tabular}{|c|c|}
\hline TSNAX|7257 & A2 \\
\hline TSPAN11|441631 & $\mathrm{A} 2$ \\
\hline TSPAN13|27075 & $\mathrm{A} 2$ \\
\hline TSPAN14|81619 & $\mathrm{A} 2$ \\
\hline TSPAN15|23555 & A2 \\
\hline TSPAN17|26262 & $\mathrm{A} 2$ \\
\hline TSPAN1|10103 & $\mathrm{A} 2$ \\
\hline TSPAN3|10099 & $\mathrm{A} 2$ \\
\hline TSPAN6|7105 & $\mathrm{A} 2$ \\
\hline TSPAN7|7102 & $\mathrm{A} 2$ \\
\hline TSSC1|7260 & $\mathrm{A} 2$ \\
\hline TSSK4|283629 & A2 \\
\hline TSTD1|100131187 & $\mathrm{A} 2$ \\
\hline TTBK2|146057 & $\mathrm{A} 2$ \\
\hline TTC13|79573 & $\mathrm{A} 2$ \\
\hline TTC33|23548 & $\mathrm{A} 2$ \\
\hline TTC $38 \mid 55020$ & $\mathrm{~A} 2$ \\
\hline TTC39A|22996 & $\mathrm{A} 2$ \\
\hline TTC9B|148014 & $\mathrm{A} 2$ \\
\hline TTC9C|283237 & $\mathrm{A} 2$ \\
\hline \begin{tabular}{l|l|} 
TTC9|23508 \\
\end{tabular} & $\mathrm{A} 2$ \\
\hline TTK|7272 & $\mathrm{A} 2$ \\
\hline TTLL10|254173 & $\mathrm{A} 2$ \\
\hline TTLL12|23170 & $\mathrm{A} 2$ \\
\hline TTYH3|80727 & $\mathrm{A} 2$ \\
\hline TUBA1B|10376 & $\mathrm{A} 2$ \\
\hline TUBB2C|10383 & $\mathrm{A} 2$ \\
\hline TUBB3|10381 & $\mathrm{A} 2$ \\
\hline TUBB8|347688 & $\mathrm{A} 2$ \\
\hline TUBB|203068 & $\mathrm{A} 2$ \\
\hline TUBG1|7283 & $\mathrm{A} 2$ \\
\hline TUBGCP6|85378 & $\mathrm{A} 2$ \\
\hline TUFM|7284 & $\mathrm{A} 2$ \\
\hline TUFT1|7286 & $\mathrm{A} 2$ \\
\hline TULP2|7288 & $\mathrm{A} 2$ \\
\hline TULP4|56995 & $\mathrm{A} 2$ \\
\hline TWF1|5756 & $\mathrm{A} 2$ \\
\hline TWIST1|7291 & $\mathrm{A} 2$ \\
\hline TWSG1|57045 & A2 \\
\hline TXNDC11|51061 & $\mathrm{A} 2$ \\
\hline TXNDC12|51060 & $\mathrm{A} 2$ \\
\hline TXNDC17|84817 & $\mathrm{A} 2$ \\
\hline TXNDC2|84203 & $\mathrm{A} 2$ \\
\hline TXNDC6|347736 & $\mathrm{A} 2$ \\
\hline TXNDC9|10190 & $\mathrm{A} 2$ \\
\hline TXNIP|10628 & $\mathrm{A} 2$ \\
\hline TXNL1|9352 & $\mathrm{A} 2$ \\
\hline TXNL4A|10907 & $\mathrm{A} 2$ \\
\hline TXN|7295 & $\mathrm{A} 2$ \\
\hline TYMP|1890 & $\mathrm{A} 2$ \\
\hline TYMS|7298 & $\mathrm{A} 2$ \\
\hline
\end{tabular}




\begin{tabular}{|c|c|}
\hline TYW3|127253 & A2 \\
\hline U2AF2|11338 & A2 \\
\hline UACA|55075 & $\mathrm{A} 2$ \\
\hline UBA1|7317 & $\mathrm{A} 2$ \\
\hline UBAP1|51271 & $\mathrm{A} 2$ \\
\hline UBAP2L|9898 & $\mathrm{A} 2$ \\
\hline UBE2A|7319 & $\mathrm{A} 2$ \\
\hline UBE2C|11065 & $\mathrm{A} 2$ \\
\hline UBE2D2|7322 & $\mathrm{A} 2$ \\
\hline UBE2E1|7324 & $\mathrm{A} 2$ \\
\hline UBE2E3|10477 & $\mathrm{A} 2$ \\
\hline UBE2F|140739 & $\mathrm{A} 2$ \\
\hline UBE2H|7328 & $\mathrm{A} 2$ \\
\hline UBE2I|7329 & $\mathrm{A} 2$ \\
\hline UBE2L6|9246 & $\mathrm{A} 2$ \\
\hline UBE2MP1|606551 & $\mathrm{A} 2$ \\
\hline UBE2N|7334 & $\mathrm{A} 2$ \\
\hline UBE2O|63893 & $\mathrm{A} 2$ \\
\hline UBE2Q1|55585 & $\mathrm{A} 2$ \\
\hline UBE2Q2P1|388165 & $\mathrm{A} 2$ \\
\hline UBE2S|27338 & $\mathrm{A} 2$ \\
\hline UBE2T|29089 & $\mathrm{A} 2$ \\
\hline UBE2V2|7336 & $\mathrm{A} 2$ \\
\hline UBE2W|55284 & $\mathrm{A} 2$ \\
\hline UBFD1|56061 & $\mathrm{A} 2$ \\
\hline UBL4A|8266 & $\mathrm{A} 2$ \\
\hline UBL5|59286 & $\mathrm{A} 2$ \\
\hline UBN1|29855 & $\mathrm{A} 2$ \\
\hline UBQLN4|56893 & $\mathrm{A} 2$ \\
\hline UBR7|55148 & $\mathrm{A} 2$ \\
\hline UBTD2|92181 & $\mathrm{A} 2$ \\
\hline UBXN4|23190 & $\mathrm{A} 2$ \\
\hline \begin{tabular}{l|l} 
UCHL5|51377 \\
\end{tabular} & $\mathrm{A} 2$ \\
\hline UCK2|7371 & $\mathrm{A} 2$ \\
\hline UFC1|51506 & $\mathrm{A} 2$ \\
\hline UFD1L|7353 & $\mathrm{A} 2$ \\
\hline UFSP2|55325 & $\mathrm{A} 2$ \\
\hline \begin{tabular}{l|l|l|} 
UGGT686 \\
\end{tabular} & $\mathrm{A} 2$ \\
\hline UHRF1|29128 & $\mathrm{A} 2$ \\
\hline UIMC1|51720 & $\mathrm{A} 2$ \\
\hline UNC119B|84747 & $\mathrm{A} 2$ \\
\hline UNC5B|219699 & $\mathrm{A} 2$ \\
\hline UNC93B1|81622 & $\mathrm{A} 2$ \\
\hline UNKL|64718 & $\mathrm{A} 2$ \\
\hline UPRT|139596 & $\mathrm{A} 2$ \\
\hline UQCRQ|27089 & $\mathrm{A} 2$ \\
\hline USF1|7391 & $\mathrm{A} 2$ \\
\hline USHBP1|83878 & $\mathrm{A} 2$ \\
\hline USP11|8237 & $\mathrm{A} 2$ \\
\hline USP14|9097 & $\mathrm{A} 2$ \\
\hline USP18|11274 & $\mathrm{A} 2$ \\
\hline
\end{tabular}




\begin{tabular}{|c|c|}
\hline USP19|10869 & $\mathrm{A} 2$ \\
\hline USP21|27005 & $\mathrm{A} 2$ \\
\hline USP25|29761 & $\mathrm{A} 2$ \\
\hline USP30|84749 & $\mathrm{A} 2$ \\
\hline USP31|57478 & $\mathrm{A} 2$ \\
\hline USP39|10713 & $\mathrm{A} 2$ \\
\hline USP53|54532 & $\mathrm{A} 2$ \\
\hline USP5|8078 & $\mathrm{A} 2$ \\
\hline USP7|7874 & $\mathrm{A} 2$ \\
\hline UST $\mid 10090$ & $\mathrm{~A} 2$ \\
\hline UTS2D|257313 & $\mathrm{A} 2$ \\
\hline UXS1|80146 & $\mathrm{A} 2$ \\
\hline VAMP3|9341 & $\mathrm{A} 2$ \\
\hline VANGL1|81839 & $\mathrm{A} 2$ \\
\hline VARS2|57176 & $\mathrm{A} 2$ \\
\hline VARS|7407 & $\mathrm{A} 2$ \\
\hline VASP|7408 & $\mathrm{A} 2$ \\
\hline VAV2|7410 & $\mathrm{A} 2$ \\
\hline VBP1|7411 & $\mathrm{A} 2$ \\
\hline $\mathrm{VCP} \mid 7415$ & $\mathrm{~A} 2$ \\
\hline VDAC1|7416 & $\mathrm{A} 2$ \\
\hline VENTX|27287 & $\mathrm{A} 2$ \\
\hline VEZT|55591 & $\mathrm{A} 2$ \\
\hline VILL|50853 & $\mathrm{A} 2$ \\
\hline VIPR1|7433 & $\mathrm{A} 2$ \\
\hline VIP|7432 & $\mathrm{A} 2$ \\
\hline VIT|5212 & $\mathrm{A} 2$ \\
\hline VKORC1L1|154807 & $\mathrm{A} 2$ \\
\hline VKORC1|79001 & $\mathrm{A} 2$ \\
\hline VLDLR|7436 & $\mathrm{A} 2$ \\
\hline VOPP1|81552 & $\mathrm{A} 2$ \\
\hline VPS11|55823 & $\mathrm{A} 2$ \\
\hline VPS13A|23230 & $\mathrm{A} 2$ \\
\hline VPS24|51652 & $\mathrm{A} 2$ \\
\hline VPS28|51160 & $\mathrm{A} 2$ \\
\hline VPS33A|65082 & $\mathrm{A} 2$ \\
\hline VPS36|51028 & $\mathrm{A} 2$ \\
\hline VPS37C|55048 & $\mathrm{A} 2$ \\
\hline VPS39|23339 & $\mathrm{A} 2$ \\
\hline VPS45|11311 & $\mathrm{A} 2$ \\
\hline VPS52|6293 & $\mathrm{A} 2$ \\
\hline VRK1|7443 & $\mathrm{A} 2$ \\
\hline VSNL1|7447 & $\mathrm{A} 2$ \\
\hline VWA1|64856 & $\mathrm{A} 2$ \\
\hline VWA5A|4013 & $\mathrm{A} 2$ \\
\hline VWCE|220001 & $\mathrm{A} 2$ \\
\hline WBP1|23559 & $\mathrm{A} 2$ \\
\hline WBP2NL|164684 & $\mathrm{A} 2$ \\
\hline WBSCR22|114049 & $\mathrm{A} 2$ \\
\hline WBSCR28|135886 & $\mathrm{A} 2$ \\
\hline WDFY2|115825 & $\mathrm{A} 2$ \\
\hline
\end{tabular}




\begin{tabular}{|c|c|}
\hline WDHD1|11169 & $\mathrm{A} 2$ \\
\hline WDR11|55717 & $\mathrm{A} 2$ \\
\hline WDR19|57728 & $\mathrm{A} 2$ \\
\hline WDR1|9948 & $\mathrm{A} 2$ \\
\hline WDR24|84219 & $\mathrm{A} 2$ \\
\hline WDR26|80232 & $\mathrm{A} 2$ \\
\hline WDR27|253769 & $\mathrm{A} 2$ \\
\hline WDR37|22884 & $\mathrm{A} 2$ \\
\hline WDR48|57599 & A2 \\
\hline WDR54|84058 & $\mathrm{A} 2$ \\
\hline WDR59|79726 & $\mathrm{A} 2$ \\
\hline WDR67|93594 & $\mathrm{A} 2$ \\
\hline WDR75|84128 & $\mathrm{A} 2$ \\
\hline WDR81|124997 & $\mathrm{A} 2$ \\
\hline WDR90|197335 & $\mathrm{A} 2$ \\
\hline WDYHV1|55093 & $\mathrm{A} 2$ \\
\hline WHAMML1|339005 & $\mathrm{A} 2$ \\
\hline WHAMM|123720 & $\mathrm{A} 2$ \\
\hline WHSC1|7468 & $\mathrm{A} 2$ \\
\hline WIBG $\mid 84305$ & $\mathrm{~A} 2$ \\
\hline WISP1|8840 & $\mathrm{A} 2$ \\
\hline WLS|79971 & $\mathrm{A} 2$ \\
\hline WNK2|65268 & $\mathrm{A} 2$ \\
\hline WNT2|7472 & $\mathrm{A} 2$ \\
\hline WNT7B|7477 & $\mathrm{A} 2$ \\
\hline WRNIP1|56897 & $\mathrm{A} 2$ \\
\hline WSCD1|23302 & $\mathrm{A} 2$ \\
\hline WTAP|9589 & $\mathrm{A} 2$ \\
\hline XIST|7503 & $\mathrm{A} 2$ \\
\hline XPA|7507 & $\mathrm{A} 2$ \\
\hline XPC|7508 & $\mathrm{A} 2$ \\
\hline XPNPEP2|7512 & $\mathrm{A} 2$ \\
\hline XPO1|7514 & $\mathrm{A} 2$ \\
\hline XPO5|57510 & $\mathrm{A} 2$ \\
\hline XPO6|23214 & $\mathrm{A} 2$ \\
\hline XPOT|11260 & $\mathrm{A} 2$ \\
\hline XRCC2|7516 & $\mathrm{A} 2$ \\
\hline XRCC4|7518 & $\mathrm{A} 2$ \\
\hline YARS2|51067 & $\mathrm{A} 2$ \\
\hline YDJC|150223 & $\mathrm{A} 2$ \\
\hline YEATS4|8089 & $\mathrm{A} 2$ \\
\hline YIF1A|10897 & $\mathrm{A} 2$ \\
\hline YIPF1|54432 & $\mathrm{A} 2$ \\
\hline YIPF2|78992 & $\mathrm{A} 2$ \\
\hline YIPF3|25844 & $\mathrm{A} 2$ \\
\hline YKT6|10652 & $\mathrm{A} 2$ \\
\hline YLPM1|56252 & $\mathrm{A} 2$ \\
\hline YME1L1|10730 & $\mathrm{A} 2$ \\
\hline YPEL4|219539 & $\mathrm{A} 2$ \\
\hline YWHAB|7529 & $\mathrm{A} 2$ \\
\hline YWHAH|7533 & $\mathrm{A} 2$ \\
\hline
\end{tabular}




\begin{tabular}{|c|c|}
\hline YWHAZ|7534 & A2 \\
\hline YY1AP1|55249 & $\mathrm{A} 2$ \\
\hline YY1|7528 & $\mathrm{A} 2$ \\
\hline YY2|404281 & $\mathrm{A} 2$ \\
\hline ZAK|51776 & A2 \\
\hline ZBED3|84327 & A2 \\
\hline ZBED4|9889 & $\mathrm{A} 2$ \\
\hline ZBTB12|221527 & $\mathrm{A} 2$ \\
\hline ZBTB16|7704 & A2 \\
\hline ZBTB1|22890 & $\mathrm{A} 2$ \\
\hline ZBTB20|26137 & $\mathrm{A} 2$ \\
\hline ZBTB34|403341 & A2 \\
\hline ZBTB40|9923 & $\mathrm{A} 2$ \\
\hline ZBTB41|360023 & $\mathrm{A} 2$ \\
\hline ZBTB42|100128927 & $\mathrm{A} 2$ \\
\hline ZBTB44|29068 & $\mathrm{A} 2$ \\
\hline ZBTB47|92999 & A2 \\
\hline ZBTB7B|51043 & $\mathrm{A} 2$ \\
\hline ZBTB8OS|339487 & A2 \\
\hline ZC3H12B|340554 & $\mathrm{A} 2$ \\
\hline ZC3H12C|85463 & $\mathrm{A} 2$ \\
\hline ZC3H13|23091 & A2 \\
\hline $\mathrm{ZC} 3 \mathrm{H} 3 \mid 23144$ & $\mathrm{~A} 2$ \\
\hline ZC3H6|376940 & $\mathrm{A} 2$ \\
\hline ZC3H7B|23264 & $\mathrm{A} 2$ \\
\hline ZCCHC10|54819 & $\mathrm{A} 2$ \\
\hline ZCCHC11|23318 & A2 \\
\hline ZCCHC12|170261 & A2 \\
\hline ZCCHC4|29063 & $\mathrm{A} 2$ \\
\hline ZCWPW1|55063 & $\mathrm{A} 2$ \\
\hline ZCWPW2|152098 & $\mathrm{A} 2$ \\
\hline $\begin{array}{l}\text { ZDHHC12|84885 } \\
\end{array}$ & $\mathrm{A} 2$ \\
\hline ZDHHC15|158866 & $\mathrm{A} 2$ \\
\hline ZDHHC16|84287 & A2 \\
\hline ZDHHC1|29800 & $\mathrm{A} 2$ \\
\hline ZDHHC24|254359 & $\mathrm{A} 2$ \\
\hline ZDHHC2|51201 & $\mathrm{A} 2$ \\
\hline ZDHHC6|64429 & A2 \\
\hline ZDHHC9|51114 & A2 \\
\hline ZER1|10444 & $\mathrm{A} 2$ \\
\hline ZFAND5|7763 & A2 \\
\hline ZFHX4|79776 & $\mathrm{A} 2$ \\
\hline ZFP161|7541 & A2 \\
\hline ZFP28|140612 & $\mathrm{A} 2$ \\
\hline ZFP2|80108 & A2 \\
\hline ZFP36|7538 & $\mathrm{A} 2$ \\
\hline ZFP37|7539 & $\mathrm{A} 2$ \\
\hline ZFP62|643836 & $\mathrm{A} 2$ \\
\hline ZFP64|55734 & A2 \\
\hline ZFP90|146198 & A2 \\
\hline ZFPM1|161882 & $\mathrm{A} 2$ \\
\hline
\end{tabular}




\begin{tabular}{|c|c|}
\hline ZFYVE1|53349 & A2 \\
\hline ZFYVE20|64145 & $\mathrm{A} 2$ \\
\hline ZFYVE9|9372 & $\mathrm{A} 2$ \\
\hline ZG16B|124220 & $\mathrm{A} 2$ \\
\hline ZHX3|23051 & A2 \\
\hline ZIC2|7546 & $\mathrm{A} 2$ \\
\hline ZMAT1|84460 & $\mathrm{A} 2$ \\
\hline ZMPSTE24|10269 & A2 \\
\hline ZMYND10|51364 & $\mathrm{A} 2$ \\
\hline ZMYND19|116225 & $\mathrm{A} 2$ \\
\hline ZMYND8|23613 & A2 \\
\hline ZNF10|7556 & $\mathrm{A} 2$ \\
\hline ZNF124|7678 & A2 \\
\hline ZNF133|7692 & $\mathrm{A} 2$ \\
\hline ZNF138|7697 & $\mathrm{A} 2$ \\
\hline ZNF146|7705 & $\mathrm{A} 2$ \\
\hline ZNF154|7710 & A2 \\
\hline ZNF157|7712 & $\mathrm{A} 2$ \\
\hline ZNF160|90338 & $\mathrm{A} 2$ \\
\hline ZNF167|55888 & $\mathrm{A} 2$ \\
\hline ZNF174|7727 & A2 \\
\hline ZNF184|7738 & $\mathrm{A} 2$ \\
\hline ZNF187|7741 & A2 \\
\hline ZNF200|7752 & $\mathrm{A} 2$ \\
\hline ZNF202|7753 & A2 \\
\hline ZNF207|7756 & A2 \\
\hline ZNF213|7760 & $\mathrm{A} 2$ \\
\hline \begin{tabular}{l|l|} 
ZNF219|51222 \\
\end{tabular} & $\mathrm{A} 2$ \\
\hline ZNF22|7570 & A2 \\
\hline ZNF239|8187 & A2 \\
\hline ZNF23|7571 & $\mathrm{A} 2$ \\
\hline ZNF248|57209 & A2 \\
\hline \begin{tabular}{l|l|l|l|} 
ZNF25|219749 \\
\end{tabular} & $\mathrm{A} 2$ \\
\hline ZNF260|339324 & $\mathrm{A} 2$ \\
\hline ZNF267|10308 & A2 \\
\hline ZNF273|10793 & $\overline{\mathrm{A} 2}$ \\
\hline ZNF276|92822 & A2 \\
\hline ZNF281|23528 & $\overline{\mathrm{A} 2}$ \\
\hline ZNF28|7576 & $\mathrm{A} 2$ \\
\hline ZNF296|162979 & $\mathrm{A} 2$ \\
\hline \begin{tabular}{l|l|} 
ZNF319|57567 \\
\end{tabular} & $\mathrm{A} 2$ \\
\hline ZNF330|27309 & $\mathrm{A} 2$ \\
\hline ZNF331|55422 & A2 \\
\hline \begin{tabular}{l|l|} 
ZNF333|84449 \\
\end{tabular} & $\mathrm{A} 2$ \\
\hline ZNF334|55713 & A2 \\
\hline ZNF337|26152 & A2 \\
\hline ZNF341|84905 & $\mathrm{A} 2$ \\
\hline ZNF345|25850 & $\mathrm{A} 2$ \\
\hline ZNF354B|117608 & $\mathrm{A} 2$ \\
\hline ZNF354C|30832 & A2 \\
\hline ZNF366|167465 & $\mathrm{A} 2$ \\
\hline
\end{tabular}




\begin{tabular}{|c|c|}
\hline ZNF367|195828 & A2 \\
\hline ZNF37B|100129482 & A2 \\
\hline ZNF383|163087 & $\mathrm{A} 2$ \\
\hline ZNF389|651302 & $\mathrm{A} 2$ \\
\hline ZNF397OS|100101467 & $\mathrm{A} 2$ \\
\hline ZNF397|84307 & A2 \\
\hline ZNF407|55628 & $\mathrm{A} 2$ \\
\hline ZNF410|57862 & $\mathrm{A} 2$ \\
\hline ZNF434|54925 & A2 \\
\hline ZNF438|220929 & $\mathrm{A} 2$ \\
\hline ZNF43|7594 & $\mathrm{A} 2$ \\
\hline ZNF443|10224 & $\mathrm{A} 2$ \\
\hline ZNF444|55311 & $\mathrm{A} 2$ \\
\hline ZNF449|203523 & $\mathrm{A} 2$ \\
\hline ZNF454|285676 & $\mathrm{A} 2$ \\
\hline \begin{tabular}{l|l|} 
ZNF462|58499 \\
\end{tabular} & $\mathrm{A} 2$ \\
\hline ZNF467|168544 & $\mathrm{A} 2$ \\
\hline ZNF468|90333 & $\mathrm{A} 2$ \\
\hline $\begin{array}{l}\text { ZNF469|84627 } \\
\end{array}$ & A2 \\
\hline ZNF470|388566 & $\mathrm{A} 2$ \\
\hline ZNF471|57573 & A2 \\
\hline ZNF480|147657 & $\mathrm{A} 2$ \\
\hline ZNF483|158399 & $\mathrm{A} 2$ \\
\hline $\begin{array}{l}\text { ZNF488|118738 } \\
\end{array}$ & $\mathrm{A} 2$ \\
\hline \begin{tabular}{l|l|} 
ZNF490|57474 \\
\end{tabular} & $\mathrm{A} 2$ \\
\hline ZNF498|221785 & $\mathrm{A} 2$ \\
\hline ZNF501|115560 & $\mathrm{A} 2$ \\
\hline ZNF507|22847 & $\mathrm{A} 2$ \\
\hline ZNF521|25925 & $\mathrm{A} 2$ \\
\hline ZNF525|170958 & $\mathrm{A} 2$ \\
\hline ZNF527|84503 & $\mathrm{A} 2$ \\
\hline ZNF540|163255 & $\mathrm{A} 2$ \\
\hline ZNF544|27300 & $\mathrm{A} 2$ \\
\hline ZNF546|339327 & A2 \\
\hline ZNF552|79818 & $\mathrm{A} 2$ \\
\hline ZNF554|115196 & $\mathrm{A} 2$ \\
\hline $\begin{array}{l}\text { ZNF559|84527 } \\
\end{array}$ & $\mathrm{A} 2$ \\
\hline \begin{tabular}{l|l|} 
ZNF561|93134 \\
\end{tabular} & $\mathrm{A} 2$ \\
\hline ZNF587|84914 & A2 \\
\hline ZNF592|9640 & $\mathrm{A} 2$ \\
\hline ZNF596|169270 & A2 \\
\hline ZNF598|90850 & $\mathrm{A} 2$ \\
\hline \begin{tabular}{l|l|} 
ZNF608|57507 &
\end{tabular} & $\mathrm{A} 2$ \\
\hline ZNF642|339559 & $\mathrm{A} 2$ \\
\hline ZNF643|65243 & A2 \\
\hline ZNF646|9726 & $\mathrm{A} 2$ \\
\hline \begin{tabular}{l|l|l|} 
ZNF655|79027 \\
\end{tabular} & $\mathrm{A} 2$ \\
\hline ZNF658|26149 & $\mathrm{A} 2$ \\
\hline ZNF662|389114 & $\mathrm{A} 2$ \\
\hline ZNF667|63934 & A2 \\
\hline ZNF668|79759 & $\mathrm{A} 2$ \\
\hline
\end{tabular}




\begin{tabular}{|c|c|}
\hline ZNF672|79894 & A2 \\
\hline ZNF673|55634 & A2 \\
\hline ZNF675|171392 & $\mathrm{A} 2$ \\
\hline ZNF677|342926 & $\mathrm{A} 2$ \\
\hline ZNF678|339500 & $\mathrm{A} 2$ \\
\hline ZNF681|148213 & $\mathrm{A} 2$ \\
\hline ZNF682|91120 & $\mathrm{A} 2$ \\
\hline ZNF687|57592 & $\mathrm{A} 2$ \\
\hline ZNF695|57116 & A2 \\
\hline ZNF699|374879 & $\mathrm{A} 2$ \\
\hline ZNF704|619279 & $\mathrm{A} 2$ \\
\hline ZNF727|442319 & A2 \\
\hline ZNF767|79970 & $\mathrm{A} 2$ \\
\hline ZNF768|79724 & $\mathrm{A} 2$ \\
\hline ZNF771|51333 & $\mathrm{A} 2$ \\
\hline ZNF778|197320 & $\mathrm{A} 2$ \\
\hline ZNF843|283933 & $\mathrm{A} 2$ \\
\hline \begin{tabular}{l|l|l|} 
ZNF853|54753 \\
\end{tabular} & $\mathrm{A} 2$ \\
\hline ZNF85|7639 & A2 \\
\hline ZNHIT2|741 & $\mathrm{A} 2$ \\
\hline ZNRF2|223082 & $\mathrm{A} 2$ \\
\hline ZRANB1|54764 & $\mathrm{A} 2$ \\
\hline ZRANB2|9406 & $\mathrm{A} 2$ \\
\hline ZSCAN12|9753 & $\mathrm{A} 2$ \\
\hline \begin{tabular}{l|l|l|} 
ZSCAN2322696 \\
\end{tabular} & $\mathrm{A} 2$ \\
\hline ZSWIM7|125150 & $\mathrm{A} 2$ \\
\hline ZWILCH|55055 & $\mathrm{A} 2$ \\
\hline ZWINT|11130 & $\mathrm{A} 2$ \\
\hline ZYG11A|440590 & $\mathrm{A} 2$ \\
\hline ZZEF1|23140 & $\mathrm{A} 2$ \\
\hline AAGAB|79719 & $\mathrm{A} 3$ \\
\hline ABCC6P1|653190 & $\mathrm{A} 3$ \\
\hline ABCC9|10060 & $\mathrm{A} 3$ \\
\hline ABHD11|83451 & $\mathrm{A} 3$ \\
\hline ABI3BP|25890 & $\mathrm{A} 3$ \\
\hline ACAA2|10449 & $\mathrm{A} 3$ \\
\hline ACADL/33 & $\mathrm{A} 3$ \\
\hline ACO1|48 & $\mathrm{A} 3$ \\
\hline ACSL4|2182 & $\mathrm{A} 3$ \\
\hline ACVRL1|94 & $\mathrm{A} 3$ \\
\hline ADAMTSL4|54507 & A3 \\
\hline ADCY3|109 & $\mathrm{A} 3$ \\
\hline ADM2|79924 & A3 \\
\hline ADM|133 & $\mathrm{A} 3$ \\
\hline ADNP|23394 & $\mathrm{A} 3$ \\
\hline ADPRH|141 & $\mathrm{A} 3$ \\
\hline AFAP1L1|134265 & $\mathrm{A} 3$ \\
\hline AFTPH|54812 & $\mathrm{A} 3$ \\
\hline AGPAT4|56895 & $\mathrm{A} 3$ \\
\hline AIFM2|84883 & A3 \\
\hline AKAP12|9590 & $\mathrm{A} 3$ \\
\hline
\end{tabular}




\begin{tabular}{|c|c|}
\hline AKIRIN2|55122 & $\mathrm{A} 3$ \\
\hline AMACR/23600 & $\mathrm{A} 3$ \\
\hline ANAPC7|51434 & $\mathrm{A} 3$ \\
\hline ANGPT1|284 & $\mathrm{A} 3$ \\
\hline ANGPT4|51378 & A3 \\
\hline ANGPTL2|23452 & A3 \\
\hline ANGPTL4|51129 & $\mathrm{A} 3$ \\
\hline ANKRD32|84250 & $\mathrm{A} 3$ \\
\hline ANO4|121601 & $\mathrm{A} 3$ \\
\hline ANO6|196527 & $\mathrm{A} 3$ \\
\hline ANTXR2|118429 & $\mathrm{A} 3$ \\
\hline ANXA9|8416 & A3 \\
\hline AOC2|314 & $\mathrm{A} 3$ \\
\hline AOC3|8639 & $\mathrm{A} 3$ \\
\hline AOX1|316 & $\mathrm{A} 3$ \\
\hline AP1AR|55435 & $\mathrm{A} 3$ \\
\hline APBB1IP|54518 & A3 \\
\hline APOL3|80833 & $\mathrm{A} 3$ \\
\hline APOLD1|81575 & A3 \\
\hline AQP7|364 & $\mathrm{A} 3$ \\
\hline ARF6|382 & $\mathrm{A} 3$ \\
\hline ARFGEF1|10565 & $\mathrm{A} 3$ \\
\hline ARHGAP11B|89839 & $\mathrm{A} 3$ \\
\hline ARHGAP17|55114 & $\mathrm{A} 3$ \\
\hline ARHGAP31|57514 & $\mathrm{A} 3$ \\
\hline ARHGEF15|22899 & $\mathrm{A} 3$ \\
\hline ARHGEF16|27237 & $\mathrm{A} 3$ \\
\hline ARID1A|8289 & A3 \\
\hline $\begin{array}{l}\text { ARMC9|80210 } \\
\end{array}$ & $\mathrm{A} 3$ \\
\hline ARRDC1|92714 & $\mathrm{A} 3$ \\
\hline ASAM|79827 & $\mathrm{A} 3$ \\
\hline ASPA|443 & $\mathrm{A} 3$ \\
\hline ASPHD2|57168 & $\mathrm{A} 3$ \\
\hline ATAD5|79915 & A3 \\
\hline ATF6|22926 & A3 \\
\hline ATN1|1822 & $\mathrm{A} 3$ \\
\hline ATP6V1A|523 & $\mathrm{A} 3$ \\
\hline ATP6V1G1|9550 & $\mathrm{A} 3$ \\
\hline ATP8B4|79895 & A3 \\
\hline ATXN2L|11273 & $\mathrm{A} 3$ \\
\hline AVL9|23080 & A3 \\
\hline B4GALNT4|338707 & $\mathrm{A} 3$ \\
\hline BACE1|23621 & A3 \\
\hline BARD1|580 & $\mathrm{A} 3$ \\
\hline BAT2L2|23215 & $\mathrm{A} 3$ \\
\hline BAZ1A|11177 & $\mathrm{A} 3$ \\
\hline BEST1|7439 & $\mathrm{A} 3$ \\
\hline BHMT2|23743 & $\mathrm{A} 3$ \\
\hline BIN3|55909 & $\mathrm{A} 3$ \\
\hline BMP2|650 & A3 \\
\hline BMP5|653 & A3 \\
\hline
\end{tabular}




\begin{tabular}{|c|c|}
\hline ВМР6|654 & A3 \\
\hline BMPR1A 657 & $\mathrm{~A} 3$ \\
\hline BRCA2|675 & $\mathrm{A} 3$ \\
\hline BRIP1|83990 & $\mathrm{A} 3$ \\
\hline BSPRY|54836 & $\mathrm{A} 3$ \\
\hline BST1|683 & $\mathrm{A} 3$ \\
\hline BTBD12|84464 & $\mathrm{A} 3$ \\
\hline BYSL|705 & $\mathrm{A} 3$ \\
\hline BZW1|9689 & A3 \\
\hline C10orf25|220979 & $\mathrm{A} 3$ \\
\hline C10orf72|196740 & $\mathrm{A} 3$ \\
\hline C10orf90|118611 & $\mathrm{A} 3$ \\
\hline C12orf59|120939 & $\mathrm{A} 3$ \\
\hline C12orf66|144577 & $\mathrm{A} 3$ \\
\hline C13orf33|84935 & $\mathrm{A} 3$ \\
\hline C14orf139|79686 & $\mathrm{A} 3$ \\
\hline C14orf49|161176 & A3 \\
\hline C16orf93|90835 & $\mathrm{A} 3$ \\
\hline C19orf12|83636 & A3 \\
\hline C19orf76|199800 & $\mathrm{A} 3$ \\
\hline C1QTNF1|114897 & $\mathrm{A} 3$ \\
\hline C1QTNF7|114905 & $\mathrm{A} 3$ \\
\hline C1R/715 & $\mathrm{A} 3$ \\
\hline C1orf123|54987 & $\mathrm{A} 3$ \\
\hline C1orf74|148304 & $\mathrm{A} 3$ \\
\hline C20orf94|128710 & $\mathrm{A} 3$ \\
\hline C21 orf58|54058 & $\mathrm{A} 3$ \\
\hline $\begin{array}{l}\mathrm{C} 2 \mathrm{CD} 2 \mid 25966 \\
\end{array}$ & $\mathrm{~A} 3$ \\
\hline $\mathrm{C} 2 \mathrm{CD} 4 \mathrm{C} \mid 126567$ & $\mathrm{~A} 3$ \\
\hline C2orf15|150590 & $\mathrm{A} 3$ \\
\hline C2orf84|653140 & $\mathrm{A} 3$ \\
\hline C3orf55|152078 & $\mathrm{A} 3$ \\
\hline $\mathrm{C} 3 \mid 718$ & $\mathrm{~A} 3$ \\
\hline C4orf52|389203 & $\mathrm{A} 3$ \\
\hline C5orf32|84418 & $\mathrm{A} 3$ \\
\hline C5orf34|375444 & $\mathrm{A} 3$ \\
\hline C6orf201|404220 & $\mathrm{A} 3$ \\
\hline C6orf72|116254 & $\mathrm{A} 3$ \\
\hline C7orf31|136895 & A3 \\
\hline C7orf41|222166 & $\mathrm{A} 3$ \\
\hline \begin{tabular}{l|l|l|} 
C7orf43 5262 \\
\end{tabular} & A3 \\
\hline $\mathrm{C} 7 \mid 730$ & A3 \\
\hline C9orf91|203197 & $\mathrm{A} 3$ \\
\hline \begin{tabular}{ll|l|} 
CA12|771 & \\
\end{tabular} & $\mathrm{A} 3$ \\
\hline $\begin{array}{l}\text { CALB2|794 } \\
\end{array}$ & A3 \\
\hline CALD1|800 & $\mathrm{A} 3$ \\
\hline CAMK1|8536 & $\mathrm{A} 3$ \\
\hline CANX|821 & $\mathrm{A} 3$ \\
\hline CAPN13|92291 & $\mathrm{A} 3$ \\
\hline $\begin{array}{l}\text { CAPN5|726 } \\
\text { CAP }\end{array}$ & A3 \\
\hline CASKIN2|57513 & $\mathrm{A} 3$ \\
\hline
\end{tabular}




\begin{tabular}{|c|c|}
\hline $\mathrm{CAT} \mid 847$ & A3 \\
\hline CCDC107|203260 & A3 \\
\hline CCDC17|149483 & $\mathrm{A} 3$ \\
\hline CCDC36|339834 & $\mathrm{A} 3$ \\
\hline CCDC3|83643 & $\mathrm{A} 3$ \\
\hline CCDC69|26112 & $\mathrm{A} 3$ \\
\hline CCDC80|151887 & $\mathrm{A} 3$ \\
\hline CCDC85A|114800 & $\mathrm{A} 3$ \\
\hline CCL21|6366 & $\mathrm{A} 3$ \\
\hline CCL23|6368 & $\mathrm{A} 3$ \\
\hline CCND3|896 & $\mathrm{A} 3$ \\
\hline CCRL1|51554 & $\mathrm{A} 3$ \\
\hline CCT2|10576 & $\mathrm{A} 3$ \\
\hline CD248|57124 & $\mathrm{A} 3$ \\
\hline CD2AP|23607 & $\mathrm{A} 3$ \\
\hline $\begin{array}{ll}\text { CD36|948 } \\
\end{array}$ & $\mathrm{A} 3$ \\
\hline CD81|975 & $\mathrm{A} 3$ \\
\hline CD93|22918 & $\mathrm{A} 3$ \\
\hline $\begin{array}{l}\text { CD99L2|83692 } \\
\end{array}$ & $\mathrm{A} 3$ \\
\hline CDC42EP2|10435 & $\mathrm{A} 3$ \\
\hline CDC6|990 & $\mathrm{A} 3$ \\
\hline CDCP1|64866 & $\mathrm{A} 3$ \\
\hline CDH13|1012 & $\mathrm{A} 3$ \\
\hline CDH5|1003 & $\mathrm{A} 3$ \\
\hline CDO1|1036 & $\mathrm{A} 3$ \\
\hline CDS1|1040 & $\mathrm{A} 3$ \\
\hline CEBPA $\mid 1050$ & $\mathrm{~A} 3$ \\
\hline CEP70|80321 & $\mathrm{A} 3$ \\
\hline CES1|1066 & $\mathrm{A} 3$ \\
\hline CFH|3075 & $\mathrm{A} 3$ \\
\hline CHML|1122 & $\mathrm{A} 3$ \\
\hline CHRDL1|91851 & $\mathrm{A} 3$ \\
\hline CHTF18|63922 & $\mathrm{A} 3$ \\
\hline CHURC1|91612 & $\mathrm{A} 3$ \\
\hline CIDEC|63924 & $\mathrm{A} 3$ \\
\hline CISH|1154 & $\mathrm{A} 3$ \\
\hline CLCNKB|1188 & $\mathrm{A} 3$ \\
\hline CLDND2|125875 & $\mathrm{A} 3$ \\
\hline CLEC14A|161198 & $\mathrm{A} 3$ \\
\hline CLEC3B|7123 & $\mathrm{A} 3$ \\
\hline CLEC4G|339390 & $\mathrm{A} 3$ \\
\hline CLIC2|1193 & $\mathrm{A} 3$ \\
\hline CLN3|1201 & $\mathrm{A} 3$ \\
\hline CMTM7|112616 & $\mathrm{A} 3$ \\
\hline \begin{tabular}{l|l|} 
CNDP2|55748 \\
\end{tabular} & $\mathrm{A} 3$ \\
\hline CNKSR2|22866 & $\mathrm{A} 3$ \\
\hline CNOT10|25904 & $\mathrm{A} 3$ \\
\hline CNOT6|57472 & $\mathrm{A} 3$ \\
\hline CNRIP1|25927 & $\mathrm{A} 3$ \\
\hline CNTFR|1271 & $\mathrm{A} 3$ \\
\hline COMMD6|170622 & $\mathrm{A} 3$ \\
\hline
\end{tabular}




\begin{tabular}{|c|c|}
\hline CPNE2|221184 & $\mathrm{A} 3$ \\
\hline CPSF6|11052 & $\mathrm{A} 3$ \\
\hline CRHR2|1395 & $\mathrm{A} 3$ \\
\hline CRNKL1|51340 & $\mathrm{A} 3$ \\
\hline CRTAP|10491 & A3 \\
\hline CSNK2A2|1459 & $\mathrm{A} 3$ \\
\hline CSRP2|1466 & $\mathrm{A} 3$ \\
\hline CSTF1|1477 & $\mathrm{A} 3$ \\
\hline CSTF3|1479 & $\mathrm{A} 3$ \\
\hline CTDSP1|58190 & $\mathrm{A} 3$ \\
\hline CXCR7|57007 & $\mathrm{A} 3$ \\
\hline CYB5R3|1727 & A3 \\
\hline CYGB|114757 & $\mathrm{A} 3$ \\
\hline CYP26B1|56603 & $\mathrm{A} 3$ \\
\hline CYP4F12|66002 & $\mathrm{A} 3$ \\
\hline CYTL1|54360 & $\mathrm{A} 3$ \\
\hline DAAM2|23500 & $\mathrm{A} 3$ \\
\hline DAB2|1601 & $\mathrm{A} 3$ \\
\hline DBR1|51163 & $\mathrm{A} 3$ \\
\hline DCAF7|10238 & $\mathrm{A} 3$ \\
\hline DCHS1|8642 & $\mathrm{A} 3$ \\
\hline DCLRE1A|9937 & $\mathrm{A} 3$ \\
\hline DCN|1634 & $\mathrm{A} 3$ \\
\hline DEF8|54849 & $\mathrm{A} 3$ \\
\hline DENND2D|79961 & $\mathrm{A} 3$ \\
\hline DENR|8562 & $\mathrm{A} 3$ \\
\hline DFNA5|1687 & A3 \\
\hline DGAT2|84649 & $\mathrm{A} 3$ \\
\hline DHDDS|79947 & $\mathrm{A} 3$ \\
\hline DHRS13|147015 & $\mathrm{A} 3$ \\
\hline DHRS3|9249 & $\mathrm{A} 3$ \\
\hline DHX34|9704 & $\mathrm{A} 3$ \\
\hline DHX9|1660 & $\mathrm{A} 3$ \\
\hline DIAPH3|81624 & $\mathrm{A} 3$ \\
\hline DLC1|10395 & $\mathrm{A} 3$ \\
\hline DLEU2|8847 & $\mathrm{A} 3$ \\
\hline DMGDH|29958 & $\mathrm{A} 3$ \\
\hline DMRT3|58524 & $\mathrm{A} 3$ \\
\hline DNHD1|144132 & $\mathrm{A} 3$ \\
\hline $\begin{array}{l}\mathrm{DOC} 2 \mathrm{~B} \mid 8447 \\
\end{array}$ & $\mathrm{~A} 3$ \\
\hline DOCK6|57572 & $\mathrm{A} 3$ \\
\hline $\mathrm{DPT} \mid 1805$ & $\mathrm{~A} 3$ \\
\hline DRP2|1821 & $\mathrm{A} 3$ \\
\hline DSEL|92126 & $\mathrm{A} 3$ \\
\hline DTX1|1840 & $\mathrm{A} 3$ \\
\hline DUS4L|11062 & $\mathrm{A} 3$ \\
\hline DUSP22|56940 & $\mathrm{A} 3$ \\
\hline EARS2|124454 & $\mathrm{A} 3$ \\
\hline EBF1|1879 & A3 \\
\hline EBF2|64641 & $\mathrm{A} 3$ \\
\hline EBF3|253738 & $\mathrm{A} 3$ \\
\hline
\end{tabular}




\begin{tabular}{|c|c|}
\hline ECE1|1889 & $\mathrm{A} 3$ \\
\hline ECM2|1842 & $\mathrm{A} 3$ \\
\hline ECSCR|641700 & A3 \\
\hline EHBP1|23301 & $\mathrm{A} 3$ \\
\hline EHD2|30846 & $\mathrm{A} 3$ \\
\hline EID3|493861 & $\mathrm{A} 3$ \\
\hline EIF3F $\mid 8665$ & $\mathrm{~A} 3$ \\
\hline EIF4E3|317649 & $\mathrm{A} 3$ \\
\hline EIF4EBP2|1979 & $\mathrm{A} 3$ \\
\hline ELTD1|64123 & $\mathrm{A} 3$ \\
\hline EMX2OS|196047 & $\mathrm{A} 3$ \\
\hline EMX2|2018 & $\mathrm{A} 3$ \\
\hline EPB42|2038 & $\mathrm{A} 3$ \\
\hline EPDR1|54749 & $\mathrm{A} 3$ \\
\hline EPHA10|284656 & $\mathrm{A} 3$ \\
\hline EPT1|85465 & $\mathrm{A} 3$ \\
\hline ERBB3|2065 & $\mathrm{A} 3$ \\
\hline ERVFRDE1|405754 & $\mathrm{A} 3$ \\
\hline ESAM $\mid 90952$ & $\mathrm{~A} 3$ \\
\hline ETS1|2113 & $\mathrm{A} 3$ \\
\hline EXTL3|2137 & $\mathrm{A} 3$ \\
\hline FAAH2|158584 & $\mathrm{A} 3$ \\
\hline FABP4|2167 & $\mathrm{A} 3$ \\
\hline FADS3|3995 & $\mathrm{A} 3$ \\
\hline FAM120A|23196 & $\mathrm{A} 3$ \\
\hline FAM124B|79843 & $\mathrm{A} 3$ \\
\hline FAM138F|641702 & A3 \\
\hline FAM160B2|64760 & $\mathrm{A} 3$ \\
\hline FAM180B|399888 & $\mathrm{A} 3$ \\
\hline FAM184A|79632 & $\mathrm{A} 3$ \\
\hline FAM199X|139231 & $\mathrm{A} 3$ \\
\hline FAM83H|286077 & $\mathrm{A} 3$ \\
\hline FAM86A|196483 & $\mathrm{A} 3$ \\
\hline FAM86C|55199 & $\mathrm{A} 3$ \\
\hline FAM89A|375061 & $\mathrm{A} 3$ \\
\hline FAM91A1|157769 & $\mathrm{A} 3$ \\
\hline FANCB $\mid 2187$ & $\mathrm{~A} 3$ \\
\hline FBLN2|2199 & $\mathrm{A} 3$ \\
\hline FBLN7|129804 & $\mathrm{A} 3$ \\
\hline FBXO22|26263 & $\mathrm{A} 3$ \\
\hline FBXO46|23403 & $\mathrm{A} 3$ \\
\hline FERMT2|10979 & $\mathrm{A} 3$ \\
\hline FEZ1|9638 & $\mathrm{A} 3$ \\
\hline FGD6|55785 & $\mathrm{A} 3$ \\
\hline FGFR1OP|11116 & $\mathrm{A} 3$ \\
\hline FHDC1|85462 & $\mathrm{A} 3$ \\
\hline FILIP1L|11259 & $\mathrm{A} 3$ \\
\hline FIP1L1|81608 & $\mathrm{A} 3$ \\
\hline FLI1|2313 & $\mathrm{A} 3$ \\
\hline FLT3LG|2323 & $\mathrm{A} 3$ \\
\hline FMO3|2328 & $\mathrm{A} 3$ \\
\hline
\end{tabular}




\begin{tabular}{|c|c|}
\hline FNDC4|64838 & A3 \\
\hline FOXD2|2306 & A3 \\
\hline FRMD1|79981 & A3 \\
\hline FSTL1|11167 & $\mathrm{A} 3$ \\
\hline FUBP1|8880 & $\mathrm{A} 3$ \\
\hline FUS|2521 & $\mathrm{A} 3$ \\
\hline FXYD6|53826 & $\mathrm{A} 3$ \\
\hline FYN|2534 & $\mathrm{A} 3$ \\
\hline FZD2|2535 & $\mathrm{A} 3$ \\
\hline FZD4|8322 & A3 \\
\hline G0S2|50486 & A3 \\
\hline GABPB1|2553 & $\mathrm{A} 3$ \\
\hline GALNT12|79695 & $\mathrm{A} 3$ \\
\hline GALNTL2|117248 & $\mathrm{A} 3$ \\
\hline GAS1|2619 & A3 \\
\hline GAS6|2621 & $\mathrm{A} 3$ \\
\hline GCOM1|145781 & $\mathrm{A} 3$ \\
\hline GDPD5|81544 & $\mathrm{A} 3$ \\
\hline GEM|2669 & A3 \\
\hline GIMAP1|170575 & $\mathrm{A} 3$ \\
\hline GIMAP7|168537 & A3 \\
\hline GINS4|84296 & $\mathrm{A} 3$ \\
\hline GJC2|57165 & $\mathrm{A} 3$ \\
\hline GK3P|2713 & A3 \\
\hline GLYAT|10249 & $\mathrm{A} 3$ \\
\hline GMPPB|29925 & $\mathrm{A} 3$ \\
\hline GNAI1|2770 & $\mathrm{A} 3$ \\
\hline GNG2|54331 & A3 \\
\hline GNL3L|54552 & $\mathrm{A} 3$ \\
\hline GNRH1|2796 & $\mathrm{A} 3$ \\
\hline GORAB|92344 & $\mathrm{A} 3$ \\
\hline GPATCH2|55105 & $\mathrm{A} 3$ \\
\hline GPBAR1|151306 & $\mathrm{A} 3$ \\
\hline GPD1|2819 & $\mathrm{A} 3$ \\
\hline GPR109A|338442 & $\mathrm{A} 3$ \\
\hline GPR116|221395 & $\mathrm{A} 3$ \\
\hline GPR125|166647 & $\mathrm{A} 3$ \\
\hline GPR133|283383 & $\mathrm{A} 3$ \\
\hline GPR34|2857 & $\mathrm{A} 3$ \\
\hline \begin{tabular}{ll|l|} 
GPX3|2878 \\
\end{tabular} & $\mathrm{A} 3$ \\
\hline GRID1|2894 & A3 \\
\hline GRK5|2869 & A3 \\
\hline GSPT1|2935 & A3 \\
\hline GSR|2936 & $\mathrm{A} 3$ \\
\hline GSTCD|79807 & $\mathrm{A} 3$ \\
\hline GTDC1|79712 & A3 \\
\hline \begin{tabular}{ll|l|} 
GYG2|8908 \\
\end{tabular} & $\mathrm{A} 3$ \\
\hline HAT1|8520 & $\mathrm{A} 3$ \\
\hline HCFC1|3054 & A3 \\
\hline HCG27|253018 & A3 \\
\hline HEPACAM|220296 & $\mathrm{A} 3$ \\
\hline
\end{tabular}




\begin{tabular}{|c|c|}
\hline HEPN1|641654 & A3 \\
\hline HIC1|3090 & $\mathrm{A} 3$ \\
\hline HMGA1|3159 & $\mathrm{A} 3$ \\
\hline HNRNPA3P1|10151 & $\mathrm{A} 3$ \\
\hline HNRNPR|10236 & $\mathrm{A} 3$ \\
\hline HOXD9|3235 & $\mathrm{A} 3$ \\
\hline HPS3|84343 & $\mathrm{A} 3$ \\
\hline HSD17B13|345275 & $\mathrm{A} 3$ \\
\hline HSPA12A|259217 & $\mathrm{A} 3$ \\
\hline HSPA14|51182 & A3 \\
\hline HYAL1|3373 & $\mathrm{A} 3$ \\
\hline ICA1|3382 & A3 \\
\hline IFFO1|25900 & $\mathrm{A} 3$ \\
\hline IGSF8|93185 & $\mathrm{A} 3$ \\
\hline IGSF9|57549 & $\mathrm{A} 3$ \\
\hline IL3RA|3563 & A3 \\
\hline INMT|11185 & $\mathrm{A} 3$ \\
\hline INPP5K|51763 & $\mathrm{A} 3$ \\
\hline \begin{tabular}{l|l|l|} 
INTS10|55174 &
\end{tabular} & A3 \\
\hline IPO4|79711 & A3 \\
\hline IQSEC3|440073 & $\mathrm{A} 3$ \\
\hline ISCA1 $\mid 81689$ & $\mathrm{~A} 3$ \\
\hline ISCU|23479 & $\mathrm{A} 3$ \\
\hline ITGA7|3679 & $\mathrm{A} 3$ \\
\hline ITGB1BP1|9270 & $\mathrm{A} 3$ \\
\hline ITM2B|9445 & $\mathrm{A} 3$ \\
\hline ITSN1|6453 & A3 \\
\hline JARID2|3720 & A3 \\
\hline $\begin{array}{l}\text { JAZF1|221895 } \\
\end{array}$ & $\mathrm{A} 3$ \\
\hline KANK2|25959 & $\mathrm{A} 3$ \\
\hline KBTBD11|9920 & $\mathrm{A} 3$ \\
\hline KCNA6|3742 & $\mathrm{A} 3$ \\
\hline KCNB1|3745 & $\mathrm{A} 3$ \\
\hline KCNIP1|30820 & A3 \\
\hline KCNIP2|30819 & A3 \\
\hline KCNJ8|3764 & $\mathrm{A} 3$ \\
\hline KCNT2|343450 & $\mathrm{A} 3$ \\
\hline KDR/3791 & A3 \\
\hline KDSR|2531 & $\mathrm{A} 3$ \\
\hline KIAA1543|57662 & $\mathrm{A} 3$ \\
\hline KIF17|57576 & A3 \\
\hline KIF20B|9585 & A3 \\
\hline KLB|152831 & A3 \\
\hline KLC1 $\mid 3831$ & $\mathrm{~A} 3$ \\
\hline KLF14|136259 & $\mathrm{A} 3$ \\
\hline KLHDC9|126823 & $\mathrm{A} 3$ \\
\hline KLHL4|56062 & $\mathrm{A} 3$ \\
\hline KRT18|3875 & $\mathrm{A} 3$ \\
\hline KRT19|3880 & A3 \\
\hline LAMA2|3908 & A3 \\
\hline LAMA4|3910 & $\mathrm{A} 3$ \\
\hline
\end{tabular}




\begin{tabular}{|c|c|}
\hline LAMB2|3913 & $\mathrm{A} 3$ \\
\hline LAMC1 13915 & $\mathrm{~A} 3$ \\
\hline LATS2|26524 & $\mathrm{A} 3$ \\
\hline LCLAT1|253558 & $\mathrm{A} 3$ \\
\hline LCN6|158062 & $\mathrm{A} 3$ \\
\hline LEO1|123169 & A3 \\
\hline LEP $\mid 3952$ & $\mathrm{~A} 3$ \\
\hline LGALS3|3958 & $\mathrm{A} 3$ \\
\hline LIG1|3978 & $\mathrm{A} 3$ \\
\hline LIG3|3980 & $\mathrm{A} 3$ \\
\hline LIMS3-LOC440895|100271835 & $\mathrm{A} 3$ \\
\hline LIMS3|96626 & $\mathrm{A} 3$ \\
\hline LIPE|3991 & $\mathrm{A} 3$ \\
\hline LMLN|89782 & $\mathrm{A} 3$ \\
\hline LMX1A|4009 & $\mathrm{A} 3$ \\
\hline \begin{tabular}{ll|l|} 
LNX2|22484 & \\
\end{tabular} & $\mathrm{A} 3$ \\
\hline LOC100128788|100128788 & $\mathrm{A} 3$ \\
\hline LOC158376|158376 & $\mathrm{A} 3$ \\
\hline LOC283731|283731 & $\mathrm{A} 3$ \\
\hline LOC284441|284441 & $\mathrm{A} 3$ \\
\hline LOC339524|339524 & $\mathrm{A} 3$ \\
\hline LOC401052|401052 & $\mathrm{A} 3$ \\
\hline LOC642597|642597 & $\mathrm{A} 3$ \\
\hline LOC80054|80054 & $\mathrm{A} 3$ \\
\hline LOC90586|90586 & $\mathrm{A} 3$ \\
\hline LPAR2|9170 & $\mathrm{A} 3$ \\
\hline LPCAT4|254531 & $\mathrm{A} 3$ \\
\hline $\begin{array}{ll}\text { LRCH2|57631 } \\
\end{array}$ & $\mathrm{A} 3$ \\
\hline LRP1|4035 & $\mathrm{A} 3$ \\
\hline LRP3|4037 & $\mathrm{A} 3$ \\
\hline LRRC16A|55604 & $\mathrm{A} 3$ \\
\hline LRRC1|55227 & $\mathrm{A} 3$ \\
\hline LRRC46|90506 & $\mathrm{A} 3$ \\
\hline LRRC59|55379 & $\mathrm{A} 3$ \\
\hline \begin{tabular}{l|l|l|} 
LRRN3|54674 \\
\end{tabular} & $\mathrm{A} 3$ \\
\hline MAP1B|4131 & $\mathrm{A} 3$ \\
\hline MAP1LC3B|81631 & $\mathrm{A} 3$ \\
\hline MAP1LC3C|440738 & $\mathrm{A} 3$ \\
\hline MAP6|4135 & $\mathrm{A} 3$ \\
\hline MAP7D1|55700 & $\mathrm{A} 3$ \\
\hline MAP7D3|79649 & $\mathrm{A} 3$ \\
\hline MARK1|4139 & $\mathrm{A} 3$ \\
\hline MARK2|2011 & $\mathrm{A} 3$ \\
\hline MARVELD2|153562 & $\mathrm{A} 3$ \\
\hline MAS1L|116511 & $\mathrm{A} 3$ \\
\hline MCAM|4162 & $\mathrm{A} 3$ \\
\hline MCM3|4172 & $\mathrm{A} 3$ \\
\hline MCTP1|79772 & $\mathrm{A} 3$ \\
\hline MDFIC|29969 & $\mathrm{A} 3$ \\
\hline MECOM|2122 & $\mathrm{A} 3$ \\
\hline MEST|4232 & $\mathrm{A} 3$ \\
\hline
\end{tabular}




\begin{tabular}{|c|c|}
\hline METTL1|4234 & $\mathrm{A} 3$ \\
\hline MFAP4|4239 & A3 \\
\hline MFNG|4242 & $\mathrm{A} 3$ \\
\hline MGLL|11343 & $\mathrm{A} 3$ \\
\hline MIPOL1|145282 & $\mathrm{A} 3$ \\
\hline MITF|4286 & A3 \\
\hline MMD|23531 & $\mathrm{A} 3$ \\
\hline MMP27|64066 & $\mathrm{A} 3$ \\
\hline MMP28|79148 & $\mathrm{A} 3$ \\
\hline MMRN1|22915 & $\mathrm{A} 3$ \\
\hline MOBKL1B|55233 & $\mathrm{A} 3$ \\
\hline MORN2|729967 & $\mathrm{A} 3$ \\
\hline MOSC1|64757 & $\mathrm{A} 3$ \\
\hline MOSPD1|56180 & $\mathrm{A} 3$ \\
\hline MRAP|56246 & $\mathrm{A} 3$ \\
\hline MRS2P2|729633 & $\mathrm{A} 3$ \\
\hline MRVI1|10335 & $\mathrm{A} 3$ \\
\hline MSX1|4487 & $\mathrm{A} 3$ \\
\hline MTA3|57504 & $\mathrm{A} 3$ \\
\hline MTL5|9633 & $\mathrm{A} 3$ \\
\hline MXD4|10608 & $\mathrm{A} 3$ \\
\hline MXRA7|439921 & $\mathrm{A} 3$ \\
\hline MYEOV|26579 & $\mathrm{A} 3$ \\
\hline MYL9|10398 & $\mathrm{A} 3$ \\
\hline MYO1C|4641 & $\mathrm{A} 3$ \\
\hline MYRIP|25924 & $\mathrm{A} 3$ \\
\hline NAGA|4668 & $\mathrm{A} 3$ \\
\hline NBN|4683 & $\mathrm{A} 3$ \\
\hline NCBP1|4686 & $\mathrm{A} 3$ \\
\hline NCOA5|57727 & $\mathrm{A} 3$ \\
\hline NCOA6|23054 & $\mathrm{A} 3$ \\
\hline NIPA2|81614 & $\mathrm{A} 3$ \\
\hline NIPSNAP1|8508 & $\mathrm{A} 3$ \\
\hline NMB|4828 & $\mathrm{A} 3$ \\
\hline NME6|10201 & $\mathrm{A} 3$ \\
\hline NMUR1|10316 & $\mathrm{A} 3$ \\
\hline NOLC1|9221 & $\mathrm{A} 3$ \\
\hline NOMO1/23420 & $\mathrm{A} 3$ \\
\hline NONO|4841 & $\mathrm{A} 3$ \\
\hline NOVA2|4858 & $\mathrm{A} 3$ \\
\hline NOV|4856 & $\mathrm{A} 3$ \\
\hline NPR1|4881 & $\mathrm{A} 3$ \\
\hline NRN1L|123904 & $\mathrm{A} 3$ \\
\hline NRP1|8829 & $\mathrm{A} 3$ \\
\hline NSF|4905 & $\mathrm{A} 3$ \\
\hline NT5E|4907 & $\mathrm{A} 3$ \\
\hline NUDT13|25961 & $\mathrm{A} 3$ \\
\hline NUP155|9631 & $\mathrm{A} 3$ \\
\hline OAF|220323 & $\mathrm{A} 3$ \\
\hline OCLN|4950 & $\mathrm{A} 3$ \\
\hline OLFML1|283298 & $\mathrm{A} 3$ \\
\hline
\end{tabular}




\begin{tabular}{|c|c|}
\hline ORC5L|5001 & A3 \\
\hline OVOL2|58495 & A3 \\
\hline P4HTM|54681 & $\mathrm{A} 3$ \\
\hline PABPC5|140886 & $\mathrm{A} 3$ \\
\hline PALB2|79728 & A3 \\
\hline PALM2-AKAP2|445815 & $\mathrm{A} 3$ \\
\hline PALM|5064 & A3 \\
\hline PAPOLA|10914 & $\mathrm{A} 3$ \\
\hline PAPOLB|56903 & $\mathrm{A} 3$ \\
\hline PARD6B $\mid 84612$ & $\mathrm{~A} 3$ \\
\hline PARP3|10039 & $\mathrm{A} 3$ \\
\hline PARVA|55742 & A3 \\
\hline PASK|23178 & $\mathrm{A} 3$ \\
\hline PCDH9|5101 & $\mathrm{A} 3$ \\
\hline PCDHB4|56131 & $\mathrm{A} 3$ \\
\hline PCDHGC3|5098 & A3 \\
\hline PCOLCE2|26577 & $\mathrm{A} 3$ \\
\hline PCSK5|5125 & $\mathrm{A} 3$ \\
\hline PCYOX1|51449 & A3 \\
\hline PDE1A|5136 & $\mathrm{A} 3$ \\
\hline PDE8B $\mid 8622$ & $\mathrm{~A} 3$ \\
\hline PDGFRL|5157 & $\mathrm{A} 3$ \\
\hline PDIK1L|149420 & A3 \\
\hline PDLIM1|9124 & $\mathrm{A} 3$ \\
\hline PDRG1|81572 & $\mathrm{A} 3$ \\
\hline PDZD2|23037 & $\mathrm{A} 3$ \\
\hline PDZRN3|23024 & A3 \\
\hline PECAM1|5175 & A3 \\
\hline PFDN6|10471 & $\mathrm{A} 3$ \\
\hline PGCP|10404 & A3 \\
\hline $\begin{array}{l}\text { PHF6|84295 } \\
\end{array}$ & $\mathrm{A} 3$ \\
\hline PHLDB1|23187 & $\mathrm{A} 3$ \\
\hline PHLDB3|653583 & $\mathrm{A} 3$ \\
\hline PI16|221476 & A3 \\
\hline PIAS3|10401 & A3 \\
\hline PIAS4|51588 & $\mathrm{A} 3$ \\
\hline PID1|55022 & $\mathrm{A} 3$ \\
\hline PIGZ|80235 & A3 \\
\hline PJA1|64219 & $\mathrm{A} 3$ \\
\hline PKDCC|91461 & $\mathrm{A} 3$ \\
\hline PKDREJ|10343 & A3 \\
\hline PKHD1L1|93035 & A3 \\
\hline PKP3|11187 & $\mathrm{A} 3$ \\
\hline \begin{tabular}{l|l|l|} 
PLA2G5322 \\
\end{tabular} & $\mathrm{A} 3$ \\
\hline PLAC9|219348 & A3 \\
\hline PLEKHA9|51054 & $\mathrm{A} 3$ \\
\hline \begin{tabular}{l|} 
PLEKHB2|55041 \\
\end{tabular} & $\mathrm{A} 3$ \\
\hline PLEKHF1|79156 & $\mathrm{A} 3$ \\
\hline PLIN1|5346 & A3 \\
\hline PLIN4|729359 & A3 \\
\hline PLXNA4|91584 & $\mathrm{A} 3$ \\
\hline
\end{tabular}




\begin{tabular}{|c|c|}
\hline PM20D1|148811 & A3 \\
\hline PMP22|5376 & $\mathrm{A} 3$ \\
\hline PMS2L5|5383 & $\mathrm{A} 3$ \\
\hline PNO1|56902 & $\mathrm{A} 3$ \\
\hline PODN|127435 & $\mathrm{A} 3$ \\
\hline POLR3GL|84265 & $\mathrm{A} 3$ \\
\hline PPARG|5468 & $\mathrm{A} 3$ \\
\hline PPAT|5471 & $\mathrm{A} 3$ \\
\hline PPM1H|57460 & $\mathrm{A} 3$ \\
\hline PPM1M|132160 & $\mathrm{A} 3$ \\
\hline PPME1|51400 & $\mathrm{A} 3$ \\
\hline PPP1R12C|54776 & A3 \\
\hline PPP1R1A|5502 & $\mathrm{A} 3$ \\
\hline PPP1R3G|648791 & $\mathrm{A} 3$ \\
\hline PRELP|5549 & $\mathrm{A} 3$ \\
\hline PRIM1|5557 & $\mathrm{A} 3$ \\
\hline PRKCZ|5590 & $\mathrm{A} 3$ \\
\hline PRLR|5618 & $\mathrm{A} 3$ \\
\hline PROCR|10544 & A3 \\
\hline PRPF40A|55660 & $\mathrm{A} 3$ \\
\hline PRPF4|9128 & $\mathrm{A} 3$ \\
\hline PRPS2|5634 & $\mathrm{A} 3$ \\
\hline PRR5|55615 & $\mathrm{A} 3$ \\
\hline PRRT4|401399 & $\mathrm{A} 3$ \\
\hline PRUNE2|158471 & $\mathrm{A} 3$ \\
\hline PSME1 15720 & $\mathrm{~A} 3$ \\
\hline PTGES3|10728 & $\mathrm{A} 3$ \\
\hline $\begin{array}{l}\text { PTGIS|5740 } \\
\text { PIS }\end{array}$ & $\mathrm{A} 3$ \\
\hline PTH1R|5745 & $\mathrm{A} 3$ \\
\hline PTK6|5753 & $\mathrm{A} 3$ \\
\hline PTPRB|5787 & $\mathrm{A} 3$ \\
\hline PTPRM|5797 & $\mathrm{A} 3$ \\
\hline \begin{tabular}{l|l|} 
PVRL3|25945 \\
\end{tabular} & $\mathrm{A} 3$ \\
\hline R3HCC1|203069 & $\mathrm{A} 3$ \\
\hline RAB19|401409 & $\mathrm{A} 3$ \\
\hline RAB6B|51560 & $\mathrm{A} 3$ \\
\hline RABEP2|79874 & $\mathrm{A} 3$ \\
\hline RAD1|5810 & $\mathrm{A} 3$ \\
\hline RAMP2|10266 & A3 \\
\hline \begin{tabular}{l|l|l|} 
RASA322821 \\
\end{tabular} & $\mathrm{A} 3$ \\
\hline RASD1|51655 & A3 \\
\hline RASL11A|387496 & A3 \\
\hline RASL12|51285 & $\mathrm{A} 3$ \\
\hline RASSF7|8045 & $\mathrm{A} 3$ \\
\hline RBBP5|5929 & $\mathrm{A} 3$ \\
\hline RBM12B|389677 & $\mathrm{A} 3$ \\
\hline RBP4|5950 & $\mathrm{A} 3$ \\
\hline RBPJ|3516 & $\mathrm{A} 3$ \\
\hline RBPMS2|348093 & $\mathrm{A} 3$ \\
\hline RCAN2|10231 & A3 \\
\hline RDH11|51109 & $\mathrm{A} 3$ \\
\hline
\end{tabular}




\begin{tabular}{|c|c|}
\hline RDH5|5959 & $\mathrm{A} 3$ \\
\hline REM1|28954 & $\mathrm{A} 3$ \\
\hline RETSAT|54884 & $\mathrm{A} 3$ \\
\hline RFC5|5985 & $\mathrm{A} 3$ \\
\hline RFWD3|55159 & $\mathrm{A} 3$ \\
\hline RFX5|5993 & $\mathrm{A} 3$ \\
\hline RGS2|5997 & $\mathrm{A} 3$ \\
\hline RHOQ|23433 & $\mathrm{A} 3$ \\
\hline RIBC2|26150 & A3 \\
\hline RILP $\mid 83547$ & $\mathrm{~A} 3$ \\
\hline RIPK2|8767 & $\mathrm{A} 3$ \\
\hline RNF130|55819 & $\mathrm{A} 3$ \\
\hline RNF2|6045 & $\mathrm{A} 3$ \\
\hline ROBO4|54538 & $\mathrm{A} 3$ \\
\hline ROD1|9991 & $\mathrm{A} 3$ \\
\hline ROGDI|79641 & $\mathrm{A} 3$ \\
\hline RPE 6120 & $\mathrm{~A} 3$ \\
\hline RPL23AP32|56969 & $\mathrm{A} 3$ \\
\hline RPS6KA2|6196 & $\mathrm{A} 3$ \\
\hline RQCD1|9125 & $\mathrm{A} 3$ \\
\hline RRAS|6237 & $\mathrm{A} 3$ \\
\hline RRP12|23223 & $\mathrm{A} 3$ \\
\hline RYK|6259 & $\mathrm{A} 3$ \\
\hline S100A4|6275 & $\mathrm{A} 3$ \\
\hline SAA1|6288 & $\mathrm{A} 3$ \\
\hline SAMD12|401474 & $\mathrm{A} 3$ \\
\hline SCARF1|8578 & A3 \\
\hline SCMH1|22955 & $\mathrm{A} 3$ \\
\hline SCN9A|6335 & $\mathrm{A} 3$ \\
\hline SCTR|6344 & $\mathrm{A} 3$ \\
\hline SCYL2|55681 & $\mathrm{A} 3$ \\
\hline \begin{tabular}{l|l|l|} 
SCYL3 57147 \\
\end{tabular} & $\mathrm{~A} 3$ \\
\hline SDSL|113675 & $\mathrm{A} 3$ \\
\hline SEC16A|9919 & $\mathrm{A} 3$ \\
\hline SEC23B|10483 & $\mathrm{A} 3$ \\
\hline $\begin{array}{l}\text { SEC61A2|55176 } \\
\end{array}$ & $\mathrm{A} 3$ \\
\hline SELP|6403 & $\mathrm{A} 3$ \\
\hline SEMA3A|10371 & $\mathrm{A} 3$ \\
\hline SERPINF1|5176 & $\mathrm{A} 3$ \\
\hline SERPINF2|5345 & $\mathrm{A} 3$ \\
\hline SERPING1|710 & $\mathrm{A} 3$ \\
\hline SET|6418 & $\mathrm{A} 3$ \\
\hline SGCB|6443 & $\mathrm{A} 3$ \\
\hline SGK2|10110 & $\mathrm{A} 3$ \\
\hline SH2D3C|10044 & $\mathrm{A} 3$ \\
\hline SH3GLB1|51100 & $\mathrm{A} 3$ \\
\hline SH3RF3|344558 & $\mathrm{A} 3$ \\
\hline SHISA3|152573 & $\mathrm{A} 3$ \\
\hline SIK2|23235 & $\mathrm{A} 3$ \\
\hline SKIV2L|6499 & $\mathrm{A} 3$ \\
\hline SLC22A3|6581 & $\mathrm{A} 3$ \\
\hline
\end{tabular}




\begin{tabular}{|c|c|}
\hline SLC25A17|10478 & $\mathrm{A} 3$ \\
\hline SLC29A4|222962 & $\mathrm{A} 3$ \\
\hline SLC39A9|55334 & $\mathrm{A} 3$ \\
\hline SLC4A5|57835 & $\mathrm{A} 3$ \\
\hline SLC9A3R1|9368 & $\mathrm{A} 3$ \\
\hline SLC9A9|285195 & A3 \\
\hline SLIT2|9353 & $\mathrm{A} 3$ \\
\hline SLIT3|6586 & $\mathrm{A} 3$ \\
\hline SMAD5OS|9597 & $\mathrm{A} 3$ \\
\hline SMAD6|4091 & $\mathrm{A} 3$ \\
\hline SOD3|6649 & $\mathrm{A} 3$ \\
\hline SORBS1|10580 & $\mathrm{A} 3$ \\
\hline SORBS3|10174 & $\mathrm{A} 3$ \\
\hline SORD|6652 & $\mathrm{A} 3$ \\
\hline SOX10|6663 & $\mathrm{A} 3$ \\
\hline SPAG1|6674 & $\mathrm{A} 3$ \\
\hline SPATA9|83890 & $\mathrm{A} 3$ \\
\hline SPNS2|124976 & $\mathrm{A} 3$ \\
\hline SPOPL|339745 & $\mathrm{A} 3$ \\
\hline SQLE|6713 & $\mathrm{A} 3$ \\
\hline SRP19|6728 & $\mathrm{A} 3$ \\
\hline SRPK1|6732 & $\mathrm{A} 3$ \\
\hline SRRT|51593 & $\mathrm{A} 3$ \\
\hline SSR1|6745 & $\mathrm{A} 3$ \\
\hline ST6GALNAC3|256435 & $\mathrm{A} 3$ \\
\hline ST6GALNAC6|30815 & $\mathrm{A} 3$ \\
\hline STAB2|55576 & $\mathrm{A} 3$ \\
\hline STARD8|9754 & $\mathrm{A} 3$ \\
\hline STK32A|202374 & $\mathrm{A} 3$ \\
\hline STK40|83931 & $\mathrm{A} 3$ \\
\hline STL|7955 & $\mathrm{A} 3$ \\
\hline STX11|8676 & $\mathrm{A} 3$ \\
\hline STX7|8417 & $\mathrm{A} 3$ \\
\hline STX8|9482 & $\mathrm{A} 3$ \\
\hline SUN1|23353 & $\mathrm{A} 3$ \\
\hline SVIP|258010 & $\mathrm{A} 3$ \\
\hline SYT8|90019 & $\mathrm{A} 3$ \\
\hline TACC1|6867 & $\mathrm{A} 3$ \\
\hline TAF1C $\mid 9013$ & $\mathrm{~A} 3$ \\
\hline TAF2 6873 & $\mathrm{~A} 3$ \\
\hline TAGLN|6876 & $\mathrm{A} 3$ \\
\hline TBC1D2B|23102 & $\mathrm{A} 3$ \\
\hline TCN2|6948 & $\mathrm{A} 3$ \\
\hline TDG|6996 & $\mathrm{A} 3$ \\
\hline TEAD1|7003 & $\mathrm{A} 3$ \\
\hline TEK|7010 & $\mathrm{A} 3$ \\
\hline TET3|200424 & $\mathrm{A} 3$ \\
\hline TFE3|7030 & $\mathrm{A} 3$ \\
\hline THSD7B|80731 & $\mathrm{A} 3$ \\
\hline THYN1|29087 & $\mathrm{A} 3$ \\
\hline TIE1|7075 & $\mathrm{A} 3$ \\
\hline
\end{tabular}




\begin{tabular}{|c|c|}
\hline TLCD1|116238 & A3 \\
\hline TLL1|7092 & $\mathrm{A} 3$ \\
\hline TLN1|7094 & $\mathrm{A} 3$ \\
\hline TMBIM1|64114 & $\mathrm{A} 3$ \\
\hline TMC2|117532 & A3 \\
\hline TMEM125|128218 & $\mathrm{A} 3$ \\
\hline TMEM132C|92293 & $\mathrm{A} 3$ \\
\hline TMEM140|55281 & $\mathrm{A} 3$ \\
\hline TMEM177|80775 & $\mathrm{A} 3$ \\
\hline TMEM184A|202915 & $\mathrm{A} 3$ \\
\hline TMEM20|159371 & $\mathrm{A} 3$ \\
\hline TMEM22|80723 & A3 \\
\hline TMEM35|59353 & $\mathrm{A} 3$ \\
\hline TMEM37|140738 & $\mathrm{A} 3$ \\
\hline TMEM41B|440026 & $\mathrm{A} 3$ \\
\hline TMEM48|55706 & $\mathrm{A} 3$ \\
\hline TMEM63C|57156 & $\mathrm{A} 3$ \\
\hline TMEM97|27346 & $\mathrm{A} 3$ \\
\hline TMPO|7112 & A3 \\
\hline TNIP1|10318 & $\mathrm{A} 3$ \\
\hline TNMD|64102 & $\mathrm{A} 3$ \\
\hline TNS1|7145 & $\mathrm{A} 3$ \\
\hline TOMM34|10953 & $\mathrm{A} 3$ \\
\hline TOP1P1|7151 & $\mathrm{A} 3$ \\
\hline TOP2A|7153 & $\mathrm{A} 3$ \\
\hline TOR1AIP2|163590 & $\mathrm{A} 3$ \\
\hline TPBG|7162 & $\mathrm{A} 3$ \\
\hline ТРРР3|51673 & $\mathrm{A} 3$ \\
\hline TPRG1L|127262 & $\mathrm{A} 3$ \\
\hline TPTE2P1|646405 & $\mathrm{A} 3$ \\
\hline TRAFD1|10906 & $\mathrm{A} 3$ \\
\hline TRERF1|55809 & $\mathrm{A} 3$ \\
\hline TRIM14|9830 & $\mathrm{A} 3$ \\
\hline TRIM62|55223 & $\overline{\mathrm{A} 3}$ \\
\hline TRIP10|9322 & $\mathrm{A} 3$ \\
\hline TRIP13|9319 & $\mathrm{A} 3$ \\
\hline TRPS1|7227 & $\mathrm{A} 3$ \\
\hline TRUB2|26995 & $\mathrm{A} 3$ \\
\hline TSEN54|283989 & A3 \\
\hline TSPAN2|10100 & $\mathrm{A} 3$ \\
\hline TSPAN4|7106 & A3 \\
\hline TTC26|79989 & A3 \\
\hline TTC7B|145567 & A3 \\
\hline TUBB2A|7280 & $\mathrm{A} 3$ \\
\hline TUBB6|84617 & $\mathrm{A} 3$ \\
\hline TUSC5|286753 & $\mathrm{A} 3$ \\
\hline TWIST2|117581 & $\mathrm{A} 3$ \\
\hline TYRO3|7301 & $\mathrm{A} 3$ \\
\hline UBE2G2|7327 & $\mathrm{A} 3$ \\
\hline UBE2K|3093 & A3 \\
\hline UBXN2B|137886 & $\mathrm{A} 3$ \\
\hline
\end{tabular}




\begin{tabular}{|c|c|}
\hline UGP2|7360 & A3 \\
\hline UMPS|7372 & $\mathrm{A} 3$ \\
\hline URB2|9816 & $\mathrm{A} 3$ \\
\hline UROD|7389 & $\mathrm{A} 3$ \\
\hline USP37|57695 & A3 \\
\hline VEGFB|7423 & $\mathrm{A} 3$ \\
\hline VEGFC|7424 & $\mathrm{A} 3$ \\
\hline VGLL3|389136 & $\mathrm{A} 3$ \\
\hline VIM|7431 & $\mathrm{A} 3$ \\
\hline VPS25|84313 & $\mathrm{A} 3$ \\
\hline VWF|7450 & $\mathrm{A} 3$ \\
\hline WDR12|55759 & A3 \\
\hline WDR13|64743 & $\mathrm{A} 3$ \\
\hline WDR55|54853 & $\mathrm{A} 3$ \\
\hline WDR76|79968 & $\mathrm{A} 3$ \\
\hline WISP2|8839 & $\mathrm{A} 3$ \\
\hline WNT9B|7484 & $\mathrm{A} 3$ \\
\hline WTIP|126374 & $\mathrm{A} 3$ \\
\hline XBP1|7494 & A3 \\
\hline XPR1|9213 & $\mathrm{A} 3$ \\
\hline XRCC5|7520 & $\mathrm{A} 3$ \\
\hline XYLB|9942 & $\mathrm{A} 3$ \\
\hline YARS|8565 & $\mathrm{A} 3$ \\
\hline YIPF6|286451 & $\mathrm{A} 3$ \\
\hline YTHDF1|54915 & $\mathrm{A} 3$ \\
\hline ZBTB9|221504 & $\mathrm{A} 3$ \\
\hline ZC3H15|55854 & $\mathrm{A} 3$ \\
\hline ZCCHC24|219654 & $\mathrm{A} 3$ \\
\hline \begin{tabular}{l|l|} 
ZDHHC13|54503 \\
\end{tabular} & $\mathrm{A} 3$ \\
\hline ZDHHC8|29801 & $\mathrm{A} 3$ \\
\hline ZEB2|9839 & $\mathrm{A} 3$ \\
\hline ZFP36L1|677 & $\mathrm{A} 3$ \\
\hline ZFPM2|23414 & $\mathrm{A} 3$ \\
\hline ZFYVE21|79038 & $\mathrm{A} 3$ \\
\hline $\begin{array}{l}\text { ZNF395|55893 } \\
\end{array}$ & $\mathrm{A} 3$ \\
\hline $\begin{array}{l}\text { ZNF423|23090 } \\
\end{array}$ & $\mathrm{A} 3$ \\
\hline \begin{tabular}{l|l} 
ZNF48|197407 \\
\end{tabular} & $\mathrm{A} 3$ \\
\hline ZNF623|9831 & $\mathrm{A} 3$ \\
\hline ZNF707|286075 & $\mathrm{A} 3$ \\
\hline $\begin{array}{l}\text { ZNF75A|7627 } \\
\end{array}$ & $\mathrm{A} 3$ \\
\hline ZNF764|92595 & A3 \\
\hline $\begin{array}{l}\text { ZNF765|91661 } \\
\end{array}$ & $\mathrm{A} 3$ \\
\hline ZNF833|401898 & A3 \\
\hline ZNF93|81931 & $\mathrm{A} 3$ \\
\hline ZSCAN12P1|221584 & $\mathrm{A} 3$ \\
\hline ZSCAN16|80345 & $\mathrm{A} 3$ \\
\hline ACAD8|27034 & A4 \\
\hline AGAP4|119016 & $\mathrm{A} 4$ \\
\hline AHSA2|130872 & A4 \\
\hline ARIH2|10425 & A4 \\
\hline C6orf174|387104 & A4 \\
\hline
\end{tabular}




\begin{tabular}{|c|c|}
\hline C7orf28B|221960 & $\mathrm{A} 4$ \\
\hline CHD2|1106 & A4 \\
\hline CMC1|152100 & $\mathrm{A} 4$ \\
\hline COX6A1|1337 & $\mathrm{A} 4$ \\
\hline CROCCL1|84809 & $\mathrm{A} 4$ \\
\hline CROCCL2|114819 & A4 \\
\hline CSE1L|1434 & $\mathrm{A} 4$ \\
\hline DEDD|9191 & A4 \\
\hline DENND2C|163259 & A4 \\
\hline DLG2|1740 & $\mathrm{A} 4$ \\
\hline DRD2|1813 & $\mathrm{A} 4$ \\
\hline EPC2|26122 & $\mathrm{A} 4$ \\
\hline FAM129A|116496 & $\mathrm{A} 4$ \\
\hline FAM21C|253725 & $\mathrm{A} 4$ \\
\hline FAM72A|729533 & $\mathrm{A} 4$ \\
\hline FLCN|201163 & $\mathrm{A} 4$ \\
\hline FLG|2312 & $\mathrm{A} 4$ \\
\hline GNB5|10681 & $\mathrm{A} 4$ \\
\hline GNPAT|8443 & A4 \\
\hline GPR113|165082 & $\mathrm{A} 4$ \\
\hline HAX1|10456 & $\mathrm{A} 4$ \\
\hline HERC1|8925 & $\mathrm{A} 4$ \\
\hline IKZF5|64376 & $\mathrm{A} 4$ \\
\hline ING5|84289 & A4 \\
\hline JMJD7-PLA2G4B|8681 & $\mathrm{A} 4$ \\
\hline KIDINS220|57498 & $\mathrm{A} 4$ \\
\hline KIF7|374654 & $\mathrm{A} 4$ \\
\hline KLHL2|11275 & $\mathrm{A} 4$ \\
\hline LAMA1|284217 & $\mathrm{A} 4$ \\
\hline LBXCOR1|390598 & $\mathrm{A} 4$ \\
\hline LOC283174|283174 & $\mathrm{A} 4$ \\
\hline MST1P2|11209 & $\mathrm{A} 4$ \\
\hline MYO9A|4649 & $\mathrm{A} 4$ \\
\hline NDUFAB1|4706 & $\mathrm{A} 4$ \\
\hline NFRKB $\mid 4798$ & $\mathrm{~A} 4$ \\
\hline PLD2|5338 & $\mathrm{A} 4$ \\
\hline RNF165|494470 & $\mathrm{A} 4$ \\
\hline RPS6KC1|26750 & $\mathrm{A} 4$ \\
\hline SEC61G|23480 & $\mathrm{A} 4$ \\
\hline SEPP1|6414 & $\mathrm{A} 4$ \\
\hline SETBP1|26040 & $\mathrm{A} 4$ \\
\hline SETDB2|83852 & $\mathrm{A} 4$ \\
\hline SGSM1|129049 & $\mathrm{A} 4$ \\
\hline SH3RF2|153769 & $\mathrm{A} 4$ \\
\hline SNORD116-4|100033416 & $\mathrm{A} 4$ \\
\hline $\begin{array}{l}\text { SYTL3|94120 } \\
\end{array}$ & $\mathrm{A} 4$ \\
\hline TNS4|84951 & $\mathrm{A} 4$ \\
\hline TRIM66|9866 & $\mathrm{A} 4$ \\
\hline TTC21B|79809 & $\mathrm{A} 4$ \\
\hline VPS72|6944 & A4 \\
\hline ZBTB49|166793 & $\mathrm{A} 4$ \\
\hline
\end{tabular}




\begin{tabular}{|c|c|}
\hline BEND6|221336 & A5 \\
\hline C2orf71|388939 & A5 \\
\hline C3orf58|205428 & $\mathrm{A} 5$ \\
\hline C9orf156|51531 & $\mathrm{A} 5$ \\
\hline C9orf66|157983 & $\mathrm{A} 5$ \\
\hline CAPZB $\mid 832$ & A5 \\
\hline CDC42EP3|10602 & $\mathrm{A} 5$ \\
\hline CDRT4|284040 & A5 \\
\hline CERCAM|51148 & A5 \\
\hline $\begin{array}{l}\text { CHST9|83539 } \\
\end{array}$ & $\mathrm{A} 5$ \\
\hline CILP2|148113 & $\mathrm{A} 5$ \\
\hline COL1A2|1278 & $\mathrm{A} 5$ \\
\hline COL3A1|1281 & $\mathrm{A} 5$ \\
\hline CPAMD8|27151 & $\mathrm{A} 5$ \\
\hline CST1|1469 & $\mathrm{A} 5$ \\
\hline DIMT1L|27292 & A5 \\
\hline FAM119B|25895 & $\mathrm{A} 5$ \\
\hline FRMD3|257019 & $\mathrm{A} 5$ \\
\hline GANC|2595 & $\mathrm{A} 5$ \\
\hline INVS|27130 & A5 \\
\hline IRS1|3667 & $\mathrm{A} 5$ \\
\hline KCTD18|130535 & $\mathrm{A} 5$ \\
\hline L3MBTL4|91133 & $\mathrm{A} 5$ \\
\hline LEPRE1|64175 & $\mathrm{A} 5$ \\
\hline MAD2L2|10459 & $\mathrm{A} 5$ \\
\hline MON2|23041 & A5 \\
\hline NFE2L2|4780 & $\mathrm{A} 5$ \\
\hline NFIX|4784 & $\mathrm{A} 5$ \\
\hline PHF3|23469 & $\mathrm{A} 5$ \\
\hline PLD1|5337 & $\mathrm{A} 5$ \\
\hline PRDXDD1P|344405 & $\mathrm{A} 5$ \\
\hline PTPRZ1|5803 & $\mathrm{A} 5$ \\
\hline RPUSD4|84881 & $\mathrm{A} 5$ \\
\hline SEMA6D|80031 & $\mathrm{A} 5$ \\
\hline SIRPB1|10326 & $\mathrm{A} 5$ \\
\hline STOX2|56977 & $\mathrm{A} 5$ \\
\hline TGFB1|7040 & $\mathrm{A} 5$ \\
\hline THBS2|7058 & $\mathrm{A} 5$ \\
\hline TRMT112|51504 & $\mathrm{A} 5$ \\
\hline UBE2M|9040 & $\mathrm{A} 5$ \\
\hline ZMAT3|64393 & $\mathrm{A} 5$ \\
\hline ZNF189|7743 & A5 \\
\hline ZNF426|79088 & $\mathrm{A} 5$ \\
\hline ZNF510|22869 & $\mathrm{A} 5$ \\
\hline ANKMY2|57037 & $\mathrm{A} 6$ \\
\hline ATP6V0C|527 & $\mathrm{A} 6$ \\
\hline BHLHE41|79365 & $\mathrm{A} 6$ \\
\hline CCL28|56477 & $\mathrm{A} 6$ \\
\hline \begin{tabular}{l|l|l|} 
CDNF|41549 \\
\end{tabular} & $\mathrm{A} 6$ \\
\hline CTTNBP2|83992 & $\mathrm{A} 6$ \\
\hline DNAJB11|51726 & $\mathrm{A} 6$ \\
\hline
\end{tabular}




\begin{tabular}{|c|c|}
\hline FAM13B|51306 & A6 \\
\hline FAT2|2196 & A6 \\
\hline KCNC4|3749 & A6 \\
\hline KCTD5|54442 & A6 \\
\hline LOC441869|441869 & A6 \\
\hline LOC728723|728723 & A6 \\
\hline LRP5|4041 & A6 \\
\hline MRPS12|6183 & A6 \\
\hline PKM2|5315 & A6 \\
\hline PMF1|11243 & A6 \\
\hline RAG1|5896 & A6 \\
\hline RHOU|58480 & A6 \\
\hline RNASE7|84659 & A6 \\
\hline SHPRH|257218 & A6 \\
\hline SMARCA2|6595 & A6 \\
\hline TBC1D14|57533 & A6 \\
\hline TTLL11|158135 & A6 \\
\hline VPS13D|55187 & $\mathrm{A} 6$ \\
\hline YIF1B|90522 & $\overline{A 6}$ \\
\hline ZBTB38|253461 & A6 \\
\hline ZDBF2|57683 & A6 \\
\hline $\mathrm{A} 2 \mathrm{M} \mid 2$ & A7 \\
\hline ACTG2|72 & A7 \\
\hline ANKAR|150709 & A7 \\
\hline AVPR2|554 & A7 \\
\hline C13orf15|28984 & A7 \\
\hline C14orf143|90141 & A7 \\
\hline C14orf28|122525 & A7 \\
\hline C18orf45|85019 & A7 \\
\hline C6orf162|57150 & A7 \\
\hline CAPZA3|93661 & A7 \\
\hline CASP12|120329 & A7 \\
\hline CCL14|6358 & A7 \\
\hline CD80|941 & A7 \\
\hline CHST3|9469 & A7 \\
\hline CTF1|1489 & A7 \\
\hline CXorf42|158801 & A7 \\
\hline CYP11A1|1583 & A7 \\
\hline CYYR1|116159 & A7 \\
\hline D4S234E|27065 & A7 \\
\hline ELAC1|55520 & A7 \\
\hline EPHB1|2047 & A7 \\
\hline FAHD2A|51011 & A7 \\
\hline FAM102A|399665 & A7 \\
\hline FAM86D|692099 & A7 \\
\hline FAP|2191 & A7 \\
\hline FBXW7|55294 & A7 \\
\hline FES|2242 & A7 \\
\hline FUT4|2526 & A7 \\
\hline HDAC4|9759 & A7 \\
\hline HOXD3|3232 & A7 \\
\hline
\end{tabular}




\begin{tabular}{|c|c|}
\hline ID4|3400 & A7 \\
\hline KCTD9|54793 & $\mathrm{A} 7$ \\
\hline KPNA2|3838 & $\mathrm{A} 7$ \\
\hline LHX6|26468 & $\mathrm{A} 7$ \\
\hline LIMK1|3984 & A7 \\
\hline LIX1L|128077 & $\mathrm{A} 7$ \\
\hline LOC100130691|100130691 & $\mathrm{A} 7$ \\
\hline LOC389705|389705 & A7 \\
\hline LRFN5|145581 & A7 \\
\hline LRRC15|131578 & $\mathrm{A} 7$ \\
\hline MAFF|23764 & $\mathrm{A} 7$ \\
\hline MBIP|51562 & $\mathrm{A} 7$ \\
\hline MEOX1|4222 & $\mathrm{A} 7$ \\
\hline MET|4233 & $\mathrm{A} 7$ \\
\hline NCALD|83988 & $\mathrm{A} 7$ \\
\hline NPFF $\mid 8620$ & $\mathrm{~A} 7$ \\
\hline NUDT7|283927 & $\mathrm{A} 7$ \\
\hline PCDHGA3|56112 & $\mathrm{A} 7$ \\
\hline PDE7B|27115 & A7 \\
\hline PPP2CA|5515 & $\mathrm{A} 7$ \\
\hline PROC|5624 & A7 \\
\hline RAB3A|5864 & $\mathrm{A} 7$ \\
\hline RBM45|129831 & $\mathrm{A} 7$ \\
\hline RBMS1|5937 & A7 \\
\hline RFXAP|5994 & $\mathrm{A} 7$ \\
\hline RIMKLB|57494 & $\mathrm{A} 7$ \\
\hline SEMA6A|57556 & $\mathrm{A} 7$ \\
\hline SFRP1|6422 & A7 \\
\hline SIRPA|140885 & $\mathrm{A} 7$ \\
\hline SLC17A7|57030 & $\mathrm{A} 7$ \\
\hline SLC23A2|9962 & $\mathrm{A} 7$ \\
\hline SOCS2|8835 & $\mathrm{A} 7$ \\
\hline SPARCL1|8404 & $\mathrm{A} 7$ \\
\hline ST7OT1|93653 & $\mathrm{A} 7$ \\
\hline TBL2|26608 & $\mathrm{A} 7$ \\
\hline TMOD2|29767 & $\mathrm{A} 7$ \\
\hline TRIOBP|11078 & $\mathrm{A} 7$ \\
\hline TUB|7275 & $\mathrm{A} 7$ \\
\hline VAMP8|8673 & $\mathrm{A} 7$ \\
\hline VAV3|10451 & $\mathrm{A} 7$ \\
\hline VCAN|1462 & A7 \\
\hline ABCA6|23460 & $\mathrm{A} 8$ \\
\hline ABCA9|10350 & $\mathrm{A} 8$ \\
\hline ABCB5|340273 & $\mathrm{A} 8$ \\
\hline ACBD6|84320 & $\mathrm{A} 8$ \\
\hline ACRC $\mid 93953$ & A8 \\
\hline ADH1C|126 & $\mathrm{A} 8$ \\
\hline ALS2CL|259173 & $\mathrm{A} 8$ \\
\hline AMY2B|280 & $\mathrm{A} 8$ \\
\hline ARHGAP10|79658 & $\mathrm{A} 8$ \\
\hline C10orf105|414152 & $\mathrm{A} 8$ \\
\hline
\end{tabular}




\begin{tabular}{|c|c|}
\hline C12orf48|55010 & A8 \\
\hline C1orf213|148898 & $\mathrm{A} 8$ \\
\hline C22orf23|84645 & $\mathrm{A} 8$ \\
\hline C22orf39|128977 & A8 \\
\hline C3orf64|285203 & $\mathrm{A} 8$ \\
\hline C4orf3|401152 & $\mathrm{A} 8$ \\
\hline C7orf51|222950 & A8 \\
\hline CACYBP|27101 & $\mathrm{A} 8$ \\
\hline CCNDBP1|23582 & $\mathrm{A} 8$ \\
\hline CELF6|60677 & $\mathrm{A} 8$ \\
\hline CENPM|79019 & $\mathrm{A} 8$ \\
\hline CENPT $\mid 80152$ & $\mathrm{~A} 8$ \\
\hline COPA|1314 & $\mathrm{A} 8$ \\
\hline CPEB1|64506 & $\mathrm{A} 8$ \\
\hline CPE|1363 & $\mathrm{A} 8$ \\
\hline CPSF3|51692 & $\mathrm{A} 8$ \\
\hline CREBL2|1389 & $\mathrm{A} 8$ \\
\hline DAP3|7818 & $\mathrm{A} 8$ \\
\hline DBF4|10926 & $\mathrm{A} 8$ \\
\hline DNASE1L3|1776 & $\mathrm{A} 8$ \\
\hline DPM2|8818 & $\mathrm{A} 8$ \\
\hline DSN1|79980 & A8 \\
\hline E2F1|1869 & $\mathrm{A} 8$ \\
\hline ECHDC2|55268 & $\mathrm{A} 8$ \\
\hline ENAH|55740 & $\mathrm{A} 8$ \\
\hline EPRS|2058 & $\mathrm{A} 8$ \\
\hline FAM160A2|84067 & $\mathrm{A} 8$ \\
\hline FAM49A|81553 & $\mathrm{A} 8$ \\
\hline FAM71D|161142 & $\mathrm{A} 8$ \\
\hline FLAD1|80308 & $\mathrm{A} 8$ \\
\hline GBAP1|2630 & $\mathrm{A} 8$ \\
\hline GEN1|348654 & $\mathrm{A} 8$ \\
\hline GOLGA6L10|647042 & $\mathrm{A} 8$ \\
\hline GPR44|11251 & $\mathrm{A} 8$ \\
\hline GPRIN1|114787 & $\mathrm{A} 8$ \\
\hline HCG2P7|80867 & $\mathrm{A} 8$ \\
\hline HPRT1|3251 & $\mathrm{A} 8$ \\
\hline IL17B $\mid 27190$ & $\mathrm{~A} 8$ \\
\hline IL6R/3570 & $\mathrm{A} 8$ \\
\hline ILF2|3608 & $\mathrm{A} 8$ \\
\hline INPP5B|3633 & $\mathrm{A} 8$ \\
\hline LENG8|114823 & $\mathrm{A} 8$ \\
\hline LEPREL1|55214 & $\mathrm{A} 8$ \\
\hline LEPR|3953 & $\mathrm{A} 8$ \\
\hline LMNB1/4001 & $\mathrm{A} 8$ \\
\hline LOC257358|257358 & $\mathrm{A} 8$ \\
\hline LOC286367|286367 & $\mathrm{A} 8$ \\
\hline $\begin{array}{l}\text { LOC728819|728819 } \\
\end{array}$ & $\mathrm{A} 8$ \\
\hline LOC91316|91316 & $\mathrm{A} 8$ \\
\hline LYNX1|66004 & $\mathrm{A} 8$ \\
\hline LYSMD4|145748 & $\mathrm{A} 8$ \\
\hline
\end{tabular}




\begin{tabular}{|c|c|}
\hline MAN2C1|4123 & A8 \\
\hline MAP2K5|5607 & A8 \\
\hline MYH11|4629 & A8 \\
\hline MYO15B|80022 & A8 \\
\hline NECAB $1 \mid 64168$ & A8 \\
\hline NTN5|126147 & A8 \\
\hline OSR1|130497 & A8 \\
\hline OTUD3|23252 & A8 \\
\hline PAQR4|124222 & A8 \\
\hline PKD1|5310 & A8 \\
\hline PPIEL|728448 & A8 \\
\hline PTGFR|5737 & A8 \\
\hline PYROXD2|84795 & A8 \\
\hline RBL2|5934 & A8 \\
\hline RBM5|10181 & A8 \\
\hline RFWD2|64326 & A8 \\
\hline RGAG4|340526 & A8 \\
\hline RNASE4|6038 & A8 \\
\hline \begin{tabular}{l|l|} 
RNPC35599 &
\end{tabular} & A8 \\
\hline RP1L1|94137 & A8 \\
\hline RYR3|6263 & A8 \\
\hline SDC3|9672 & A8 \\
\hline SDHC|6391 & A8 \\
\hline $\begin{array}{l}\text { SFRS5|6430 } \\
\text { SF }\end{array}$ & A8 \\
\hline SKA1|220134 & A8 \\
\hline SLC37A1|54020 & A8 \\
\hline SLC7A14|57709 & A8 \\
\hline SMAD3|4088 & A8 \\
\hline SMG6|23293 & A8 \\
\hline SNRPD1|6632 & A8 \\
\hline SNX1|6642 & A8 \\
\hline \begin{tabular}{l|l|l|} 
SNX33 & 257364 \\
\end{tabular} & A8 \\
\hline STIM2|57620 & A8 \\
\hline STX6|10228 & A8 \\
\hline TADA1|117143 & A8 \\
\hline TBC1D13|54662 & A8 \\
\hline TBC1D7|51256 & A8 \\
\hline TESC|54997 & A8 \\
\hline TFB2M|64216 & A8 \\
\hline \begin{tabular}{l|l|} 
TPCN12373 \\
\end{tabular} & A8 \\
\hline ТРPP|11076 & A8 \\
\hline TRIM68|55128 & A8 \\
\hline TSSK3|81629 & A8 \\
\hline TTC23|64927 & A8 \\
\hline TTLL3|26140 & A8 \\
\hline WASF3|10810 & A8 \\
\hline WDR34|89891 & $\mathrm{A} 8$ \\
\hline WDTC1|23038 & A8 \\
\hline WSB1|26118 & A8 \\
\hline ?|100133144 & B2 \\
\hline$? \mid 100134869$ & $\mathrm{~B} 2$ \\
\hline
\end{tabular}




\begin{tabular}{|c|c|}
\hline ?|10357 & B2 \\
\hline$? \mid 155060$ & $\mathrm{~B} 2$ \\
\hline$? 26823$ & $\mathrm{~B} 2$ \\
\hline$? \mid 340602$ & $\mathrm{~B} 2$ \\
\hline$? 388795$ & $\mathrm{~B} 2$ \\
\hline$? 390284$ & $\mathrm{~B} 2$ \\
\hline$? 391343$ & $\mathrm{~B} 2$ \\
\hline$? \mid 391714$ & $\mathrm{~B} 2$ \\
\hline$? \mid 442388$ & B2 \\
\hline$? 553137$ & $\mathrm{~B} 2$ \\
\hline ?57714 & $\mathrm{B} 2$ \\
\hline$? 645851$ & $\mathrm{~B} 2$ \\
\hline$? 652919$ & $\mathrm{~B} 2$ \\
\hline$? 728603$ & $\mathrm{~B} 2$ \\
\hline$? 728788$ & $\mathrm{~B} 2$ \\
\hline$?$ ?29884 & $\mathrm{B} 2$ \\
\hline$? \mid 8225$ & $\mathrm{~B} 2$ \\
\hline$? 90288$ & $\mathrm{~B} 2$ \\
\hline A1CF|29974 & B2 \\
\hline A2BP1|54715 & $\mathrm{B} 2$ \\
\hline A2LD1|87769 & $\mathrm{B} 2$ \\
\hline A2ML1|144568 & $\mathrm{B} 2$ \\
\hline A4GALT|53947 & $\mathrm{B} 2$ \\
\hline A4GNT|51146 & $\mathrm{B} 2$ \\
\hline AAA1|404744 & $\mathrm{B} 2$ \\
\hline AAAS|8086 & $\mathrm{B} 2$ \\
\hline AACSL|729522 & $\mathrm{B} 2$ \\
\hline AACS|65985 & $\mathrm{B} 2$ \\
\hline AADACL2|344752 & $\mathrm{B} 2$ \\
\hline AADACL3|126767 & $\mathrm{B} 2$ \\
\hline AADACL4|343066 & $\mathrm{B} 2$ \\
\hline AADAT|51166 & $\mathrm{B} 2$ \\
\hline AAK1|22848 & $\mathrm{B} 2$ \\
\hline AAMP|14 & $\mathrm{B} 2$ \\
\hline AANAT|15 & $\mathrm{B} 2$ \\
\hline AARS2|57505 & $\mathrm{B} 2$ \\
\hline AARSD1|80755 & $\mathrm{B} 2$ \\
\hline AARS|16 & $\mathrm{B} 2$ \\
\hline AASDHPPT|60496 & $\mathrm{B} 2$ \\
\hline AASDH|132949 & $\mathrm{B} 2$ \\
\hline AATF|26574 & B2 \\
\hline AATK|9625 & $\mathrm{B} 2$ \\
\hline ABAT|18 & $\mathrm{B} 2$ \\
\hline ABCA11P|79963 & $\mathrm{B} 2$ \\
\hline ABCA12|26154 & $\mathrm{B} 2$ \\
\hline ABCA13|154664 & B2 \\
\hline ABCA17P 650655 & $\mathrm{~B} 2$ \\
\hline ABCA2|20 & $\mathrm{B} 2$ \\
\hline \begin{tabular}{ll|l}
$\mathrm{ABCA} 3$ & 21 \\
\end{tabular} & $\mathrm{~B} 2$ \\
\hline ABCA4|24 & B2 \\
\hline ABCA7|10347 & $\mathrm{B} 2$ \\
\hline
\end{tabular}




\begin{tabular}{|c|c|}
\hline ABCB11|8647 & B2 \\
\hline ABCB4|5244 & B2 \\
\hline ABCB6|10058 & $\mathrm{B} 2$ \\
\hline ABCB7|22 & $\mathrm{B} 2$ \\
\hline ABCC $10 \mid 89845$ & B2 \\
\hline ABCC11|85320 & B2 \\
\hline ABCC12|94160 & $\mathrm{B} 2$ \\
\hline ABCC13|150000 & $\mathrm{B} 2$ \\
\hline $\mathrm{ABCC} 1 \mid 4363$ & $\mathrm{~B} 2$ \\
\hline $\mathrm{ABCC} 2 \mid 1244$ & $\mathrm{~B} 2$ \\
\hline ABCC3|8714 & $\mathrm{B} 2$ \\
\hline ABCC4|10257 & B2 \\
\hline ABCC6Р2|730013 & $\mathrm{B} 2$ \\
\hline ABCC $8 \mid 6833$ & $\mathrm{~B} 2$ \\
\hline $\mathrm{ABCD} 1 \mid 215$ & $\mathrm{~B} 2$ \\
\hline ABCE1|6059 & $\mathrm{B} 2$ \\
\hline ABCF2|10061 & $\mathrm{B} 2$ \\
\hline ABCG2|9429 & $\mathrm{B} 2$ \\
\hline ABCG4|64137 & B2 \\
\hline ABCG5|64240 & $\mathrm{B} 2$ \\
\hline ABCG8|64241 & $\mathrm{B} 2$ \\
\hline ABHD10|55347 & $\mathrm{B} 2$ \\
\hline ABHD12B|145447 & $\mathrm{B} 2$ \\
\hline ABHD13|84945 & $\mathrm{B} 2$ \\
\hline ABHD14A|25864 & $\mathrm{B} 2$ \\
\hline ABHD15|116236 & $\mathrm{B} 2$ \\
\hline ABHD2|11057 & B2 \\
\hline ABHD3|171586 & B2 \\
\hline ABHD4|63874 & $\mathrm{B} 2$ \\
\hline \begin{tabular}{l|l|} 
ABHD5|51099 \\
\end{tabular} & $\mathrm{B} 2$ \\
\hline ABHD8|79575 & $\mathrm{B} 2$ \\
\hline ABI1|10006 & $\mathrm{B} 2$ \\
\hline ABI2|10152 & $\mathrm{B} 2$ \\
\hline ABI3|51225 & B2 \\
\hline ABLIM2|84448 & $\mathrm{B} 2$ \\
\hline $\mathrm{ABO} \mid 28$ & $\mathrm{~B} 2$ \\
\hline ABP1|26 & $\mathrm{B} 2$ \\
\hline ABRA|137735 & $\mathrm{B} 2$ \\
\hline ABT1|29777 & $\mathrm{B} 2$ \\
\hline ABTB2|25841 & $\mathrm{B} 2$ \\
\hline ACAA1|30 & B2 \\
\hline ACACA|31 & $\mathrm{B} 2$ \\
\hline ACAD10|80724 & B2 \\
\hline ACAD9|28976 & $\mathrm{B} 2$ \\
\hline ACADM|34 & $\mathrm{B} 2$ \\
\hline ACADSB|36 & $\mathrm{B} 2$ \\
\hline ACAN|176 & $\mathrm{B} 2$ \\
\hline ACAP1|9744 & $\mathrm{B} 2$ \\
\hline ACAP2|23527 & $\mathrm{B} 2$ \\
\hline ACAP3|116983 & $\mathrm{B} 2$ \\
\hline ACAT1|38 & $\mathrm{B} 2$ \\
\hline
\end{tabular}




\begin{tabular}{|c|c|}
\hline ACBD5|91452 & B2 \\
\hline ACBD7|414149 & $\mathrm{B} 2$ \\
\hline ACCN1|40 & $\mathrm{B} 2$ \\
\hline ACCN2|41 & $\mathrm{B} 2$ \\
\hline ACCN3|9311 & $\mathrm{B} 2$ \\
\hline ACCN4|55515 & B2 \\
\hline ACCN5|51802 & $\mathrm{B} 2$ \\
\hline ACCSL|390110 & $\mathrm{B} 2$ \\
\hline $\mathrm{ACD} \mid 65057$ & $\mathrm{~B} 2$ \\
\hline ACE2|59272 & $\mathrm{B} 2$ \\
\hline ACER1|125981 & $\mathrm{B} 2$ \\
\hline ACER2|340485 & B2 \\
\hline \begin{tabular}{l|l|} 
ACER3 55331 \\
\end{tabular} & $\mathrm{~B} 2$ \\
\hline ACE $\mid 1636$ & $\mathrm{~B} 2$ \\
\hline ACHE|43 & $\mathrm{B} 2$ \\
\hline ACIN1|22985 & $\mathrm{B} 2$ \\
\hline ACLY|47 & $\mathrm{B} 2$ \\
\hline ACMSD|130013 & $\mathrm{B} 2$ \\
\hline ACN9|57001 & B2 \\
\hline $\mathrm{ACO} 2 \mid 50$ & $\mathrm{~B} 2$ \\
\hline ACOT11|26027 & $\mathrm{B} 2$ \\
\hline ACOT12|134526 & $\mathrm{B} 2$ \\
\hline ACOT13|55856 & $\mathrm{B} 2$ \\
\hline ACOT1|641371 & B2 \\
\hline ACOT2|10965 & $\mathrm{B} 2$ \\
\hline ACOT4|122970 & $\mathrm{B} 2$ \\
\hline ACOT6|641372 & B2 \\
\hline ACOX1|51 & B2 \\
\hline ACOX2|8309 & $\mathrm{B} 2$ \\
\hline ACOX3|8310 & $\mathrm{B} 2$ \\
\hline ACOXL|55289 & $\mathrm{B} 2$ \\
\hline ACP5|54 & $\mathrm{B} 2$ \\
\hline ACP6|51205 & $\mathrm{B} 2$ \\
\hline ACPL2|92370 & B2 \\
\hline ACPP 55 & $\mathrm{~B} 2$ \\
\hline ACPT|93650 & $\mathrm{B} 2$ \\
\hline ACRBP|84519 & $\mathrm{B} 2$ \\
\hline ACRV1|56 & $\mathrm{B} 2$ \\
\hline ACSBG1|23205 & $\mathrm{B} 2$ \\
\hline ACSF2|80221 & $\mathrm{B} 2$ \\
\hline ACSF3|197322 & B2 \\
\hline ACSL3|2181 & $\mathrm{B} 2$ \\
\hline ACSL6|23305 & B2 \\
\hline ACSM1|116285 & $\mathrm{B} 2$ \\
\hline ACSM2A|123876 & $\mathrm{B} 2$ \\
\hline ACSM2B|348158 & $\mathrm{B} 2$ \\
\hline ACSM3|6296 & $\mathrm{B} 2$ \\
\hline ACSM4|341392 & $\mathrm{B} 2$ \\
\hline ACSS1|84532 & $\mathrm{B} 2$ \\
\hline ACSS3|79611 & $\mathrm{B} 2$ \\
\hline ACTA1|58 & $\mathrm{B} 2$ \\
\hline
\end{tabular}




\begin{tabular}{|c|c|}
\hline ACTBL2|345651 & B2 \\
\hline ACTC1|70 & B2 \\
\hline ACTL6B|51412 & $\mathrm{B} 2$ \\
\hline ACTL7A|10881 & $\mathrm{B} 2$ \\
\hline ACTL7B|10880 & $\mathrm{B} 2$ \\
\hline ACTL8|81569 & $\mathrm{B} 2$ \\
\hline ACTL9|284382 & $\mathrm{B} 2$ \\
\hline ACTN1|87 & $\mathrm{B} 2$ \\
\hline ACTN2|88 & $\mathrm{B} 2$ \\
\hline ACTN3|89 & $\mathrm{B} 2$ \\
\hline ACTN4|81 & $\mathrm{B} 2$ \\
\hline ACTR10|55860 & $\mathrm{B} 2$ \\
\hline ACTR1A|10121 & $\mathrm{B} 2$ \\
\hline ACTR3B|57180 & $\mathrm{B} 2$ \\
\hline ACTR3C|653857 & $\mathrm{B} 2$ \\
\hline ACTR5|79913 & $\mathrm{B} 2$ \\
\hline ACTR6|64431 & $\mathrm{B} 2$ \\
\hline $\begin{array}{l}\text { ACTR8|93973 } \\
\end{array}$ & $\mathrm{B} 2$ \\
\hline ACTRT1|139741 & B2 \\
\hline ACTRT2|140625 & $\mathrm{B} 2$ \\
\hline ACVR1B|91 & $\mathrm{B} 2$ \\
\hline ACVR1|90 & $\mathrm{B} 2$ \\
\hline ACVR2B|93 & $\mathrm{B} 2$ \\
\hline ACY1|95 & B2 \\
\hline ACY3|91703 & $\mathrm{B} 2$ \\
\hline ACYP1|97 & $\mathrm{B} 2$ \\
\hline ACYP2|98 & $\mathrm{B} 2$ \\
\hline ADAD1|132612 & B2 \\
\hline ADAL|161823 & $\mathrm{B} 2$ \\
\hline ADAM10|102 & $\mathrm{B} 2$ \\
\hline ADAM11|4185 & $\mathrm{B} 2$ \\
\hline ADAM17|6868 & $\mathrm{B} 2$ \\
\hline ADAM18|8749 & $\mathrm{B} 2$ \\
\hline ADAM20|8748 & B2 \\
\hline ADAM21P1|145241 & $\mathrm{B} 2$ \\
\hline ADAM21|8747 & $\mathrm{B} 2$ \\
\hline ADAM22|53616 & $\mathrm{B} 2$ \\
\hline ADAM23|8745 & $\mathrm{B} 2$ \\
\hline ADAM28|10863 & B2 \\
\hline ADAM29|11086 & $\mathrm{B} 2$ \\
\hline ADAM2|2515 & B2 \\
\hline ADAM30|11085 & $\mathrm{B} 2$ \\
\hline ADAM32|203102 & B2 \\
\hline ADAM3A|1587 & $\mathrm{B} 2$ \\
\hline ADAM5P|255926 & $\mathrm{B} 2$ \\
\hline ADAM6|8755 & $\mathrm{B} 2$ \\
\hline ADAM9|8754 & $\mathrm{B} 2$ \\
\hline ADAMDEC1|27299 & $\mathrm{B} 2$ \\
\hline ADAMTS10|81794 & $\mathrm{B} 2$ \\
\hline ADAMTS13|11093 & $\mathrm{B} 2$ \\
\hline ADAMTS15|170689 & $\mathrm{B} 2$ \\
\hline
\end{tabular}




\begin{tabular}{|c|c|}
\hline ADAMTS16|170690 & B2 \\
\hline ADAMTS17|170691 & $\mathrm{B} 2$ \\
\hline ADAMTS19|171019 & $\mathrm{B} 2$ \\
\hline ADAMTS20|80070 & $\mathrm{B} 2$ \\
\hline ADAMTS2|9509 & B2 \\
\hline ADAMTS4|9507 & $\mathrm{B} 2$ \\
\hline ADAMTS8|11095 & $\mathrm{B} 2$ \\
\hline ADAMTSL1|92949 & $\mathrm{B} 2$ \\
\hline ADAMTSL3|57188 & $\mathrm{B} 2$ \\
\hline ADAMTSL5|339366 & $\mathrm{B} 2$ \\
\hline ADAP2|55803 & $\mathrm{B} 2$ \\
\hline ADARB2|105 & B2 \\
\hline ADAT1|23536 & $\mathrm{B} 2$ \\
\hline ADAT3|113179 & $\mathrm{B} 2$ \\
\hline ADA|100 & $\mathrm{B} 2$ \\
\hline ADCK1|57143 & $\mathrm{B} 2$ \\
\hline ADCK4|79934 & B2 \\
\hline ADCY10|55811 & $\mathrm{B} 2$ \\
\hline ADCY1|107 & B2 \\
\hline ADCY2|108 & $\mathrm{B} 2$ \\
\hline ADCY7|113 & $\mathrm{B} 2$ \\
\hline ADCY8|114 & $\mathrm{B} 2$ \\
\hline ADCY9|115 & $\mathrm{B} 2$ \\
\hline ADCYAP1|116 & $\mathrm{B} 2$ \\
\hline ADD2|119 & $\mathrm{B} 2$ \\
\hline ADH7|131 & $\mathrm{B} 2$ \\
\hline ADI1|55256 & $\mathrm{B} 2$ \\
\hline ADIG|149685 & $\mathrm{B} 2$ \\
\hline ADIPOR2|79602 & $\mathrm{B} 2$ \\
\hline ADK|132 & $\mathrm{B} 2$ \\
\hline ADNP2|22850 & $\mathrm{B} 2$ \\
\hline ADORA1|134 & $\mathrm{B} 2$ \\
\hline ADORA2B|136 & $\mathrm{B} 2$ \\
\hline $\mathrm{ADO} \mid 84890$ & B2 \\
\hline ADPGK|83440 & $\mathrm{B} 2$ \\
\hline ADPRHL1|113622 & $\mathrm{B} 2$ \\
\hline ADRA1B|147 & $\mathrm{B} 2$ \\
\hline ADRA1D|146 & $\mathrm{B} 2$ \\
\hline ADRA2B|151 & $\mathrm{B} 2$ \\
\hline ADRA2C|152 & $\mathrm{B} 2$ \\
\hline ADRB1|153 & B2 \\
\hline ADRB3|155 & $\mathrm{B} 2$ \\
\hline ADRBK1|156 & B2 \\
\hline ADSL|158 & $\mathrm{B} 2$ \\
\hline ADSSL1|122622 & $\mathrm{B} 2$ \\
\hline AEBP2|121536 & $\mathrm{B} 2$ \\
\hline AEN|64782 & $\mathrm{B} 2$ \\
\hline AES|166 & $\mathrm{B} 2$ \\
\hline AFAP1L2|84632 & $\mathrm{B} 2$ \\
\hline AFAP1|60312 & B2 \\
\hline AFARP1|246182 & $\mathrm{B} 2$ \\
\hline
\end{tabular}




\begin{tabular}{|c|c|}
\hline AFF2|2334 & B2 \\
\hline AFF3|3899 & B2 \\
\hline AFF4|27125 & $\mathrm{B} 2$ \\
\hline AFG3L1|172 & $\mathrm{B} 2$ \\
\hline AFG3L2|10939 & B2 \\
\hline AFMID|125061 & B2 \\
\hline AFM|173 & $\mathrm{B} 2$ \\
\hline AFP|174 & B2 \\
\hline AGAP1|116987 & $\mathrm{B} 2$ \\
\hline AGAP2|116986 & $\mathrm{B} 2$ \\
\hline AGAP3|116988 & $\mathrm{B} 2$ \\
\hline AGAP5|729092 & B2 \\
\hline AGAP6|414189 & $\mathrm{B} 2$ \\
\hline AGAP7|653268 & $\mathrm{B} 2$ \\
\hline AGAP8|728404 & $\mathrm{B} 2$ \\
\hline AGA|175 & $\mathrm{B} 2$ \\
\hline AGBL1|123624 & $\mathrm{B} 2$ \\
\hline $\begin{array}{l}\text { AGBL2|79841 } \\
\end{array}$ & $\mathrm{B} 2$ \\
\hline AGBL3|340351 & B2 \\
\hline AGBL4|84871 & $\mathrm{B} 2$ \\
\hline AGBL5|60509 & $\mathrm{B} 2$ \\
\hline AGFG1|3267 & $\mathrm{B} 2$ \\
\hline AGGF1|55109 & $\mathrm{B} 2$ \\
\hline AGK|55750 & $\mathrm{B} 2$ \\
\hline AGL|178 & $\mathrm{B} 2$ \\
\hline AGMAT|79814 & $\mathrm{B} 2$ \\
\hline AGPAT1|10554 & B2 \\
\hline \begin{tabular}{l|l} 
AGPAT3|56894 \\
\end{tabular} & B2 \\
\hline AGPAT5|55326 & $\mathrm{B} 2$ \\
\hline AGPAT6|137964 & $\mathrm{B} 2$ \\
\hline AGPAT9|84803 & $\mathrm{B} 2$ \\
\hline AGPHD1|123688 & $\mathrm{B} 2$ \\
\hline AGPS $\mid 8540$ & $\mathrm{~B} 2$ \\
\hline AGR2|10551 & B2 \\
\hline AGRN|375790 & $\mathrm{B} 2$ \\
\hline AGRP|181 & $\mathrm{B} 2$ \\
\hline AGTPBP1|23287 & $\mathrm{B} 2$ \\
\hline AGTR1|185 & $\mathrm{B} 2$ \\
\hline AGTR2|186 & $\mathrm{B} 2$ \\
\hline AGT|183 & $\mathrm{B} 2$ \\
\hline AGXT2L1|64850 & B2 \\
\hline AGXT2|64902 & $\mathrm{B} 2$ \\
\hline AGXT|189 & B2 \\
\hline AHCYL1|10768 & $\mathrm{B} 2$ \\
\hline AHCYL2|23382 & $\mathrm{B} 2$ \\
\hline AHI1|54806 & $\mathrm{B} 2$ \\
\hline AHNAK2|113146 & $\mathrm{B} 2$ \\
\hline AHRR|57491 & $\mathrm{B} 2$ \\
\hline AHR|196 & $\mathrm{B} 2$ \\
\hline AHSG|197 & $\mathrm{B} 2$ \\
\hline AHSP|51327 & $\mathrm{B} 2$ \\
\hline
\end{tabular}




\begin{tabular}{|c|c|}
\hline AICDA|57379 & B2 \\
\hline AIDA|64853 & B2 \\
\hline AIF1|199 & $\mathrm{B} 2$ \\
\hline AIFM3|150209 & $\mathrm{B} 2$ \\
\hline AIM1L|55057 & $\mathrm{B} 2$ \\
\hline AIM1|202 & $\mathrm{B} 2$ \\
\hline AIM2|9447 & $\mathrm{B} 2$ \\
\hline AIMP1|9255 & $\mathrm{B} 2$ \\
\hline AIPL1|23746 & $\mathrm{B} 2$ \\
\hline AIP $\mid 9049$ & $\mathrm{~B} 2$ \\
\hline AIRE|326 & $\mathrm{B} 2$ \\
\hline AK1|203 & B2 \\
\hline AK2|204 & $\mathrm{B} 2$ \\
\hline AK3L1|205 & $\mathrm{B} 2$ \\
\hline AK7|122481 & $\mathrm{B} 2$ \\
\hline AKAP10|11216 & $\mathrm{B} 2$ \\
\hline AKAP14|158798 & B2 \\
\hline AKAP4|8852 & $\mathrm{B} 2$ \\
\hline AKAP8L|26993 & B2 \\
\hline AKAP8|10270 & $\mathrm{B} 2$ \\
\hline AKAP9|10142 & $\mathrm{B} 2$ \\
\hline AKD1|221264 & $\mathrm{B} 2$ \\
\hline AKIRIN1|79647 & $\mathrm{B} 2$ \\
\hline AKNAD1|254268 & $\mathrm{B} 2$ \\
\hline AKNA|80709 & $\mathrm{B} 2$ \\
\hline AKR1A1|10327 & $\mathrm{B} 2$ \\
\hline AKR1B10|57016 & $\mathrm{B} 2$ \\
\hline AKR1B15|441282 & B2 \\
\hline AKR1B1|231 & $\mathrm{B} 2$ \\
\hline AKR1C4|1109 & $\mathrm{B} 2$ \\
\hline AKR1CL1|340811 & $\mathrm{B} 2$ \\
\hline AKR1D1|6718 & $\mathrm{B} 2$ \\
\hline AKR1E2|83592 & $\mathrm{B} 2$ \\
\hline AKR7A2|8574 & $\mathrm{B} 2$ \\
\hline AKR7A3|22977 & $\mathrm{B} 2$ \\
\hline AKR7L|246181 & $\mathrm{B} 2$ \\
\hline AKT1S1|84335 & $\mathrm{B} 2$ \\
\hline AKT2|208 & $\mathrm{B} 2$ \\
\hline AKT3|10000 & $\mathrm{B} 2$ \\
\hline AKTIP|64400 & $\mathrm{B} 2$ \\
\hline ALAS1|211 & B2 \\
\hline ALAS2|212 & $\mathrm{B} 2$ \\
\hline ALB 213 & B2 \\
\hline ALCAM|214 & $\mathrm{B} 2$ \\
\hline ALDH16A1|126133 & $\mathrm{B} 2$ \\
\hline ALDH1L2|160428 & $\mathrm{B} 2$ \\
\hline ALDH3A1|218 & $\mathrm{B} 2$ \\
\hline ALDH3B1|221 & $\mathrm{B} 2$ \\
\hline ALDH3B2|222 & $\mathrm{B} 2$ \\
\hline ALDH4A1|8659 & B2 \\
\hline ALDH5A1|7915 & $\mathrm{B} 2$ \\
\hline
\end{tabular}




\begin{tabular}{|c|c|}
\hline ALDH6A1|4329 & B2 \\
\hline ALDH8A1|64577 & $\mathrm{B} 2$ \\
\hline ALDH9A1|223 & $\mathrm{B} 2$ \\
\hline ALDOA|226 & $\mathrm{B} 2$ \\
\hline ALDOB|229 & B2 \\
\hline ALG10B|144245 & $\mathrm{B} 2$ \\
\hline ALG10|84920 & $\mathrm{B} 2$ \\
\hline ALG12|79087 & $\mathrm{B} 2$ \\
\hline ALG13|79868 & $\mathrm{B} 2$ \\
\hline ALG14|199857 & $\mathrm{B} 2$ \\
\hline ALG1L2|644974 & $\mathrm{B} 2$ \\
\hline ALG1L|200810 & B2 \\
\hline ALG2|85365 & $\mathrm{B} 2$ \\
\hline ALG5|29880 & $\mathrm{B} 2$ \\
\hline ALG8|79053 & $\mathrm{B} 2$ \\
\hline ALG9|79796 & $\mathrm{B} 2$ \\
\hline ALKBH1|8846 & $\mathrm{B} 2$ \\
\hline ALKBH2|121642 & $\mathrm{B} 2$ \\
\hline ALKBH4|54784 & B2 \\
\hline ALKBH5|54890 & $\mathrm{B} 2$ \\
\hline ALKBH7|84266 & $\mathrm{B} 2$ \\
\hline ALKBH8|91801 & $\mathrm{B} 2$ \\
\hline ALK|238 & $\mathrm{B} 2$ \\
\hline ALLC|55821 & $\mathrm{B} 2$ \\
\hline ALMS1P|200420 & $\mathrm{B} 2$ \\
\hline ALMS1|7840 & $\mathrm{B} 2$ \\
\hline ALOX12B|242 & $\mathrm{B} 2$ \\
\hline ALOX12P2|245 & B2 \\
\hline ALOX12|239 & $\mathrm{B} 2$ \\
\hline ALOX15B|247 & $\mathrm{B} 2$ \\
\hline ALOX15|246 & $\mathrm{B} 2$ \\
\hline ALOX5AP|241 & $\mathrm{B} 2$ \\
\hline ALOX5|240 & $\mathrm{B} 2$ \\
\hline ALOXE3|59344 & B2 \\
\hline ALPI|248 & $\mathrm{B} 2$ \\
\hline ALPK1|80216 & $\mathrm{B} 2$ \\
\hline ALPK2|115701 & $\mathrm{B} 2$ \\
\hline ALPK3|57538 & $\mathrm{B} 2$ \\
\hline ALPPL2|251 & $\mathrm{B} 2$ \\
\hline ALPP $\mid 250$ & $\mathrm{~B} 2$ \\
\hline ALS2CR11|151254 & B2 \\
\hline ALS2CR12|130540 & $\mathrm{B} 2$ \\
\hline ALS2|57679 & B2 \\
\hline ALX1|8092 & $\mathrm{B} 2$ \\
\hline ALX3|257 & $\mathrm{B} 2$ \\
\hline AMAC1L2|83650 & $\mathrm{B} 2$ \\
\hline AMAC1L3|643664 & $\mathrm{B} 2$ \\
\hline AMAC1|146861 & $\mathrm{B} 2$ \\
\hline AMBN|258 & $\mathrm{B} 2$ \\
\hline AMBP|259 & $\mathrm{B} 2$ \\
\hline AMBRA1|55626 & $\mathrm{B} 2$ \\
\hline
\end{tabular}




\begin{tabular}{|c|c|}
\hline AMD1|262 & B2 \\
\hline AMDHD1|144193 & $\mathrm{B} 2$ \\
\hline AMDHD2|51005 & $\mathrm{B} 2$ \\
\hline AMELX|265 & $\mathrm{B} 2$ \\
\hline AMFR|267 & B2 \\
\hline AMHR2|269 & $\mathrm{B} 2$ \\
\hline AMH 268 & $\mathrm{~B} 2$ \\
\hline AMICA1|120425 & $\mathrm{B} 2$ \\
\hline AMMECR1L|83607 & $\mathrm{B} 2$ \\
\hline AMN1|196394 & $\mathrm{B} 2$ \\
\hline AMN|81693 & $\mathrm{B} 2$ \\
\hline AMOT|154796 & B2 \\
\hline AMPD1|270 & $\mathrm{B} 2$ \\
\hline AMPD2|271 & B2 \\
\hline AMPD3|272 & $\mathrm{B} 2$ \\
\hline AMPH|273 & B2 \\
\hline AMTN|401138 & B2 \\
\hline AMY1A|276 & $\mathrm{B} 2$ \\
\hline AMY2A|279 & B2 \\
\hline AMZ1|155185 & $\mathrm{B} 2$ \\
\hline AMZ2P1|201283 & B2 \\
\hline ANAPC10|10393 & $\mathrm{B} 2$ \\
\hline ANAPC13|25847 & $\mathrm{B} 2$ \\
\hline ANAPC16|119504 & $\mathrm{B} 2$ \\
\hline ANAPC1|64682 & $\mathrm{B} 2$ \\
\hline ANAPC2|29882 & $\mathrm{B} 2$ \\
\hline ANAPC5|51433 & $\mathrm{B} 2$ \\
\hline ANGEL1|23357 & B2 \\
\hline ANGPT2|285 & $\mathrm{B} 2$ \\
\hline ANGPTL3|27329 & $\mathrm{B} 2$ \\
\hline ANGPTL5|253935 & $\mathrm{B} 2$ \\
\hline ANK1|286 & $\mathrm{B} 2$ \\
\hline ANK3|288 & $\mathrm{B} 2$ \\
\hline ANKFN1|162282 & B2 \\
\hline ANKHD1-EIF4EBP3|404734 & $\mathrm{B} 2$ \\
\hline ANKHD1|54882 & $\mathrm{B} 2$ \\
\hline ANKH|56172 & $\mathrm{B} 2$ \\
\hline ANKIB1|54467 & $\mathrm{B} 2$ \\
\hline ANKLE1|126549 & $\mathrm{B} 2$ \\
\hline ANKLE2|23141 & $\mathrm{B} 2$ \\
\hline ANKMY1|51281 & B2 \\
\hline ANKRA2|57763 & $\mathrm{B} 2$ \\
\hline ANKRD10|55608 & B2 \\
\hline ANKRD12|23253 & $\mathrm{B} 2$ \\
\hline ANKRD13A|88455 & $\mathrm{B} 2$ \\
\hline ANKRD13B|124930 & $\mathrm{B} 2$ \\
\hline ANKRD13C|81573 & $\mathrm{B} 2$ \\
\hline ANKRD13D|338692 & $\mathrm{B} 2$ \\
\hline ANKRD16|54522 & $\mathrm{B} 2$ \\
\hline ANKRD17|26057 & B2 \\
\hline ANKRD19|138649 & $\mathrm{B} 2$ \\
\hline
\end{tabular}




\begin{tabular}{|c|c|}
\hline ANKRD1|27063 & B2 \\
\hline ANKRD20A3|441425 & B2 \\
\hline ANKRD20A4|728747 & $\mathrm{B} 2$ \\
\hline ANKRD20B|729171 & $\mathrm{B} 2$ \\
\hline ANKRD23|200539 & B2 \\
\hline ANKRD24|170961 & $\mathrm{B} 2$ \\
\hline ANKRD26P1|124149 & $\mathrm{B} 2$ \\
\hline ANKRD26|22852 & $\mathrm{B} 2$ \\
\hline ANKRD27|84079 & $\mathrm{B} 2$ \\
\hline ANKRD28|23243 & $\mathrm{B} 2$ \\
\hline ANKRD2|26287 & $\mathrm{B} 2$ \\
\hline ANKRD30A|91074 & B2 \\
\hline ANKRD30B|374860 & $\mathrm{B} 2$ \\
\hline ANKRD31|256006 & $\mathrm{B} 2$ \\
\hline ANKRD33|341405 & $\mathrm{B} 2$ \\
\hline ANKRD34A|284615 & $\mathrm{B} 2$ \\
\hline ANKRD34B|340120 & $\mathrm{B} 2$ \\
\hline ANKRD34C|390616 & $\mathrm{B} 2$ \\
\hline ANKRD36BP1|84832 & B2 \\
\hline ANKRD36B|57730 & $\mathrm{B} 2$ \\
\hline ANKRD37|353322 & $\mathrm{B} 2$ \\
\hline ANKRD39|51239 & $\mathrm{B} 2$ \\
\hline ANKRD42|338699 & $\mathrm{B} 2$ \\
\hline ANKRD43|134548 & $\mathrm{B} 2$ \\
\hline ANKRD44|91526 & $\mathrm{B} 2$ \\
\hline ANKRD45|339416 & $\mathrm{B} 2$ \\
\hline ANKRD46|157567 & $\mathrm{B} 2$ \\
\hline ANKRD49|54851 & B2 \\
\hline ANKRD50|57182 & $\mathrm{B} 2$ \\
\hline ANKRD52|283373 & $\mathrm{B} 2$ \\
\hline ANKRD54|129138 & $\mathrm{B} 2$ \\
\hline ANKRD55|79722 & $\mathrm{B} 2$ \\
\hline ANKRD56|345079 & $\mathrm{B} 2$ \\
\hline ANKRD57|65124 & B2 \\
\hline ANKRD7|56311 & $\mathrm{B} 2$ \\
\hline ANKRD9|122416 & $\mathrm{B} 2$ \\
\hline ANKS1B|56899 & $\mathrm{B} 2$ \\
\hline ANKS3|124401 & $\mathrm{B} 2$ \\
\hline ANKS4B|257629 & $\mathrm{B} 2$ \\
\hline ANKS6|203286 & $\mathrm{B} 2$ \\
\hline ANKZF1|55139 & B2 \\
\hline ANO10|55129 & $\mathrm{B} 2$ \\
\hline ANO1|55107 & B2 \\
\hline ANO3|63982 & $\mathrm{B} 2$ \\
\hline ANO5|203859 & $\mathrm{B} 2$ \\
\hline ANO7|50636 & $\mathrm{B} 2$ \\
\hline ANO8|57719 & $\mathrm{B} 2$ \\
\hline ANP32A|8125 & $\mathrm{B} 2$ \\
\hline ANP32B|10541 & $\mathrm{B} 2$ \\
\hline ANP32C|23520 & $\mathrm{B} 2$ \\
\hline ANP32D|23519 & B2 \\
\hline
\end{tabular}




\begin{tabular}{|c|c|}
\hline ANPEP|290 & $\mathrm{B} 2$ \\
\hline ANTXR1|84168 & $\mathrm{B} 2$ \\
\hline ANTXRL|195977 & $\mathrm{B} 2$ \\
\hline ANUBL1 93550 & $\mathrm{~B} 2$ \\
\hline ANXA10|11199 & B2 \\
\hline ANXA13|312 & B2 \\
\hline ANXA2P1|303 & $\mathrm{B} 2$ \\
\hline ANXA2P2|304 & B2 \\
\hline ANXA2P3|305 & $\mathrm{B} 2$ \\
\hline ANXA2|302 & $\mathrm{B} 2$ \\
\hline ANXA4|307 & $\mathrm{B} 2$ \\
\hline ANXA5|308 & $\mathrm{B} 2$ \\
\hline ANXA7|310 & $\mathrm{B} 2$ \\
\hline ANXA8L1|728113 & $\mathrm{B} 2$ \\
\hline ANXA8L2|244 & $\mathrm{B} 2$ \\
\hline ANXA8|653145 & $\mathrm{B} 2$ \\
\hline $\mathrm{AOAH} \mid 313$ & $\mathrm{~B} 2$ \\
\hline AOX2P|344454 & $\mathrm{B} 2$ \\
\hline AP1B1|162 & $\mathrm{B} 2$ \\
\hline AP1G1|164 & $\mathrm{B} 2$ \\
\hline AP1M1|8907 & $\mathrm{B} 2$ \\
\hline AP1S2|8905 & $\mathrm{B} 2$ \\
\hline AP2A1|160 & $\mathrm{B} 2$ \\
\hline AP2B1|163 & $\mathrm{B} 2$ \\
\hline AP3B2|8120 & $\mathrm{B} 2$ \\
\hline AP3M1|26985 & $\mathrm{B} 2$ \\
\hline AP3M2|10947 & $\mathrm{B} 2$ \\
\hline AP3S1|1176 & $\mathrm{B} 2$ \\
\hline AP3S2|10239 & $\mathrm{B} 2$ \\
\hline AP4B1|10717 & $\mathrm{B} 2$ \\
\hline AP4E1|23431 & $\mathrm{B} 2$ \\
\hline AP4S1|11154 & $\mathrm{B} 2$ \\
\hline APAF1|317 & $\mathrm{B} 2$ \\
\hline APBA2|321 & $\mathrm{B} 2$ \\
\hline APBA3|9546 & $\mathrm{B} 2$ \\
\hline APBB2|323 & $\mathrm{B} 2$ \\
\hline APBB3|10307 & $\mathrm{B} 2$ \\
\hline APC2|10297 & $\mathrm{B} 2$ \\
\hline APC|324 & $\mathrm{B} 2$ \\
\hline APEX1|328 & $\mathrm{B} 2$ \\
\hline APH1B|83464 & $\mathrm{B} 2$ \\
\hline API5|8539 & $\mathrm{B} 2$ \\
\hline APIP|51074 & $\mathrm{B} 2$ \\
\hline APLNR|187 & $\mathrm{B} 2$ \\
\hline APLN|8862 & $\mathrm{B} 2$ \\
\hline APLP1|333 & $\mathrm{B} 2$ \\
\hline APLP2|334 & $\mathrm{B} 2$ \\
\hline APOA1|335 & $\mathrm{B} 2$ \\
\hline APOA2|336 & $\mathrm{B} 2$ \\
\hline APOA4|337 & $\mathrm{B} 2$ \\
\hline APOA5|116519 & $\mathrm{B} 2$ \\
\hline
\end{tabular}




\begin{tabular}{|c|c|}
\hline APOBEC1|339 & B2 \\
\hline APOBEC2|10930 & $\mathrm{B} 2$ \\
\hline APOBEC3A|200315 & $\mathrm{B} 2$ \\
\hline APOBEC3B|9582 & $\mathrm{B} 2$ \\
\hline APOBEC3D|140564 & B2 \\
\hline APOBEC3F|200316 & $\mathrm{B} 2$ \\
\hline APOBEC3G|60489 & $\mathrm{B} 2$ \\
\hline APOBEC3H|164668 & $\mathrm{B} 2$ \\
\hline APOBEC4|403314 & $\mathrm{B} 2$ \\
\hline APOC1P1|342 & $\mathrm{B} 2$ \\
\hline APOC1|341 & $\mathrm{B} 2$ \\
\hline APOC3|345 & B2 \\
\hline APOC4|346 & $\mathrm{B} 2$ \\
\hline APOD|347 & $\mathrm{B} 2$ \\
\hline APOE|348 & $\mathrm{B} 2$ \\
\hline APOF|319 & $\mathrm{B} 2$ \\
\hline APOH 350 & $\mathrm{~B} 2$ \\
\hline APOL1|8542 & $\mathrm{B} 2$ \\
\hline APOL2|23780 & B2 \\
\hline APOL4|80832 & $\mathrm{B} 2$ \\
\hline APOL5|80831 & $\mathrm{B} 2$ \\
\hline APOL6|80830 & $\mathrm{B} 2$ \\
\hline APOM|55937 & $\mathrm{B} 2$ \\
\hline APOOL|139322 & $\mathrm{B} 2$ \\
\hline APPBP2|10513 & $\mathrm{B} 2$ \\
\hline APPL1|26060 & $\mathrm{B} 2$ \\
\hline $\mathrm{APP} \mid 351$ & $\mathrm{~B} 2$ \\
\hline APRT|353 & B2 \\
\hline AQP10|89872 & $\mathrm{B} 2$ \\
\hline AQP11|282679 & $\mathrm{B} 2$ \\
\hline AQP12A|375318 & $\mathrm{B} 2$ \\
\hline AQP12B|653437 & $\mathrm{B} 2$ \\
\hline AQP2|359 & $\mathrm{B} 2$ \\
\hline AQP3|360 & B2 \\
\hline AQP4|361 & $\mathrm{B} 2$ \\
\hline AQP5|362 & $\mathrm{B} 2$ \\
\hline AQP6|363 & $\mathrm{B} 2$ \\
\hline AQP8|343 & $\mathrm{B} 2$ \\
\hline AQP9|366 & $\mathrm{B} 2$ \\
\hline AQR|9716 & $\mathrm{B} 2$ \\
\hline ARAF|369 & B2 \\
\hline ARAP2|116984 & $\mathrm{B} 2$ \\
\hline ARCN1|372 & B2 \\
\hline AREG|374 & $\mathrm{B} 2$ \\
\hline ARFGAP2|84364 & $\mathrm{B} 2$ \\
\hline ARFGAP3|26286 & $\mathrm{B} 2$ \\
\hline ARFGEF2|10564 & $\mathrm{B} 2$ \\
\hline ARFIP1|27236 & $\mathrm{B} 2$ \\
\hline ARFRP1|10139 & $\mathrm{B} 2$ \\
\hline ARG1|383 & $\mathrm{B} 2$ \\
\hline ARGFXP2|503640 & $\mathrm{B} 2$ \\
\hline
\end{tabular}




\begin{tabular}{|c|c|}
\hline ARGFX|503582 & B2 \\
\hline ARHGAP12|94134 & B2 \\
\hline ARHGAP15|55843 & $\mathrm{B} 2$ \\
\hline ARHGAP18|93663 & $\mathrm{B} 2$ \\
\hline ARHGAP1|392 & B2 \\
\hline ARHGAP22|58504 & $\mathrm{B} 2$ \\
\hline ARHGAP25|9938 & $\mathrm{B} 2$ \\
\hline ARHGAP27|201176 & $\mathrm{B} 2$ \\
\hline ARHGAP28|79822 & $\mathrm{B} 2$ \\
\hline ARHGAP30|257106 & $\mathrm{B} 2$ \\
\hline ARHGAP33|115703 & $\mathrm{B} 2$ \\
\hline ARHGAP36|158763 & B2 \\
\hline ARHGAP4|393 & $\mathrm{B} 2$ \\
\hline ARHGAP8|23779 & $\mathrm{B} 2$ \\
\hline ARHGAP9|64333 & $\mathrm{B} 2$ \\
\hline ARHGDIA|396 & $\mathrm{B} 2$ \\
\hline ARHGDIG|398 & $\mathrm{B} 2$ \\
\hline ARHGEF10L|55160 & $\mathrm{B} 2$ \\
\hline ARHGEF12|23365 & B2 \\
\hline ARHGEF18|23370 & $\mathrm{B} 2$ \\
\hline ARHGEF19|128272 & $\mathrm{B} 2$ \\
\hline ARHGEF1|9138 & $\mathrm{B} 2$ \\
\hline ARHGEF2|9181 & $\mathrm{B} 2$ \\
\hline ARHGEF33|100271715 & $\mathrm{B} 2$ \\
\hline ARHGEF35|445328 & $\mathrm{B} 2$ \\
\hline ARHGEF38|54848 & $\mathrm{B} 2$ \\
\hline ARHGEF3|50650 & $\mathrm{B} 2$ \\
\hline ARHGEF4|50649 & B2 \\
\hline $\begin{array}{l}\text { ARHGEF5|7984 } \\
\end{array}$ & $\mathrm{B} 2$ \\
\hline ARHGEF9|23229 & $\mathrm{B} 2$ \\
\hline ARID2|196528 & $\mathrm{B} 2$ \\
\hline ARID3B|10620 & $\mathrm{B} 2$ \\
\hline ARID3C|138715 & $\mathrm{B} 2$ \\
\hline ARID4B|51742 & B2 \\
\hline ARIH1|25820 & $\mathrm{B} 2$ \\
\hline ARL13A|392509 & $\mathrm{B} 2$ \\
\hline ARL13B|200894 & $\mathrm{B} 2$ \\
\hline ARL14|80117 & $\mathrm{B} 2$ \\
\hline ARL15|54622 & $\mathrm{B} 2$ \\
\hline \begin{tabular}{l|l|} 
ARL16|339231 \\
\end{tabular} & $\mathrm{B} 2$ \\
\hline ARL17A|51326 & B2 \\
\hline ARL17B|641522 & $\mathrm{B} 2$ \\
\hline ARL2BP|23568 & B2 \\
\hline ARL4D|379 & $\mathrm{B} 2$ \\
\hline ARL5A|26225 & $\mathrm{B} 2$ \\
\hline ARL5B|221079 & $\mathrm{B} 2$ \\
\hline ARL5C|390790 & $\mathrm{B} 2$ \\
\hline ARL6IP4|51329 & $\mathrm{B} 2$ \\
\hline ARL6IP5|10550 & $\mathrm{B} 2$ \\
\hline ARL6IP6|151188 & $\mathrm{B} 2$ \\
\hline ARL6|84100 & $\mathrm{B} 2$ \\
\hline
\end{tabular}




\begin{tabular}{|c|c|}
\hline ARL9|132946 & B2 \\
\hline ARMC2|84071 & B2 \\
\hline $\begin{array}{l}\text { ARMC3|219681 } \\
\end{array}$ & $\mathrm{B} 2$ \\
\hline ARMC4|55130 & $\mathrm{B} 2$ \\
\hline ARMC5|79798 & $\mathrm{B} 2$ \\
\hline ARMC8|25852 & B2 \\
\hline ARMCX2|9823 & $\mathrm{B} 2$ \\
\hline ARMCX3|51566 & $\mathrm{B} 2$ \\
\hline ARMCX5|64860 & $\mathrm{B} 2$ \\
\hline ARMCX6|54470 & $\mathrm{B} 2$ \\
\hline ARMS2|387715 & $\mathrm{B} 2$ \\
\hline ARNTL2|56938 & B2 \\
\hline ARNTL|406 & $\mathrm{B} 2$ \\
\hline ARNT|405 & $\mathrm{B} 2$ \\
\hline ARPC1A|10552 & $\mathrm{B} 2$ \\
\hline ARPM1|84517 & $\mathrm{B} 2$ \\
\hline ARPP19|10776 & $\mathrm{B} 2$ \\
\hline ARPP21|10777 & $\mathrm{B} 2$ \\
\hline ARR3|407 & B2 \\
\hline ARRB2|409 & B2 \\
\hline ARRDC4|91947 & $\mathrm{B} 2$ \\
\hline ARRDC5|645432 & $\mathrm{B} 2$ \\
\hline ARSA|410 & $\mathrm{B} 2$ \\
\hline ARSD|414 & $\mathrm{B} 2$ \\
\hline ARSE|415 & $\mathrm{B} 2$ \\
\hline ARSF|416 & $\mathrm{B} 2$ \\
\hline ARSG|22901 & $\mathrm{B} 2$ \\
\hline ARSH|347527 & B2 \\
\hline ARSJ|79642 & $\mathrm{B} 2$ \\
\hline ARSK|153642 & $\mathrm{B} 2$ \\
\hline ART1|417 & $\mathrm{B} 2$ \\
\hline ART3|419 & $\mathrm{B} 2$ \\
\hline ART5|116969 & $\mathrm{B} 2$ \\
\hline ARTN|9048 & B2 \\
\hline ARVCF|421 & $\mathrm{B} 2$ \\
\hline ARX|170302 & $\mathrm{B} 2$ \\
\hline $\mathrm{AR} \mid 367$ & $\mathrm{~B} 2$ \\
\hline AS3MT|57412 & $\mathrm{B} 2$ \\
\hline ASAH1|427 & $\mathrm{B} 2$ \\
\hline ASAH2B|653308 & $\mathrm{B} 2$ \\
\hline \begin{tabular}{l|l|l|} 
ASAH2 & 16624
\end{tabular} & B2 \\
\hline ASAP1IT1|29065 & $\mathrm{B} 2$ \\
\hline ASAP1|50807 & $\mathrm{B} 2$ \\
\hline ASAP2|8853 & $\mathrm{B} 2$ \\
\hline ASAP3|55616 & $\mathrm{B} 2$ \\
\hline ASB10|136371 & $\mathrm{B} 2$ \\
\hline ASB11|140456 & $\mathrm{B} 2$ \\
\hline ASB12|142689 & $\mathrm{B} 2$ \\
\hline ASB14|142686 & $\mathrm{B} 2$ \\
\hline ASB15|142685 & B2 \\
\hline ASB16|92591 & $\mathrm{B} 2$ \\
\hline
\end{tabular}




\begin{tabular}{|c|c|}
\hline ASB17|127247 & B2 \\
\hline ASB18|401036 & $\mathrm{B} 2$ \\
\hline ASB2|51676 & $\mathrm{B} 2$ \\
\hline ASB3|51130 & $\mathrm{B} 2$ \\
\hline ASB4|51666 & B2 \\
\hline ASB5|140458 & $\mathrm{B} 2$ \\
\hline ASB6|140459 & $\mathrm{B} 2$ \\
\hline ASB7|140460 & $\mathrm{B} 2$ \\
\hline ASB8|140461 & $\mathrm{B} 2$ \\
\hline ASB9|140462 & $\mathrm{B} 2$ \\
\hline ASCC1|51008 & $\mathrm{B} 2$ \\
\hline ASCC2|84164 & B2 \\
\hline ASCC3|10973 & $\mathrm{B} 2$ \\
\hline ASCL1|429 & $\mathrm{B} 2$ \\
\hline ASCL2|430 & $\mathrm{B} 2$ \\
\hline ASCL3|56676 & B2 \\
\hline ASCL4|121549 & B2 \\
\hline ASF1A|25842 & $\mathrm{B} 2$ \\
\hline ASFMR1|100126270 & B2 \\
\hline ASGR1|432 & $\mathrm{B} 2$ \\
\hline ASGR2|433 & $\mathrm{B} 2$ \\
\hline ASH1L|55870 & $\mathrm{B} 2$ \\
\hline ASH2L $\mid 9070$ & $\mathrm{~B} 2$ \\
\hline ASIP|434 & $\mathrm{B} 2$ \\
\hline ASL|435 & $\mathrm{B} 2$ \\
\hline ASMTL|8623 & $\mathrm{B} 2$ \\
\hline ASMT 438 & $\mathrm{~B} 2$ \\
\hline ASNSD1|54529 & $\mathrm{B} 2$ \\
\hline ASNS|440 & $\mathrm{B} 2$ \\
\hline ASPDH|554235 & $\mathrm{B} 2$ \\
\hline ASPG|374569 & $\mathrm{B} 2$ \\
\hline ASPHD1|253982 & $\mathrm{B} 2$ \\
\hline ASRGL1|80150 & $\mathrm{B} 2$ \\
\hline ASS1|445 & $\mathrm{B} 2$ \\
\hline $\begin{array}{l}\text { ASTE1|28990 } \\
\end{array}$ & $\mathrm{B} 2$ \\
\hline ASTL|431705 & $\mathrm{B} 2$ \\
\hline ASTN2|23245 & $\mathrm{B} 2$ \\
\hline ASXL2|55252 & $\mathrm{B} 2$ \\
\hline ASZ1|136991 & B2 \\
\hline ATAD1|84896 & $\mathrm{B} 2$ \\
\hline ATAD2B|54454 & B2 \\
\hline ATAD3A|55210 & $\mathrm{B} 2$ \\
\hline ATAD3B|83858 & B2 \\
\hline ATAD3C|219293 & $\mathrm{B} 2$ \\
\hline ATCAY $\mid 85300$ & $\mathrm{~B} 2$ \\
\hline ATE1|11101 & $\mathrm{B} 2$ \\
\hline ATF1|466 & $\mathrm{B} 2$ \\
\hline ATF2|1386 & $\mathrm{B} 2$ \\
\hline ATF4|468 & $\mathrm{B} 2$ \\
\hline ATF7IP2|80063 & B2 \\
\hline ATF7IP|55729 & $\mathrm{B} 2$ \\
\hline
\end{tabular}




\begin{tabular}{|c|c|}
\hline ATG10|83734 & B2 \\
\hline ATG12|9140 & $\mathrm{B} 2$ \\
\hline ATG16L2|89849 & B2 \\
\hline ATG2A|23130 & B2 \\
\hline ATG3|64422 & B2 \\
\hline ATG4A|115201 & $\mathrm{B} 2$ \\
\hline ATG4B|23192 & B2 \\
\hline ATG5|9474 & B2 \\
\hline ATG7|10533 & B2 \\
\hline ATG9A|79065 & $\mathrm{B} 2$ \\
\hline ATHL1|80162 & B2 \\
\hline ATL2|64225 & B2 \\
\hline ATL3|25923 & $\mathrm{B} 2$ \\
\hline ATMIN|23300 & B2 \\
\hline ATOH7|220202 & $\mathrm{B} 2$ \\
\hline ATP10A|57194 & B2 \\
\hline ATP10B|23120 & $\mathrm{B} 2$ \\
\hline ATP10D|57205 & B2 \\
\hline ATP11A|23250 & B2 \\
\hline ATP11B|23200 & B2 \\
\hline ATP11C|286410 & B2 \\
\hline ATP12A|479 & $\mathrm{B} 2$ \\
\hline ATP13A3|79572 & B2 \\
\hline ATP13A4|84239 & B2 \\
\hline ATP13A5|344905 & $\mathrm{B} 2$ \\
\hline ATP1A1|476 & B2 \\
\hline ATP1A2|477 & B2 \\
\hline \begin{tabular}{l|l|l|} 
ATP1A3|478 &
\end{tabular} & B2 \\
\hline ATP1A4|480 & B2 \\
\hline ATP1B1|481 & B2 \\
\hline ATP1B3|483 & B2 \\
\hline ATP1B4|23439 & $\mathrm{B} 2$ \\
\hline ATP2A1|487 & $\mathrm{B} 2$ \\
\hline ATP2A2|488 & B2 \\
\hline 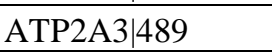 & B2 \\
\hline ATP2B1|490 & B2 \\
\hline ATP2B2|491 & $\mathrm{B} 2$ \\
\hline ATP2B3|492 & B2 \\
\hline ATP2C1|27032 & $\mathrm{B} 2$ \\
\hline ATP4A|495 & $\mathrm{B} 2$ \\
\hline ATP4B|496 & B2 \\
\hline ATP5A1|498 & B2 \\
\hline ATP5B|506 & B2 \\
\hline ATP5D|513 & $\mathrm{B} 2$ \\
\hline ATP5EP2|432369 & B2 \\
\hline ATP5E|514 & B2 \\
\hline ATP5F1|515 & $\mathrm{B} 2$ \\
\hline ATP5G1|516 & $\mathrm{B} 2$ \\
\hline ATP5G2|517 & B2 \\
\hline \begin{tabular}{l|l} 
ATP5G3|518 \\
\end{tabular} & B2 \\
\hline ATP5H|10476 & B2 \\
\hline
\end{tabular}




\begin{tabular}{|c|c|}
\hline ATP5I|521 & B2 \\
\hline ATP5J2|9551 & $\mathrm{B} 2$ \\
\hline ATP5J|522 & $\mathrm{B} 2$ \\
\hline ATP5L2|267020 & $\mathrm{B} 2$ \\
\hline ATP5L|10632 & $\mathrm{B} 2$ \\
\hline ATP5O|539 & $\mathrm{B} 2$ \\
\hline ATP5SL|55101 & $\mathrm{B} 2$ \\
\hline ATP5S|27109 & $\mathrm{B} 2$ \\
\hline ATP6AP1L|92270 & $\mathrm{B} 2$ \\
\hline ATP6V0A1|535 & $\mathrm{B} 2$ \\
\hline ATP6V0A2|23545 & $\mathrm{B} 2$ \\
\hline ATP6V0A4|50617 & B2 \\
\hline ATP6V0D1|9114 & $\mathrm{B} 2$ \\
\hline ATP6V0D2|245972 & $\mathrm{B} 2$ \\
\hline ATP6V0E1|8992 & $\mathrm{B} 2$ \\
\hline ATP6V0E2|155066 & $\mathrm{B} 2$ \\
\hline ATP6V1B1|525 & $\mathrm{B} 2$ \\
\hline ATP6V1B2|526 & $\mathrm{B} 2$ \\
\hline ATP6V1C1|528 & B2 \\
\hline ATP6V1C2|245973 & $\mathrm{B} 2$ \\
\hline ATP6V1E1|529 & $\mathrm{B} 2$ \\
\hline ATP6V1E2|90423 & $\mathrm{B} 2$ \\
\hline ATP6V1G2|534 & $\mathrm{B} 2$ \\
\hline ATP6V1G3|127124 & $\mathrm{B} 2$ \\
\hline ATP6V1H|51606 & $\mathrm{B} 2$ \\
\hline ATP7A|538 & $\mathrm{B} 2$ \\
\hline ATP8A1|10396 & $\mathrm{B} 2$ \\
\hline ATP8A2|51761 & $\mathrm{B} 2$ \\
\hline ATP8B2|57198 & $\mathrm{B} 2$ \\
\hline ATP8B3|148229 & $\mathrm{B} 2$ \\
\hline ATP8B5P|158381 & $\mathrm{B} 2$ \\
\hline ATP9A|10079 & $\mathrm{B} 2$ \\
\hline ATP9B|374868 & $\mathrm{B} 2$ \\
\hline ATPAF1|64756 & B2 \\
\hline ATPAF2|91647 & $\mathrm{B} 2$ \\
\hline ATPBD4|89978 & $\mathrm{B} 2$ \\
\hline ATRNL1|26033 & $\mathrm{B} 2$ \\
\hline ATRN|8455 & $\mathrm{B} 2$ \\
\hline ATRX|546 & B2 \\
\hline ATR|545 & $\mathrm{B} 2$ \\
\hline ATXN10|25814 & B2 \\
\hline ATXN1|6310 & $\mathrm{B} 2$ \\
\hline ATXN2|6311 & B2 \\
\hline ATXN3L|92552 & $\mathrm{B} 2$ \\
\hline ATXN7L1|222255 & $\mathrm{B} 2$ \\
\hline ATXN7L2|127002 & $\mathrm{B} 2$ \\
\hline ATXN7L3B|552889 & $\mathrm{B} 2$ \\
\hline ATXN7|6314 & $\mathrm{B} 2$ \\
\hline ATXN8OS|6315 & $\mathrm{B} 2$ \\
\hline AURKAIP1|54998 & B2 \\
\hline AURKC|6795 & $\mathrm{B} 2$ \\
\hline
\end{tabular}




\begin{tabular}{|c|c|}
\hline AUTS2|26053 & B2 \\
\hline AVEN|57099 & B2 \\
\hline AVIL|10677 & $\mathrm{B} 2$ \\
\hline AVPR1B|553 & $\mathrm{B} 2$ \\
\hline AVP|551 & B2 \\
\hline AWAT1|158833 & $\mathrm{B} 2$ \\
\hline AWAT2|158835 & $\mathrm{B} 2$ \\
\hline AZGP1|563 & $\mathrm{B} 2$ \\
\hline AZI1|22994 & $\mathrm{B} 2$ \\
\hline AZIN1|51582 & $\mathrm{B} 2$ \\
\hline AZU1|566 & $\mathrm{B} 2$ \\
\hline B2M|567 & B2 \\
\hline B3GALT4|8705 & $\mathrm{B} 2$ \\
\hline B3GALT5|10317 & $\mathrm{B} 2$ \\
\hline B3GALT6|126792 & $\mathrm{B} 2$ \\
\hline B3GALTL|145173 & $\mathrm{B} 2$ \\
\hline B3GAT1|27087 & $\mathrm{B} 2$ \\
\hline B3GAT2|135152 & $\mathrm{B} 2$ \\
\hline B3GNT2|10678 & B2 \\
\hline B3GNT3|10331 & $\mathrm{B} 2$ \\
\hline B3GNT5|84002 & $\mathrm{B} 2$ \\
\hline B3GNT6|192134 & $\mathrm{B} 2$ \\
\hline B3GNT7|93010 & $\mathrm{B} 2$ \\
\hline B3GNT8|374907 & $\mathrm{B} 2$ \\
\hline B3GNT9|84752 & $\mathrm{B} 2$ \\
\hline B3GNTL1|146712 & $\mathrm{B} 2$ \\
\hline B4GALNT1|2583 & $\mathrm{B} 2$ \\
\hline B4GALNT2|124872 & $\mathrm{B} 2$ \\
\hline B4GALT2|8704 & $\mathrm{B} 2$ \\
\hline B4GALT5|9334 & $\mathrm{B} 2$ \\
\hline B4GALT7|11285 & $\mathrm{B} 2$ \\
\hline B9D1|27077 & $\mathrm{B} 2$ \\
\hline B9D2|80776 & $\mathrm{B} 2$ \\
\hline BAALC|79870 & $\mathrm{B} 2$ \\
\hline BAAT 570 & $\mathrm{~B} 2$ \\
\hline BACE2|25825 & $\mathrm{B} 2$ \\
\hline BACH1|571 & $\mathrm{B} 2$ \\
\hline $\mathrm{BAD} \mid 572$ & $\mathrm{~B} 2$ \\
\hline BAG1|573 & B2 \\
\hline BAG2|9532 & $\mathrm{B} 2$ \\
\hline $\begin{array}{l}\text { BAG3|9531 } \\
\end{array}$ & B2 \\
\hline BAG4|9530 & $\mathrm{B} 2$ \\
\hline BAG5|9529 & B2 \\
\hline BAGE2|85319 & $\mathrm{B} 2$ \\
\hline BAGE|574 & $\mathrm{B} 2$ \\
\hline BAHCC1|57597 & $\mathrm{B} 2$ \\
\hline BAHD1|22893 & $\mathrm{B} 2$ \\
\hline BAI1|575 & $\mathrm{B} 2$ \\
\hline BAIAP2L2|80115 & $\mathrm{B} 2$ \\
\hline BAIAP3|8938 & B2 \\
\hline BAMBI|25805 & B2 \\
\hline
\end{tabular}




\begin{tabular}{|c|c|}
\hline BANF2|140836 & B2 \\
\hline BANK1|55024 & B2 \\
\hline $\begin{array}{l}\text { BANP|54971 } \\
\text { | }\end{array}$ & $\mathrm{B} 2$ \\
\hline BAP1|8314 & $\mathrm{B} 2$ \\
\hline BARHL1|56751 & $\mathrm{B} 2$ \\
\hline BARHL2|343472 & $\mathrm{B} 2$ \\
\hline BARX1|56033 & $\mathrm{B} 2$ \\
\hline BARX2|8538 & $\mathrm{B} 2$ \\
\hline BASE|317716 & $\mathrm{B} 2$ \\
\hline BAT1|7919 & $\mathrm{B} 2$ \\
\hline BAT2L1|84726 & $\mathrm{B} 2$ \\
\hline BAT2|7916 & $\mathrm{B} 2$ \\
\hline BAT3|7917 & $\mathrm{B} 2$ \\
\hline BAT4|7918 & $\mathrm{B} 2$ \\
\hline BATF2|116071 & $\mathrm{B} 2$ \\
\hline 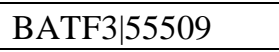 & $\mathrm{B} 2$ \\
\hline BATF|10538 & $\mathrm{B} 2$ \\
\hline BAZ1B|9031 & $\mathrm{B} 2$ \\
\hline BAZ2A|11176 & $\mathrm{B} 2$ \\
\hline BBS1|582 & $\mathrm{B} 2$ \\
\hline BBS4|585 & $\mathrm{B} 2$ \\
\hline BBS5|129880 & $\mathrm{B} 2$ \\
\hline BBS9|27241 & $\mathrm{B} 2$ \\
\hline BBX|56987 & $\mathrm{B} 2$ \\
\hline BCAM|4059 & $\mathrm{B} 2$ \\
\hline BCAN|63827 & $\mathrm{B} 2$ \\
\hline BCAP29|55973 & $\mathrm{B} 2$ \\
\hline BCAP31|10134 & $\mathrm{B} 2$ \\
\hline BCAR1|9564 & $\mathrm{B} 2$ \\
\hline BCAR3|8412 & $\mathrm{B} 2$ \\
\hline BCAR4|400500 & $\mathrm{B} 2$ \\
\hline BCAS1|8537 & $\mathrm{B} 2$ \\
\hline BCAS2|10286 & $\mathrm{B} 2$ \\
\hline $\begin{array}{l}\text { BCAS3|54828 } \\
\end{array}$ & $\mathrm{B} 2$ \\
\hline BCAS4|55653 & $\mathrm{B} 2$ \\
\hline BCAT1|586 & $\mathrm{B} 2$ \\
\hline BCAT2|587 & $\mathrm{B} 2$ \\
\hline BCCIP|56647 & $\mathrm{B} 2$ \\
\hline BCDIN3D|144233 & $\mathrm{B} 2$ \\
\hline BCKDHA|593 & $\mathrm{B} 2$ \\
\hline BCKDHB|594 & $\mathrm{B} 2$ \\
\hline BCL10|8915 & $\mathrm{B} 2$ \\
\hline BCL11A|53335 & $\mathrm{B} 2$ \\
\hline BCL11B|64919 & $\mathrm{B} 2$ \\
\hline BCL2A1|597 & $\mathrm{B} 2$ \\
\hline BCL2L10|10017 & $\mathrm{B} 2$ \\
\hline BCL2L11|10018 & $\mathrm{B} 2$ \\
\hline BCL2L13|23786 & $\mathrm{B} 2$ \\
\hline BCL2L14|79370 & $\mathrm{B} 2$ \\
\hline BCL2L15|440603 & $\mathrm{B} 2$ \\
\hline BCL2|596 & $\mathrm{B} 2$ \\
\hline
\end{tabular}




\begin{tabular}{|c|c|}
\hline BCL6B $\mid 255877$ & B2 \\
\hline BCL7A $\mid 605$ & B2 \\
\hline BCL7B $\mid 9275$ & $\mathrm{~B} 2$ \\
\hline BCL7C|9274 & $\mathrm{B} 2$ \\
\hline BCL8|606 & B2 \\
\hline BCL9L|283149 & $\mathrm{B} 2$ \\
\hline BCLAF1|9774 & $\mathrm{B} 2$ \\
\hline BCMO1|53630 & $\mathrm{B} 2$ \\
\hline $\mathrm{BCO} 2 \mid 83875$ & $\mathrm{~B} 2$ \\
\hline BCORL1|63035 & $\mathrm{B} 2$ \\
\hline BCR|613 & $\mathrm{B} 2$ \\
\hline BCS1L|617 & $\mathrm{B} 2$ \\
\hline BCYRN1|618 & $\mathrm{B} 2$ \\
\hline BDH1|622 & $\mathrm{B} 2$ \\
\hline BDKRB1|623 & $\mathrm{B} 2$ \\
\hline BDKRB2|624 & $\mathrm{B} 2$ \\
\hline BDNF|627 & $\mathrm{B} 2$ \\
\hline BDP1|55814 & $\mathrm{B} 2$ \\
\hline BEAN|146227 & $\mathrm{B} 2$ \\
\hline BECN1|8678 & $\mathrm{B} 2$ \\
\hline BEND2|139105 & $\mathrm{B} 2$ \\
\hline BEND4|389206 & $\mathrm{B} 2$ \\
\hline BEST2|54831 & $\mathrm{B} 2$ \\
\hline BEST3|144453 & $\mathrm{B} 2$ \\
\hline BEST4|266675 & $\mathrm{B} 2$ \\
\hline BET1L|51272 & $\mathrm{B} 2$ \\
\hline BET1|10282 & $\mathrm{B} 2$ \\
\hline BET3L|100128327 & $\mathrm{B} 2$ \\
\hline BEX1|55859 & $\mathrm{B} 2$ \\
\hline BEX2|84707 & $\mathrm{B} 2$ \\
\hline BEX4|56271 & $\mathrm{B} 2$ \\
\hline \begin{tabular}{l|l|} 
BEX5 340542 \\
\end{tabular} & $\mathrm{~B} 2$ \\
\hline BEYLA|497634 & $\mathrm{B} 2$ \\
\hline BFSP1|631 & $\mathrm{B} 2$ \\
\hline BFSP2|8419 & $\mathrm{B} 2$ \\
\hline BGLAP|632 & $\mathrm{B} 2$ \\
\hline BHLHA15|168620 & $\mathrm{B} 2$ \\
\hline BHLHB9|80823 & $\mathrm{B} 2$ \\
\hline BHLHE22|27319 & $\mathrm{B} 2$ \\
\hline BHLHE40|8553 & $\mathrm{B} 2$ \\
\hline BHMT|635 & $\mathrm{B} 2$ \\
\hline BICC1|80114 & $\mathrm{B} 2$ \\
\hline BICD1|636 & $\mathrm{B} 2$ \\
\hline $\mathrm{BID} \mid 637$ & $\mathrm{~B} 2$ \\
\hline BIN1|274 & $\mathrm{B} 2$ \\
\hline BIN2|51411 & $\mathrm{B} 2$ \\
\hline BIRC2|329 & $\mathrm{B} 2$ \\
\hline BIRC3|330 & $\mathrm{B} 2$ \\
\hline BIRC6|57448 & $\mathrm{B} 2$ \\
\hline BIRC7|79444 & $\mathrm{B} 2$ \\
\hline BIRC8|112401 & $\mathrm{B} 2$ \\
\hline
\end{tabular}




\begin{tabular}{|c|c|}
\hline BLID|414899 & B2 \\
\hline BLK $\mid 640$ & B2 \\
\hline BLMH|642 & $\mathrm{B} 2$ \\
\hline BLM|641 & $\mathrm{B} 2$ \\
\hline BLOC1S1|2647 & B2 \\
\hline BLVRA|644 & $\mathrm{B} 2$ \\
\hline BLVRB|645 & $\mathrm{B} 2$ \\
\hline BMF|90427 & $\mathrm{B} 2$ \\
\hline BMP10|27302 & $\mathrm{B} 2$ \\
\hline BMP15|9210 & $\mathrm{B} 2$ \\
\hline BMP1|649 & $\mathrm{B} 2$ \\
\hline BMP2K|55589 & $\mathrm{B} 2$ \\
\hline BMP3|651 & $\mathrm{B} 2$ \\
\hline BMP4|652 & $\mathrm{B} 2$ \\
\hline BMP7|655 & $\mathrm{B} 2$ \\
\hline BMP8B|656 & $\mathrm{B} 2$ \\
\hline BMPR1B|658 & $\mathrm{B} 2$ \\
\hline BMPR2|659 & $\mathrm{B} 2$ \\
\hline BMS1P4|729096 & $\mathrm{B} 2$ \\
\hline BMS1|9790 & $\mathrm{B} 2$ \\
\hline BNC1|646 & $\mathrm{B} 2$ \\
\hline BNC2|54796 & $\mathrm{B} 2$ \\
\hline BNIP3|664 & $\mathrm{B} 2$ \\
\hline BNIPL|149428 & $\mathrm{B} 2$ \\
\hline BOD1|91272 & $\mathrm{B} 2$ \\
\hline BOLL|66037 & $\mathrm{B} 2$ \\
\hline BPESC1|60467 & $\mathrm{B} 2$ \\
\hline BPGM|669 & $\mathrm{B} 2$ \\
\hline BPHL $\mid 670$ & $\mathrm{~B} 2$ \\
\hline BPIL1|80341 & $\mathrm{B} 2$ \\
\hline BPIL2|254240 & $\mathrm{B} 2$ \\
\hline BPIL3|128859 & $\mathrm{B} 2$ \\
\hline BPI|671 & $\mathrm{B} 2$ \\
\hline BPTF|2186 & $\mathrm{B} 2$ \\
\hline BRAF|673 & $\mathrm{B} 2$ \\
\hline BRAP|8315 & $\mathrm{B} 2$ \\
\hline BRD1|23774 & $\mathrm{B} 2$ \\
\hline BRD2|6046 & $\mathrm{B} 2$ \\
\hline BRD3|8019 & $\mathrm{B} 2$ \\
\hline BRD4|23476 & $\mathrm{B} 2$ \\
\hline BRD7P3|23629 & $\mathrm{B} 2$ \\
\hline BRD7|29117 & $\mathrm{B} 2$ \\
\hline BRD8|10902 & $\mathrm{B} 2$ \\
\hline BRD9|65980 & $\mathrm{B} 2$ \\
\hline BRDT|676 & $\mathrm{B} 2$ \\
\hline BREA2|286076 & $\mathrm{B} 2$ \\
\hline BRE|9577 & $\mathrm{B} 2$ \\
\hline BRF1|2972 & $\mathrm{B} 2$ \\
\hline BRF2|55290 & $\mathrm{B} 2$ \\
\hline BRI3|25798 & $\mathrm{B} 2$ \\
\hline BRP44L|51660 & $\mathrm{B} 2$ \\
\hline
\end{tabular}




\begin{tabular}{|c|c|}
\hline BRP44|25874 & B2 \\
\hline BRPF1|7862 & B2 \\
\hline BRPF3|27154 & $\mathrm{B} 2$ \\
\hline BRS3|680 & $\mathrm{B} 2$ \\
\hline BRSK1|84446 & $\mathrm{B} 2$ \\
\hline BRSK2|9024 & $\mathrm{B} 2$ \\
\hline BRWD1|54014 & $\mathrm{B} 2$ \\
\hline BSDC1|55108 & $\mathrm{B} 2$ \\
\hline BSG|682 & $\mathrm{B} 2$ \\
\hline BSND|7809 & $\mathrm{B} 2$ \\
\hline BSN|8927 & $\mathrm{B} 2$ \\
\hline BTBD16|118663 & $\mathrm{B} 2$ \\
\hline BTBD17|388419 & $\mathrm{B} 2$ \\
\hline BTBD18|643376 & $\mathrm{B} 2$ \\
\hline BTBD19|149478 & $\mathrm{B} 2$ \\
\hline BTBD1|53339 & $\mathrm{B} 2$ \\
\hline BTBD2|55643 & $\mathrm{B} 2$ \\
\hline BTBD3|22903 & $\mathrm{B} 2$ \\
\hline BTBD6|90135 & $\mathrm{B} 2$ \\
\hline BTBD9|114781 & $\mathrm{B} 2$ \\
\hline BTC|685 & $\mathrm{B} 2$ \\
\hline BTD|686 & $\mathrm{B} 2$ \\
\hline BTF3L1|690 & $\mathrm{B} 2$ \\
\hline BTF3|689 & $\mathrm{B} 2$ \\
\hline BTG1|694 & $\mathrm{B} 2$ \\
\hline BTG2|7832 & $\mathrm{B} 2$ \\
\hline BTG3|10950 & $\mathrm{B} 2$ \\
\hline BTG4|54766 & $\mathrm{B} 2$ \\
\hline BTK|695 & $\mathrm{B} 2$ \\
\hline BTLA|151888 & $\mathrm{B} 2$ \\
\hline BTN1A1|696 & $\mathrm{B} 2$ \\
\hline BTN2A2|10385 & $\mathrm{B} 2$ \\
\hline BTN3A1|11119 & $\mathrm{B} 2$ \\
\hline BTN3A2|11118 & $\mathrm{B} 2$ \\
\hline BTN3A3|10384 & $\mathrm{B} 2$ \\
\hline BTNL2|56244 & $\mathrm{B} 2$ \\
\hline \begin{tabular}{l|l|l|} 
BTNL3 & 10917 \\
\end{tabular} & $\mathrm{~B} 2$ \\
\hline \begin{tabular}{l|l|} 
BTNL89908 \\
\end{tabular} & $\mathrm{B} 2$ \\
\hline BTRC|8945 & $\mathrm{B} 2$ \\
\hline BVES|11149 & $\mathrm{B} 2$ \\
\hline C10orf107|219621 & $\mathrm{B} 2$ \\
\hline C10orf110|55853 & $\mathrm{B} 2$ \\
\hline C10orf111|221060 & $\mathrm{B} 2$ \\
\hline $\begin{array}{l}\text { C10orf113|387638 } \\
\end{array}$ & $\mathrm{B} 2$ \\
\hline C10orf114|399726 & $\mathrm{B} 2$ \\
\hline C10orf118|55088 & $\mathrm{B} 2$ \\
\hline C10orf119|79892 & $\mathrm{B} 2$ \\
\hline C10orf122|387718 & $\mathrm{B} 2$ \\
\hline C10orf125|282969 & $\mathrm{B} 2$ \\
\hline C10orf129|142827 & $\mathrm{B} 2$ \\
\hline C10orf12|26148 & $\mathrm{B} 2$ \\
\hline
\end{tabular}




\begin{tabular}{|c|c|}
\hline C10orf131|100127889 & B2 \\
\hline C10orf137|26098 & B2 \\
\hline C10orf140|387640 & $\mathrm{B} 2$ \\
\hline C10orf26|54838 & $\mathrm{B} 2$ \\
\hline C10orf27|219793 & $\mathrm{B} 2$ \\
\hline C10orf28|27291 & $\mathrm{B} 2$ \\
\hline C10orf2|56652 & $\mathrm{B} 2$ \\
\hline C10orf32|119032 & $\mathrm{B} 2$ \\
\hline C10orf40|283025 & $\mathrm{B} 2$ \\
\hline C10orf41|100131213 & $\mathrm{B} 2$ \\
\hline C10orf47|254427 & $\mathrm{B} 2$ \\
\hline C10orf55|414236 & $\mathrm{B} 2$ \\
\hline C10orf62|414157 & $\mathrm{B} 2$ \\
\hline C10orf67|256815 & $\mathrm{B} 2$ \\
\hline C10orf68|79741 & B2 \\
\hline C10orf71|118461 & $\mathrm{B} 2$ \\
\hline C10orf75|90271 & $\mathrm{B} 2$ \\
\hline C10orf76|79591 & $\mathrm{B} 2$ \\
\hline C10orf78|119392 & $\mathrm{B} 2$ \\
\hline C10orf79|80217 & $\mathrm{B} 2$ \\
\hline C10orf81|79949 & $\mathrm{B} 2$ \\
\hline C10orf82|143379 & $\mathrm{B} 2$ \\
\hline C10orf84|63877 & $\mathrm{B} 2$ \\
\hline C10orf88|80007 & $\mathrm{B} 2$ \\
\hline C10orf91|170393 & $\mathrm{B} 2$ \\
\hline C10orf93|255352 & $\mathrm{B} 2$ \\
\hline C10orf96|374355 & $\mathrm{B} 2$ \\
\hline C10orf99|387695 & $\mathrm{B} 2$ \\
\hline C11orf16|56673 & $\mathrm{B} 2$ \\
\hline C11orf17|56672 & $\mathrm{B} 2$ \\
\hline C11orf1|64776 & $\mathrm{B} 2$ \\
\hline C11 orf20|25858 & $\mathrm{B} 2$ \\
\hline C11orf21|29125 & $\mathrm{B} 2$ \\
\hline C11orf30|56946 & $\mathrm{B} 2$ \\
\hline C11orf34|349633 & $\mathrm{B} 2$ \\
\hline C11orf35|256329 & $\mathrm{B} 2$ \\
\hline C11orf41|25758 & $\mathrm{B} 2$ \\
\hline C11orf42|160298 & $\mathrm{B} 2$ \\
\hline C11orf45|219833 & $\mathrm{B} 2$ \\
\hline C11orf46|120534 & $\mathrm{B} 2$ \\
\hline C11orf51|25906 & $\mathrm{B} 2$ \\
\hline C11orf52|91894 & $\mathrm{B} 2$ \\
\hline C11orf54|28970 & $\mathrm{B} 2$ \\
\hline C11orf57|55216 & $\mathrm{B} 2$ \\
\hline C11orf58|10944 & $\mathrm{B} 2$ \\
\hline C11orf59|55004 & $\mathrm{B} 2$ \\
\hline C11orf65|160140 & $\mathrm{B} 2$ \\
\hline C11orf67|28971 & $\mathrm{B} 2$ \\
\hline C11orf68|83638 & $\mathrm{B} 2$ \\
\hline $\begin{array}{l}\text { C11orf70|85016 } \\
\end{array}$ & $\mathrm{B} 2$ \\
\hline C11orf71|54494 & $\mathrm{B} 2$ \\
\hline
\end{tabular}




\begin{tabular}{|c|c|}
\hline C11orf73|51501 & B2 \\
\hline C11orf74|119710 & B2 \\
\hline C11orf75|56935 & $\mathrm{B} 2$ \\
\hline C11orf80|79703 & $\mathrm{B} 2$ \\
\hline C11orf85|283129 & $\mathrm{B} 2$ \\
\hline C11orf86|254439 & $\mathrm{B} 2$ \\
\hline C11orf87|399947 & $\mathrm{B} 2$ \\
\hline C11orf88|399949 & $\mathrm{B} 2$ \\
\hline C11orf90|387804 & $\mathrm{B} 2$ \\
\hline C11orf92|399948 & $\mathrm{B} 2$ \\
\hline C11orf94|143678 & $\mathrm{B} 2$ \\
\hline C11orf9|745 & $\mathrm{B} 2$ \\
\hline C12orf10|60314 & $\mathrm{B} 2$ \\
\hline C12orf24|29902 & $\mathrm{B} 2$ \\
\hline C12orf27|283460 & B2 \\
\hline C12orf29|91298 & $\mathrm{B} 2$ \\
\hline C12orf35|55196 & $\mathrm{B} 2$ \\
\hline C12orf36|283422 & $\mathrm{B} 2$ \\
\hline C12orf39|80763 & $\mathrm{B} 2$ \\
\hline C12orf40|283461 & $\mathrm{B} 2$ \\
\hline C12orf41|54934 & $\mathrm{B} 2$ \\
\hline C12orf42|374470 & B2 \\
\hline C12orf45|121053 & $\mathrm{B} 2$ \\
\hline C12orf47|51275 & $\mathrm{B} 2$ \\
\hline C12orf49|79794 & $\mathrm{B} 2$ \\
\hline C12orf4|57102 & $\mathrm{B} 2$ \\
\hline C12orf50|160419 & $\mathrm{B} 2$ \\
\hline C12orf51|283450 & $\mathrm{B} 2$ \\
\hline C12orf53|196500 & $\mathrm{B} 2$ \\
\hline C12orf54|121273 & $\mathrm{B} 2$ \\
\hline C12orf56|115749 & $\mathrm{B} 2$ \\
\hline C12orf57|113246 & $\mathrm{B} 2$ \\
\hline \begin{tabular}{l|l|l|} 
C12orf5 & \\
\end{tabular} & $\mathrm{B} 2$ \\
\hline C12orf60|144608 & $\mathrm{B} 2$ \\
\hline C12orf61|283416 & $\mathrm{B} 2$ \\
\hline C12orf62|84987 & $\mathrm{B} 2$ \\
\hline C12orf63|374467 & $\mathrm{B} 2$ \\
\hline C12orf65|91574 & $\mathrm{B} 2$ \\
\hline C12orf69|440087 & $\mathrm{B} 2$ \\
\hline C12orf71|728858 & $\mathrm{B} 2$ \\
\hline C12orf72|254013 & $\mathrm{B} 2$ \\
\hline C12orf73|728568 & $\mathrm{B} 2$ \\
\hline C12orf74|338809 & $\mathrm{B} 2$ \\
\hline C12orf75|387882 & $\mathrm{B} 2$ \\
\hline C12orf76|400073 & $\mathrm{B} 2$ \\
\hline C12orf77|196415 & $\mathrm{B} 2$ \\
\hline C13orf16|121793 & $\mathrm{B} 2$ \\
\hline C13orf18|80183 & $\mathrm{B} 2$ \\
\hline C13orf1|57213 & $\mathrm{B} 2$ \\
\hline $\begin{array}{l}\text { C13orf23|80209 } \\
\end{array}$ & $\mathrm{B} 2$ \\
\hline C13orf26|122046 & $\mathrm{B} 2$ \\
\hline
\end{tabular}




\begin{tabular}{|c|c|}
\hline C13orf27|93081 & B2 \\
\hline C13orf29|283487 & B2 \\
\hline C13orf30|144809 & $\mathrm{B} 2$ \\
\hline C13orf31|144811 & $\mathrm{B} 2$ \\
\hline C13orf35|400165 & $\mathrm{B} 2$ \\
\hline C13orf38|728591 & $\mathrm{B} 2$ \\
\hline C13orf39|196541 & $\mathrm{B} 2$ \\
\hline C14orf101|54916 & $\mathrm{B} 2$ \\
\hline C14orf102|55051 & $\mathrm{B} 2$ \\
\hline C14orf104|55172 & $\mathrm{B} 2$ \\
\hline C14orf105|55195 & $\mathrm{B} 2$ \\
\hline C14orf106|55320 & $\mathrm{B} 2$ \\
\hline C14orf109|26175 & $\mathrm{B} 2$ \\
\hline C14orf115|55237 & $\mathrm{B} 2$ \\
\hline C14orf126|112487 & B2 \\
\hline C14orf135|64430 & $\mathrm{B} 2$ \\
\hline C14orf138|79609 & $\mathrm{B} 2$ \\
\hline C14orf145|145508 & $\mathrm{B} 2$ \\
\hline C14orf147|171546 & $\mathrm{B} 2$ \\
\hline C14orf148|122945 & $\mathrm{B} 2$ \\
\hline C14orf153|84334 & $\mathrm{B} 2$ \\
\hline C14orf162|56936 & B2 \\
\hline C14orf165|414767 & $\mathrm{B} 2$ \\
\hline C14orf166B|145497 & $\mathrm{B} 2$ \\
\hline C14orf166|51637 & $\mathrm{B} 2$ \\
\hline C14orf167|55449 & $\mathrm{B} 2$ \\
\hline C14orf176|643382 & $\mathrm{B} 2$ \\
\hline C14orf178|283579 & $\mathrm{B} 2$ \\
\hline C14orf179|112752 & $\mathrm{B} 2$ \\
\hline C14orf181|400223 & $\mathrm{B} 2$ \\
\hline C14orf182|283551 & $\mathrm{B} 2$ \\
\hline C14orf183|196913 & $\mathrm{B} 2$ \\
\hline C14orf184|650662 & $\mathrm{B} 2$ \\
\hline C14orf19|280655 & $\mathrm{B} 2$ \\
\hline C14orf21|161424 & $\mathrm{B} 2$ \\
\hline C14orf23|387978 & $\mathrm{B} 2$ \\
\hline C14orf2|9556 & $\mathrm{B} 2$ \\
\hline C14orf33|100129075 & $\mathrm{B} 2$ \\
\hline C14orf34|645687 & $\mathrm{B} 2$ \\
\hline C14orf37|145407 & $\mathrm{B} 2$ \\
\hline C14orf39|317761 & $\mathrm{B} 2$ \\
\hline C14orf43|91748 & $\mathrm{B} 2$ \\
\hline C14orf45|80127 & $\mathrm{B} 2$ \\
\hline C14orf48|256369 & $\mathrm{B} 2$ \\
\hline C14orf4|64207 & $\mathrm{B} 2$ \\
\hline C14orf50|145376 & $\mathrm{B} 2$ \\
\hline C14orf53|440184 & $\mathrm{B} 2$ \\
\hline C14orf68|283600 & $\mathrm{B} 2$ \\
\hline C14orf72|145200 & $\mathrm{B} 2$ \\
\hline C14orf73|91828 & $\mathrm{B} 2$ \\
\hline C14orf79|122616 & $\mathrm{B} 2$ \\
\hline
\end{tabular}




\begin{tabular}{|c|c|}
\hline C14orf86|283592 & B2 \\
\hline C14orf93|60686 & B2 \\
\hline C15orf24|56851 & $\mathrm{B} 2$ \\
\hline C15orf26|161502 & $\mathrm{B} 2$ \\
\hline C15orf27|123591 & $\mathrm{B} 2$ \\
\hline C15orf28|80035 & $\mathrm{B} 2$ \\
\hline C15orf2|23742 & $\mathrm{B} 2$ \\
\hline C15orf33|196951 & $\mathrm{B} 2$ \\
\hline C15orf34|80072 & $\mathrm{B} 2$ \\
\hline C15orf37|283687 & $\mathrm{B} 2$ \\
\hline C15orf38|348110 & $\mathrm{B} 2$ \\
\hline C15orf39|56905 & $\mathrm{B} 2$ \\
\hline C15orf41|84529 & $\mathrm{B} 2$ \\
\hline C15orf43|145645 & $\mathrm{B} 2$ \\
\hline C15orf44|81556 & B2 \\
\hline C15orf48|84419 & $\mathrm{B} 2$ \\
\hline C15orf50|414926 & $\mathrm{B} 2$ \\
\hline C15orf53|400359 & $\mathrm{B} 2$ \\
\hline C15orf55|256646 & $\mathrm{B} 2$ \\
\hline C15orf56|644809 & $\mathrm{B} 2$ \\
\hline C15orf57|90416 & $\mathrm{B} 2$ \\
\hline C15orf58|390637 & $\mathrm{B} 2$ \\
\hline C15orf59|388135 & $\mathrm{B} 2$ \\
\hline $\begin{array}{l}\text { C15orf5|81698 } \\
\end{array}$ & $\mathrm{B} 2$ \\
\hline C15orf60|283677 & $\mathrm{B} 2$ \\
\hline C15orf61|145853 & $\mathrm{B} 2$ \\
\hline C15orf62|643338 & $\mathrm{B} 2$ \\
\hline C16orf11|146325 & $\mathrm{B} 2$ \\
\hline C16orf3|750 & $\mathrm{B} 2$ \\
\hline C16orf42|115939 & $\mathrm{B} 2$ \\
\hline C16orf45|89927 & $\mathrm{B} 2$ \\
\hline C16orf46|123775 & $\mathrm{B} 2$ \\
\hline C16orf48|84080 & $\mathrm{B} 2$ \\
\hline C16orf52|730094 & $\mathrm{B} 2$ \\
\hline C16orf54|283897 & $\mathrm{B} 2$ \\
\hline C16orf57|79650 & $\mathrm{B} 2$ \\
\hline C16orf62|57020 & $\mathrm{B} 2$ \\
\hline C16orf63|123811 & $\mathrm{B} 2$ \\
\hline C16orf68|79091 & $\mathrm{B} 2$ \\
\hline $\begin{array}{l}\text { C16orf73|254528 } \\
\end{array}$ & $\mathrm{B} 2$ \\
\hline C16orf74|404550 & $\mathrm{B} 2$ \\
\hline C16orf78|123970 & $\mathrm{B} 2$ \\
\hline C16orf79|283870 & $\mathrm{B} 2$ \\
\hline C16orf7|9605 & $\mathrm{B} 2$ \\
\hline C16orf80|29105 & $\mathrm{B} 2$ \\
\hline C16orf81|283860 & $\mathrm{B} 2$ \\
\hline C16orf82|162083 & $\mathrm{B} 2$ \\
\hline C16orf87|388272 & $\mathrm{B} 2$ \\
\hline C16orf89|146556 & $\mathrm{B} 2$ \\
\hline C16orf90|646174 & $\mathrm{B} 2$ \\
\hline C16orf92|146378 & $\mathrm{B} 2$ \\
\hline
\end{tabular}




\begin{tabular}{|c|c|}
\hline C17orf101|79701 & B2 \\
\hline C17orf102|400591 & B2 \\
\hline C17orf104|284071 & $\mathrm{B} 2$ \\
\hline C17orf105|284067 & $\mathrm{B} 2$ \\
\hline C17orf106|100134934 & $\mathrm{B} 2$ \\
\hline C17orf37|84299 & $\mathrm{B} 2$ \\
\hline C17orf42|79736 & $\mathrm{B} 2$ \\
\hline C17orf44|284029 & $\mathrm{B} 2$ \\
\hline C17orf46|124783 & $\mathrm{B} 2$ \\
\hline C17orf47|284083 & $\mathrm{B} 2$ \\
\hline C17orf49|124944 & $\mathrm{B} 2$ \\
\hline C17orf50|146853 & $\mathrm{B} 2$ \\
\hline C17orf53|78995 & $\mathrm{B} 2$ \\
\hline C17orf54|283982 & $\mathrm{B} 2$ \\
\hline C17orf55|284185 & B2 \\
\hline C17orf56|146705 & $\mathrm{B} 2$ \\
\hline C17orf58|284018 & $\mathrm{B} 2$ \\
\hline C17orf59|54785 & $\mathrm{B} 2$ \\
\hline C17orf60|284021 & $\mathrm{B} 2$ \\
\hline C17orf61|254863 & $\mathrm{B} 2$ \\
\hline C17orf63|55731 & $\mathrm{B} 2$ \\
\hline C17orf64|124773 & B2 \\
\hline C17orf66|256957 & $\mathrm{B} 2$ \\
\hline C17orf67|339210 & $\mathrm{B} 2$ \\
\hline C17orf68|80169 & $\mathrm{B} 2$ \\
\hline C17orf71|55181 & $\mathrm{B} 2$ \\
\hline C17orf72|92340 & $\mathrm{B} 2$ \\
\hline $\begin{array}{l}\text { C17orf73|55018 } \\
\end{array}$ & $\mathrm{B} 2$ \\
\hline C17orf74|201243 & $\mathrm{B} 2$ \\
\hline C17orf75|64149 & $\mathrm{B} 2$ \\
\hline C17orf76|388341 & $\mathrm{B} 2$ \\
\hline C17orf77|146723 & $\mathrm{B} 2$ \\
\hline C17orf78|284099 & $\mathrm{B} 2$ \\
\hline C17orf79|55352 & $\mathrm{B} 2$ \\
\hline C17orf80|55028 & $\mathrm{B} 2$ \\
\hline C17orf82|388407 & $\mathrm{B} 2$ \\
\hline C17orf86|654434 & $\mathrm{B} 2$ \\
\hline C17orf87|388325 & $\mathrm{B} 2$ \\
\hline C17orf88|23591 & $\mathrm{B} 2$ \\
\hline C17orf89|284184 & $\mathrm{B} 2$ \\
\hline C17orf93|360205 & $\mathrm{B} 2$ \\
\hline C17orf95|124512 & $\mathrm{B} 2$ \\
\hline C17orf96|100170841 & $\mathrm{B} 2$ \\
\hline C17orf97|400566 & $\mathrm{B} 2$ \\
\hline C17orf98|388381 & $\mathrm{B} 2$ \\
\hline C17orf99|100141515 & $\mathrm{B} 2$ \\
\hline C18orf10|25941 & $\mathrm{B} 2$ \\
\hline C18orf16|147429 & $\mathrm{B} 2$ \\
\hline C18orf19|125228 & $\mathrm{B} 2$ \\
\hline C18orf1|753 & $\mathrm{B} 2$ \\
\hline C18orf20|221241 & $\mathrm{B} 2$ \\
\hline
\end{tabular}




\begin{tabular}{|c|c|}
\hline C18orf21|83608 & B2 \\
\hline C18orf22|79863 & B2 \\
\hline C18orf25|147339 & B2 \\
\hline C18orf26|284254 & $\mathrm{B} 2$ \\
\hline C18orf2|56651 & $\mathrm{B} 2$ \\
\hline C18orf54|162681 & $\mathrm{B} 2$ \\
\hline C18orf55|29090 & $\mathrm{B} 2$ \\
\hline C18orf62|284274 & $\mathrm{B} 2$ \\
\hline C18orf8|29919 & B2 \\
\hline C19orf18|147685 & $\mathrm{B} 2$ \\
\hline C19orf20|91978 & B2 \\
\hline C19orf23|148046 & $\mathrm{B} 2$ \\
\hline C19orf25|148223 & $\mathrm{B} 2$ \\
\hline C19orf26|255057 & B2 \\
\hline C19orf29|58509 & $\mathrm{B} 2$ \\
\hline C19orf2|8725 & $\mathrm{B} 2$ \\
\hline C19orf30|284424 & $\mathrm{B} 2$ \\
\hline C19orf33|64073 & $\mathrm{B} 2$ \\
\hline C19orf34|255193 & $\mathrm{B} 2$ \\
\hline C19orf35|374872 & $\mathrm{B} 2$ \\
\hline C19orf36|113177 & $\mathrm{B} 2$ \\
\hline C19orf38|255809 & $\mathrm{B} 2$ \\
\hline C19orf41|126123 & $\mathrm{B} 2$ \\
\hline C19orf42|79086 & $\mathrm{B} 2$ \\
\hline C19orf43|79002 & $\mathrm{B} 2$ \\
\hline C19orf44|84167 & $\mathrm{B} 2$ \\
\hline C19orf47|126526 & B2 \\
\hline C19orf50|79036 & $\mathrm{B} 2$ \\
\hline C19orf54|284325 & $\mathrm{B} 2$ \\
\hline C19orf55|148137 & $\mathrm{B} 2$ \\
\hline C19orf56|51398 & $\mathrm{B} 2$ \\
\hline C19orf57|79173 & $\mathrm{B} 2$ \\
\hline C19orf59|199675 & $\mathrm{B} 2$ \\
\hline C19orf60|55049 & $\mathrm{B} 2$ \\
\hline C19orf62|29086 & $\mathrm{B} 2$ \\
\hline C19orf66|55337 & $\mathrm{B} 2$ \\
\hline C19orf6|91304 & $\mathrm{B} 2$ \\
\hline C19orf70|125988 & $\mathrm{B} 2$ \\
\hline C19orf71|100128569 & $\mathrm{B} 2$ \\
\hline C19orf73|55150 & $\mathrm{B} 2$ \\
\hline C19orf75|284369 & $\mathrm{B} 2$ \\
\hline C19orf77|284422 & $\mathrm{B} 2$ \\
\hline C1D|10438 & $\mathrm{B} 2$ \\
\hline C1GALT1|56913 & $\mathrm{B} 2$ \\
\hline C1QA|712 & $\mathrm{B} 2$ \\
\hline C1QBP|708 & $\mathrm{B} 2$ \\
\hline C1QB|713 & $\mathrm{B} 2$ \\
\hline C1QC|714 & $\mathrm{B} 2$ \\
\hline C1QL1|10882 & $\mathrm{B} 2$ \\
\hline C1QL3|389941 & B2 \\
\hline C1QL4|338761 & B2 \\
\hline
\end{tabular}




\begin{tabular}{|c|c|}
\hline C1QTNF3|114899 & B2 \\
\hline C1QTNF4|114900 & B2 \\
\hline C1QTNF8|390664 & $\mathrm{B} 2$ \\
\hline C1orf100|200159 & $\mathrm{B} 2$ \\
\hline C1orf101|257044 & $\mathrm{B} 2$ \\
\hline C1orf104|284618 & $\mathrm{B} 2$ \\
\hline C1orf105|92346 & $\mathrm{B} 2$ \\
\hline C1orf106|55765 & $\mathrm{B} 2$ \\
\hline C1orf107|27042 & $\mathrm{B} 2$ \\
\hline C1orf109|54955 & $\mathrm{B} 2$ \\
\hline C1orf110|339512 & $\mathrm{B} 2$ \\
\hline C1orf111|284680 & $\mathrm{B} 2$ \\
\hline C1orf113|79729 & $\mathrm{B} 2$ \\
\hline C1orf114|57821 & $\mathrm{B} 2$ \\
\hline C1orf116|79098 & B2 \\
\hline C1orf125|126859 & $\mathrm{B} 2$ \\
\hline C1orf126|200197 & $\mathrm{B} 2$ \\
\hline C1orf127|148345 & $\mathrm{B} 2$ \\
\hline C1orf128|57095 & $\mathrm{B} 2$ \\
\hline C1orf129|80133 & $\mathrm{B} 2$ \\
\hline C1orf133|574036 & $\mathrm{B} 2$ \\
\hline C1orf141|400757 & $\mathrm{B} 2$ \\
\hline C1orf144|26099 & $\mathrm{B} 2$ \\
\hline C1orf14|81626 & $\mathrm{B} 2$ \\
\hline C1orf150|148823 & $\mathrm{B} 2$ \\
\hline C1orf151|440574 & $\mathrm{B} 2$ \\
\hline C1orf152|767846 & $\mathrm{B} 2$ \\
\hline C1orf157|284573 & $\mathrm{B} 2$ \\
\hline C1orf158|93190 & $\mathrm{B} 2$ \\
\hline C1orf159|54991 & $\mathrm{B} 2$ \\
\hline C1orf161|126868 & $\mathrm{B} 2$ \\
\hline C1orf162|128346 & $\mathrm{B} 2$ \\
\hline C1orf163|65260 & $\mathrm{B} 2$ \\
\hline C1orf168|199920 & $\mathrm{B} 2$ \\
\hline C1orf170|84808 & $\mathrm{B} 2$ \\
\hline C1orf172|126695 & $\mathrm{B} 2$ \\
\hline C1orf173|127254 & $\mathrm{B} 2$ \\
\hline C1orf174|339448 & $\mathrm{B} 2$ \\
\hline C1orf177|163747 & $\mathrm{B} 2$ \\
\hline C1orf180|439927 & $\mathrm{B} 2$ \\
\hline C1orf185|284546 & $\mathrm{B} 2$ \\
\hline C1orf186|440712 & $\mathrm{B} 2$ \\
\hline C1orf187|374946 & $\mathrm{B} 2$ \\
\hline C1orf189|388701 & $\mathrm{B} 2$ \\
\hline C1orf192|257177 & $\mathrm{B} 2$ \\
\hline C1orf194|127003 & $\mathrm{B} 2$ \\
\hline C1orf198|84886 & $\mathrm{B} 2$ \\
\hline C1orf200|644997 & $\mathrm{B} 2$ \\
\hline C1orf201|90529 & $\mathrm{B} 2$ \\
\hline $\begin{array}{l}\text { C1orf203|84852 } \\
\end{array}$ & $\mathrm{B} 2$ \\
\hline C1orf204|284677 & $\mathrm{B} 2$ \\
\hline
\end{tabular}




\begin{tabular}{|c|c|}
\hline C1orf210|149466 & B2 \\
\hline C1orf212|113444 & B2 \\
\hline C1orf216|127703 & $\mathrm{B} 2$ \\
\hline C1orf21|81563 & $\mathrm{B} 2$ \\
\hline C1orf223|374973 & $\mathrm{B} 2$ \\
\hline C1orf226|400793 & $\mathrm{B} 2$ \\
\hline C1orf227|149643 & $\mathrm{B} 2$ \\
\hline C1orf228|339541 & $\mathrm{B} 2$ \\
\hline C1orf229|388759 & $\mathrm{B} 2$ \\
\hline C1orf230|284485 & $\mathrm{B} 2$ \\
\hline C1orf25|81627 & $\mathrm{B} 2$ \\
\hline C1orf26|54823 & $\mathrm{B} 2$ \\
\hline C1orf38|9473 & $\mathrm{B} 2$ \\
\hline \begin{tabular}{l|l|} 
C1orf49|84066 \\
\end{tabular} & $\mathrm{B} 2$ \\
\hline C1orf50|79078 & B2 \\
\hline C1orf51|148523 & $\mathrm{B} 2$ \\
\hline C1orf54|79630 & $\mathrm{B} 2$ \\
\hline C1orf55|163859 & $\mathrm{B} 2$ \\
\hline C1orf59|113802 & $\mathrm{B} 2$ \\
\hline C1orf61|10485 & $\mathrm{B} 2$ \\
\hline $\begin{array}{l}\text { C1orf63|57035 } \\
\end{array}$ & $\mathrm{B} 2$ \\
\hline C1orf64|149563 & $\mathrm{B} 2$ \\
\hline C1orf65|164127 & $\mathrm{B} 2$ \\
\hline C1orf66|51093 & $\mathrm{B} 2$ \\
\hline C1orf68|100129271 & $\mathrm{B} 2$ \\
\hline C1orf69|200205 & $\mathrm{B} 2$ \\
\hline C1orf70|339453 & $\mathrm{B} 2$ \\
\hline C1orf83|127428 & $\mathrm{B} 2$ \\
\hline C1orf84|149469 & $\mathrm{B} 2$ \\
\hline C1orf86|199990 & $\mathrm{B} 2$ \\
\hline C1orf87|127795 & $\mathrm{B} 2$ \\
\hline C1orf88|128344 & $\mathrm{B} 2$ \\
\hline C1orf89|79363 & $\mathrm{B} 2$ \\
\hline C1orf91|56063 & $\mathrm{B} 2$ \\
\hline C1orf92|149499 & $\mathrm{B} 2$ \\
\hline C1orf94|84970 & $\mathrm{B} 2$ \\
\hline C1orf95|375057 & $\mathrm{B} 2$ \\
\hline C1orf96|126731 & $\mathrm{B} 2$ \\
\hline C20orf106|200232 & $\mathrm{B} 2$ \\
\hline C20orf107|388799 & $\mathrm{B} 2$ \\
\hline C20orf108|116151 & $\mathrm{B} 2$ \\
\hline C20orf111|51526 & $\mathrm{B} 2$ \\
\hline C20orf114|92747 & $\mathrm{B} 2$ \\
\hline C20orf117|140710 & $\mathrm{B} 2$ \\
\hline C20orf118|140711 & $\mathrm{B} 2$ \\
\hline C20orf11|54994 & $\mathrm{B} 2$ \\
\hline C20orf123|128506 & $\mathrm{B} 2$ \\
\hline C20orf12|55184 & $\mathrm{B} 2$ \\
\hline C20orf134|170487 & $\mathrm{B} 2$ \\
\hline C20orf135|140701 & $\mathrm{B} 2$ \\
\hline C20orf141|128653 & B2 \\
\hline
\end{tabular}




\begin{tabular}{|c|c|}
\hline C20orf144|128864 & B2 \\
\hline C20orf152|140894 & B2 \\
\hline C20orf166|128826 & $\mathrm{B} 2$ \\
\hline C20orf173|140873 & $\mathrm{B} 2$ \\
\hline C20orf177|63939 & $\mathrm{B} 2$ \\
\hline C20orf186|149954 & $\mathrm{B} 2$ \\
\hline C20orf191|149934 & $\mathrm{B} 2$ \\
\hline C20orf195|79025 & $\mathrm{B} 2$ \\
\hline C20orf196|149840 & $\mathrm{B} 2$ \\
\hline C20orf197|284756 & $\mathrm{B} 2$ \\
\hline C20orf199|441951 & $\mathrm{B} 2$ \\
\hline C20orf200|253868 & $\mathrm{B} 2$ \\
\hline C20orf201|198437 & $\mathrm{B} 2$ \\
\hline C20orf202|400831 & $\mathrm{B} 2$ \\
\hline C20orf26|26074 & B2 \\
\hline C20orf27|54976 & $\mathrm{B} 2$ \\
\hline C20orf29|55317 & $\mathrm{B} 2$ \\
\hline C20orf30|29058 & $\mathrm{B} 2$ \\
\hline $\begin{array}{l}\text { C20orf3|57136 } \\
\end{array}$ & $\mathrm{B} 2$ \\
\hline C20orf43|51507 & $\mathrm{B} 2$ \\
\hline $\begin{array}{l}\text { C20orf46|55321 } \\
\end{array}$ & $\mathrm{B} 2$ \\
\hline C20orf4|25980 & B2 \\
\hline C20orf56|140828 & $\mathrm{B} 2$ \\
\hline C20orf70|140683 & $\mathrm{B} 2$ \\
\hline C20orf72|92667 & $\mathrm{B} 2$ \\
\hline C20orf7|79133 & $\mathrm{B} 2$ \\
\hline C20orf85|128602 & $\mathrm{B} 2$ \\
\hline C20orf96|140680 & $\mathrm{B} 2$ \\
\hline C21orf119|84996 & $\mathrm{B} 2$ \\
\hline C21orf121|150142 & $\mathrm{B} 2$ \\
\hline C21orf122|728039 & $\mathrm{B} 2$ \\
\hline C21orf125|284836 & $\mathrm{B} 2$ \\
\hline C21orf128|150147 & $\mathrm{B} 2$ \\
\hline C21orf129|150135 & $\mathrm{B} 2$ \\
\hline C21orf130|284835 & $\mathrm{B} 2$ \\
\hline $\begin{array}{l}\text { C21orf15|54094 } \\
\end{array}$ & $\mathrm{B} 2$ \\
\hline C21orf29|54084 & $\mathrm{B} 2$ \\
\hline $\begin{array}{l}\text { C21 orf33|8209 } \\
\end{array}$ & $\mathrm{B} 2$ \\
\hline $\begin{array}{l}\text { C21orf56|84221 } \\
\end{array}$ & $\mathrm{B} 2$ \\
\hline C21 orf57|54059 & $\mathrm{B} 2$ \\
\hline C21orf67|84536 & $\mathrm{B} 2$ \\
\hline C21 orf70|85395 & $\mathrm{B} 2$ \\
\hline C21orf71|282566 & $\mathrm{B} 2$ \\
\hline C21orf7|56911 & $\mathrm{B} 2$ \\
\hline C21orf81|391267 & $\mathrm{B} 2$ \\
\hline C21orf82|114036 & $\mathrm{B} 2$ \\
\hline C21orf84|114038 & $\mathrm{B} 2$ \\
\hline C21orf88|114041 & $\mathrm{B} 2$ \\
\hline C21orf90|114043 & $\mathrm{B} 2$ \\
\hline C21orf91|54149 & $\mathrm{B} 2$ \\
\hline C21orf96|80215 & $\mathrm{B} 2$ \\
\hline
\end{tabular}




\begin{tabular}{|c|c|}
\hline C21orf99|149992 & B2 \\
\hline C22orf13|83606 & B2 \\
\hline C22orf15|150248 & $\mathrm{B} 2$ \\
\hline C22orf24|25775 & $\mathrm{B} 2$ \\
\hline C22orf25|128989 & $\mathrm{B} 2$ \\
\hline C22orf26|55267 & $\mathrm{B} 2$ \\
\hline C22orf27|150291 & $\mathrm{B} 2$ \\
\hline C22orf28|51493 & $\mathrm{B} 2$ \\
\hline C22orf29|79680 & $\mathrm{B} 2$ \\
\hline C22orf30|253143 & $\mathrm{B} 2$ \\
\hline C22orf31|25770 & $\mathrm{B} 2$ \\
\hline C22orf32|91689 & $\mathrm{B} 2$ \\
\hline C22orf34|348645 & $\mathrm{B} 2$ \\
\hline C22orf36|388886 & $\mathrm{B} 2$ \\
\hline C22orf40|150383 & B2 \\
\hline C22orf41|644186 & $\mathrm{B} 2$ \\
\hline C22orf43|51233 & $\mathrm{B} 2$ \\
\hline C22orf45|646023 & $\mathrm{B} 2$ \\
\hline C22orf9|23313 & $\mathrm{B} 2$ \\
\hline $\begin{array}{l}\mathrm{C} 2 \mathrm{CD} 3 \mid 26005 \\
\end{array}$ & $\mathrm{~B} 2$ \\
\hline C2CD4A|145741 & $\mathrm{B} 2$ \\
\hline C2CD4B 388125 & $\mathrm{~B} 2$ \\
\hline C2CD4D|100191040 & $\mathrm{B} 2$ \\
\hline C2orf14|100132708 & $\mathrm{B} 2$ \\
\hline C2orf16|84226 & $\mathrm{B} 2$ \\
\hline C2orf24|27013 & $\mathrm{B} 2$ \\
\hline C2orf27A|29798 & $\mathrm{B} 2$ \\
\hline C2orf27B|408029 & $\mathrm{B} 2$ \\
\hline C2orf28|51374 & $\mathrm{B} 2$ \\
\hline C2orf34|79823 & $\mathrm{B} 2$ \\
\hline C2orf39|92749 & $\mathrm{B} 2$ \\
\hline C2orf3|6936 & $\mathrm{B} 2$ \\
\hline C2orf42|54980 & $\mathrm{B} 2$ \\
\hline C2orf43|60526 & $\mathrm{B} 2$ \\
\hline C2orf44|80304 & $\mathrm{B} 2$ \\
\hline C2orf47|79568 & $\mathrm{B} 2$ \\
\hline $\begin{array}{l}\text { C2orf49|79074 } \\
\end{array}$ & $\mathrm{B} 2$ \\
\hline C2orf50|130813 & $\mathrm{B} 2$ \\
\hline C2orf51|200523 & $\mathrm{B} 2$ \\
\hline C2orf52|151477 & $\mathrm{B} 2$ \\
\hline C2orf53|339779 & $\mathrm{B} 2$ \\
\hline C2orf54|79919 & $\mathrm{B} 2$ \\
\hline C2orf55|343990 & $\mathrm{B} 2$ \\
\hline C2orf56|55471 & $\mathrm{B} 2$ \\
\hline C2orf57|165100 & $\mathrm{B} 2$ \\
\hline C2orf60|129450 & $\mathrm{B} 2$ \\
\hline C2orf61|285051 & $\mathrm{B} 2$ \\
\hline C2orf62|375307 & $\mathrm{B} 2$ \\
\hline C2orf63|130162 & $\mathrm{B} 2$ \\
\hline C2orf64|493753 & $\mathrm{B} 2$ \\
\hline C2orf65|130951 & $\mathrm{B} 2$ \\
\hline
\end{tabular}




\begin{tabular}{|c|c|}
\hline C2orf66|401027 & B2 \\
\hline C2orf68|388969 & $\mathrm{B} 2$ \\
\hline C2orf69|205327 & B2 \\
\hline C2orf70|339778 & $\mathrm{B} 2$ \\
\hline C2orf72|257407 & B2 \\
\hline C2orf73|129852 & $\mathrm{B} 2$ \\
\hline C2orf76|130355 & $\mathrm{B} 2$ \\
\hline C2orf77|129881 & $\mathrm{B} 2$ \\
\hline C2orf78|388960 & B2 \\
\hline C2orf79|391356 & B2 \\
\hline C2orf7|84279 & B2 \\
\hline C2orf80|389073 & $\mathrm{B} 2$ \\
\hline C2orf81|388963 & $\mathrm{B} 2$ \\
\hline C2orf82|389084 & B2 \\
\hline C2orf83|56918 & $\mathrm{B} 2$ \\
\hline C2orf85|285093 & B2 \\
\hline C2orf89|129293 & $\mathrm{B} 2$ \\
\hline $\begin{array}{l}\mathrm{C} 2 \mid 717 \\
\end{array}$ & $\mathrm{~B} 2$ \\
\hline C3AR1|719 & $\mathrm{B} 2$ \\
\hline C3orf10|55845 & $\mathrm{B} 2$ \\
\hline C3orf15|89876 & $\mathrm{B} 2$ \\
\hline C3orf16|389161 & $\mathrm{B} 2$ \\
\hline C3orf17|25871 & $\mathrm{B} 2$ \\
\hline C3orf19|51244 & $\mathrm{B} 2$ \\
\hline C3orf1|51300 & $\mathrm{B} 2$ \\
\hline C3orf20|84077 & $\mathrm{B} 2$ \\
\hline C3orf22|152065 & $\mathrm{B} 2$ \\
\hline C3orf23|285343 & $\mathrm{B} 2$ \\
\hline C3orf24|115795 & B2 \\
\hline C3orf26|84319 & $\mathrm{B} 2$ \\
\hline C3orf27|23434 & $\mathrm{B} 2$ \\
\hline C3orf30|152405 & $\mathrm{B} 2$ \\
\hline C3orf31|132001 & $\mathrm{B} 2$ \\
\hline C3orf32|51066 & $\mathrm{B} 2$ \\
\hline C3orf33|285315 & $\mathrm{B} 2$ \\
\hline C3orf34|84984 & $\mathrm{B} 2$ \\
\hline C3orf35|339883 & B2 \\
\hline C3orf37|56941 & $\mathrm{B} 2$ \\
\hline C3orf39|84892 & $\mathrm{B} 2$ \\
\hline C3orf43|255798 & $\mathrm{B} 2$ \\
\hline C3orf45|132228 & B2 \\
\hline C3orf48|151649 & $\mathrm{B} 2$ \\
\hline C3orf49|132200 & $\mathrm{B} 2$ \\
\hline C3orf51|711 & $\mathrm{B} 2$ \\
\hline C3orf57|165679 & $\mathrm{B} 2$ \\
\hline C3orf59|151963 & $\mathrm{B} 2$ \\
\hline C3orf62|375341 & B2 \\
\hline C3orf63|23272 & B2 \\
\hline C3orf65|646600 & $\mathrm{B} 2$ \\
\hline C3orf66|677779 & $\mathrm{B} 2$ \\
\hline C3orf70|285382 & B2 \\
\hline
\end{tabular}




\begin{tabular}{|c|c|}
\hline C3orf71|646450 & B2 \\
\hline C3orf72|401089 & B2 \\
\hline C3orf74|100128378 & $\mathrm{B} 2$ \\
\hline C3orf75|54859 & $\mathrm{B} 2$ \\
\hline C3orf79|152118 & $\mathrm{B} 2$ \\
\hline C4BPA|722 & $\mathrm{B} 2$ \\
\hline C4BPB|725 & $\mathrm{B} 2$ \\
\hline C4orf10|317648 & $\mathrm{B} 2$ \\
\hline C4orf14|84273 & $\mathrm{B} 2$ \\
\hline C4orf17|84103 & $\mathrm{B} 2$ \\
\hline C4orf19|55286 & $\mathrm{B} 2$ \\
\hline C4orf21|55345 & $\mathrm{B} 2$ \\
\hline C4orf22|255119 & $\mathrm{B} 2$ \\
\hline C4orf26|152816 & $\mathrm{B} 2$ \\
\hline C4orf27|54969 & B2 \\
\hline \begin{tabular}{l|l|} 
C4orf29|80167 \\
\end{tabular} & $\mathrm{B} 2$ \\
\hline C4orf31|79625 & $\mathrm{B} 2$ \\
\hline C4orf32|132720 & $\mathrm{B} 2$ \\
\hline C4orf33|132321 & $\mathrm{B} 2$ \\
\hline $\begin{array}{l}\text { C4orf35|85438 } \\
\end{array}$ & $\mathrm{B} 2$ \\
\hline C4orf36|132989 & $\mathrm{B} 2$ \\
\hline C4orf37|285555 & $\mathrm{B} 2$ \\
\hline C4orf39|152756 & $\mathrm{B} 2$ \\
\hline C4orf40|401137 & $\mathrm{B} 2$ \\
\hline C4orf41|60684 & $\mathrm{B} 2$ \\
\hline $\begin{array}{l}\text { C4orf43|55319 } \\
\end{array}$ & $\mathrm{B} 2$ \\
\hline C4orf44|345222 & $\mathrm{B} 2$ \\
\hline C4orf45|152940 & $\mathrm{B} 2$ \\
\hline C4orf47|441054 & $\mathrm{B} 2$ \\
\hline C4orf50|389197 & $\mathrm{B} 2$ \\
\hline C4orf51|646603 & $\mathrm{B} 2$ \\
\hline C4orf6|10141 & $\mathrm{B} 2$ \\
\hline C4orf7|260436 & $\mathrm{B} 2$ \\
\hline C5AR1|728 & $\mathrm{B} 2$ \\
\hline C5orf20|140947 & $\mathrm{B} 2$ \\
\hline \begin{tabular}{l|l|l|} 
C5orf23 & 79614 \\
\end{tabular} & $\mathrm{~B} 2$ \\
\hline C5orf24|134553 & $\mathrm{B} 2$ \\
\hline C5orf25|375484 & $\mathrm{B} 2$ \\
\hline C5orf27|202299 & $\mathrm{B} 2$ \\
\hline C5orf28|64417 & $\mathrm{B} 2$ \\
\hline C5orf33|133686 & $\mathrm{B} 2$ \\
\hline C5orf35|133383 & $\mathrm{B} 2$ \\
\hline C5orf36|285600 & $\mathrm{B} 2$ \\
\hline C5orf38|153571 & $\mathrm{B} 2$ \\
\hline C5orf39|389289 & $\mathrm{B} 2$ \\
\hline C5orf40|408263 & $\mathrm{B} 2$ \\
\hline C5orf42|65250 & $\mathrm{B} 2$ \\
\hline C5orf44|80006 & $\mathrm{B} 2$ \\
\hline C5orf46|389336 & $\mathrm{B} 2$ \\
\hline C5orf47|133491 & $\mathrm{B} 2$ \\
\hline C5orf48|389320 & B2 \\
\hline
\end{tabular}




\begin{tabular}{|c|c|}
\hline C5orf51|285636 & B2 \\
\hline C5orf52|100190949 & B2 \\
\hline C5orf54|63920 & $\mathrm{B} 2$ \\
\hline C5orf55|116349 & $\mathrm{B} 2$ \\
\hline C5orf58|133874 & $\mathrm{B} 2$ \\
\hline C5orf60|285679 & $\mathrm{B} 2$ \\
\hline $\mathrm{C} 5 \mid 727$ & $\mathrm{~B} 2$ \\
\hline C6orf103|79747 & $\mathrm{B} 2$ \\
\hline C6orf106|64771 & $\mathrm{B} 2$ \\
\hline C6orf108|10591 & $\mathrm{B} 2$ \\
\hline C6orf10|10665 & $\mathrm{B} 2$ \\
\hline C6orf114|85411 & $\mathrm{B} 2$ \\
\hline C6orf118|168090 & $\mathrm{B} 2$ \\
\hline C6orf124|653483 & $\mathrm{B} 2$ \\
\hline C6orf130|221443 & $\mathrm{B} 2$ \\
\hline C6orf132|647024 & $\mathrm{B} 2$ \\
\hline C6orf134|79969 & $\mathrm{B} 2$ \\
\hline C6orf136|221545 & $\mathrm{B} 2$ \\
\hline C6orf138|442213 & $\mathrm{B} 2$ \\
\hline C6orf141|135398 & $\mathrm{B} 2$ \\
\hline C6orf142|90523 & $\mathrm{B} 2$ \\
\hline C6orf146|222826 & B2 \\
\hline C6orf147|387097 & $\mathrm{B} 2$ \\
\hline C6orf155|79940 & $\mathrm{B} 2$ \\
\hline C6orf15|29113 & $\mathrm{B} 2$ \\
\hline C6orf165|154313 & $\mathrm{B} 2$ \\
\hline C6orf167|253714 & $\mathrm{B} 2$ \\
\hline C6orf168|84553 & $\mathrm{B} 2$ \\
\hline C6orf170|221322 & $\mathrm{B} 2$ \\
\hline $\begin{array}{l}\text { C6orf176|90632 } \\
\end{array}$ & $\mathrm{B} 2$ \\
\hline C6orf182|285753 & $\mathrm{B} 2$ \\
\hline C6orf186|728464 & $\mathrm{B} 2$ \\
\hline \begin{tabular}{l|l|l|} 
C6orf192|16843 \\
\end{tabular} & $\mathrm{B} 2$ \\
\hline C6orf195|154386 & $\mathrm{B} 2$ \\
\hline C6orf203|51250 & $\mathrm{B} 2$ \\
\hline C6orf208|80069 & $\mathrm{B} 2$ \\
\hline C6orf211|79624 & $\mathrm{B} 2$ \\
\hline C6orf217|100131814 & $\mathrm{B} 2$ \\
\hline C6orf218|221718 & $\mathrm{B} 2$ \\
\hline C6orf221|154288 & $\mathrm{B} 2$ \\
\hline C6orf222|389384 & $\mathrm{B} 2$ \\
\hline C6orf223|221416 & $\mathrm{B} 2$ \\
\hline C6orf225|619208 & $\mathrm{B} 2$ \\
\hline C6orf226|441150 & $\mathrm{B} 2$ \\
\hline C6orf227|401253 & $\mathrm{B} 2$ \\
\hline C6orf25|80739 & $\mathrm{B} 2$ \\
\hline C6orf26|401251 & $\mathrm{B} 2$ \\
\hline C6orf27|80737 & $\mathrm{B} 2$ \\
\hline C6orf41|100133205 & $\mathrm{B} 2$ \\
\hline C6orf47|57827 & $\mathrm{B} 2$ \\
\hline C6orf48|50854 & $\mathrm{B} 2$ \\
\hline
\end{tabular}




\begin{tabular}{|c|c|}
\hline C6orf52|347744 & B2 \\
\hline C6orf58|352999 & $\mathrm{B} 2$ \\
\hline C6orf70|55780 & B2 \\
\hline C6orf81|221481 & $\mathrm{B} 2$ \\
\hline C6orf94|153918 & B2 \\
\hline C6orf97|80129 & $\mathrm{B} 2$ \\
\hline C6|729 & B2 \\
\hline C7orf10|79783 & $\mathrm{B} 2$ \\
\hline C7orf13|129790 & $\mathrm{B} 2$ \\
\hline C7orf16|10842 & $\mathrm{B} 2$ \\
\hline C7orf23|79161 & B2 \\
\hline C7orf25|79020 & $\mathrm{B} 2$ \\
\hline C7orf26|79034 & $\mathrm{B} 2$ \\
\hline C7orf27|221927 & $\mathrm{B} 2$ \\
\hline C7orf29|113763 & $\mathrm{B} 2$ \\
\hline C7orf30|115416 & $\mathrm{B} 2$ \\
\hline C7orf33|202865 & B2 \\
\hline C7orf34|135927 & $\mathrm{B} 2$ \\
\hline C7orf36|57002 & $\mathrm{B} 2$ \\
\hline C7orf40|285958 & $\mathrm{B} 2$ \\
\hline C7orf42|55069 & $\mathrm{B} 2$ \\
\hline C7orf44|55744 & $\mathrm{B} 2$ \\
\hline C7orf45|136263 & $\mathrm{B} 2$ \\
\hline C7orf46|340277 & $\mathrm{B} 2$ \\
\hline C7orf49|78996 & $\mathrm{B} 2$ \\
\hline C7orf4|64433 & $\mathrm{B} 2$ \\
\hline C7orf50|84310 & $\mathrm{B} 2$ \\
\hline C7orf52|375607 & $\mathrm{B} 2$ \\
\hline C7orf53|286006 & $\mathrm{B} 2$ \\
\hline C7orf54|27099 & B2 \\
\hline C7orf55|154791 & $\mathrm{B} 2$ \\
\hline C7orf57|136288 & $\mathrm{B} 2$ \\
\hline C7orf59|389541 & $\mathrm{B} 2$ \\
\hline C7orf60|154743 & $\mathrm{B} 2$ \\
\hline C7orf61|402573 & $\mathrm{B} 2$ \\
\hline C7orf63|79846 & $\mathrm{B} 2$ \\
\hline C7orf64|84060 & B2 \\
\hline C7orf65|401335 & $\mathrm{B} 2$ \\
\hline C7orf68|29923 & $\mathrm{B} 2$ \\
\hline C7orf69|80099 & $\mathrm{B} 2$ \\
\hline C7orf71|285941 & $\mathrm{B} 2$ \\
\hline C7orf72|100130988 & $\mathrm{B} 2$ \\
\hline $\mathrm{C} 8 \mathrm{~A} \mid 731$ & $\mathrm{~B} 2$ \\
\hline $\mathrm{C} 8 \mathrm{~B} \mid 732$ & $\mathrm{~B} 2$ \\
\hline C8G|733 & $\mathrm{B} 2$ \\
\hline C8ORFK29|340393 & $\mathrm{B} 2$ \\
\hline C8orf12|83656 & $\mathrm{B} 2$ \\
\hline C8orf22|492307 & $\mathrm{B} 2$ \\
\hline C8orf31|286122 & $\mathrm{B} 2$ \\
\hline C8orf33|65265 & B2 \\
\hline C8orf34|116328 & B2 \\
\hline
\end{tabular}




\begin{tabular}{|c|c|}
\hline C8orf37|157657 & B2 \\
\hline C8orf39|55472 & $\mathrm{B} 2$ \\
\hline C8orf40|114926 & B2 \\
\hline C8orf41|80185 & $\mathrm{B} 2$ \\
\hline C8orf42|157695 & B2 \\
\hline C8orf44|56260 & $\mathrm{B} 2$ \\
\hline C8orf45|157777 & $\mathrm{B} 2$ \\
\hline C8orf46|254778 & $\mathrm{B} 2$ \\
\hline C8orf47|203111 & $\mathrm{B} 2$ \\
\hline C8orf48|157773 & $\mathrm{B} 2$ \\
\hline C8orf4|56892 & B2 \\
\hline C8orf55|51337 & $\mathrm{B} 2$ \\
\hline C8orf56|157556 & $\mathrm{B} 2$ \\
\hline C8orf59|401466 & $\mathrm{B} 2$ \\
\hline C8orf73|642475 & $\mathrm{B} 2$ \\
\hline C8orf75|619351 & $\mathrm{B} 2$ \\
\hline C8orf77|286103 & B2 \\
\hline C8orf80|389643 & $\mathrm{B} 2$ \\
\hline C8orf84|157869 & $\mathrm{B} 2$ \\
\hline C8orf85|441376 & $\mathrm{B} 2$ \\
\hline C8orf86|389649 & $\mathrm{B} 2$ \\
\hline C9orf103|414328 & $\mathrm{B} 2$ \\
\hline C9orf106|414318 & $\mathrm{B} 2$ \\
\hline C9orf109|286333 & $\mathrm{B} 2$ \\
\hline C9orf110|100128385 & $\mathrm{B} 2$ \\
\hline C9orf114|51490 & $\mathrm{B} 2$ \\
\hline C9orf117|286207 & $\mathrm{B} 2$ \\
\hline C9orf122|158228 & $\mathrm{B} 2$ \\
\hline C9orf123|90871 & $\mathrm{B} 2$ \\
\hline C9orf128|392307 & $\mathrm{B} 2$ \\
\hline C9orf129|445577 & $\mathrm{B} 2$ \\
\hline C9orf135|138255 & $\mathrm{B} 2$ \\
\hline C9orf139|401563 & $\mathrm{B} 2$ \\
\hline C9orf144|389715 & $\mathrm{B} 2$ \\
\hline C9orf150|286343 & $\mathrm{B} 2$ \\
\hline C9orf152|401546 & $\mathrm{B} 2$ \\
\hline C9orf153|389766 & $\mathrm{B} 2$ \\
\hline C9orf167|54863 & $\mathrm{B} 2$ \\
\hline C9orf169|375791 & $\mathrm{B} 2$ \\
\hline C9orf16|79095 & $\mathrm{B} 2$ \\
\hline C9orf170|401535 & $\mathrm{B} 2$ \\
\hline C9orf171|389799 & $\mathrm{B} 2$ \\
\hline C9orf173|441476 & $\mathrm{B} 2$ \\
\hline C9orf23|138716 & $\mathrm{B} 2$ \\
\hline C9orf24|84688 & $\mathrm{B} 2$ \\
\hline C9orf25|203259 & $\mathrm{B} 2$ \\
\hline C9orf37|85026 & $\mathrm{B} 2$ \\
\hline C9orf3|84909 & $\mathrm{B} 2$ \\
\hline C9orf40|55071 & $\mathrm{B} 2$ \\
\hline C9orf41|138199 & B2 \\
\hline C9orf43|257169 & B2 \\
\hline
\end{tabular}




\begin{tabular}{|c|c|}
\hline C9orf44|158314 & B2 \\
\hline C9orf45|81571 & B2 \\
\hline C9orf47|286223 & $\mathrm{B} 2$ \\
\hline C9orf4|23732 & $\mathrm{B} 2$ \\
\hline C9orf50|375759 & $\mathrm{B} 2$ \\
\hline C9orf53|51198 & $\mathrm{B} 2$ \\
\hline C9orf57|138240 & $\mathrm{B} 2$ \\
\hline C9orf68|55064 & $\mathrm{B} 2$ \\
\hline C9orf69|90120 & $\mathrm{B} 2$ \\
\hline C9orf6|54942 & $\mathrm{B} 2$ \\
\hline C9orf70|84850 & $\mathrm{B} 2$ \\
\hline C9orf71|169693 & $\mathrm{B} 2$ \\
\hline C9orf78|51759 & $\mathrm{B} 2$ \\
\hline C9orf79|286234 & $\mathrm{B} 2$ \\
\hline C9orf80|58493 & $\mathrm{B} 2$ \\
\hline C9orf82|79886 & $\mathrm{B} 2$ \\
\hline C9orf84|158401 & $\mathrm{B} 2$ \\
\hline C9orf86|55684 & $\mathrm{B} 2$ \\
\hline C9orf93|203238 & $\mathrm{B} 2$ \\
\hline C9orf96|169436 & $\mathrm{B} 2$ \\
\hline C9orf9|11092 & $\mathrm{B} 2$ \\
\hline C9|735 & $\mathrm{B} 2$ \\
\hline CA10|56934 & $\mathrm{B} 2$ \\
\hline CA13|377677 & $\mathrm{B} 2$ \\
\hline CA14|23632 & $\mathrm{B} 2$ \\
\hline CA1|759 & $\mathrm{B} 2$ \\
\hline CA2 2760 & $\mathrm{~B} 2$ \\
\hline CA3|761 & $\mathrm{B} 2$ \\
\hline CA5A|763 & $\mathrm{B} 2$ \\
\hline CA5BP|340591 & $\mathrm{B} 2$ \\
\hline CA6|765 & $\mathrm{B} 2$ \\
\hline CA7|766 & $\mathrm{B} 2$ \\
\hline CA8|767 & $\mathrm{B} 2$ \\
\hline CA9|768 & $\mathrm{B} 2$ \\
\hline CAB39|51719 & $\mathrm{B} 2$ \\
\hline \begin{tabular}{l|l|l|} 
CABC1|5997 \\
\end{tabular} & $\mathrm{B} 2$ \\
\hline CABIN1|23523 & $\mathrm{B} 2$ \\
\hline CABLES1|91768 & $\mathrm{B} 2$ \\
\hline CABP4|57010 & $\mathrm{B} 2$ \\
\hline \begin{tabular}{l|l|} 
CABP5 56344 \\
\end{tabular} & $\mathrm{~B} 2$ \\
\hline CABP7|164633 & $\mathrm{B} 2$ \\
\hline CACNA1A|773 & $\mathrm{B} 2$ \\
\hline CACNA1B|774 & $\mathrm{B} 2$ \\
\hline CACNA1E|777 & $\mathrm{B} 2$ \\
\hline CACNA1F|778 & $\mathrm{B} 2$ \\
\hline CACNA1G|8913 & $\mathrm{B} 2$ \\
\hline CACNA1H|8912 & $\mathrm{B} 2$ \\
\hline CACNA1I|8911 & $\mathrm{B} 2$ \\
\hline CACNA1S|779 & $\mathrm{B} 2$ \\
\hline CACNA2D1|781 & $\mathrm{B} 2$ \\
\hline CACNA2D2|9254 & $\mathrm{B} 2$ \\
\hline
\end{tabular}




\begin{tabular}{|c|c|}
\hline CACNA2D3|55799 & B2 \\
\hline CACNA2D4|93589 & $\mathrm{B} 2$ \\
\hline CACNB1|782 & $\mathrm{B} 2$ \\
\hline CACNB2|783 & $\mathrm{B} 2$ \\
\hline CACNB4|785 & B2 \\
\hline CACNG1|786 & $\mathrm{B} 2$ \\
\hline CACNG2|10369 & $\mathrm{B} 2$ \\
\hline CACNG3|10368 & $\mathrm{B} 2$ \\
\hline CACNG5|27091 & $\mathrm{B} 2$ \\
\hline CACNG6|59285 & $\mathrm{B} 2$ \\
\hline CACNG7|59284 & $\mathrm{B} 2$ \\
\hline CACNG8|59283 & $\mathrm{B} 2$ \\
\hline CADM1|23705 & $\mathrm{B} 2$ \\
\hline CADM2|253559 & $\mathrm{B} 2$ \\
\hline CADM4|199731 & $\mathrm{B} 2$ \\
\hline CADPS2|93664 & $\mathrm{B} 2$ \\
\hline CAD|790 & $\mathrm{B} 2$ \\
\hline CAGE1|285782 & $\mathrm{B} 2$ \\
\hline CALB1|793 & $\mathrm{B} 2$ \\
\hline CALCA|796 & $\mathrm{B} 2$ \\
\hline CALCB|797 & $\mathrm{B} 2$ \\
\hline CALCOCO2|10241 & $\mathrm{B} 2$ \\
\hline CALCRL|10203 & $\mathrm{B} 2$ \\
\hline CALCR|799 & $\mathrm{B} 2$ \\
\hline CALHM1|255022 & $\mathrm{B} 2$ \\
\hline CALHM2|51063 & $\mathrm{B} 2$ \\
\hline CALHM3|119395 & $\mathrm{B} 2$ \\
\hline CALM1|801 & $\mathrm{B} 2$ \\
\hline CALM3|808 & $\mathrm{B} 2$ \\
\hline CALML3|810 & $\mathrm{B} 2$ \\
\hline CALML4|91860 & $\mathrm{B} 2$ \\
\hline CALML5|51806 & $\mathrm{B} 2$ \\
\hline CALML6|163688 & $\mathrm{B} 2$ \\
\hline CALR3|125972 & $\mathrm{B} 2$ \\
\hline CALY|50632 & $\mathrm{B} 2$ \\
\hline CAMK1D|57118 & $\mathrm{B} 2$ \\
\hline CAMK1G|57172 & $\mathrm{B} 2$ \\
\hline CAMK2A|815 & $\mathrm{B} 2$ \\
\hline CAMK2B $\mid 816$ & B2 \\
\hline CAMK2D|817 & $\mathrm{B} 2$ \\
\hline CAMK2G|818 & $\mathrm{B} 2$ \\
\hline CAMK2N1|55450 & $\mathrm{B} 2$ \\
\hline CAMK4|814 & $\mathrm{B} 2$ \\
\hline CAMKK1|84254 & $\mathrm{B} 2$ \\
\hline CAMKV|79012 & $\mathrm{B} 2$ \\
\hline CAMLG|819 & $\mathrm{B} 2$ \\
\hline CAMP $\mid 820$ & $\mathrm{~B} 2$ \\
\hline CAMSAP1L1|23271 & $\mathrm{B} 2$ \\
\hline CAMSAP1|157922 & $\mathrm{B} 2$ \\
\hline CAMTA1|23261 & $\mathrm{B} 2$ \\
\hline CAND2|23066 & $\mathrm{B} 2$ \\
\hline
\end{tabular}




\begin{tabular}{|c|c|}
\hline CAP2|10486 & B2 \\
\hline CAPN10|11132 & $\mathrm{B} 2$ \\
\hline CAPN12|147968 & $\mathrm{B} 2$ \\
\hline CAPN14|440854 & $\mathrm{B} 2$ \\
\hline CAPN2|824 & $\mathrm{B} 2$ \\
\hline CAPN7|23473 & $\mathrm{B} 2$ \\
\hline CAPN8|388743 & $\mathrm{B} 2$ \\
\hline CAPN9|10753 & $\mathrm{B} 2$ \\
\hline CAPNS1|826 & $\mathrm{B} 2$ \\
\hline CAPNS2|84290 & $\mathrm{B} 2$ \\
\hline CAPSL|133690 & $\mathrm{B} 2$ \\
\hline CAPZA2|830 & $\mathrm{B} 2$ \\
\hline CARD11|84433 & $\mathrm{B} 2$ \\
\hline CARD14|79092 & $\mathrm{B} 2$ \\
\hline CARD16|114769 & $\mathrm{B} 2$ \\
\hline CARD17|440068 & $\mathrm{B} 2$ \\
\hline CARD18|59082 & $\mathrm{B} 2$ \\
\hline CARD9|64170 & $\mathrm{B} 2$ \\
\hline CARHSP1|23589 & $\mathrm{B} 2$ \\
\hline CARKD|55739 & $\mathrm{B} 2$ \\
\hline CARNS1|57571 & $\mathrm{B} 2$ \\
\hline CARS2|79587 & $\mathrm{B} 2$ \\
\hline CARS|833 & $\mathrm{B} 2$ \\
\hline CARTPT|9607 & $\mathrm{B} 2$ \\
\hline CASC1|55259 & $\mathrm{B} 2$ \\
\hline CASC2|255082 & $\mathrm{B} 2$ \\
\hline CASC3|22794 & $\mathrm{B} 2$ \\
\hline CASC4|113201 & $\mathrm{B} 2$ \\
\hline CASD1|64921 & $\mathrm{B} 2$ \\
\hline CASKIN1|57524 & $\mathrm{B} 2$ \\
\hline CASK|8573 & $\mathrm{B} 2$ \\
\hline CASP10|843 & $\mathrm{B} 2$ \\
\hline CASP14|23581 & $\mathrm{B} 2$ \\
\hline CASP4|837 & $\mathrm{B} 2$ \\
\hline CASP5|838 & $\mathrm{B} 2$ \\
\hline CASP7|840 & $\mathrm{B} 2$ \\
\hline $\begin{array}{l}\text { CASP8AP2|9994 } \\
\end{array}$ & B2 \\
\hline CASP8|841 & $\mathrm{B} 2$ \\
\hline CASP9|842 & $\mathrm{B} 2$ \\
\hline CASQ1|844 & $\mathrm{B} 2$ \\
\hline CASQ2|845 & $\mathrm{B} 2$ \\
\hline CASR $\mid 846$ & $\mathrm{~B} 2$ \\
\hline CASS4|57091 & $\mathrm{B} 2$ \\
\hline CAST|831 & $\mathrm{B} 2$ \\
\hline $\begin{array}{l}\text { CASZ1|54897 } \\
\end{array}$ & $\mathrm{B} 2$ \\
\hline CATSPER2P1|440278 & $\mathrm{B} 2$ \\
\hline CATSPER3|347732 & $\mathrm{B} 2$ \\
\hline CATSPER4|378807 & $\mathrm{B} 2$ \\
\hline CATSPERB|79820 & $\mathrm{B} 2$ \\
\hline CATSPERG|57828 & $\mathrm{B} 2$ \\
\hline CAV3|859 & $\mathrm{B} 2$ \\
\hline
\end{tabular}




\begin{tabular}{|c|c|}
\hline CBARA1|10367 & $\mathrm{B} 2$ \\
\hline CBFA2T2|9139 & $\mathrm{B} 2$ \\
\hline CBFA2T3|863 & B2 \\
\hline CBLB $\mid 868$ & $\mathrm{~B} 2$ \\
\hline CBLL1|79872 & $\mathrm{B} 2$ \\
\hline CBLN1|869 & B2 \\
\hline CBLN2|147381 & $\mathrm{B} 2$ \\
\hline CBLN3|643866 & $\mathrm{B} 2$ \\
\hline CBLN4|140689 & $\mathrm{B} 2$ \\
\hline CBR1|873 & $\mathrm{B} 2$ \\
\hline CBR3|874 & $\mathrm{B} 2$ \\
\hline CBR4|84869 & $\mathrm{B} 2$ \\
\hline CBS $\mid 875$ & $\mathrm{~B} 2$ \\
\hline CBWD1|55871 & $\mathrm{B} 2$ \\
\hline CBWD2|150472 & $\mathrm{B} 2$ \\
\hline CBWD3|445571 & $\mathrm{B} 2$ \\
\hline CBWD5|220869 & $\mathrm{B} 2$ \\
\hline CBWD6|644019 & $\mathrm{B} 2$ \\
\hline CBX5|23468 & $\mathrm{B} 2$ \\
\hline CBX8|57332 & $\mathrm{B} 2$ \\
\hline CBY1|25776 & $\mathrm{B} 2$ \\
\hline CC2D1A|54862 & $\mathrm{B} 2$ \\
\hline CC2D1B|200014 & $\mathrm{B} 2$ \\
\hline CCBL1|883 & $\mathrm{B} 2$ \\
\hline \begin{tabular}{l|l|l|} 
CCBL2 56267 \\
\end{tabular} & $\mathrm{~B} 2$ \\
\hline CCBP2|1238 & $\mathrm{B} 2$ \\
\hline CCDC101|112869 & $\mathrm{B} 2$ \\
\hline CCDC102B|79839 & $\mathrm{B} 2$ \\
\hline CCDC103|388389 & $\mathrm{B} 2$ \\
\hline CCDC104|112942 & $\mathrm{B} 2$ \\
\hline CCDC106|29903 & $\mathrm{B} 2$ \\
\hline CCDC108|255101 & $\mathrm{B} 2$ \\
\hline CCDC109A|90550 & $\mathrm{B} 2$ \\
\hline CCDC110|256309 & $\mathrm{B} 2$ \\
\hline CCDC113|29070 & $\mathrm{B} 2$ \\
\hline CCDC114|93233 & $\mathrm{B} 2$ \\
\hline CCDC115|84317 & $\mathrm{B} 2$ \\
\hline CCDC117|150275 & $\mathrm{B} 2$ \\
\hline CCDC11|220136 & $\mathrm{B} 2$ \\
\hline CCDC120|90060 & $\mathrm{B} 2$ \\
\hline CCDC122|160857 & $\mathrm{B} 2$ \\
\hline CCDC124|115098 & $\mathrm{B} 2$ \\
\hline CCDC126|90693 & $\mathrm{B} 2$ \\
\hline CCDC127|133957 & $\mathrm{B} 2$ \\
\hline CCDC129|223075 & $\mathrm{B} 2$ \\
\hline CCDC12|151903 & $\mathrm{B} 2$ \\
\hline CCDC130|81576 & $\mathrm{B} 2$ \\
\hline CCDC132|55610 & $\mathrm{B} 2$ \\
\hline CCDC134|79879 & $\mathrm{B} 2$ \\
\hline CCDC135|84229 & $\mathrm{B} 2$ \\
\hline CCDC13|152206 & $\mathrm{B} 2$ \\
\hline
\end{tabular}




\begin{tabular}{|c|c|}
\hline CCDC140|151278 & B2 \\
\hline CCDC141|285025 & B2 \\
\hline CCDC142|84865 & $\mathrm{B} 2$ \\
\hline CCDC144A|9720 & $\mathrm{B} 2$ \\
\hline CCDC144B|284047 & $\mathrm{B} 2$ \\
\hline CCDC144C|348254 & $\mathrm{B} 2$ \\
\hline CCDC144NL|339184 & $\mathrm{B} 2$ \\
\hline CCDC146|57639 & $\mathrm{B} 2$ \\
\hline CCDC147|159686 & $\mathrm{B} 2$ \\
\hline CCDC148|130940 & $\mathrm{B} 2$ \\
\hline CCDC149|91050 & $\mathrm{B} 2$ \\
\hline CCDC14|64770 & $\mathrm{B} 2$ \\
\hline CCDC151|115948 & $\mathrm{B} 2$ \\
\hline CCDC153|283152 & $\mathrm{B} 2$ \\
\hline CCDC154|645811 & B2 \\
\hline CCDC155|147872 & $\mathrm{B} 2$ \\
\hline CCDC157|550631 & $\mathrm{B} 2$ \\
\hline CCDC159|126075 & $\mathrm{B} 2$ \\
\hline CCDC15|80071 & $\mathrm{B} 2$ \\
\hline CCDC160|347475 & $\mathrm{B} 2$ \\
\hline CCDC163P|126661 & $\mathrm{B} 2$ \\
\hline CCDC18|343099 & $\mathrm{B} 2$ \\
\hline CCDC21|64793 & $\mathrm{B} 2$ \\
\hline \begin{tabular}{ll|l|} 
CCDC23 374969 \\
\end{tabular} & $\mathrm{~B} 2$ \\
\hline CCDC27|148870 & $\mathrm{B} 2$ \\
\hline CCDC28B|79140 & $\mathrm{B} 2$ \\
\hline CCDC30|728621 & $\mathrm{B} 2$ \\
\hline CCDC33|80125 & $\mathrm{B} 2$ \\
\hline CCDC34|91057 & $\mathrm{B} 2$ \\
\hline CCDC37|348807 & $\mathrm{B} 2$ \\
\hline CCDC38|120935 & $\mathrm{B} 2$ \\
\hline \begin{tabular}{l|l|} 
CCDC39|339829 \\
\end{tabular} & $\mathrm{B} 2$ \\
\hline CCDC40|55036 & $\mathrm{B} 2$ \\
\hline CCDC41|51134 & $\mathrm{B} 2$ \\
\hline CCDC42B|387885 & $\mathrm{B} 2$ \\
\hline \begin{tabular}{ll|l|} 
CCDC42|146849 \\
\end{tabular} & $\mathrm{B} 2$ \\
\hline CCDC45|90799 & $\mathrm{B} 2$ \\
\hline CCDC47|57003 & $\mathrm{B} 2$ \\
\hline CCDC48|79825 & $\mathrm{B} 2$ \\
\hline CCDC53|51019 & $\mathrm{B} 2$ \\
\hline CCDC55|84081 & $\mathrm{B} 2$ \\
\hline CCDC56|28958 & $\mathrm{B} 2$ \\
\hline CCDC57|284001 & $\mathrm{B} 2$ \\
\hline CCDC59|29080 & $\mathrm{B} 2$ \\
\hline CCDC60|160777 & $\mathrm{B} 2$ \\
\hline CCDC61|729440 & $\mathrm{B} 2$ \\
\hline $\begin{array}{l}\text { CCDC62|84660 } \\
\end{array}$ & $\mathrm{B} 2$ \\
\hline CCDC63|160762 & $\mathrm{B} 2$ \\
\hline CCDC64B|146439 & $\mathrm{B} 2$ \\
\hline $\begin{array}{l}\text { CCDC65|85478 } \\
\end{array}$ & $\mathrm{B} 2$ \\
\hline CCDC66|285331 & $\mathrm{B} 2$ \\
\hline
\end{tabular}




\begin{tabular}{|c|c|}
\hline CCDC67|159989 & B2 \\
\hline CCDC6 $\mid 8030$ & B2 \\
\hline CCDC70|83446 & $\mathrm{B} 2$ \\
\hline CCDC71|64925 & $\mathrm{B} 2$ \\
\hline CCDC72|51372 & $\mathrm{B} 2$ \\
\hline CCDC73|493860 & $\mathrm{B} 2$ \\
\hline CCDC74A|90557 & $\mathrm{B} 2$ \\
\hline CCDC74B|91409 & $\mathrm{B} 2$ \\
\hline CCDC77|84318 & $\mathrm{B} 2$ \\
\hline CCDC79|283847 & $\mathrm{B} 2$ \\
\hline CCDC7|221016 & $\mathrm{B} 2$ \\
\hline CCDC81|60494 & $\mathrm{B} 2$ \\
\hline $\begin{array}{ll}\text { CCDC83|220047 } \\
\end{array}$ & $\mathrm{B} 2$ \\
\hline CCDC84|338657 & $\mathrm{B} 2$ \\
\hline CCDC85B|11007 & B2 \\
\hline CCDC85C|317762 & $\mathrm{B} 2$ \\
\hline CCDC86|79080 & $\mathrm{B} 2$ \\
\hline CCDC88A|55704 & $\mathrm{B} 2$ \\
\hline $\begin{array}{l}\text { CCDC88B|283234 } \\
\end{array}$ & $\mathrm{B} 2$ \\
\hline CCDC89|220388 & $\mathrm{B} 2$ \\
\hline CCDC90A|63933 & $\mathrm{B} 2$ \\
\hline CCDC90B $\mid 60492$ & $\mathrm{~B} 2$ \\
\hline CCDC91|55297 & $\mathrm{B} 2$ \\
\hline CCDC94|55702 & $\mathrm{B} 2$ \\
\hline CCDC97|90324 & $\mathrm{B} 2$ \\
\hline CCDC9|26093 & $\mathrm{B} 2$ \\
\hline CCHCR1|54535 & $\mathrm{B} 2$ \\
\hline CCIN|881 & $\mathrm{B} 2$ \\
\hline CCKAR|886 & $\mathrm{B} 2$ \\
\hline CCKBR|887 & $\mathrm{B} 2$ \\
\hline CCK $\mid 885$ & $\mathrm{~B} 2$ \\
\hline CCL11|6356 & $\mathrm{B} 2$ \\
\hline CCL13|6357 & $\mathrm{B} 2$ \\
\hline CCL14-CCL15|348249 & $\mathrm{B} 2$ \\
\hline CCL15|6359 & $\mathrm{B} 2$ \\
\hline CCL17|6361 & $\mathrm{B} 2$ \\
\hline CCL18|6362 & $\mathrm{B} 2$ \\
\hline CCL19|6363 & $\mathrm{B} 2$ \\
\hline CCL1|6346 & $\mathrm{B} 2$ \\
\hline CCL20|6364 & $\mathrm{B} 2$ \\
\hline CCL22|6367 & $\mathrm{B} 2$ \\
\hline CCL24|6369 & $\mathrm{B} 2$ \\
\hline CCL25|6370 & $\mathrm{B} 2$ \\
\hline CCL26|10344 & $\mathrm{B} 2$ \\
\hline CCL27|10850 & $\mathrm{B} 2$ \\
\hline \begin{tabular}{ll|l|} 
CCL2 6347 \\
\end{tabular} & $\mathrm{~B} 2$ \\
\hline CCL3L1|6349 & $\mathrm{B} 2$ \\
\hline CCL3L3|414062 & $\mathrm{B} 2$ \\
\hline CCL3|6348 & $\mathrm{B} 2$ \\
\hline CCL4L2|388372 & $\mathrm{B} 2$ \\
\hline CCL4|6351 & $\mathrm{B} 2$ \\
\hline
\end{tabular}




\begin{tabular}{|c|c|}
\hline CCL5|6352 & B2 \\
\hline CCL7|6354 & B2 \\
\hline CCL8|6355 & $\mathrm{B} 2$ \\
\hline CCM2|83605 & $\mathrm{B} 2$ \\
\hline CCNA1 $\mid 8900$ & $\mathrm{~B} 2$ \\
\hline CCNB3|85417 & $\mathrm{B} 2$ \\
\hline $\mathrm{CCNC} \mid 892$ & $\mathrm{~B} 2$ \\
\hline CCND1|595 & B2 \\
\hline CCNG2|901 & $\mathrm{B} 2$ \\
\hline $\mathrm{CCNH} \mid 902$ & $\mathrm{~B} 2$ \\
\hline CCNI2|645121 & $\mathrm{B} 2$ \\
\hline CCNI|10983 & $\mathrm{B} 2$ \\
\hline CCNJL|79616 & $\mathrm{B} 2$ \\
\hline CCNJ|54619 & $\mathrm{B} 2$ \\
\hline CCNL2|81669 & B2 \\
\hline CCNT1|904 & $\mathrm{B} 2$ \\
\hline CCNT2|905 & $\mathrm{B} 2$ \\
\hline CCNYL1|151195 & $\mathrm{B} 2$ \\
\hline CCNY|219771 & $\mathrm{B} 2$ \\
\hline CCPG1|9236 & $\mathrm{B} 2$ \\
\hline CCR1|1230 & $\mathrm{B} 2$ \\
\hline CCR2|729230 & $\mathrm{B} 2$ \\
\hline CCR3|1232 & $\mathrm{B} 2$ \\
\hline CCR4|1233 & $\mathrm{B} 2$ \\
\hline CCR5|1234 & $\mathrm{B} 2$ \\
\hline CCR6|1235 & $\mathrm{B} 2$ \\
\hline CCR7|1236 & $\mathrm{B} 2$ \\
\hline CCR9|10803 & $\mathrm{B} 2$ \\
\hline CCRL2|9034 & $\mathrm{B} 2$ \\
\hline CCRN4L|25819 & $\mathrm{B} 2$ \\
\hline CCS|9973 & $\mathrm{B} 2$ \\
\hline CCT4|10575 & $\mathrm{B} 2$ \\
\hline CCT6A|908 & $\mathrm{B} 2$ \\
\hline CCT6B|10693 & $\mathrm{B} 2$ \\
\hline CCT6P1|643253 & $\mathrm{B} 2$ \\
\hline CCT8L2|150160 & $\mathrm{B} 2$ \\
\hline CD101|9398 & $\mathrm{B} 2$ \\
\hline CD109|135228 & $\mathrm{B} 2$ \\
\hline CD14|929 & $\mathrm{B} 2$ \\
\hline CD151|977 & $\mathrm{B} 2$ \\
\hline CD163L1|283316 & $\mathrm{B} 2$ \\
\hline CD163|9332 & $\mathrm{B} 2$ \\
\hline CD164|8763 & $\mathrm{B} 2$ \\
\hline CD177|57126 & $\mathrm{B} 2$ \\
\hline CD180|4064 & $\mathrm{B} 2$ \\
\hline CD19|930 & $\mathrm{B} 2$ \\
\hline CD1A|909 & $\mathrm{B} 2$ \\
\hline CD1B $\mid 910$ & $\mathrm{~B} 2$ \\
\hline CD1C|911 & $\mathrm{B} 2$ \\
\hline CD1D|912 & $\mathrm{B} 2$ \\
\hline CD1E|913 & $\mathrm{B} 2$ \\
\hline
\end{tabular}




\begin{tabular}{|c|c|}
\hline CD200R1L|344807 & $\mathrm{B} 2$ \\
\hline CD200R1|131450 & $\mathrm{B} 2$ \\
\hline CD207|50489 & $\mathrm{B} 2$ \\
\hline CD226|10666 & $\mathrm{B} 2$ \\
\hline CD22|933 & $\mathrm{B} 2$ \\
\hline CD244|51744 & B2 \\
\hline CD247|919 & $\mathrm{B} 2$ \\
\hline CD24|100133941 & $\mathrm{B} 2$ \\
\hline CD274|29126 & B2 \\
\hline CD27|939 & $\mathrm{B} 2$ \\
\hline CD28|940 & $\mathrm{B} 2$ \\
\hline CD2BP2|10421 & $\mathrm{B} 2$ \\
\hline CD2|914 & $\mathrm{B} 2$ \\
\hline CD300A|11314 & $\mathrm{B} 2$ \\
\hline CD300C|10871 & $\mathrm{B} 2$ \\
\hline CD300E|342510 & B2 \\
\hline CD300LB|124599 & $\mathrm{B} 2$ \\
\hline CD300LD|100131439 & $\mathrm{B} 2$ \\
\hline CD300LF|146722 & $\mathrm{B} 2$ \\
\hline $\begin{array}{l}\mathrm{CD} 320 \mid 51293 \\
\end{array}$ & $\mathrm{~B} 2$ \\
\hline CD33|945 & $\mathrm{B} 2$ \\
\hline CD37|951 & $\mathrm{B} 2$ \\
\hline CD38|952 & $\mathrm{B} 2$ \\
\hline CD3D|915 & $\mathrm{B} 2$ \\
\hline CD3EAP|10849 & $\mathrm{B} 2$ \\
\hline CD3E 916 & $\mathrm{~B} 2$ \\
\hline CD3G|917 & $\mathrm{B} 2$ \\
\hline CD40LG|959 & $\mathrm{B} 2$ \\
\hline CD40|958 & $\mathrm{B} 2$ \\
\hline CD44|960 & $\mathrm{B} 2$ \\
\hline CD47|961 & $\mathrm{B} 2$ \\
\hline CD48|962 & $\mathrm{B} 2$ \\
\hline CD4|920 & $\mathrm{B} 2$ \\
\hline CD52|1043 & $\mathrm{B} 2$ \\
\hline CD53|963 & $\mathrm{B} 2$ \\
\hline CD55|1604 & $\mathrm{B} 2$ \\
\hline CD58|965 & $\mathrm{B} 2$ \\
\hline CD59|966 & $\mathrm{B} 2$ \\
\hline CD5L|922 & $\mathrm{B} 2$ \\
\hline $\begin{array}{l}\text { CD5 } 5921 \\
\end{array}$ & $\mathrm{~B} 2$ \\
\hline CD63|967 & $\mathrm{B} 2$ \\
\hline CD68|968 & $\mathrm{B} 2$ \\
\hline CD69|969 & $\mathrm{B} 2$ \\
\hline CD6|923 & $\mathrm{B} 2$ \\
\hline CD70|970 & $\mathrm{B} 2$ \\
\hline CD72|971 & $\mathrm{B} 2$ \\
\hline CD74|972 & $\mathrm{B} 2$ \\
\hline CD79A|973 & $\mathrm{B} 2$ \\
\hline CD79B|974 & $\mathrm{B} 2$ \\
\hline CD7|924 & $\mathrm{B} 2$ \\
\hline CD82|3732 & $\mathrm{B} 2$ \\
\hline
\end{tabular}




\begin{tabular}{|c|c|}
\hline CD83|9308 & B2 \\
\hline CD84|8832 & B2 \\
\hline CD8A|925 & B2 \\
\hline CD8B|926 & $\mathrm{B} 2$ \\
\hline CD96|10225 & B2 \\
\hline CD97|976 & $\mathrm{B} 2$ \\
\hline CD99|4267 & B2 \\
\hline CDAN1|146059 & $\mathrm{B} 2$ \\
\hline CDA|978 & B2 \\
\hline CDC14A|8556 & B2 \\
\hline CDC16|8881 & B2 \\
\hline CDC20B|166979 & B2 \\
\hline CDC23|8697 & $\mathrm{B} 2$ \\
\hline CDC25B|994 & B2 \\
\hline CDC26|246184 & $\mathrm{B} 2$ \\
\hline CDC27|996 & B2 \\
\hline CDC34|997 & $\mathrm{B} 2$ \\
\hline CDC37L1|55664 & B2 \\
\hline CDC37|11140 & $\mathrm{B} 2$ \\
\hline CDC40|51362 & $\mathrm{B} 2$ \\
\hline CDC42BPA|8476 & B2 \\
\hline CDC42BPB|9578 & $\mathrm{B} 2$ \\
\hline CDC42BPG|55561 & $\mathrm{B} 2$ \\
\hline CDC42EP1|11135 & B2 \\
\hline CDC42EP5|148170 & $\mathrm{B} 2$ \\
\hline CDC42SE2|56990 & $\mathrm{B} 2$ \\
\hline CDC5L|988 & B2 \\
\hline CDCA7L|55536 & $\mathrm{B} 2$ \\
\hline CDCA7|83879 & B2 \\
\hline CDH10|1008 & $\mathrm{B} 2$ \\
\hline CDH15|1013 & $\mathrm{B} 2$ \\
\hline CDH16|1014 & $\mathrm{B} 2$ \\
\hline CDH17|1015 & $\mathrm{B} 2$ \\
\hline CDH18|1016 & $\mathrm{B} 2$ \\
\hline \begin{tabular}{ll|l|} 
CDH19|28513 \\
\end{tabular} & $\mathrm{B} 2$ \\
\hline CDH1|999 & B2 \\
\hline CDH22|64405 & $\mathrm{B} 2$ \\
\hline CDH24|64403 & $\mathrm{B} 2$ \\
\hline CDH26|60437 & $\mathrm{B} 2$ \\
\hline CDH2|1000 & $\mathrm{B} 2$ \\
\hline CDH3|1001 & $\mathrm{B} 2$ \\
\hline CDH6|1004 & $\mathrm{B} 2$ \\
\hline CDH7|1005 & $\mathrm{B} 2$ \\
\hline CDH8|1006 & B2 \\
\hline CDH9|1007 & $\mathrm{B} 2$ \\
\hline CDHR1|92211 & $\mathrm{B} 2$ \\
\hline CDHR2|54825 & $\mathrm{B} 2$ \\
\hline CDHR3|222256 & $\mathrm{B} 2$ \\
\hline CDHR4|389118 & $\mathrm{B} 2$ \\
\hline CDHR5|53841 & B2 \\
\hline CDIPT|10423 & B2 \\
\hline
\end{tabular}




\begin{tabular}{|c|c|}
\hline CDK11A|728642 & B2 \\
\hline CDK11B|984 & $\mathrm{B} 2$ \\
\hline CDK12|51755 & B2 \\
\hline CDK13|8621 & B2 \\
\hline CDK14|5218 & B2 \\
\hline CDK15|65061 & $\mathrm{B} 2$ \\
\hline CDK17|5128 & B2 \\
\hline CDK18|5129 & B2 \\
\hline CDK19|23097 & B2 \\
\hline CDK20|23552 & $\mathrm{B} 2$ \\
\hline CDK2AP1|8099 & B2 \\
\hline CDK5R1|8851 & B2 \\
\hline CDK5R2|8941 & $\mathrm{B} 2$ \\
\hline CDK5RAP1|51654 & B2 \\
\hline CDK5RAP2|55755 & B2 \\
\hline CDK5RAP3|80279 & $\mathrm{B} 2$ \\
\hline CDK8|1024 & $\mathrm{B} 2$ \\
\hline CDK9|1025 & B2 \\
\hline CDKAL1|54901 & $\mathrm{B} 2$ \\
\hline CDKL1 $\mid 8814$ & $\mathrm{~B} 2$ \\
\hline CDKL3|51265 & $\mathrm{B} 2$ \\
\hline CDKL4|344387 & $\mathrm{B} 2$ \\
\hline CDKL5|6792 & $\mathrm{B} 2$ \\
\hline CDKN1A|1026 & $\mathrm{B} 2$ \\
\hline CDKN1B|1027 & $\mathrm{B} 2$ \\
\hline CDKN2AIPNL|91368 & $\mathrm{B} 2$ \\
\hline CDKN2A|1029 & $\mathrm{B} 2$ \\
\hline CDKN2B|1030 & $\mathrm{B} 2$ \\
\hline CDON|50937 & B2 \\
\hline CDR1|1038 & $\mathrm{B} 2$ \\
\hline CDRT15P|94158 & $\mathrm{B} 2$ \\
\hline CDRT15|146822 & $\mathrm{B} 2$ \\
\hline CDRT1|374286 & B2 \\
\hline CDS2 $\mid 8760$ & $\mathrm{~B} 2$ \\
\hline CDSN|1041 & $\mathrm{B} 2$ \\
\hline CDV3|55573 & B2 \\
\hline CDX1|1044 & $\mathrm{B} 2$ \\
\hline CDX2|1045 & $\mathrm{B} 2$ \\
\hline CDYL2|124359 & $\mathrm{B} 2$ \\
\hline CDYL $\mid 9425$ & $\mathrm{~B} 2$ \\
\hline CEACAM16|388551 & $\mathrm{B} 2$ \\
\hline CEACAM19|56971 & $\mathrm{B} 2$ \\
\hline CEACAM1|634 & $\mathrm{B} 2$ \\
\hline CEACAM20|125931 & $\mathrm{B} 2$ \\
\hline CEACAM21|90273 & $\mathrm{B} 2$ \\
\hline CEACAM22P|388550 & $\mathrm{B} 2$ \\
\hline CEACAM3|1084 & $\mathrm{B} 2$ \\
\hline CEACAM4|1089 & B2 \\
\hline CEACAM5|1048 & $\mathrm{B} 2$ \\
\hline CEACAM6|4680 & $\mathrm{B} 2$ \\
\hline CEACAM7|1087 & B2 \\
\hline
\end{tabular}




\begin{tabular}{|c|c|}
\hline CEACAM8|1088 & B2 \\
\hline CEBPB $\mid 1051$ & $\mathrm{~B} 2$ \\
\hline CEBPE|1053 & $\mathrm{B} 2$ \\
\hline CEBPG|1054 & $\mathrm{B} 2$ \\
\hline CEBPZ|10153 & $\mathrm{B} 2$ \\
\hline CECR1|51816 & B2 \\
\hline CECR2|27443 & $\mathrm{B} 2$ \\
\hline CECR4|100130717 & $\mathrm{B} 2$ \\
\hline CECR5|27440 & $\mathrm{B} 2$ \\
\hline \begin{tabular}{l|l|} 
CECR6|27439 \\
\end{tabular} & $\mathrm{B} 2$ \\
\hline CELA1|1990 & $\mathrm{B} 2$ \\
\hline CELA2B|51032 & $\mathrm{B} 2$ \\
\hline CELA3B|23436 & $\mathrm{B} 2$ \\
\hline CELF1|10658 & $\mathrm{B} 2$ \\
\hline CELF3|11189 & $\mathrm{B} 2$ \\
\hline CELF4|56853 & $\mathrm{B} 2$ \\
\hline CELF5|60680 & $\mathrm{B} 2$ \\
\hline CELP|1057 & $\mathrm{B} 2$ \\
\hline CELSR2|1952 & $\mathrm{B} 2$ \\
\hline CEL|1056 & $\mathrm{B} 2$ \\
\hline CEMP1|752014 & $\mathrm{B} 2$ \\
\hline CEND1|51286 & $\mathrm{B} 2$ \\
\hline CENPBD1|92806 & $\mathrm{B} 2$ \\
\hline CENPB|1059 & $\mathrm{B} 2$ \\
\hline CENPJ|55835 & $\mathrm{B} 2$ \\
\hline CENPQ|55166 & $\mathrm{B} 2$ \\
\hline CENPV|201161 & $\mathrm{B} 2$ \\
\hline CENPW|387103 & $\mathrm{B} 2$ \\
\hline CEP110|11064 & $\mathrm{B} 2$ \\
\hline CEP135|9662 & $\mathrm{B} 2$ \\
\hline CEP152|22995 & $\mathrm{B} 2$ \\
\hline CEP170L|645455 & $\mathrm{B} 2$ \\
\hline CEP170|9859 & $\mathrm{B} 2$ \\
\hline CEP192|55125 & $\mathrm{B} 2$ \\
\hline CEP250|11190 & $\mathrm{B} 2$ \\
\hline CEP290|80184 & $\mathrm{B} 2$ \\
\hline CEP63|80254 & $\mathrm{B} 2$ \\
\hline CEP72|55722 & $\mathrm{B} 2$ \\
\hline CEP76|79959 & $\mathrm{B} 2$ \\
\hline CEPT1|10390 & $\mathrm{B} 2$ \\
\hline CER1|9350 & $\mathrm{B} 2$ \\
\hline CERK|64781 & $\mathrm{B} 2$ \\
\hline CES3|23491 & $\mathrm{B} 2$ \\
\hline CES7|221223 & $\mathrm{B} 2$ \\
\hline CES8|283848 & $\mathrm{B} 2$ \\
\hline CETN3|1070 & $\mathrm{B} 2$ \\
\hline CETP|1071 & $\mathrm{B} 2$ \\
\hline CFC1B|653275 & $\mathrm{B} 2$ \\
\hline CFDP1|10428 & $\mathrm{B} 2$ \\
\hline CFHR1|3078 & $\mathrm{B} 2$ \\
\hline \begin{tabular}{l|l|l|} 
CFHR3 10878 \\
\end{tabular} & B2 \\
\hline
\end{tabular}




\begin{tabular}{|c|c|}
\hline CFHR4|10877 & B2 \\
\hline $\begin{array}{l}\text { CFHR5|81494 } \\
\end{array}$ & B2 \\
\hline CFL2|1073 & $\mathrm{B} 2$ \\
\hline CFP|5199 & $\mathrm{B} 2$ \\
\hline CFTR $\mid 1080$ & $\mathrm{~B} 2$ \\
\hline CGA|1081 & $\mathrm{B} 2$ \\
\hline CGB1|114335 & $\mathrm{B} 2$ \\
\hline CGB2|114336 & $\mathrm{B} 2$ \\
\hline CGB5|93659 & $\mathrm{B} 2$ \\
\hline CGB8|94115 & $\mathrm{B} 2$ \\
\hline CGB|1082 & $\mathrm{B} 2$ \\
\hline CGGBP1|8545 & $\mathrm{B} 2$ \\
\hline CGREF1|10669 & $\mathrm{B} 2$ \\
\hline CHAC1|79094 & $\mathrm{B} 2$ \\
\hline CHADL $\mid 150356$ & B2 \\
\hline CHAD|1101 & $\mathrm{B} 2$ \\
\hline CHCHD10|400916 & $\mathrm{B} 2$ \\
\hline CHCHD2|51142 & $\mathrm{B} 2$ \\
\hline \begin{tabular}{ll|l|} 
CHCHD3|54927 \\
\end{tabular} & $\mathrm{B} 2$ \\
\hline CHCHD4|131474 & $\mathrm{B} 2$ \\
\hline CHCHD6|84303 & $\mathrm{B} 2$ \\
\hline CHCHD7|79145 & B2 \\
\hline CHD1L|9557 & $\mathrm{B} 2$ \\
\hline CHD1|1105 & $\mathrm{B} 2$ \\
\hline CHD3|1107 & $\mathrm{B} 2$ \\
\hline CHD5|26038 & $\mathrm{B} 2$ \\
\hline CHD6|84181 & $\mathrm{B} 2$ \\
\hline CHD7|55636 & $\mathrm{B} 2$ \\
\hline CHD8|57680 & $\mathrm{B} 2$ \\
\hline CHDH|55349 & $\mathrm{B} 2$ \\
\hline CHERP|10523 & $\mathrm{B} 2$ \\
\hline CHGA|1113 & $\mathrm{B} 2$ \\
\hline CHGB|1114 & $\mathrm{B} 2$ \\
\hline CHI3L1|1116 & $\mathrm{B} 2$ \\
\hline CHI3L2|1117 & $\mathrm{B} 2$ \\
\hline CHIA|27159 & $\mathrm{B} 2$ \\
\hline CHIC1|53344 & $\mathrm{B} 2$ \\
\hline CHID1|66005 & $\mathrm{B} 2$ \\
\hline CHIT1|1118 & $\mathrm{B} 2$ \\
\hline CHKA|1119 & $\mathrm{B} 2$ \\
\hline CHKB $\mid 1120$ & $\mathrm{~B} 2$ \\
\hline CHMP1A|5119 & $\mathrm{B} 2$ \\
\hline CHMP1B|57132 & $\mathrm{B} 2$ \\
\hline CHMP2A|27243 & $\mathrm{B} 2$ \\
\hline CHMP2B 25978 & $\mathrm{~B} 2$ \\
\hline CHMP4A|29082 & $\mathrm{B} 2$ \\
\hline CHMP4C $\mid 92421$ & $\mathrm{~B} 2$ \\
\hline CHMP6|79643 & $\mathrm{B} 2$ \\
\hline CHMP7|91782 & $\mathrm{B} 2$ \\
\hline CHM|1121 & $\mathrm{B} 2$ \\
\hline CHN1|1123 & $\mathrm{B} 2$ \\
\hline
\end{tabular}




\begin{tabular}{|c|c|}
\hline CHN2|1124 & B2 \\
\hline CHODL|140578 & B2 \\
\hline CHP2|63928 & B2 \\
\hline CHPT1|56994 & B2 \\
\hline CHP $\mid 11261$ & B2 \\
\hline CHRDL2|25884 & $\mathrm{B} 2$ \\
\hline CHRD|8646 & B2 \\
\hline CHRM1|1128 & $\mathrm{B} 2$ \\
\hline CHRM2|1129 & B2 \\
\hline CHRM3|1131 & B2 \\
\hline CHRM4|1132 & B2 \\
\hline CHRM5|1133 & B2 \\
\hline CHRNA10|57053 & $\mathrm{B} 2$ \\
\hline CHRNA1|1134 & B2 \\
\hline CHRNA2|1135 & $\mathrm{B} 2$ \\
\hline CHRNA3|1136 & B2 \\
\hline CHRNA4|1137 & $\mathrm{B} 2$ \\
\hline CHRNA5|1138 & B2 \\
\hline \begin{tabular}{l|l|} 
CHRNA95584 \\
\end{tabular} & $\mathrm{B} 2$ \\
\hline CHRNB1|1140 & $\mathrm{B} 2$ \\
\hline CHRNB2|1141 & B2 \\
\hline CHRNB3|1142 & $\mathrm{B} 2$ \\
\hline CHRNB4|1143 & $\mathrm{B} 2$ \\
\hline CHRND|1144 & B2 \\
\hline CHRNE|1145 & B2 \\
\hline CHRNG|1146 & $\mathrm{B} 2$ \\
\hline CHST10|9486 & $\mathrm{B} 2$ \\
\hline CHST12|55501 & $\mathrm{B} 2$ \\
\hline CHST13|166012 & B2 \\
\hline CHST14|113189 & $\mathrm{B} 2$ \\
\hline CHST1|8534 & $\mathrm{B} 2$ \\
\hline CHST4|10164 & $\mathrm{B} 2$ \\
\hline \begin{tabular}{l|l|} 
CHST5|23563 \\
\end{tabular} & $\mathrm{B} 2$ \\
\hline CHST8|64377 & $\mathrm{B} 2$ \\
\hline CHSY1|22856 & $\mathrm{B} 2$ \\
\hline CHTF8|54921 & B2 \\
\hline CHUK|1147 & $\mathrm{B} 2$ \\
\hline CIAO1|9391 & $\mathrm{B} 2$ \\
\hline CIAPIN1|57019 & $\mathrm{B} 2$ \\
\hline CIB1|10519 & $\mathrm{B} 2$ \\
\hline CIB2|10518 & $\mathrm{B} 2$ \\
\hline CIB4|130106 & $\mathrm{B} 2$ \\
\hline CIC|23152 & $\mathrm{B} 2$ \\
\hline CIDEB|27141 & B2 \\
\hline CIDECP $\mid 152302$ & $\mathrm{~B} 2$ \\
\hline CIITA|4261 & $\mathrm{B} 2$ \\
\hline CILP $\mid 8483$ & B2 \\
\hline CINP|51550 & B2 \\
\hline CIR1|9541 & B2 \\
\hline CIRH1A|84916 & B2 \\
\hline CISD1|55847 & B2 \\
\hline
\end{tabular}




\begin{tabular}{|c|c|}
\hline CISD3|284106 & B2 \\
\hline CITED1|4435 & $\mathrm{B} 2$ \\
\hline CITED4|163732 & $\mathrm{B} 2$ \\
\hline CIZ1|25792 & $\mathrm{B} 2$ \\
\hline CKB|1152 & $\mathrm{B} 2$ \\
\hline CKMT1A|548596 & $\mathrm{B} 2$ \\
\hline CKMT1B|1159 & $\mathrm{B} 2$ \\
\hline CKMT2|1160 & $\mathrm{B} 2$ \\
\hline CKM|1158 & $\mathrm{B} 2$ \\
\hline CLASP2|23122 & $\mathrm{B} 2$ \\
\hline CLCA1|1179 & $\mathrm{B} 2$ \\
\hline CLCA2 $\mid 9635$ & $\mathrm{~B} 2$ \\
\hline CLCA3P|9629 & $\mathrm{B} 2$ \\
\hline CLCC1|23155 & $\mathrm{B} 2$ \\
\hline CLCF1|23529 & B2 \\
\hline CLCN1|1180 & $\mathrm{B} 2$ \\
\hline CLCN3|1182 & $\mathrm{B} 2$ \\
\hline CLCN4|1183 & $\mathrm{B} 2$ \\
\hline CLCN5|1184 & $\mathrm{B} 2$ \\
\hline CLCNKA|1187 & $\mathrm{B} 2$ \\
\hline CLC|1178 & $\mathrm{B} 2$ \\
\hline CLDN10|9071 & B2 \\
\hline CLDN12|9069 & $\mathrm{B} 2$ \\
\hline CLDN16|10686 & $\mathrm{B} 2$ \\
\hline CLDN17|26285 & $\mathrm{B} 2$ \\
\hline CLDN1|9076 & $\mathrm{B} 2$ \\
\hline CLDN20|49861 & $\mathrm{B} 2$ \\
\hline CLDN23|137075 & $\mathrm{B} 2$ \\
\hline CLDN25|644672 & $\mathrm{B} 2$ \\
\hline CLDN2|9075 & $\mathrm{B} 2$ \\
\hline CLDN3|1365 & $\mathrm{B} 2$ \\
\hline CLDN4|1364 & $\mathrm{B} 2$ \\
\hline CLDN6|9074 & $\mathrm{B} 2$ \\
\hline CLDN8|9073 & $\mathrm{B} 2$ \\
\hline CLDN9 90080 & $\mathrm{~B} 2$ \\
\hline CLDND1|56650 & $\mathrm{B} 2$ \\
\hline CLEC10A|10462 & $\mathrm{B} 2$ \\
\hline CLEC12A|160364 & $\mathrm{B} 2$ \\
\hline CLEC12B|387837 & $\mathrm{B} 2$ \\
\hline CLEC16A|23274 & $\mathrm{B} 2$ \\
\hline CLEC17A|388512 & $\mathrm{B} 2$ \\
\hline CLEC18A|348174 & $\mathrm{B} 2$ \\
\hline CLEC18B|497190 & $\mathrm{B} 2$ \\
\hline CLEC18C|283971 & $\mathrm{B} 2$ \\
\hline CLEC1B|51266 & $\mathrm{B} 2$ \\
\hline CLEC2A|387836 & $\mathrm{B} 2$ \\
\hline CLEC2B|9976 & $\mathrm{B} 2$ \\
\hline CLEC2D|29121 & $\mathrm{B} 2$ \\
\hline CLEC3A|10143 & $\mathrm{B} 2$ \\
\hline CLEC4A|50856 & $\mathrm{B} 2$ \\
\hline CLEC4C|170482 & $\mathrm{B} 2$ \\
\hline
\end{tabular}




\begin{tabular}{|c|c|}
\hline CLEC4D|338339 & B2 \\
\hline CLEC4E|26253 & B2 \\
\hline CLEC4M|10332 & $\mathrm{B} 2$ \\
\hline CLEC6A|93978 & $\mathrm{B} 2$ \\
\hline CLEC7A|64581 & $\mathrm{B} 2$ \\
\hline CLEC9A|283420 & $\mathrm{B} 2$ \\
\hline CLECL1|160365 & $\mathrm{B} 2$ \\
\hline CLIC3|9022 & $\mathrm{B} 2$ \\
\hline CLIC4|25932 & $\mathrm{B} 2$ \\
\hline CLIC5|53405 & $\mathrm{B} 2$ \\
\hline CLIC6|54102 & $\mathrm{B} 2$ \\
\hline CLINT1|9685 & $\mathrm{B} 2$ \\
\hline CLIP1|6249 & $\mathrm{B} 2$ \\
\hline CLIP2|7461 & $\mathrm{B} 2$ \\
\hline CLK2P|1197 & $\mathrm{B} 2$ \\
\hline CLK2|1196 & $\mathrm{B} 2$ \\
\hline CLLU1OS|574016 & $\mathrm{B} 2$ \\
\hline CLLU1|574028 & $\mathrm{B} 2$ \\
\hline CLMN|79789 & $\mathrm{B} 2$ \\
\hline CLN5|1203 & $\mathrm{B} 2$ \\
\hline CLNK|116449 & $\mathrm{B} 2$ \\
\hline CLNS1A|1207 & B2 \\
\hline CLOCK $\mid 9575$ & $\mathrm{~B} 2$ \\
\hline CLP1|10978 & $\mathrm{B} 2$ \\
\hline CLPS|1208 & $\mathrm{B} 2$ \\
\hline CLPX|10845 & $\mathrm{B} 2$ \\
\hline CLRN1OS|116933 & $\mathrm{B} 2$ \\
\hline CLRN1|7401 & $\mathrm{B} 2$ \\
\hline CLRN3|119467 & $\mathrm{B} 2$ \\
\hline CLSTN1|22883 & $\mathrm{B} 2$ \\
\hline CLSTN2|64084 & $\mathrm{B} 2$ \\
\hline CLSTN3|9746 & $\mathrm{B} 2$ \\
\hline CLTA|1211 & $\mathrm{B} 2$ \\
\hline CLTB|1212 & $\mathrm{B} 2$ \\
\hline \begin{tabular}{l|l|} 
CLTCL1|8218 \\
\end{tabular} & $\mathrm{B} 2$ \\
\hline CLTC|1213 & $\mathrm{B} 2$ \\
\hline CLUAP1|23059 & $\mathrm{B} 2$ \\
\hline $\begin{array}{l}\text { CLUL1|27098 } \\
\end{array}$ & $\mathrm{B} 2$ \\
\hline CLU|1191 & $\mathrm{B} 2$ \\
\hline CLVS2|134829 & $\mathrm{B} 2$ \\
\hline CMAH|8418 & $\mathrm{B} 2$ \\
\hline CMAS|55907 & $\mathrm{B} 2$ \\
\hline CMBL|134147 & $\mathrm{B} 2$ \\
\hline CMIP|80790 & $\mathrm{B} 2$ \\
\hline CMKLR1|1240 & $\mathrm{B} 2$ \\
\hline CMPK2|129607 & $\mathrm{B} 2$ \\
\hline CMTM1|113540 & $\mathrm{B} 2$ \\
\hline CMTM2|146225 & $\mathrm{B} 2$ \\
\hline CMTM3|123920 & $\mathrm{B} 2$ \\
\hline CMTM4|146223 & $\mathrm{B} 2$ \\
\hline CMTM5|116173 & $\mathrm{B} 2$ \\
\hline
\end{tabular}




\begin{tabular}{|c|c|}
\hline CMTM6|54918 & B2 \\
\hline CMTM8|152189 & B2 \\
\hline CMYA5|202333 & $\mathrm{B} 2$ \\
\hline CN5H6.4|150384 & $\mathrm{B} 2$ \\
\hline CNBD1|168975 & $\mathrm{B} 2$ \\
\hline CNBP|7555 & $\mathrm{B} 2$ \\
\hline CNDP1|84735 & $\mathrm{B} 2$ \\
\hline CNFN|84518 & $\mathrm{B} 2$ \\
\hline CNGA1|1259 & $\mathrm{B} 2$ \\
\hline CNGA2|1260 & $\mathrm{B} 2$ \\
\hline CNGA3|1261 & $\mathrm{B} 2$ \\
\hline CNGA4|1262 & $\mathrm{B} 2$ \\
\hline CNGB1|1258 & $\mathrm{B} 2$ \\
\hline \begin{tabular}{ll|l|} 
CNGB3 54714 \\
\end{tabular} & $\mathrm{~B} 2$ \\
\hline CNIH3|149111 & B2 \\
\hline CNKSR1|10256 & $\mathrm{B} 2$ \\
\hline CNKSR3|154043 & $\mathrm{B} 2$ \\
\hline CNN2|1265 & $\mathrm{B} 2$ \\
\hline CNN3|1266 & $\mathrm{B} 2$ \\
\hline CNNM1|26507 & $\mathrm{B} 2$ \\
\hline CNNM2|54805 & $\mathrm{B} 2$ \\
\hline CNNM3|26505 & $\mathrm{B} 2$ \\
\hline CNOT1|23019 & $\mathrm{B} 2$ \\
\hline CNOT2|4848 & $\mathrm{B} 2$ \\
\hline CNOT4 44850 & $\mathrm{~B} 2$ \\
\hline CNOT7|29883 & $\mathrm{B} 2$ \\
\hline CNOT8|9337 & $\mathrm{B} 2$ \\
\hline CNPY1|285888 & $\mathrm{B} 2$ \\
\hline CNPY4|245812 & $\mathrm{B} 2$ \\
\hline $\begin{array}{l}\mathrm{CNP} \mid 1267 \\
\end{array}$ & $\mathrm{~B} 2$ \\
\hline CNR1|1268 & $\mathrm{B} 2$ \\
\hline CNR2|1269 & $\mathrm{B} 2$ \\
\hline CNST|163882 & $\mathrm{B} 2$ \\
\hline CNTD1|124817 & $\mathrm{B} 2$ \\
\hline CNTD2|79935 & $\mathrm{B} 2$ \\
\hline CNTF|1270 & $\mathrm{B} 2$ \\
\hline CNTLN|54875 & $\mathrm{B} 2$ \\
\hline CNTN1|1272 & $\mathrm{B} 2$ \\
\hline CNTN2|6900 & $\mathrm{B} 2$ \\
\hline CNTN3|5067 & $\mathrm{B} 2$ \\
\hline CNTN4|152330 & $\mathrm{B} 2$ \\
\hline $\begin{array}{l}\text { CNTN5|53942 } \\
\end{array}$ & $\mathrm{B} 2$ \\
\hline CNTN6|27255 & $\mathrm{B} 2$ \\
\hline CNTNAP1|8506 & $\mathrm{B} 2$ \\
\hline CNTNAP2|26047 & $\mathrm{B} 2$ \\
\hline CNTNAP4|85445 & $\mathrm{B} 2$ \\
\hline \begin{tabular}{l|l|} 
CNTNAP5|129684 \\
\end{tabular} & $\mathrm{B} 2$ \\
\hline CNTROB|116840 & $\mathrm{B} 2$ \\
\hline COASY|80347 & $\mathrm{B} 2$ \\
\hline COBL|23242 & $\mathrm{B} 2$ \\
\hline COBRA1|25920 & $\mathrm{B} 2$ \\
\hline
\end{tabular}




\begin{tabular}{|c|c|}
\hline $\mathrm{COCH} \mid 1690$ & B2 \\
\hline COG1|9382 & $\mathrm{B} 2$ \\
\hline COG3|83548 & $\mathrm{B} 2$ \\
\hline COG4|25839 & $\mathrm{B} 2$ \\
\hline COG5|10466 & $\mathrm{B} 2$ \\
\hline COG6|57511 & B2 \\
\hline COG8 $\mid 84342$ & $\mathrm{~B} 2$ \\
\hline COL11A2|1302 & $\mathrm{B} 2$ \\
\hline COL13A1|1305 & $\mathrm{B} 2$ \\
\hline COL16A1|1307 & $\mathrm{B} 2$ \\
\hline COL18A1|80781 & $\mathrm{B} 2$ \\
\hline COL19A1|1310 & $\mathrm{B} 2$ \\
\hline COL20A1|57642 & $\mathrm{B} 2$ \\
\hline COL21A1|81578 & $\mathrm{B} 2$ \\
\hline COL22A1|169044 & $\mathrm{B} 2$ \\
\hline COL27A1|85301 & $\mathrm{B} 2$ \\
\hline COL28A1|340267 & $\mathrm{B} 2$ \\
\hline COL29A1|256076 & $\mathrm{B} 2$ \\
\hline COL2A1|1280 & $\mathrm{B} 2$ \\
\hline COL4A1|1282 & $\mathrm{B} 2$ \\
\hline COL4A2|1284 & $\mathrm{B} 2$ \\
\hline COL4A3|1285 & $\mathrm{B} 2$ \\
\hline COL4A4|1286 & $\mathrm{B} 2$ \\
\hline COL4A5|1287 & $\mathrm{B} 2$ \\
\hline COL4A6|1288 & $\mathrm{B} 2$ \\
\hline COL5A3|50509 & $\mathrm{B} 2$ \\
\hline COL6A1|1291 & $\mathrm{B} 2$ \\
\hline COL6A2|1292 & $\mathrm{B} 2$ \\
\hline COL6A4P2|646300 & $\mathrm{B} 2$ \\
\hline COL7A1|1294 & $\mathrm{B} 2$ \\
\hline COL8A2|1296 & $\mathrm{B} 2$ \\
\hline COL9A1|1297 & $\mathrm{B} 2$ \\
\hline COL9A2|1298 & $\mathrm{B} 2$ \\
\hline COL9A3|1299 & $\mathrm{B} 2$ \\
\hline COLEC10|10584 & $\mathrm{B} 2$ \\
\hline COLEC12|81035 & $\mathrm{B} 2$ \\
\hline COLQ|8292 & $\mathrm{B} 2$ \\
\hline COMMD1|150684 & $\mathrm{B} 2$ \\
\hline COMMD2|51122 & $\mathrm{B} 2$ \\
\hline COMMD8|54951 & $\mathrm{B} 2$ \\
\hline COMMD9|29099 & $\mathrm{B} 2$ \\
\hline COMT|1312 & $\mathrm{B} 2$ \\
\hline COPG2|26958 & $\mathrm{B} 2$ \\
\hline COPS3|8533 & $\mathrm{B} 2$ \\
\hline COPS4|51138 & $\mathrm{B} 2$ \\
\hline COPS6|10980 & $\mathrm{B} 2$ \\
\hline COPS7A|50813 & $\mathrm{B} 2$ \\
\hline COPS7B|64708 & $\mathrm{B} 2$ \\
\hline COPZ2|51226 & $\mathrm{B} 2$ \\
\hline COQ10A|93058 & $\mathrm{B} 2$ \\
\hline COQ10B|80219 & B2 \\
\hline
\end{tabular}




\begin{tabular}{|c|c|}
\hline COQ2|27235 & B2 \\
\hline COQ3|51805 & $\mathrm{B} 2$ \\
\hline COQ4|51117 & B2 \\
\hline COQ5|84274 & $\mathrm{B} 2$ \\
\hline COQ6551004 & B2 \\
\hline COQ7|10229 & $\mathrm{B} 2$ \\
\hline COQ9|57017 & B2 \\
\hline CORO1A|11151 & $\mathrm{B} 2$ \\
\hline CORO6|84940 & B2 \\
\hline CORT|1325 & B2 \\
\hline COTL1|23406 & B2 \\
\hline COX10|1352 & $\mathrm{B} 2$ \\
\hline COX11|1353 & $\mathrm{B} 2$ \\
\hline COX15|1355 & B2 \\
\hline COX18|285521 & B2 \\
\hline COX19|90639 & B2 \\
\hline COX4I1|1327 & $\mathrm{B} 2$ \\
\hline COX4NB|10328 & $\mathrm{B} 2$ \\
\hline COX5A|9377 & $\mathrm{B} 2$ \\
\hline COX5B|1329 & $\mathrm{B} 2$ \\
\hline COX6A2|1339 & $\mathrm{B} 2$ \\
\hline COX6B1|1340 & $\mathrm{B} 2$ \\
\hline COX6B2|125965 & $\mathrm{B} 2$ \\
\hline COX7A1|1346 & $\mathrm{B} 2$ \\
\hline COX7A2L|9167 & $\mathrm{B} 2$ \\
\hline COX7B2|170712 & $\mathrm{B} 2$ \\
\hline COX7B|1349 & $\mathrm{B} 2$ \\
\hline COX7C|1350 & $\mathrm{B} 2$ \\
\hline COX8C|341947 & B2 \\
\hline CP110|9738 & $\mathrm{B} 2$ \\
\hline CPA2|1358 & $\mathrm{B} 2$ \\
\hline CPA3|1359 & $\mathrm{B} 2$ \\
\hline CPA4|51200 & $\mathrm{B} 2$ \\
\hline CPA5|93979 & $\mathrm{B} 2$ \\
\hline CPA6|57094 & $\mathrm{B} 2$ \\
\hline CPB1|1360 & $\mathrm{B} 2$ \\
\hline CPB2|1361 & B2 \\
\hline CPD|1362 & $\mathrm{B} 2$ \\
\hline CPEB2|132864 & $\mathrm{B} 2$ \\
\hline CPEB3|22849 & $\mathrm{B} 2$ \\
\hline CPEB4|80315 & B2 \\
\hline CPLX1|10815 & $\mathrm{B} 2$ \\
\hline CPLX2|10814 & $\mathrm{B} 2$ \\
\hline CPLX3|594855 & $\mathrm{B} 2$ \\
\hline CPLX4|339302 & $\mathrm{B} 2$ \\
\hline CPN1|1369 & $\mathrm{B} 2$ \\
\hline CPN2|1370 & B2 \\
\hline CPNE1|8904 & B2 \\
\hline CPNE4|131034 & B2 \\
\hline \begin{tabular}{l|l|} 
CPNE5 57699 \\
\end{tabular} & B2 \\
\hline CPNE6|9362 & $\mathrm{B} 2$ \\
\hline
\end{tabular}




\begin{tabular}{|c|c|}
\hline CPNE7|27132 & B2 \\
\hline CPNE9|151835 & B2 \\
\hline CPOX|1371 & $\mathrm{B} 2$ \\
\hline CPO|130749 & $\mathrm{B} 2$ \\
\hline CPPED1|55313 & B2 \\
\hline CPSF1|29894 & $\mathrm{B} 2$ \\
\hline CPSF3L|54973 & $\mathrm{B} 2$ \\
\hline CPSF4L|642843 & $\mathrm{B} 2$ \\
\hline CPSF4|10898 & B2 \\
\hline CPSF7|79869 & $\mathrm{B} 2$ \\
\hline CPT1A|1374 & $\mathrm{B} 2$ \\
\hline CPT1B|1375 & $\mathrm{B} 2$ \\
\hline CPT1C|126129 & $\mathrm{B} 2$ \\
\hline CPT2|1376 & $\mathrm{B} 2$ \\
\hline CPXCR1|53336 & $\mathrm{B} 2$ \\
\hline CPXM1|56265 & $\mathrm{B} 2$ \\
\hline CPZ|8532 & B2 \\
\hline $\mathrm{CP} \mid 1356$ & $\mathrm{~B} 2$ \\
\hline CR1L|1379 & $\mathrm{B} 2$ \\
\hline CR2|1380 & $\mathrm{B} 2$ \\
\hline CRABP1|1381 & $\mathrm{B} 2$ \\
\hline CRAMP1L|57585 & $\mathrm{B} 2$ \\
\hline CRAT|1384 & $\mathrm{B} 2$ \\
\hline CRB1|23418 & $\mathrm{B} 2$ \\
\hline \begin{tabular}{l|l|} 
CRCT1|54544 \\
\end{tabular} & $\mathrm{B} 2$ \\
\hline CREB1|1385 & $\mathrm{B} 2$ \\
\hline CREB3L2|64764 & $\mathrm{B} 2$ \\
\hline CREB3L3|84699 & $\mathrm{B} 2$ \\
\hline \begin{tabular}{l|l|l|} 
CREB3 & 10488 \\
\end{tabular} & $\mathrm{~B} 2$ \\
\hline CREBBP|1387 & $\mathrm{B} 2$ \\
\hline CREBZF|58487 & $\mathrm{B} 2$ \\
\hline CREG1|8804 & $\mathrm{B} 2$ \\
\hline CREG2|200407 & $\mathrm{B} 2$ \\
\hline CREM|1390 & $\mathrm{B} 2$ \\
\hline CRHR1|1394 & $\mathrm{B} 2$ \\
\hline CRH|1392 & $\mathrm{B} 2$ \\
\hline CRIP2|1397 & $\mathrm{B} 2$ \\
\hline CRIP3|401262 & $\mathrm{B} 2$ \\
\hline CRIPAK|285464 & $\mathrm{B} 2$ \\
\hline CRIPT|9419 & $\mathrm{B} 2$ \\
\hline CRISP1|167 & $\mathrm{B} 2$ \\
\hline CRISP2|7180 & $\mathrm{B} 2$ \\
\hline CRISP3|10321 & $\mathrm{B} 2$ \\
\hline CRISPLD1|83690 & $\mathrm{B} 2$ \\
\hline CRISPLD2|83716 & $\mathrm{B} 2$ \\
\hline CRKL|1399 & $\mathrm{B} 2$ \\
\hline CRLF1|9244 & $\mathrm{B} 2$ \\
\hline CRLF2|64109 & $\mathrm{B} 2$ \\
\hline CRLF3|51379 & $\mathrm{B} 2$ \\
\hline CRLS1|54675 & $\mathrm{B} 2$ \\
\hline CRMP1|1400 & $\mathrm{B} 2$ \\
\hline
\end{tabular}




\begin{tabular}{|c|c|}
\hline CRNN|49860 & B2 \\
\hline CROCC 9696 & $\mathrm{~B} 2$ \\
\hline CROT|54677 & $\mathrm{B} 2$ \\
\hline CRP|1401 & $\mathrm{B} 2$ \\
\hline CRTAM|56253 & $\mathrm{B} 2$ \\
\hline CRTC1|23373 & $\mathrm{B} 2$ \\
\hline \begin{tabular}{l|l|l|} 
CRTC3|64784 \\
\end{tabular} & $\mathrm{B} 2$ \\
\hline CRX|1406 & $\mathrm{B} 2$ \\
\hline CRY1|1407 & $\mathrm{B} 2$ \\
\hline CRYAA|1409 & $\mathrm{B} 2$ \\
\hline CRYBA1|1411 & $\mathrm{B} 2$ \\
\hline CRYBA2|1412 & $\mathrm{B} 2$ \\
\hline CRYBA4|1413 & $\mathrm{B} 2$ \\
\hline CRYBB1|1414 & $\mathrm{B} 2$ \\
\hline CRYBB2|1415 & $\mathrm{B} 2$ \\
\hline CRYBB3|1417 & $\mathrm{B} 2$ \\
\hline CRYGC|1420 & $\mathrm{B} 2$ \\
\hline \begin{tabular}{l|l|l|} 
CRYGD|1421 \\
\end{tabular} & $\mathrm{B} 2$ \\
\hline CRYGN|155051 & $\mathrm{B} 2$ \\
\hline CRYM|1428 & $\mathrm{B} 2$ \\
\hline CRYZ|1429 & $\mathrm{B} 2$ \\
\hline CSAG1|158511 & $\mathrm{B} 2$ \\
\hline CSAG2|728461 & $\mathrm{B} 2$ \\
\hline CSAG3|389903 & $\mathrm{B} 2$ \\
\hline \begin{tabular}{l|l|} 
CSDC2 27254 \\
\end{tabular} & $\mathrm{~B} 2$ \\
\hline CSF1R|1436 & $\mathrm{B} 2$ \\
\hline CSF2RA|1438 & $\mathrm{B} 2$ \\
\hline CSF2RB|1439 & $\mathrm{B} 2$ \\
\hline CSF2|1437 & $\mathrm{B} 2$ \\
\hline CSF3R/1441 & $\mathrm{B} 2$ \\
\hline CSF3|1440 & $\mathrm{B} 2$ \\
\hline CSGALNACT2|55454 & $\mathrm{B} 2$ \\
\hline CSH2|1443 & $\mathrm{B} 2$ \\
\hline CSMD1|64478 & $\mathrm{B} 2$ \\
\hline CSMD3|114788 & $\mathrm{B} 2$ \\
\hline CSN1S1|1446 & $\mathrm{B} 2$ \\
\hline CSN1S2A|286828 & $\mathrm{B} 2$ \\
\hline CSN2|1447 & $\mathrm{B} 2$ \\
\hline \begin{tabular}{ll|l|l|} 
CSN3|148 \\
\end{tabular} & $\mathrm{B} 2$ \\
\hline CSNK1A1L|122011 & $\mathrm{B} 2$ \\
\hline CSNK1A1P|161635 & $\mathrm{B} 2$ \\
\hline CSNK1A1|1452 & $\mathrm{B} 2$ \\
\hline CSNK1D|1453 & $\mathrm{B} 2$ \\
\hline CSNK1E|1454 & $\mathrm{B} 2$ \\
\hline CSNK1G1|53944 & $\mathrm{B} 2$ \\
\hline CSNK1G2|1455 & $\mathrm{B} 2$ \\
\hline CSNK1G3|1456 & $\mathrm{B} 2$ \\
\hline CSNK2A1P|283106 & $\mathrm{B} 2$ \\
\hline CSNK2A1|1457 & $\mathrm{B} 2$ \\
\hline CSPG4|1464 & $\mathrm{B} 2$ \\
\hline \begin{tabular}{l|l|l|} 
CSPG5 & 10675 \\
\end{tabular} & $\mathrm{~B} 2$ \\
\hline
\end{tabular}




\begin{tabular}{|c|c|}
\hline CSPP1|79848 & $\mathrm{B} 2$ \\
\hline CSRP2BP|57325 & $\mathrm{B} 2$ \\
\hline CSRP3|8048 & $\mathrm{B} 2$ \\
\hline CST11|140880 & $\mathrm{B} 2$ \\
\hline CST3|1471 & $\mathrm{B} 2$ \\
\hline CST4|1472 & B2 \\
\hline CST5|1473 & $\mathrm{B} 2$ \\
\hline CST6|1474 & $\mathrm{B} 2$ \\
\hline CST7|8530 & $\mathrm{B} 2$ \\
\hline CST9L|128821 & $\mathrm{B} 2$ \\
\hline CST9|128822 & $\mathrm{B} 2$ \\
\hline CSTA|1475 & $\mathrm{B} 2$ \\
\hline CSTB|1476 & $\mathrm{B} 2$ \\
\hline CSTF2T|23283 & $\mathrm{B} 2$ \\
\hline CSTL1|128817 & $\mathrm{B} 2$ \\
\hline CSTT|164380 & $\mathrm{B} 2$ \\
\hline $\mathrm{CS} \mid 1431$ & $\mathrm{~B} 2$ \\
\hline CT45A1|541466 & $\mathrm{B} 2$ \\
\hline CT45A2|728911 & $\mathrm{B} 2$ \\
\hline CT45A4|441520 & $\mathrm{B} 2$ \\
\hline CT45A5|441521 & $\mathrm{B} 2$ \\
\hline CT62|196993 & $\mathrm{B} 2$ \\
\hline CTAG1B|1485 & $\mathrm{B} 2$ \\
\hline \begin{tabular}{ll|} 
CTAG2|30848 \\
\end{tabular} & B2 \\
\hline CTAGE1|64693 & $\mathrm{B} 2$ \\
\hline CTAGE4|100128553 & $\mathrm{B} 2$ \\
\hline CTAGE6|340307 & $\mathrm{B} 2$ \\
\hline CTAGE9|643854 & $\mathrm{B} 2$ \\
\hline CTBP1|1487 & $\mathrm{B} 2$ \\
\hline CTBP2|1488 & $\mathrm{B} 2$ \\
\hline CTBS|1486 & $\mathrm{B} 2$ \\
\hline CTCFL|140690 & $\mathrm{B} 2$ \\
\hline CTCF|10664 & $\mathrm{B} 2$ \\
\hline CTDP1|9150 & $\mathrm{B} 2$ \\
\hline CTDSPL2|51496 & $\mathrm{B} 2$ \\
\hline CTGF|1490 & $\mathrm{B} 2$ \\
\hline CTH|1491 & $\mathrm{B} 2$ \\
\hline CTLA4|1493 & $\mathrm{B} 2$ \\
\hline CTNNA1|1495 & $\mathrm{B} 2$ \\
\hline CTNNA2|1496 & $\mathrm{B} 2$ \\
\hline CTNNA3|29119 & $\mathrm{B} 2$ \\
\hline CTNNB1|1499 & $\mathrm{B} 2$ \\
\hline CTNNBIP1|56998 & $\mathrm{B} 2$ \\
\hline CTNNBL1|56259 & $\mathrm{B} 2$ \\
\hline CTNND1|1500 & $\mathrm{B} 2$ \\
\hline CTNND2|1501 & $\mathrm{B} 2$ \\
\hline CTNS|1497 & $\mathrm{B} 2$ \\
\hline CTR9|9646 & $\mathrm{B} 2$ \\
\hline CTRB1|1504 & $\mathrm{B} 2$ \\
\hline CTRB2|440387 & $\mathrm{B} 2$ \\
\hline CTRC|11330 & $\mathrm{B} 2$ \\
\hline
\end{tabular}




\begin{tabular}{|c|c|}
\hline CTRL|1506 & $\mathrm{B} 2$ \\
\hline CTSA|5476 & $\mathrm{B} 2$ \\
\hline CTSB|1508 & $\mathrm{B} 2$ \\
\hline CTSC|1075 & $\mathrm{B} 2$ \\
\hline CTSD|1509 & $\mathrm{B} 2$ \\
\hline CTSE|1510 & $\mathrm{B} 2$ \\
\hline CTSF|8722 & $\mathrm{B} 2$ \\
\hline CTSH|1512 & $\mathrm{B} 2$ \\
\hline CTSK|1513 & $\mathrm{B} 2$ \\
\hline CTSL1|1514 & $\mathrm{B} 2$ \\
\hline CTSL2|1515 & $\mathrm{B} 2$ \\
\hline CTSO|1519 & $\mathrm{B} 2$ \\
\hline CTSS|1520 & $\mathrm{B} 2$ \\
\hline CTSW|1521 & $\mathrm{B} 2$ \\
\hline CTSZ|1522 & $\mathrm{B} 2$ \\
\hline CTTNBP2NL|55917 & $\mathrm{B} 2$ \\
\hline CTTN|2017 & $\mathrm{B} 2$ \\
\hline CTU1|90353 & $\mathrm{B} 2$ \\
\hline CTU2|348180 & $\mathrm{B} 2$ \\
\hline CTXN2|399697 & $\mathrm{B} 2$ \\
\hline CTXN3|613212 & $\mathrm{B} 2$ \\
\hline CUEDC1|404093 & $\mathrm{B} 2$ \\
\hline CUEDC2|79004 & $\mathrm{B} 2$ \\
\hline CUL1|8454 & $\mathrm{B} 2$ \\
\hline CUL2|8453 & $\mathrm{B} 2$ \\
\hline CUL3|8452 & $\mathrm{B} 2$ \\
\hline CUL4A|8451 & $\mathrm{B} 2$ \\
\hline CUL4B $\mid 8450$ & $\mathrm{~B} 2$ \\
\hline CUL5|8065 & $\mathrm{B} 2$ \\
\hline CUL7|9820 & $\mathrm{B} 2$ \\
\hline CUL9|23113 & $\mathrm{B} 2$ \\
\hline CUTC|51076 & $\mathrm{B} 2$ \\
\hline CUX1|1523 & $\mathrm{B} 2$ \\
\hline CUX2|23316 & $\mathrm{B} 2$ \\
\hline CUZD1|50624 & $\mathrm{B} 2$ \\
\hline CWC15|51503 & $\mathrm{B} 2$ \\
\hline CWC25|54883 & $\mathrm{B} 2$ \\
\hline CWC27|10283 & $\mathrm{B} 2$ \\
\hline CWF19L1|55280 & $\mathrm{B} 2$ \\
\hline CWH43|80157 & $\mathrm{B} 2$ \\
\hline CXADRP2|646243 & $\mathrm{B} 2$ \\
\hline CXADRP3|440224 & $\mathrm{B} 2$ \\
\hline CXADR $\mid 1525$ & $\mathrm{~B} 2$ \\
\hline CXCL10|3627 & $\mathrm{B} 2$ \\
\hline CXCL11|6373 & $\mathrm{B} 2$ \\
\hline CXCL13|10563 & B2 \\
\hline CXCL14|9547 & $\mathrm{B} 2$ \\
\hline CXCL16|58191 & $\mathrm{B} 2$ \\
\hline CXCL17|284340 & $\mathrm{B} 2$ \\
\hline CXCL1|2919 & $\mathrm{B} 2$ \\
\hline CXCL5|6374 & B2 \\
\hline
\end{tabular}




\begin{tabular}{|c|c|}
\hline CXCL6|6372 & B2 \\
\hline CXCL9|4283 & B2 \\
\hline CXCR1|3577 & $\mathrm{B} 2$ \\
\hline CXCR2P1|3580 & B2 \\
\hline CXCR2|3579 & B2 \\
\hline CXCR3|2833 & B2 \\
\hline CXCR4|7852 & B2 \\
\hline CXCR5|643 & $\mathrm{B} 2$ \\
\hline CXCR6|10663 & $\mathrm{B} 2$ \\
\hline CXXC1|30827 & $\mathrm{B} 2$ \\
\hline CXXC4|80319 & $\mathrm{B} 2$ \\
\hline CXorf1|9142 & $\mathrm{B} 2$ \\
\hline CXorf21|80231 & $\mathrm{B} 2$ \\
\hline CXorf22|170063 & $\mathrm{B} 2$ \\
\hline CXorf23|256643 & B2 \\
\hline CXorf26|51260 & $\mathrm{B} 2$ \\
\hline CXorf27|25763 & B2 \\
\hline CXorf30|645090 & B2 \\
\hline CXorf38|159013 & B2 \\
\hline CXorf41|139212 & $\mathrm{B} 2$ \\
\hline CXorf48|54967 & $\mathrm{B} 2$ \\
\hline CXorf49B|100132994 & $\mathrm{B} 2$ \\
\hline CXorf50B|653687 & $\mathrm{B} 2$ \\
\hline CXorf56|63932 & $\mathrm{B} 2$ \\
\hline CXorf57|55086 & $\mathrm{B} 2$ \\
\hline CXorf58|254158 & B2 \\
\hline CXorf59|286464 & B2 \\
\hline CXorf61|203413 & $\mathrm{B} 2$ \\
\hline CXorf64|100130613 & $\mathrm{B} 2$ \\
\hline CXorf65|158830 & $\mathrm{B} 2$ \\
\hline CYB5A|1528 & $\mathrm{B} 2$ \\
\hline CYB5B|80777 & $\mathrm{B} 2$ \\
\hline CYB5D1|124637 & $\mathrm{B} 2$ \\
\hline CYB5R1|51706 & $\mathrm{B} 2$ \\
\hline CYB5R2|51700 & $\mathrm{B} 2$ \\
\hline CYB5R4|51167 & $\mathrm{B} 2$ \\
\hline CYB5RL|606495 & B2 \\
\hline CYBASC3|220002 & B2 \\
\hline CYBA|1535 & $\mathrm{B} 2$ \\
\hline CYBB $\mid 1536$ & B2 \\
\hline CYC1|1537 & B2 \\
\hline CYCSP52|360155 & B2 \\
\hline CYFIP1|23191 & $\mathrm{B} 2$ \\
\hline CYHR1|50626 & B2 \\
\hline CYLD|1540 & $\mathrm{B} 2$ \\
\hline CYP11B1|1584 & $\mathrm{B} 2$ \\
\hline CYP17A1|1586 & $\mathrm{B} 2$ \\
\hline CYP19A1|1588 & B2 \\
\hline CYP1A1|1543 & B2 \\
\hline CYP1A2|1544 & $\mathrm{B} 2$ \\
\hline CYP1B1|1545 & B2 \\
\hline
\end{tabular}




\begin{tabular}{|c|c|}
\hline CYP21A2|1589 & $\mathrm{B} 2$ \\
\hline CYP24A1|1591 & $\mathrm{B} 2$ \\
\hline CYP26A1|1592 & $\mathrm{B} 2$ \\
\hline CYP26C1|340665 & $\mathrm{B} 2$ \\
\hline CYP27A1|1593 & $\mathrm{B} 2$ \\
\hline CYP27B1|1594 & $\mathrm{B} 2$ \\
\hline CYP27C1|339761 & $\mathrm{B} 2$ \\
\hline CYP2A13|1553 & $\mathrm{B} 2$ \\
\hline CYP2A6|1548 & $\mathrm{B} 2$ \\
\hline CYP2A7|1549 & $\mathrm{B} 2$ \\
\hline CYP2B6|1555 & $\mathrm{B} 2$ \\
\hline CYP2C18|1562 & $\mathrm{B} 2$ \\
\hline CYP2C19|1557 & $\mathrm{B} 2$ \\
\hline CYP2C8|1558 & $\mathrm{B} 2$ \\
\hline \begin{tabular}{l|l|} 
CYP2C9|1559 \\
\end{tabular} & $\mathrm{B} 2$ \\
\hline CYP2D6|1565 & $\mathrm{B} 2$ \\
\hline CYP2D7P1|1564 & $\mathrm{B} 2$ \\
\hline CYP2E1|1571 & $\mathrm{B} 2$ \\
\hline CYP2F1|1572 & $\mathrm{B} 2$ \\
\hline CYP2J2|1573 & $\mathrm{B} 2$ \\
\hline CYP2R1|120227 & $\mathrm{B} 2$ \\
\hline CYP2S1|29785 & $\mathrm{B} 2$ \\
\hline CYP2W1|54905 & $\mathrm{B} 2$ \\
\hline CYP3A43|64816 & $\mathrm{B} 2$ \\
\hline CYP3A4|1576 & $\mathrm{B} 2$ \\
\hline CYP3A5|1577 & $\mathrm{B} 2$ \\
\hline CYP3A7|1551 & $\mathrm{B} 2$ \\
\hline CYP4A11|1579 & $\mathrm{B} 2$ \\
\hline CYP4A22|284541 & $\mathrm{B} 2$ \\
\hline CYP4B1|1580 & $\mathrm{B} 2$ \\
\hline CYP4F11|57834 & $\mathrm{B} 2$ \\
\hline CYP4F22|126410 & $\mathrm{B} 2$ \\
\hline CYP4F2|8529 & $\mathrm{B} 2$ \\
\hline \begin{tabular}{ll|} 
CYP4F3|4051 \\
\end{tabular} & $\mathrm{B} 2$ \\
\hline CYP4F8|11283 & $\mathrm{B} 2$ \\
\hline CYP4V2|285440 & $\mathrm{B} 2$ \\
\hline CYP4X1|260293 & $\mathrm{B} 2$ \\
\hline CYP4Z1|199974 & $\mathrm{B} 2$ \\
\hline CYP4Z2P|163720 & $\mathrm{B} 2$ \\
\hline CYP7B1|9420 & $\mathrm{B} 2$ \\
\hline CYP8B1|1582 & $\mathrm{B} 2$ \\
\hline CYS1|192668 & $\mathrm{B} 2$ \\
\hline CYSLTR1|10800 & $\mathrm{B} 2$ \\
\hline CYSLTR2|57105 & $\mathrm{B} 2$ \\
\hline CYTH1|9267 & $\mathrm{B} 2$ \\
\hline CYTH2|9266 & $\mathrm{B} 2$ \\
\hline CYTH4|27128 & $\mathrm{B} 2$ \\
\hline CYTIP|9595 & $\mathrm{B} 2$ \\
\hline CYTSA|23384 & $\mathrm{B} 2$ \\
\hline CYTSB|92521 & $\mathrm{B} 2$ \\
\hline CYorf15A|246126 & $\mathrm{B} 2$ \\
\hline
\end{tabular}




\begin{tabular}{|c|c|}
\hline CYorf15B|84663 & B2 \\
\hline D2HGDH|728294 & B2 \\
\hline DAAM1|23002 & $\mathrm{B} 2$ \\
\hline DAB1|1600 & $\mathrm{B} 2$ \\
\hline DACH1|1602 & $\mathrm{B} 2$ \\
\hline DACH2|117154 & $\mathrm{B} 2$ \\
\hline DACT3|147906 & $\mathrm{B} 2$ \\
\hline DAD1L|56286 & $\mathrm{B} 2$ \\
\hline DAG1|1605 & $\mathrm{B} 2$ \\
\hline DAGLA|747 & $\mathrm{B} 2$ \\
\hline DAK|26007 & $\mathrm{B} 2$ \\
\hline DALRD3|55152 & $\mathrm{B} 2$ \\
\hline DAND5|199699 & $\mathrm{B} 2$ \\
\hline DAO|1610 & $\mathrm{B} 2$ \\
\hline DAPK1|1612 & $\mathrm{B} 2$ \\
\hline DAPK3|1613 & $\mathrm{B} 2$ \\
\hline DAPL1|92196 & $\mathrm{B} 2$ \\
\hline DAPP1|27071 & $\mathrm{B} 2$ \\
\hline DARS|1615 & $\mathrm{B} 2$ \\
\hline DAZAP2|9802 & $\mathrm{B} 2$ \\
\hline DAZL $\mid 1618$ & $\mathrm{~B} 2$ \\
\hline DBF4B $\mid 80174$ & $\mathrm{~B} 2$ \\
\hline $\mathrm{DBH} \mid 1621$ & $\mathrm{~B} 2$ \\
\hline DBI|1622 & $\mathrm{B} 2$ \\
\hline DBN1|1627 & $\mathrm{B} 2$ \\
\hline DBNDD2|55861 & $\mathrm{B} 2$ \\
\hline DBNL|28988 & $\mathrm{B} 2$ \\
\hline DBP $\mid 1628$ & $\mathrm{~B} 2$ \\
\hline DBT|1629 & $\mathrm{B} 2$ \\
\hline DBX1|120237 & $\mathrm{B} 2$ \\
\hline DCAF11|80344 & $\mathrm{B} 2$ \\
\hline DCAF12L1|139170 & $\mathrm{B} 2$ \\
\hline DCAF12L2|340578 & $\mathrm{B} 2$ \\
\hline DCAF15|90379 & $\mathrm{B} 2$ \\
\hline DCAF16|54876 & $\mathrm{B} 2$ \\
\hline DCAF17|80067 & $\mathrm{B} 2$ \\
\hline DCAF4L1|285429 & $\mathrm{B} 2$ \\
\hline DCAF4L2|138009 & $\mathrm{B} 2$ \\
\hline DCAF4|26094 & $\mathrm{B} 2$ \\
\hline DCAF6|55827 & $\mathrm{B} 2$ \\
\hline DCAF8L2|347442 & $\mathrm{B} 2$ \\
\hline DCAF8|50717 & $\mathrm{B} 2$ \\
\hline DCC $\mid 1630$ & $\mathrm{~B} 2$ \\
\hline DCDC1|341019 & $\mathrm{B} 2$ \\
\hline DCDC2B|149069 & $\mathrm{B} 2$ \\
\hline DCDC2|51473 & $\mathrm{B} 2$ \\
\hline DCD|117159 & $\mathrm{B} 2$ \\
\hline DCI|1632 & $\mathrm{B} 2$ \\
\hline DCLK1|9201 & $\mathrm{B} 2$ \\
\hline DCLK2|166614 & $\mathrm{B} 2$ \\
\hline \begin{tabular}{l|l|} 
DCLK3|85443 \\
\end{tabular} & $\mathrm{B} 2$ \\
\hline
\end{tabular}




\begin{tabular}{|c|c|}
\hline DCLRE1B|64858 & B2 \\
\hline DCLRE1C|64421 & B2 \\
\hline DCP1B|196513 & B2 \\
\hline DCP2|167227 & $\mathrm{B} 2$ \\
\hline DCPS|28960 & $\mathrm{B} 2$ \\
\hline DCST1|149095 & $\mathrm{B} 2$ \\
\hline DCST2|127579 & $\mathrm{B} 2$ \\
\hline DCTD|1635 & $\mathrm{B} 2$ \\
\hline DCTN1|1639 & $\mathrm{B} 2$ \\
\hline DCTN2|10540 & $\mathrm{B} 2$ \\
\hline DCTN3|11258 & $\mathrm{B} 2$ \\
\hline DCTN4|51164 & $\mathrm{B} 2$ \\
\hline DCTN6|10671 & $\mathrm{B} 2$ \\
\hline DCT|1638 & $\mathrm{B} 2$ \\
\hline DCUN1D1|54165 & $\mathrm{B} 2$ \\
\hline DCUN1D2|55208 & $\mathrm{B} 2$ \\
\hline DCXR|51181 & $\mathrm{B} 2$ \\
\hline DCX $\mid 1641$ & $\mathrm{~B} 2$ \\
\hline DDA1|79016 & $\mathrm{B} 2$ \\
\hline DDAH2|23564 & $\mathrm{B} 2$ \\
\hline DDC|1644 & B2 \\
\hline DDHD1|80821 & $\mathrm{B} 2$ \\
\hline DDHD2|23259 & $\mathrm{B} 2$ \\
\hline DDI1|414301 & $\mathrm{B} 2$ \\
\hline DDI2|84301 & $\mathrm{B} 2$ \\
\hline DDIT3|1649 & $\mathrm{B} 2$ \\
\hline DDIT4L|115265 & $\mathrm{B} 2$ \\
\hline DDIT4|54541 & $\mathrm{B} 2$ \\
\hline DDN|23109 & $\mathrm{B} 2$ \\
\hline DDRGK1|65992 & $\mathrm{B} 2$ \\
\hline DDTL|100037417 & B2 \\
\hline DDT|1652 & $\mathrm{B} 2$ \\
\hline DDX10|1662 & $\mathrm{B} 2$ \\
\hline DDX11L2|84771 & $\mathrm{B} 2$ \\
\hline DDX11|1663 & $\mathrm{B} 2$ \\
\hline DDX12|440081 & $\mathrm{B} 2$ \\
\hline DDX18|8886 & $\mathrm{B} 2$ \\
\hline DDX1|1653 & $\mathrm{B} 2$ \\
\hline DDX20|11218 & $\mathrm{B} 2$ \\
\hline DDX21|9188 & $\mathrm{B} 2$ \\
\hline DDX27|55661 & $\mathrm{B} 2$ \\
\hline $\begin{array}{l}\text { DDX28|55794 } \\
\end{array}$ & $\mathrm{B} 2$ \\
\hline DDX31|64794 & $\mathrm{B} 2$ \\
\hline DDX3Y|8653 & $\mathrm{B} 2$ \\
\hline DDX42|11325 & B2 \\
\hline DDX43|55510 & $\mathrm{B} 2$ \\
\hline DDX46|9879 & $\mathrm{B} 2$ \\
\hline DDX47|51202 & $\mathrm{B} 2$ \\
\hline DDX4|54514 & $\mathrm{B} 2$ \\
\hline DDX50|79009 & $\mathrm{B} 2$ \\
\hline DDX51|317781 & $\mathrm{B} 2$ \\
\hline
\end{tabular}




\begin{tabular}{|c|c|}
\hline DDX52|11056 & B2 \\
\hline DDX53|168400 & B2 \\
\hline DDX54|79039 & $\mathrm{B} 2$ \\
\hline DDX55|57696 & $\mathrm{B} 2$ \\
\hline DDX59|83479 & $\mathrm{B} 2$ \\
\hline DDX5|1655 & $\mathrm{B} 2$ \\
\hline DDX60L|91351 & $\mathrm{B} 2$ \\
\hline $\begin{array}{l}\text { DDX60|55601 } \\
\end{array}$ & $\mathrm{B} 2$ \\
\hline DDX6|1656 & $\mathrm{B} 2$ \\
\hline DEAF1|10522 & $\mathrm{B} 2$ \\
\hline DEC1|50514 & $\mathrm{B} 2$ \\
\hline DECR1|1666 & $\mathrm{B} 2$ \\
\hline DECR2|26063 & $\mathrm{B} 2$ \\
\hline DEF6|50619 & $\mathrm{B} 2$ \\
\hline DEFA1B|728358 & $\mathrm{B} 2$ \\
\hline DEFA4|1669 & $\mathrm{B} 2$ \\
\hline DEFB103B|414325 & $\mathrm{B} 2$ \\
\hline DEFB109P1B|641517 & $\mathrm{B} 2$ \\
\hline DEFB118|117285 & $\mathrm{B} 2$ \\
\hline DEFB119|245932 & $\mathrm{B} 2$ \\
\hline DEFB124|245937 & $\mathrm{B} 2$ \\
\hline DEFB125|245938 & $\mathrm{B} 2$ \\
\hline DEFB126|81623 & $\mathrm{B} 2$ \\
\hline DEFB131|644414 & $\mathrm{B} 2$ \\
\hline DEFB132|400830 & $\mathrm{B} 2$ \\
\hline DEGS1|8560 & $\mathrm{B} 2$ \\
\hline DEK|7913 & $\mathrm{B} 2$ \\
\hline DENND1A|57706 & $\mathrm{B} 2$ \\
\hline DENND1B|163486 & $\mathrm{B} 2$ \\
\hline DENND1C|79958 & $\mathrm{B} 2$ \\
\hline DENND4A|10260 & $\mathrm{B} 2$ \\
\hline DENND4B|9909 & $\mathrm{B} 2$ \\
\hline DEPDC4|120863 & $\mathrm{B} 2$ \\
\hline DEPDC5|9681 & $\mathrm{B} 2$ \\
\hline DEPDC6|64798 & $\mathrm{B} 2$ \\
\hline DERA|51071 & $\mathrm{B} 2$ \\
\hline \begin{tabular}{l|l|l|} 
DERL2|51009 \\
\end{tabular} & $\mathrm{B} 2$ \\
\hline \begin{tabular}{l|l|} 
DERL3|91319 \\
\end{tabular} & $\mathrm{B} 2$ \\
\hline DES|1674 & $\mathrm{B} 2$ \\
\hline DEXI|28955 & $\mathrm{B} 2$ \\
\hline DFFA|1676 & $\mathrm{B} 2$ \\
\hline DFFB|1677 & $\mathrm{B} 2$ \\
\hline DFNB31|25861 & $\mathrm{B} 2$ \\
\hline DGAT1|8694 & $\mathrm{B} 2$ \\
\hline DGAT2L6|347516 & $\mathrm{B} 2$ \\
\hline DGCR10|26222 & $\mathrm{B} 2$ \\
\hline DGCR11|25786 & $\mathrm{B} 2$ \\
\hline DGCR14|8220 & $\mathrm{B} 2$ \\
\hline DGCR2|9993 & $\mathrm{B} 2$ \\
\hline \begin{tabular}{l|l|} 
DGCR5|26220 \\
\end{tabular} & $\mathrm{B} 2$ \\
\hline DGCR6L|85359 & $\mathrm{B} 2$ \\
\hline
\end{tabular}




\begin{tabular}{|c|c|}
\hline DGCR6|8214 & B2 \\
\hline DGCR8|54487 & B2 \\
\hline DGCR9|25787 & $\mathrm{B} 2$ \\
\hline DGKA|1606 & $\mathrm{B} 2$ \\
\hline DGKB|1607 & B2 \\
\hline DGKD|8527 & $\mathrm{B} 2$ \\
\hline DGKE|8526 & $\mathrm{B} 2$ \\
\hline DGKG|1608 & $\mathrm{B} 2$ \\
\hline DGKH|160851 & $\mathrm{B} 2$ \\
\hline DGKI|9162 & $\mathrm{B} 2$ \\
\hline DGKK|139189 & $\mathrm{B} 2$ \\
\hline DGKQ|1609 & $\mathrm{B} 2$ \\
\hline DGKZ|8525 & $\mathrm{B} 2$ \\
\hline DHCR7|1717 & $\mathrm{B} 2$ \\
\hline DHDH|27294 & $\mathrm{B} 2$ \\
\hline DHFRL1|200895 & $\mathrm{B} 2$ \\
\hline DHFR|1719 & B2 \\
\hline DHH|50846 & $\mathrm{B} 2$ \\
\hline DHODH|1723 & $\mathrm{B} 2$ \\
\hline DHPS|1725 & $\mathrm{B} 2$ \\
\hline DHRS11|79154 & $\mathrm{B} 2$ \\
\hline DHRS1|115817 & $\mathrm{B} 2$ \\
\hline DHRS2|10202 & $\mathrm{B} 2$ \\
\hline DHRS4L1|728635 & $\mathrm{B} 2$ \\
\hline DHRS4L2|317749 & $\mathrm{B} 2$ \\
\hline DHRS4|10901 & $\mathrm{B} 2$ \\
\hline DHRS7B|25979 & $\mathrm{B} 2$ \\
\hline DHRS7C|201140 & $\mathrm{B} 2$ \\
\hline DHRS7|51635 & $\mathrm{B} 2$ \\
\hline \begin{tabular}{l|l|} 
DHRS9|10170 \\
\end{tabular} & $\mathrm{B} 2$ \\
\hline DHRSX|207063 & $\mathrm{B} 2$ \\
\hline DHX16|8449 & $\mathrm{B} 2$ \\
\hline DHX30|22907 & $\mathrm{B} 2$ \\
\hline \begin{tabular}{l|l|} 
DHX32|55760 &
\end{tabular} & $\mathrm{B} 2$ \\
\hline 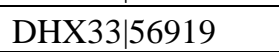 & $\mathrm{B} 2$ \\
\hline DHX35|60625 & $\mathrm{B} 2$ \\
\hline DHX36|170506 & $\mathrm{B} 2$ \\
\hline DHX37|57647 & $\mathrm{B} 2$ \\
\hline DHX40P1|653645 & $\mathrm{B} 2$ \\
\hline DHX40|79665 & $\mathrm{B} 2$ \\
\hline DHX57|90957 & $\mathrm{B} 2$ \\
\hline DHX58|79132 & $\mathrm{B} 2$ \\
\hline DHX8|1659 & $\mathrm{B} 2$ \\
\hline DIAPH1|1729 & $\mathrm{B} 2$ \\
\hline DICER1|23405 & $\mathrm{B} 2$ \\
\hline DIDO1|11083 & $\mathrm{B} 2$ \\
\hline DIO1|1733 & $\mathrm{B} 2$ \\
\hline DIO2|1734 & $\mathrm{B} 2$ \\
\hline DIO3|1735 & $\mathrm{B} 2$ \\
\hline DIP2A|23181 & $\mathrm{B} 2$ \\
\hline $\begin{array}{l}\text { DIP2B } 57609 \\
\end{array}$ & $\mathrm{~B} 2$ \\
\hline
\end{tabular}




\begin{tabular}{|c|c|}
\hline DIRAS1|148252 & B2 \\
\hline DIRAS2|54769 & B2 \\
\hline DIRAS3|9077 & B2 \\
\hline DIRC3|729582 & $\mathrm{B} 2$ \\
\hline DIS3L2|129563 & B2 \\
\hline DIS3L|115752 & $\mathrm{B} 2$ \\
\hline DIS3|22894 & $\mathrm{B} 2$ \\
\hline DISC2|27184 & $\mathrm{B} 2$ \\
\hline DISP1|84976 & B2 \\
\hline DISP2|85455 & $\mathrm{B} 2$ \\
\hline DKFZP434H168|26077 & $\mathrm{B} 2$ \\
\hline DKFZP434K028|26070 & $\mathrm{B} 2$ \\
\hline DKFZP586I1420|222161 & $\mathrm{B} 2$ \\
\hline DKFZP686I15217|401232 & $\mathrm{B} 2$ \\
\hline DKFZp434J0226|93429 & $\mathrm{B} 2$ \\
\hline DKFZp434L192|222029 & $\mathrm{B} 2$ \\
\hline DKFZp566F0947|94023 & B2 \\
\hline DKFZp686A1627|266695 & $\mathrm{B} 2$ \\
\hline DKFZp686O24166|374383 & $\mathrm{B} 2$ \\
\hline DKFZp761E198|91056 & $\mathrm{B} 2$ \\
\hline DKK1|22943 & $\mathrm{B} 2$ \\
\hline DKK2|27123 & $\mathrm{B} 2$ \\
\hline DKK4|27121 & $\mathrm{B} 2$ \\
\hline DKKL1|27120 & $\mathrm{B} 2$ \\
\hline DLAT|1737 & $\mathrm{B} 2$ \\
\hline DLD|1738 & $\mathrm{B} 2$ \\
\hline DLEU2L|79469 & $\mathrm{B} 2$ \\
\hline DLEU7|220107 & $\mathrm{B} 2$ \\
\hline DLG1|1739 & $\mathrm{B} 2$ \\
\hline DLG4|1742 & $\mathrm{B} 2$ \\
\hline $\begin{array}{l}\text { DLG5|9231 } \\
\end{array}$ & $\mathrm{B} 2$ \\
\hline DLGAP1|9229 & $\mathrm{B} 2$ \\
\hline DLGAP3|58512 & $\mathrm{B} 2$ \\
\hline DLGAP4|22839 & $\mathrm{B} 2$ \\
\hline DLK1 $\mid 8788$ & $\mathrm{~B} 2$ \\
\hline DLL3|10683 & $\mathrm{B} 2$ \\
\hline DLX1|1745 & $\mathrm{B} 2$ \\
\hline DLX2|1746 & $\mathrm{B} 2$ \\
\hline DLX3|1747 & B2 \\
\hline DLX4|1748 & $\mathrm{B} 2$ \\
\hline DLX5|1749 & $\mathrm{B} 2$ \\
\hline DLX6AS|285987 & $\mathrm{B} 2$ \\
\hline DLX6|1750 & $\mathrm{B} 2$ \\
\hline DMAP1|55929 & $\mathrm{B} 2$ \\
\hline DMBT1|1755 & $\mathrm{B} 2$ \\
\hline DMBX1|127343 & $\mathrm{B} 2$ \\
\hline DMC1|11144 & $\mathrm{B} 2$ \\
\hline DMKN|93099 & $\mathrm{B} 2$ \\
\hline DMP1|1758 & $\mathrm{B} 2$ \\
\hline DMPK|1760 & $\mathrm{B} 2$ \\
\hline DMRT1|1761 & $\mathrm{B} 2$ \\
\hline
\end{tabular}




\begin{tabular}{|c|c|}
\hline DMRTA1|63951 & B2 \\
\hline DMRTA2|63950 & B2 \\
\hline DMRTB1|63948 & $\mathrm{B} 2$ \\
\hline DMRTC1B|728656 & B2 \\
\hline DMRTC1|63947 & $\mathrm{B} 2$ \\
\hline DMRTC2|63946 & B2 \\
\hline DMTF1|9988 & $\mathrm{B} 2$ \\
\hline DMWD|1762 & $\mathrm{B} 2$ \\
\hline DMXL2|23312 & B2 \\
\hline DNAH10|196385 & $\mathrm{B} 2$ \\
\hline DNAH11|8701 & B2 \\
\hline DNAH12|201625 & $\mathrm{B} 2$ \\
\hline DNAH17|8632 & $\mathrm{B} 2$ \\
\hline DNAH2|146754 & $\mathrm{B} 2$ \\
\hline DNAH3|55567 & $\mathrm{B} 2$ \\
\hline DNAH5|1767 & B2 \\
\hline DNAH7|56171 & $\mathrm{B} 2$ \\
\hline DNAH8|1769 & $\mathrm{B} 2$ \\
\hline DNAI1|27019 & $\mathrm{B} 2$ \\
\hline DNAI2|64446 & $\mathrm{B} 2$ \\
\hline DNAJA2|10294 & $\mathrm{B} 2$ \\
\hline DNAJB12|54788 & $\mathrm{B} 2$ \\
\hline DNAJB13|374407 & B2 \\
\hline DNAJB14|79982 & $\mathrm{B} 2$ \\
\hline DNAJB1|3337 & $\mathrm{B} 2$ \\
\hline DNAJB2|3300 & $\mathrm{B} 2$ \\
\hline DNAJB3|414061 & $\mathrm{B} 2$ \\
\hline DNAJB5|25822 & $\mathrm{B} 2$ \\
\hline DNAJB6|10049 & $\mathrm{B} 2$ \\
\hline DNAJB7|150353 & $\mathrm{B} 2$ \\
\hline DNAJB8|165721 & $\mathrm{B} 2$ \\
\hline DNAJB9|4189 & $\mathrm{B} 2$ \\
\hline DNAJC11|55735 & $\mathrm{B} 2$ \\
\hline DNAJC12|56521 & $\mathrm{B} 2$ \\
\hline DNAJC13|23317 & $\mathrm{B} 2$ \\
\hline DNAJC15|29103 & $\mathrm{B} 2$ \\
\hline DNAJC16|23341 & $\mathrm{B} 2$ \\
\hline DNAJC17|55192 & $\mathrm{B} 2$ \\
\hline DNAJC19|131118 & $\mathrm{B} 2$ \\
\hline DNAJC21|134218 & $\mathrm{B} 2$ \\
\hline DNAJC24|120526 & $\mathrm{B} 2$ \\
\hline DNAJC25-GNG10|552891 & $\mathrm{B} 2$ \\
\hline DNAJC25|548645 & $\mathrm{B} 2$ \\
\hline DNAJC28|54943 & $\mathrm{B} 2$ \\
\hline DNAJC30|84277 & $\mathrm{B} 2$ \\
\hline DNAJC3|5611 & $\mathrm{B} 2$ \\
\hline DNAJC4|3338 & $\mathrm{B} 2$ \\
\hline DNAJC5B|85479 & $\mathrm{B} 2$ \\
\hline DNAJC5G|285126 & $\mathrm{B} 2$ \\
\hline DNAJC5|80331 & $\mathrm{B} 2$ \\
\hline DNAJC6 9829 & $\mathrm{~B} 2$ \\
\hline
\end{tabular}




\begin{tabular}{|c|c|}
\hline DNAJC8|22826 & B2 \\
\hline DNAL1|83544 & B2 \\
\hline DNAL4|10126 & B2 \\
\hline DNALI1|7802 & $\mathrm{B} 2$ \\
\hline DNASE1L1|1774 & $\mathrm{B} 2$ \\
\hline DNASE2B|58511 & $\mathrm{B} 2$ \\
\hline DND1|373863 & $\mathrm{B} 2$ \\
\hline DNER|92737 & $\mathrm{B} 2$ \\
\hline DNLZ|728489 & $\mathrm{B} 2$ \\
\hline DNM1L|10059 & $\mathrm{B} 2$ \\
\hline DNM1P35|100128285 & $\mathrm{B} 2$ \\
\hline DNM1|1759 & $\mathrm{B} 2$ \\
\hline DNM2|1785 & $\mathrm{B} 2$ \\
\hline DNMBP 23268 & $\mathrm{~B} 2$ \\
\hline DNMT3L|29947 & $\mathrm{B} 2$ \\
\hline DNTTIP1|116092 & $\mathrm{B} 2$ \\
\hline DNTTIP2|30836 & $\mathrm{B} 2$ \\
\hline DNTT|1791 & $\mathrm{B} 2$ \\
\hline DOC2A|8448 & $\mathrm{B} 2$ \\
\hline DOCK10|55619 & $\mathrm{B} 2$ \\
\hline DOCK2|1794 & $\mathrm{B} 2$ \\
\hline DOCK3|1795 & $\mathrm{B} 2$ \\
\hline DOCK5|80005 & $\mathrm{B} 2$ \\
\hline DOCK7|85440 & $\mathrm{B} 2$ \\
\hline DOHH $\mid 83475$ & $\mathrm{~B} 2$ \\
\hline DOK1|1796 & $\mathrm{B} 2$ \\
\hline DOK2|9046 & $\mathrm{B} 2$ \\
\hline DOK4|55715 & $\mathrm{B} 2$ \\
\hline DOK5|55816 & $\mathrm{B} 2$ \\
\hline DOK6|220164 & $\mathrm{B} 2$ \\
\hline DOK7|285489 & $\mathrm{B} 2$ \\
\hline DOM3Z|1797 & $\mathrm{B} 2$ \\
\hline DOT1L|84444 & $\mathrm{B} 2$ \\
\hline DPCR1|135656 & $\mathrm{B} 2$ \\
\hline DPEP2|64174 & $\mathrm{B} 2$ \\
\hline DPEP3|64180 & $\mathrm{B} 2$ \\
\hline DPF1|8193 & $\mathrm{B} 2$ \\
\hline DPF2|5977 & $\mathrm{B} 2$ \\
\hline DPF3|8110 & $\mathrm{B} 2$ \\
\hline DPH1|1801 & $\mathrm{B} 2$ \\
\hline DPH3B|100132911 & $\mathrm{B} 2$ \\
\hline DPH3|285381 & $\mathrm{B} 2$ \\
\hline DPH5|51611 & $\mathrm{B} 2$ \\
\hline DPM3|54344 & $\mathrm{B} 2$ \\
\hline DPP10|57628 & $\mathrm{B} 2$ \\
\hline DPP7|29952 & $\mathrm{B} 2$ \\
\hline DPP8|54878 & $\mathrm{B} 2$ \\
\hline DPPA2|151871 & $\mathrm{B} 2$ \\
\hline DPPA3|359787 & $\mathrm{B} 2$ \\
\hline DPPA4|55211 & $\mathrm{B} 2$ \\
\hline \begin{tabular}{l|l|} 
DPPA5 340168 \\
\end{tabular} & $\mathrm{~B} 2$ \\
\hline
\end{tabular}




\begin{tabular}{|c|c|}
\hline DPRXP4|503645 & B2 \\
\hline DPY19L1|23333 & B2 \\
\hline DPY19L2P1|554236 & $\mathrm{B} 2$ \\
\hline DPY19L2P2|349152 & $\mathrm{B} 2$ \\
\hline DPY19L2P4|442523 & B2 \\
\hline DPY19L3|147991 & $\mathrm{B} 2$ \\
\hline DPY19L4|286148 & $\mathrm{B} 2$ \\
\hline DPYSL3|1809 & $\mathrm{B} 2$ \\
\hline DPYSL4|10570 & B2 \\
\hline DPYSL5|56896 & $\mathrm{B} 2$ \\
\hline DPYS|1807 & $\mathrm{B} 2$ \\
\hline DQX1|165545 & $\mathrm{B} 2$ \\
\hline DR1|1810 & $\mathrm{B} 2$ \\
\hline DRAM1|55332 & $\mathrm{B} 2$ \\
\hline DRAM2|128338 & B2 \\
\hline DRD3|1814 & $\mathrm{B} 2$ \\
\hline DRD5|1816 & $\mathrm{B} 2$ \\
\hline DRG1|4733 & $\mathrm{B} 2$ \\
\hline DRG2|1819 & B2 \\
\hline DRGX|644168 & $\mathrm{B} 2$ \\
\hline DSC2|1824 & $\mathrm{B} 2$ \\
\hline DSC3|1825 & $\mathrm{B} 2$ \\
\hline DSCAML1|57453 & $\mathrm{B} 2$ \\
\hline DSCAM|1826 & $\mathrm{B} 2$ \\
\hline DSCR3|10311 & $\mathrm{B} 2$ \\
\hline DSCR4|10281 & $\mathrm{B} 2$ \\
\hline DSCR8|84677 & $\mathrm{B} 2$ \\
\hline DSCR9|257203 & B2 \\
\hline DSG1|1828 & $\mathrm{B} 2$ \\
\hline DSG2|1829 & $\mathrm{B} 2$ \\
\hline DSG3|1830 & $\mathrm{B} 2$ \\
\hline DSG4|147409 & $\mathrm{B} 2$ \\
\hline DSPP|1834 & $\mathrm{B} 2$ \\
\hline DSP|1832 & $\mathrm{B} 2$ \\
\hline DSTN|11034 & $\mathrm{B} 2$ \\
\hline DSTYK|25778 & $\mathrm{B} 2$ \\
\hline DTD1|92675 & $\mathrm{B} 2$ \\
\hline DTHD1|401124 & $\mathrm{B} 2$ \\
\hline DTNA|1837 & $\mathrm{B} 2$ \\
\hline DTNBP1|84062 & $\mathrm{B} 2$ \\
\hline DTNB|1838 & B2 \\
\hline DTWD2|285605 & $\mathrm{B} 2$ \\
\hline DTX3|196403 & B2 \\
\hline DTX4|23220 & $\mathrm{B} 2$ \\
\hline DULLARD|23399 & B2 \\
\hline DUOX1|53905 & $\mathrm{B} 2$ \\
\hline DUOX2|50506 & $\mathrm{B} 2$ \\
\hline DUOXA1|90527 & $\mathrm{B} 2$ \\
\hline DUOXA2|405753 & $\mathrm{B} 2$ \\
\hline DUPD1|338599 & $\mathrm{B} 2$ \\
\hline DUS1L|64118 & $\mathrm{B} 2$ \\
\hline
\end{tabular}




\begin{tabular}{|c|c|}
\hline DUS2L|54920 & B2 \\
\hline DUS3L|56931 & B2 \\
\hline DUSP10|11221 & $\mathrm{B} 2$ \\
\hline DUSP11|8446 & $\mathrm{B} 2$ \\
\hline DUSP13|51207 & $\mathrm{B} 2$ \\
\hline DUSP15|128853 & $\mathrm{B} 2$ \\
\hline DUSP16|80824 & $\mathrm{B} 2$ \\
\hline DUSP18|150290 & $\mathrm{B} 2$ \\
\hline DUSP23|54935 & $\mathrm{B} 2$ \\
\hline DUSP26|78986 & $\mathrm{B} 2$ \\
\hline DUSP27|92235 & $\mathrm{B} 2$ \\
\hline DUSP28|285193 & $\mathrm{B} 2$ \\
\hline DUSP2|1844 & $\mathrm{B} 2$ \\
\hline DUSP3|1845 & $\mathrm{B} 2$ \\
\hline DUSP4|1846 & B2 \\
\hline DUSP5P|574029 & $\mathrm{B} 2$ \\
\hline DUSP5|1847 & $\mathrm{B} 2$ \\
\hline DUSP8|1850 & $\mathrm{B} 2$ \\
\hline DUSP9|1852 & $\mathrm{B} 2$ \\
\hline DUT|1854 & $\mathrm{B} 2$ \\
\hline DUXA|503835 & $\mathrm{B} 2$ \\
\hline DVL1|1855 & $\mathrm{B} 2$ \\
\hline DVL2|1856 & $\mathrm{B} 2$ \\
\hline DVWA|344875 & $\mathrm{B} 2$ \\
\hline DYDC1|143241 & $\mathrm{B} 2$ \\
\hline DYDC2|84332 & $\mathrm{B} 2$ \\
\hline DYM|54808 & $\mathrm{B} 2$ \\
\hline DYNC1H1|1778 & $\mathrm{B} 2$ \\
\hline DYNC1I1|1780 & $\mathrm{B} 2$ \\
\hline DYNC1I2|1781 & $\mathrm{B} 2$ \\
\hline DYNC2H1|79659 & $\mathrm{B} 2$ \\
\hline DYNC2LI1 51626 & $\mathrm{~B} 2$ \\
\hline DYNLL2|140735 & $\mathrm{B} 2$ \\
\hline DYNLRB1|83658 & $\mathrm{B} 2$ \\
\hline DYNLRB2|83657 & $\mathrm{B} 2$ \\
\hline DYNLT3|6990 & $\mathrm{B} 2$ \\
\hline DYRK1A|1859 & $\mathrm{B} 2$ \\
\hline DYRK1B|9149 & $\mathrm{B} 2$ \\
\hline DYRK2|8445 & $\mathrm{B} 2$ \\
\hline DYRK3|8444 & $\mathrm{B} 2$ \\
\hline DYRK4|8798 & $\mathrm{B} 2$ \\
\hline DYSFIP1|116729 & $\mathrm{B} 2$ \\
\hline DYSF|8291 & $\mathrm{B} 2$ \\
\hline DYTN|391475 & $\mathrm{B} 2$ \\
\hline DZIP3|9666 & $\mathrm{B} 2$ \\
\hline E2F4|1874 & $\mathrm{B} 2$ \\
\hline E2F6|1876 & $\mathrm{B} 2$ \\
\hline EAF2|55840 & $\mathrm{B} 2$ \\
\hline EAPP|55837 & $\mathrm{B} 2$ \\
\hline EBAG9|9166 & $\mathrm{B} 2$ \\
\hline EBF4|57593 & $\mathrm{B} 2$ \\
\hline
\end{tabular}




\begin{tabular}{|c|c|}
\hline EBI3|10148 & B2 \\
\hline EBPL|84650 & B2 \\
\hline ECD|11319 & B2 \\
\hline ECEL1|9427 & $\mathrm{B} 2$ \\
\hline ECH1|1891 & $\mathrm{B} 2$ \\
\hline ECHS1|1892 & $\mathrm{B} 2$ \\
\hline ECM1|1893 & $\mathrm{B} 2$ \\
\hline ECSIT|51295 & $\mathrm{B} 2$ \\
\hline ECT2L|345930 & $\mathrm{B} 2$ \\
\hline EDARADD|128178 & $\mathrm{B} 2$ \\
\hline EDAR|10913 & $\mathrm{B} 2$ \\
\hline EDC3|80153 & $\mathrm{B} 2$ \\
\hline EDC4|23644 & $\mathrm{B} 2$ \\
\hline EDDM3A|10876 & $\mathrm{B} 2$ \\
\hline EDDM3B|64184 & $\mathrm{B} 2$ \\
\hline EDEM1|9695 & $\mathrm{B} 2$ \\
\hline EDF1|8721 & $\mathrm{B} 2$ \\
\hline EDIL3|10085 & $\mathrm{B} 2$ \\
\hline EDNRA|1909 & $\mathrm{B} 2$ \\
\hline EED $\mid 8726$ & $\mathrm{~B} 2$ \\
\hline EEF1A2|1917 & B2 \\
\hline EEF1DP3|196549 & $\mathrm{B} 2$ \\
\hline EEF1D|1936 & $\mathrm{B} 2$ \\
\hline EEF1G|1937 & $\mathrm{B} 2$ \\
\hline EEFSEC|60678 & B2 \\
\hline EFCAB10|100130771 & $\mathrm{B} 2$ \\
\hline EFCAB2|84288 & $\mathrm{B} 2$ \\
\hline EFCAB3|146779 & $\mathrm{B} 2$ \\
\hline EFCAB4A|283229 & $\mathrm{B} 2$ \\
\hline EFCAB4B|84766 & $\mathrm{B} 2$ \\
\hline EFCAB5|374786 & $\mathrm{B} 2$ \\
\hline EFCAB6|64800 & $\mathrm{B} 2$ \\
\hline EFCAB7|84455 & $\mathrm{B} 2$ \\
\hline EFEMP2|30008 & $\mathrm{B} 2$ \\
\hline EFHA1|221154 & $\mathrm{B} 2$ \\
\hline EFHB|151651 & $\mathrm{B} 2$ \\
\hline EFHC1|114327 & $\mathrm{B} 2$ \\
\hline EFHC2|80258 & $\mathrm{B} 2$ \\
\hline EFHD1|80303 & $\mathrm{B} 2$ \\
\hline EFHD2|79180 & $\mathrm{B} 2$ \\
\hline EFNA2|1943 & $\mathrm{B} 2$ \\
\hline EFNA5|1946 & $\mathrm{B} 2$ \\
\hline EFNB2|1948 & $\mathrm{B} 2$ \\
\hline $\begin{array}{l}\text { EFR3B|22979 } \\
\end{array}$ & $\mathrm{B} 2$ \\
\hline EFS|10278 & $\mathrm{B} 2$ \\
\hline EFTUD1|79631 & $\mathrm{B} 2$ \\
\hline EGFL6|25975 & $\mathrm{B} 2$ \\
\hline EGFR|1956 & $\mathrm{B} 2$ \\
\hline EGF|1950 & $\mathrm{B} 2$ \\
\hline EGLN1|54583 & $\mathrm{B} 2$ \\
\hline EGLN2|112398 & $\mathrm{B} 2$ \\
\hline
\end{tabular}




\begin{tabular}{|c|c|}
\hline EGLN3|112399 & B2 \\
\hline EGOT|100126791 & B2 \\
\hline EGR4|1961 & B2 \\
\hline EHBP1L1|254102 & B2 \\
\hline EHD1|10938 & B2 \\
\hline EHD4|30844 & $\mathrm{B} 2$ \\
\hline EHF|26298 & $\mathrm{B} 2$ \\
\hline EHMT1|79813 & B2 \\
\hline EI24|9538 & B2 \\
\hline EID2|163126 & $\mathrm{B} 2$ \\
\hline EIF1AX|1964 & B2 \\
\hline EIF1AY|9086 & B2 \\
\hline EIF2AK4|440275 & B2 \\
\hline EIF2A|83939 & $\mathrm{B} 2$ \\
\hline EIF2B1|1967 & $\mathrm{B} 2$ \\
\hline EIF2B2|8892 & $\mathrm{B} 2$ \\
\hline EIF2B4|8890 & $\mathrm{B} 2$ \\
\hline EIF2B5|8893 & $\mathrm{B} 2$ \\
\hline \begin{tabular}{l|l|} 
EIF2C1|26523 \\
\end{tabular} & B2 \\
\hline \begin{tabular}{l|l|} 
EIF2C2|27161 \\
\end{tabular} & $\mathrm{B} 2$ \\
\hline \begin{tabular}{l|l|} 
EIF2C3|192669 \\
\end{tabular} & $\mathrm{B} 2$ \\
\hline EIF2S1|1965 & B2 \\
\hline EIF2S3|1968 & $\mathrm{B} 2$ \\
\hline EIF3A|8661 & B2 \\
\hline EIF3B|8662 & $\mathrm{B} 2$ \\
\hline EIF3CL|728689 & B2 \\
\hline EIF3C|8663 & B2 \\
\hline EIF3E|3646 & B2 \\
\hline EIF3G|8666 & $\mathrm{B} 2$ \\
\hline EIF3IP1|442720 & $\mathrm{B} 2$ \\
\hline EIF3I|8668 & B2 \\
\hline EIF3J|8669 & $\mathrm{B} 2$ \\
\hline EIF3K|27335 & B2 \\
\hline EIF3M|10480 & B2 \\
\hline EIF4A1|1973 & B2 \\
\hline EIF4E1B|253314 & $\mathrm{B} 2$ \\
\hline EIF4E2|9470 & B2 \\
\hline EIF4EBP1|1978 & $\mathrm{B} 2$ \\
\hline EIF4EBP3|8637 & B2 \\
\hline EIF4ENIF1|56478 & $\mathrm{B} 2$ \\
\hline EIF4G2|1982 & B2 \\
\hline \begin{tabular}{l|l|} 
EIF4G3|8672 &
\end{tabular} & B2 \\
\hline EIF4H|7458 & B2 \\
\hline \begin{tabular}{l|l|l|} 
EIF5A2|56648 &
\end{tabular} & B2 \\
\hline $\begin{array}{l}\text { EIF5|1983 } \\
\end{array}$ & $\mathrm{B} 2$ \\
\hline $\begin{array}{l}\text { ELAC2|60528 } \\
\end{array}$ & B2 \\
\hline ELAVL2|1993 & $\mathrm{B} 2$ \\
\hline ELAVL3|1995 & B2 \\
\hline ELAVL4|1996 & $\mathrm{B} 2$ \\
\hline ELF1|1997 & B2 \\
\hline ELF2|1998 & $\mathrm{B} 2$ \\
\hline
\end{tabular}




\begin{tabular}{|c|c|}
\hline ELF3|1999 & B2 \\
\hline ELFN1|392617 & B2 \\
\hline ELFN2|114794 & $\mathrm{B} 2$ \\
\hline ELK3|2004 & $\mathrm{B} 2$ \\
\hline ELK4|2005 & $\mathrm{B} 2$ \\
\hline ELL2|22936 & $\mathrm{B} 2$ \\
\hline ELL|8178 & B2 \\
\hline ELMO3|79767 & $\mathrm{B} 2$ \\
\hline ELMOD1|55531 & $\mathrm{B} 2$ \\
\hline ELN|2006 & $\mathrm{B} 2$ \\
\hline ELOF1|84337 & $\mathrm{B} 2$ \\
\hline ELOVL1|64834 & $\mathrm{B} 2$ \\
\hline \begin{tabular}{l|l|} 
ELOVL2|54898 \\
\end{tabular} & $\mathrm{B} 2$ \\
\hline ELOVL3|83401 & $\mathrm{B} 2$ \\
\hline ELOVL4|6785 & $\mathrm{B} 2$ \\
\hline ELOVL5|60481 & $\mathrm{B} 2$ \\
\hline ELOVL6|79071 & $\mathrm{B} 2$ \\
\hline ELOVL7|79993 & $\mathrm{B} 2$ \\
\hline ELP2P|100131454 & $\mathrm{B} 2$ \\
\hline ELP2|55250 & $\mathrm{B} 2$ \\
\hline $\begin{array}{l}\text { ELP3|55140 } \\
\end{array}$ & $\mathrm{B} 2$ \\
\hline ELP4|26610 & B2 \\
\hline ELSPBP1|64100 & $\mathrm{B} 2$ \\
\hline EMB|133418 & $\mathrm{B} 2$ \\
\hline EMD|2010 & $\mathrm{B} 2$ \\
\hline EME2|197342 & $\mathrm{B} 2$ \\
\hline EMG1|10436 & $\mathrm{B} 2$ \\
\hline EMID1|129080 & $\mathrm{B} 2$ \\
\hline EMID2|136227 & $\mathrm{B} 2$ \\
\hline EMILIN1|11117 & $\mathrm{B} 2$ \\
\hline EML1|2009 & $\mathrm{B} 2$ \\
\hline EML2|24139 & $\mathrm{B} 2$ \\
\hline EML4|27436 & $\mathrm{B} 2$ \\
\hline \begin{tabular}{l|l|} 
EML6|40054 \\
\end{tabular} & $\mathrm{B} 2$ \\
\hline EMP3|2014 & $\mathrm{B} 2$ \\
\hline EMR1|2015 & $\mathrm{B} 2$ \\
\hline EMR2|30817 & $\mathrm{B} 2$ \\
\hline EMR3|84658 & $\mathrm{B} 2$ \\
\hline EMR4P|326342 & $\mathrm{B} 2$ \\
\hline EMX1|2016 & $\mathrm{B} 2$ \\
\hline EN1|2019 & $\mathrm{B} 2$ \\
\hline EN2|2020 & $\mathrm{B} 2$ \\
\hline ENAM|10117 & $\mathrm{B} 2$ \\
\hline ENDOD1|23052 & $\mathrm{B} 2$ \\
\hline ENDOG|2021 & $\mathrm{B} 2$ \\
\hline ENHO|375704 & $\mathrm{B} 2$ \\
\hline ENKUR|219670 & $\mathrm{B} 2$ \\
\hline ENO1|2023 & $\mathrm{B} 2$ \\
\hline \begin{tabular}{ll|l|} 
ENO3|2027 & \\
\end{tabular} & $\mathrm{B} 2$ \\
\hline ENOSF1|55556 & $\mathrm{B} 2$ \\
\hline ENOX1|55068 & $\mathrm{B} 2$ \\
\hline
\end{tabular}




\begin{tabular}{|c|c|}
\hline ENOX2|10495 & B2 \\
\hline ENPEP|2028 & B2 \\
\hline ENPP1|5167 & $\mathrm{B} 2$ \\
\hline ENPP3|5169 & $\mathrm{B} 2$ \\
\hline ENPP4|22875 & B2 \\
\hline ENPP5|59084 & $\mathrm{B} 2$ \\
\hline ENPP7|339221 & $\mathrm{B} 2$ \\
\hline ENTHD1|150350 & $\mathrm{B} 2$ \\
\hline ENTPD1|953 & $\mathrm{B} 2$ \\
\hline ENTPD2|954 & $\mathrm{B} 2$ \\
\hline ENTPD3|956 & $\mathrm{B} 2$ \\
\hline ENTPD4|9583 & B2 \\
\hline ENTPD5|957 & $\mathrm{B} 2$ \\
\hline ENTPD6|955 & $\mathrm{B} 2$ \\
\hline ENTPD8|377841 & $\mathrm{B} 2$ \\
\hline EOMES|8320 & $\mathrm{B} 2$ \\
\hline EP300|2033 & B2 \\
\hline EP400NL|347918 & $\mathrm{B} 2$ \\
\hline EP400|57634 & B2 \\
\hline EPB41L1|2036 & $\mathrm{B} 2$ \\
\hline EPB41L3|23136 & $\mathrm{B} 2$ \\
\hline EPB41L4A|64097 & $\mathrm{B} 2$ \\
\hline EPB41L5|57669 & $\mathrm{B} 2$ \\
\hline EPB41|2035 & $\mathrm{B} 2$ \\
\hline EPGN|255324 & $\mathrm{B} 2$ \\
\hline EPHA1|2041 & $\mathrm{B} 2$ \\
\hline EPHA3|2042 & $\mathrm{B} 2$ \\
\hline EPHA4|2043 & B2 \\
\hline EPHA5|2044 & $\mathrm{B} 2$ \\
\hline EPHA6|285220 & $\mathrm{B} 2$ \\
\hline EPHA7|2045 & $\mathrm{B} 2$ \\
\hline EPHA8|2046 & $\mathrm{B} 2$ \\
\hline EPHB2|2048 & $\mathrm{B} 2$ \\
\hline EPHB3|2049 & B2 \\
\hline EPHB4|2050 & $\mathrm{B} 2$ \\
\hline EPHX1|2052 & $\mathrm{B} 2$ \\
\hline \begin{tabular}{l|l|l|} 
EPHX3 & 79852 \\
\end{tabular} & B2 \\
\hline EPHX4|253152 & $\mathrm{B} 2$ \\
\hline EPM2AIP1|9852 & B2 \\
\hline EPM2A|7957 & $\mathrm{B} 2$ \\
\hline EPN1|29924 & B2 \\
\hline EPN2|22905 & $\mathrm{B} 2$ \\
\hline EPOR|2057 & B2 \\
\hline EPO|2056 & $\mathrm{B} 2$ \\
\hline EPPK1|83481 & B2 \\
\hline EPS8L2|64787 & $\mathrm{B} 2$ \\
\hline $\begin{array}{l}\text { EPS8L3|79574 } \\
\end{array}$ & $\mathrm{B} 2$ \\
\hline EPX|8288 & B2 \\
\hline EPYC|1833 & $\mathrm{B} 2$ \\
\hline ERAL1|26284 & B2 \\
\hline ERAP2|64167 & $\mathrm{B} 2$ \\
\hline
\end{tabular}




\begin{tabular}{|c|c|}
\hline ERAS|3266 & B2 \\
\hline ERBB2|2064 & $\mathrm{B} 2$ \\
\hline ERBB4|2066 & B2 \\
\hline ERC1|23085 & $\mathrm{B} 2$ \\
\hline ERC2|26059 & $\mathrm{B} 2$ \\
\hline ERCC1|2067 & $\mathrm{B} 2$ \\
\hline ERCC2|2068 & $\mathrm{B} 2$ \\
\hline ERCC3|2071 & $\mathrm{B} 2$ \\
\hline ERCC4|2072 & $\mathrm{B} 2$ \\
\hline ERCC6|2074 & $\mathrm{B} 2$ \\
\hline ERCC8|1161 & $\mathrm{B} 2$ \\
\hline EREG|2069 & $\mathrm{B} 2$ \\
\hline ERF|2077 & $\mathrm{B} 2$ \\
\hline $\begin{array}{l}\text { ERGIC3|51614 } \\
\end{array}$ & $\mathrm{B} 2$ \\
\hline ERI1|90459 & $\mathrm{B} 2$ \\
\hline ERLIN1|10613 & $\mathrm{B} 2$ \\
\hline ERLIN2|11160 & $\mathrm{B} 2$ \\
\hline ERN1|2081 & $\mathrm{B} 2$ \\
\hline ERN2|10595 & $\mathrm{B} 2$ \\
\hline ERO1LB|56605 & $\mathrm{B} 2$ \\
\hline ERP29|10961 & $\mathrm{B} 2$ \\
\hline ERP44|23071 & $\mathrm{B} 2$ \\
\hline ESCO1|114799 & $\mathrm{B} 2$ \\
\hline ESF1|51575 & $\mathrm{B} 2$ \\
\hline ESPNL|339768 & $\mathrm{B} 2$ \\
\hline \begin{tabular}{l|l|} 
ESPNP|28429 &
\end{tabular} & $\mathrm{B} 2$ \\
\hline ESRRA|2101 & $\mathrm{B} 2$ \\
\hline \begin{tabular}{l|l|l|} 
ESRRB| $\mid 2103$ \\
\end{tabular} & $\mathrm{~B} 2$ \\
\hline ESRRG|2104 & $\mathrm{B} 2$ \\
\hline ESX1|80712 & $\mathrm{B} 2$ \\
\hline $\begin{array}{l}\text { ESYT3|83850 } \\
\end{array}$ & $\mathrm{B} 2$ \\
\hline ETAA1|54465 & $\mathrm{B} 2$ \\
\hline ETF1|2107 & $\mathrm{B} 2$ \\
\hline ETFA|2108 & $\mathrm{B} 2$ \\
\hline \begin{tabular}{l|l|} 
ETHE1|23474 \\
\end{tabular} & $\mathrm{B} 2$ \\
\hline $\begin{array}{l}\text { ETNK1|55500 } \\
\end{array}$ & $\mathrm{B} 2$ \\
\hline ETV2|2116 & $\mathrm{B} 2$ \\
\hline ETV3|2117 & $\mathrm{B} 2$ \\
\hline ETV4|2118 & $\mathrm{B} 2$ \\
\hline ETV6|2120 & $\mathrm{B} 2$ \\
\hline EVC|2121 & $\mathrm{B} 2$ \\
\hline EVI2A|2123 & $\mathrm{B} 2$ \\
\hline EVI2B|2124 & $\mathrm{B} 2$ \\
\hline EVI5L|115704 & $\mathrm{B} 2$ \\
\hline EVPLL|645027 & $\mathrm{B} 2$ \\
\hline EVX1|2128 & $\mathrm{B} 2$ \\
\hline EWSR1|2130 & $\mathrm{B} 2$ \\
\hline EXD1|161829 & $\mathrm{B} 2$ \\
\hline EXD2|55218 & $\mathrm{B} 2$ \\
\hline EXD3|54932 & $\mathrm{B} 2$ \\
\hline EXOC1|55763 & $\mathrm{B} 2$ \\
\hline
\end{tabular}




\begin{tabular}{|c|c|}
\hline EXOC2|55770 & B2 \\
\hline EXOC3L2|90332 & B2 \\
\hline EXOC3L|283849 & B2 \\
\hline EXOC4|60412 & B2 \\
\hline EXOC5|10640 & B2 \\
\hline EXOC7|23265 & B2 \\
\hline EXOC8|149371 & B2 \\
\hline EXOG|9941 & $\mathrm{B} 2$ \\
\hline EXOSC10|5394 & B2 \\
\hline EXOSC2|23404 & B2 \\
\hline EXOSC5|56915 & B2 \\
\hline EXOSC6|118460 & B2 \\
\hline EXOSC7|23016 & B2 \\
\hline EXOSC8|11340 & $\mathrm{B} 2$ \\
\hline \begin{tabular}{l|l|} 
EXOSC9|5393 \\
\end{tabular} & B2 \\
\hline $\begin{array}{l}\text { EXPH5|23086 } \\
\end{array}$ & $\mathrm{B} 2$ \\
\hline EXT1|2131 & B2 \\
\hline EXT2|2132 & $\mathrm{B} 2$ \\
\hline EXTL1|2134 & B2 \\
\hline EYA1|2138 & $\mathrm{B} 2$ \\
\hline EYA2|2139 & $\mathrm{B} 2$ \\
\hline EYA3|2140 & B2 \\
\hline EYA4|2070 & $\mathrm{B} 2$ \\
\hline EYS|346007 & B2 \\
\hline F11|2160 & B2 \\
\hline F13A1|2162 & $\mathrm{B} 2$ \\
\hline $\mathrm{F} 13 \mathrm{~B} \mid 2165$ & B2 \\
\hline F2RL1|2150 & B2 \\
\hline F2RL3|9002 & $\mathrm{B} 2$ \\
\hline F2|2147 & $\mathrm{B} 2$ \\
\hline F5|2153 & $\mathrm{B} 2$ \\
\hline F8A1|8263 & $\mathrm{B} 2$ \\
\hline F9|2158 & B2 \\
\hline FA2H|79152 & $\mathrm{B} 2$ \\
\hline FABP1|2168 & B2 \\
\hline FABP2|2169 & $\mathrm{B} 2$ \\
\hline FABP3|2170 & B2 \\
\hline FABP5L3|220832 & $\mathrm{B} 2$ \\
\hline FABP5|2171 & $\mathrm{B} 2$ \\
\hline FABP6|2172 & $\mathrm{B} 2$ \\
\hline FABP7|2173 & B2 \\
\hline FABP9|646480 & $\mathrm{B} 2$ \\
\hline FADS1|3992 & B2 \\
\hline FADS2|9415 & B2 \\
\hline FADS6|283985 & $\mathrm{B} 2$ \\
\hline FAF1|11124 & $\mathrm{B} 2$ \\
\hline FAHD2B|151313 & B2 \\
\hline FAH|2184 & $\mathrm{B} 2$ \\
\hline FAIM2|23017 & B2 \\
\hline FAIM3|9214 & B2 \\
\hline FAIM|55179 & $\mathrm{B} 2$ \\
\hline
\end{tabular}




\begin{tabular}{|c|c|}
\hline FAM100A|124402 & B2 \\
\hline FAM100B|283991 & $\mathrm{B} 2$ \\
\hline FAM101A|144347 & $\mathrm{B} 2$ \\
\hline FAM101B|359845 & $\mathrm{B} 2$ \\
\hline FAM102B|284611 & B2 \\
\hline FAM104B|90736 & $\mathrm{B} 2$ \\
\hline FAM105A|54491 & $\mathrm{B} 2$ \\
\hline FAM105B|90268 & $\mathrm{B} 2$ \\
\hline FAM106A|80039 & B2 \\
\hline FAM106C|100129396 & $\mathrm{B} 2$ \\
\hline FAM107B|83641 & $\mathrm{B} 2$ \\
\hline FAM108A1|81926 & B2 \\
\hline FAM108B1|51104 & $\mathrm{B} 2$ \\
\hline FAM109A|144717 & $\mathrm{B} 2$ \\
\hline FAM109B|150368 & B2 \\
\hline FAM110C|642273 & $\mathrm{B} 2$ \\
\hline FAM111A|63901 & B2 \\
\hline FAM113A|64773 & $\mathrm{B} 2$ \\
\hline \begin{tabular}{l|l|l|} 
FAM113B|91523 & \\
\end{tabular} & B2 \\
\hline FAM114A2|10827 & $\mathrm{B} 2$ \\
\hline FAM115A|9747 & $\mathrm{B} 2$ \\
\hline FAM115C|285966 & $\mathrm{B} 2$ \\
\hline FAM116A|201627 & $\mathrm{B} 2$ \\
\hline FAM116B|414918 & $\mathrm{B} 2$ \\
\hline FAM117B|150864 & $\mathrm{B} 2$ \\
\hline FAM118A|55007 & $\mathrm{B} 2$ \\
\hline FAM118B|79607 & $\mathrm{B} 2$ \\
\hline FAM119A|151194 & B2 \\
\hline FAM120AOS|158293 & $\mathrm{B} 2$ \\
\hline FAM120B $\mid 84498$ & $\mathrm{~B} 2$ \\
\hline FAM120C|54954 & $\mathrm{B} 2$ \\
\hline FAM122C|159091 & B2 \\
\hline FAM123A|219287 & $\mathrm{B} 2$ \\
\hline FAM123B|139285 & $\mathrm{B} 2$ \\
\hline FAM123C|205147 & $\mathrm{B} 2$ \\
\hline FAM125A|93343 & $\mathrm{B} 2$ \\
\hline FAM126B|285172 & $\mathrm{B} 2$ \\
\hline FAM127B|26071 & $\mathrm{B} 2$ \\
\hline FAM127C|441518 & $\mathrm{B} 2$ \\
\hline FAM128B|80097 & $\mathrm{B} 2$ \\
\hline FAM129B|64855 & B2 \\
\hline FAM129C|199786 & $\mathrm{B} 2$ \\
\hline FAM131B|9715 & B2 \\
\hline FAM131C|348487 & $\mathrm{B} 2$ \\
\hline FAM132A|388581 & $\mathrm{B} 2$ \\
\hline FAM133A|286499 & $\mathrm{B} 2$ \\
\hline FAM133B|257415 & $\mathrm{B} 2$ \\
\hline FAM134A|79137 & $\mathrm{B} 2$ \\
\hline FAM134B|54463 & $\mathrm{B} 2$ \\
\hline FAM134C|162427 & $\mathrm{B} 2$ \\
\hline FAM135B|51059 & $\mathrm{B} 2$ \\
\hline
\end{tabular}




\begin{tabular}{|c|c|}
\hline FAM136B|387071 & B2 \\
\hline FAM138B|654412 & $\mathrm{B} 2$ \\
\hline FAM138D|677784 & $\mathrm{B} 2$ \\
\hline FAM138E|100124412 & $\mathrm{B} 2$ \\
\hline FAM149B1|317662 & B2 \\
\hline FAM150A|389658 & $\mathrm{B} 2$ \\
\hline FAM150B|285016 & $\mathrm{B} 2$ \\
\hline FAM151A|338094 & $\mathrm{B} 2$ \\
\hline FAM153B|202134 & $\mathrm{B} 2$ \\
\hline FAM153C|653316 & $\mathrm{B} 2$ \\
\hline FAM154A|158297 & $\mathrm{B} 2$ \\
\hline FAM154B 283726 & B2 \\
\hline FAM155A|728215 & $\mathrm{B} 2$ \\
\hline FAM155B|27112 & $\mathrm{B} 2$ \\
\hline FAM157A|728262 & $\mathrm{B} 2$ \\
\hline FAM157B|100132403 & $\mathrm{B} 2$ \\
\hline FAM159A|348378 & B2 \\
\hline FAM160A1|729830 & $\mathrm{B} 2$ \\
\hline FAM160B1|57700 & B2 \\
\hline FAM161A|84140 & $\mathrm{B} 2$ \\
\hline FAM161B|145483 & $\mathrm{B} 2$ \\
\hline FAM163A|148753 & $\mathrm{B} 2$ \\
\hline FAM163B|642968 & $\mathrm{B} 2$ \\
\hline FAM164A|51101 & $\mathrm{B} 2$ \\
\hline FAM164C|79696 & $\mathrm{B} 2$ \\
\hline FAM165B|54065 & $\mathrm{B} 2$ \\
\hline FAM166A|401565 & $\mathrm{B} 2$ \\
\hline FAM166B|730112 & $\mathrm{B} 2$ \\
\hline FAM167A|83648 & $\mathrm{B} 2$ \\
\hline FAM167B|84734 & $\mathrm{B} 2$ \\
\hline FAM168A|23201 & $\mathrm{B} 2$ \\
\hline FAM169A|26049 & B2 \\
\hline FAM169B|283777 & $\mathrm{B} 2$ \\
\hline FAM170A|340069 & $\mathrm{B} 2$ \\
\hline FAM171A2|284069 & $\mathrm{B} 2$ \\
\hline FAM171B|165215 & $\mathrm{B} 2$ \\
\hline FAM173B|134145 & $\mathrm{B} 2$ \\
\hline FAM174A|345757 & $\mathrm{B} 2$ \\
\hline FAM175B|23172 & B2 \\
\hline FAM176A|84141 & $\mathrm{B} 2$ \\
\hline \begin{tabular}{l|l|l|} 
FAM176B 55194 \\
\end{tabular} & B2 \\
\hline FAM177A1|283635 & $\mathrm{B} 2$ \\
\hline FAM177B|400823 & B2 \\
\hline FAM178A|55719 & $\mathrm{B} 2$ \\
\hline FAM178B|51252 & $\mathrm{B} 2$ \\
\hline FAM179A|165186 & $\mathrm{B} 2$ \\
\hline FAM179B|23116 & $\mathrm{B} 2$ \\
\hline FAM181B|220382 & $\mathrm{B} 2$ \\
\hline FAM182A|284800 & $\mathrm{B} 2$ \\
\hline $\begin{array}{l}\text { FAM182B|728882 } \\
\text { | } 1828\end{array}$ & B2 \\
\hline FAM183A|440585 & $\mathrm{B} 2$ \\
\hline
\end{tabular}




\begin{tabular}{|c|c|}
\hline FAM183B|340286 & B2 \\
\hline FAM184B|27146 & $\mathrm{B} 2$ \\
\hline FAM185A|222234 & $\mathrm{B} 2$ \\
\hline FAM186A|121006 & $\mathrm{B} 2$ \\
\hline FAM187B|148109 & B2 \\
\hline FAM188A|80013 & $\mathrm{B} 2$ \\
\hline FAM188B|84182 & $\mathrm{B} 2$ \\
\hline FAM189A1|23359 & $\mathrm{B} 2$ \\
\hline FAM18B2|201158 & $\mathrm{B} 2$ \\
\hline FAM18B|51030 & $\mathrm{B} 2$ \\
\hline FAM190A|401145 & $\mathrm{B} 2$ \\
\hline FAM192A|80011 & B2 \\
\hline FAM193A|8603 & $\mathrm{B} 2$ \\
\hline FAM194A|131831 & $\mathrm{B} 2$ \\
\hline FAM194B|220081 & $\mathrm{B} 2$ \\
\hline FAM195B|348262 & $\mathrm{B} 2$ \\
\hline FAM196A|642938 & $\mathrm{B} 2$ \\
\hline FAM198A|729085 & $\mathrm{B} 2$ \\
\hline \begin{tabular}{l|l|l|} 
FAM198B 51313 \\
\end{tabular} & B2 \\
\hline FAM19A2|338811 & $\mathrm{B} 2$ \\
\hline FAM19A3|284467 & $\mathrm{B} 2$ \\
\hline FAM19A4|151647 & $\mathrm{B} 2$ \\
\hline FAM19A5|25817 & $\mathrm{B} 2$ \\
\hline FAM200A|221786 & $\mathrm{B} 2$ \\
\hline FAM20A|54757 & $\mathrm{B} 2$ \\
\hline $\begin{array}{l}\text { FAM20B|9917 } \\
\end{array}$ & $\mathrm{B} 2$ \\
\hline FAM20C|56975 & $\mathrm{B} 2$ \\
\hline FAM21A|387680 & B2 \\
\hline FAM21B|55747 & $\mathrm{B} 2$ \\
\hline FAM22A|728118 & $\mathrm{B} 2$ \\
\hline FAM22D|728130 & $\mathrm{B} 2$ \\
\hline FAM22F|54754 & $\mathrm{B} 2$ \\
\hline FAM22G|441457 & $\mathrm{B} 2$ \\
\hline FAM24B|196792 & B2 \\
\hline FAM25A|643161 & $\mathrm{B} 2$ \\
\hline FAM25B|100132929 & $\mathrm{B} 2$ \\
\hline FAM26E|254228 & $\mathrm{B} 2$ \\
\hline FAM26F|441168 & $\mathrm{B} 2$ \\
\hline FAM27A|548321 & B2 \\
\hline FAM27B|100133121 & $\mathrm{B} 2$ \\
\hline FAM27C|100132948 & B2 \\
\hline FAM32A|26017 & $\mathrm{B} 2$ \\
\hline FAM35A|54537 & B2 \\
\hline FAM35B2|439965 & $\mathrm{B} 2$ \\
\hline FAM35B|414241 & $\mathrm{B} 2$ \\
\hline FAM38A|9780 & $\mathrm{B} 2$ \\
\hline FAM38B|63895 & $\mathrm{B} 2$ \\
\hline FAM3A|60343 & $\mathrm{B} 2$ \\
\hline FAM3B|54097 & $\mathrm{B} 2$ \\
\hline FAM3C|10447 & $\mathrm{B} 2$ \\
\hline FAM3D|131177 & $\mathrm{B} 2$ \\
\hline
\end{tabular}




\begin{tabular}{|c|c|}
\hline FAM40A|85369 & B2 \\
\hline FAM40B|57464 & $\mathrm{B} 2$ \\
\hline FAM41C|284593 & $\mathrm{B} 2$ \\
\hline FAM43B|163933 & $\mathrm{B} 2$ \\
\hline FAM45A|404636 & B2 \\
\hline FAM45B|55855 & $\mathrm{B} 2$ \\
\hline FAM46A|55603 & $\mathrm{B} 2$ \\
\hline FAM46B|115572 & $\mathrm{B} 2$ \\
\hline FAM46C|54855 & $\mathrm{B} 2$ \\
\hline FAM46D|169966 & $\mathrm{B} 2$ \\
\hline FAM47A|158724 & $\mathrm{B} 2$ \\
\hline FAM47B|170062 & B2 \\
\hline FAM47C|442444 & $\mathrm{B} 2$ \\
\hline FAM48A|55578 & $\mathrm{B} 2$ \\
\hline FAM48B1|100130302 & B2 \\
\hline FAM48B2|170067 & $\mathrm{B} 2$ \\
\hline FAM50A|9130 & $\mathrm{B} 2$ \\
\hline FAM50B|26240 & $\mathrm{B} 2$ \\
\hline FAM53A|152877 & B2 \\
\hline FAM53B|9679 & $\mathrm{B} 2$ \\
\hline FAM55A|120400 & $\mathrm{B} 2$ \\
\hline FAM55B|120406 & $\mathrm{B} 2$ \\
\hline FAM55C|91775 & $\mathrm{B} 2$ \\
\hline FAM57A|79850 & $\mathrm{B} 2$ \\
\hline FAM57B|83723 & $\mathrm{B} 2$ \\
\hline FAM58A|92002 & $\mathrm{B} 2$ \\
\hline FAM58B|339521 & $\mathrm{B} 2$ \\
\hline FAM59A|64762 & B2 \\
\hline FAM5B|57795 & $\mathrm{B} 2$ \\
\hline FAM5C|339479 & $\mathrm{B} 2$ \\
\hline FAM60A|58516 & $\mathrm{B} 2$ \\
\hline FAM63A|55793 & $\mathrm{B} 2$ \\
\hline FAM63B|54629 & $\mathrm{B} 2$ \\
\hline $\begin{array}{l}\text { FAM65B|9750 } \\
\end{array}$ & B2 \\
\hline FAM65C|140876 & $\mathrm{B} 2$ \\
\hline FAM66C|440078 & $\mathrm{B} 2$ \\
\hline FAM66D|100132923 & $\mathrm{B} 2$ \\
\hline FAM66E|100132103 & $\mathrm{B} 2$ \\
\hline FAM69B|138311 & B2 \\
\hline FAM69C|125704 & $\mathrm{B} 2$ \\
\hline FAM70B|348013 & B2 \\
\hline FAM71A|149647 & $\mathrm{B} 2$ \\
\hline FAM71B|153745 & B2 \\
\hline FAM71C|196472 & $\mathrm{B} 2$ \\
\hline FAM71E1|112703 & $\mathrm{B} 2$ \\
\hline FAM71E2|284418 & $\mathrm{B} 2$ \\
\hline FAM71F1|84691 & $\mathrm{B} 2$ \\
\hline FAM71F2|346653 & $\mathrm{B} 2$ \\
\hline FAM73A|374986 & $\mathrm{B} 2$ \\
\hline FAM73B|84895 & $\mathrm{B} 2$ \\
\hline FAM74A1|401507 & $\mathrm{B} 2$ \\
\hline
\end{tabular}




\begin{tabular}{|c|c|}
\hline FAM74A3|728495 & B2 \\
\hline FAM74A44401508 & $\mathrm{B} 2$ \\
\hline FAM75A2|642265 & $\mathrm{B} 2$ \\
\hline FAM75A3|727830 & $\mathrm{B} 2$ \\
\hline FAM75A5|727905 & B2 \\
\hline FAM75A6|389730 & B2 \\
\hline FAM75C1|441452 & $\mathrm{B} 2$ \\
\hline FAM76B|143684 & $\mathrm{B} 2$ \\
\hline FAM78A|286336 & $\mathrm{B} 2$ \\
\hline FAM78B|149297 & $\mathrm{B} 2$ \\
\hline FAM7A3|89837 & $\mathrm{B} 2$ \\
\hline FAM81A|145773 & B2 \\
\hline FAM81B|153643 & $\mathrm{B} 2$ \\
\hline FAM82A2|55177 & $\mathrm{B} 2$ \\
\hline FAM82B|51115 & $\mathrm{B} 2$ \\
\hline FAM83A|84985 & $\mathrm{B} 2$ \\
\hline FAM83B|222584 & $\mathrm{B} 2$ \\
\hline FAM83C|128876 & $\mathrm{B} 2$ \\
\hline FAM83E|54854 & B2 \\
\hline FAM83F|113828 & $\mathrm{B} 2$ \\
\hline FAM83G|644815 & $\mathrm{B} 2$ \\
\hline FAM84A|151354 & $\mathrm{B} 2$ \\
\hline FAM84B|157638 & $\mathrm{B} 2$ \\
\hline FAM86B1|85002 & $\mathrm{B} 2$ \\
\hline FAM86B2|653333 & $\mathrm{B} 2$ \\
\hline FAM89B|23625 & $\mathrm{B} 2$ \\
\hline FAM8A1|51439 & B2 \\
\hline FAM90A1|55138 & B2 \\
\hline FAM90A7|441317 & $\mathrm{B} 2$ \\
\hline FAM92A1|137392 & $\mathrm{B} 2$ \\
\hline FAM92A3|403315 & $\mathrm{B} 2$ \\
\hline FAM92B|339145 & $\mathrm{B} 2$ \\
\hline FAM96B|51647 & $\mathrm{B} 2$ \\
\hline FAM98A|25940 & B2 \\
\hline FAM98B|283742 & $\mathrm{B} 2$ \\
\hline FAM98C|147965 & $\mathrm{B} 2$ \\
\hline FAM9A|171482 & $\mathrm{B} 2$ \\
\hline FAM9B|171483 & $\mathrm{B} 2$ \\
\hline FAM9C|171484 & B2 \\
\hline FANCC|2176 & $\mathrm{B} 2$ \\
\hline FANCE|2178 & B2 \\
\hline FANCL 55120 & $\mathrm{~B} 2$ \\
\hline FANCM|57697 & B2 \\
\hline FANK1|92565 & $\mathrm{B} 2$ \\
\hline FAR1|84188 & B2 \\
\hline \begin{tabular}{l|l|l|} 
FAR2|55711 \\
\end{tabular} & $\mathrm{B} 2$ \\
\hline FARP2|9855 & $\mathrm{B} 2$ \\
\hline FARS2|10667 & $\mathrm{B} 2$ \\
\hline FASLG|356 & $\mathrm{B} 2$ \\
\hline FASN|2194 & $\mathrm{B} 2$ \\
\hline FASTKD1|79675 & $\mathrm{B} 2$ \\
\hline
\end{tabular}




\begin{tabular}{|c|c|}
\hline FASTKD2|22868 & B2 \\
\hline FASTKD5|60493 & $\mathrm{B} 2$ \\
\hline FASTK|10922 & $\mathrm{B} 2$ \\
\hline FAS 355 & $\mathrm{~B} 2$ \\
\hline FAT3|120114 & $\mathrm{B} 2$ \\
\hline FATE1|89885 & $\mathrm{B} 2$ \\
\hline FAU|2197 & $\mathrm{B} 2$ \\
\hline FBF1|85302 & $\mathrm{B} 2$ \\
\hline FBLIM1|54751 & B2 \\
\hline FBLL1|345630 & $\mathrm{B} 2$ \\
\hline FBLN1|2192 & $\mathrm{B} 2$ \\
\hline FBL|2091 & $\mathrm{B} 2$ \\
\hline FBN1|2200 & $\mathrm{B} 2$ \\
\hline FBN2|2201 & $\mathrm{B} 2$ \\
\hline FBN3|84467 & $\mathrm{B} 2$ \\
\hline FBP2|8789 & $\mathrm{B} 2$ \\
\hline FBRSL1|57666 & $\mathrm{B} 2$ \\
\hline FBXL12|54850 & $\mathrm{B} 2$ \\
\hline FBXL13|222235 & $\mathrm{B} 2$ \\
\hline FBXL15|79176 & $\mathrm{B} 2$ \\
\hline FBXL17|64839 & $\mathrm{B} 2$ \\
\hline FBXL18|80028 & $\mathrm{B} 2$ \\
\hline FBXL20|84961 & $\mathrm{B} 2$ \\
\hline FBXL21|26223 & $\mathrm{B} 2$ \\
\hline $\begin{array}{l}\text { FBXL2|25827 } \\
\end{array}$ & $\mathrm{B} 2$ \\
\hline \begin{tabular}{l|l|l|} 
FBXL3|26224 & \\
\end{tabular} & $\mathrm{B} 2$ \\
\hline FBXL4|26235 & $\mathrm{B} 2$ \\
\hline FBXL7|23194 & $\mathrm{B} 2$ \\
\hline FBXL8|55336 & $\mathrm{B} 2$ \\
\hline FBXO10|26267 & $\mathrm{B} 2$ \\
\hline FBXO11|80204 & $\mathrm{B} 2$ \\
\hline FBXO15|201456 & $\mathrm{B} 2$ \\
\hline FBXO18|84893 & $\mathrm{B} 2$ \\
\hline FBXO21|23014 & $\mathrm{B} 2$ \\
\hline FBXO24|26261 & $\mathrm{B} 2$ \\
\hline FBXO25|26260 & $\mathrm{B} 2$ \\
\hline FBXO27|126433 & $\mathrm{B} 2$ \\
\hline FBXO2|26232 & $\mathrm{B} 2$ \\
\hline FBXO30|84085 & $\mathrm{B} 2$ \\
\hline FBXO32|114907 & $\mathrm{B} 2$ \\
\hline FBXO34|55030 & $\mathrm{B} 2$ \\
\hline FBXO36|130888 & $\mathrm{B} 2$ \\
\hline FBXO38|81545 & $\mathrm{B} 2$ \\
\hline FBXO39|162517 & $\mathrm{B} 2$ \\
\hline FBXO3|26273 & $\mathrm{B} 2$ \\
\hline FBXO40|51725 & $\mathrm{B} 2$ \\
\hline FBXO44|93611 & $\mathrm{B} 2$ \\
\hline FBXO47|494188 & $\mathrm{B} 2$ \\
\hline FBXO4|26272 & $\mathrm{B} 2$ \\
\hline FBXO7|25793 & $\mathrm{B} 2$ \\
\hline FBXO9|26268 & $\mathrm{B} 2$ \\
\hline
\end{tabular}




\begin{tabular}{|c|c|}
\hline FBXW10|10517 & B2 \\
\hline FBXW11|23291 & B2 \\
\hline FBXW12|285231 & $\mathrm{B} 2$ \\
\hline FBXW2|26190 & $\mathrm{B} 2$ \\
\hline FBXW4|6468 & B2 \\
\hline FBXW5|54461 & B2 \\
\hline FBXW8|26259 & $\mathrm{B} 2$ \\
\hline FBXW9|84261 & $\mathrm{B} 2$ \\
\hline FCAMR|83953 & $\mathrm{B} 2$ \\
\hline FCAR/2204 & $\mathrm{B} 2$ \\
\hline FCER1A|2205 & $\mathrm{B} 2$ \\
\hline FCER1G|2207 & B2 \\
\hline FCER2|2208 & $\mathrm{B} 2$ \\
\hline FCF1|51077 & $\mathrm{B} 2$ \\
\hline FCGBP|8857 & $\mathrm{B} 2$ \\
\hline FCGR2A|2212 & $\mathrm{B} 2$ \\
\hline FCGR2B|2213 & B2 \\
\hline FCGR2C|9103 & $\mathrm{B} 2$ \\
\hline FCGR3B|2215 & B2 \\
\hline FCHO1|23149 & $\mathrm{B} 2$ \\
\hline FCHO2|115548 & $\mathrm{B} 2$ \\
\hline FCHSD1|89848 & $\mathrm{B} 2$ \\
\hline FCHSD2|9873 & $\mathrm{B} 2$ \\
\hline FCN1|2219 & $\mathrm{B} 2$ \\
\hline FCN2|2220 & $\mathrm{B} 2$ \\
\hline FCN3|8547 & $\mathrm{B} 2$ \\
\hline FCRL1|115350 & B2 \\
\hline FCRL2|79368 & B2 \\
\hline \begin{tabular}{l|l|l|} 
FCRL3|15352 \\
\end{tabular} & $\mathrm{B} 2$ \\
\hline FCRL4|83417 & $\mathrm{B} 2$ \\
\hline FCRL5|83416 & $\mathrm{B} 2$ \\
\hline FCRL6|343413 & B2 \\
\hline FCRLA|84824 & $\mathrm{B} 2$ \\
\hline FCRLB|127943 & B2 \\
\hline FDFT1|2222 & $\mathrm{B} 2$ \\
\hline FDPSL2A|619190 & $\mathrm{B} 2$ \\
\hline FDX1L|112812 & $\mathrm{B} 2$ \\
\hline FDX1|2230 & B2 \\
\hline FDXACB1|91893 & B2 \\
\hline FECH|2235 & $\mathrm{B} 2$ \\
\hline FEM1A|55527 & B2 \\
\hline FEM1B|10116 & $\mathrm{B} 2$ \\
\hline \begin{tabular}{l|l|} 
FEM1C 56929 \\
\end{tabular} & B2 \\
\hline FER1L4|80307 & $\mathrm{B} 2$ \\
\hline $\begin{array}{l}\text { FER1L5|90342 } \\
\end{array}$ & B2 \\
\hline FER1L6|654463 & $\mathrm{B} 2$ \\
\hline FERD3L|222894 & $\mathrm{B} 2$ \\
\hline FERMT1|55612 & $\mathrm{B} 2$ \\
\hline FERMT3|83706 & $\mathrm{B} 2$ \\
\hline FETUB|26998 & $\mathrm{B} 2$ \\
\hline FEV|54738 & $\mathrm{B} 2$ \\
\hline
\end{tabular}




\begin{tabular}{|c|c|}
\hline FEZF1|389549 & B2 \\
\hline FEZF2|55079 & B2 \\
\hline FFAR1|2864 & $\mathrm{B} 2$ \\
\hline FFAR2|2867 & $\mathrm{B} 2$ \\
\hline FFAR3|2865 & B2 \\
\hline FGA|2243 & $\mathrm{B} 2$ \\
\hline FGB|2244 & $\mathrm{B} 2$ \\
\hline FGD2|221472 & $\mathrm{B} 2$ \\
\hline FGF11|2256 & B2 \\
\hline FGF12|2257 & $\mathrm{B} 2$ \\
\hline FGF13|2258 & $\mathrm{B} 2$ \\
\hline FGF14|2259 & $\mathrm{B} 2$ \\
\hline FGF16|8823 & $\mathrm{B} 2$ \\
\hline FGF18|8817 & $\mathrm{B} 2$ \\
\hline FGF19|9965 & $\mathrm{B} 2$ \\
\hline $\begin{array}{l}\text { FGF20|26281 } \\
\end{array}$ & $\mathrm{B} 2$ \\
\hline FGF21|26291 & $\mathrm{B} 2$ \\
\hline FGF22|27006 & $\mathrm{B} 2$ \\
\hline \begin{tabular}{l|l|} 
FGF23|8074 &
\end{tabular} & $\mathrm{B} 2$ \\
\hline FGF3|2248 & $\mathrm{B} 2$ \\
\hline FGF4|2249 & $\mathrm{B} 2$ \\
\hline FGF5|2250 & $\mathrm{B} 2$ \\
\hline FGFBP1|9982 & $\mathrm{B} 2$ \\
\hline \begin{tabular}{l|l|} 
FGFBP2|83888 & \\
\end{tabular} & $\mathrm{B} 2$ \\
\hline FGFR2|2263 & $\mathrm{B} 2$ \\
\hline FGFR3|2261 & $\mathrm{B} 2$ \\
\hline FGFR4|2264 & $\mathrm{B} 2$ \\
\hline FGFRL1|53834 & $\mathrm{B} 2$ \\
\hline FGGY|55277 & $\mathrm{B} 2$ \\
\hline FGG|2266 & $\mathrm{B} 2$ \\
\hline FGL1|2267 & $\mathrm{B} 2$ \\
\hline \begin{tabular}{l|l|} 
FGR & 2268 \\
\end{tabular} & $\mathrm{~B} 2$ \\
\hline FHIT|2272 & $\mathrm{B} 2$ \\
\hline FHL1|2273 & $\mathrm{B} 2$ \\
\hline \begin{tabular}{l|l|} 
FHL2 2274 \\
\end{tabular} & $\mathrm{~B} 2$ \\
\hline FHL3|2275 & $\mathrm{B} 2$ \\
\hline FHOD1|29109 & $\mathrm{B} 2$ \\
\hline FIBCD1|84929 & $\mathrm{B} 2$ \\
\hline FIBIN|387758 & B2 \\
\hline FICD|11153 & $\mathrm{B} 2$ \\
\hline FIG4|9896 & $\mathrm{B} 2$ \\
\hline FIGLA|344018 & $\mathrm{B} 2$ \\
\hline \begin{tabular}{l|l|} 
FIGNL1|63979 &
\end{tabular} & $\mathrm{B} 2$ \\
\hline FILIP1|27145 & $\mathrm{B} 2$ \\
\hline FIS1|51024 & $\mathrm{B} 2$ \\
\hline FITM1|161247 & B2 \\
\hline FITM2|128486 & $\mathrm{B} 2$ \\
\hline FIZ1|84922 & $\mathrm{B} 2$ \\
\hline FJX1|24147 & $\mathrm{B} 2$ \\
\hline FKBP11|51303 & $\mathrm{B} 2$ \\
\hline FKBP14|55033 & B2 \\
\hline
\end{tabular}




\begin{tabular}{|c|c|}
\hline FKBP15|23307 & B2 \\
\hline FKBP1AP1|2282 & $\mathrm{B} 2$ \\
\hline FKBP1A|2280 & $\mathrm{B} 2$ \\
\hline FKBP2|2286 & $\mathrm{B} 2$ \\
\hline FKBP5|2289 & B2 \\
\hline FKBP6|8468 & $\mathrm{B} 2$ \\
\hline FKBP7|51661 & $\mathrm{B} 2$ \\
\hline FKBP8|23770 & $\mathrm{B} 2$ \\
\hline FKBP9L|360132 & $\mathrm{B} 2$ \\
\hline FKBP9|11328 & $\mathrm{B} 2$ \\
\hline FKRP|79147 & $\mathrm{B} 2$ \\
\hline FKSG29|100131561 & B2 \\
\hline FKSG83|83954 & $\mathrm{B} 2$ \\
\hline FLG2|388698 & $\mathrm{B} 2$ \\
\hline FLII $\mid 2314$ & $\mathrm{~B} 2$ \\
\hline FLJ10213|55096 & $\mathrm{B} 2$ \\
\hline FLJ10661|286042 & B2 \\
\hline FLJ11235|54508 & $\mathrm{B} 2$ \\
\hline FLJ12825|440101 & B2 \\
\hline FLJ13224|79857 & $\mathrm{B} 2$ \\
\hline FLJ14107|80094 & $\mathrm{B} 2$ \\
\hline FLJ16779|100192386 & $\mathrm{B} 2$ \\
\hline FLJ22536|401237 & $\mathrm{B} 2$ \\
\hline FLJ23867|200058 & $\mathrm{B} 2$ \\
\hline FLJ25328|148231 & $\mathrm{B} 2$ \\
\hline FLJ25758|497049 & $\mathrm{B} 2$ \\
\hline FLJ30679|146512 & $\mathrm{B} 2$ \\
\hline FLJ32063|150538 & B2 \\
\hline FLJ33630|644873 & $\mathrm{B} 2$ \\
\hline FLJ34503|285759 & $\mathrm{B} 2$ \\
\hline FLJ35220|284131 & $\mathrm{B} 2$ \\
\hline FLJ35390|255031 & $\mathrm{B} 2$ \\
\hline FLJ35776|649446 & $\mathrm{B} 2$ \\
\hline FLJ36000|284124 & B2 \\
\hline FLJ36031|168455 & $\mathrm{B} 2$ \\
\hline FLJ37201|283011 & $\mathrm{B} 2$ \\
\hline FLJ37307|283521 & $\mathrm{B} 2$ \\
\hline $\begin{array}{l}\text { FLJ37453|729614 } \\
\end{array}$ & $\mathrm{B} 2$ \\
\hline FLJ39609|100130417 & B2 \\
\hline FLJ39739|388685 & $\mathrm{B} 2$ \\
\hline FLJ40292|643210 & B2 \\
\hline FLJ40330|645784 & $\mathrm{B} 2$ \\
\hline FLJ40504|284085 & B2 \\
\hline FLJ40852|285962 & $\mathrm{B} 2$ \\
\hline FLJ41941|100192420 & $\mathrm{B} 2$ \\
\hline FLJ42289|388182 & $\mathrm{B} 2$ \\
\hline FLJ42393|401105 & $\mathrm{B} 2$ \\
\hline FLJ42627|645644 & $\mathrm{B} 2$ \\
\hline FLJ42709|441094 & $\mathrm{B} 2$ \\
\hline FLJ43859|389761 & $\mathrm{B} 2$ \\
\hline FLJ43860|389690 & $\mathrm{B} 2$ \\
\hline
\end{tabular}




\begin{tabular}{|c|c|}
\hline FLJ43950|347127 & B2 \\
\hline FLJ44054|643365 & $\mathrm{B} 2$ \\
\hline FLJ44082|389762 & $\mathrm{B} 2$ \\
\hline FLJ44606|401207 & $\mathrm{B} 2$ \\
\hline FLJ45079|400624 & B2 \\
\hline FLJ45244|400242 & B2 \\
\hline FLJ45445|399844 & $\mathrm{B} 2$ \\
\hline FLJ46111|283102 & B2 \\
\hline FLJ46321|389763 & $\mathrm{B} 2$ \\
\hline FLJ90757|440465 & $\mathrm{B} 2$ \\
\hline FLNA|2316 & $\mathrm{B} 2$ \\
\hline FLNB|2317 & B2 \\
\hline FLNC|2318 & $\mathrm{B} 2$ \\
\hline FLOT1|10211 & $\mathrm{B} 2$ \\
\hline FLOT2|2319 & $\mathrm{B} 2$ \\
\hline FLRT1|23769 & $\mathrm{B} 2$ \\
\hline FLRT3|23767 & B2 \\
\hline FLT1|2321 & $\mathrm{B} 2$ \\
\hline FLT3|2322 & B2 \\
\hline FLVCR2|55640 & $\mathrm{B} 2$ \\
\hline FLYWCH1|84256 & $\mathrm{B} 2$ \\
\hline FMN1|342184 & $\mathrm{B} 2$ \\
\hline FMN2|56776 & $\mathrm{B} 2$ \\
\hline FMNL1|752 & $\mathrm{B} 2$ \\
\hline \begin{tabular}{l|l|l|} 
FMNL3|91010 \\
\end{tabular} & $\mathrm{B} 2$ \\
\hline FMO1|2326 & $\mathrm{B} 2$ \\
\hline FMO5|2330 & B2 \\
\hline FMO6P|388714 & B2 \\
\hline FMO9P|116123 & $\mathrm{B} 2$ \\
\hline FMR1NB|158521 & $\mathrm{B} 2$ \\
\hline FMR1|2332 & $\mathrm{B} 2$ \\
\hline FN3KRP|79672 & $\mathrm{B} 2$ \\
\hline FN3K|64122 & $\mathrm{B} 2$ \\
\hline FNBP1L|54874 & B2 \\
\hline FNBP1|23048 & $\mathrm{B} 2$ \\
\hline FNBP4|23360 & $\mathrm{B} 2$ \\
\hline FNDC3B|64778 & $\mathrm{B} 2$ \\
\hline FNDC5|252995 & $\mathrm{B} 2$ \\
\hline FNDC7|163479 & B2 \\
\hline \begin{tabular}{l|l|} 
FNDC8 & 54752 \\
\end{tabular} & $\mathrm{~B} 2$ \\
\hline FNIP1|96459 & B2 \\
\hline FNTA|2339 & $\mathrm{B} 2$ \\
\hline \begin{tabular}{l|l|} 
FNTB 2342 \\
\end{tabular} & B2 \\
\hline FOLH1B|219595 & $\mathrm{B} 2$ \\
\hline FOLH1|2346 & $\mathrm{B} 2$ \\
\hline FOLR1|2348 & $\mathrm{B} 2$ \\
\hline FOLR3|2352 & $\mathrm{B} 2$ \\
\hline FOLR4|390243 & $\mathrm{B} 2$ \\
\hline FOSL1|8061 & $\mathrm{B} 2$ \\
\hline FOSL2|2355 & $\mathrm{B} 2$ \\
\hline FOXA2|3170 & $\mathrm{B} 2$ \\
\hline
\end{tabular}




\begin{tabular}{|c|c|}
\hline FOXA3|3171 & B2 \\
\hline FOXB1|27023 & B2 \\
\hline FOXB2|442425 & $\mathrm{B} 2$ \\
\hline FOXC1|2296 & $\mathrm{B} 2$ \\
\hline FOXC2|2303 & B2 \\
\hline FOXD1|2297 & $\mathrm{B} 2$ \\
\hline FOXD3|27022 & $\mathrm{B} 2$ \\
\hline FOXD4L2|100036519 & $\mathrm{B} 2$ \\
\hline FOXD4L3|286380 & $\mathrm{B} 2$ \\
\hline FOXD4L5|653427 & $\mathrm{B} 2$ \\
\hline FOXD4L6|653404 & $\mathrm{B} 2$ \\
\hline FOXD4|2298 & B2 \\
\hline FOXE1|2304 & $\mathrm{B} 2$ \\
\hline FOXE3|2301 & $\mathrm{B} 2$ \\
\hline FOXF1|2294 & $\mathrm{B} 2$ \\
\hline FOXF2|2295 & $\mathrm{B} 2$ \\
\hline FOXG1|2290 & $\mathrm{B} 2$ \\
\hline FOXI1|2299 & $\mathrm{B} 2$ \\
\hline FOXI2|399823 & $\mathrm{B} 2$ \\
\hline FOXI3|344167 & $\mathrm{B} 2$ \\
\hline FOXJ1|2302 & $\mathrm{B} 2$ \\
\hline FOXJ2|55810 & $\mathrm{B} 2$ \\
\hline FOXJ3|22887 & $\mathrm{B} 2$ \\
\hline FOXK1|221937 & $\mathrm{B} 2$ \\
\hline FOXL1|2300 & $\mathrm{B} 2$ \\
\hline FOXL2|668 & $\mathrm{B} 2$ \\
\hline FOXN1|8456 & $\mathrm{B} 2$ \\
\hline FOXN4|121643 & $\mathrm{B} 2$ \\
\hline FOXO3B|2310 & $\mathrm{B} 2$ \\
\hline FOXO3|2309 & $\mathrm{B} 2$ \\
\hline FOXP1|27086 & $\mathrm{B} 2$ \\
\hline FOXP4|116113 & $\mathrm{B} 2$ \\
\hline FOXQ1|94234 & $\mathrm{B} 2$ \\
\hline FOXR1|283150 & $\mathrm{B} 2$ \\
\hline FOXR2|139628 & $\mathrm{B} 2$ \\
\hline FOXRED1|55572 & $\mathrm{B} 2$ \\
\hline FPGS|2356 & $\mathrm{B} 2$ \\
\hline FPGT|8790 & $\mathrm{B} 2$ \\
\hline FPR1|2357 & $\mathrm{B} 2$ \\
\hline \begin{tabular}{l|l|l|} 
FPR2 2358 \\
\end{tabular} & $\mathrm{~B} 2$ \\
\hline \begin{tabular}{l|l|l|} 
FPR3 2359 \\
\end{tabular} & $\mathrm{~B} 2$ \\
\hline FRAS1|80144 & $\mathrm{B} 2$ \\
\hline FRAT1|10023 & $\mathrm{B} 2$ \\
\hline FREM2|341640 & $\mathrm{B} 2$ \\
\hline FRG2B|441581 & $\mathrm{B} 2$ \\
\hline FRG2C|100288801 & $\mathrm{B} 2$ \\
\hline FRG2|448831 & $\mathrm{B} 2$ \\
\hline FRMD5|84978 & $\mathrm{B} 2$ \\
\hline FRMD6|122786 & $\mathrm{B} 2$ \\
\hline FRMD7|90167 & $\mathrm{B} 2$ \\
\hline FRMD8|83786 & $\mathrm{B} 2$ \\
\hline
\end{tabular}




\begin{tabular}{|c|c|}
\hline FRMPD2L1|728798 & B2 \\
\hline FRMPD2|143162 & $\mathrm{B} 2$ \\
\hline FRRS1|391059 & $\mathrm{B} 2$ \\
\hline FRS2|10818 & $\mathrm{B} 2$ \\
\hline FRS3|10817 & B2 \\
\hline FRYL|285527 & $\mathrm{B} 2$ \\
\hline FRZB|2487 & $\mathrm{B} 2$ \\
\hline FSCB $\mid 84075$ & $\mathrm{~B} 2$ \\
\hline FSCN1|6624 & $\mathrm{B} 2$ \\
\hline \begin{tabular}{l|l|l|} 
FSCN25794 \\
\end{tabular} & $\mathrm{B} 2$ \\
\hline FSCN3|29999 & $\mathrm{B} 2$ \\
\hline FSD1L|83856 & B2 \\
\hline FSD1|79187 & $\mathrm{B} 2$ \\
\hline FSD2|123722 & $\mathrm{B} 2$ \\
\hline FSHB|2488 & $\mathrm{B} 2$ \\
\hline FSHR|2492 & $\mathrm{B} 2$ \\
\hline FSTL3|10272 & B2 \\
\hline FSTL4|23105 & $\mathrm{B} 2$ \\
\hline FSTL5|56884 & B2 \\
\hline FST $\mid 10468$ & $\mathrm{~B} 2$ \\
\hline FTCD|10841 & $\mathrm{B} 2$ \\
\hline FTH1|2495 & $\mathrm{B} 2$ \\
\hline FTHL3|2498 & $\mathrm{B} 2$ \\
\hline FTL|2512 & $\mathrm{B} 2$ \\
\hline FTMT|94033 & $\mathrm{B} 2$ \\
\hline FTSJ3|117246 & $\mathrm{B} 2$ \\
\hline FTSJD1|55783 & $\mathrm{B} 2$ \\
\hline FTSJD2|23070 & B2 \\
\hline $\begin{array}{ll}\text { FUBP3|8939 } \\
\end{array}$ & $\mathrm{B} 2$ \\
\hline FUCA1|2517 & $\mathrm{B} 2$ \\
\hline FUCA2|2519 & $\mathrm{B} 2$ \\
\hline FUK|197258 & B2 \\
\hline FUNDC2P2|388965 & $\mathrm{B} 2$ \\
\hline FUNDC2|65991 & B2 \\
\hline FURIN|5045 & $\mathrm{B} 2$ \\
\hline FUT11|170384 & $\mathrm{B} 2$ \\
\hline FUT1|2523 & $\mathrm{B} 2$ \\
\hline FUT3|2525 & $\mathrm{B} 2$ \\
\hline FUT5|2527 & B2 \\
\hline FUT6|2528 & $\mathrm{B} 2$ \\
\hline FUT7|2529 & B2 \\
\hline FUT9|10690 & $\mathrm{B} 2$ \\
\hline FUZ|80199 & B2 \\
\hline FXC1|26515 & $\mathrm{B} 2$ \\
\hline FXN|2395 & $\mathrm{B} 2$ \\
\hline FXR1|8087 & $\mathrm{B} 2$ \\
\hline FXR2|9513 & $\mathrm{B} 2$ \\
\hline FXYD4|53828 & $\mathrm{B} 2$ \\
\hline $\begin{array}{l}\text { FXYD5|53827 } \\
\end{array}$ & $\mathrm{B} 2$ \\
\hline FXYD7|53822 & $\mathrm{B} 2$ \\
\hline FYB $\mid 2533$ & B2 \\
\hline
\end{tabular}




\begin{tabular}{|c|c|}
\hline FYCO1|79443 & B2 \\
\hline FYTTD1|84248 & $\mathrm{B} 2$ \\
\hline FZD10|11211 & B2 \\
\hline FZD1|8321 & $\mathrm{B} 2$ \\
\hline FZD3|7976 & $\mathrm{B} 2$ \\
\hline FZD6|8323 & B2 \\
\hline FZD8|8325 & B2 \\
\hline FZD9|8326 & $\mathrm{B} 2$ \\
\hline FZR1|51343 & B2 \\
\hline G2E3|55632 & $\mathrm{B} 2$ \\
\hline G3BP2|9908 & B2 \\
\hline G6PC2|57818 & $\mathrm{B} 2$ \\
\hline G6PC|2538 & $\mathrm{B} 2$ \\
\hline G6PD|2539 & $\mathrm{B} 2$ \\
\hline GAA|2548 & $\mathrm{B} 2$ \\
\hline GAB1|2549 & $\mathrm{B} 2$ \\
\hline GAB2|9846 & $\mathrm{B} 2$ \\
\hline GAB4|128954 & $\mathrm{B} 2$ \\
\hline GABARAPL2|11345 & $\mathrm{B} 2$ \\
\hline GABARAP|11337 & $\mathrm{B} 2$ \\
\hline GABBR2|9568 & $\mathrm{B} 2$ \\
\hline GABPB2|126626 & $\mathrm{B} 2$ \\
\hline GABRA1|2554 & $\mathrm{B} 2$ \\
\hline GABRA3|2556 & $\mathrm{B} 2$ \\
\hline GABRA5|2558 & $\mathrm{B} 2$ \\
\hline GABRB1|2560 & $\mathrm{B} 2$ \\
\hline GABRB2|2561 & $\mathrm{B} 2$ \\
\hline GABRB3|2562 & $\mathrm{B} 2$ \\
\hline GABRG2|2566 & $\mathrm{B} 2$ \\
\hline GABRG3|2567 & $\mathrm{B} 2$ \\
\hline GABRP|2568 & $\mathrm{B} 2$ \\
\hline GABRQ|55879 & $\mathrm{B} 2$ \\
\hline GABRR1|2569 & $\mathrm{B} 2$ \\
\hline $\begin{array}{l}\text { GABRR2|2570 } \\
\text { GABR }\end{array}$ & B2 \\
\hline GABRR3|200959 & $\mathrm{B} 2$ \\
\hline GAD1|2571 & $\mathrm{B} 2$ \\
\hline GAD2|2572 & $\mathrm{B} 2$ \\
\hline GADD45A|1647 & $\mathrm{B} 2$ \\
\hline GADD45B|4616 & $\mathrm{B} 2$ \\
\hline GADD45GIP1|90480 & $\mathrm{B} 2$ \\
\hline GADD45G|10912 & $\mathrm{B} 2$ \\
\hline GADL1|339896 & $\mathrm{B} 2$ \\
\hline GAGE10|643832 & $\mathrm{B} 2$ \\
\hline GAGE12D|100132399 & $\mathrm{B} 2$ \\
\hline GAGE12J|729396 & B2 \\
\hline GAGE1|2543 & $\mathrm{B} 2$ \\
\hline GAGE2A|729447 & $\mathrm{B} 2$ \\
\hline GAGE4|2576 & $\mathrm{B} 2$ \\
\hline GAGE8|100101629 & $\mathrm{B} 2$ \\
\hline GAL3ST1|9514 & $\mathrm{B} 2$ \\
\hline GAL3ST2|64090 & B2 \\
\hline
\end{tabular}




\begin{tabular}{|c|c|}
\hline GALC $\mid 2581$ & B2 \\
\hline GALK1|2584 & $\mathrm{B} 2$ \\
\hline GALK2|2585 & B2 \\
\hline GALM|130589 & $\mathrm{B} 2$ \\
\hline GALNT13|114805 & $\mathrm{B} 2$ \\
\hline GALNT14|79623 & $\mathrm{B} 2$ \\
\hline GALNT2|2590 & $\mathrm{B} 2$ \\
\hline GALNT3|2591 & B2 \\
\hline GALNT4|8693 & $\mathrm{B} 2$ \\
\hline GALNT5|11227 & B2 \\
\hline GALNT8|26290 & $\mathrm{B} 2$ \\
\hline GALNT9|50614 & $\mathrm{B} 2$ \\
\hline GALNTL4|374378 & $\mathrm{B} 2$ \\
\hline GALNTL6|442117 & $\mathrm{B} 2$ \\
\hline GALP $\mid 85569$ & B2 \\
\hline GALR2|8811 & $\mathrm{B} 2$ \\
\hline GALR3|8484 & $\mathrm{B} 2$ \\
\hline GAL|51083 & B2 \\
\hline GAMT|2593 & $\mathrm{B} 2$ \\
\hline GAN|8139 & $\mathrm{B} 2$ \\
\hline GAP43|2596 & B2 \\
\hline GAPDHS|26330 & $\mathrm{B} 2$ \\
\hline GAPDH|2597 & $\mathrm{B} 2$ \\
\hline GAPVD1|26130 & $\mathrm{B} 2$ \\
\hline GAR1|54433 & B2 \\
\hline GARNL3|84253 & $\mathrm{B} 2$ \\
\hline GAS2L1|10634 & $\mathrm{B} 2$ \\
\hline GAS5|60674 & $\mathrm{B} 2$ \\
\hline GAS8|2622 & $\mathrm{B} 2$ \\
\hline GAST 2520 & $\mathrm{~B} 2$ \\
\hline GATA1|2623 & $\mathrm{B} 2$ \\
\hline GATA2|2624 & $\mathrm{B} 2$ \\
\hline GATA4|2626 & $\mathrm{B} 2$ \\
\hline GATA5|140628 & $\mathrm{B} 2$ \\
\hline GATAD1|57798 & $\mathrm{B} 2$ \\
\hline $\begin{array}{l}\text { GATAD2B|57459 } \\
\end{array}$ & $\mathrm{B} 2$ \\
\hline GATC|283459 & $\mathrm{B} 2$ \\
\hline GATM|2628 & $\mathrm{B} 2$ \\
\hline GATSL1|389523 & $\mathrm{B} 2$ \\
\hline \begin{tabular}{l|l|} 
GATSL2|729438 \\
\end{tabular} & $\mathrm{B} 2$ \\
\hline $\begin{array}{l}\text { GATSL3|652968 } \\
\end{array}$ & $\mathrm{B} 2$ \\
\hline GATS|352954 & $\mathrm{B} 2$ \\
\hline GBA2|57704 & $\mathrm{B} 2$ \\
\hline GBA3|57733 & B2 \\
\hline GBAS|2631 & $\mathrm{B} 2$ \\
\hline GBGT1|26301 & $\mathrm{B} 2$ \\
\hline GBP1|2633 & $\mathrm{B} 2$ \\
\hline GBP2|2634 & $\mathrm{B} 2$ \\
\hline GBP3|2635 & $\mathrm{B} 2$ \\
\hline GBP4|115361 & $\mathrm{B} 2$ \\
\hline GBP5|115362 & $\mathrm{B} 2$ \\
\hline
\end{tabular}




\begin{tabular}{|c|c|}
\hline GBP6|163351 & B2 \\
\hline GBP7|388646 & B2 \\
\hline GBX1|2636 & B2 \\
\hline GBX2|2637 & $\mathrm{B} 2$ \\
\hline GCA|25801 & B2 \\
\hline GCC1|79571 & $\mathrm{B} 2$ \\
\hline GCC2|9648 & B2 \\
\hline GCDH|2639 & $\mathrm{B} 2$ \\
\hline GCET2|257144 & B2 \\
\hline GCFC1|94104 & B2 \\
\hline GCGR|2642 & B2 \\
\hline GCG|2641 & B2 \\
\hline GCHFR|2644 & $\mathrm{B} 2$ \\
\hline GCKR|2646 & B2 \\
\hline GCK|2645 & $\mathrm{B} 2$ \\
\hline GCLC 2729 & B2 \\
\hline GCLM|2730 & $\mathrm{B} 2$ \\
\hline GCM1|8521 & B2 \\
\hline GCM2|9247 & $\mathrm{B} 2$ \\
\hline GCN1L1|10985 & $\mathrm{B} 2$ \\
\hline GCNT1|2650 & B2 \\
\hline GCNT2|2651 & $\mathrm{B} 2$ \\
\hline GCNT3|9245 & $\mathrm{B} 2$ \\
\hline GCNT7|140687 & B2 \\
\hline GCSH|2653 & $\mathrm{B} 2$ \\
\hline GC|2638 & $\mathrm{B} 2$ \\
\hline GDAP1L1|78997 & $\mathrm{B} 2$ \\
\hline GDAP1|54332 & B2 \\
\hline GDAP2|54834 & $\mathrm{B} 2$ \\
\hline GDA|9615 & $\mathrm{B} 2$ \\
\hline GDEP|118425 & $\mathrm{B} 2$ \\
\hline GDF11|10220 & $\mathrm{B} 2$ \\
\hline GDF15|9518 & $\mathrm{B} 2$ \\
\hline GDF1|2657 & $\mathrm{B} 2$ \\
\hline GDF2|2658 & $\mathrm{B} 2$ \\
\hline GDF3|9573 & B2 \\
\hline GDF5|8200 & $\mathrm{B} 2$ \\
\hline GDF6|392255 & $\mathrm{B} 2$ \\
\hline GDF7|151449 & $\mathrm{B} 2$ \\
\hline GDF9|2661 & $\mathrm{B} 2$ \\
\hline GDNF|2668 & $\mathrm{B} 2$ \\
\hline GDPD1|284161 & $\mathrm{B} 2$ \\
\hline GDPD2|54857 & $\mathrm{B} 2$ \\
\hline GDPD3|79153 & $\mathrm{B} 2$ \\
\hline GDPD4|220032 & $\mathrm{B} 2$ \\
\hline $\begin{array}{l}\text { GEMIN5|25929 } \\
\end{array}$ & $\mathrm{B} 2$ \\
\hline GEMIN8P4|492303 & $\mathrm{B} 2$ \\
\hline GEMIN8|54960 & $\mathrm{B} 2$ \\
\hline GET4|51608 & $\mathrm{B} 2$ \\
\hline GFI1B|8328 & B2 \\
\hline GFI1|2672 & B2 \\
\hline
\end{tabular}




\begin{tabular}{|c|c|}
\hline GFM2|84340 & B2 \\
\hline GFOD2|81577 & $\mathrm{B} 2$ \\
\hline GFPT1|2673 & $\mathrm{B} 2$ \\
\hline GFPT2|9945 & $\mathrm{B} 2$ \\
\hline GFRA1|2674 & B2 \\
\hline GFRA3|2676 & $\mathrm{B} 2$ \\
\hline GFRAL|389400 & $\mathrm{B} 2$ \\
\hline GGA1|26088 & $\mathrm{B} 2$ \\
\hline GGA2|23062 & B2 \\
\hline GGA3|23163 & $\mathrm{B} 2$ \\
\hline GGCX|2677 & $\mathrm{B} 2$ \\
\hline GGH|8836 & B2 \\
\hline GGNBP1|449520 & $\mathrm{B} 2$ \\
\hline GGNBP2|79893 & $\mathrm{B} 2$ \\
\hline GGN|199720 & B2 \\
\hline GGT1|2678 & $\mathrm{B} 2$ \\
\hline GGT3P|2679 & $\mathrm{B} 2$ \\
\hline 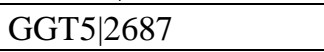 & $\mathrm{B} 2$ \\
\hline GGT6|124975 & B2 \\
\hline GGT8P|645367 & $\mathrm{B} 2$ \\
\hline GGTLC1|92086 & $\mathrm{B} 2$ \\
\hline GGTLC2|91227 & $\mathrm{B} 2$ \\
\hline GH1|2688 & $\mathrm{B} 2$ \\
\hline 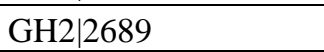 & $\mathrm{B} 2$ \\
\hline GHDC|84514 & $\mathrm{B} 2$ \\
\hline GHITM|27069 & $\mathrm{B} 2$ \\
\hline GHRHR|2692 & $\mathrm{B} 2$ \\
\hline GHRH|2691 & B2 \\
\hline GHRLOS|100126793 & $\mathrm{B} 2$ \\
\hline GHRL|51738 & $\mathrm{B} 2$ \\
\hline GHSR|2693 & $\mathrm{B} 2$ \\
\hline GIF|2694 & $\mathrm{B} 2$ \\
\hline GIGYF2|26058 & $\mathrm{B} 2$ \\
\hline GIMAP2|26157 & B2 \\
\hline GIN1|54826 & $\mathrm{B} 2$ \\
\hline GIPC3|126326 & $\mathrm{B} 2$ \\
\hline GIPR/2696 & $\mathrm{B} 2$ \\
\hline GIP|2695 & $\mathrm{B} 2$ \\
\hline GIT1|28964 & B2 \\
\hline GJA10|84694 & $\mathrm{B} 2$ \\
\hline GJA1|2697 & B2 \\
\hline GJA4|2701 & $\mathrm{B} 2$ \\
\hline GJA5|2702 & B2 \\
\hline GJA8|2703 & $\mathrm{B} 2$ \\
\hline $\begin{array}{ll}\text { GJA9|81025 } \\
\end{array}$ & B2 \\
\hline GJB1|2705 & $\mathrm{B} 2$ \\
\hline GJB3|2707 & $\mathrm{B} 2$ \\
\hline GJB4|127534 & $\mathrm{B} 2$ \\
\hline GJB5|2709 & $\mathrm{B} 2$ \\
\hline GJB6|10804 & $\mathrm{B} 2$ \\
\hline GJB7|375519 & $\mathrm{B} 2$ \\
\hline
\end{tabular}




\begin{tabular}{|c|c|}
\hline GJC1|10052 & B2 \\
\hline GJC3|349149 & $\mathrm{B} 2$ \\
\hline GJD4|219770 & $\mathrm{B} 2$ \\
\hline GK5|256356 & $\mathrm{B} 2$ \\
\hline GKAP1|80318 & $\mathrm{B} 2$ \\
\hline GKN1|56287 & B2 \\
\hline GKN2|200504 & $\mathrm{B} 2$ \\
\hline GLA|2717 & $\mathrm{B} 2$ \\
\hline GLB1L2|89944 & B2 \\
\hline GLB1L3|112937 & $\mathrm{B} 2$ \\
\hline GLB1L|79411 & $\mathrm{B} 2$ \\
\hline GLCCI1|113263 & $\mathrm{B} 2$ \\
\hline GLCE|26035 & $\mathrm{B} 2$ \\
\hline GLDC|2731 & $\mathrm{B} 2$ \\
\hline GLI2|2736 & B2 \\
\hline GLI3|2737 & $\mathrm{B} 2$ \\
\hline GLI4|2738 & $\mathrm{B} 2$ \\
\hline GLIPR1L1|256710 & $\mathrm{B} 2$ \\
\hline GLIPR1L2|144321 & B2 \\
\hline GLIPR1|11010 & $\mathrm{B} 2$ \\
\hline GLIPR2|152007 & $\mathrm{B} 2$ \\
\hline GLIS1|148979 & $\mathrm{B} 2$ \\
\hline GLMN|11146 & $\mathrm{B} 2$ \\
\hline GLOD4|51031 & $\mathrm{B} 2$ \\
\hline GLOD5|392465 & $\mathrm{B} 2$ \\
\hline GLP1R|2740 & $\mathrm{B} 2$ \\
\hline GLRA1|2741 & $\mathrm{B} 2$ \\
\hline GLRA2|2742 & B2 \\
\hline GLRA3|8001 & $\mathrm{B} 2$ \\
\hline GLRB|2743 & $\mathrm{B} 2$ \\
\hline \begin{tabular}{l|l|l|} 
GLRX5|51218 \\
\end{tabular} & $\mathrm{B} 2$ \\
\hline GLRX|2745 & $\mathrm{B} 2$ \\
\hline GLS2|27165 & $\mathrm{B} 2$ \\
\hline GLS|2744 & $\mathrm{B} 2$ \\
\hline GLT1D1|144423 & $\mathrm{B} 2$ \\
\hline GLT8D1|55830 & $\mathrm{B} 2$ \\
\hline \begin{tabular}{l|l|} 
GLT8D2|83468 \\
\end{tabular} & $\mathrm{B} 2$ \\
\hline GLTPD1|80772 & $\mathrm{B} 2$ \\
\hline GLTPD2|388323 & $\mathrm{B} 2$ \\
\hline GLTP|51228 & $\mathrm{B} 2$ \\
\hline GLTSCR1|29998 & B2 \\
\hline GLUD1|2746 & $\mathrm{B} 2$ \\
\hline GLUD2|2747 & B2 \\
\hline GLUL|2752 & $\mathrm{B} 2$ \\
\hline GLYATL1|92292 & B2 \\
\hline GLYATL2|219970 & $\mathrm{B} 2$ \\
\hline GLYATL3|389396 & $\mathrm{B} 2$ \\
\hline GLYCAM1|644076 & $\mathrm{B} 2$ \\
\hline GLYCTK|132158 & $\mathrm{B} 2$ \\
\hline GM2A|2760 & $\mathrm{B} 2$ \\
\hline GMCL1L|64396 & $\mathrm{B} 2$ \\
\hline
\end{tabular}




\begin{tabular}{|c|c|}
\hline GMCL1|64395 & B2 \\
\hline GMEB1|10691 & B2 \\
\hline GMEB2|26205 & B2 \\
\hline GMFB|2764 & $\mathrm{B} 2$ \\
\hline GMFG|9535 & $\mathrm{B} 2$ \\
\hline GML $\mid 2765$ & $\mathrm{~B} 2$ \\
\hline GMNN|51053 & $\mathrm{B} 2$ \\
\hline GMPR2|51292 & $\mathrm{B} 2$ \\
\hline GMPR|2766 & $\mathrm{B} 2$ \\
\hline GNA12|2768 & $\mathrm{B} 2$ \\
\hline GNA13|10672 & $\mathrm{B} 2$ \\
\hline GNA14|9630 & $\mathrm{B} 2$ \\
\hline GNA15|2769 & $\mathrm{B} 2$ \\
\hline GNAI2|2771 & $\mathrm{B} 2$ \\
\hline GNAO1|2775 & $\mathrm{B} 2$ \\
\hline GNASAS|149775 & $\mathrm{B} 2$ \\
\hline GNAS|2778 & $\mathrm{B} 2$ \\
\hline GNAT1|2779 & $\mathrm{B} 2$ \\
\hline GNAT2|2780 & $\mathrm{B} 2$ \\
\hline GNAT3|346562 & $\mathrm{B} 2$ \\
\hline GNB1L|54584 & $\mathrm{B} 2$ \\
\hline GNB1|2782 & $\mathrm{B} 2$ \\
\hline GNB2L1|10399 & $\mathrm{B} 2$ \\
\hline GNB3|2784 & $\mathrm{B} 2$ \\
\hline GNB4|59345 & $\mathrm{B} 2$ \\
\hline GNG10|2790 & $\mathrm{B} 2$ \\
\hline GNG13|51764 & $\mathrm{B} 2$ \\
\hline $\begin{array}{l}\text { GNG3|2785 } \\
\end{array}$ & $\mathrm{B} 2$ \\
\hline GNG4|2786 & $\mathrm{B} 2$ \\
\hline GNG8|94235 & $\mathrm{B} 2$ \\
\hline GNGT1|2792 & $\mathrm{B} 2$ \\
\hline GNGT2|2793 & $\mathrm{B} 2$ \\
\hline GNL3|26354 & $\mathrm{B} 2$ \\
\hline GNLY|10578 & $\mathrm{B} 2$ \\
\hline GNMT|27232 & $\mathrm{B} 2$ \\
\hline GNPDA2|132789 & $\mathrm{B} 2$ \\
\hline GNPTAB|79158 & $\mathrm{B} 2$ \\
\hline GNPTG|84572 & $\mathrm{B} 2$ \\
\hline \begin{tabular}{l|l|l|} 
GNRH2 2797 \\
\end{tabular} & $\mathrm{~B} 2$ \\
\hline GNRHR2|114814 & $\mathrm{B} 2$ \\
\hline GNRHR|2798 & $\mathrm{B} 2$ \\
\hline GNS|2799 & $\mathrm{B} 2$ \\
\hline GOLGA1|2800 & $\mathrm{B} 2$ \\
\hline GOLGA2B|55592 & $\mathrm{B} 2$ \\
\hline GOLGA3|2802 & $\mathrm{B} 2$ \\
\hline GOLGA4|2803 & $\mathrm{B} 2$ \\
\hline GOLGA6A|342096 & $\mathrm{B} 2$ \\
\hline GOLGA6B|55889 & $\mathrm{B} 2$ \\
\hline GOLGA6C|653641 & $\mathrm{B} 2$ \\
\hline GOLGA6L1|283767 & $\mathrm{B} 2$ \\
\hline GOLGA6L6|727832 & $\mathrm{B} 2$ \\
\hline
\end{tabular}




\begin{tabular}{|c|c|}
\hline GOLGA6L9|440295 & B2 \\
\hline GOLGA7B|401647 & $\mathrm{B} 2$ \\
\hline GOLGA7|51125 & $\mathrm{B} 2$ \\
\hline GOLGA8DP|100132979 & $\mathrm{B} 2$ \\
\hline GOLGA8E|390535 & $\mathrm{B} 2$ \\
\hline GOLGA8G|283768 & $\mathrm{B} 2$ \\
\hline GOLGB1|2804 & $\mathrm{B} 2$ \\
\hline GOLIM4|27333 & $\mathrm{B} 2$ \\
\hline GOPC|57120 & B2 \\
\hline GOSR1|9527 & $\mathrm{B} 2$ \\
\hline GOSR2|9570 & $\mathrm{B} 2$ \\
\hline GOT1L1|137362 & $\mathrm{B} 2$ \\
\hline GOT1|2805 & $\mathrm{B} 2$ \\
\hline GOT2|2806 & $\mathrm{B} 2$ \\
\hline GP1BA|2811 & B2 \\
\hline GP2|2813 & $\mathrm{B} 2$ \\
\hline GP5|2814 & $\mathrm{B} 2$ \\
\hline GP6|51206 & $\mathrm{B} 2$ \\
\hline GP9|2815 & B2 \\
\hline GPA33|10223 & $\mathrm{B} 2$ \\
\hline GPAT2|150763 & $\mathrm{B} 2$ \\
\hline GPATCH1|55094 & $\mathrm{B} 2$ \\
\hline GPATCH3|63906 & $\mathrm{B} 2$ \\
\hline GPATCH8|23131 & $\mathrm{B} 2$ \\
\hline GPBP1L1|60313 & $\mathrm{B} 2$ \\
\hline GPBP1|65056 & $\mathrm{B} 2$ \\
\hline GPC1|2817 & $\mathrm{B} 2$ \\
\hline GPC2|221914 & B2 \\
\hline GPC5|2262 & $\mathrm{B} 2$ \\
\hline GPC6|10082 & $\mathrm{B} 2$ \\
\hline GPCPD1|56261 & $\mathrm{B} 2$ \\
\hline GPD1L|23171 & $\mathrm{B} 2$ \\
\hline GPD2|2820 & $\mathrm{B} 2$ \\
\hline GPHA2|170589 & $\mathrm{B} 2$ \\
\hline GPHN|10243 & $\mathrm{B} 2$ \\
\hline GPI|2821 & $\mathrm{B} 2$ \\
\hline GPKOW|27238 & $\mathrm{B} 2$ \\
\hline GPM6A|2823 & $\mathrm{B} 2$ \\
\hline GPN2|54707 & $\mathrm{B} 2$ \\
\hline GPN3|51184 & $\mathrm{B} 2$ \\
\hline GPNMB|10457 & B2 \\
\hline GPR101|83550 & $\mathrm{B} 2$ \\
\hline GPR107|57720 & B2 \\
\hline GPR108|56927 & $\mathrm{B} 2$ \\
\hline GPR110|266977 & B2 \\
\hline GPR111|222611 & $\mathrm{B} 2$ \\
\hline GPR114|221188 & $\mathrm{B} 2$ \\
\hline GPR115|221393 & $\mathrm{B} 2$ \\
\hline \begin{tabular}{|l|l|} 
GPR123|84435 \\
\end{tabular} & $\mathrm{B} 2$ \\
\hline GPR126|57211 & $\mathrm{B} 2$ \\
\hline GPR128|84873 & $\mathrm{B} 2$ \\
\hline
\end{tabular}




\begin{tabular}{|c|c|}
\hline GPR12|2835 & B2 \\
\hline GPR132|29933 & B2 \\
\hline GPR139|124274 & B2 \\
\hline GPR141|353345 & $\mathrm{B} 2$ \\
\hline GPR150|285601 & $\mathrm{B} 2$ \\
\hline GPR151|134391 & $\mathrm{B} 2$ \\
\hline GPR152|390212 & $\mathrm{B} 2$ \\
\hline GPR153|387509 & $\mathrm{B} 2$ \\
\hline GPR155|151556 & $\mathrm{B} 2$ \\
\hline GPR156|165829 & $\mathrm{B} 2$ \\
\hline GPR157|80045 & $\mathrm{B} 2$ \\
\hline GPR158|57512 & $\mathrm{B} 2$ \\
\hline GPR15|2838 & $\mathrm{B} 2$ \\
\hline GPR161|23432 & $\mathrm{B} 2$ \\
\hline GPR162|27239 & $\mathrm{B} 2$ \\
\hline GPR171|29909 & $\mathrm{B} 2$ \\
\hline GPR172B|55065 & $\mathrm{B} 2$ \\
\hline GPR173|54328 & $\mathrm{B} 2$ \\
\hline GPR174|84636 & $\mathrm{B} 2$ \\
\hline GPR176|11245 & $\mathrm{B} 2$ \\
\hline GPR179|440435 & $\mathrm{B} 2$ \\
\hline GPR180|160897 & $\mathrm{B} 2$ \\
\hline GPR183|1880 & $\mathrm{B} 2$ \\
\hline GPR18|2841 & $\mathrm{B} 2$ \\
\hline GPR1|2825 & $\mathrm{B} 2$ \\
\hline GPR20|2843 & $\mathrm{B} 2$ \\
\hline GPR21|2844 & $\mathrm{B} 2$ \\
\hline GPR22|2845 & $\mathrm{B} 2$ \\
\hline GPR25|2848 & $\mathrm{B} 2$ \\
\hline GPR26|2849 & $\mathrm{B} 2$ \\
\hline GPR27|2850 & $\mathrm{B} 2$ \\
\hline GPR31|2853 & $\mathrm{B} 2$ \\
\hline GPR32|2854 & $\mathrm{B} 2$ \\
\hline GPR35|2859 & $\mathrm{B} 2$ \\
\hline GPR37L1|9283 & $\mathrm{B} 2$ \\
\hline GPR37|2861 & $\mathrm{B} 2$ \\
\hline GPR39|2863 & $\mathrm{B} 2$ \\
\hline GPR3|2827 & $\mathrm{B} 2$ \\
\hline GPR4|2828 & $\mathrm{B} 2$ \\
\hline GPR50|9248 & $\mathrm{B} 2$ \\
\hline GPR52|9293 & $\mathrm{B} 2$ \\
\hline GPR55|9290 & $\mathrm{B} 2$ \\
\hline GPR56|9289 & $\mathrm{B} 2$ \\
\hline GPR61|83873 & $\mathrm{B} 2$ \\
\hline GPR63|81491 & $\mathrm{B} 2$ \\
\hline GPR64|10149 & $\mathrm{B} 2$ \\
\hline GPR65|8477 & $\mathrm{B} 2$ \\
\hline GPR6|2830 & $\mathrm{B} 2$ \\
\hline $\begin{array}{l}\text { GPR78|27201 } \\
\end{array}$ & $\mathrm{B} 2$ \\
\hline GPR82|27197 & $\mathrm{B} 2$ \\
\hline GPR83|10888 & $\mathrm{B} 2$ \\
\hline
\end{tabular}




\begin{tabular}{|c|c|}
\hline GPR85|54329 & B2 \\
\hline GPR87|53836 & B2 \\
\hline GPR89B|51463 & $\mathrm{B} 2$ \\
\hline GPR89C|728932 & $\mathrm{B} 2$ \\
\hline GPR98|84059 & B2 \\
\hline GPRC5C|55890 & $\mathrm{B} 2$ \\
\hline GPRC5D|55507 & $\mathrm{B} 2$ \\
\hline GPRC6A|222545 & $\mathrm{B} 2$ \\
\hline GPRIN2|9721 & B2 \\
\hline GPRIN3|285513 & $\mathrm{B} 2$ \\
\hline GPS1|2873 & $\mathrm{B} 2$ \\
\hline GPS2|2874 & B2 \\
\hline GPSM1|26086 & $\mathrm{B} 2$ \\
\hline GPSM3|63940 & $\mathrm{B} 2$ \\
\hline GPT2|84706 & $\mathrm{B} 2$ \\
\hline GPT|2875 & $\mathrm{B} 2$ \\
\hline GPX1|2876 & $\mathrm{B} 2$ \\
\hline GPX2|2877 & $\mathrm{B} 2$ \\
\hline GPX4|2879 & $\mathrm{B} 2$ \\
\hline GPX5|2880 & B2 \\
\hline GPX7|2882 & $\mathrm{B} 2$ \\
\hline GRAMD1B|57476 & $\mathrm{B} 2$ \\
\hline GRAMD1C|54762 & $\mathrm{B} 2$ \\
\hline GRAMD2|196996 & $\mathrm{B} 2$ \\
\hline GRAMD4|23151 & $\mathrm{B} 2$ \\
\hline GRAP2|9402 & $\mathrm{B} 2$ \\
\hline GRAPL|400581 & $\mathrm{B} 2$ \\
\hline GRAP|10750 & $\mathrm{B} 2$ \\
\hline GRB10|2887 & $\mathrm{B} 2$ \\
\hline GRB14|2888 & $\mathrm{B} 2$ \\
\hline GRB7|2886 & $\mathrm{B} 2$ \\
\hline GREB1L|80000 & $\mathrm{B} 2$ \\
\hline GREB1|9687 & $\mathrm{B} 2$ \\
\hline GREM1|26585 & $\mathrm{B} 2$ \\
\hline GRHL1|29841 & $\mathrm{B} 2$ \\
\hline GRHL3|57822 & $\mathrm{B} 2$ \\
\hline GRHPR|9380 & $\mathrm{B} 2$ \\
\hline GRIA1|2890 & $\mathrm{B} 2$ \\
\hline GRIA2|2891 & $\mathrm{B} 2$ \\
\hline GRIA3|2892 & $\mathrm{B} 2$ \\
\hline GRID2IP|392862 & $\mathrm{B} 2$ \\
\hline GRID2|2895 & B2 \\
\hline GRIK1|2897 & $\mathrm{B} 2$ \\
\hline GRIK2|2898 & $\mathrm{B} 2$ \\
\hline GRIK3|2899 & $\mathrm{B} 2$ \\
\hline GRIK4|2900 & $\mathrm{B} 2$ \\
\hline GRIN1|2902 & $\mathrm{B} 2$ \\
\hline GRIN2A|2903 & $\mathrm{B} 2$ \\
\hline GRIN2C|2905 & $\mathrm{B} 2$ \\
\hline GRIN3A|116443 & $\mathrm{B} 2$ \\
\hline GRIN3B|116444 & $\mathrm{B} 2$ \\
\hline
\end{tabular}




\begin{tabular}{|c|c|}
\hline GRINA|2907 & B2 \\
\hline GRIP1|23426 & $\mathrm{B} 2$ \\
\hline GRIP2|80852 & $\mathrm{B} 2$ \\
\hline GRIPAP1|56850 & $\mathrm{B} 2$ \\
\hline GRK1|6011 & $\mathrm{B} 2$ \\
\hline GRK4|2868 & B2 \\
\hline GRK6|2870 & $\mathrm{B} 2$ \\
\hline GRK7|131890 & $\mathrm{B} 2$ \\
\hline GRLF1|2909 & $\mathrm{B} 2$ \\
\hline GRM1|2911 & $\mathrm{B} 2$ \\
\hline GRM2|2912 & $\mathrm{B} 2$ \\
\hline GRM3|2913 & B2 \\
\hline GRM4|2914 & $\mathrm{B} 2$ \\
\hline $\begin{array}{l}\text { GRM5|2915 } \\
\end{array}$ & $\mathrm{B} 2$ \\
\hline GRM6|2916 & $\mathrm{B} 2$ \\
\hline GRPEL1|80273 & $\mathrm{B} 2$ \\
\hline GRPR|2925 & $\mathrm{B} 2$ \\
\hline \begin{tabular}{l|l|} 
GRP & 2922 \\
\end{tabular} & $\mathrm{~B} 2$ \\
\hline GRSF1|2926 & B2 \\
\hline GRWD1|83743 & $\mathrm{B} 2$ \\
\hline GRXCR2|643226 & $\mathrm{B} 2$ \\
\hline GSC|145258 & $\mathrm{B} 2$ \\
\hline GSDMA|284110 & $\mathrm{B} 2$ \\
\hline GSDMB|55876 & $\mathrm{B} 2$ \\
\hline GSDMC|56169 & $\mathrm{B} 2$ \\
\hline GSDMD|79792 & $\mathrm{B} 2$ \\
\hline GSG1L|146395 & B2 \\
\hline GSG1|83445 & B2 \\
\hline GSS|2937 & $\mathrm{B} 2$ \\
\hline GSTA1|2938 & $\mathrm{B} 2$ \\
\hline GSTA2|2939 & $\mathrm{B} 2$ \\
\hline GSTA3|2940 & B2 \\
\hline GSTA4|2941 & $\mathrm{B} 2$ \\
\hline GSTA5|221357 & B2 \\
\hline GSTK1|373156 & $\mathrm{B} 2$ \\
\hline GSTM1|2944 & $\mathrm{B} 2$ \\
\hline GSTM2P1|442245 & $\mathrm{B} 2$ \\
\hline GSTM2|2946 & $\mathrm{B} 2$ \\
\hline GSTM3|2947 & B2 \\
\hline GSTM4|2948 & $\mathrm{B} 2$ \\
\hline GSTM5|2949 & B2 \\
\hline GSTO1|9446 & $\mathrm{B} 2$ \\
\hline GSTO2|119391 & B2 \\
\hline GSTP1|2950 & $\mathrm{B} 2$ \\
\hline GSTT1|2952 & $\mathrm{B} 2$ \\
\hline GSTT2|2953 & $\mathrm{B} 2$ \\
\hline GSTTP2|653399 & $\mathrm{B} 2$ \\
\hline GSTZ1|2954 & $\mathrm{B} 2$ \\
\hline GSX2|170825 & B2 \\
\hline GTF2A1L|11036 & $\mathrm{B} 2$ \\
\hline \begin{tabular}{l|l|} 
GTF2A1|2957 \\
\end{tabular} & $\mathrm{B} 2$ \\
\hline
\end{tabular}




\begin{tabular}{|c|c|}
\hline GTF2A2|2958 & B2 \\
\hline GTF2B|2959 & B2 \\
\hline GTF2E2|2961 & $\mathrm{B} 2$ \\
\hline GTF2F1|2962 & $\mathrm{B} 2$ \\
\hline GTF2F2|2963 & B2 \\
\hline GTF2H2B|653238 & $\mathrm{B} 2$ \\
\hline GTF2H2C|728340 & $\mathrm{B} 2$ \\
\hline GTF2H2|2966 & $\mathrm{B} 2$ \\
\hline GTF2H3|2967 & B2 \\
\hline GTF2H4|2968 & $\mathrm{B} 2$ \\
\hline GTF2H5|404672 & $\mathrm{B} 2$ \\
\hline GTF2IP1|2970 & $\mathrm{B} 2$ \\
\hline GTF2I|2969 & $\mathrm{B} 2$ \\
\hline GTF3A|2971 & $\mathrm{B} 2$ \\
\hline \begin{tabular}{ll|l|l|} 
GTF3C3|9330 \\
\end{tabular} & $\mathrm{B} 2$ \\
\hline GTF3C4|9329 & $\mathrm{B} 2$ \\
\hline GTF3C5|9328 & B2 \\
\hline GTF3C6|112495 & $\mathrm{B} 2$ \\
\hline GTPBP10|85865 & $\mathrm{B} 2$ \\
\hline GTPBP1|9567 & $\mathrm{B} 2$ \\
\hline GTPBP2|54676 & $\mathrm{B} 2$ \\
\hline GTPBP5|26164 & $\mathrm{B} 2$ \\
\hline GTPBP8|29083 & $\mathrm{B} 2$ \\
\hline GTSF1L|149699 & $\mathrm{B} 2$ \\
\hline GTSF1|121355 & $\mathrm{B} 2$ \\
\hline GUCA1B|2979 & $\mathrm{B} 2$ \\
\hline GUCA1C $\mid 9626$ & $\mathrm{~B} 2$ \\
\hline GUCA2A|2980 & $\mathrm{B} 2$ \\
\hline GUCA2B|2981 & $\mathrm{B} 2$ \\
\hline GUCY1A2|2977 & $\mathrm{B} 2$ \\
\hline GUCY1A3|2982 & $\mathrm{B} 2$ \\
\hline GUCY1B2|2974 & $\mathrm{B} 2$ \\
\hline GUCY1B3|2983 & $\mathrm{B} 2$ \\
\hline GUCY2C|2984 & $\mathrm{B} 2$ \\
\hline GUCY2D|3000 & $\mathrm{B} 2$ \\
\hline GUCY2E|390226 & $\mathrm{B} 2$ \\
\hline GUCY2F|2986 & $\mathrm{B} 2$ \\
\hline GUCY2GP|390003 & $\mathrm{B} 2$ \\
\hline GUF1 60558 & B2 \\
\hline GULP1|51454 & $\mathrm{B} 2$ \\
\hline GUSBL1|387036 & $\mathrm{B} 2$ \\
\hline GUSBL2|375513 & $\mathrm{B} 2$ \\
\hline GUSBP1|728411 & $\mathrm{B} 2$ \\
\hline GUSBP3|653188 & $\mathrm{B} 2$ \\
\hline GUSB|2990 & $\mathrm{B} 2$ \\
\hline GVIN1|387751 & $\mathrm{B} 2$ \\
\hline GXYLT1|283464 & $\mathrm{B} 2$ \\
\hline GXYLT2|727936 & $\mathrm{B} 2$ \\
\hline GYG1|2992 & $\mathrm{B} 2$ \\
\hline GYLTL1B|120071 & $\mathrm{B} 2$ \\
\hline GYPA|2993 & $\mathrm{B} 2$ \\
\hline
\end{tabular}




\begin{tabular}{|c|c|}
\hline GYPB|2994 & B2 \\
\hline GYS1|2997 & $\mathrm{B} 2$ \\
\hline GYS2|2998 & $\mathrm{B} 2$ \\
\hline GZF1|64412 & $\mathrm{B} 2$ \\
\hline GZMA|3001 & $\mathrm{B} 2$ \\
\hline GZMB|3002 & B2 \\
\hline GZMH|2999 & $\mathrm{B} 2$ \\
\hline GZMK|3003 & $\mathrm{B} 2$ \\
\hline GZMM|3004 & $\mathrm{B} 2$ \\
\hline H19|283120 & $\mathrm{B} 2$ \\
\hline H1FNT|341567 & $\mathrm{B} 2$ \\
\hline H1FOO|132243 & $\mathrm{B} 2$ \\
\hline H1FX|8971 & $\mathrm{B} 2$ \\
\hline H2AFB1|474382 & $\mathrm{B} 2$ \\
\hline H2AFJ|55766 & $\mathrm{B} 2$ \\
\hline H2AFY2|55506 & $\mathrm{B} 2$ \\
\hline H2BFM|286436 & $\mathrm{B} 2$ \\
\hline H2BFWT|158983 & $\mathrm{B} 2$ \\
\hline H2BFXP|767811 & $\mathrm{B} 2$ \\
\hline H3F3B|3021 & $\mathrm{B} 2$ \\
\hline H3F3C|440093 & $\mathrm{B} 2$ \\
\hline HAAO|23498 & $\mathrm{B} 2$ \\
\hline HABP2|3026 & $\mathrm{B} 2$ \\
\hline HACE1|57531 & $\mathrm{B} 2$ \\
\hline HADHB|3032 & $\mathrm{B} 2$ \\
\hline HAGH|3029 & $\mathrm{B} 2$ \\
\hline HAL|3034 & $\mathrm{B} 2$ \\
\hline HAMP|57817 & $\mathrm{B} 2$ \\
\hline HAND1|9421 & $\mathrm{B} 2$ \\
\hline HAO1|54363 & $\mathrm{B} 2$ \\
\hline HAO2|51179 & $\mathrm{B} 2$ \\
\hline HAPLN1|1404 & $\mathrm{B} 2$ \\
\hline HAPLN2|60484 & $\mathrm{B} 2$ \\
\hline HAPLN3|145864 & $\mathrm{B} 2$ \\
\hline HAR1A|768096 & $\mathrm{B} 2$ \\
\hline HAR1B|768097 & $\mathrm{B} 2$ \\
\hline HARS|3035 & $\mathrm{B} 2$ \\
\hline HAS1|3036 & $\mathrm{B} 2$ \\
\hline HAS2AS|594842 & $\mathrm{B} 2$ \\
\hline HAS2|3037 & $\mathrm{B} 2$ \\
\hline HAUS2|55142 & $\mathrm{B} 2$ \\
\hline \begin{tabular}{l|l|} 
HAUS3|79441 \\
\end{tabular} & $\mathrm{B} 2$ \\
\hline HAUS5|23354 & $\mathrm{B} 2$ \\
\hline HAUS6|54801 & $\mathrm{B} 2$ \\
\hline HAUS7|55559 & $\mathrm{B} 2$ \\
\hline HAVCR1|26762 & $\mathrm{B} 2$ \\
\hline \begin{tabular}{l|l|} 
HAVCR2|84868 \\
\end{tabular} & $\mathrm{B} 2$ \\
\hline HBA1|3039 & $\mathrm{B} 2$ \\
\hline HBA2|3040 & $\mathrm{B} 2$ \\
\hline HBBP1|3044 & $\mathrm{B} 2$ \\
\hline HBB 3043 & $\mathrm{~B} 2$ \\
\hline
\end{tabular}




\begin{tabular}{|c|c|}
\hline HBD|3045 & B2 \\
\hline HBE1|3046 & B2 \\
\hline HBG1|3047 & $\mathrm{B} 2$ \\
\hline HBG2|3048 & $\mathrm{B} 2$ \\
\hline HBM|3042 & $\mathrm{B} 2$ \\
\hline HBQ1|3049 & B2 \\
\hline HBS1L|10767 & $\mathrm{B} 2$ \\
\hline HBXIP|10542 & $\mathrm{B} 2$ \\
\hline HBZ|3050 & $\mathrm{B} 2$ \\
\hline HCCS $\mid 3052$ & $\mathrm{~B} 2$ \\
\hline HCFC1R1|54985 & $\mathrm{B} 2$ \\
\hline HCG18|414777 & $\mathrm{B} 2$ \\
\hline HCG26|352961 & $\mathrm{B} 2$ \\
\hline HCG4P6|80868 & $\mathrm{B} 2$ \\
\hline HCG4|54435 & $\mathrm{B} 2$ \\
\hline HCG9|10255 & $\mathrm{B} 2$ \\
\hline HCK 3055 & $\mathrm{~B} 2$ \\
\hline HCLS1|3059 & $\mathrm{B} 2$ \\
\hline HCN1|348980 & $\mathrm{B} 2$ \\
\hline HCN2|610 & $\mathrm{B} 2$ \\
\hline HCN4|10021 & $\mathrm{B} 2$ \\
\hline HCP5|10866 & B2 \\
\hline HCRTR2|3062 & $\mathrm{B} 2$ \\
\hline HCRT|3060 & B2 \\
\hline HCST $\mid 10870$ & $\mathrm{~B} 2$ \\
\hline HDAC10|83933 & B2 \\
\hline HDAC11|79885 & $\mathrm{B} 2$ \\
\hline HDAC2|3066 & $\mathrm{B} 2$ \\
\hline HDAC3 $\mid 8841$ & $\mathrm{~B} 2$ \\
\hline HDAC9|9734 & $\mathrm{B} 2$ \\
\hline HDC|3067 & $\mathrm{B} 2$ \\
\hline HDDC2|51020 & $\mathrm{B} 2$ \\
\hline HDDC3|374659 & B2 \\
\hline HDGFL1|154150 & $\mathrm{B} 2$ \\
\hline HDGFRP2|84717 & $\mathrm{B} 2$ \\
\hline HDGFRP3|50810 & $\mathrm{B} 2$ \\
\hline HDHD1A|8226 & $\mathrm{B} 2$ \\
\hline HDHD2|84064 & $\mathrm{B} 2$ \\
\hline HDHD3|81932 & $\mathrm{B} 2$ \\
\hline HDLBP|3069 & $\mathrm{B} 2$ \\
\hline HDX|139324 & $\mathrm{B} 2$ \\
\hline HEATR1|55127 & $\mathrm{B} 2$ \\
\hline HEATR4|399671 & $\mathrm{B} 2$ \\
\hline HEATR5A|25938 & B2 \\
\hline HEATR5B|54497 & $\mathrm{B} 2$ \\
\hline HEATR6|63897 & $\mathrm{B} 2$ \\
\hline HEATR7A|727957 & $\mathrm{B} 2$ \\
\hline HEATR7B2|133558 & $\mathrm{B} 2$ \\
\hline HEBP1|50865 & $\mathrm{B} 2$ \\
\hline HEBP2|23593 & $\mathrm{B} 2$ \\
\hline HECA|51696 & $\mathrm{B} 2$ \\
\hline
\end{tabular}




\begin{tabular}{|c|c|}
\hline HECTD1|25831 & B2 \\
\hline HECTD2|143279 & B2 \\
\hline HECTD3|79654 & $\mathrm{B} 2$ \\
\hline HECW1|23072 & $\mathrm{B} 2$ \\
\hline HECW2|57520 & B2 \\
\hline HEG1|57493 & $\mathrm{B} 2$ \\
\hline HELB|92797 & $\mathrm{B} 2$ \\
\hline HELT $\mid 391723$ & $\mathrm{~B} 2$ \\
\hline HELZ|9931 & $\mathrm{B} 2$ \\
\hline HEMGN|55363 & $\mathrm{B} 2$ \\
\hline HEMK1|51409 & $\mathrm{B} 2$ \\
\hline HEPACAM2|253012 & $\mathrm{B} 2$ \\
\hline HEPHL1|341208 & $\mathrm{B} 2$ \\
\hline HEPH|9843 & $\mathrm{B} 2$ \\
\hline HERC2P4|440362 & $\mathrm{B} 2$ \\
\hline HERC3|8916 & $\mathrm{B} 2$ \\
\hline HERC4|26091 & $\mathrm{B} 2$ \\
\hline \begin{tabular}{l|l|l|} 
HERC5 51191 \\
\end{tabular} & $\mathrm{~B} 2$ \\
\hline HERC6|55008 & $\mathrm{B} 2$ \\
\hline HERPUD1|9709 & $\mathrm{B} 2$ \\
\hline HES1|3280 & $\mathrm{B} 2$ \\
\hline HES2|54626 & $\mathrm{B} 2$ \\
\hline HES4|57801 & $\mathrm{B} 2$ \\
\hline HES5|388585 & $\mathrm{B} 2$ \\
\hline HES7|84667 & B2 \\
\hline HESRG|790952 & $\mathrm{B} 2$ \\
\hline HESX1|8820 & $\mathrm{B} 2$ \\
\hline HEXA|3073 & $\mathrm{B} 2$ \\
\hline HEXB|3074 & $\mathrm{B} 2$ \\
\hline HEXDC|284004 & $\mathrm{B} 2$ \\
\hline HEXIM1|10614 & $\mathrm{B} 2$ \\
\hline HEXIM2|124790 & $\mathrm{B} 2$ \\
\hline HEY1|23462 & $\mathrm{B} 2$ \\
\hline HEY2|23493 & $\mathrm{B} 2$ \\
\hline HFE2|148738 & $\mathrm{B} 2$ \\
\hline HFE|3077 & $\mathrm{B} 2$ \\
\hline HGC6.3|100128124 & $\mathrm{B} 2$ \\
\hline HGD|3081 & $\mathrm{B} 2$ \\
\hline HGFAC|3083 & $\mathrm{B} 2$ \\
\hline HGSNAT|138050 & $\mathrm{B} 2$ \\
\hline HGS|9146 & $\mathrm{B} 2$ \\
\hline HHATL|57467 & $\mathrm{B} 2$ \\
\hline HHEX|3087 & $\mathrm{B} 2$ \\
\hline HHIPL1|84439 & $\mathrm{B} 2$ \\
\hline HHIPL2|79802 & $\mathrm{B} 2$ \\
\hline HHIP 64399 & $\mathrm{~B} 2$ \\
\hline HHLA1|10086 & $\mathrm{B} 2$ \\
\hline HHLA2|11148 & $\mathrm{B} 2$ \\
\hline HHLA3|11147 & $\mathrm{B} 2$ \\
\hline HIAT1|64645 & $\mathrm{B} 2$ \\
\hline HIATL2|84278 & $\mathrm{B} 2$ \\
\hline
\end{tabular}




\begin{tabular}{|c|c|}
\hline HIBCH|26275 & B2 \\
\hline HIC2|23119 & B2 \\
\hline HIF1AN|55662 & B2 \\
\hline HIF1A|3091 & $\mathrm{B} 2$ \\
\hline HIGD1A|25994 & $\mathrm{B} 2$ \\
\hline HIGD1B|51751 & $\mathrm{B} 2$ \\
\hline HIGD1C|613227 & $\mathrm{B} 2$ \\
\hline HIGD2A|192286 & $\mathrm{B} 2$ \\
\hline HIGD2B|123346 & $\mathrm{B} 2$ \\
\hline HILS1|373861 & $\mathrm{B} 2$ \\
\hline HINFP|25988 & $\mathrm{B} 2$ \\
\hline HINT1|3094 & $\mathrm{B} 2$ \\
\hline HINT2|84681 & $\mathrm{B} 2$ \\
\hline HINT3|135114 & $\mathrm{B} 2$ \\
\hline HIP1R|9026 & $\mathrm{B} 2$ \\
\hline HIP1|3092 & $\mathrm{B} 2$ \\
\hline HIPK1|204851 & $\mathrm{B} 2$ \\
\hline HIPK4|147746 & $\mathrm{B} 2$ \\
\hline HIRA|7290 & $\mathrm{B} 2$ \\
\hline HIRIP3|8479 & $\mathrm{B} 2$ \\
\hline HIST1H1A|3024 & $\mathrm{B} 2$ \\
\hline HIST1H1B|3009 & $\mathrm{B} 2$ \\
\hline HIST1H1D|3007 & $\mathrm{B} 2$ \\
\hline HIST1H1E|3008 & $\mathrm{B} 2$ \\
\hline HIST1H2AB|8335 & $\mathrm{B} 2$ \\
\hline HIST1H2AE|3012 & $\mathrm{B} 2$ \\
\hline HIST1H2AG|8969 & $\mathrm{B} 2$ \\
\hline HIST1H2AH|85235 & $\mathrm{B} 2$ \\
\hline HIST1H2AK|8330 & $\mathrm{B} 2$ \\
\hline HIST1H2AL|8332 & $\mathrm{B} 2$ \\
\hline HIST1H2BB|3018 & $\mathrm{B} 2$ \\
\hline HIST1H2BF|8343 & $\mathrm{B} 2$ \\
\hline HIST1H2BI|8346 & $\mathrm{B} 2$ \\
\hline HIST1H2BL|8340 & $\mathrm{B} 2$ \\
\hline HIST1H2BM|8342 & $\mathrm{B} 2$ \\
\hline HIST1H3A|8350 & $\mathrm{B} 2$ \\
\hline HIST1H3B $\mid 8358$ & $\mathrm{~B} 2$ \\
\hline HIST1H3C $\mid 8352$ & $\mathrm{~B} 2$ \\
\hline HIST1H3G|8355 & $\mathrm{B} 2$ \\
\hline HIST1H3I|8354 & $\mathrm{B} 2$ \\
\hline HIST1H4B|8366 & $\mathrm{B} 2$ \\
\hline HIST1H4C|8364 & $\mathrm{B} 2$ \\
\hline HIST1H4E|8367 & $\mathrm{B} 2$ \\
\hline HIST1H4F|8361 & $\mathrm{B} 2$ \\
\hline HIST1H4G|8369 & $\mathrm{B} 2$ \\
\hline HIST1H4H|8365 & $\mathrm{B} 2$ \\
\hline HIST1H4K|8362 & $\mathrm{B} 2$ \\
\hline HIST1H4L|8368 & $\mathrm{B} 2$ \\
\hline HIST2H2AB|317772 & $\mathrm{B} 2$ \\
\hline HIST2H2BA|337875 & $\mathrm{B} 2$ \\
\hline HIST2H3C|126961 & $\mathrm{B} 2$ \\
\hline
\end{tabular}




\begin{tabular}{|c|c|}
\hline HIST2H3D|653604 & $\mathrm{B} 2$ \\
\hline HIST2H4A|8370 & B2 \\
\hline HIST3H2A|92815 & $\mathrm{B} 2$ \\
\hline HIST3H3|8290 & $\mathrm{B} 2$ \\
\hline HIST4H4|121504 & $\mathrm{B} 2$ \\
\hline HIVEP1|3096 & $\mathrm{B} 2$ \\
\hline HIVEP3|59269 & $\mathrm{B} 2$ \\
\hline HK2|3099 & $\mathrm{B} 2$ \\
\hline HK3|3101 & $\mathrm{B} 2$ \\
\hline HKDC1|80201 & $\mathrm{B} 2$ \\
\hline HKR1|284459 & $\mathrm{B} 2$ \\
\hline HLA-A|3105 & $\mathrm{B} 2$ \\
\hline HLA-B|3106 & $\mathrm{B} 2$ \\
\hline HLA-DMA|3108 & $\mathrm{B} 2$ \\
\hline HLA-DMB|3109 & $\mathrm{B} 2$ \\
\hline HLA-DOA|3111 & $\mathrm{B} 2$ \\
\hline HLA-DOB 3112 & B2 \\
\hline HLA-DPA1|3113 & $\mathrm{B} 2$ \\
\hline HLA-DPB1|3115 & $\mathrm{B} 2$ \\
\hline HLA-DPB2|3116 & $\mathrm{B} 2$ \\
\hline HLA-DQA1|3117 & $\mathrm{B} 2$ \\
\hline HLA-DQA2|3118 & $\mathrm{B} 2$ \\
\hline HLA-DQB1|3119 & $\mathrm{B} 2$ \\
\hline HLA-DQB2|3120 & $\mathrm{B} 2$ \\
\hline HLA-DRA|3122 & $\mathrm{B} 2$ \\
\hline HLA-DRB1|3123 & $\mathrm{B} 2$ \\
\hline HLA-DRB5|3127 & $\mathrm{B} 2$ \\
\hline HLA-DRB6|3128 & $\mathrm{B} 2$ \\
\hline HLA-F|3134 & $\mathrm{B} 2$ \\
\hline HLA-G|3135 & $\mathrm{B} 2$ \\
\hline HLA-H|3136 & $\mathrm{B} 2$ \\
\hline HLA-J/3137 & $\mathrm{B} 2$ \\
\hline HLA-L|3139 & $\mathrm{B} 2$ \\
\hline HLX 3142 & $\mathrm{~B} 2$ \\
\hline HMBOX1|79618 & $\mathrm{B} 2$ \\
\hline HMCN1|83872 & $\mathrm{B} 2$ \\
\hline HMG20A|10363 & $\mathrm{B} 2$ \\
\hline HMGB1|3146 & $\mathrm{B} 2$ \\
\hline HMGB3L1|128872 & $\mathrm{B} 2$ \\
\hline HMGB4|127540 & $\mathrm{B} 2$ \\
\hline HMGCL|3155 & $\mathrm{B} 2$ \\
\hline HMGCS1|3157 & $\mathrm{B} 2$ \\
\hline HMGCS2|3158 & $\mathrm{B} 2$ \\
\hline HMGN4|10473 & $\mathrm{B} 2$ \\
\hline HMGN5|79366 & $\mathrm{B} 2$ \\
\hline HMGXB3|22993 & $\mathrm{B} 2$ \\
\hline HMGXB4|10042 & $\mathrm{B} 2$ \\
\hline HMHA1|23526 & $\mathrm{B} 2$ \\
\hline HMHB1|57824 & $\mathrm{B} 2$ \\
\hline HMOX1|3162 & $\mathrm{B} 2$ \\
\hline HMP19|51617 & $\mathrm{B} 2$ \\
\hline
\end{tabular}




\begin{tabular}{|c|c|}
\hline HMSD|284293 & B2 \\
\hline HMX1|3166 & B2 \\
\hline HMX2|3167 & B2 \\
\hline HMX3|340784 & $\mathrm{B} 2$ \\
\hline HNF1A|6927 & $\mathrm{B} 2$ \\
\hline HNF1B $\mid 6928$ & $\mathrm{~B} 2$ \\
\hline HNF4A 3172 & $\mathrm{~B} 2$ \\
\hline HNF4G|3174 & $\mathrm{B} 2$ \\
\hline HNRNPA0|10949 & $\mathrm{B} 2$ \\
\hline HNRNPA1L2|144983 & $\mathrm{B} 2$ \\
\hline HNRNPA1|3178 & $\mathrm{B} 2$ \\
\hline HNRNPA3|220988 & $\mathrm{B} 2$ \\
\hline HNRNPD|3184 & $\mathrm{B} 2$ \\
\hline HNRNPH1|3187 & $\mathrm{B} 2$ \\
\hline HNRNPH2|3188 & $\mathrm{B} 2$ \\
\hline HNRNPH3|3189 & $\mathrm{B} 2$ \\
\hline HNRNPM $\mid 4670$ & $\mathrm{~B} 2$ \\
\hline HNRNPUL2|221092 & $\mathrm{B} 2$ \\
\hline HNRPLL|92906 & $\mathrm{B} 2$ \\
\hline HOMER1|9456 & $\mathrm{B} 2$ \\
\hline HOMER2|9455 & $\mathrm{B} 2$ \\
\hline HOMEZ|57594 & $\mathrm{B} 2$ \\
\hline HOOK1|51361 & $\mathrm{B} 2$ \\
\hline HORMAD1|84072 & $\mathrm{B} 2$ \\
\hline HORMAD2|150280 & $\mathrm{B} 2$ \\
\hline HOXA11AS|221883 & $\mathrm{B} 2$ \\
\hline HOXA11|3207 & $\mathrm{B} 2$ \\
\hline HOXA13|3209 & $\mathrm{B} 2$ \\
\hline HOXA1|3198 & $\mathrm{B} 2$ \\
\hline HOXB13|10481 & $\mathrm{B} 2$ \\
\hline HOXB1|3211 & $\mathrm{B} 2$ \\
\hline HOXB2|3212 & $\mathrm{B} 2$ \\
\hline HOXB3|3213 & $\mathrm{B} 2$ \\
\hline HOXB4|3214 & $\mathrm{B} 2$ \\
\hline HOXB5|3215 & $\mathrm{B} 2$ \\
\hline HOXB6|3216 & $\mathrm{B} 2$ \\
\hline HOXB7|3217 & $\mathrm{B} 2$ \\
\hline HOXB8|3218 & $\mathrm{B} 2$ \\
\hline HOXB9|3219 & $\mathrm{B} 2$ \\
\hline HOXC12|3228 & $\mathrm{B} 2$ \\
\hline HOXC5|3222 & $\mathrm{B} 2$ \\
\hline HOXC6|3223 & $\mathrm{B} 2$ \\
\hline HOXC8|3224 & $\mathrm{B} 2$ \\
\hline HOXC9|3225 & $\mathrm{B} 2$ \\
\hline HOXD11|3237 & $\mathrm{B} 2$ \\
\hline HOXD12|3238 & $\mathrm{B} 2$ \\
\hline HOXD13|3239 & $\mathrm{B} 2$ \\
\hline HOXD1|3231 & $\mathrm{B} 2$ \\
\hline HOXD8|3234 & $\mathrm{B} 2$ \\
\hline HP1BP3|50809 & $\mathrm{B} 2$ \\
\hline HPCAL1|3241 & B2 \\
\hline
\end{tabular}




\begin{tabular}{|c|c|}
\hline HPCAL4|51440 & B2 \\
\hline HPCA|3208 & B2 \\
\hline HPDL|84842 & $\mathrm{B} 2$ \\
\hline HPD|3242 & $\mathrm{B} 2$ \\
\hline HPGDS|27306 & $\mathrm{B} 2$ \\
\hline HPGD|3248 & $\mathrm{B} 2$ \\
\hline HPR/3250 & $\mathrm{B} 2$ \\
\hline HPS6|79803 & $\mathrm{B} 2$ \\
\hline HPSE|10855 & $\mathrm{B} 2$ \\
\hline HPVC1|3262 & $\mathrm{B} 2$ \\
\hline HPX|3263 & $\mathrm{B} 2$ \\
\hline HPYR1|93668 & $\mathrm{B} 2$ \\
\hline $\mathrm{HP} \mid 3240$ & $\mathrm{~B} 2$ \\
\hline HRASLS2|54979 & $\mathrm{B} 2$ \\
\hline HRASLS|57110 & $\mathrm{B} 2$ \\
\hline HRAS|3265 & $\mathrm{B} 2$ \\
\hline HRCT1|646962 & $\mathrm{B} 2$ \\
\hline HRC $\mid 3270$ & $\mathrm{~B} 2$ \\
\hline HRG|3273 & $\mathrm{B} 2$ \\
\hline HRH1|3269 & $\mathrm{B} 2$ \\
\hline HRH2|3274 & $\mathrm{B} 2$ \\
\hline HRH3|11255 & $\mathrm{B} 2$ \\
\hline HRK|8739 & $\mathrm{B} 2$ \\
\hline HRNBP3|146713 & $\mathrm{B} 2$ \\
\hline HRSP12|10247 & B2 \\
\hline HR|55806 & $\mathrm{B} 2$ \\
\hline HS1BP3|64342 & $\mathrm{B} 2$ \\
\hline HS2ST1|9653 & $\mathrm{B} 2$ \\
\hline HS3ST1|9957 & $\mathrm{B} 2$ \\
\hline HS3ST2|9956 & $\mathrm{B} 2$ \\
\hline HS3ST3B1|9953 & $\mathrm{B} 2$ \\
\hline HS3ST5|222537 & B2 \\
\hline HS3ST6|64711 & $\mathrm{B} 2$ \\
\hline HS6ST2|90161 & $\mathrm{B} 2$ \\
\hline HSBP1L1|440498 & $\mathrm{B} 2$ \\
\hline HSBP1|3281 & $\mathrm{B} 2$ \\
\hline HSCB|150274 & $\mathrm{B} 2$ \\
\hline HSD11B1L|374875 & $\mathrm{B} 2$ \\
\hline HSD11B2|3291 & $\mathrm{B} 2$ \\
\hline HSD17B12|51144 & $\mathrm{B} 2$ \\
\hline HSD17B14|51171 & $\mathrm{B} 2$ \\
\hline HSD17B1|3292 & $\mathrm{B} 2$ \\
\hline HSD17B2|3294 & $\mathrm{B} 2$ \\
\hline HSD17B3|3293 & $\mathrm{B} 2$ \\
\hline HSD17B4|3295 & $\mathrm{B} 2$ \\
\hline HSD17B7P2|158160 & $\mathrm{B} 2$ \\
\hline HSD17B8|7923 & $\mathrm{B} 2$ \\
\hline HSD3B1|3283 & $\mathrm{B} 2$ \\
\hline HSD3B2|3284 & $\mathrm{B} 2$ \\
\hline HSD3B7|80270 & $\mathrm{B} 2$ \\
\hline $\begin{array}{l}\text { HSDL1 } 183693 \\
\end{array}$ & $\mathrm{~B} 2$ \\
\hline
\end{tabular}




\begin{tabular}{|c|c|}
\hline HSF1|3297 & B2 \\
\hline HSF2|3298 & B2 \\
\hline HSF5|124535 & B2 \\
\hline HSFX2|100130086 & $\mathrm{B} 2$ \\
\hline HSFYL1|27437 & $\mathrm{B} 2$ \\
\hline HSN2|378465 & B2 \\
\hline HSP90AB2P|391634 & B2 \\
\hline HSP90AB4P|664618 & $\mathrm{B} 2$ \\
\hline HSP90B3P|343477 & B2 \\
\hline HSPA1A|3303 & $\mathrm{B} 2$ \\
\hline HSPA1B|3304 & B2 \\
\hline HSPA1L|3305 & B2 \\
\hline HSPA2|3306 & $\mathrm{B} 2$ \\
\hline HSPA4L|22824 & $\mathrm{B} 2$ \\
\hline HSPA6|3310 & $\mathrm{B} 2$ \\
\hline HSPA7|3311 & $\mathrm{B} 2$ \\
\hline HSPB3|8988 & $\mathrm{B} 2$ \\
\hline HSPB7|27129 & $\mathrm{B} 2$ \\
\hline \begin{tabular}{l|l|} 
HSPB8 26353 \\
\end{tabular} & $\mathrm{~B} 2$ \\
\hline HSPB9|94086 & $\mathrm{B} 2$ \\
\hline HSPBAP1|79663 & $\mathrm{B} 2$ \\
\hline HSPC072|29075 & $\mathrm{B} 2$ \\
\hline $\begin{array}{l}\text { HSPC159|29094 } \\
\end{array}$ & B2 \\
\hline HSPG2|3339 & $\mathrm{B} 2$ \\
\hline HTATSF1|27336 & $\mathrm{B} 2$ \\
\hline HTA|283902 & $\mathrm{B} 2$ \\
\hline HTR1A|3350 & B2 \\
\hline HTR1B|3351 & $\mathrm{B} 2$ \\
\hline HTR1E|3354 & $\mathrm{B} 2$ \\
\hline HTR1F|3355 & $\mathrm{B} 2$ \\
\hline HTR2B|3357 & $\mathrm{B} 2$ \\
\hline HTR2C|3358 & B2 \\
\hline HTR3A|3359 & $\mathrm{B} 2$ \\
\hline HTR3B|9177 & $\mathrm{B} 2$ \\
\hline HTR3D|200909 & $\mathrm{B} 2$ \\
\hline HTR3E|285242 & $\mathrm{B} 2$ \\
\hline HTR5A|3361 & $\mathrm{B} 2$ \\
\hline HTR6|3362 & $\mathrm{B} 2$ \\
\hline HTR7P1|93164 & $\mathrm{B} 2$ \\
\hline HTR7|3363 & $\mathrm{B} 2$ \\
\hline HTRA1|5654 & $\mathrm{B} 2$ \\
\hline HTRA2|27429 & $\mathrm{B} 2$ \\
\hline $\begin{array}{l}\text { HTRA3|94031 } \\
\text { HTR }\end{array}$ & $\mathrm{B} 2$ \\
\hline HTRA4|203100 & $\mathrm{B} 2$ \\
\hline HTT|3064 & B2 \\
\hline HULC|728655 & $\mathrm{B} 2$ \\
\hline HUNK|30811 & $\mathrm{B} 2$ \\
\hline HUS1B|135458 & $\mathrm{B} 2$ \\
\hline HUWE1|10075 & $\mathrm{B} 2$ \\
\hline HVCN1|84329 & $\mathrm{B} 2$ \\
\hline HYAL2|8692 & $\mathrm{B} 2$ \\
\hline
\end{tabular}




\begin{tabular}{|c|c|}
\hline HYAL4|23553 & $\mathrm{B} 2$ \\
\hline HYDIN|54768 & $\mathrm{B} 2$ \\
\hline HYI $\mid 81888$ & $\mathrm{~B} 2$ \\
\hline IAH1|285148 & $\mathrm{B} 2$ \\
\hline IAPP|3375 & $\mathrm{B} 2$ \\
\hline IBSP|3381 & $\mathrm{B} 2$ \\
\hline IBTK|25998 & $\mathrm{B} 2$ \\
\hline ICA1L|130026 & $\mathrm{B} 2$ \\
\hline ICAM1|3383 & $\mathrm{B} 2$ \\
\hline ICAM3|3385 & $\mathrm{B} 2$ \\
\hline ICAM4|3386 & $\mathrm{B} 2$ \\
\hline ICAM5|7087 & $\mathrm{B} 2$ \\
\hline ICK|22858 & $\mathrm{B} 2$ \\
\hline ICMT|23463 & $\mathrm{B} 2$ \\
\hline ICOSLG|23308 & $\mathrm{B} 2$ \\
\hline ICOS|29851 & $\mathrm{B} 2$ \\
\hline ID2B $\mid 84099$ & B2 \\
\hline ID2|3398 & $\mathrm{B} 2$ \\
\hline ID3|3399 & $\mathrm{B} 2$ \\
\hline IDH1|3417 & $\mathrm{B} 2$ \\
\hline IDH2|3418 & $\mathrm{B} 2$ \\
\hline IDH3A|3419 & $\mathrm{B} 2$ \\
\hline IDH3B|3420 & $\mathrm{B} 2$ \\
\hline IDH3G|3421 & $\mathrm{B} 2$ \\
\hline IDI1|3422 & $\mathrm{B} 2$ \\
\hline \begin{tabular}{l|l} 
IDI2|91734 \\
\end{tabular} & $\mathrm{B} 2$ \\
\hline IDO1|3620 & $\mathrm{B} 2$ \\
\hline IDO2|169355 & $\mathrm{B} 2$ \\
\hline IDS|3423 & $\mathrm{B} 2$ \\
\hline IDUA|3425 & $\mathrm{B} 2$ \\
\hline IER2|9592 & $\mathrm{B} 2$ \\
\hline IER3|8870 & $\mathrm{B} 2$ \\
\hline \begin{tabular}{l|l|l|l|} 
IER5 &
\end{tabular} & $\mathrm{B} 2$ \\
\hline IFI16|3428 & $\mathrm{B} 2$ \\
\hline IFI27L1|122509 & $\mathrm{B} 2$ \\
\hline IFI27L2|83982 & $\mathrm{B} 2$ \\
\hline IFI27|3429 & $\mathrm{B} 2$ \\
\hline IFI44L|10964 & $\mathrm{B} 2$ \\
\hline IFI44|10561 & $\mathrm{B} 2$ \\
\hline IFIH1|64135 & $\mathrm{B} 2$ \\
\hline IFIT1B|439996 & $\mathrm{B} 2$ \\
\hline IFIT1|3434 & $\mathrm{B} 2$ \\
\hline IFIT2|3433 & $\mathrm{B} 2$ \\
\hline IFIT3|3437 & $\mathrm{B} 2$ \\
\hline IFIT5|24138 & $\mathrm{B} 2$ \\
\hline IFITM1|8519 & $\mathrm{B} 2$ \\
\hline IFITM2|10581 & $\mathrm{B} 2$ \\
\hline IFITM3|10410 & $\mathrm{B} 2$ \\
\hline IFITM4P|340198 & $\mathrm{B} 2$ \\
\hline IFITM5|387733 & $\mathrm{B} 2$ \\
\hline IFLTD1|160492 & $\mathrm{B} 2$ \\
\hline
\end{tabular}




\begin{tabular}{|c|c|}
\hline IFNA10|3446 & B2 \\
\hline IFNA13|3447 & B2 \\
\hline IFNA17|3451 & B2 \\
\hline IFNA1|3439 & B2 \\
\hline IFNA21|3452 & $\mathrm{B} 2$ \\
\hline IFNA2|3440 & $\mathrm{B} 2$ \\
\hline IFNA5|3442 & $\mathrm{B} 2$ \\
\hline IFNA6|3443 & $\mathrm{B} 2$ \\
\hline IFNA8|3445 & $\mathrm{B} 2$ \\
\hline IFNAR1|3454 & $\mathrm{B} 2$ \\
\hline IFNAR2|3455 & $\mathrm{B} 2$ \\
\hline IFNB1|3456 & $\mathrm{B} 2$ \\
\hline IFNGR2|3460 & $\mathrm{B} 2$ \\
\hline IFNG|3458 & $\mathrm{B} 2$ \\
\hline IFNK|56832 & $\mathrm{B} 2$ \\
\hline IFNW1|3467 & $\mathrm{B} 2$ \\
\hline IFRD1|3475 & $\mathrm{B} 2$ \\
\hline $\begin{array}{l}\text { IFRD2|7866 } \\
\end{array}$ & $\mathrm{B} 2$ \\
\hline \begin{tabular}{l|l|l|} 
IFT122 55764 \\
\end{tabular} & $\mathrm{~B} 2$ \\
\hline IFT140|9742 & $\mathrm{B} 2$ \\
\hline IFT172|26160 & $\mathrm{B} 2$ \\
\hline IFT20|90410 & $\mathrm{B} 2$ \\
\hline IFT27|11020 & $\mathrm{B} 2$ \\
\hline IFT46|56912 & $\mathrm{B} 2$ \\
\hline IFT52|51098 & $\mathrm{B} 2$ \\
\hline IFT57|55081 & $\mathrm{B} 2$ \\
\hline IFT74|80173 & $\mathrm{B} 2$ \\
\hline IFT80|57560 & $\mathrm{B} 2$ \\
\hline IFT81|28981 & $\mathrm{B} 2$ \\
\hline IFT88|8100 & $\mathrm{B} 2$ \\
\hline IGBP1|3476 & $\mathrm{B} 2$ \\
\hline IGDCC3|9543 & $\mathrm{B} 2$ \\
\hline IGDCC4|57722 & $\mathrm{B} 2$ \\
\hline IGF1R/3480 & $\mathrm{B} 2$ \\
\hline IGF2AS|51214 & $\mathrm{B} 2$ \\
\hline IGF2BP1|10642 & $\mathrm{B} 2$ \\
\hline IGF2BP3|10643 & $\mathrm{B} 2$ \\
\hline IGF2R/3482 & $\mathrm{B} 2$ \\
\hline IGFALS|3483 & $\mathrm{B} 2$ \\
\hline IGFBP1|3484 & $\mathrm{B} 2$ \\
\hline IGFBP2|3485 & $\mathrm{B} 2$ \\
\hline IGFBP3|3486 & $\mathrm{B} 2$ \\
\hline IGFBP4|3487 & $\mathrm{B} 2$ \\
\hline IGFBP5|3488 & $\mathrm{B} 2$ \\
\hline IGFBP7|3490 & $\mathrm{B} 2$ \\
\hline IGFL1|374918 & $\mathrm{B} 2$ \\
\hline \begin{tabular}{l|l|l|} 
IGFL2|147920 &
\end{tabular} & $\mathrm{B} 2$ \\
\hline \begin{tabular}{l|l|l|} 
IGFL3 388555 \\
\end{tabular} & $\mathrm{~B} 2$ \\
\hline IGFL4|444882 & $\mathrm{B} 2$ \\
\hline IGFN1|91156 & $\mathrm{B} 2$ \\
\hline IGHMBP2|3508 & $\mathrm{B} 2$ \\
\hline
\end{tabular}




\begin{tabular}{|c|c|}
\hline IGLL1|3543 & B2 \\
\hline IGLL3|91353 & B2 \\
\hline IGLON5|402665 & $\mathrm{B} 2$ \\
\hline IGSF11|152404 & $\mathrm{B} 2$ \\
\hline IGSF1|3547 & $\mathrm{B} 2$ \\
\hline IGSF21|84966 & $\mathrm{B} 2$ \\
\hline IGSF22|283284 & $\mathrm{B} 2$ \\
\hline IGSF3|3321 & $\mathrm{B} 2$ \\
\hline IGSF5|150084 & $\mathrm{B} 2$ \\
\hline IGSF6|10261 & $\mathrm{B} 2$ \\
\hline IGSF9B|22997 & $\mathrm{B} 2$ \\
\hline IHH|3549 & $\mathrm{B} 2$ \\
\hline IKBIP|121457 & $\mathrm{B} 2$ \\
\hline IKBKAP|8518 & $\mathrm{B} 2$ \\
\hline IKBKB $\mid 3551$ & $\mathrm{~B} 2$ \\
\hline IKBKG|8517 & $\mathrm{B} 2$ \\
\hline IKZF1|10320 & B2 \\
\hline IKZF2|22807 & $\mathrm{B} 2$ \\
\hline IKZF3|22806 & $\mathrm{B} 2$ \\
\hline $\mathrm{IK} \mid 3550$ & $\mathrm{~B} 2$ \\
\hline IL10RA|3587 & $\mathrm{B} 2$ \\
\hline IL10RB|3588 & $\mathrm{B} 2$ \\
\hline IL10|3586 & $\mathrm{B} 2$ \\
\hline IL12A|3592 & $\mathrm{B} 2$ \\
\hline IL12B $\mid 3593$ & $\mathrm{~B} 2$ \\
\hline IL12RB1|3594 & $\mathrm{B} 2$ \\
\hline IL12RB2|3595 & $\mathrm{B} 2$ \\
\hline IL13RA1|3597 & $\mathrm{B} 2$ \\
\hline IL13RA2|3598 & $\mathrm{B} 2$ \\
\hline IL13|3596 & $\mathrm{B} 2$ \\
\hline IL15|3600 & $\mathrm{B} 2$ \\
\hline IL16|3603 & $\mathrm{B} 2$ \\
\hline IL17A|3605 & $\mathrm{B} 2$ \\
\hline IL17C|27189 & $\mathrm{B} 2$ \\
\hline IL17D|53342 & $\mathrm{B} 2$ \\
\hline IL17F|112744 & $\mathrm{B} 2$ \\
\hline IL17RB|55540 & $\mathrm{B} 2$ \\
\hline IL17RC|84818 & $\mathrm{B} 2$ \\
\hline IL17REL|400935 & $\mathrm{B} 2$ \\
\hline IL17RE|132014 & $\mathrm{B} 2$ \\
\hline IL18BP|10068 & $\mathrm{B} 2$ \\
\hline IL18R1|8809 & $\mathrm{B} 2$ \\
\hline IL18RAP|8807 & $\mathrm{B} 2$ \\
\hline IL19|29949 & $\mathrm{B} 2$ \\
\hline IL1A|3552 & $\mathrm{B} 2$ \\
\hline IL1B|3553 & $\mathrm{B} 2$ \\
\hline IL1F10|84639 & $\mathrm{B} 2$ \\
\hline IL1F5|26525 & $\mathrm{B} 2$ \\
\hline IL1F6|27179 & $\mathrm{B} 2$ \\
\hline IL1F7|27178 & $\mathrm{B} 2$ \\
\hline IL1F8|27177 & $\mathrm{B} 2$ \\
\hline
\end{tabular}




\begin{tabular}{|l|l|} 
IL1F9|56300 & B2 \\
\hline IL1R2|7850 & B2 \\
\hline IL1RAPL1|11141 & B2 \\
\hline IL1RAPL2|26280 & B2 \\
\hline IL1RAP|3556 & B2 \\
\hline IL1RL1|9173 & B2 \\
\hline IL1RN|3557 & B2 \\
\hline IL20RA|53832 & B2 \\
\hline IL20RB|53833 & B2 \\
\hline IL20|50604 & B2 \\
\hline IL21|59067 & B2 \\
\hline IL22RA2|116379 & B2 \\
\hline IL22|50616 & B2 \\
\hline IL23A|51561 & B2 \\
\hline IL23R|149233 & B2 \\
\hline IL24|11009 & B2 \\
\hline IL25|64806 & B2 \\
\hline IL26|55801 & B2 \\
\hline IL27RA|9466 & B2 \\
\hline IL27|246778 & B2 \\
\hline IL28A|282616 & B2 \\
\hline IL28B|282617 & B2 \\
\hline IL29|282618 & B2 \\
\hline IL2RA|3559 & B2 \\
\hline IL2RB|3560 & B2 \\
\hline IL2RG|3561 & B2 \\
\hline IL2|3558 & B2 \\
\hline IL31RA|133396 & B2 \\
\hline IL32|9235 & B2 \\
\hline IL34|146433 & B2 \\
\hline IL4|3565 & B2 \\
\hline IL5RA|3568 & B2 \\
\hline IL5|3567 & B2 \\
\hline IL6ST|3572 & B2 \\
\hline IL6|3569 & B2 \\
\hline IL7R|3575 & B2 \\
\hline IL7|3574 & B2 \\
\hline IL8|3576 & B2 \\
\hline IL9|3578 & B2 \\
\hline ILF3|3609 & B2 \\
\hline ILKAP|80895 & B2 \\
\hline ILVBL|10994 & IMMP1L|196294 \\
\hline IMMT|10989 & IMP3|55272 \\
\hline IMP4|92856 & IMP5|162540 \\
\hline IMPA1|3612 & IMPA2|3613 \\
\hline IMPACT|55364 & \\
\hline
\end{tabular}




\begin{tabular}{|c|c|}
\hline IMPDH1|3614 & B2 \\
\hline IMPDH2|3615 & B2 \\
\hline IMPG1|3617 & B2 \\
\hline IMPG2|50939 & $\mathrm{B} 2$ \\
\hline INADL|10207 & $\mathrm{B} 2$ \\
\hline INA|9118 & $\mathrm{B} 2$ \\
\hline INCA1|388324 & $\mathrm{B} 2$ \\
\hline INE2|8551 & $\mathrm{B} 2$ \\
\hline INF2|64423 & $\mathrm{B} 2$ \\
\hline ING1|3621 & $\mathrm{B} 2$ \\
\hline ING2|3622 & $\mathrm{B} 2$ \\
\hline ING4|51147 & $\mathrm{B} 2$ \\
\hline INGX|27160 & $\mathrm{B} 2$ \\
\hline INHA|3623 & $\mathrm{B} 2$ \\
\hline INHBC $\mid 3626$ & $\mathrm{~B} 2$ \\
\hline INHBE|83729 & $\mathrm{B} 2$ \\
\hline INO80B $\mid 83444$ & $\mathrm{~B} 2$ \\
\hline INO80C|125476 & $\mathrm{B} 2$ \\
\hline INO80D|54891 & $\mathrm{B} 2$ \\
\hline INO80E|283899 & $\mathrm{B} 2$ \\
\hline INPP4A|3631 & $\mathrm{B} 2$ \\
\hline INPP4B $\mid 8821$ & $\mathrm{~B} 2$ \\
\hline INPP5D|3635 & $\mathrm{B} 2$ \\
\hline INPP5E|56623 & $\mathrm{B} 2$ \\
\hline INPP5F|22876 & $\mathrm{B} 2$ \\
\hline INPP5J|27124 & $\mathrm{B} 2$ \\
\hline INPPL1|3636 & $\mathrm{B} 2$ \\
\hline INS-IGF2|723961 & $\mathrm{B} 2$ \\
\hline INSC|387755 & $\mathrm{B} 2$ \\
\hline INSIG1|3638 & $\mathrm{B} 2$ \\
\hline \begin{tabular}{l|l|} 
INSIG2|51141 \\
\end{tabular} & $\mathrm{B} 2$ \\
\hline INSL3|3640 & $\mathrm{B} 2$ \\
\hline INSL4|3641 & $\mathrm{B} 2$ \\
\hline INSL5|10022 & $\mathrm{B} 2$ \\
\hline INSL6|11172 & $\mathrm{B} 2$ \\
\hline INSM1|3642 & $\mathrm{B} 2$ \\
\hline INSM2|84684 & $\mathrm{B} 2$ \\
\hline INSRR|3645 & $\mathrm{B} 2$ \\
\hline INSR|3643 & $\mathrm{B} 2$ \\
\hline INTS12|57117 & $\mathrm{B} 2$ \\
\hline INTS1|26173 & $\mathrm{B} 2$ \\
\hline INTS2|57508 & $\mathrm{B} 2$ \\
\hline INTS3|65123 & $\mathrm{B} 2$ \\
\hline INTS4L1|285905 & $\mathrm{B} 2$ \\
\hline INTS4L2|644619 & $\mathrm{B} 2$ \\
\hline INTS4|92105 & $\mathrm{B} 2$ \\
\hline INTS5|80789 & $\mathrm{B} 2$ \\
\hline INTS6|26512 & $\mathrm{B} 2$ \\
\hline INTS9|55756 & $\mathrm{B} 2$ \\
\hline IP6K2|51447 & $\mathrm{B} 2$ \\
\hline \begin{tabular}{l|l|l|} 
IP6K3|17283 \\
\end{tabular} & $\mathrm{B} 2$ \\
\hline
\end{tabular}




\begin{tabular}{|c|c|}
\hline IPCEF1|26034 & B2 \\
\hline IPMK|253430 & B2 \\
\hline IPO11|51194 & B2 \\
\hline IPO13|9670 & $\mathrm{B} 2$ \\
\hline IPO5|3843 & $\mathrm{B} 2$ \\
\hline IPO7|10527 & $\mathrm{B} 2$ \\
\hline IPO8|10526 & $\mathrm{B} 2$ \\
\hline IPP $\mid 3652$ & $\mathrm{~B} 2$ \\
\hline IQCA1|79781 & $\mathrm{B} 2$ \\
\hline IQCB1|9657 & $\mathrm{B} 2$ \\
\hline IQCC|55721 & $\mathrm{B} 2$ \\
\hline IQCE|23288 & $\mathrm{B} 2$ \\
\hline IQCF1|132141 & $\mathrm{B} 2$ \\
\hline IQCF2|389123 & $\mathrm{B} 2$ \\
\hline IQCF3|401067 & $\mathrm{B} 2$ \\
\hline IQCF6|440956 & $\mathrm{B} 2$ \\
\hline IQCG $\mid 84223$ & $\mathrm{~B} 2$ \\
\hline IQCH|64799 & $\mathrm{B} 2$ \\
\hline IQCJ|654502 & $\mathrm{B} 2$ \\
\hline IQGAP1|8826 & $\mathrm{B} 2$ \\
\hline IQGAP2|10788 & $\mathrm{B} 2$ \\
\hline IQSEC2|23096 & $\mathrm{B} 2$ \\
\hline IQUB|154865 & $\mathrm{B} 2$ \\
\hline IRAK1BP1|134728 & $\mathrm{B} 2$ \\
\hline IRAK1|3654 & $\mathrm{B} 2$ \\
\hline IRAK3|11213 & $\mathrm{B} 2$ \\
\hline IRAK4|51135 & $\mathrm{B} 2$ \\
\hline IREB2|3658 & $\mathrm{B} 2$ \\
\hline IRF1|3659 & $\mathrm{B} 2$ \\
\hline IRF2BP1|26145 & $\mathrm{B} 2$ \\
\hline IRF2BP2|359948 & $\mathrm{B} 2$ \\
\hline IRF2|3660 & $\mathrm{B} 2$ \\
\hline \begin{tabular}{ll|l} 
IRF3 & 3661 \\
\end{tabular} & $\mathrm{~B} 2$ \\
\hline IRF4|3662 & $\mathrm{B} 2$ \\
\hline IRF5|3663 & $\mathrm{B} 2$ \\
\hline IRF6|3664 & $\mathrm{B} 2$ \\
\hline IRF8|3394 & $\mathrm{B} 2$ \\
\hline IRGC|56269 & $\mathrm{B} 2$ \\
\hline IRGM|345611 & $\mathrm{B} 2$ \\
\hline IRGQ|126298 & $\mathrm{B} 2$ \\
\hline IRS4|8471 & $\mathrm{B} 2$ \\
\hline IRX2|153572 & $\mathrm{B} 2$ \\
\hline IRX3|79191 & $\mathrm{B} 2$ \\
\hline IRX4|50805 & $\mathrm{B} 2$ \\
\hline ISCA1P1|389293 & $\mathrm{B} 2$ \\
\hline ISCA2|122961 & $\mathrm{B} 2$ \\
\hline ISG20|3669 & $\mathrm{B} 2$ \\
\hline \begin{tabular}{l|l|l|} 
ISL1670 & \\
\end{tabular} & $\mathrm{B} 2$ \\
\hline ISL2|64843 & $\mathrm{B} 2$ \\
\hline ISLR|3671 & $\mathrm{B} 2$ \\
\hline ISM2|145501 & $\mathrm{B} 2$ \\
\hline
\end{tabular}




\begin{tabular}{|c|c|}
\hline ISOC1|51015 & B2 \\
\hline ISPD|729920 & B2 \\
\hline ISX|91464 & B2 \\
\hline ISY1|57461 & $\mathrm{B} 2$ \\
\hline ISYNA1|51477 & $\mathrm{B} 2$ \\
\hline ITCH $\mid 83737$ & $\mathrm{~B} 2$ \\
\hline ITFG1 $\mid 81533$ & $\mathrm{~B} 2$ \\
\hline ITFG3|83986 & $\mathrm{B} 2$ \\
\hline ITGA10|8515 & $\mathrm{B} 2$ \\
\hline ITGA11|22801 & $\mathrm{B} 2$ \\
\hline ITGA2B|3674 & $\mathrm{B} 2$ \\
\hline ITGA2|3673 & $\mathrm{B} 2$ \\
\hline ITGA4|3676 & $\mathrm{B} 2$ \\
\hline ITGA5|3678 & $\mathrm{B} 2$ \\
\hline ITGA8 85516 & $\mathrm{~B} 2$ \\
\hline ITGAD|3681 & $\mathrm{B} 2$ \\
\hline ITGAE|3682 & $\mathrm{B} 2$ \\
\hline ITGAL|3683 & $\mathrm{B} 2$ \\
\hline ITGAM|3684 & $\mathrm{B} 2$ \\
\hline ITGAV|3685 & $\mathrm{B} 2$ \\
\hline ITGAX|3687 & $\mathrm{B} 2$ \\
\hline ITGB1BP2|26548 & $\mathrm{B} 2$ \\
\hline ITGB1BP3|27231 & $\mathrm{B} 2$ \\
\hline ITGB2|3689 & $\mathrm{B} 2$ \\
\hline ITGB3BP|23421 & $\mathrm{B} 2$ \\
\hline ITGB3|3690 & $\mathrm{B} 2$ \\
\hline ITGB4|3691 & $\mathrm{B} 2$ \\
\hline ITGB5|3693 & $\mathrm{B} 2$ \\
\hline ITGB6|3694 & $\mathrm{B} 2$ \\
\hline ITGB7|3695 & $\mathrm{B} 2$ \\
\hline ITGB8|3696 & $\mathrm{B} 2$ \\
\hline ITGBL1|9358 & $\mathrm{B} 2$ \\
\hline ITIH1|3697 & $\mathrm{B} 2$ \\
\hline ITIH2|3698 & $\mathrm{B} 2$ \\
\hline ITIH3|3699 & $\mathrm{B} 2$ \\
\hline ITIH4|3700 & $\mathrm{B} 2$ \\
\hline ITIH5L|347365 & $\mathrm{B} 2$ \\
\hline ITK|3702 & $\mathrm{B} 2$ \\
\hline ITLN1 55600 & $\mathrm{~B} 2$ \\
\hline ITLN2|142683 & $\mathrm{B} 2$ \\
\hline ITM2C|81618 & $\mathrm{B} 2$ \\
\hline ITPA|3704 & $\mathrm{B} 2$ \\
\hline ITPK1|3705 & $\mathrm{B} 2$ \\
\hline ITPKA|3706 & $\mathrm{B} 2$ \\
\hline ITPKB|3707 & $\mathrm{B} 2$ \\
\hline ITPKC|80271 & $\mathrm{B} 2$ \\
\hline ITPR2|3709 & $\mathrm{B} 2$ \\
\hline ITPRIPL2|162073 & $\mathrm{B} 2$ \\
\hline $\begin{array}{l}\text { ITPRIP|85450 } \\
\end{array}$ & $\mathrm{B} 2$ \\
\hline ITSN2|50618 & $\mathrm{B} 2$ \\
\hline IVD|3712 & $\mathrm{B} 2$ \\
\hline
\end{tabular}




\begin{tabular}{|c|c|}
\hline IVL/3713 & B2 \\
\hline IVNS1ABP|10625 & $\mathrm{B} 2$ \\
\hline IWS1|55677 & $\mathrm{B} 2$ \\
\hline IYD|389434 & $\mathrm{B} 2$ \\
\hline IZUMO1|284359 & $\mathrm{B} 2$ \\
\hline JAGN1|84522 & B2 \\
\hline JAK2|3717 & $\mathrm{B} 2$ \\
\hline JAK3|3718 & $\mathrm{B} 2$ \\
\hline JAKMIP2|9832 & $\mathrm{B} 2$ \\
\hline JAKMIP3|282973 & $\mathrm{B} 2$ \\
\hline JHDM1D|80853 & $\mathrm{B} 2$ \\
\hline JKAMP|51528 & B2 \\
\hline $\begin{array}{l}\text { JMJD5|79831 } \\
\end{array}$ & $\mathrm{B} 2$ \\
\hline JMJD6|23210 & $\mathrm{B} 2$ \\
\hline JMJD8|339123 & $\mathrm{B} 2$ \\
\hline JOSD1|9929 & $\mathrm{B} 2$ \\
\hline JOSD2|126119 & $\mathrm{B} 2$ \\
\hline JPH1|56704 & $\mathrm{B} 2$ \\
\hline JPH3|57338 & B2 \\
\hline JSRP1|126306 & $\mathrm{B} 2$ \\
\hline JUB|84962 & $\mathrm{B} 2$ \\
\hline JUNB|3726 & $\mathrm{B} 2$ \\
\hline JUP|3728 & $\mathrm{B} 2$ \\
\hline KAAG1|353219 & $\mathrm{B} 2$ \\
\hline KAL1|3730 & $\mathrm{B} 2$ \\
\hline KANK4|163782 & $\mathrm{B} 2$ \\
\hline KARS|3735 & B2 \\
\hline KAT2A|2648 & B2 \\
\hline KAT5|10524 & $\mathrm{B} 2$ \\
\hline KATNA1|11104 & $\mathrm{B} 2$ \\
\hline KATNAL2|83473 & $\mathrm{B} 2$ \\
\hline KATNB1|10300 & $\mathrm{B} 2$ \\
\hline KAZALD1|81621 & $\mathrm{B} 2$ \\
\hline KAZ|23254 & B2 \\
\hline KBTBD10|10324 & $\mathrm{B} 2$ \\
\hline KBTBD12|166348 & $\mathrm{B} 2$ \\
\hline KBTBD13|390594 & $\mathrm{B} 2$ \\
\hline KBTBD2|25948 & $\mathrm{B} 2$ \\
\hline KBTBD4|55709 & B2 \\
\hline KBTBD5|131377 & $\mathrm{B} 2$ \\
\hline KBTBD7|84078 & B2 \\
\hline KBTBD8|84541 & $\mathrm{B} 2$ \\
\hline KC6|641516 & B2 \\
\hline KCMF1|56888 & $\mathrm{B} 2$ \\
\hline KCNA10|3744 & $\mathrm{B} 2$ \\
\hline KCNA3|3738 & $\mathrm{B} 2$ \\
\hline KCNA5|3741 & $\mathrm{B} 2$ \\
\hline KCNA7|3743 & $\mathrm{B} 2$ \\
\hline KCNAB2|8514 & $\mathrm{B} 2$ \\
\hline KCNAB3|9196 & $\mathrm{B} 2$ \\
\hline KCNB2|9312 & $\mathrm{B} 2$ \\
\hline
\end{tabular}




\begin{tabular}{|c|c|}
\hline KCNC1|3746 & B2 \\
\hline KCNC2|3747 & $\mathrm{B} 2$ \\
\hline KCNC3|3748 & $\mathrm{B} 2$ \\
\hline KCND1 13750 & $\mathrm{~B} 2$ \\
\hline KCND2|3751 & $\mathrm{B} 2$ \\
\hline KCND3|3752 & $\mathrm{B} 2$ \\
\hline KCNE1L|23630 & $\mathrm{B} 2$ \\
\hline KCNE2|9992 & $\mathrm{B} 2$ \\
\hline KCNE3|10008 & $\mathrm{B} 2$ \\
\hline KCNE4|23704 & $\mathrm{B} 2$ \\
\hline KCNF1|3754 & $\mathrm{B} 2$ \\
\hline KCNG1|3755 & $\mathrm{B} 2$ \\
\hline KCNG2|26251 & $\mathrm{B} 2$ \\
\hline KCNG3|170850 & $\mathrm{B} 2$ \\
\hline KCNG4|93107 & $\mathrm{B} 2$ \\
\hline KCNH2|3757 & $\mathrm{B} 2$ \\
\hline KCNH4|23415 & B2 \\
\hline KCNH6|81033 & $\mathrm{B} 2$ \\
\hline KCNH7|90134 & $\mathrm{B} 2$ \\
\hline KCNJ10|3766 & $\mathrm{B} 2$ \\
\hline KCNJ11|3767 & $\mathrm{B} 2$ \\
\hline KCNJ12|3768 & $\mathrm{B} 2$ \\
\hline $\begin{array}{l}\text { KCNJ13|3769 } \\
\end{array}$ & $\mathrm{B} 2$ \\
\hline KCNJ14|3770 & $\mathrm{B} 2$ \\
\hline KCNJ15|3772 & $\mathrm{B} 2$ \\
\hline KCNJ16|3773 & $\mathrm{B} 2$ \\
\hline KCNJ1|3758 & $\mathrm{B} 2$ \\
\hline KCNJ3|3760 & $\mathrm{B} 2$ \\
\hline KCNJ4|3761 & $\mathrm{B} 2$ \\
\hline KCNJ5|3762 & $\mathrm{B} 2$ \\
\hline KCNJ6|3763 & $\mathrm{B} 2$ \\
\hline KCNJ9|3765 & $\mathrm{B} 2$ \\
\hline KCNK10|54207 & $\mathrm{B} 2$ \\
\hline KCNK12|56660 & $\mathrm{B} 2$ \\
\hline KCNK13|56659 & $\mathrm{B} 2$ \\
\hline KCNK16|83795 & $\mathrm{B} 2$ \\
\hline KCNK17|89822 & $\mathrm{B} 2$ \\
\hline KCNK2|3776 & $\mathrm{B} 2$ \\
\hline KCNK3|3777 & $\mathrm{B} 2$ \\
\hline KCNK4|50801 & $\mathrm{B} 2$ \\
\hline KCNK5|8645 & $\mathrm{B} 2$ \\
\hline KCNK7|10089 & $\mathrm{B} 2$ \\
\hline KCNK9|51305 & $\mathrm{B} 2$ \\
\hline KCNMA1|3778 & $\mathrm{B} 2$ \\
\hline KCNMB2|10242 & $\mathrm{B} 2$ \\
\hline KCNMB3|27094 & $\mathrm{B} 2$ \\
\hline KCNMB4|27345 & $\mathrm{B} 2$ \\
\hline KCNN1|3780 & $\mathrm{B} 2$ \\
\hline KCNN2|3781 & $\mathrm{B} 2$ \\
\hline KCNN3|3782 & $\mathrm{B} 2$ \\
\hline KCNN4|3783 & $\mathrm{B} 2$ \\
\hline
\end{tabular}




\begin{tabular}{|c|c|}
\hline KCNQ1DN|55539 & $\mathrm{B} 2$ \\
\hline KCNQ1OT1|10984 & $\mathrm{B} 2$ \\
\hline KCNQ1/3784 & $\mathrm{B} 2$ \\
\hline KCNQ2|3785 & $\mathrm{B} 2$ \\
\hline KCNQ3|3786 & $\mathrm{B} 2$ \\
\hline KCNQ4|9132 & $\mathrm{B} 2$ \\
\hline KCNQ5|56479 & $\mathrm{B} 2$ \\
\hline KCNRG|283518 & $\mathrm{B} 2$ \\
\hline KCNS1|3787 & $\mathrm{B} 2$ \\
\hline KCNS2|3788 & $\mathrm{B} 2$ \\
\hline KCNT1|57582 & $\mathrm{B} 2$ \\
\hline KCNU1|157855 & $\mathrm{B} 2$ \\
\hline KCNV1|27012 & $\mathrm{B} 2$ \\
\hline KCNV2|169522 & $\mathrm{B} 2$ \\
\hline $\mathrm{KCP} \mid 375616$ & $\mathrm{~B} 2$ \\
\hline KCTD11|147040 & $\mathrm{B} 2$ \\
\hline KCTD16|57528 & $\mathrm{B} 2$ \\
\hline KCTD17|79734 & $\mathrm{B} 2$ \\
\hline KCTD19|146212 & $\mathrm{B} 2$ \\
\hline KCTD1|284252 & $\mathrm{B} 2$ \\
\hline KCTD20|222658 & $\mathrm{B} 2$ \\
\hline KCTD21|283219 & $\mathrm{B} 2$ \\
\hline KCTD8|386617 & $\mathrm{B} 2$ \\
\hline KDELC2|143888 & $\mathrm{B} 2$ \\
\hline KDM2A|22992 & $\mathrm{B} 2$ \\
\hline KDM2B|84678 & $\mathrm{B} 2$ \\
\hline KDM3A|55818 & $\mathrm{B} 2$ \\
\hline KDM3B|51780 & $\mathrm{B} 2$ \\
\hline KDM4A|9682 & $\mathrm{B} 2$ \\
\hline KDM4C|23081 & $\mathrm{B} 2$ \\
\hline KDM4DL|390245 & $\mathrm{B} 2$ \\
\hline KDM4D|55693 & $\mathrm{B} 2$ \\
\hline KDM5A|5927 & $\mathrm{B} 2$ \\
\hline KDM5C $\mid 8242$ & $\mathrm{~B} 2$ \\
\hline \begin{tabular}{l|l|l|} 
KDM5D| & 8284 \\
\end{tabular} & $\mathrm{~B} 2$ \\
\hline KDM6A|7403 & $\mathrm{B} 2$ \\
\hline KEAP1|9817 & $\mathrm{B} 2$ \\
\hline KEL|3792 & $\mathrm{B} 2$ \\
\hline KERA|11081 & $\mathrm{B} 2$ \\
\hline KGFLP1|387628 & $\mathrm{B} 2$ \\
\hline KGFLP2|654466 & $\mathrm{B} 2$ \\
\hline KHDC1L|100129128 & $\mathrm{B} 2$ \\
\hline KHDC1|80759 & $\mathrm{B} 2$ \\
\hline KHDRBS2|202559 & $\mathrm{B} 2$ \\
\hline KHDRBS3|10656 & $\mathrm{B} 2$ \\
\hline KHK|3795 & $\mathrm{B} 2$ \\
\hline KHNYN|23351 & $\mathrm{B} 2$ \\
\hline KHSRP $\mid 8570$ & $\mathrm{~B} 2$ \\
\hline KIAA0020|9933 & $\mathrm{B} 2$ \\
\hline KIAA0087|9808 & $\mathrm{B} 2$ \\
\hline KIAA0090|23065 & $\mathrm{B} 2$ \\
\hline
\end{tabular}




\begin{tabular}{|c|c|}
\hline KIAA0100|9703 & B2 \\
\hline KIAA0114|57291 & B2 \\
\hline KIAA0125|9834 & B2 \\
\hline KIAA0146|23514 & $\mathrm{B} 2$ \\
\hline KIAA0174|9798 & B2 \\
\hline KIAA0182|23199 & $\mathrm{B} 2$ \\
\hline KIAA0195|9772 & $\mathrm{B} 2$ \\
\hline KIAA0232|9778 & B2 \\
\hline KIAA0247|9766 & $\mathrm{B} 2$ \\
\hline KIAA0284|283638 & B2 \\
\hline KIAA0317|9870 & B2 \\
\hline KIAA0319L|79932 & $\mathrm{B} 2$ \\
\hline KIAA0319|9856 & $\mathrm{B} 2$ \\
\hline KIAA0368|23392 & B2 \\
\hline KIAA0415|9907 & $\mathrm{B} 2$ \\
\hline KIAA0513|9764 & B2 \\
\hline KIAA0528|9847 & $\mathrm{B} 2$ \\
\hline KIAA0562|9731 & $\mathrm{B} 2$ \\
\hline KIAA0564|23078 & $\mathrm{B} 2$ \\
\hline KIAA0586|9786 & $\mathrm{B} 2$ \\
\hline KIAA0649|9858 & B2 \\
\hline KIAA0652|9776 & $\mathrm{B} 2$ \\
\hline KIAA0664P3|100132341 & $\mathrm{B} 2$ \\
\hline KIAA0664|23277 & $\mathrm{B} 2$ \\
\hline KIAA0748|9840 & B2 \\
\hline KIAA0753|9851 & B2 \\
\hline KIAA0776|23376 & $\mathrm{B} 2$ \\
\hline KIAA0802|23255 & $\mathrm{B} 2$ \\
\hline KIAA0892|23383 & $\mathrm{B} 2$ \\
\hline KIAA0895L|653319 & B2 \\
\hline KIAA0907|22889 & $\mathrm{B} 2$ \\
\hline KIAA0922|23240 & B2 \\
\hline KIAA0947|23379 & B2 \\
\hline KIAA1012|22878 & $\mathrm{B} 2$ \\
\hline KIAA1024|23251 & $\mathrm{B} 2$ \\
\hline KIAA1033|23325 & B2 \\
\hline KIAA1147|57189 & $\mathrm{B} 2$ \\
\hline KIAA1161|57462 & $\mathrm{B} 2$ \\
\hline KIAA1191|57179 & $\mathrm{B} 2$ \\
\hline KIAA1210|57481 & $\mathrm{B} 2$ \\
\hline KIAA1217|56243 & $\mathrm{B} 2$ \\
\hline KIAA1239|57495 & $\mathrm{B} 2$ \\
\hline KIAA1244|57221 & $\mathrm{B} 2$ \\
\hline KIAA1257|57501 & $\mathrm{B} 2$ \\
\hline KIAA1267|284058 & $\mathrm{B} 2$ \\
\hline KIAA1274|27143 & $\mathrm{B} 2$ \\
\hline KIAA1279|26128 & B2 \\
\hline KIAA1310|55683 & $\mathrm{B} 2$ \\
\hline KIAA1324L|222223 & $\mathrm{B} 2$ \\
\hline KIAA1324|57535 & $\mathrm{B} 2$ \\
\hline KIAA1370|56204 & $\mathrm{B} 2$ \\
\hline
\end{tabular}




\begin{tabular}{|c|c|}
\hline KIAA1377|57562 & B2 \\
\hline KIAA1383|54627 & B2 \\
\hline KIAA1407|57577 & B2 \\
\hline KIAA1409|57578 & $\mathrm{B} 2$ \\
\hline KIAA1429|25962 & $\mathrm{B} 2$ \\
\hline KIAA1430|57587 & $\mathrm{B} 2$ \\
\hline KIAA1432|57589 & $\mathrm{B} 2$ \\
\hline KIAA1462|57608 & $\mathrm{B} 2$ \\
\hline KIAA1467|57613 & $\mathrm{B} 2$ \\
\hline KIAA1468|57614 & B2 \\
\hline KIAA1486|57624 & $\mathrm{B} 2$ \\
\hline KIAA1529|57653 & $\mathrm{B} 2$ \\
\hline KIAA1530|57654 & $\mathrm{B} 2$ \\
\hline KIAA1539|80256 & $\mathrm{B} 2$ \\
\hline KIAA1549|57670 & B2 \\
\hline KIAA1586|57691 & $\mathrm{B} 2$ \\
\hline KIAA1609|57707 & $\mathrm{B} 2$ \\
\hline KIAA1632|57724 & $\mathrm{B} 2$ \\
\hline KIAA1644|85352 & $\mathrm{B} 2$ \\
\hline KIAA1704|55425 & $\mathrm{B} 2$ \\
\hline KIAA1712|80817 & B2 \\
\hline KIAA1715|80856 & $\mathrm{B} 2$ \\
\hline KIAA1731|85459 & $\mathrm{B} 2$ \\
\hline KIAA1737|85457 & $\mathrm{B} 2$ \\
\hline KIAA1751|85452 & B2 \\
\hline KIAA1755|85449 & $\mathrm{B} 2$ \\
\hline KIAA1797|54914 & $\mathrm{B} 2$ \\
\hline KIAA1804|84451 & $\mathrm{B} 2$ \\
\hline KIAA1826|84437 & $\mathrm{B} 2$ \\
\hline KIAA1875|340390 & $\mathrm{B} 2$ \\
\hline KIAA1919|91749 & $\mathrm{B} 2$ \\
\hline KIAA1949|170954 & $\mathrm{B} 2$ \\
\hline KIAA1958|158405 & $\mathrm{B} 2$ \\
\hline KIAA1967|57805 & $\mathrm{B} 2$ \\
\hline KIAA1984|84960 & $\mathrm{B} 2$ \\
\hline KIAA2013|90231 & $\mathrm{B} 2$ \\
\hline KIAA2018|205717 & $\mathrm{B} 2$ \\
\hline KIAA2022|340533 & $\mathrm{B} 2$ \\
\hline KIAA2026|158358 & $\mathrm{B} 2$ \\
\hline KIF12|113220 & $\mathrm{B} 2$ \\
\hline KIF13B|23303 & $\mathrm{B} 2$ \\
\hline \begin{tabular}{l|l|} 
KIF16B & 55614 \\
\end{tabular} & $\mathrm{~B} 2$ \\
\hline KIF19|124602 & $\mathrm{B} 2$ \\
\hline KIF1A|547 & $\mathrm{B} 2$ \\
\hline KIF1B|23095 & $\mathrm{B} 2$ \\
\hline KIF21B|23046 & $\mathrm{B} 2$ \\
\hline KIF27|55582 & $\mathrm{B} 2$ \\
\hline KIF2A|3796 & $\mathrm{B} 2$ \\
\hline KIF3A|11127 & $\mathrm{B} 2$ \\
\hline KIF3C|3797 & $\mathrm{B} 2$ \\
\hline KIF5A|3798 & $\mathrm{B} 2$ \\
\hline
\end{tabular}




\begin{tabular}{|c|c|}
\hline KIF5B|3799 & B2 \\
\hline KIF5C|3800 & B2 \\
\hline KIF6|221458 & $\mathrm{B} 2$ \\
\hline KIF9|64147 & $\mathrm{B} 2$ \\
\hline KIFC3|3801 & B2 \\
\hline KILLIN|100144748 & B2 \\
\hline KIN|22944 & $\mathrm{B} 2$ \\
\hline KIR2DL1|3802 & B2 \\
\hline KIR2DL3|3804 & $\mathrm{B} 2$ \\
\hline KIR2DL4|3805 & $\mathrm{B} 2$ \\
\hline KIR2DS4|3809 & $\mathrm{B} 2$ \\
\hline KIR3DL1|3811 & B2 \\
\hline KIR3DL2|3812 & B2 \\
\hline KIR3DL3|115653 & $\mathrm{B} 2$ \\
\hline KIR3DX1|90011 & $\mathrm{B} 2$ \\
\hline KIRREL2|84063 & $\mathrm{B} 2$ \\
\hline KIRREL3|84623 & B2 \\
\hline KISS1R|84634 & $\mathrm{B} 2$ \\
\hline KISS1|3814 & B2 \\
\hline KITLG|4254 & $\mathrm{B} 2$ \\
\hline KLC3|147700 & $\mathrm{B} 2$ \\
\hline KLC4|89953 & B2 \\
\hline KLF12|11278 & $\mathrm{B} 2$ \\
\hline KLF16|83855 & B2 \\
\hline KLF17|128209 & $\mathrm{B} 2$ \\
\hline KLF5|688 & B2 \\
\hline KLF7|8609 & B2 \\
\hline KLHDC10|23008 & B2 \\
\hline KLHDC3|116138 & $\mathrm{B} 2$ \\
\hline KLHDC4|54758 & $\mathrm{B} 2$ \\
\hline KLHDC5|57542 & $\mathrm{B} 2$ \\
\hline KLHDC7A|127707 & B2 \\
\hline KLHDC7B|113730 & B2 \\
\hline KLHDC8A|55220 & $\mathrm{B} 2$ \\
\hline KLHL11|55175 & B2 \\
\hline KLHL14|57565 & $\mathrm{B} 2$ \\
\hline KLHL17|339451 & B2 \\
\hline KLHL18|23276 & $\mathrm{B} 2$ \\
\hline KLHL1|57626 & B2 \\
\hline KLHL20|27252 & $\mathrm{B} 2$ \\
\hline KLHL22|84861 & B2 \\
\hline KLHL23|151230 & $\mathrm{B} 2$ \\
\hline KLHL24|54800 & B2 \\
\hline KLHL25|64410 & B2 \\
\hline KLHL26|55295 & B2 \\
\hline KLHL28|54813 & B2 \\
\hline KLHL30|377007 & $\mathrm{B} 2$ \\
\hline KLHL31|401265 & B2 \\
\hline KLHL33|123103 & B2 \\
\hline KLHL34|257240 & B2 \\
\hline KLHL35|283212 & $\mathrm{B} 2$ \\
\hline
\end{tabular}




\begin{tabular}{|c|c|}
\hline KLHL36|79786 & $\mathrm{B} 2$ \\
\hline KLHL38|340359 & $\mathrm{B} 2$ \\
\hline KLHL5|51088 & $\mathrm{B} 2$ \\
\hline KLHL6|89857 & $\mathrm{B} 2$ \\
\hline KLHL7|55975 & $\mathrm{B} 2$ \\
\hline KLHL8|57563 & $\mathrm{B} 2$ \\
\hline KLHL9|55958 & $\mathrm{B} 2$ \\
\hline KLK10|5655 & $\mathrm{B} 2$ \\
\hline KLK11|11012 & B2 \\
\hline KLK12|43849 & $\mathrm{B} 2$ \\
\hline KLK13|26085 & $\mathrm{B} 2$ \\
\hline KLK14|43847 & $\mathrm{B} 2$ \\
\hline KLK15|55554 & $\mathrm{B} 2$ \\
\hline KLK1|3816 & $\mathrm{B} 2$ \\
\hline KLK2|3817 & $\mathrm{B} 2$ \\
\hline KLK3|354 & $\mathrm{B} 2$ \\
\hline KLK5|25818 & $\mathrm{B} 2$ \\
\hline KLK6|5653 & $\mathrm{B} 2$ \\
\hline KLK7|5650 & $\mathrm{B} 2$ \\
\hline KLK8|11202 & $\mathrm{B} 2$ \\
\hline KLK9|284366 & $\mathrm{B} 2$ \\
\hline KLKB1|3818 & $\mathrm{B} 2$ \\
\hline KLRA1|10748 & $\mathrm{B} 2$ \\
\hline KLRB1|3820 & $\mathrm{B} 2$ \\
\hline KLRC1|3821 & $\mathrm{B} 2$ \\
\hline KLRC2|3822 & $\mathrm{B} 2$ \\
\hline KLRC3|3823 & $\mathrm{B} 2$ \\
\hline KLRC4 $\mid 8302$ & $\mathrm{~B} 2$ \\
\hline KLRD1|3824 & $\mathrm{B} 2$ \\
\hline KLRG1|10219 & $\mathrm{B} 2$ \\
\hline KLRG2|346689 & $\mathrm{B} 2$ \\
\hline KLRK1|22914 & $\mathrm{B} 2$ \\
\hline KMO|8564 & $\mathrm{B} 2$ \\
\hline KNCN|148930 & $\mathrm{B} 2$ \\
\hline KNDC1|85442 & $\mathrm{B} 2$ \\
\hline KNG1/3827 & $\mathrm{B} 2$ \\
\hline KPNA1|3836 & $\mathrm{B} 2$ \\
\hline KPNA3|3839 & $\mathrm{B} 2$ \\
\hline KPNA4|3840 & $\mathrm{B} 2$ \\
\hline KPNA6|23633 & $\mathrm{B} 2$ \\
\hline KPNA7|402569 & $\mathrm{B} 2$ \\
\hline KPRP|448834 & $\mathrm{B} 2$ \\
\hline KRBA1|84626 & $\mathrm{B} 2$ \\
\hline KRCC1|51315 & $\mathrm{B} 2$ \\
\hline KREMEN2|79412 & $\mathrm{B} 2$ \\
\hline KRI1|65095 & $\mathrm{B} 2$ \\
\hline KRIT1|889 & $\mathrm{B} 2$ \\
\hline KRR1|11103 & $\mathrm{B} 2$ \\
\hline KRT10|3858 & $\mathrm{B} 2$ \\
\hline KRT12|3859 & $\mathrm{B} 2$ \\
\hline KRT13|3860 & $\mathrm{B} 2$ \\
\hline
\end{tabular}




\begin{tabular}{|c|c|}
\hline KRT16|3868 & B2 \\
\hline KRT1|3848 & B2 \\
\hline KRT20|54474 & $\mathrm{B} 2$ \\
\hline KRT222|125113 & $\mathrm{B} 2$ \\
\hline KRT23|25984 & $\mathrm{B} 2$ \\
\hline KRT24|192666 & $\mathrm{B} 2$ \\
\hline KRT25|147183 & $\mathrm{B} 2$ \\
\hline KRT27|342574 & $\mathrm{B} 2$ \\
\hline KRT2|3849 & $\mathrm{B} 2$ \\
\hline KRT31|3881 & $\mathrm{B} 2$ \\
\hline KRT32|3882 & $\mathrm{B} 2$ \\
\hline KRT33A|3883 & $\mathrm{B} 2$ \\
\hline KRT33B|3884 & $\mathrm{B} 2$ \\
\hline KRT34|3885 & $\mathrm{B} 2$ \\
\hline KRT35|3886 & $\mathrm{B} 2$ \\
\hline KRT36|8689 & $\mathrm{B} 2$ \\
\hline KRT37|8688 & $\mathrm{B} 2$ \\
\hline KRT38|8687 & $\mathrm{B} 2$ \\
\hline KRT39|390792 & $\mathrm{B} 2$ \\
\hline KRT3|3850 & $\mathrm{B} 2$ \\
\hline KRT40|125115 & $\mathrm{B} 2$ \\
\hline KRT4|3851 & $\mathrm{B} 2$ \\
\hline KRT6A|3853 & $\mathrm{B} 2$ \\
\hline KRT6B|3854 & $\mathrm{B} 2$ \\
\hline KRT6C|286887 & $\mathrm{B} 2$ \\
\hline KRT71|112802 & $\mathrm{B} 2$ \\
\hline KRT72|140807 & $\mathrm{B} 2$ \\
\hline KRT73|319101 & $\mathrm{B} 2$ \\
\hline KRT74|121391 & $\mathrm{B} 2$ \\
\hline KRT75|9119 & $\mathrm{B} 2$ \\
\hline KRT76|51350 & $\mathrm{B} 2$ \\
\hline KRT77|374454 & $\mathrm{B} 2$ \\
\hline KRT78|196374 & $\mathrm{B} 2$ \\
\hline KRT79|338785 & $\mathrm{B} 2$ \\
\hline KRT7|3855 & $\mathrm{B} 2$ \\
\hline KRT80|144501 & $\mathrm{B} 2$ \\
\hline KRT81|3887 & $\mathrm{B} 2$ \\
\hline KRT82|3888 & $\mathrm{B} 2$ \\
\hline KRT83|3889 & $\mathrm{B} 2$ \\
\hline KRT84|3890 & $\mathrm{B} 2$ \\
\hline KRT85|3891 & $\mathrm{B} 2$ \\
\hline KRT86|3892 & $\mathrm{B} 2$ \\
\hline $\begin{array}{l}\text { KRT9|3857 } \\
\end{array}$ & $\mathrm{B} 2$ \\
\hline KRTAP1-1|81851 & $\mathrm{B} 2$ \\
\hline KRTAP1-3|81850 & $\mathrm{B} 2$ \\
\hline KRTAP1-5|83895 & $\mathrm{B} 2$ \\
\hline KRTAP10-12|386685 & $\mathrm{B} 2$ \\
\hline KRTAP10-2|386679 & $\mathrm{B} 2$ \\
\hline KRTAP10-4|386672 & $\mathrm{B} 2$ \\
\hline KRTAP11-1|337880 & $\mathrm{B} 2$ \\
\hline KRTAP12-1|353332 & $\mathrm{B} 2$ \\
\hline
\end{tabular}




\begin{tabular}{|c|c|}
\hline KRTAP13-2|337959 & B2 \\
\hline KRTAP17-1|83902 & $\mathrm{B} 2$ \\
\hline KRTAP2-1|81872 & B2 \\
\hline KRTAP2-2|728279 & $\mathrm{B} 2$ \\
\hline KRTAP21-2|337978 & B2 \\
\hline KRTAP3-1|83896 & B2 \\
\hline KRTAP3-2|83897 & $\mathrm{B} 2$ \\
\hline KRTAP3-3|85293 & $\mathrm{B} 2$ \\
\hline KRTAP4-11|653240 & $\mathrm{B} 2$ \\
\hline KRTAP4-1|85285 & $\mathrm{B} 2$ \\
\hline KRTAP4-4|84616 & B2 \\
\hline KRTAP5-10|387273 & B2 \\
\hline KRTAP5-11|440051 & $\mathrm{B} 2$ \\
\hline KRTAP5-1|387264 & $\mathrm{B} 2$ \\
\hline KRTAP5-2|440021 & $\mathrm{B} 2$ \\
\hline KRTAP5-3|387266 & $\mathrm{B} 2$ \\
\hline KRTAP5-4|387267 & $\mathrm{B} 2$ \\
\hline KRTAP5-5|439915 & $\mathrm{B} 2$ \\
\hline KRTAP5-6|440023 & $\mathrm{B} 2$ \\
\hline KRTAP5-7|440050 & $\mathrm{B} 2$ \\
\hline KRTAP5-8|57830 & $\mathrm{B} 2$ \\
\hline KRTAP5-9|3846 & $\mathrm{B} 2$ \\
\hline KRTAP7-1|337878 & $\mathrm{B} 2$ \\
\hline KRTAP9-4|85280 & $\mathrm{B} 2$ \\
\hline KRTCAP3|200634 & $\mathrm{B} 2$ \\
\hline KRTDAP|388533 & $\mathrm{B} 2$ \\
\hline KSR2|283455 & $\mathrm{B} 2$ \\
\hline KTELC1|56983 & $\mathrm{B} 2$ \\
\hline KTI12|112970 & $\mathrm{B} 2$ \\
\hline KTN1|3895 & $\mathrm{B} 2$ \\
\hline KYNU|8942 & $\mathrm{B} 2$ \\
\hline L1CAM|3897 & $\mathrm{B} 2$ \\
\hline L1TD1|54596 & $\mathrm{B} 2$ \\
\hline L2HGDH|79944 & B2 \\
\hline L3MBTL2|83746 & $\mathrm{B} 2$ \\
\hline L3MBTL3|84456 & $\mathrm{B} 2$ \\
\hline LACE1|246269 & $\mathrm{B} 2$ \\
\hline LACRT $\mid 90070$ & $\mathrm{~B} 2$ \\
\hline LACTB2|51110 & $\mathrm{B} 2$ \\
\hline LACTB|114294 & $\mathrm{B} 2$ \\
\hline LAD1|3898 & $\mathrm{B} 2$ \\
\hline LAG3|3902 & $\mathrm{B} 2$ \\
\hline LAIR1|3903 & $\mathrm{B} 2$ \\
\hline LAIR2|3904 & $\mathrm{B} 2$ \\
\hline LALBA|3906 & B2 \\
\hline LAMA5|3911 & $\mathrm{B} 2$ \\
\hline LAMB1|3912 & $\mathrm{B} 2$ \\
\hline LAMB4|22798 & $\mathrm{B} 2$ \\
\hline LAMP1|3916 & $\mathrm{B} 2$ \\
\hline LAMP2|3920 & $\mathrm{B} 2$ \\
\hline \begin{tabular}{l|l|l|} 
LAMP3|27074 \\
\end{tabular} & $\mathrm{B} 2$ \\
\hline
\end{tabular}




\begin{tabular}{|c|c|}
\hline LANCL2|55915 & B2 \\
\hline LANCL3|347404 & $\mathrm{B} 2$ \\
\hline LAP3|51056 & $\mathrm{B} 2$ \\
\hline LAPTM4A|9741 & B2 \\
\hline LAPTM5|7805 & B2 \\
\hline LARGE|9215 & B2 \\
\hline LARP1|23367 & B2 \\
\hline LARP7|51574 & $\mathrm{B} 2$ \\
\hline LARS2|23395 & $\mathrm{B} 2$ \\
\hline LARS|51520 & $\mathrm{B} 2$ \\
\hline LASP1|3927 & B2 \\
\hline LASS1|10715 & $\mathrm{B} 2$ \\
\hline LASS3|204219 & $\mathrm{B} 2$ \\
\hline LASS4|79603 & $\mathrm{B} 2$ \\
\hline LASS5|91012 & $\mathrm{B} 2$ \\
\hline LATS1|9113 & $\mathrm{B} 2$ \\
\hline LAT 27040 & $\mathrm{~B} 2$ \\
\hline LAX1|54900 & $\mathrm{B} 2$ \\
\hline LAYN|143903 & $\mathrm{B} 2$ \\
\hline $\mathrm{LBH} \mid 81606$ & $\mathrm{~B} 2$ \\
\hline LBP|3929 & $\mathrm{B} 2$ \\
\hline LBR/3930 & $\mathrm{B} 2$ \\
\hline LBX1|10660 & $\mathrm{B} 2$ \\
\hline LBX2|85474 & $\mathrm{B} 2$ \\
\hline LCE1B|353132 & $\mathrm{B} 2$ \\
\hline LCE1C|353133 & $\mathrm{B} 2$ \\
\hline LCE1E|353135 & $\mathrm{B} 2$ \\
\hline LCE2A|353139 & $\mathrm{B} 2$ \\
\hline LCE3A|353142 & $\mathrm{B} 2$ \\
\hline LCE3D|84648 & $\mathrm{B} 2$ \\
\hline LCE3E|353145 & $\mathrm{B} 2$ \\
\hline LCE5A|254910 & $\mathrm{B} 2$ \\
\hline LCK|3932 & $\mathrm{B} 2$ \\
\hline LCMT2|9836 & $\mathrm{B} 2$ \\
\hline LCN12|286256 & $\mathrm{B} 2$ \\
\hline LCN15|389812 & $\mathrm{B} 2$ \\
\hline LCN1|3933 & $\mathrm{B} 2$ \\
\hline LCN2|3934 & $\mathrm{B} 2$ \\
\hline \begin{tabular}{ll|l|} 
LCN8|138307 \\
\end{tabular} & $\mathrm{B} 2$ \\
\hline LCNL1|401562 & $\mathrm{B} 2$ \\
\hline LCORL|254251 & $\mathrm{B} 2$ \\
\hline LCOR $\mid 84458$ & $\mathrm{~B} 2$ \\
\hline LCP1|3936 & $\mathrm{B} 2$ \\
\hline LCP2|3937 & $\mathrm{B} 2$ \\
\hline LCTL|197021 & $\mathrm{B} 2$ \\
\hline LCT|3938 & $\mathrm{B} 2$ \\
\hline LDB1|8861 & $\mathrm{B} 2$ \\
\hline LDB3|11155 & $\mathrm{B} 2$ \\
\hline LDHAL6A|160287 & $\mathrm{B} 2$ \\
\hline LDHAL6B|92483 & B2 \\
\hline LDHC|3948 & B2 \\
\hline
\end{tabular}




\begin{tabular}{|c|c|}
\hline LDLRAD1|388633 & B2 \\
\hline LDLRAD3|143458 & $\mathrm{B} 2$ \\
\hline LDLRAP1|26119 & $\mathrm{B} 2$ \\
\hline LDLR/3949 & $\mathrm{B} 2$ \\
\hline LDOC1L|84247 & $\mathrm{B} 2$ \\
\hline LDOC1|23641 & $\mathrm{B} 2$ \\
\hline LEAP2|116842 & $\mathrm{B} 2$ \\
\hline LECT1|11061 & $\mathrm{B} 2$ \\
\hline LECT2|3950 & B2 \\
\hline LEFTY1|10637 & $\mathrm{B} 2$ \\
\hline LEFTY2|7044 & $\mathrm{B} 2$ \\
\hline LEKR1|389170 & $\mathrm{B} 2$ \\
\hline LEMD1|93273 & $\mathrm{B} 2$ \\
\hline LEMD3|23592 & $\mathrm{B} 2$ \\
\hline LENEP|55891 & B2 \\
\hline LENG1|79165 & $\mathrm{B} 2$ \\
\hline LENG9|94059 & $\mathrm{B} 2$ \\
\hline LEPROTL1|23484 & $\mathrm{B} 2$ \\
\hline LETM2|137994 & B2 \\
\hline LFNG|3955 & $\mathrm{B} 2$ \\
\hline LGALS14|56891 & $\mathrm{B} 2$ \\
\hline LGALS1|3956 & $\mathrm{B} 2$ \\
\hline LGALS2|3957 & $\mathrm{B} 2$ \\
\hline LGALS3BP|3959 & $\mathrm{B} 2$ \\
\hline LGALS4|3960 & $\mathrm{B} 2$ \\
\hline LGALS7B|653499 & $\mathrm{B} 2$ \\
\hline LGALS7|3963 & $\mathrm{B} 2$ \\
\hline LGALS9B|284194 & B2 \\
\hline LGALS9C|654346 & $\mathrm{B} 2$ \\
\hline $\begin{array}{l}\text { LGALS9|3965 } \\
\end{array}$ & $\mathrm{B} 2$ \\
\hline LGI1|9211 & $\mathrm{B} 2$ \\
\hline LGI2|55203 & B2 \\
\hline LGI3|203190 & $\mathrm{B} 2$ \\
\hline LGMN|5641 & B2 \\
\hline LGR5|8549 & $\mathrm{B} 2$ \\
\hline LGSN|51557 & $\mathrm{B} 2$ \\
\hline LGTN|1939 & $\mathrm{B} 2$ \\
\hline LHB|3972 & $\mathrm{B} 2$ \\
\hline LHFPL1|340596 & $\mathrm{B} 2$ \\
\hline LHFPL2|10184 & $\mathrm{B} 2$ \\
\hline LHFPL3|375612 & B2 \\
\hline LHFPL4|375323 & $\mathrm{B} 2$ \\
\hline LHFPL5|222662 & B2 \\
\hline LHPP|64077 & $\mathrm{B} 2$ \\
\hline LHX1|3975 & $\mathrm{B} 2$ \\
\hline LHX2|9355 & $\mathrm{B} 2$ \\
\hline LHX3|8022 & $\mathrm{B} 2$ \\
\hline LHX4|89884 & $\mathrm{B} 2$ \\
\hline LHX5|64211 & $\mathrm{B} 2$ \\
\hline LHX8|431707 & $\mathrm{B} 2$ \\
\hline LHX9|56956 & $\mathrm{B} 2$ \\
\hline
\end{tabular}




\begin{tabular}{|c|c|}
\hline LIG4|3981 & B2 \\
\hline LILRA1|11024 & $\mathrm{B} 2$ \\
\hline LILRA2|11027 & $\mathrm{B} 2$ \\
\hline LILRA3|11026 & $\mathrm{B} 2$ \\
\hline LILRA4|23547 & $\mathrm{B} 2$ \\
\hline LILRA5|353514 & B2 \\
\hline LILRA6|79168 & $\mathrm{B} 2$ \\
\hline LILRB1|10859 & $\mathrm{B} 2$ \\
\hline LILRB2|10288 & $\mathrm{B} 2$ \\
\hline LILRB3|11025 & $\mathrm{B} 2$ \\
\hline LILRB4|11006 & $\mathrm{B} 2$ \\
\hline LILRP2|79166 & B2 \\
\hline LIM2|3982 & $\mathrm{B} 2$ \\
\hline LIMCH1|22998 & $\mathrm{B} 2$ \\
\hline LIMD1|8994 & $\mathrm{B} 2$ \\
\hline LIMD2|80774 & $\mathrm{B} 2$ \\
\hline LIME1|54923 & $\mathrm{B} 2$ \\
\hline LIMK2|3985 & $\mathrm{B} 2$ \\
\hline LIMS1|3987 & B2 \\
\hline LIN28A|79727 & $\mathrm{B} 2$ \\
\hline LIN28B|389421 & B2 \\
\hline LIN37|55957 & $\mathrm{B} 2$ \\
\hline LIN52|91750 & $\mathrm{B} 2$ \\
\hline LIN54|132660 & $\mathrm{B} 2$ \\
\hline LIN7A|8825 & $\mathrm{B} 2$ \\
\hline LIN7B|64130 & $\mathrm{B} 2$ \\
\hline LIN7C|55327 & $\mathrm{B} 2$ \\
\hline LINGO1|84894 & B2 \\
\hline LINGO2|158038 & $\mathrm{B} 2$ \\
\hline LINGO3|645191 & $\mathrm{B} 2$ \\
\hline LINGO4|339398 & $\mathrm{B} 2$ \\
\hline LINS1|55180 & $\mathrm{B} 2$ \\
\hline LIPA|3988 & $\mathrm{B} 2$ \\
\hline LIPF $\mid 8513$ & B2 \\
\hline LIPG|9388 & $\mathrm{B} 2$ \\
\hline LIPH|200879 & $\mathrm{B} 2$ \\
\hline LIPI|149998 & $\mathrm{B} 2$ \\
\hline LIPJ|142910 & $\mathrm{B} 2$ \\
\hline LIPK|643414 & $\mathrm{B} 2$ \\
\hline LIPM|340654 & $\mathrm{B} 2$ \\
\hline LIPN|643418 & B2 \\
\hline LIPT2|387787 & $\mathrm{B} 2$ \\
\hline LITAF $\mid 9516$ & B2 \\
\hline LIX1|167410 & $\mathrm{B} 2$ \\
\hline LLGL1|3996 & $\mathrm{B} 2$ \\
\hline LMAN1L|79748 & $\mathrm{B} 2$ \\
\hline LMAN2L|81562 & $\mathrm{B} 2$ \\
\hline LMBR1L|55716 & $\mathrm{B} 2$ \\
\hline LMBRD2|92255 & $\mathrm{B} 2$ \\
\hline LMCD1|29995 & B2 \\
\hline LMF1|64788 & $\mathrm{B} 2$ \\
\hline
\end{tabular}




\begin{tabular}{|l|l|}
\hline LMF2|91289 & B2 \\
\hline LMNA|4000 & B2 \\
\hline LMO1|4004 & B2 \\
\hline LMO3|55885 & B2 \\
\hline LMO4|8543 & B2 \\
\hline LMO7|4008 & B2 \\
\hline LMOD2|442721 & B2 \\
\hline LMOD3|56203 & B2 \\
\hline LMTK2|22853 & B2 \\
\hline LMTK3|114783 & B2 \\
\hline LMX1B|4010 & B2 \\
\hline LNP1|348801 & B2 \\
\hline LNPEP|4012 & B2 \\
\hline LNX1|84708 & B2 \\
\hline LOC100009676|100009676 & B2 \\
\hline LOC100101266|100101266 & B2 \\
\hline LOC100101938|100101938 & B2 \\
\hline LOC100124692|100124692 & B2 \\
\hline LOC100125556|100125556 & B2 \\
\hline LOC100127888|100127888 & B2 \\
\hline LOC100128023|100128023 & B2 \\
\hline LOC100128076|100128076 & B2 \\
\hline LOC100128239|100128239 & B2 \\
\hline LOC100128292|100128292 & B2 \\
\hline LOC100128542|100128542 & B2 \\
\hline LOC100128554|100128554 & B2 \\
\hline LOC100128573|100128573 & B2 \\
\hline LOC100128811|100128811 & B2 \\
\hline LOC100128822|100128822 & B2 \\
\hline LOC100128842|100128842 & B2 \\
\hline LOC100128977|100128977 & B2 \\
\hline LOC100129034|100129034 & B2 \\
\hline LOC100129055|100129055 & B2 \\
\hline LOC100129534|100129534 & B2 \\
\hline LOC100129716|100129716 & B2 \\
\hline LOC100130093|100130093 & B2 \\
\hline LOC100130148|100130148 & B2 \\
\hline LOC100130238|100130238 & B2 \\
\hline LOC100130264|100130264 & B2 \\
\hline LOC100130274|100130274 & B2 \\
\hline LOC100130331|100130331 & B2 \\
\hline LOC100130386|100130386 & B2 \\
\hline LOC100130522|100130522 & B2 \\
\hline LOC100130557|100130557 & B2 \\
\hline LOC100130581|100130581 & B2 \\
\hline LOC100130776|100130776 & B2 \\
\hline LOC100130872|100130872 $\mid 100130933$ & B2 \\
\hline LOC100131551|100131551 & B2 \\
\hline LOC100131691|100131691 & B2 \\
\hline
\end{tabular}




\begin{tabular}{|c|c|}
\hline LOC100131726|100131726 & $\mathrm{B} 2$ \\
\hline LOC100132111|100132111 & B2 \\
\hline LOC100132215|100132215 & B2 \\
\hline LOC100132247|100132247 & $\mathrm{B} 2$ \\
\hline LOC100132287|100132287 & $\mathrm{B} 2$ \\
\hline LOC100132288|100132288 & $\mathrm{B} 2$ \\
\hline LOC100132354|100132354 & $\mathrm{B} 2$ \\
\hline LOC100132831|100132831 & $\mathrm{B} 2$ \\
\hline LOC100132832|100132832 & $\mathrm{B} 2$ \\
\hline LOC100133050|100133050 & B2 \\
\hline LOC100133161|100133161 & $\mathrm{B} 2$ \\
\hline LOC100133308|100133308 & $\mathrm{B} 2$ \\
\hline LOC100133331|100133331 & B2 \\
\hline LOC100133469|100133469 & $\mathrm{B} 2$ \\
\hline LOC100133612|100133612 & $\mathrm{B} 2$ \\
\hline LOC100133669|100133669 & B2 \\
\hline LOC100133893|100133893 & $\mathrm{B} 2$ \\
\hline LOC100133920|100133920 & $\mathrm{B} 2$ \\
\hline LOC100133957|100133957 & $\mathrm{B} 2$ \\
\hline LOC100133985|100133985 & $\mathrm{B} 2$ \\
\hline LOC100133991|100133991 & $\mathrm{B} 2$ \\
\hline LOC100134229|100134229 & B2 \\
\hline LOC100134368|100134368 & $\mathrm{B} 2$ \\
\hline LOC100134868|100134868 & $\mathrm{B} 2$ \\
\hline LOC100144604|100144604 & $\mathrm{B} 2$ \\
\hline LOC100170939|100170939 & $\mathrm{B} 2$ \\
\hline LOC100188947|100188947 & $\mathrm{B} 2$ \\
\hline LOC100188949|100188949 & $\mathrm{B} 2$ \\
\hline LOC100189589|100189589 & $\mathrm{B} 2$ \\
\hline LOC100190940|100190940 & $\mathrm{B} 2$ \\
\hline LOC100190986|100190986 & $\mathrm{B} 2$ \\
\hline LOC100192378|100192378 & $\mathrm{B} 2$ \\
\hline LOC100192379|100192379 & $\mathrm{B} 2$ \\
\hline LOC100192426|100192426 & $\mathrm{B} 2$ \\
\hline LOC100216001|100216001 & $\mathrm{B} 2$ \\
\hline LOC100216545|100216545 & $\mathrm{B} 2$ \\
\hline LOC100233209|100233209 & $\mathrm{B} 2$ \\
\hline LOC100240734|100240734 & B2 \\
\hline LOC100268168|100268168 & $\mathrm{B} 2$ \\
\hline LOC100270710|100270710 & $\mathrm{B} 2$ \\
\hline LOC100270746|100270746 & $\mathrm{B} 2$ \\
\hline LOC100270804|100270804 & $\mathrm{B} 2$ \\
\hline LOC100271831|100271831 & $\mathrm{B} 2$ \\
\hline LOC100271832|100271832 & $\mathrm{B} 2$ \\
\hline LOC100271836|100271836 & B2 \\
\hline LOC100272146|100272146 & $\mathrm{B} 2$ \\
\hline LOC100272216|100272216 & $\mathrm{B} 2$ \\
\hline LOC100272217|100272217 & $\mathrm{B} 2$ \\
\hline LOC100272228|100272228 & $\mathrm{B} 2$ \\
\hline LOC100286793|100286793 & B2 \\
\hline LOC100286844|100286844 & $\mathrm{B} 2$ \\
\hline
\end{tabular}




\begin{tabular}{|c|c|}
\hline LOC100287227|100287227 & B2 \\
\hline LOC100287718|100287718 & B2 \\
\hline LOC100288778|100288778 & B2 \\
\hline LOC100289341|100289341 & B2 \\
\hline LOC100302401|100302401 & $\mathrm{B} 2$ \\
\hline LOC100302640|100302640 & $\mathrm{B} 2$ \\
\hline LOC100303728|100303728 & $\mathrm{B} 2$ \\
\hline LOC115110|115110 & $\mathrm{B} 2$ \\
\hline LOC116437|116437 & $\mathrm{B} 2$ \\
\hline LOC121838|121838 & B2 \\
\hline LOC121952|121952 & $\mathrm{B} 2$ \\
\hline LOC126536|126536 & $\mathrm{B} 2$ \\
\hline LOC127841|127841 & B2 \\
\hline LOC143666|143666 & $\mathrm{B} 2$ \\
\hline LOC144438|144438 & $\mathrm{B} 2$ \\
\hline LOC144486|144486 & B2 \\
\hline LOC144742|144742 & $\mathrm{B} 2$ \\
\hline LOC145474|145474 & $\mathrm{B} 2$ \\
\hline LOC145783|145783 & $\mathrm{B} 2$ \\
\hline LOC145837|145837 & $\mathrm{B} 2$ \\
\hline LOC145845|145845 & $\mathrm{B} 2$ \\
\hline LOC146336|146336 & $\mathrm{B} 2$ \\
\hline LOC146481|146481 & $\mathrm{B} 2$ \\
\hline LOC146880|146880 & $\mathrm{B} 2$ \\
\hline LOC147804|147804 & $\mathrm{B} 2$ \\
\hline LOC148189|148189 & $\mathrm{B} 2$ \\
\hline LOC148413|148413 & $\mathrm{B} 2$ \\
\hline LOC148696|148696 & $\mathrm{B} 2$ \\
\hline LOC148824|148824 & $\mathrm{B} 2$ \\
\hline LOC149134|149134 & $\mathrm{B} 2$ \\
\hline LOC149620|149620 & B2 \\
\hline LOC149837|149837 & $\mathrm{B} 2$ \\
\hline LOC150185|150185 & $\mathrm{B} 2$ \\
\hline LOC150197|150197 & $\mathrm{B} 2$ \\
\hline LOC150381|150381 & $\mathrm{B} 2$ \\
\hline LOC150527|150527 & $\mathrm{B} 2$ \\
\hline LOC150568|150568 & $\mathrm{B} 2$ \\
\hline LOC150622|150622 & B2 \\
\hline LOC150776|150776 & $\mathrm{B} 2$ \\
\hline LOC150786|150786 & $\mathrm{B} 2$ \\
\hline LOC151162|151162 & $\mathrm{B} 2$ \\
\hline LOC151174|151174 & $\mathrm{B} 2$ \\
\hline LOC151534|151534 & $\mathrm{B} 2$ \\
\hline LOC151658|151658 & $\mathrm{B} 2$ \\
\hline LOC152024|152024 & B2 \\
\hline LOC152217|152217 & $\mathrm{B} 2$ \\
\hline LOC152225|152225 & $\mathrm{B} 2$ \\
\hline LOC153328|153328 & $\mathrm{B} 2$ \\
\hline LOC153684|153684 & $\mathrm{B} 2$ \\
\hline LOC153910|153910 & B2 \\
\hline LOC154449|154449 & $\mathrm{B} 2$ \\
\hline
\end{tabular}




\begin{tabular}{|c|c|}
\hline LOC154761|154761 & B2 \\
\hline LOC154822|154822 & $\mathrm{B} 2$ \\
\hline LOC157381|157381 & $\mathrm{B} 2$ \\
\hline LOC157627|157627 & $\mathrm{B} 2$ \\
\hline LOC158572|158572 & B2 \\
\hline LOC162632|162632 & B2 \\
\hline LOC168474|168474 & $\mathrm{B} 2$ \\
\hline LOC200030|200030 & $\mathrm{B} 2$ \\
\hline LOC200726|200726 & B2 \\
\hline LOC202181|202181 & $\mathrm{B} 2$ \\
\hline LOC202781|202781 & $\mathrm{B} 2$ \\
\hline LOC219347|219347 & B2 \\
\hline LOC220429|220429 & $\mathrm{B} 2$ \\
\hline LOC220594|220594 & $\mathrm{B} 2$ \\
\hline LOC220729|220729 & B2 \\
\hline LOC220930|220930 & $\mathrm{B} 2$ \\
\hline LOC221710|221710 & $\mathrm{B} 2$ \\
\hline LOC222699|222699 & $\mathrm{B} 2$ \\
\hline LOC253039|253039 & B2 \\
\hline LOC253724|253724 & $\mathrm{B} 2$ \\
\hline LOC254312|254312 & $\mathrm{B} 2$ \\
\hline LOC254559|254559 & $\mathrm{B} 2$ \\
\hline LOC255167|255167 & $\mathrm{B} 2$ \\
\hline LOC256880|256880 & $\mathrm{B} 2$ \\
\hline LOC25845|25845 & $\mathrm{B} 2$ \\
\hline LOC26102|26102 & $\mathrm{B} 2$ \\
\hline LOC282997|282997 & $\mathrm{B} 2$ \\
\hline LOC283050|283050 & B2 \\
\hline LOC283070|283070 & $\mathrm{B} 2$ \\
\hline LOC283267|283267 & $\mathrm{B} 2$ \\
\hline LOC283314|283314 & $\mathrm{B} 2$ \\
\hline LOC283332|283332 & $\mathrm{B} 2$ \\
\hline LOC283404|283404 & $\mathrm{B} 2$ \\
\hline LOC283663|283663 & $\mathrm{B} 2$ \\
\hline LOC283761|283761 & $\mathrm{B} 2$ \\
\hline LOC283999|283999 & $\mathrm{B} 2$ \\
\hline LOC284023|284023 & $\mathrm{B} 2$ \\
\hline LOC284100|284100 & $\mathrm{B} 2$ \\
\hline LOC284232|284232 & B2 \\
\hline LOC284233|284233 & $\mathrm{B} 2$ \\
\hline LOC284379|284379 & B2 \\
\hline LOC284551|284551 & $\mathrm{B} 2$ \\
\hline LOC284578|284578 & B2 \\
\hline LOC284632|284632 & $\mathrm{B} 2$ \\
\hline LOC284688|284688 & $\mathrm{B} 2$ \\
\hline LOC284749|284749 & B2 \\
\hline LOC284798|284798 & $\mathrm{B} 2$ \\
\hline LOC284837|284837 & $\mathrm{B} 2$ \\
\hline LOC284900|284900 & $\mathrm{B} 2$ \\
\hline LOC285033|285033 & $\mathrm{B} 2$ \\
\hline LOC285045|285045 & $\mathrm{B} 2$ \\
\hline
\end{tabular}




\begin{tabular}{|c|c|}
\hline LOC285194|285194 & B2 \\
\hline LOC285359|285359 & B2 \\
\hline LOC285370|285370 & $\mathrm{B} 2$ \\
\hline LOC285375|285375 & B2 \\
\hline LOC285401|285401 & B2 \\
\hline LOC285456|285456 & B2 \\
\hline LOC285501|285501 & B2 \\
\hline LOC285548|285548 & $\mathrm{B} 2$ \\
\hline LOC285593|285593 & $\mathrm{B} 2$ \\
\hline LOC285627|285627 & B2 \\
\hline LOC285629|285629 & B2 \\
\hline LOC285692|285692 & B2 \\
\hline LOC285696|285696 & $\mathrm{B} 2$ \\
\hline LOC285733|285733 & $\mathrm{B} 2$ \\
\hline LOC285735|285735 & $\mathrm{B} 2$ \\
\hline LOC285740|285740 & B2 \\
\hline LOC285768|285768 & $\mathrm{B} 2$ \\
\hline LOC285780|285780 & $\mathrm{B} 2$ \\
\hline LOC285847|285847 & $\mathrm{B} 2$ \\
\hline LOC285954|285954 & $\mathrm{B} 2$ \\
\hline LOC286002|286002 & $\mathrm{B} 2$ \\
\hline LOC286135|286135 & $\mathrm{B} 2$ \\
\hline LOC286238|286238 & $\mathrm{B} 2$ \\
\hline LOC286359|286359 & B2 \\
\hline LOC286467|286467 & $\mathrm{B} 2$ \\
\hline LOC29034|29034 & $\mathrm{B} 2$ \\
\hline LOC338651|338651 & B2 \\
\hline LOC338799|338799 & $\mathrm{B} 2$ \\
\hline LOC339047|339047 & $\mathrm{B} 2$ \\
\hline LOC339240|339240 & $\mathrm{B} 2$ \\
\hline LOC339535|339535 & $\mathrm{B} 2$ \\
\hline LOC339788|339788 & $\mathrm{B} 2$ \\
\hline LOC340017|340017 & $\mathrm{B} 2$ \\
\hline LOC340074|340074 & $\mathrm{B} 2$ \\
\hline LOC340357|340357 & $\mathrm{B} 2$ \\
\hline LOC340508|340508 & $\mathrm{B} 2$ \\
\hline LOC344595|344595 & $\mathrm{B} 2$ \\
\hline LOC347376|347376 & $\mathrm{B} 2$ \\
\hline LOC348021|348021 & $\mathrm{B} 2$ \\
\hline LOC348840|348840 & $\mathrm{B} 2$ \\
\hline LOC349114|349114 & $\mathrm{B} 2$ \\
\hline LOC360030|360030 & $\mathrm{B} 2$ \\
\hline LOC374491|374491 & $\mathrm{B} 2$ \\
\hline LOC387646|387646 & $\mathrm{B} 2$ \\
\hline LOC388152|388152 & $\mathrm{B} 2$ \\
\hline LOC388242|388242 & B2 \\
\hline LOC388387|388387 & $\mathrm{B} 2$ \\
\hline LOC388428|388428 & $\mathrm{B} 2$ \\
\hline LOC388588|388588 & $\mathrm{B} 2$ \\
\hline LOC388692|388692 & $\mathrm{B} 2$ \\
\hline LOC388789|388789 & B2 \\
\hline
\end{tabular}




\begin{tabular}{|c|c|}
\hline LOC388796|388796 & B2 \\
\hline LOC389033|389033 & $\mathrm{B} 2$ \\
\hline LOC389332|389332 & B2 \\
\hline LOC389333|389333 & B2 \\
\hline LOC389458|389458 & B2 \\
\hline LOC389634|389634 & B2 \\
\hline LOC389791|389791 & B2 \\
\hline LOC391322|391322 & $\mathrm{B} 2$ \\
\hline LOC392196|392196 & B2 \\
\hline LOC399744|399744 & B2 \\
\hline LOC400043|400043 & B2 \\
\hline LOC400657|400657 & B2 \\
\hline LOC400696|400696 & B2 \\
\hline LOC400752|400752 & B2 \\
\hline LOC400759|400759 & B2 \\
\hline LOC400794|400794 & B2 \\
\hline LOC400891|400891 & B2 \\
\hline LOC400927|400927 & B2 \\
\hline LOC400940|400940 & B2 \\
\hline LOC401387|401387 & B2 \\
\hline LOC401397|401397 & B2 \\
\hline LOC401463|401463 & $\mathrm{B} 2$ \\
\hline LOC401588|401588 & B2 \\
\hline LOC402377|402377 & $\mathrm{B} 2$ \\
\hline LOC402644|402644 & B2 \\
\hline LOC407835|407835 & $\mathrm{B} 2$ \\
\hline LOC440040|440040 & B2 \\
\hline LOC440173|440173 & B2 \\
\hline LOC440354|440354 & B2 \\
\hline LOC440356|440356 & B2 \\
\hline LOC440461|440461 & B2 \\
\hline LOC440563|440563 & B2 \\
\hline LOC440896|440896 & B2 \\
\hline LOC440905|440905 & $\mathrm{B} 2$ \\
\hline LOC440925|440925 & B2 \\
\hline LOC440944|440944 & B2 \\
\hline LOC440957|440957 & $\mathrm{B} 2$ \\
\hline LOC441046|441046 & B2 \\
\hline LOC441089|441089 & B2 \\
\hline LOC441177|441177 & B2 \\
\hline LOC441204|441204 & B2 \\
\hline LOC441208|441208 & B2 \\
\hline LOC441294|441294 & B2 \\
\hline LOC441454|441454 & B2 \\
\hline LOC441455|441455 & B2 \\
\hline LOC441601|441601 & B2 \\
\hline LOC441666|441666 & B2 \\
\hline LOC442308|442308 & B2 \\
\hline LOC442421|442421 & B2 \\
\hline LOC442454|442454 & B2 \\
\hline LOC442459|442459 & B2 \\
\hline
\end{tabular}




\begin{tabular}{|c|c|}
\hline LOC494141|494141 & B2 \\
\hline LOC541473|541473 & $\mathrm{B} 2$ \\
\hline LOC550112|550112 & $\mathrm{B} 2$ \\
\hline LOC554202|554202 & $\mathrm{B} 2$ \\
\hline LOC595101|595101 & B2 \\
\hline LOC606724|606724 & B2 \\
\hline LOC613037|613037 & $\mathrm{B} 2$ \\
\hline LOC619207|619207 & $\mathrm{B} 2$ \\
\hline LOC641298|641298 & B2 \\
\hline LOC641367|641367 & $\mathrm{B} 2$ \\
\hline LOC642846|642846 & $\mathrm{B} 2$ \\
\hline LOC642852|642852 & B2 \\
\hline LOC643008|643008 & $\mathrm{B} 2$ \\
\hline LOC643677|643677 & $\mathrm{B} 2$ \\
\hline LOC643719|643719 & B2 \\
\hline LOC643763|643763 & $\mathrm{B} 2$ \\
\hline LOC643837|643837 & $\mathrm{B} 2$ \\
\hline LOC643923|643923 & $\mathrm{B} 2$ \\
\hline LOC644145|644145 & B2 \\
\hline LOC644165|644165 & $\mathrm{B} 2$ \\
\hline LOC644172|644172 & $\mathrm{B} 2$ \\
\hline LOC644669|644669 & $\mathrm{B} 2$ \\
\hline LOC644936|644936 & $\mathrm{B} 2$ \\
\hline LOC645166|645166 & $\mathrm{B} 2$ \\
\hline LOC645323|645323 & $\mathrm{B} 2$ \\
\hline LOC645332|645332 & $\mathrm{B} 2$ \\
\hline LOC645431|645431 & $\mathrm{B} 2$ \\
\hline LOC645676|645676 & B2 \\
\hline LOC645752|645752 & $\mathrm{B} 2$ \\
\hline LOC646214|646214 & $\mathrm{B} 2$ \\
\hline LOC646471|646471 & $\mathrm{B} 2$ \\
\hline LOC646627|646627 & $\mathrm{B} 2$ \\
\hline LOC646762|646762 & $\mathrm{B} 2$ \\
\hline LOC646851|646851 & $\mathrm{B} 2$ \\
\hline LOC646999|646999 & $\mathrm{B} 2$ \\
\hline LOC647121|647121 & $\mathrm{B} 2$ \\
\hline LOC647288|647288 & $\mathrm{B} 2$ \\
\hline LOC647859|647859 & $\mathrm{B} 2$ \\
\hline LOC647946|647946 & B2 \\
\hline LOC647979|647979 & $\mathrm{B} 2$ \\
\hline LOC648691|648691 & B2 \\
\hline LOC649330|649330 & $\mathrm{B} 2$ \\
\hline LOC651250|651250 & B2 \\
\hline LOC652276|652276 & $\mathrm{B} 2$ \\
\hline LOC653113|653113 & $\mathrm{B} 2$ \\
\hline LOC653544|653544 & $\mathrm{B} 2$ \\
\hline LOC653653|653653 & $\mathrm{B} 2$ \\
\hline LOC653786|653786 & $\mathrm{B} 2$ \\
\hline LOC654433|654433 & $\mathrm{B} 2$ \\
\hline LOC678655|678655 & $\mathrm{B} 2$ \\
\hline LOC723809|723809 & B2 \\
\hline
\end{tabular}




\begin{tabular}{|c|c|}
\hline LOC723972|723972 & B2 \\
\hline LOC727677|727677 & $\mathrm{B} 2$ \\
\hline LOC727896|727896 & B2 \\
\hline LOC727924|727924 & B2 \\
\hline LOC728024|728024 & B2 \\
\hline LOC728190|728190 & B2 \\
\hline LOC728276|728276 & B2 \\
\hline LOC728323|728323 & $\mathrm{B} 2$ \\
\hline LOC728606|728606 & $\mathrm{B} 2$ \\
\hline LOC728613|728613 & B2 \\
\hline LOC728640|728640 & B2 \\
\hline LOC728643|728643 & B2 \\
\hline LOC728743|728743 & $\mathrm{B} 2$ \\
\hline LOC728758|728758 & $\mathrm{B} 2$ \\
\hline LOC728855|728855 & $\mathrm{B} 2$ \\
\hline LOC728875|728875 & B2 \\
\hline LOC728989|728989 & B2 \\
\hline LOC729020|729020 & B2 \\
\hline LOC729082|729082 & B2 \\
\hline LOC729156|729156 & B2 \\
\hline LOC729176|729176 & $\mathrm{B} 2$ \\
\hline LOC729234|729234 & $\mathrm{B} 2$ \\
\hline LOC729375|729375 & B2 \\
\hline LOC729603|729603 & $\mathrm{B} 2$ \\
\hline LOC729609|729609 & B2 \\
\hline LOC729668|729668 & $\mathrm{B} 2$ \\
\hline LOC729678|729678 & B2 \\
\hline LOC729991-MEF2B|4207 & B2 \\
\hline LOC729991|729991 & B2 \\
\hline LOC730101|730101 & $\mathrm{B} 2$ \\
\hline LOC730668|730668 & B2 \\
\hline LOC731779|731779 & B2 \\
\hline LOC731789|731789 & $\mathrm{B} 2$ \\
\hline LOC80154|80154 & $\mathrm{B} 2$ \\
\hline LOC81691|81691 & $\mathrm{B} 2$ \\
\hline LOC84740|84740 & B2 \\
\hline LOC84856|84856 & $\mathrm{B} 2$ \\
\hline LOC84931|84931 & B2 \\
\hline LOC84989|84989 & B2 \\
\hline LOC90110|90110 & B2 \\
\hline LOC90246|90246 & B2 \\
\hline LOC91450|91450 & B2 \\
\hline LOC91948|91948 & B2 \\
\hline LOC92249|92249 & $\mathrm{B} 2$ \\
\hline LOC92973|92973 & $\mathrm{B} 2$ \\
\hline LOC93432|93432 & B2 \\
\hline LOC93622|93622 & B2 \\
\hline LOC96610|96610 & B2 \\
\hline LOH12CR1|118426 & B2 \\
\hline LONP1|9361 & B2 \\
\hline LONP2|83752 & B2 \\
\hline
\end{tabular}




\begin{tabular}{|c|c|}
\hline LONRF2|164832 & B2 \\
\hline LOR|4014 & $\mathrm{B} 2$ \\
\hline LOXHD1|125336 & $\mathrm{B} 2$ \\
\hline LOXL2|4017 & $\mathrm{B} 2$ \\
\hline LOXL3|84695 & B2 \\
\hline LOXL4|84171 & B2 \\
\hline LOX|4015 & $\mathrm{B} 2$ \\
\hline LPAL2|80350 & $\mathrm{B} 2$ \\
\hline LPAR3|23566 & B2 \\
\hline LPAR4|2846 & $\mathrm{B} 2$ \\
\hline LPA|4018 & $\mathrm{B} 2$ \\
\hline LPCAT3|10162 & B2 \\
\hline LPGAT1|9926 & $\mathrm{B} 2$ \\
\hline LPHN1|22859 & $\mathrm{B} 2$ \\
\hline LPIN1|23175 & $\mathrm{B} 2$ \\
\hline LPIN2|9663 & $\mathrm{B} 2$ \\
\hline LPIN3|64900 & B2 \\
\hline LPO|4025 & $\mathrm{B} 2$ \\
\hline LPPR3|79948 & B2 \\
\hline LPP $\mid 4026$ & $\mathrm{~B} 2$ \\
\hline LPXN|9404 & $\mathrm{B} 2$ \\
\hline LQK1|642946 & $\mathrm{B} 2$ \\
\hline LRAT|9227 & $\mathrm{B} 2$ \\
\hline 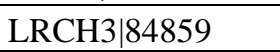 & $\mathrm{B} 2$ \\
\hline LRCH4|4034 & $\mathrm{B} 2$ \\
\hline LRDD|55367 & $\mathrm{B} 2$ \\
\hline LRFN3|79414 & B2 \\
\hline LRFN4|78999 & B2 \\
\hline LRG1|116844 & $\mathrm{B} 2$ \\
\hline LRGUK|136332 & $\mathrm{B} 2$ \\
\hline LRIG1|26018 & $\mathrm{B} 2$ \\
\hline LRIT1|26103 & B2 \\
\hline LRIT2|340745 & $\mathrm{B} 2$ \\
\hline LRIT3|345193 & B2 \\
\hline LRP10|26020 & $\mathrm{B} 2$ \\
\hline LRP11|84918 & $\mathrm{B} 2$ \\
\hline LRP12|29967 & $\mathrm{B} 2$ \\
\hline LRP1B|53353 & $\mathrm{B} 2$ \\
\hline LRP2BP|55805 & B2 \\
\hline LRP2|4036 & $\mathrm{B} 2$ \\
\hline LRP5L|91355 & B2 \\
\hline LRP6|4040 & $\mathrm{B} 2$ \\
\hline LRPAP1|4043 & B2 \\
\hline LRPPRC|10128 & $\mathrm{B} 2$ \\
\hline LRRC10B|390205 & $\mathrm{B} 2$ \\
\hline LRRC10|376132 & $\mathrm{B} 2$ \\
\hline LRRC14B|389257 & $\mathrm{B} 2$ \\
\hline LRRC14|9684 & $\mathrm{B} 2$ \\
\hline $\begin{array}{l}\text { LRRC16B|90668 } \\
\end{array}$ & $\mathrm{B} 2$ \\
\hline LRRC17|10234 & $\mathrm{B} 2$ \\
\hline LRRC18|474354 & $\mathrm{B} 2$ \\
\hline
\end{tabular}




\begin{tabular}{|c|c|}
\hline LRRC19|64922 & B2 \\
\hline LRRC20|55222 & $\mathrm{B} 2$ \\
\hline LRRC23|10233 & $\mathrm{B} 2$ \\
\hline LRRC24|441381 & $\mathrm{B} 2$ \\
\hline LRRC25|126364 & B2 \\
\hline LRRC26|389816 & $\mathrm{B} 2$ \\
\hline LRRC27|80313 & $\mathrm{B} 2$ \\
\hline LRRC28|123355 & $\mathrm{B} 2$ \\
\hline LRRC2|79442 & $\mathrm{B} 2$ \\
\hline LRRC31|79782 & $\mathrm{B} 2$ \\
\hline LRRC32|2615 & $\mathrm{B} 2$ \\
\hline LRRC36|55282 & B2 \\
\hline LRRC37A2|474170 & $\mathrm{B} 2$ \\
\hline LRRC37A3|374819 & $\mathrm{B} 2$ \\
\hline LRRC37A|9884 & $\mathrm{B} 2$ \\
\hline LRRC37B2|147172 & $\mathrm{B} 2$ \\
\hline LRRC37B|114659 & B2 \\
\hline LRRC39|127495 & $\mathrm{B} 2$ \\
\hline LRRC40|55631 & B2 \\
\hline LRRC41|10489 & $\mathrm{B} 2$ \\
\hline $\begin{array}{l}\text { LRRC43|254050 } \\
\end{array}$ & $\mathrm{B} 2$ \\
\hline LRRC47|57470 & $\mathrm{B} 2$ \\
\hline LRRC48|83450 & $\mathrm{B} 2$ \\
\hline LRRC49|54839 & $\mathrm{B} 2$ \\
\hline LRRC4C|57689 & $\mathrm{B} 2$ \\
\hline LRRC4|64101 & $\mathrm{B} 2$ \\
\hline LRRC50|123872 & $\mathrm{B} 2$ \\
\hline LRRC52|440699 & B2 \\
\hline \begin{tabular}{l|l|} 
LRRC55|219527 \\
\end{tabular} & $\mathrm{B} 2$ \\
\hline LRRC57|255252 & $\mathrm{B} 2$ \\
\hline LRRC58|116064 & $\mathrm{B} 2$ \\
\hline LRRC66|339977 & B2 \\
\hline LRRC67|286187 & $\mathrm{B} 2$ \\
\hline LRRC69|100130742 & $\mathrm{B} 2$ \\
\hline LRRC7|57554 & $\mathrm{B} 2$ \\
\hline LRRC8A|56262 & $\mathrm{B} 2$ \\
\hline LRRC8B|23507 & $\mathrm{B} 2$ \\
\hline LRRC8D|55144 & $\mathrm{B} 2$ \\
\hline LRRFIP1|9208 & $\mathrm{B} 2$ \\
\hline LRRFIP2|9209 & $\mathrm{B} 2$ \\
\hline LRRIQ1|84125 & B2 \\
\hline LRRIQ3|127255 & $\mathrm{B} 2$ \\
\hline LRRIQ4|344657 & B2 \\
\hline LRRK1|79705 & $\mathrm{B} 2$ \\
\hline LRRK2|120892 & $\mathrm{B} 2$ \\
\hline LRRN1|57633 & $\mathrm{B} 2$ \\
\hline LRRN4|164312 & $\mathrm{B} 2$ \\
\hline LRRTM3|347731 & $\mathrm{B} 2$ \\
\hline LRRTM4|80059 & $\mathrm{B} 2$ \\
\hline LRTM1|57408 & $\mathrm{B} 2$ \\
\hline LRTM2|654429 & $\mathrm{B} 2$ \\
\hline
\end{tabular}




\begin{tabular}{|c|c|}
\hline LRTOMT|220074 & B2 \\
\hline LSAMP 4045 & $\mathrm{~B} 2$ \\
\hline LSG1|55341 & $\mathrm{B} 2$ \\
\hline LSM10|84967 & $\mathrm{B} 2$ \\
\hline LSM11|134353 & $\mathrm{B} 2$ \\
\hline LSM14A|26065 & $\mathrm{B} 2$ \\
\hline LSM1|27257 & $\mathrm{B} 2$ \\
\hline LSM6|11157 & $\mathrm{B} 2$ \\
\hline LSM7|51690 & B2 \\
\hline LSMD1|84316 & $\mathrm{B} 2$ \\
\hline LSP1|4046 & $\mathrm{B} 2$ \\
\hline LSS 4047 & B2 \\
\hline LST-3TM12|338821 & $\mathrm{B} 2$ \\
\hline LST1|7940 & $\mathrm{B} 2$ \\
\hline LTA4H|4048 & B2 \\
\hline LTA|4049 & $\mathrm{B} 2$ \\
\hline LTB4R2|56413 & $\mathrm{B} 2$ \\
\hline LTB4R|1241 & $\mathrm{B} 2$ \\
\hline LTBP1|4052 & B2 \\
\hline LTBP3|4054 & $\mathrm{B} 2$ \\
\hline LTBR/4055 & $\mathrm{B} 2$ \\
\hline LTB $\mid 4050$ & $\mathrm{~B} 2$ \\
\hline LTF 4057 & $\mathrm{~B} 2$ \\
\hline LTK $\mid 4058$ & $\mathrm{~B} 2$ \\
\hline LTV1|84946 & $\mathrm{B} 2$ \\
\hline LUC7L2|51631 & $\mathrm{B} 2$ \\
\hline LUC7L3|51747 & $\mathrm{B} 2$ \\
\hline LUC7L|55692 & B2 \\
\hline LUM|4060 & $\mathrm{B} 2$ \\
\hline LUZP4|51213 & $\mathrm{B} 2$ \\
\hline LUZP6|767558 & $\mathrm{B} 2$ \\
\hline LXN|56925 & B2 \\
\hline LY6D|8581 & $\mathrm{B} 2$ \\
\hline LY6E|4061 & $\mathrm{B} 2$ \\
\hline LY6G5C|80741 & $\mathrm{B} 2$ \\
\hline LY6G6C|80740 & $\mathrm{B} 2$ \\
\hline LY6G6D|58530 & $\mathrm{B} 2$ \\
\hline LY6G6E|79136 & $\mathrm{B} 2$ \\
\hline LY6G6F|259215 & B2 \\
\hline LY6H|4062 & $\mathrm{B} 2$ \\
\hline LY6K|54742 & B2 \\
\hline LY86|9450 & $\mathrm{B} 2$ \\
\hline LY96|23643 & B2 \\
\hline LY9|4063 & $\mathrm{B} 2$ \\
\hline LYAR|55646 & $\mathrm{B} 2$ \\
\hline LYG1|129530 & $\mathrm{B} 2$ \\
\hline \begin{tabular}{l|l|} 
LYG254773 \\
\end{tabular} & $\mathrm{B} 2$ \\
\hline LYL1|4066 & $\mathrm{B} 2$ \\
\hline LYN|4067 & $\mathrm{B} 2$ \\
\hline LYPD2|137797 & $\mathrm{B} 2$ \\
\hline LYPD3|27076 & $\mathrm{B} 2$ \\
\hline
\end{tabular}




\begin{tabular}{|c|c|}
\hline LYPD4|147719 & B2 \\
\hline LYPD5|284348 & $\mathrm{B} 2$ \\
\hline LYPD6B|130576 & $\mathrm{B} 2$ \\
\hline LYPD6|130574 & B2 \\
\hline LYRM2|57226 & B2 \\
\hline LYRM4|57128 & B2 \\
\hline LYRM5|144363 & B2 \\
\hline LYSMD2|256586 & $\mathrm{B} 2$ \\
\hline LYSMD3|116068 & B2 \\
\hline LYST|1130 & $\mathrm{B} 2$ \\
\hline LYZL1|84569 & B2 \\
\hline LYZL2|119180 & $\mathrm{B} 2$ \\
\hline LYZL4|131375 & $\mathrm{B} 2$ \\
\hline LYZ|4069 & $\mathrm{B} 2$ \\
\hline LZIC|84328 & $\mathrm{B} 2$ \\
\hline LZTFL1|54585 & $\mathrm{B} 2$ \\
\hline LZTR1|8216 & $\mathrm{B} 2$ \\
\hline LZTS1|11178 & $\mathrm{B} 2$ \\
\hline LZTS2|84445 & $\mathrm{B} 2$ \\
\hline M6PR|4074 & $\mathrm{B} 2$ \\
\hline MAB21L2|10586 & $\mathrm{B} 2$ \\
\hline MACC1|346389 & $\mathrm{B} 2$ \\
\hline MACF1|23499 & $\mathrm{B} 2$ \\
\hline MACROD1|28992 & $\mathrm{B} 2$ \\
\hline MACROD2|140733 & $\mathrm{B} 2$ \\
\hline MAD1L1|8379 & $\mathrm{B} 2$ \\
\hline MADCAM1 $\mid 8174$ & $\mathrm{~B} 2$ \\
\hline MADD|8567 & $\mathrm{B} 2$ \\
\hline MAEA|10296 & $\mathrm{B} 2$ \\
\hline MAEL|84944 & B2 \\
\hline MAF1|84232 & $\mathrm{B} 2$ \\
\hline MAFA|389692 & $\mathrm{B} 2$ \\
\hline MAFB|9935 & $\mathrm{B} 2$ \\
\hline MAGEA10|4109 & $\mathrm{B} 2$ \\
\hline MAGEA11|4110 & $\mathrm{B} 2$ \\
\hline MAGEA12|4111 & $\mathrm{B} 2$ \\
\hline MAGEA1|4100 & $\mathrm{B} 2$ \\
\hline MAGEA2|4101 & $\mathrm{B} 2$ \\
\hline MAGEA3|4102 & $\mathrm{B} 2$ \\
\hline MAGEA4|4103 & $\mathrm{B} 2$ \\
\hline MAGEA5|4104 & $\mathrm{B} 2$ \\
\hline MAGEA6|4105 & $\mathrm{B} 2$ \\
\hline MAGEA8|4107 & $\mathrm{B} 2$ \\
\hline MAGEA9B|728269 & $\mathrm{B} 2$ \\
\hline MAGEB10|139422 & $\mathrm{B} 2$ \\
\hline MAGEB16|139604 & $\mathrm{B} 2$ \\
\hline MAGEB18|286514 & $\mathrm{B} 2$ \\
\hline MAGEB1|4112 & $\mathrm{B} 2$ \\
\hline MAGEB2|4113 & $\mathrm{B} 2$ \\
\hline MAGEB4|4115 & $\mathrm{B} 2$ \\
\hline MAGEB6|158809 & B2 \\
\hline
\end{tabular}




\begin{tabular}{|c|c|}
\hline MAGEC1|9947 & B2 \\
\hline MAGEC2|51438 & $\mathrm{B} 2$ \\
\hline MAGEC3|139081 & $\mathrm{B} 2$ \\
\hline MAGED4B|81557 & $\mathrm{B} 2$ \\
\hline MAGED4|728239 & B2 \\
\hline MAGEE1|57692 & $\mathrm{B} 2$ \\
\hline MAGEH1|28986 & $\mathrm{B} 2$ \\
\hline MAGEL2|54551 & $\mathrm{B} 2$ \\
\hline MAGI2|9863 & $\mathrm{B} 2$ \\
\hline MAGI3|260425 & $\mathrm{B} 2$ \\
\hline MAGOH|4116 & $\mathrm{B} 2$ \\
\hline MAGT1|84061 & B2 \\
\hline MAG|4099 & $\mathrm{B} 2$ \\
\hline MAK16|84549 & $\mathrm{B} 2$ \\
\hline MAK|4117 & $\mathrm{B} 2$ \\
\hline MALAT1|378938 & $\mathrm{B} 2$ \\
\hline MALL|7851 & B2 \\
\hline MALT1|10892 & $\mathrm{B} 2$ \\
\hline MAL|4118 & B2 \\
\hline MAMDC4|158056 & $\mathrm{B} 2$ \\
\hline MAML1|9794 & $\mathrm{B} 2$ \\
\hline MAMLD1|10046 & B2 \\
\hline MAMSTR|284358 & $\mathrm{B} 2$ \\
\hline MAN1A1|4121 & $\mathrm{B} 2$ \\
\hline MAN1A2|10905 & $\mathrm{B} 2$ \\
\hline MAN1B1|11253 & $\mathrm{B} 2$ \\
\hline MAN1C1|57134 & $\mathrm{B} 2$ \\
\hline MAN2A1|4124 & B2 \\
\hline MAN2B2|23324 & $\mathrm{B} 2$ \\
\hline MANBAL|63905 & $\mathrm{B} 2$ \\
\hline MANBA|4126 & $\mathrm{B} 2$ \\
\hline MANEA|79694 & $\mathrm{B} 2$ \\
\hline MANSC1|54682 & $\mathrm{B} 2$ \\
\hline MAOB $\mid 4129$ & $\mathrm{~B} 2$ \\
\hline MAP1LC3A|84557 & $\mathrm{B} 2$ \\
\hline MAP1LC3B2|643246 & $\mathrm{B} 2$ \\
\hline MAP1S|55201 & $\mathrm{B} 2$ \\
\hline MAP2K1|5604 & $\mathrm{B} 2$ \\
\hline MAP2K2|5605 & B2 \\
\hline MAP2K3|5606 & $\mathrm{B} 2$ \\
\hline MAP2K4|6416 & B2 \\
\hline MAP2K7|5609 & $\mathrm{B} 2$ \\
\hline MAP3K10|4294 & $\mathrm{B} 2$ \\
\hline MAP3K11|4296 & $\mathrm{B} 2$ \\
\hline MAP3K12|7786 & $\mathrm{B} 2$ \\
\hline MAP3K13|9175 & $\mathrm{B} 2$ \\
\hline MAP3K15|389840 & $\mathrm{B} 2$ \\
\hline MAP3K1|4214 & $\mathrm{B} 2$ \\
\hline MAP3K2|10746 & $\mathrm{B} 2$ \\
\hline MAP3K7|6885 & $\mathrm{B} 2$ \\
\hline MAP3K9|4293 & $\mathrm{B} 2$ \\
\hline
\end{tabular}




\begin{tabular}{|c|c|}
\hline MAP4K1|11184 & B2 \\
\hline MAP4K3|8491 & $\mathrm{B} 2$ \\
\hline MAP4K4|9448 & $\mathrm{B} 2$ \\
\hline MAP4K5|11183 & $\mathrm{B} 2$ \\
\hline MAP4|4134 & B2 \\
\hline MAP6D1|79929 & $\mathrm{B} 2$ \\
\hline MAP7D2|256714 & $\mathrm{B} 2$ \\
\hline MAP9|79884 & $\mathrm{B} 2$ \\
\hline MAPK12|6300 & $\mathrm{B} 2$ \\
\hline MAPK14|1432 & $\mathrm{B} 2$ \\
\hline MAPK1IP1L|93487 & $\mathrm{B} 2$ \\
\hline MAPK1|5594 & B2 \\
\hline MAPK3|5595 & $\mathrm{B} 2$ \\
\hline MAPK4|5596 & $\mathrm{B} 2$ \\
\hline MAPK6|5597 & $\mathrm{B} 2$ \\
\hline MAPK7|5598 & $\mathrm{B} 2$ \\
\hline MAPK8IP1|9479 & B2 \\
\hline MAPK8IP3|23162 & $\mathrm{B} 2$ \\
\hline MAPK8|5599 & B2 \\
\hline MAPKAP1|79109 & $\mathrm{B} 2$ \\
\hline MAPKAPK3|7867 & $\mathrm{B} 2$ \\
\hline MAPKSP1|8649 & B2 \\
\hline MAPRE2|10982 & $\mathrm{B} 2$ \\
\hline MAPRE3|22924 & $\mathrm{B} 2$ \\
\hline MAPT|4137 & $\mathrm{B} 2$ \\
\hline MARCH10|162333 & $\mathrm{B} 2$ \\
\hline MARCH11|441061 & $\mathrm{B} 2$ \\
\hline MARCH1|55016 & B2 \\
\hline MARCH2|51257 & $\mathrm{B} 2$ \\
\hline MARCH3|115123 & $\mathrm{B} 2$ \\
\hline MARCH5|54708 & $\mathrm{B} 2$ \\
\hline MARCH6|10299 & $\mathrm{B} 2$ \\
\hline MARCH7|64844 & $\mathrm{B} 2$ \\
\hline MARCH8|220972 & B2 \\
\hline MARCKS|4082 & $\mathrm{B} 2$ \\
\hline MARCO|8685 & $\mathrm{B} 2$ \\
\hline MARK3|4140 & $\mathrm{B} 2$ \\
\hline MARK4|57787 & $\mathrm{B} 2$ \\
\hline MARS2|92935 & B2 \\
\hline MARVELD1|83742 & $\mathrm{B} 2$ \\
\hline MARVELD3|91862 & B2 \\
\hline MAS1|4142 & $\mathrm{B} 2$ \\
\hline MAST1|22983 & $\mathrm{B} 2$ \\
\hline MAST2|23139 & $\mathrm{B} 2$ \\
\hline MAST3|23031 & $\mathrm{B} 2$ \\
\hline MAST4|375449 & $\mathrm{B} 2$ \\
\hline MAT1A|4143 & $\mathrm{B} 2$ \\
\hline MAT2A|4144 & $\mathrm{B} 2$ \\
\hline MATK|4145 & $\mathrm{B} 2$ \\
\hline MATN1|4146 & $\mathrm{B} 2$ \\
\hline MATN4|8785 & $\mathrm{B} 2$ \\
\hline
\end{tabular}




\begin{tabular}{|c|c|}
\hline MATR3|9782 & B2 \\
\hline MAX|4149 & B2 \\
\hline MBD2|8932 & $\mathrm{B} 2$ \\
\hline MBD3L2|125997 & $\mathrm{B} 2$ \\
\hline MBD3L5|284428 & $\mathrm{B} 2$ \\
\hline MBD3|53615 & $\mathrm{B} 2$ \\
\hline MBD5|55777 & $\mathrm{B} 2$ \\
\hline MBD6|114785 & $\mathrm{B} 2$ \\
\hline MBL2|4153 & $\mathrm{B} 2$ \\
\hline MBLAC1|255374 & $\mathrm{B} 2$ \\
\hline MBOAT1|154141 & $\mathrm{B} 2$ \\
\hline MBOAT4|619373 & B2 \\
\hline MBTD1|54799 & $\mathrm{B} 2$ \\
\hline MBTPS1|8720 & $\mathrm{B} 2$ \\
\hline MBTPS2|51360 & $\mathrm{B} 2$ \\
\hline $\mathrm{MB} \mid 4151$ & $\mathrm{~B} 2$ \\
\hline MC2R $\mid 4158$ & B2 \\
\hline MC3R/4159 & $\mathrm{B} 2$ \\
\hline MC4R|4160 & B2 \\
\hline MC5R|4161 & $\mathrm{B} 2$ \\
\hline MCART1|92014 & $\mathrm{B} 2$ \\
\hline MCART2|147407 & $\mathrm{B} 2$ \\
\hline MCART3P|442229 & $\mathrm{B} 2$ \\
\hline MCART6|401612 & $\mathrm{B} 2$ \\
\hline MCAT|27349 & $\mathrm{B} 2$ \\
\hline MCCC1|56922 & $\mathrm{B} 2$ \\
\hline MCCC2|64087 & $\mathrm{B} 2$ \\
\hline MCCD1|401250 & B2 \\
\hline MCEE|84693 & $\mathrm{B} 2$ \\
\hline MCF2L2|23101 & $\mathrm{B} 2$ \\
\hline MCF2L|23263 & $\mathrm{B} 2$ \\
\hline MCF2|4168 & B2 \\
\hline MCHR1|2847 & $\mathrm{B} 2$ \\
\hline MCHR2|84539 & B2 \\
\hline MCL1|4170 & $\mathrm{B} 2$ \\
\hline MCM3APAS|114044 & $\mathrm{B} 2$ \\
\hline MCM3AP|8888 & $\mathrm{B} 2$ \\
\hline MCM5|4174 & $\mathrm{B} 2$ \\
\hline MCM7|4176 & $\mathrm{B} 2$ \\
\hline MCM8|84515 & $\mathrm{B} 2$ \\
\hline \begin{tabular}{l|l|} 
MCM9|254394 \\
\end{tabular} & B2 \\
\hline MCOLN1|57192 & $\mathrm{B} 2$ \\
\hline MCOLN3|55283 & B2 \\
\hline MCRS1|10445 & $\mathrm{B} 2$ \\
\hline \begin{tabular}{l|l|l|} 
MCTP2|55784 \\
\end{tabular} & B2 \\
\hline MDC1|9656 & $\mathrm{B} 2$ \\
\hline MDFI|4188 & $\mathrm{B} 2$ \\
\hline MDGA1|266727 & $\mathrm{B} 2$ \\
\hline MDGA2|161357 & $\mathrm{B} 2$ \\
\hline MDH1B|130752 & $\mathrm{B} 2$ \\
\hline MDH1|4190 & $\mathrm{B} 2$ \\
\hline
\end{tabular}




\begin{tabular}{|c|c|}
\hline MDH2|4191 & B2 \\
\hline MDK|4192 & B2 \\
\hline MDM1|56890 & $\mathrm{B} 2$ \\
\hline MDM2|4193 & $\mathrm{B} 2$ \\
\hline MDM4|4194 & $\mathrm{B} 2$ \\
\hline MDP1|145553 & $\mathrm{B} 2$ \\
\hline MDS2|259283 & $\mathrm{B} 2$ \\
\hline ME1|4199 & $\mathrm{B} 2$ \\
\hline ME2|4200 & $\mathrm{B} 2$ \\
\hline MECR|51102 & $\mathrm{B} 2$ \\
\hline MED10|84246 & $\mathrm{B} 2$ \\
\hline MED11|400569 & B2 \\
\hline MED12L|116931 & $\mathrm{B} 2$ \\
\hline MED12|9968 & $\mathrm{B} 2$ \\
\hline MED13L|23389 & $\mathrm{B} 2$ \\
\hline MED13|9969 & $\mathrm{B} 2$ \\
\hline MED15|51586 & B2 \\
\hline MED16|10025 & $\mathrm{B} 2$ \\
\hline MED17|9440 & B2 \\
\hline MED18|54797 & $\mathrm{B} 2$ \\
\hline MED19|219541 & $\mathrm{B} 2$ \\
\hline MED1|5469 & $\mathrm{B} 2$ \\
\hline MED21|9412 & $\mathrm{B} 2$ \\
\hline MED22|6837 & $\mathrm{B} 2$ \\
\hline MED23|9439 & $\mathrm{B} 2$ \\
\hline MED24|9862 & $\mathrm{B} 2$ \\
\hline MED26|9441 & $\mathrm{B} 2$ \\
\hline MED27|9442 & B2 \\
\hline MED28|80306 & $\mathrm{B} 2$ \\
\hline \begin{tabular}{l|l|} 
MED29|55588 \\
\end{tabular} & $\mathrm{B} 2$ \\
\hline MED30|90390 & $\mathrm{B} 2$ \\
\hline MED31|51003 & $\mathrm{B} 2$ \\
\hline MED4|29079 & $\mathrm{B} 2$ \\
\hline MED6|10001 & B2 \\
\hline MED7|9443 & $\mathrm{B} 2$ \\
\hline MEF2A|4205 & $\mathrm{B} 2$ \\
\hline MEF2B|100271849 & $\mathrm{B} 2$ \\
\hline MEF2C|4208 & $\mathrm{B} 2$ \\
\hline MEF2D 4209 & $\mathrm{~B} 2$ \\
\hline MEFV|4210 & $\mathrm{B} 2$ \\
\hline MEG8|79104 & B2 \\
\hline MEGF10|84466 & $\mathrm{B} 2$ \\
\hline MEGF11|84465 & B2 \\
\hline MEGF6|1953 & $\mathrm{B} 2$ \\
\hline MEGF9|1955 & $\mathrm{B} 2$ \\
\hline MEI1|150365 & $\mathrm{B} 2$ \\
\hline MEIG1|644890 & $\mathrm{B} 2$ \\
\hline MEP1A|4224 & $\mathrm{B} 2$ \\
\hline MEP1B $\mid 4225$ & $\mathrm{~B} 2$ \\
\hline MEPCE|56257 & B2 \\
\hline MEPE|56955 & $\mathrm{B} 2$ \\
\hline
\end{tabular}




\begin{tabular}{|c|c|}
\hline MESDC1|59274 & B2 \\
\hline MESP1|55897 & $\mathrm{B} 2$ \\
\hline MESP2|145873 & $\mathrm{B} 2$ \\
\hline METAP1|23173 & $\mathrm{B} 2$ \\
\hline METAP2|10988 & B2 \\
\hline METRNL|284207 & $\mathrm{B} 2$ \\
\hline METT11D1|64745 & $\mathrm{B} 2$ \\
\hline METT5D1|196074 & $\mathrm{B} 2$ \\
\hline METTL10|399818 & $\mathrm{B} 2$ \\
\hline METTL11A|28989 & $\mathrm{B} 2$ \\
\hline METTL12|751071 & $\mathrm{B} 2$ \\
\hline METTL14|57721 & B2 \\
\hline METTL2B|55798 & $\mathrm{B} 2$ \\
\hline METTL3|56339 & $\mathrm{B} 2$ \\
\hline METTL4|64863 & $\mathrm{B} 2$ \\
\hline METTL5|29081 & $\mathrm{B} 2$ \\
\hline METTL7B|196410 & $\mathrm{B} 2$ \\
\hline METTL8|79828 & $\mathrm{B} 2$ \\
\hline METTL9|51108 & $\mathrm{B} 2$ \\
\hline MEX3B|84206 & $\mathrm{B} 2$ \\
\hline MEX3C|51320 & $\mathrm{B} 2$ \\
\hline MFAP1|4236 & $\mathrm{B} 2$ \\
\hline MFAP3L|9848 & $\mathrm{B} 2$ \\
\hline MFAP3|4238 & $\mathrm{B} 2$ \\
\hline MFF|56947 & $\mathrm{B} 2$ \\
\hline MFGE8|4240 & $\mathrm{B} 2$ \\
\hline MFHAS1|9258 & $\mathrm{B} 2$ \\
\hline MFI2|4241 & $\mathrm{B} 2$ \\
\hline MFN1|55669 & $\mathrm{B} 2$ \\
\hline MFN2|9927 & $\mathrm{B} 2$ \\
\hline MFRP|83552 & $\mathrm{B} 2$ \\
\hline MFSD11|79157 & B2 \\
\hline MFSD1|64747 & $\mathrm{B} 2$ \\
\hline MFSD2A|84879 & B2 \\
\hline MFSD2B|388931 & $\mathrm{B} 2$ \\
\hline MFSD4|148808 & $\mathrm{B} 2$ \\
\hline MFSD6L|162387 & $\mathrm{B} 2$ \\
\hline MFSD6|54842 & $\mathrm{B} 2$ \\
\hline MFSD7|84179 & B2 \\
\hline MFSD8|256471 & $\mathrm{B} 2$ \\
\hline MFSD9|84804 & B2 \\
\hline MGAM|8972 & $\mathrm{B} 2$ \\
\hline MGAT1|4245 & B2 \\
\hline MGAT4C|25834 & $\mathrm{B} 2$ \\
\hline MGAT5B|146664 & $\mathrm{B} 2$ \\
\hline MGAT5|4249 & $\mathrm{B} 2$ \\
\hline MGA|23269 & $\mathrm{B} 2$ \\
\hline MGC12916|84815 & $\mathrm{B} 2$ \\
\hline MGC12982|84793 & $\mathrm{B} 2$ \\
\hline MGC14436|84983 & B2 \\
\hline MGC16025|85009 & $\mathrm{B} 2$ \\
\hline
\end{tabular}




\begin{tabular}{|c|c|}
\hline MGC16121|84848 & B2 \\
\hline MGC16142|84849 & B2 \\
\hline MGC16275|85001 & $\mathrm{B} 2$ \\
\hline MGC16384|114130 & $\mathrm{B} 2$ \\
\hline MGC16703|113691 & B2 \\
\hline MGC23270|196872 & $\mathrm{B} 2$ \\
\hline MGC26647|219557 & $\mathrm{B} 2$ \\
\hline MGC27382|149047 & $\mathrm{B} 2$ \\
\hline MGC2752|65996 & B2 \\
\hline MGC2889|84789 & $\mathrm{B} 2$ \\
\hline MGC29506|51237 & $\mathrm{B} 2$ \\
\hline MGC34034|154089 & B2 \\
\hline MGC3771|81854 & $\mathrm{B} 2$ \\
\hline MGC42105|167359 & $\mathrm{B} 2$ \\
\hline MGC4473|79100 & $\mathrm{B} 2$ \\
\hline MGC45800|90768 & $\mathrm{B} 2$ \\
\hline MGC57346|401884 & $\mathrm{B} 2$ \\
\hline MGC70857|414919 & $\mathrm{B} 2$ \\
\hline MGC72080|389538 & $\mathrm{B} 2$ \\
\hline MGEA5|10724 & $\mathrm{B} 2$ \\
\hline MGMT|4255 & $\mathrm{B} 2$ \\
\hline MGP|4256 & $\mathrm{B} 2$ \\
\hline MGST1|4257 & $\mathrm{B} 2$ \\
\hline MGST2|4258 & $\mathrm{B} 2$ \\
\hline MGST3|4259 & $\mathrm{B} 2$ \\
\hline MIA2|117153 & $\mathrm{B} 2$ \\
\hline MIA3 375056 & $\mathrm{~B} 2$ \\
\hline MIAT|440823 & B2 \\
\hline MIA $\mid 8190$ & $\mathrm{~B} 2$ \\
\hline MIB1|57534 & B2 \\
\hline MIB2|142678 & $\mathrm{B} 2$ \\
\hline MICAL1|64780 & $\mathrm{B} 2$ \\
\hline MICALCL|84953 & $\mathrm{B} 2$ \\
\hline MICALL1|85377 & B2 \\
\hline MICA|4276 & $\mathrm{B} 2$ \\
\hline MID1IP1|58526 & $\mathrm{B} 2$ \\
\hline MID2|11043 & $\mathrm{B} 2$ \\
\hline MIDN|90007 & $\mathrm{B} 2$ \\
\hline MIER1|57708 & $\mathrm{B} 2$ \\
\hline MIER2|54531 & $\mathrm{B} 2$ \\
\hline MIER3|166968 & B2 \\
\hline MIF4GD|57409 & $\mathrm{B} 2$ \\
\hline MIIP|60672 & $\mathrm{B} 2$ \\
\hline MIMT1|100073347 & $\mathrm{B} 2$ \\
\hline MINA|84864 & $\mathrm{B} 2$ \\
\hline MIOS|54468 & $\mathrm{B} 2$ \\
\hline MIOX|55586 & $\mathrm{B} 2$ \\
\hline MIPEP|4285 & $\mathrm{B} 2$ \\
\hline MIP|4284 & $\mathrm{B} 2$ \\
\hline MIR155HG|114614 & B2 \\
\hline MIS12|79003 & $\mathrm{B} 2$ \\
\hline
\end{tabular}




\begin{tabular}{|c|c|}
\hline MITD1|129531 & $\mathrm{B} 2$ \\
\hline MIXL1|83881 & B2 \\
\hline MKKS|8195 & $\mathrm{B} 2$ \\
\hline MKL1|57591 & $\mathrm{B} 2$ \\
\hline MKL2|57496 & $\mathrm{B} 2$ \\
\hline MKNK2|2872 & $\mathrm{B} 2$ \\
\hline MKRN1|23608 & $\mathrm{B} 2$ \\
\hline MKRN2|23609 & $\mathrm{B} 2$ \\
\hline MKRN3|7681 & $\mathrm{B} 2$ \\
\hline MKS1|54903 & $\mathrm{B} 2$ \\
\hline MKX|283078 & $\mathrm{B} 2$ \\
\hline MLANA|2315 & $\mathrm{B} 2$ \\
\hline MLC1|23209 & $\mathrm{B} 2$ \\
\hline MLEC $\mid 9761$ & $\mathrm{~B} 2$ \\
\hline MLF1|4291 & $\mathrm{B} 2$ \\
\hline MLH1|4292 & $\mathrm{B} 2$ \\
\hline MLH3|27030 & $\mathrm{B} 2$ \\
\hline MLKL|197259 & $\mathrm{B} 2$ \\
\hline MLL2|8085 & $\mathrm{B} 2$ \\
\hline MLL3|58508 & $\mathrm{B} 2$ \\
\hline MLL4|9757 & $\mathrm{B} 2$ \\
\hline MLL5|55904 & $\mathrm{B} 2$ \\
\hline MLLT10|8028 & $\mathrm{B} 2$ \\
\hline MLLT1|4298 & $\mathrm{B} 2$ \\
\hline MLLT3|4300 & B2 \\
\hline MLLT4|4301 & $\mathrm{B} 2$ \\
\hline MLNR|2862 & $\mathrm{B} 2$ \\
\hline MLXIP|22877 & $\mathrm{B} 2$ \\
\hline MLX|6945 & $\mathrm{B} 2$ \\
\hline MMAA|166785 & $\mathrm{B} 2$ \\
\hline MMAB|326625 & $\mathrm{B} 2$ \\
\hline MMACHC|25974 & B2 \\
\hline MMADHC|27249 & $\mathrm{B} 2$ \\
\hline MMD2|221938 & $\mathrm{B} 2$ \\
\hline MMEL1|79258 & $\mathrm{B} 2$ \\
\hline MMP10|4319 & $\mathrm{B} 2$ \\
\hline MMP12|4321 & $\mathrm{B} 2$ \\
\hline MMP13|4322 & $\mathrm{B} 2$ \\
\hline MMP15|4324 & $\mathrm{B} 2$ \\
\hline MMP16|4325 & $\mathrm{B} 2$ \\
\hline MMP17|4326 & $\mathrm{B} 2$ \\
\hline MMP1|4312 & $\mathrm{B} 2$ \\
\hline MMP20|9313 & $\mathrm{B} 2$ \\
\hline MMP21|118856 & $\mathrm{B} 2$ \\
\hline MMP23A|8511 & $\mathrm{B} 2$ \\
\hline MMP23B $\mid 8510$ & $\mathrm{~B} 2$ \\
\hline MMP24|10893 & $\mathrm{B} 2$ \\
\hline MMP25|64386 & $\mathrm{B} 2$ \\
\hline MMP26|56547 & $\mathrm{B} 2$ \\
\hline MMP2|4313 & $\mathrm{B} 2$ \\
\hline MMP7|4316 & $\mathrm{B} 2$ \\
\hline
\end{tabular}




\begin{tabular}{|c|c|}
\hline MMP8|4317 & B2 \\
\hline MMP9|4318 & B2 \\
\hline MMS19|64210 & $\mathrm{B} 2$ \\
\hline MN1|4330 & $\mathrm{B} 2$ \\
\hline MNAT1|4331 & B2 \\
\hline MNDA|4332 & $\mathrm{B} 2$ \\
\hline MNS1|55329 & $\mathrm{B} 2$ \\
\hline MNT|4335 & $\mathrm{B} 2$ \\
\hline MNX1|3110 & $\mathrm{B} 2$ \\
\hline MOAP1|64112 & $\mathrm{B} 2$ \\
\hline MOB2|81532 & $\mathrm{B} 2$ \\
\hline MOBKL1A|92597 & B2 \\
\hline MOBKL2A|126308 & $\mathrm{B} 2$ \\
\hline MOBKL3|25843 & $\mathrm{B} 2$ \\
\hline MOBP|4336 & $\mathrm{B} 2$ \\
\hline MOCOS|55034 & $\mathrm{B} 2$ \\
\hline MOCS2|4338 & B2 \\
\hline MOGAT1|116255 & $\mathrm{B} 2$ \\
\hline MOGAT2|80168 & B2 \\
\hline MOGAT3|346606 & $\mathrm{B} 2$ \\
\hline MOG|4340 & $\mathrm{B} 2$ \\
\hline MON1A|84315 & $\mathrm{B} 2$ \\
\hline MON1B|22879 & $\mathrm{B} 2$ \\
\hline MORC1|27136 & $\mathrm{B} 2$ \\
\hline MORC3|23515 & $\mathrm{B} 2$ \\
\hline MORC4|79710 & $\mathrm{B} 2$ \\
\hline MORF4L1|10933 & $\mathrm{B} 2$ \\
\hline MORF4|10934 & B2 \\
\hline MORN1|79906 & $\mathrm{B} 2$ \\
\hline MORN3|283385 & B2 \\
\hline MORN4|118812 & $\mathrm{B} 2$ \\
\hline MORN5|254956 & B2 \\
\hline MOSC2|54996 & $\mathrm{B} 2$ \\
\hline MOSPD2|158747 & B2 \\
\hline MOV10L1|54456 & $\mathrm{B} 2$ \\
\hline MPEG1|219972 & $\mathrm{B} 2$ \\
\hline MPG|4350 & $\mathrm{B} 2$ \\
\hline MPHOSPH10|10199 & $\mathrm{B} 2$ \\
\hline MPHOSPH6|10200 & $\mathrm{B} 2$ \\
\hline MPI|4351 & $\mathrm{B} 2$ \\
\hline MPND|84954 & B2 \\
\hline MPO|4353 & $\mathrm{B} 2$ \\
\hline MPP1|4354 & $\mathrm{B} 2$ \\
\hline MPP2|4355 & $\mathrm{B} 2$ \\
\hline MPP3|4356 & $\mathrm{B} 2$ \\
\hline MPP4|58538 & $\mathrm{B} 2$ \\
\hline MPP5|64398 & $\mathrm{B} 2$ \\
\hline MPP7|143098 & $\mathrm{B} 2$ \\
\hline MPPE1|65258 & $\mathrm{B} 2$ \\
\hline MPPED1|758 & B2 \\
\hline MPST|4357 & $\mathrm{B} 2$ \\
\hline
\end{tabular}




\begin{tabular}{|c|c|}
\hline MPV17L|255027 & B2 \\
\hline MPZL1|9019 & $\mathrm{B} 2$ \\
\hline MPZL2|10205 & $\mathrm{B} 2$ \\
\hline MPZL3|196264 & $\mathrm{B} 2$ \\
\hline MRC2|9902 & B2 \\
\hline MRE11A|4361 & $\mathrm{B} 2$ \\
\hline MREG|55686 & $\mathrm{B} 2$ \\
\hline MRFAP1L1|114932 & $\mathrm{B} 2$ \\
\hline MRFAP1|93621 & $\mathrm{B} 2$ \\
\hline MRGPRD|116512 & $\mathrm{B} 2$ \\
\hline MRGPRE|116534 & $\mathrm{B} 2$ \\
\hline MRGPRX4|117196 & B2 \\
\hline MRI1|84245 & $\mathrm{B} 2$ \\
\hline MRM1|79922 & $\mathrm{B} 2$ \\
\hline MRO| 83876 & $\mathrm{~B} 2$ \\
\hline MRP63|78988 & $\mathrm{B} 2$ \\
\hline MRPL10|124995 & $\mathrm{B} 2$ \\
\hline MRPL11|65003 & $\mathrm{B} 2$ \\
\hline MRPL16|54948 & B2 \\
\hline MRPL18|29074 & $\mathrm{B} 2$ \\
\hline MRPL1|65008 & $\mathrm{B} 2$ \\
\hline MRPL20|55052 & $\mathrm{B} 2$ \\
\hline MRPL21|219927 & $\mathrm{B} 2$ \\
\hline MRPL23|6150 & $\mathrm{B} 2$ \\
\hline MRPL27|51264 & $\mathrm{B} 2$ \\
\hline MRPL2|51069 & $\mathrm{B} 2$ \\
\hline MRPL30|51263 & $\mathrm{B} 2$ \\
\hline MRPL32|64983 & B2 \\
\hline MRPL33|9553 & $\mathrm{B} 2$ \\
\hline MRPL34|64981 & $\mathrm{B} 2$ \\
\hline MRPL35|51318 & $\mathrm{B} 2$ \\
\hline MRPL37|51253 & $\mathrm{B} 2$ \\
\hline MRPL38|64978 & $\mathrm{B} 2$ \\
\hline MRPL39|54148 & B2 \\
\hline MRPL40|64976 & $\mathrm{B} 2$ \\
\hline MRPL41|64975 & $\mathrm{B} 2$ \\
\hline MRPL42P5|359821 & $\mathrm{B} 2$ \\
\hline MRPL43|84545 & $\mathrm{B} 2$ \\
\hline MRPL44|65080 & $\mathrm{B} 2$ \\
\hline MRPL45|84311 & $\mathrm{B} 2$ \\
\hline MRPL46|26589 & B2 \\
\hline MRPL48|51642 & $\mathrm{B} 2$ \\
\hline MRPL4|51073 & B2 \\
\hline MRPL50|54534 & $\mathrm{B} 2$ \\
\hline MRPL52|122704 & B2 \\
\hline MRPL53|116540 & $\mathrm{B} 2$ \\
\hline MRPL54|116541 & $\mathrm{B} 2$ \\
\hline MRPS11|64963 & $\mathrm{B} 2$ \\
\hline MRPS15|64960 & $\mathrm{B} 2$ \\
\hline MRPS17|51373 & $\mathrm{B} 2$ \\
\hline MRPS18A|55168 & $\mathrm{B} 2$ \\
\hline
\end{tabular}




\begin{tabular}{|c|c|}
\hline MRPS18B|28973 & B2 \\
\hline MRPS18C|51023 & B2 \\
\hline MRPS22|56945 & $\mathrm{B} 2$ \\
\hline MRPS24|64951 & $\mathrm{B} 2$ \\
\hline MRPS25|64432 & B2 \\
\hline MRPS26|64949 & B2 \\
\hline MRPS27|23107 & $\mathrm{B} 2$ \\
\hline MRPS28|28957 & $\mathrm{B} 2$ \\
\hline MRPS2|51116 & $\mathrm{B} 2$ \\
\hline MRPS30|10884 & $\mathrm{B} 2$ \\
\hline MRPS33|51650 & $\mathrm{B} 2$ \\
\hline MRPS36|92259 & B2 \\
\hline MRPS5|64969 & $\mathrm{B} 2$ \\
\hline MRPS6|64968 & $\mathrm{B} 2$ \\
\hline MRPS7|51081 & $\mathrm{B} 2$ \\
\hline MRPS9|64965 & $\mathrm{B} 2$ \\
\hline MRRF|92399 & $\mathrm{B} 2$ \\
\hline MRS2|57380 & $\mathrm{B} 2$ \\
\hline MRTO4|51154 & B2 \\
\hline MS4A10|341116 & $\mathrm{B} 2$ \\
\hline MS4A12|54860 & $\mathrm{B} 2$ \\
\hline MS4A14|84689 & $\mathrm{B} 2$ \\
\hline MS4A15|219995 & $\mathrm{B} 2$ \\
\hline MS4A1|931 & $\mathrm{B} 2$ \\
\hline MS4A2|2206 & $\mathrm{B} 2$ \\
\hline MS4A3|932 & $\mathrm{B} 2$ \\
\hline MS4A4A|51338 & B2 \\
\hline MS4A6A|64231 & B2 \\
\hline MS4A6E|245802 & $\mathrm{B} 2$ \\
\hline MS4A7|58475 & $\mathrm{B} 2$ \\
\hline MS4A8B|83661 & $\mathrm{B} 2$ \\
\hline MSC|9242 & $\mathrm{B} 2$ \\
\hline MSGN1|343930 & $\mathrm{B} 2$ \\
\hline MSH3|4437 & B2 \\
\hline MSH4|4438 & $\mathrm{B} 2$ \\
\hline MSH5|4439 & $\mathrm{B} 2$ \\
\hline MSI1|4440 & $\mathrm{B} 2$ \\
\hline MSL1|339287 & $\mathrm{B} 2$ \\
\hline MSL2|55167 & $\mathrm{B} 2$ \\
\hline MSL3L2|151507 & $\mathrm{B} 2$ \\
\hline MSL3|10943 & B2 \\
\hline MSLNL|401827 & $\mathrm{B} 2$ \\
\hline MSLN|10232 & $\mathrm{B} 2$ \\
\hline MSMB|4477 & $\mathrm{B} 2$ \\
\hline MSMP|692094 & $\mathrm{B} 2$ \\
\hline MSN|4478 & B2 \\
\hline MSRB2|22921 & $\mathrm{B} 2$ \\
\hline MST1|4485 & $\mathrm{B} 2$ \\
\hline MST4|51765 & $\mathrm{B} 2$ \\
\hline MSTN|2660 & $\mathrm{B} 2$ \\
\hline MSTO1|55154 & $\mathrm{B} 2$ \\
\hline
\end{tabular}




\begin{tabular}{|c|c|}
\hline MSTO2P|100129405 & B2 \\
\hline MSX2P1|55545 & B2 \\
\hline MSX2|4488 & $\mathrm{B} 2$ \\
\hline MT1A|4489 & $\mathrm{B} 2$ \\
\hline MT1DP|326343 & $\mathrm{B} 2$ \\
\hline MT1F|4494 & $\mathrm{B} 2$ \\
\hline MT1G|4495 & $\mathrm{B} 2$ \\
\hline MT1H|4496 & $\mathrm{B} 2$ \\
\hline MT1IP|644314 & $\mathrm{B} 2$ \\
\hline MT2A|4502 & $\mathrm{B} 2$ \\
\hline MT3|4504 & $\mathrm{B} 2$ \\
\hline MT4|84560 & B2 \\
\hline MTA1|9112 & $\mathrm{B} 2$ \\
\hline MTA2|9219 & $\mathrm{B} 2$ \\
\hline MTAP|4507 & $\mathrm{B} 2$ \\
\hline MTCH1|23787 & $\mathrm{B} 2$ \\
\hline MTCP1NB|100272147 & $\mathrm{B} 2$ \\
\hline MTCP1|4515 & $\mathrm{B} 2$ \\
\hline MTERFD3|80298 & B2 \\
\hline MTERF|7978 & $\mathrm{B} 2$ \\
\hline MTF1|4520 & $\mathrm{B} 2$ \\
\hline MTF2|22823 & $\mathrm{B} 2$ \\
\hline MTFMT|123263 & $\mathrm{B} 2$ \\
\hline MTG1|92170 & $\mathrm{B} 2$ \\
\hline MTHFD1L|25902 & $\mathrm{B} 2$ \\
\hline MTHFD1|4522 & $\mathrm{B} 2$ \\
\hline MTHFD2L|441024 & $\mathrm{B} 2$ \\
\hline MTHFR|4524 & B2 \\
\hline \begin{tabular}{l|l|l|} 
MTHFSD 64779 \\
\end{tabular} & $\mathrm{~B} 2$ \\
\hline MTHFS|10588 & $\mathrm{B} 2$ \\
\hline MTIF2|4528 & $\mathrm{B} 2$ \\
\hline \begin{tabular}{l|l|l|} 
MTIF3|219402 \\
\end{tabular} & B2 \\
\hline MTM1|4534 & $\mathrm{B} 2$ \\
\hline MTMR11|10903 & B2 \\
\hline MTMR14|64419 & $\mathrm{B} 2$ \\
\hline MTMR1|8776 & $\mathrm{B} 2$ \\
\hline MTMR2|8898 & $\mathrm{B} 2$ \\
\hline MTMR4|9110 & $\mathrm{B} 2$ \\
\hline MTMR6|9107 & $\mathrm{B} 2$ \\
\hline MTMR7|9108 & $\mathrm{B} 2$ \\
\hline MTNR1A|4543 & B2 \\
\hline MTNR1B|4544 & $\mathrm{B} 2$ \\
\hline MTO1|25821 & B2 \\
\hline MTOR|2475 & $\mathrm{B} 2$ \\
\hline MTPAP|55149 & $\mathrm{B} 2$ \\
\hline MTRF1L|54516 & $\mathrm{B} 2$ \\
\hline MTRF1|9617 & $\mathrm{B} 2$ \\
\hline MTRR|4552 & $\mathrm{B} 2$ \\
\hline MTR|4548 & $\mathrm{B} 2$ \\
\hline MTSS1L|92154 & $\mathrm{B} 2$ \\
\hline MTSS1|9788 & $\mathrm{B} 2$ \\
\hline
\end{tabular}




\begin{tabular}{|c|c|}
\hline МTTP|4547 & B2 \\
\hline MTUS2|23281 & $\mathrm{B} 2$ \\
\hline MTVR2|246754 & $\mathrm{B} 2$ \\
\hline MTX3|345778 & B2 \\
\hline MUC12|10071 & B2 \\
\hline MUC13|56667 & B2 \\
\hline MUC15|143662 & $\mathrm{B} 2$ \\
\hline MUC16|94025 & $\mathrm{B} 2$ \\
\hline MUC17|140453 & $\mathrm{B} 2$ \\
\hline MUC20|200958 & $\mathrm{B} 2$ \\
\hline MUC21|394263 & B2 \\
\hline MUC2|4583 & B2 \\
\hline MUC4|4585 & B2 \\
\hline MUC5B|727897 & $\mathrm{B} 2$ \\
\hline MUC6|4588 & $\mathrm{B} 2$ \\
\hline MUC7|4589 & $\mathrm{B} 2$ \\
\hline MUCL1|118430 & $\mathrm{B} 2$ \\
\hline MUDENG|55745 & $\mathrm{B} 2$ \\
\hline MUL1|79594 & B2 \\
\hline MUM1L1|139221 & $\mathrm{B} 2$ \\
\hline MURC|347273 & $\mathrm{B} 2$ \\
\hline MUS81|80198 & $\mathrm{B} 2$ \\
\hline MUSK|4593 & $\mathrm{B} 2$ \\
\hline MUSTN1|389125 & B2 \\
\hline MUTED|63915 & $\mathrm{B} 2$ \\
\hline MUTYH|4595 & B2 \\
\hline MUT|4594 & $\mathrm{B} 2$ \\
\hline MVD 4597 & B2 \\
\hline MVK|4598 & $\mathrm{B} 2$ \\
\hline MX2|4600 & $\mathrm{B} 2$ \\
\hline MXD1|4084 & B2 \\
\hline MXRA8|54587 & $\mathrm{B} 2$ \\
\hline MYADML2|255275 & B2 \\
\hline MYADML|151325 & $\mathrm{B} 2$ \\
\hline MYBBP1A|10514 & B2 \\
\hline MYBL1|4603 & $\mathrm{B} 2$ \\
\hline MYBPC1|4604 & $\mathrm{B} 2$ \\
\hline MYBPC2|4606 & $\mathrm{B} 2$ \\
\hline MYBPC3|4607 & $\mathrm{B} 2$ \\
\hline MYBPHL|343263 & $\mathrm{B} 2$ \\
\hline MYBPH|4608 & B2 \\
\hline MYCBPAP|84073 & $\mathrm{B} 2$ \\
\hline MYCL1|4610 & B2 \\
\hline MYCNOS|10408 & B2 \\
\hline MYCN|4613 & $\mathrm{B} 2$ \\
\hline MYEOV2|150678 & B2 \\
\hline MYF5|4617 & $\mathrm{B} 2$ \\
\hline MYF6|4618 & B2 \\
\hline MYH13|8735 & $\mathrm{B} 2$ \\
\hline MYH14|79784 & B2 \\
\hline MYH15|22989 & B2 \\
\hline
\end{tabular}




\begin{tabular}{|c|c|}
\hline MYH16|84176 & B2 \\
\hline MYH1|4619 & $\mathrm{B} 2$ \\
\hline MYH2|4620 & $\mathrm{B} 2$ \\
\hline MYH4|4622 & $\mathrm{B} 2$ \\
\hline MYH6|4624 & $\mathrm{B} 2$ \\
\hline MYH7|4625 & B2 \\
\hline MYH8|4626 & $\mathrm{B} 2$ \\
\hline MYH9|4627 & $\mathrm{B} 2$ \\
\hline MYL10|93408 & $\mathrm{B} 2$ \\
\hline MYL12A|10627 & $\mathrm{B} 2$ \\
\hline MYL12B|103910 & B2 \\
\hline MYL1|4632 & B2 \\
\hline MYL2|4633 & B2 \\
\hline MYL3|4634 & $\mathrm{B} 2$ \\
\hline MYL4|4635 & $\mathrm{B} 2$ \\
\hline MYL5|4636 & $\mathrm{B} 2$ \\
\hline MYL6B|140465 & $\mathrm{B} 2$ \\
\hline MYL6|4637 & $\mathrm{B} 2$ \\
\hline MYL7|58498 & B2 \\
\hline MYLIP|29116 & B2 \\
\hline MYLK2|85366 & B2 \\
\hline MYLK3|91807 & B2 \\
\hline MYLK4|340156 & B2 \\
\hline MYLPF|29895 & B2 \\
\hline MYNN|55892 & B2 \\
\hline MYO10|4651 & B2 \\
\hline MYO15A|51168 & B2 \\
\hline MYO16|23026 & B2 \\
\hline MYO18A|399687 & $\mathrm{B} 2$ \\
\hline MYO18B|84700 & $\mathrm{B} 2$ \\
\hline MYO1A|4640 & $\mathrm{B} 2$ \\
\hline MYO1B|4430 & B2 \\
\hline MYO1E|4643 & B2 \\
\hline MYO1F|4542 & $\mathrm{B} 2$ \\
\hline MYO1G|64005 & B2 \\
\hline MYO3A|53904 & $\mathrm{B} 2$ \\
\hline MYO3B|140469 & B2 \\
\hline MYO5A|4644 & $\mathrm{B} 2$ \\
\hline MYO5C|55930 & $\mathrm{B} 2$ \\
\hline MYO7A|4647 & $\mathrm{B} 2$ \\
\hline MYO7B|4648 & B2 \\
\hline MYO9B|4650 & B2 \\
\hline MYOD1|4654 & B2 \\
\hline MYOF|26509 & $\mathrm{B} 2$ \\
\hline MYOG|4656 & B2 \\
\hline MYOM1|8736 & B2 \\
\hline MYOM2|9172 & $\mathrm{B} 2$ \\
\hline MYOM3|127294 & B2 \\
\hline MYOT|9499 & B2 \\
\hline MYOZ1|58529 & B2 \\
\hline MYOZ2|51778 & $\mathrm{B} 2$ \\
\hline
\end{tabular}




\begin{tabular}{|c|c|}
\hline MYOZ3|91977 & $\mathrm{B} 2$ \\
\hline MYPN|84665 & $\mathrm{B} 2$ \\
\hline MYPOP|339344 & $\mathrm{B} 2$ \\
\hline MYSM1|114803 & $\mathrm{B} 2$ \\
\hline MYST1|84148 & $\mathrm{B} 2$ \\
\hline MYST2|11143 & $\mathrm{B} 2$ \\
\hline MYST3|7994 & $\mathrm{B} 2$ \\
\hline MYST4|23522 & $\mathrm{B} 2$ \\
\hline MYT1L|23040 & $\mathrm{B} 2$ \\
\hline MYT1|4661 & $\mathrm{B} 2$ \\
\hline MZF1|7593 & $\mathrm{B} 2$ \\
\hline N4BP1|9683 & $\mathrm{B} 2$ \\
\hline N4BP2|55728 & $\mathrm{B} 2$ \\
\hline N6AMT1|29104 & $\mathrm{B} 2$ \\
\hline N6AMT2|221143 & $\mathrm{B} 2$ \\
\hline NAA10|8260 & $\mathrm{B} 2$ \\
\hline NAA11|84779 & $\mathrm{B} 2$ \\
\hline NAA25|80018 & $\mathrm{B} 2$ \\
\hline NAA30|122830 & $\mathrm{B} 2$ \\
\hline NAA40|79829 & $\mathrm{B} 2$ \\
\hline NAA50|80218 & $\mathrm{B} 2$ \\
\hline NAAA|27163 & $\mathrm{B} 2$ \\
\hline NAALADL2|254827 & $\mathrm{B} 2$ \\
\hline NAB2|4665 & $\mathrm{B} 2$ \\
\hline NACA2|342538 & $\mathrm{B} 2$ \\
\hline NACAP1|83955 & $\mathrm{B} 2$ \\
\hline NACC2|138151 & $\mathrm{B} 2$ \\
\hline NADK|65220 & $\mathrm{B} 2$ \\
\hline NADSYN1|55191 & $\mathrm{B} 2$ \\
\hline NAE1|8883 & $\mathrm{B} 2$ \\
\hline NAF1|92345 & $\mathrm{B} 2$ \\
\hline NAGLU|4669 & $\mathrm{B} 2$ \\
\hline NAGPA|51172 & $\mathrm{B} 2$ \\
\hline NAGS|162417 & $\mathrm{B} 2$ \\
\hline NAIF1|203245 & $\mathrm{B} 2$ \\
\hline NAIP|4671 & $\mathrm{B} 2$ \\
\hline NALCN|259232 & $\mathrm{B} 2$ \\
\hline NAMPT|10135 & $\mathrm{B} 2$ \\
\hline NANOG|79923 & $\mathrm{B} 2$ \\
\hline NANOS1|340719 & $\mathrm{B} 2$ \\
\hline NANOS2|339345 & $\mathrm{B} 2$ \\
\hline NANOS3|342977 & $\mathrm{B} 2$ \\
\hline NANP|140838 & $\mathrm{B} 2$ \\
\hline NAP1L1|4673 & $\mathrm{B} 2$ \\
\hline \begin{tabular}{l|l|l|} 
NAP1L3|4675 \\
\end{tabular} & $\mathrm{B} 2$ \\
\hline NAP1L4|4676 & $\mathrm{B} 2$ \\
\hline NAP1L6|645996 & $\mathrm{B} 2$ \\
\hline NAPB|63908 & $\mathrm{B} 2$ \\
\hline NAPEPLD|222236 & $\mathrm{B} 2$ \\
\hline NAPG|8774 & $\mathrm{B} 2$ \\
\hline NAPRT1|93100 & $\mathrm{B} 2$ \\
\hline
\end{tabular}




\begin{tabular}{|c|c|}
\hline NAPSA|9476 & B2 \\
\hline NAPSB|256236 & B2 \\
\hline NARF|26502 & $\mathrm{B} 2$ \\
\hline NARG2|79664 & $\mathrm{B} 2$ \\
\hline NARS2|79731 & B2 \\
\hline NASP|4678 & $\mathrm{B} 2$ \\
\hline NAT10|55226 & $\mathrm{B} 2$ \\
\hline NAT1 $\mid 9$ & $\mathrm{~B} 2$ \\
\hline NAT2|10 & $\mathrm{B} 2$ \\
\hline NAT8B|51471 & B2 \\
\hline NAT8|9027 & $\mathrm{B} 2$ \\
\hline NAT9|26151 & B2 \\
\hline NAV1|89796 & $\mathrm{B} 2$ \\
\hline NBAS|51594 & $\mathrm{B} 2$ \\
\hline NBEAL2|23218 & $\mathrm{B} 2$ \\
\hline NBEA|26960 & B2 \\
\hline NBL1|4681 & B2 \\
\hline NBPF10|100132406 & B2 \\
\hline NBPF14|25832 & B2 \\
\hline NBPF15|284565 & $\mathrm{B} 2$ \\
\hline NBPF16|728936 & $\mathrm{B} 2$ \\
\hline NBPF1|55672 & $\mathrm{B} 2$ \\
\hline NBPF22P|285622 & $\mathrm{B} 2$ \\
\hline $\begin{array}{l}\text { NBPF3|84224 } \\
\end{array}$ & $\mathrm{B} 2$ \\
\hline NBPF4|148545 & $\mathrm{B} 2$ \\
\hline NBPF6|653149 & $\mathrm{B} 2$ \\
\hline NBPF7|343505 & $\mathrm{B} 2$ \\
\hline NBPF9|400818 & B2 \\
\hline NBR1|4077 & $\mathrm{B} 2$ \\
\hline NBR2|10230 & $\mathrm{B} 2$ \\
\hline NCAM2|4685 & $\mathrm{B} 2$ \\
\hline NCAN|1463 & B2 \\
\hline NCAPH2|29781 & $\mathrm{B} 2$ \\
\hline NCCRP1|342897 & B2 \\
\hline NCF1B|654816 & $\mathrm{B} 2$ \\
\hline NCF1C 654817 & $\mathrm{~B} 2$ \\
\hline NCF1|653361 & $\mathrm{B} 2$ \\
\hline NCF2|4688 & $\mathrm{B} 2$ \\
\hline NCF4|4689 & B2 \\
\hline NCK1|4690 & $\mathrm{B} 2$ \\
\hline NCK2|8440 & B2 \\
\hline NCKAP1L|3071 & $\mathrm{B} 2$ \\
\hline NCKAP1|10787 & B2 \\
\hline NCKAP5|344148 & $\mathrm{B} 2$ \\
\hline NCKIPSD|51517 & $\mathrm{B} 2$ \\
\hline NCL|4691 & $\mathrm{B} 2$ \\
\hline \begin{tabular}{l|l|l|} 
NCOA2|10499 \\
\end{tabular} & $\mathrm{B} 2$ \\
\hline NCOA3|8202 & B2 \\
\hline NCOR1|9611 & $\mathrm{B} 2$ \\
\hline NCOR2|9612 & B2 \\
\hline NCR1|9437 & B2 \\
\hline
\end{tabular}




\begin{tabular}{|c|c|}
\hline NCR2|9436 & B2 \\
\hline \begin{tabular}{l|l|} 
NCR3 & 259197 \\
\end{tabular} & $\mathrm{~B} 2$ \\
\hline NCRNA00028|140875 & $\mathrm{B} 2$ \\
\hline NCRNA00051|619434 & $\mathrm{B} 2$ \\
\hline NCRNA00052|145978 & B2 \\
\hline NCRNA00081|92482 & $\mathrm{B} 2$ \\
\hline NCRNA00085|147650 & $\mathrm{B} 2$ \\
\hline NCRNA00093|100188954 & $\mathrm{B} 2$ \\
\hline NCRNA00094|266655 & $\mathrm{B} 2$ \\
\hline NCRNA00095|283932 & $\mathrm{B} 2$ \\
\hline NCRNA00099|359822 & $\mathrm{B} 2$ \\
\hline NCRNA00105|80161 & $\mathrm{B} 2$ \\
\hline NCRNA00107|283981 & $\mathrm{B} 2$ \\
\hline NCRNA00110|642976 & $\mathrm{B} 2$ \\
\hline NCRNA00111|54090 & $\mathrm{B} 2$ \\
\hline NCRNA00112|54089 & $\mathrm{B} 2$ \\
\hline NCRNA00113|54088 & $\mathrm{B} 2$ \\
\hline NCRNA00114|400866 & $\mathrm{B} 2$ \\
\hline NCRNA00115|79854 & $\mathrm{B} 2$ \\
\hline NCRNA00116|205251 & $\mathrm{B} 2$ \\
\hline NCRNA00119|348808 & $\mathrm{B} 2$ \\
\hline NCRNA00157|54075 & $\mathrm{B} 2$ \\
\hline NCRNA00158|54072 & $\mathrm{B} 2$ \\
\hline NCRNA00159|54070 & $\mathrm{B} 2$ \\
\hline NCRNA00160|54064 & $\mathrm{B} 2$ \\
\hline NCRNA00161|118421 & $\mathrm{B} 2$ \\
\hline NCRNA00162|378825 & $\mathrm{B} 2$ \\
\hline NCRNA00164|554226 & $\mathrm{B} 2$ \\
\hline NCRNA00167|440072 & $\mathrm{B} 2$ \\
\hline NCRNA00169|400508 & $\mathrm{B} 2$ \\
\hline NCRNA00171|80862 & $\mathrm{B} 2$ \\
\hline NCRNA00173|100287569 & $\mathrm{B} 2$ \\
\hline NCRNA00174|285908 & $\mathrm{B} 2$ \\
\hline NCRNA00175|378832 & $\mathrm{B} 2$ \\
\hline NCRNA00181|503538 & $\mathrm{B} 2$ \\
\hline NCRNA00183|554203 & $\mathrm{B} 2$ \\
\hline NCRNA00189|193629 & $\mathrm{B} 2$ \\
\hline NCRNA00200|399706 & $\mathrm{B} 2$ \\
\hline NCRNA00201|284702 & $\mathrm{B} 2$ \\
\hline NCRNA00202|387644 & $\mathrm{B} 2$ \\
\hline NCRNA00203|319085 & $\mathrm{B} 2$ \\
\hline NCRNA00235|64493 & $\mathrm{B} 2$ \\
\hline NCS1|23413 & $\mathrm{B} 2$ \\
\hline NDFIP1|80762 & $\mathrm{B} 2$ \\
\hline NDFIP2|54602 & $\mathrm{B} 2$ \\
\hline \begin{tabular}{l|l|} 
NDNL2 & 56160 \\
\end{tabular} & $\mathrm{~B} 2$ \\
\hline NDP|4693 & B2 \\
\hline NDRG1|10397 & $\mathrm{B} 2$ \\
\hline NDRG4|65009 & $\mathrm{B} 2$ \\
\hline NDST2|8509 & $\mathrm{B} 2$ \\
\hline NDST3|9348 & $\mathrm{B} 2$ \\
\hline
\end{tabular}




\begin{tabular}{|c|c|}
\hline NDST4|64579 & B2 \\
\hline NDUFA10|4705 & B2 \\
\hline NDUFA11|126328 & $\mathrm{B} 2$ \\
\hline NDUFA12|55967 & $\mathrm{B} 2$ \\
\hline NDUFA1|4694 & B2 \\
\hline NDUFA2|4695 & B2 \\
\hline NDUFA3|4696 & $\mathrm{B} 2$ \\
\hline NDUFA4L2|56901 & $\mathrm{B} 2$ \\
\hline NDUFA4|4697 & $\mathrm{B} 2$ \\
\hline NDUFA5|4698 & $\mathrm{B} 2$ \\
\hline NDUFA6|4700 & $\mathrm{B} 2$ \\
\hline NDUFA8|4702 & B2 \\
\hline NDUFA9|4704 & $\mathrm{B} 2$ \\
\hline NDUFAF1|51103 & $\mathrm{B} 2$ \\
\hline NDUFAF2|91942 & $\mathrm{B} 2$ \\
\hline NDUFAF3|25915 & $\mathrm{B} 2$ \\
\hline NDUFAF4|29078 & B2 \\
\hline NDUFB10|4716 & $\mathrm{B} 2$ \\
\hline NDUFB11|54539 & B2 \\
\hline NDUFB1|4707 & $\mathrm{B} 2$ \\
\hline NDUFB2|4708 & $\mathrm{B} 2$ \\
\hline NDUFB3|4709 & $\mathrm{B} 2$ \\
\hline NDUFB5|4711 & $\mathrm{B} 2$ \\
\hline NDUFB6|4712 & $\mathrm{B} 2$ \\
\hline NDUFB7|4713 & $\mathrm{B} 2$ \\
\hline NDUFB8|4714 & $\mathrm{B} 2$ \\
\hline NDUFC1|4717 & $\mathrm{B} 2$ \\
\hline NDUFC2|4718 & B2 \\
\hline NDUFS1|4719 & $\mathrm{B} 2$ \\
\hline NDUFS3|4722 & $\mathrm{B} 2$ \\
\hline NDUFS5|4725 & $\mathrm{B} 2$ \\
\hline NDUFS6|4726 & $\mathrm{B} 2$ \\
\hline NDUFS7|374291 & $\mathrm{B} 2$ \\
\hline NDUFS8|4728 & B2 \\
\hline NDUFV1|4723 & $\mathrm{B} 2$ \\
\hline NDUFV3|4731 & $\mathrm{B} 2$ \\
\hline NEAT1|283131 & $\mathrm{B} 2$ \\
\hline NEBL/10529 & $\mathrm{B} 2$ \\
\hline NEB $\mid 4703$ & B2 \\
\hline NECAB2|54550 & $\mathrm{B} 2$ \\
\hline NECAP2|55707 & B2 \\
\hline NEDD1|121441 & $\mathrm{B} 2$ \\
\hline NEDD4L|23327 & B2 \\
\hline NEDD4|4734 & $\mathrm{B} 2$ \\
\hline NEDD8|4738 & $\mathrm{B} 2$ \\
\hline NEFH|4744 & $\mathrm{B} 2$ \\
\hline NEFL|4747 & $\mathrm{B} 2$ \\
\hline NEFM|4741 & $\mathrm{B} 2$ \\
\hline NEGR1|257194 & $\mathrm{B} 2$ \\
\hline 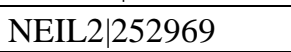 & B2 \\
\hline NEK10|152110 & $\mathrm{B} 2$ \\
\hline
\end{tabular}




\begin{tabular}{|c|c|}
\hline NEK11|79858 & $\mathrm{B} 2$ \\
\hline NEK4|6787 & $\mathrm{B} 2$ \\
\hline NEK6|10783 & $\mathrm{B} 2$ \\
\hline NEK8|284086 & $\mathrm{B} 2$ \\
\hline NELF|26012 & $\mathrm{B} 2$ \\
\hline NELL1|4745 & $\mathrm{B} 2$ \\
\hline NELL2|4753 & $\mathrm{B} 2$ \\
\hline NENF|29937 & $\mathrm{B} 2$ \\
\hline NEO1|4756 & $\mathrm{B} 2$ \\
\hline NET1|10276 & $\mathrm{B} 2$ \\
\hline NETO1|81832 & $\mathrm{B} 2$ \\
\hline NETO2|81831 & $\mathrm{B} 2$ \\
\hline NEU3|10825 & $\mathrm{B} 2$ \\
\hline NEU4|129807 & $\mathrm{B} 2$ \\
\hline NEURL1B|54492 & $\mathrm{B} 2$ \\
\hline NEURL3|93082 & $\mathrm{B} 2$ \\
\hline NEURL|9148 & $\mathrm{B} 2$ \\
\hline NEUROD1|4760 & $\mathrm{B} 2$ \\
\hline NEUROD2|4761 & $\mathrm{B} 2$ \\
\hline NEUROD4|58158 & $\mathrm{B} 2$ \\
\hline NEUROG3|50674 & $\mathrm{B} 2$ \\
\hline NEXN|91624 & $\mathrm{B} 2$ \\
\hline NF1 4763 & $\mathrm{~B} 2$ \\
\hline NF2|4771 & $\mathrm{B} 2$ \\
\hline NFAM1|150372 & $\mathrm{B} 2$ \\
\hline NFASC|23114 & $\mathrm{B} 2$ \\
\hline NFATC1|4772 & $\mathrm{B} 2$ \\
\hline NFATC2IP|84901 & $\mathrm{B} 2$ \\
\hline NFATC4|4776 & $\mathrm{B} 2$ \\
\hline NFE2L1|4779 & $\mathrm{B} 2$ \\
\hline NFE2L3|9603 & $\mathrm{B} 2$ \\
\hline NFE2|4778 & $\mathrm{B} 2$ \\
\hline NFIA|4774 & $\mathrm{B} 2$ \\
\hline NFIC|4782 & $\mathrm{B} 2$ \\
\hline NFKB1|4790 & $\mathrm{B} 2$ \\
\hline NFKB2|4791 & $\mathrm{B} 2$ \\
\hline NFKBIA|4792 & $\mathrm{B} 2$ \\
\hline NFKBIB|4793 & $\mathrm{B} 2$ \\
\hline NFKBID|84807 & $\mathrm{B} 2$ \\
\hline NFKBIL1|4795 & $\mathrm{B} 2$ \\
\hline NFKBIZ|64332 & $\mathrm{B} 2$ \\
\hline NFS1|9054 & $\mathrm{B} 2$ \\
\hline NFX1|4799 & $\mathrm{B} 2$ \\
\hline NFXL1|152518 & $\mathrm{B} 2$ \\
\hline NFYB|4801 & $\mathrm{B} 2$ \\
\hline NFYC|4802 & $\mathrm{B} 2$ \\
\hline \begin{tabular}{l|l|} 
NGB & 58157 \\
\end{tabular} & $\mathrm{~B} 2$ \\
\hline NGDN|25983 & $\mathrm{B} 2$ \\
\hline NGEF|25791 & $\mathrm{B} 2$ \\
\hline NGFRAP1|27018 & $\mathrm{B} 2$ \\
\hline NGLY1|55768 & $\mathrm{B} 2$ \\
\hline
\end{tabular}




\begin{tabular}{|c|c|}
\hline NGRN|51335 & B2 \\
\hline NHEDC1|150159 & B2 \\
\hline NHEDC2|133308 & $\mathrm{B} 2$ \\
\hline NHEG1|100294720 & $\mathrm{B} 2$ \\
\hline NHLH1 $\mid 4807$ & $\mathrm{~B} 2$ \\
\hline NHLH2|4808 & $\mathrm{B} 2$ \\
\hline NHLRC1|378884 & $\mathrm{B} 2$ \\
\hline NHLRC2|374354 & $\mathrm{B} 2$ \\
\hline NHLRC4|283948 & $\mathrm{B} 2$ \\
\hline NHP2L1|4809 & $\mathrm{B} 2$ \\
\hline NHSL1|57224 & $\mathrm{B} 2$ \\
\hline NHSL2|340527 & B2 \\
\hline NHS|4810 & $\mathrm{B} 2$ \\
\hline NID1|4811 & $\mathrm{B} 2$ \\
\hline NID2|22795 & $\mathrm{B} 2$ \\
\hline NIF3L1|60491 & B2 \\
\hline NINJ2|4815 & B2 \\
\hline NINL|22981 & $\mathrm{B} 2$ \\
\hline NIN|51199 & B2 \\
\hline NIP7|51388 & $\mathrm{B} 2$ \\
\hline NIPAL1|152519 & $\mathrm{B} 2$ \\
\hline NIPAL2|79815 & $\mathrm{B} 2$ \\
\hline NIPAL3|57185 & $\mathrm{B} 2$ \\
\hline NIPAL4|348938 & $\mathrm{B} 2$ \\
\hline NIPBL|25836 & $\mathrm{B} 2$ \\
\hline NIPSNAP3A|25934 & $\mathrm{B} 2$ \\
\hline NIT1 $\mid 4817$ & $\mathrm{~B} 2$ \\
\hline NKAIN3|286183 & $\mathrm{B} 2$ \\
\hline NKAIN4|128414 & $\mathrm{B} 2$ \\
\hline NKD1 $\mid 85407$ & $\mathrm{~B} 2$ \\
\hline NKG7|4818 & $\mathrm{B} 2$ \\
\hline NKIRAS1|28512 & $\mathrm{B} 2$ \\
\hline NKIRAS2|28511 & $\mathrm{B} 2$ \\
\hline NKRF|55922 & B2 \\
\hline NKX1-2|390010 & $\mathrm{B} 2$ \\
\hline NKX2-1/7080 & $\mathrm{B} 2$ \\
\hline NKX2-2|4821 & $\mathrm{B} 2$ \\
\hline NKX2-3|159296 & $\mathrm{B} 2$ \\
\hline NKX2-5|1482 & B2 \\
\hline NKX2-6|137814 & $\mathrm{B} 2$ \\
\hline NKX2-8|26257 & B2 \\
\hline NKX3-1|4824 & $\mathrm{B} 2$ \\
\hline NKX6-1|4825 & B2 \\
\hline NKX6-3|157848 & $\mathrm{B} 2$ \\
\hline NLE1|54475 & $\mathrm{B} 2$ \\
\hline NLGN2|57555 & $\mathrm{B} 2$ \\
\hline NLGN4Y|22829 & $\mathrm{B} 2$ \\
\hline NLRC3|197358 & $\mathrm{B} 2$ \\
\hline NLRC4|58484 & $\mathrm{B} 2$ \\
\hline NLRC5|84166 & B2 \\
\hline NLRP10|338322 & B2 \\
\hline
\end{tabular}




\begin{tabular}{|c|c|}
\hline NLRP11|204801 & $\mathrm{B} 2$ \\
\hline NLRP12|91662 & $\mathrm{B} 2$ \\
\hline NLRP13|126204 & $\mathrm{B} 2$ \\
\hline NLRP14|338323 & $\mathrm{B} 2$ \\
\hline NLRP2|55655 & B2 \\
\hline NLRP3|114548 & $\mathrm{B} 2$ \\
\hline NLRP4|147945 & $\mathrm{B} 2$ \\
\hline NLRP5|126206 & $\mathrm{B} 2$ \\
\hline NLRP6|171389 & $\mathrm{B} 2$ \\
\hline NLRP7|199713 & $\mathrm{B} 2$ \\
\hline NLRP8|126205 & $\mathrm{B} 2$ \\
\hline NLRP9|338321 & $\mathrm{B} 2$ \\
\hline NMD3|51068 & $\mathrm{B} 2$ \\
\hline NME1-NME2|654364 & $\mathrm{B} 2$ \\
\hline NME2P1|283458 & $\mathrm{B} 2$ \\
\hline NME2|4831 & $\mathrm{B} 2$ \\
\hline NME5|8382 & B2 \\
\hline NMI|9111 & $\mathrm{B} 2$ \\
\hline NMNAT1|64802 & $\mathrm{B} 2$ \\
\hline NMUR2|56923 & $\mathrm{B} 2$ \\
\hline NMU|10874 & $\mathrm{B} 2$ \\
\hline NNAT|4826 & $\mathrm{B} 2$ \\
\hline NNT|23530 & $\mathrm{B} 2$ \\
\hline NOC3L|64318 & $\mathrm{B} 2$ \\
\hline NOD2|64127 & $\mathrm{B} 2$ \\
\hline NOG|9241 & $\mathrm{B} 2$ \\
\hline NOL11|25926 & $\mathrm{B} 2$ \\
\hline NOL12|79159 & $\mathrm{B} 2$ \\
\hline NOL3|8996 & $\mathrm{B} 2$ \\
\hline NOL4 $\mid 8715$ & $\mathrm{~B} 2$ \\
\hline NOL6 $\mid 65083$ & $\mathrm{~B} 2$ \\
\hline NOL7|51406 & B2 \\
\hline NOL8|55035 & $\mathrm{B} 2$ \\
\hline NOL9|79707 & $\mathrm{B} 2$ \\
\hline NOM1|64434 & $\mathrm{B} 2$ \\
\hline NOP10|55505 & $\mathrm{B} 2$ \\
\hline NOP14|8602 & $\mathrm{B} 2$ \\
\hline NOS1AP|9722 & $\mathrm{B} 2$ \\
\hline NOS1|4842 & $\mathrm{B} 2$ \\
\hline NOS2|4843 & $\mathrm{B} 2$ \\
\hline NOS3|4846 & $\mathrm{B} 2$ \\
\hline NOSIP|51070 & $\mathrm{B} 2$ \\
\hline NOSTRIN|115677 & $\mathrm{B} 2$ \\
\hline NOTCH1|4851 & $\mathrm{B} 2$ \\
\hline NOTCH2NL|388677 & $\mathrm{B} 2$ \\
\hline NOTCH2|4853 & $\mathrm{B} 2$ \\
\hline NOTCH3|4854 & $\mathrm{B} 2$ \\
\hline NOTO|344022 & $\mathrm{B} 2$ \\
\hline NOTUM|147111 & $\mathrm{B} 2$ \\
\hline NOVA1|4857 & $\mathrm{B} 2$ \\
\hline NOX1|27035 & $\mathrm{B} 2$ \\
\hline
\end{tabular}




\begin{tabular}{|c|c|}
\hline NOX4|50507 & B2 \\
\hline NOX5|79400 & $\mathrm{B} 2$ \\
\hline NOXA1|10811 & $\mathrm{B} 2$ \\
\hline NOXO1|124056 & $\mathrm{B} 2$ \\
\hline NPAS1|4861 & $\mathrm{B} 2$ \\
\hline NPAS2|4862 & B2 \\
\hline NPAT 4863 & $\mathrm{~B} 2$ \\
\hline NPBWR1|2831 & B2 \\
\hline NPB|256933 & $\mathrm{B} 2$ \\
\hline NPC1L1|29881 & $\mathrm{B} 2$ \\
\hline NPC1|4864 & B2 \\
\hline NPC2|10577 & B2 \\
\hline NPDC1|56654 & B2 \\
\hline NPEPL1|79716 & $\mathrm{B} 2$ \\
\hline NPEPPS|9520 & $\mathrm{B} 2$ \\
\hline NPFFR1|64106 & $\mathrm{B} 2$ \\
\hline NPFFR2|10886 & $\mathrm{B} 2$ \\
\hline NPHP1|4867 & $\mathrm{B} 2$ \\
\hline NPHP4|261734 & B2 \\
\hline NPHS1|4868 & $\mathrm{B} 2$ \\
\hline NPHS2|7827 & B2 \\
\hline NPIPL3|23117 & $\mathrm{B} 2$ \\
\hline NPLOC4|55666 & B2 \\
\hline NPL|80896 & $\mathrm{B} 2$ \\
\hline NPM1|4869 & $\mathrm{B} 2$ \\
\hline NPM3|10360 & B2 \\
\hline NPPA|4878 & B2 \\
\hline NPPB|4879 & B2 \\
\hline NPPC|4880 & $\mathrm{B} 2$ \\
\hline NPR3|4883 & $\mathrm{B} 2$ \\
\hline NPRL3|8131 & $\mathrm{B} 2$ \\
\hline NPTN|27020 & $\mathrm{B} 2$ \\
\hline NPTX1|4884 & B2 \\
\hline NPTX2|4885 & B2 \\
\hline NPTXR|23467 & B2 \\
\hline NPW|283869 & $\mathrm{B} 2$ \\
\hline NPY1R|4886 & $\mathrm{B} 2$ \\
\hline NPY5R|4889 & $\mathrm{B} 2$ \\
\hline NPY6R|4888 & $\mathrm{B} 2$ \\
\hline NPY|4852 & $\mathrm{B} 2$ \\
\hline NQO1|1728 & B2 \\
\hline NQO2|4835 & $\mathrm{B} 2$ \\
\hline NR0B1|190 & B2 \\
\hline \begin{tabular}{l|l|} 
NR0B2|8431 &
\end{tabular} & B2 \\
\hline NR1D1|9572 & B2 \\
\hline NR1H2|7376 & B2 \\
\hline NR1H4|9971 & $\mathrm{B} 2$ \\
\hline NR1I2|8856 & B2 \\
\hline NR1I3|9970 & B2 \\
\hline NR2E1|7101 & B2 \\
\hline NR2E3|10002 & $\mathrm{B} 2$ \\
\hline
\end{tabular}




\begin{tabular}{|c|c|}
\hline NR2F1|7025 & B2 \\
\hline NR2F2|7026 & B2 \\
\hline NR4A2|4929 & $\mathrm{B} 2$ \\
\hline NR5A1|2516 & $\mathrm{B} 2$ \\
\hline NR6A1|2649 & B2 \\
\hline NRADDP|100129354 & $\mathrm{B} 2$ \\
\hline NRAP|4892 & $\mathrm{B} 2$ \\
\hline NRARP|441478 & $\mathrm{B} 2$ \\
\hline NRBF2|29982 & B2 \\
\hline NRCAM|4897 & $\mathrm{B} 2$ \\
\hline NRD1|4898 & $\mathrm{B} 2$ \\
\hline NRG3|10718 & $\mathrm{B} 2$ \\
\hline NRG4|145957 & $\mathrm{B} 2$ \\
\hline NRGN|4900 & $\mathrm{B} 2$ \\
\hline NRIP1|8204 & B2 \\
\hline NRIP3|56675 & $\mathrm{B} 2$ \\
\hline NRK|203447 & $\mathrm{B} 2$ \\
\hline NRL|4901 & $\mathrm{B} 2$ \\
\hline NRP2|8828 & B2 \\
\hline NRSN1|140767 & $\mathrm{B} 2$ \\
\hline NRTN|4902 & $\mathrm{B} 2$ \\
\hline NRXN1|9378 & $\mathrm{B} 2$ \\
\hline NRXN2|9379 & $\mathrm{B} 2$ \\
\hline NRXN3|9369 & $\mathrm{B} 2$ \\
\hline NSA2|10412 & $\mathrm{B} 2$ \\
\hline NSD1|64324 & $\mathrm{B} 2$ \\
\hline NSFL1C|55968 & $\mathrm{B} 2$ \\
\hline NSMAF|8439 & B2 \\
\hline NSMCE4A|54780 & $\mathrm{B} 2$ \\
\hline NSUN2|54888 & $\mathrm{B} 2$ \\
\hline \begin{tabular}{l|l|l|} 
NSUN3 63899 \\
\end{tabular} & $\mathrm{~B} 2$ \\
\hline NSUN4|387338 & B2 \\
\hline NSUN5P1|155400 & $\mathrm{B} 2$ \\
\hline NSUN5P2|260294 & $\mathrm{B} 2$ \\
\hline NSUN6|221078 & $\mathrm{B} 2$ \\
\hline NSUN7|79730 & $\mathrm{B} 2$ \\
\hline NT5C1A|84618 & $\mathrm{B} 2$ \\
\hline NT5C1B|93034 & $\mathrm{B} 2$ \\
\hline NT5C2|22978 & $\mathrm{B} 2$ \\
\hline NT5C3L|115024 & $\mathrm{B} 2$ \\
\hline NT5C|30833 & B2 \\
\hline NT5DC1|221294 & $\mathrm{B} 2$ \\
\hline NT5DC2|64943 & B2 \\
\hline NT5M|56953 & $\mathrm{B} 2$ \\
\hline NTAN1|123803 & $\mathrm{B} 2$ \\
\hline NTM|50863 & $\mathrm{B} 2$ \\
\hline NTN1|9423 & $\mathrm{B} 2$ \\
\hline NTN3|4917 & $\mathrm{B} 2$ \\
\hline NTN4|59277 & $\mathrm{B} 2$ \\
\hline NTNG1|22854 & $\mathrm{B} 2$ \\
\hline NTRK1|4914 & $\mathrm{B} 2$ \\
\hline
\end{tabular}




\begin{tabular}{|c|c|}
\hline NTSR1|4923 & B2 \\
\hline NTSR2|23620 & B2 \\
\hline NTS|4922 & $\mathrm{B} 2$ \\
\hline NUAK1|9891 & $\mathrm{B} 2$ \\
\hline NUB1|51667 & B2 \\
\hline NUBPL|80224 & B2 \\
\hline NUCB1|4924 & $\mathrm{B} 2$ \\
\hline NUCKS1|64710 & B2 \\
\hline NUDCD1|84955 & $\mathrm{B} 2$ \\
\hline NUDCD2|134492 & B2 \\
\hline NUDCD3|23386 & $\mathrm{B} 2$ \\
\hline NUDC|10726 & B2 \\
\hline NUDT10|170685 & B2 \\
\hline NUDT12|83594 & $\mathrm{B} 2$ \\
\hline NUDT14|256281 & B2 \\
\hline NUDT15|55270 & B2 \\
\hline NUDT16P1|152195 & B2 \\
\hline NUDT17|200035 & $\mathrm{B} 2$ \\
\hline NUDT18|79873 & B2 \\
\hline NUDT21|11051 & B2 \\
\hline NUDT2|318 & B2 \\
\hline NUDT4|11163 & $\mathrm{B} 2$ \\
\hline NUDT6|11162 & B2 \\
\hline NUDT8|254552 & B2 \\
\hline \begin{tabular}{l|l|l|} 
NUDT9 5343 \\
\end{tabular} & $\mathrm{~B} 2$ \\
\hline NUFIP1|26747 & $\mathrm{B} 2$ \\
\hline NUFIP2|57532 & B2 \\
\hline NUMA1|4926 & B2 \\
\hline NUMBL $\mid 9253$ & $\mathrm{~B} 2$ \\
\hline NUMB $\mid 8650$ & B2 \\
\hline NUP153|9972 & $\mathrm{B} 2$ \\
\hline NUP160|23279 & B2 \\
\hline NUP188|23511 & B2 \\
\hline NUP210L|91181 & B2 \\
\hline NUP214|8021 & $\mathrm{B} 2$ \\
\hline NUP35|129401 & $\mathrm{B} 2$ \\
\hline NUP43|348995 & $\mathrm{B} 2$ \\
\hline NUP50|10762 & B2 \\
\hline NUP54|53371 & B2 \\
\hline NUP62CL|54830 & $\mathrm{B} 2$ \\
\hline NUP88|4927 & B2 \\
\hline NUP93|9688 & B2 \\
\hline NUP98|4928 & B2 \\
\hline NUPL2|11097 & $\mathrm{B} 2$ \\
\hline NUPR1|26471 & B2 \\
\hline NUS1|116150 & B2 \\
\hline NWD1|284434 & $\mathrm{B} 2$ \\
\hline NXF1|10482 & B2 \\
\hline NXF2|56001 & B2 \\
\hline NXF3|56000 & B2 \\
\hline NXF4|55999 & B2 \\
\hline
\end{tabular}




\begin{tabular}{|c|c|}
\hline NXF5|55998 & B2 \\
\hline NXNL2|158046 & B2 \\
\hline NXN|64359 & B2 \\
\hline NXPH1|30010 & B2 \\
\hline NXPH2|11249 & B2 \\
\hline NXPH3|11248 & B2 \\
\hline NXPH4|11247 & B2 \\
\hline NXT1|29107 & $\mathrm{B} 2$ \\
\hline NXT2|55916 & B2 \\
\hline NYX|60506 & B2 \\
\hline OASL|8638 & B2 \\
\hline OAT|4942 & B2 \\
\hline OAZ1|4946 & B2 \\
\hline OAZ2|4947 & $\mathrm{B} 2$ \\
\hline OBFC2A|64859 & $\mathrm{B} 2$ \\
\hline OBP2A|29991 & $\mathrm{B} 2$ \\
\hline OBP2B|29989 & B2 \\
\hline OBSCN|84033 & $\mathrm{B} 2$ \\
\hline \begin{tabular}{l|} 
OBSL1|23363 \\
\end{tabular} & B2 \\
\hline OC90|729330 & $\mathrm{B} 2$ \\
\hline OCEL1|79629 & $\mathrm{B} 2$ \\
\hline OCIAD1|54940 & B2 \\
\hline OCLM|10896 & $\mathrm{B} 2$ \\
\hline OCM|654231 & B2 \\
\hline OCRL|4952 & B2 \\
\hline ODAM|54959 & $\mathrm{B} 2$ \\
\hline ODC1|4953 & B2 \\
\hline ODF1|4956 & B2 \\
\hline ODF2L|57489 & $\mathrm{B} 2$ \\
\hline ODF3B|440836 & B2 \\
\hline ODF3L2|284451 & $\mathrm{B} 2$ \\
\hline ODF3|113746 & $\mathrm{B} 2$ \\
\hline ODF4|146852 & B2 \\
\hline ODZ3|55714 & $\mathrm{B} 2$ \\
\hline ODZ4|26011 & B2 \\
\hline OFD1|8481 & $\mathrm{B} 2$ \\
\hline OGDHL|55753 & B2 \\
\hline OGDH|4967 & $\mathrm{B} 2$ \\
\hline OGFOD1|55239 & $\mathrm{B} 2$ \\
\hline OGFOD2|79676 & $\mathrm{B} 2$ \\
\hline OGFRL1|79627 & B2 \\
\hline OGFR|11054 & $\mathrm{B} 2$ \\
\hline OGG1|4968 & B2 \\
\hline OIT3|170392 & B2 \\
\hline OLAH|55301 & $\mathrm{B} 2$ \\
\hline OLFM1|10439 & $\mathrm{B} 2$ \\
\hline OLFM2|93145 & B2 \\
\hline OLFM3|118427 & $\mathrm{B} 2$ \\
\hline OLFM4|10562 & $\mathrm{B} 2$ \\
\hline OLFML3|56944 & B2 \\
\hline OLIG1|116448 & $\mathrm{B} 2$ \\
\hline
\end{tabular}




\begin{tabular}{|c|c|}
\hline OLIG2|10215 & B2 \\
\hline \begin{tabular}{l|l|l|} 
OLIG3 & 167826 \\
\end{tabular} & $\mathrm{~B} 2$ \\
\hline OMA1|115209 & $\mathrm{B} 2$ \\
\hline OMD|4958 & $\mathrm{B} 2$ \\
\hline OMG|4974 & B2 \\
\hline OMP|4975 & B2 \\
\hline ONECUT1|3175 & $\mathrm{B} 2$ \\
\hline ONECUT2|9480 & $\mathrm{B} 2$ \\
\hline OOEP|441161 & B2 \\
\hline OPA1|4976 & $\mathrm{B} 2$ \\
\hline OPA3|80207 & $\mathrm{B} 2$ \\
\hline OPALIN|93377 & $\mathrm{B} 2$ \\
\hline OPCML|4978 & $\mathrm{B} 2$ \\
\hline OPHN1|4983 & $\mathrm{B} 2$ \\
\hline OPLAH|26873 & B2 \\
\hline OPN1LW|5956 & $\mathrm{B} 2$ \\
\hline OPN1MW|2652 & B2 \\
\hline OPN1SW|611 & $\mathrm{B} 2$ \\
\hline OPN3|23596 & B2 \\
\hline OPN4|94233 & $\mathrm{B} 2$ \\
\hline OPN5|221391 & $\mathrm{B} 2$ \\
\hline OPRD1|4985 & $\mathrm{B} 2$ \\
\hline OPRK1|4986 & $\mathrm{B} 2$ \\
\hline OPRL1|4987 & $\mathrm{B} 2$ \\
\hline OPRM1|4988 & $\mathrm{B} 2$ \\
\hline OPTC|26254 & $\mathrm{B} 2$ \\
\hline OR10A2|341276 & B2 \\
\hline OR10A4|283297 & B2 \\
\hline OR10AD1|121275 & $\mathrm{B} 2$ \\
\hline OR10G2|26534 & $\mathrm{B} 2$ \\
\hline OR10G4|390264 & $\mathrm{B} 2$ \\
\hline OR10H1|26539 & B2 \\
\hline OR10H2|26538 & $\mathrm{B} 2$ \\
\hline OR10H4|126541 & B2 \\
\hline OR10H5|284433 & $\mathrm{B} 2$ \\
\hline OR10J3|441911 & $\mathrm{B} 2$ \\
\hline \begin{tabular}{l|l|} 
OR10Q19960 \\
\end{tabular} & B2 \\
\hline OR10R2|343406 & $\mathrm{B} 2$ \\
\hline OR10W1|81341 & $\mathrm{B} 2$ \\
\hline OR11A1|26531 & $\mathrm{B} 2$ \\
\hline OR11G2|390439 & B2 \\
\hline OR11H12|440153 & $\mathrm{B} 2$ \\
\hline OR11H4|390442 & B2 \\
\hline OR11H6|122748 & $\mathrm{B} 2$ \\
\hline OR11L1|391189 & $\mathrm{B} 2$ \\
\hline OR12D2|26529 & $\mathrm{B} 2$ \\
\hline OR12D3|81797 & $\mathrm{B} 2$ \\
\hline OR13A1|79290 & $\mathrm{B} 2$ \\
\hline OR13C2|392376 & $\mathrm{B} 2$ \\
\hline OR13C5|138799 & $\mathrm{B} 2$ \\
\hline OR13D1|286365 & $\mathrm{B} 2$ \\
\hline
\end{tabular}




\begin{tabular}{|c|c|}
\hline OR13F1|138805 & B2 \\
\hline OR13G1|441933 & B2 \\
\hline OR13H1|347468 & $\mathrm{B} 2$ \\
\hline OR13J1|392309 & $\mathrm{B} 2$ \\
\hline OR14A16|284532 & B2 \\
\hline OR14C36|127066 & $\mathrm{B} 2$ \\
\hline OR14I1|401994 & $\mathrm{B} 2$ \\
\hline OR1B1|347169 & $\mathrm{B} 2$ \\
\hline OR1C1|26188 & $\mathrm{B} 2$ \\
\hline OR1D4|8385 & $\mathrm{B} 2$ \\
\hline OR1E1|8387 & $\mathrm{B} 2$ \\
\hline OR1E2|8388 & B2 \\
\hline OR1F1|4992 & $\mathrm{B} 2$ \\
\hline OR1F2P|26184 & $\mathrm{B} 2$ \\
\hline OR1G1|8390 & $\mathrm{B} 2$ \\
\hline OR1J1|347168 & B2 \\
\hline OR1J2|26740 & $\mathrm{B} 2$ \\
\hline OR1J4|26219 & $\mathrm{B} 2$ \\
\hline OR1K1|392392 & $\mathrm{B} 2$ \\
\hline OR1L1|26737 & $\mathrm{B} 2$ \\
\hline OR1L3|26735 & $\mathrm{B} 2$ \\
\hline OR1L4|254973 & $\mathrm{B} 2$ \\
\hline OR1L6|392390 & $\mathrm{B} 2$ \\
\hline OR1L8|138881 & $\mathrm{B} 2$ \\
\hline OR1M1|125963 & $\mathrm{B} 2$ \\
\hline OR1N1|138883 & $\mathrm{B} 2$ \\
\hline OR1N2|138882 & $\mathrm{B} 2$ \\
\hline OR1Q1|158131 & $\mathrm{B} 2$ \\
\hline OR2A12|346525 & $\mathrm{B} 2$ \\
\hline OR2A14|135941 & $\mathrm{B} 2$ \\
\hline OR2A25|392138 & $\mathrm{B} 2$ \\
\hline OR2A2|442361 & $\mathrm{B} 2$ \\
\hline OR2A4|79541 & $\mathrm{B} 2$ \\
\hline OR2A5|393046 & $\mathrm{B} 2$ \\
\hline OR2A7|401427 & $\mathrm{B} 2$ \\
\hline OR2AE1|81392 & $\mathrm{B} 2$ \\
\hline OR2AG1|144125 & $\mathrm{B} 2$ \\
\hline OR2AG2|338755 & $\mathrm{B} 2$ \\
\hline OR2AK2|391191 & $\mathrm{B} 2$ \\
\hline OR2AT4|341152 & $\mathrm{B} 2$ \\
\hline OR2B11|127623 & $\mathrm{B} 2$ \\
\hline OR2B2|81697 & B2 \\
\hline OR2C1|4993 & $\mathrm{B} 2$ \\
\hline OR2C3|81472 & $\mathrm{B} 2$ \\
\hline OR2D2|120776 & $\mathrm{B} 2$ \\
\hline OR2F1|26211 & $\mathrm{B} 2$ \\
\hline OR2F2|135948 & $\mathrm{B} 2$ \\
\hline OR2H1|26716 & $\mathrm{B} 2$ \\
\hline OR2H2|7932 & B2 \\
\hline OR2J2|26707 & $\mathrm{B} 2$ \\
\hline OR2J3|442186 & $\mathrm{B} 2$ \\
\hline
\end{tabular}




\begin{tabular}{|c|c|}
\hline OR2K2|26248 & B2 \\
\hline OR2L1P|26247 & $\mathrm{B} 2$ \\
\hline OR2L2|26246 & B2 \\
\hline OR2L8|391190 & $\mathrm{B} 2$ \\
\hline OR2M3|127062 & B2 \\
\hline OR2M4|26245 & $\mathrm{B} 2$ \\
\hline OR2S2|56656 & $\mathrm{B} 2$ \\
\hline OR2T33|391195 & $\mathrm{B} 2$ \\
\hline OR2T3|343173 & $\mathrm{B} 2$ \\
\hline OR2T4|127074 & $\mathrm{B} 2$ \\
\hline OR2T6|254879 & B2 \\
\hline OR2T8|343172 & $\mathrm{B} 2$ \\
\hline OR2V2|285659 & $\mathrm{B} 2$ \\
\hline OR2W3|343171 & $\mathrm{B} 2$ \\
\hline OR2W5|441932 & $\mathrm{B} 2$ \\
\hline OR3A1|4994 & $\mathrm{B} 2$ \\
\hline OR3A2|4995 & $\mathrm{B} 2$ \\
\hline OR3A3|8392 & $\mathrm{B} 2$ \\
\hline OR3A4|390756 & $\mathrm{B} 2$ \\
\hline OR4C11|219429 & $\mathrm{B} 2$ \\
\hline OR4C3|256144 & $\mathrm{B} 2$ \\
\hline OR4C45|403257 & $\mathrm{B} 2$ \\
\hline OR4C6|219432 & $\mathrm{B} 2$ \\
\hline OR4D10|390197 & $\mathrm{B} 2$ \\
\hline OR4D1|26689 & $\mathrm{B} 2$ \\
\hline OR4D2|124538 & $\mathrm{B} 2$ \\
\hline OR4D6|219983 & $\mathrm{B} 2$ \\
\hline \begin{tabular}{l|l|} 
OR4E2|26686 &
\end{tabular} & $\mathrm{B} 2$ \\
\hline OR4F15|390649 & $\mathrm{B} 2$ \\
\hline OR4F21|441308 & $\mathrm{B} 2$ \\
\hline OR4F29|729759 & $\mathrm{B} 2$ \\
\hline $\begin{array}{l}\text { OR4F5|79501 } \\
\end{array}$ & $\mathrm{B} 2$ \\
\hline OR4F6|390648 & $\mathrm{B} 2$ \\
\hline OR4K15|81127 & $\mathrm{B} 2$ \\
\hline OR4K1|79544 & $\mathrm{B} 2$ \\
\hline OR4M1|441670 & $\mathrm{B} 2$ \\
\hline OR4M2|390538 & $\mathrm{B} 2$ \\
\hline OR4N2|390429 & $\mathrm{B} 2$ \\
\hline OR4N3P|390539 & $\mathrm{B} 2$ \\
\hline OR4N4|283694 & $\mathrm{B} 2$ \\
\hline OR51B2|79345 & $\mathrm{B} 2$ \\
\hline OR51B4|79339 & $\mathrm{B} 2$ \\
\hline OR51B5|282763 & $\mathrm{B} 2$ \\
\hline OR51B6|390058 & $\mathrm{B} 2$ \\
\hline OR51E1|143503 & $\mathrm{B} 2$ \\
\hline OR51E2|81285 & $\mathrm{B} 2$ \\
\hline OR51I1|390063 & $\mathrm{B} 2$ \\
\hline OR51I2|390064 & $\mathrm{B} 2$ \\
\hline OR51M1|390059 & $\mathrm{B} 2$ \\
\hline OR51Q1|390061 & $\mathrm{B} 2$ \\
\hline OR52B2|255725 & $\mathrm{B} 2$ \\
\hline
\end{tabular}




\begin{tabular}{|c|c|}
\hline OR52B4|143496 & B2 \\
\hline OR52B6|340980 & $\mathrm{B} 2$ \\
\hline OR52D1|390066 & B2 \\
\hline OR52E4|390081 & $\mathrm{B} 2$ \\
\hline OR52E6|390078 & B2 \\
\hline OR52E8|390079 & $\mathrm{B} 2$ \\
\hline OR52H1|390067 & $\mathrm{B} 2$ \\
\hline OR52I1|390037 & $\mathrm{B} 2$ \\
\hline OR52I2|143502 & $\mathrm{B} 2$ \\
\hline OR52K1|390036 & $\mathrm{B} 2$ \\
\hline OR52K2|119774 & B2 \\
\hline OR52L1|338751 & $\mathrm{B} 2$ \\
\hline OR52M1|119772 & $\mathrm{B} 2$ \\
\hline OR52N1|79473 & $\mathrm{B} 2$ \\
\hline OR52N2|390077 & $\mathrm{B} 2$ \\
\hline OR52N5|390075 & $\mathrm{B} 2$ \\
\hline OR52W1|120787 & $\mathrm{B} 2$ \\
\hline OR56A3|390083 & $\mathrm{B} 2$ \\
\hline OR56A4|120793 & $\mathrm{B} 2$ \\
\hline OR56A5|390084 & $\mathrm{B} 2$ \\
\hline OR56B1|387748 & $\mathrm{B} 2$ \\
\hline OR56B4|196335 & $\mathrm{B} 2$ \\
\hline OR5AK2|390181 & $\mathrm{B} 2$ \\
\hline OR5AN1|390195 & $\mathrm{B} 2$ \\
\hline OR5AU1|390445 & $\mathrm{B} 2$ \\
\hline OR5B12|390191 & $\mathrm{B} 2$ \\
\hline OR5B21|219968 & $\mathrm{B} 2$ \\
\hline OR5B2|390190 & $\mathrm{B} 2$ \\
\hline OR5C1|392391 & $\mathrm{B} 2$ \\
\hline OR5E1P|26343 & $\mathrm{B} 2$ \\
\hline OR5H2|79310 & $\mathrm{B} 2$ \\
\hline OR5M11|219487 & $\mathrm{B} 2$ \\
\hline \begin{tabular}{l|l|} 
OR5M8|219484 \\
\end{tabular} & $\mathrm{B} 2$ \\
\hline OR5P2|120065 & $\mathrm{B} 2$ \\
\hline OR5P3|120066 & $\mathrm{B} 2$ \\
\hline OR6A2|8590 & $\mathrm{B} 2$ \\
\hline OR6B1|135946 & $\mathrm{B} 2$ \\
\hline OR6B2|389090 & $\mathrm{B} 2$ \\
\hline OR6B3|150681 & $\mathrm{B} 2$ \\
\hline OR6C2|341416 & $\mathrm{B} 2$ \\
\hline OR6C70|390327 & $\mathrm{B} 2$ \\
\hline OR6F1|343169 & $\mathrm{B} 2$ \\
\hline OR6K3|391114 & $\mathrm{B} 2$ \\
\hline OR6T1|219874 & $\mathrm{B} 2$ \\
\hline OR6V1|346517 & $\mathrm{B} 2$ \\
\hline OR6W1P|89883 & $\mathrm{B} 2$ \\
\hline $\begin{array}{l}\text { OR7A5|26659 } \\
\end{array}$ & $\mathrm{B} 2$ \\
\hline \begin{tabular}{l|l|} 
OR7C1|26664 & \\
\end{tabular} & $\mathrm{B} 2$ \\
\hline OR7D2|162998 & $\mathrm{B} 2$ \\
\hline OR7D4|125958 & $\mathrm{B} 2$ \\
\hline OR7E156P|283491 & $\mathrm{B} 2$ \\
\hline
\end{tabular}




\begin{tabular}{|c|c|}
\hline OR7E24|26648 & B2 \\
\hline OR7E37P|26636 & $\mathrm{B} 2$ \\
\hline OR7E5P|219445 & $\mathrm{B} 2$ \\
\hline OR7E91P|79315 & $\mathrm{B} 2$ \\
\hline OR8A1|390275 & B2 \\
\hline OR8B12|219858 & $\mathrm{B} 2$ \\
\hline OR8B3|390271 & $\mathrm{B} 2$ \\
\hline OR8D1|283159 & $\mathrm{B} 2$ \\
\hline OR8D4|338662 & B2 \\
\hline OR8G5|219865 & $\mathrm{B} 2$ \\
\hline OR8S1|341568 & $\mathrm{B} 2$ \\
\hline OR9A2|135924 & $\mathrm{B} 2$ \\
\hline OR9A4|130075 & $\mathrm{B} 2$ \\
\hline $\begin{array}{l}\text { OR9Q1|219956 } \\
\end{array}$ & $\mathrm{B} 2$ \\
\hline ORAI1|84876 & B2 \\
\hline \begin{tabular}{l|l|l|} 
ORAI3|93129 \\
\end{tabular} & $\mathrm{B} 2$ \\
\hline ORAOV1|220064 & $\mathrm{B} 2$ \\
\hline ORC2L|4999 & $\mathrm{B} 2$ \\
\hline ORC3L|23595 & B2 \\
\hline ORC4L|5000 & $\mathrm{B} 2$ \\
\hline ORM1|5004 & $\mathrm{B} 2$ \\
\hline ORM2|5005 & $\mathrm{B} 2$ \\
\hline ORMDL1|94101 & $\mathrm{B} 2$ \\
\hline ORMDL3|94103 & $\mathrm{B} 2$ \\
\hline OS9|10956 & $\mathrm{B} 2$ \\
\hline OSBP2|23762 & $\mathrm{B} 2$ \\
\hline OSBPL10|114884 & $\mathrm{B} 2$ \\
\hline OSBPL11|114885 & B2 \\
\hline OSBPL2|9885 & $\mathrm{B} 2$ \\
\hline OSBPL3|26031 & $\mathrm{B} 2$ \\
\hline OSBPL5|114879 & $\mathrm{B} 2$ \\
\hline OSBPL6|114880 & B2 \\
\hline OSBPL7|114881 & $\mathrm{B} 2$ \\
\hline OSBPL8|114882 & $\mathrm{B} 2$ \\
\hline OSBP|5007 & $\mathrm{B} 2$ \\
\hline OSCAR|126014 & $\mathrm{B} 2$ \\
\hline OSCP1|127700 & $\mathrm{B} 2$ \\
\hline OSGEPL1|64172 & $\mathrm{B} 2$ \\
\hline $\begin{array}{l}\text { OSGEP|55644 } \\
\end{array}$ & $\mathrm{B} 2$ \\
\hline OSGIN1|29948 & $\mathrm{B} 2$ \\
\hline OSGIN2|734 & B2 \\
\hline OSMR $\mid 9180$ & $\mathrm{~B} 2$ \\
\hline OSM|5008 & B2 \\
\hline OSR2|116039 & $\mathrm{B} 2$ \\
\hline OSTBETA|123264 & $\mathrm{B} 2$ \\
\hline OSTCL|202459 & $\mathrm{B} 2$ \\
\hline OSTF1|26578 & $\mathrm{B} 2$ \\
\hline OSTM1|28962 & $\mathrm{B} 2$ \\
\hline OSTN|344901 & $\mathrm{B} 2$ \\
\hline OSTalpha|200931 & $\mathrm{B} 2$ \\
\hline OTC|5009 & $\mathrm{B} 2$ \\
\hline
\end{tabular}




\begin{tabular}{|c|c|}
\hline OTOA|146183 & B2 \\
\hline OTOF|9381 & B2 \\
\hline OTOL1|131149 & $\mathrm{B} 2$ \\
\hline OTOP1|133060 & $\mathrm{B} 2$ \\
\hline OTOP2|92736 & B2 \\
\hline OTOP3|347741 & $\mathrm{B} 2$ \\
\hline OTOR|56914 & $\mathrm{B} 2$ \\
\hline OTOS|150677 & $\mathrm{B} 2$ \\
\hline OTP $\mid 23440$ & B2 \\
\hline OTUD1|220213 & $\mathrm{B} 2$ \\
\hline OTUD4|54726 & $\mathrm{B} 2$ \\
\hline OTUD5|55593 & B2 \\
\hline OTUD6A|139562 & $\mathrm{B} 2$ \\
\hline OTUD6B|51633 & $\mathrm{B} 2$ \\
\hline OTX1|5013 & $\mathrm{B} 2$ \\
\hline OTX2|5015 & $\mathrm{B} 2$ \\
\hline OVCH1|341350 & $\mathrm{B} 2$ \\
\hline OVGP1|5016 & $\mathrm{B} 2$ \\
\hline OXA1L|5018 & $\mathrm{B} 2$ \\
\hline OXCT2|64064 & $\mathrm{B} 2$ \\
\hline OXER1|165140 & $\mathrm{B} 2$ \\
\hline OXNAD1|92106 & $\mathrm{B} 2$ \\
\hline OXR1|55074 & $\mathrm{B} 2$ \\
\hline OXSR1|9943 & $\mathrm{B} 2$ \\
\hline OXT|5020 & $\mathrm{B} 2$ \\
\hline P2RX1|5023 & $\mathrm{B} 2$ \\
\hline P2RX2|22953 & B2 \\
\hline P2RX3|5024 & $\mathrm{B} 2$ \\
\hline P2RX5|5026 & $\mathrm{B} 2$ \\
\hline P2RX6|9127 & $\mathrm{B} 2$ \\
\hline P2RX7|5027 & $\mathrm{B} 2$ \\
\hline P2RY10|27334 & $\mathrm{B} 2$ \\
\hline P2RY11|5032 & $\mathrm{B} 2$ \\
\hline P2RY1|5028 & $\mathrm{B} 2$ \\
\hline P2RY2|5029 & $\mathrm{B} 2$ \\
\hline P2RY4|5030 & $\mathrm{B} 2$ \\
\hline P2RY6|5031 & $\mathrm{B} 2$ \\
\hline P2RY8|286530 & $\mathrm{B} 2$ \\
\hline P4HA1|5033 & $\mathrm{B} 2$ \\
\hline P704P|641455 & $\mathrm{B} 2$ \\
\hline PA2G4|5036 & $\mathrm{B} 2$ \\
\hline PAAF1|80227 & B2 \\
\hline PABPC1L2A|340529 & $\mathrm{B} 2$ \\
\hline PABPC1L2B|645974 & $\mathrm{B} 2$ \\
\hline PABPC1L|80336 & $\mathrm{B} 2$ \\
\hline PABPC1P2|728773 & $\mathrm{B} 2$ \\
\hline PABPC1|26986 & $\mathrm{B} 2$ \\
\hline PABPC3|5042 & $\mathrm{B} 2$ \\
\hline PABPC4|8761 & $\mathrm{B} 2$ \\
\hline PABPN1L|390748 & $\mathrm{B} 2$ \\
\hline PABPN1|8106 & $\mathrm{B} 2$ \\
\hline
\end{tabular}




\begin{tabular}{|c|c|}
\hline PACS $1 \mid 55690$ & B2 \\
\hline PACS2|23241 & $\mathrm{B} 2$ \\
\hline PACSIN2|11252 & B2 \\
\hline PACSIN3|29763 & B2 \\
\hline PADI1|29943 & B2 \\
\hline PADI2|11240 & B2 \\
\hline PADI3|51702 & B2 \\
\hline PADI4|23569 & $\mathrm{B} 2$ \\
\hline PADI6|353238 & B2 \\
\hline PAEP|5047 & B2 \\
\hline PAF1|54623 & B2 \\
\hline PAFAH1B2|5049 & B2 \\
\hline PAG1|55824 & B2 \\
\hline PAGE2B|389860 & B2 \\
\hline PAGE2|203569 & B2 \\
\hline PAGE4|9506 & $\mathrm{B} 2$ \\
\hline PAGE5|90737 & B2 \\
\hline PAH|5053 & $\mathrm{B} 2$ \\
\hline PAIP2B|400961 & B2 \\
\hline PAIP2|51247 & $\mathrm{B} 2$ \\
\hline PAK1IP1|55003 & $\mathrm{B} 2$ \\
\hline PAK1|5058 & B2 \\
\hline PAK6|56924 & $\mathrm{B} 2$ \\
\hline PALLD|23022 & B2 \\
\hline PALM2|114299 & B2 \\
\hline PALM3|342979 & $\mathrm{B} 2$ \\
\hline PAM|5066 & B2 \\
\hline \begin{tabular}{l|l|} 
PAN3|255967 &
\end{tabular} & B2 \\
\hline PANK1|53354 & $\mathrm{B} 2$ \\
\hline PANK2|80025 & B2 \\
\hline PANK4|55229 & $\mathrm{B} 2$ \\
\hline PANX2|56666 & $\mathrm{B} 2$ \\
\hline PANX3|116337 & B2 \\
\hline PAOX|196743 & $\mathrm{B} 2$ \\
\hline PAPD4|167153 & B2 \\
\hline PAPD5|64282 & $\mathrm{B} 2$ \\
\hline PAPD7|11044 & B2 \\
\hline PAPL|390928 & $\mathrm{B} 2$ \\
\hline PAPOLG|64895 & $\mathrm{B} 2$ \\
\hline PAPPA|5069 & $\mathrm{B} 2$ \\
\hline PAPSS1|9061 & B2 \\
\hline PAPSS2|9060 & $\mathrm{B} 2$ \\
\hline PAQR3|152559 & B2 \\
\hline PAQR5|54852 & B2 \\
\hline PAQR6|79957 & B2 \\
\hline PAQR7|164091 & $\mathrm{B} 2$ \\
\hline PAQR8|85315 & B2 \\
\hline PAQR9|344838 & $\mathrm{B} 2$ \\
\hline PAR4|347745 & B2 \\
\hline PARD3|56288 & B2 \\
\hline PARD6A|50855 & $\mathrm{B} 2$ \\
\hline
\end{tabular}




\begin{tabular}{|c|c|}
\hline |PARD6G|84552 & B2 \\
\hline PARG|8505 & $\mathrm{B} 2$ \\
\hline PARK7|11315 & B2 \\
\hline PARL|55486 & $\mathrm{B} 2$ \\
\hline \begin{tabular}{|l|l|} 
PARM1|25849 &
\end{tabular} & B2 \\
\hline PARP10|84875 & B2 \\
\hline PARP11|57097 & B2 \\
\hline PARP15|165631 & $\mathrm{B} 2$ \\
\hline PARP16|54956 & B2 \\
\hline PARP2|10038 & $\mathrm{B} 2$ \\
\hline PARP4|143 & B2 \\
\hline \begin{tabular}{|l|} 
PARP6|56965 \\
\end{tabular} & B2 \\
\hline \begin{tabular}{|l|} 
PARP8|79668 \\
\end{tabular} & $\mathrm{B} 2$ \\
\hline \begin{tabular}{|l|} 
PARS2|25973 \\
\end{tabular} & $\mathrm{B} 2$ \\
\hline \begin{tabular}{|l|} 
PART1|25859 \\
\end{tabular} & $\mathrm{B} 2$ \\
\hline PARVB|29780 & $\mathrm{B} 2$ \\
\hline PARVG|64098 & $\mathrm{B} 2$ \\
\hline PASD1|139135 & $\mathrm{B} 2$ \\
\hline PATE1|160065 & $\mathrm{B} 2$ \\
\hline \begin{tabular}{|l|l|} 
PATE2|399967 \\
\end{tabular} & $\mathrm{B} 2$ \\
\hline \begin{tabular}{|l|} 
PATE3|100169851 \\
\end{tabular} & $\mathrm{B} 2$ \\
\hline PATE4|399968 & $\mathrm{B} 2$ \\
\hline \begin{tabular}{|l|} 
PATL1|219988 \\
\end{tabular} & $\mathrm{B} 2$ \\
\hline \begin{tabular}{|l|l|} 
PATL2|197135 \\
\end{tabular} & $\mathrm{B} 2$ \\
\hline PAWR|5074 & $\mathrm{B} 2$ \\
\hline \begin{tabular}{|l|l|} 
PAX1|5075 \\
\end{tabular} & $\mathrm{B} 2$ \\
\hline PAX3|5077 & $\mathrm{B} 2$ \\
\hline PAX5|5079 & $\mathrm{B} 2$ \\
\hline PAX6|5080 & $\mathrm{B} 2$ \\
\hline PAX7|5081 & $\mathrm{B} 2$ \\
\hline PAX8|7849 & $\mathrm{B} 2$ \\
\hline PBLD|64081 & $\mathrm{B} 2$ \\
\hline PBOV1|59351 & $\mathrm{B} 2$ \\
\hline PBRM1|55193 & $\mathrm{B} 2$ \\
\hline PBX2|5089 & $\mathrm{B} 2$ \\
\hline PBX3|5090 & $\mathrm{B} 2$ \\
\hline PBX4|80714 & $\mathrm{B} 2$ \\
\hline PBXIP1|57326 & $\mathrm{B} 2$ \\
\hline PCA3|50652 & B2 \\
\hline PCBD1|5092 & $\mathrm{B} 2$ \\
\hline PCBD2|84105 & $\mathrm{B} 2$ \\
\hline PCBP1|5093 & $\mathrm{B} 2$ \\
\hline PCBP2|5094 & $\mathrm{B} 2$ \\
\hline PCBP4|57060 & $\mathrm{B} 2$ \\
\hline PCCB 5096 & B2 \\
\hline PCDH10|57575 & $\mathrm{B} 2$ \\
\hline PCDH11X|27328 & $\mathrm{B} 2$ \\
\hline PCDH11Y|83259 & $\mathrm{B} 2$ \\
\hline PCDH12|51294 & $\mathrm{B} 2$ \\
\hline PCDH15|65217 & $\mathrm{B} 2$ \\
\hline PCDH1|5097 & $\mathrm{B} 2$ \\
\hline
\end{tabular}




\begin{tabular}{|c|c|}
\hline PCDH20|64881 & B2 \\
\hline PCDH7|5099 & $\mathrm{B} 2$ \\
\hline PCDH8|5100 & B2 \\
\hline PCDHA10|56139 & $\mathrm{B} 2$ \\
\hline PCDHA11|56138 & $\mathrm{B} 2$ \\
\hline PCDHA12|56137 & B2 \\
\hline PCDHA13|56136 & B2 \\
\hline PCDHA1|56147 & $\mathrm{B} 2$ \\
\hline PCDHA2|56146 & B2 \\
\hline PCDHA3|56145 & $\mathrm{B} 2$ \\
\hline PCDHA4|56144 & B2 \\
\hline PCDHA5|56143 & $\mathrm{B} 2$ \\
\hline PCDHA6|56142 & $\mathrm{B} 2$ \\
\hline PCDHA7|56141 & $\mathrm{B} 2$ \\
\hline PCDHA8|56140 & $\mathrm{B} 2$ \\
\hline PCDHA9|9752 & $\mathrm{B} 2$ \\
\hline PCDHAC1|56135 & $\mathrm{B} 2$ \\
\hline \begin{tabular}{l|l|} 
PCDHAC2|56134 & \\
\end{tabular} & $\mathrm{B} 2$ \\
\hline PCDHB10|56126 & $\mathrm{B} 2$ \\
\hline PCDHB11|56125 & $\mathrm{B} 2$ \\
\hline PCDHB13|56123 & $\mathrm{B} 2$ \\
\hline PCDHB14|56122 & $\mathrm{B} 2$ \\
\hline PCDHB16|57717 & $\mathrm{B} 2$ \\
\hline PCDHB17|54661 & $\mathrm{B} 2$ \\
\hline PCDHB1|29930 & $\mathrm{B} 2$ \\
\hline PCDHB2|56133 & $\mathrm{B} 2$ \\
\hline PCDHB3|56132 & $\mathrm{B} 2$ \\
\hline PCDHB5|26167 & $\mathrm{B} 2$ \\
\hline PCDHB6|56130 & $\mathrm{B} 2$ \\
\hline PCDHB8|56128 & $\mathrm{B} 2$ \\
\hline PCDHB9|56127 & $\mathrm{B} 2$ \\
\hline PCDHGA10|56106 & $\mathrm{B} 2$ \\
\hline PCDHGA12|26025 & $\mathrm{B} 2$ \\
\hline PCDHGA1|56114 & B2 \\
\hline PCDHGA4|56111 & $\mathrm{B} 2$ \\
\hline PCDHGA5|56110 & $\mathrm{B} 2$ \\
\hline PCDHGA7|56108 & $\mathrm{B} 2$ \\
\hline PCDHGA8|9708 & $\mathrm{B} 2$ \\
\hline PCDHGB1|56104 & B2 \\
\hline PCDHGB2|56103 & $\mathrm{B} 2$ \\
\hline PCDHGB3|56102 & $\mathrm{B} 2$ \\
\hline PCDHGB4|8641 & $\mathrm{B} 2$ \\
\hline PCDHGB5|56101 & $\mathrm{B} 2$ \\
\hline PCDHGB8P|56120 & $\mathrm{B} 2$ \\
\hline PCDHGC4|56098 & B2 \\
\hline PCDHGC5|56097 & $\mathrm{B} 2$ \\
\hline PCDP1|200373 & $\mathrm{B} 2$ \\
\hline PCF11|51585 & $\mathrm{B} 2$ \\
\hline PCGEM1|64002 & $\mathrm{B} 2$ \\
\hline PCGF1|84759 & $\mathrm{B} 2$ \\
\hline PCGF2|7703 & $\mathrm{B} 2$ \\
\hline
\end{tabular}




\begin{tabular}{|c|c|}
\hline PCGF3|10336 & B2 \\
\hline PCGF6|84108 & B2 \\
\hline PCID2|55795 & B2 \\
\hline PCIF1|63935 & $\mathrm{B} 2$ \\
\hline PCK2|5106 & $\mathrm{B} 2$ \\
\hline PCLO|27445 & $\mathrm{B} 2$ \\
\hline PCM1|5108 & $\mathrm{B} 2$ \\
\hline PCMT1|5110 & $\mathrm{B} 2$ \\
\hline PCMTD1|115294 & $\mathrm{B} 2$ \\
\hline PCMTD2|55251 & $\mathrm{B} 2$ \\
\hline PCNAP1|359806 & B2 \\
\hline PCNP|57092 & $\mathrm{B} 2$ \\
\hline PCNT 5116 & $\mathrm{~B} 2$ \\
\hline PCNXL2|80003 & $\mathrm{B} 2$ \\
\hline PCOLCE|5118 & $\mathrm{B} 2$ \\
\hline PCOTH|542767 & $\mathrm{B} 2$ \\
\hline PCP2|126006 & $\mathrm{B} 2$ \\
\hline PCP4L1|654790 & $\mathrm{B} 2$ \\
\hline PCP4|5121 & $\mathrm{B} 2$ \\
\hline PCSK1N|27344 & $\mathrm{B} 2$ \\
\hline PCSK1|5122 & $\mathrm{B} 2$ \\
\hline PCSK2|5126 & $\mathrm{B} 2$ \\
\hline $\begin{array}{l}\text { PCSK4|54760 } \\
\end{array}$ & $\mathrm{B} 2$ \\
\hline PCSK6|5046 & $\mathrm{B} 2$ \\
\hline PCSK7|9159 & $\mathrm{B} 2$ \\
\hline PCSK9|255738 & $\mathrm{B} 2$ \\
\hline PCTP|58488 & $\mathrm{B} 2$ \\
\hline PCYT1A|5130 & $\mathrm{B} 2$ \\
\hline PCYT2|5833 & $\mathrm{B} 2$ \\
\hline PDAP1|11333 & $\mathrm{B} 2$ \\
\hline PDCD11|22984 & $\mathrm{B} 2$ \\
\hline PDCD1LG2|80380 & $\mathrm{B} 2$ \\
\hline PDCD1|5133 & $\mathrm{B} 2$ \\
\hline PDCD2L|84306 & $\mathrm{B} 2$ \\
\hline PDCD2|5134 & $\mathrm{B} 2$ \\
\hline PDCD4|27250 & $\mathrm{B} 2$ \\
\hline PDCD5|9141 & $\mathrm{B} 2$ \\
\hline PDCD6IP|10015 & $\mathrm{B} 2$ \\
\hline PDCD7|10081 & $\mathrm{B} 2$ \\
\hline PDCL|5082 & $\mathrm{B} 2$ \\
\hline PDC|5132 & $\mathrm{B} 2$ \\
\hline PDDC $1 \mid 347862$ & $\mathrm{~B} 2$ \\
\hline PDE10A|10846 & $\mathrm{B} 2$ \\
\hline PDE12|201626 & $\mathrm{B} 2$ \\
\hline PDE3A|5139 & $\mathrm{B} 2$ \\
\hline PDE4A|5141 & $\mathrm{B} 2$ \\
\hline PDE4B|5142 & $\mathrm{B} 2$ \\
\hline PDE4C|5143 & $\mathrm{B} 2$ \\
\hline PDE4DIP|9659 & $\mathrm{B} 2$ \\
\hline PDE4D|5144 & $\mathrm{B} 2$ \\
\hline PDE6A|5145 & $\mathrm{B} 2$ \\
\hline
\end{tabular}




\begin{tabular}{|c|c|}
\hline PDE6B|5158 & B2 \\
\hline PDE6C|5146 & B2 \\
\hline PDE6G|5148 & $\mathrm{B} 2$ \\
\hline PDE6H|5149 & $\mathrm{B} 2$ \\
\hline PDE7A|5150 & B2 \\
\hline PDE9A|5152 & $\mathrm{B} 2$ \\
\hline PDGFB|5155 & $\mathrm{B} 2$ \\
\hline PDGFC|56034 & $\mathrm{B} 2$ \\
\hline PDGFRB|5159 & $\mathrm{B} 2$ \\
\hline PDHA1 $\mid 5160$ & $\mathrm{~B} 2$ \\
\hline PDHA2|5161 & $\mathrm{B} 2$ \\
\hline PDHB|5162 & $\mathrm{B} 2$ \\
\hline PDHX $\mid 8050$ & $\mathrm{~B} 2$ \\
\hline PDIA2|64714 & $\mathrm{B} 2$ \\
\hline PDILT|204474 & $\mathrm{B} 2$ \\
\hline PDK1|5163 & B2 \\
\hline PDLIM2|64236 & $\mathrm{B} 2$ \\
\hline PDLIM3|27295 & $\mathrm{B} 2$ \\
\hline PDLIM5|10611 & $\mathrm{B} 2$ \\
\hline PDLIM7|9260 & $\mathrm{B} 2$ \\
\hline PDP1|54704 & $\mathrm{B} 2$ \\
\hline PDPK1|5170 & $\mathrm{B} 2$ \\
\hline PDPN|10630 & $\mathrm{B} 2$ \\
\hline PDS5A|23244 & $\mathrm{B} 2$ \\
\hline \begin{tabular}{l|l|l|} 
PDS5B|23047 \\
\end{tabular} & $\mathrm{B} 2$ \\
\hline PDSS2|57107 & $\mathrm{B} 2$ \\
\hline PDX1|3651 & $\mathrm{B} 2$ \\
\hline PDXDC2|283970 & $\mathrm{B} 2$ \\
\hline PDXK|8566 & $\mathrm{B} 2$ \\
\hline PDYN|5173 & $\mathrm{B} 2$ \\
\hline PDZD3|79849 & $\mathrm{B} 2$ \\
\hline PDZD7|79955 & $\mathrm{B} 2$ \\
\hline PDZD8|118987 & $\mathrm{B} 2$ \\
\hline $\begin{array}{l}\text { PDZD9|255762 } \\
\end{array}$ & $\mathrm{B} 2$ \\
\hline PDZK1IP1|10158 & $\mathrm{B} 2$ \\
\hline PDZK1P1|728939 & $\mathrm{B} 2$ \\
\hline PDZK1|5174 & $\mathrm{B} 2$ \\
\hline PDZRN4|29951 & $\mathrm{B} 2$ \\
\hline PEA15|8682 & $\mathrm{B} 2$ \\
\hline PEBP1|5037 & $\mathrm{B} 2$ \\
\hline PEBP4|157310 & $\mathrm{B} 2$ \\
\hline PECI $\mid 10455$ & $\mathrm{~B} 2$ \\
\hline PECR|55825 & $\mathrm{B} 2$ \\
\hline PEF1|553115 & $\mathrm{B} 2$ \\
\hline PEG10|23089 & $\mathrm{B} 2$ \\
\hline PEG3AS|100169890 & $\mathrm{B} 2$ \\
\hline PEG3|5178 & $\mathrm{B} 2$ \\
\hline \begin{tabular}{l|l|} 
PELI3|246330 \\
\end{tabular} & $\mathrm{B} 2$ \\
\hline PELO|53918 & $\mathrm{B} 2$ \\
\hline PELP1|27043 & $\mathrm{B} 2$ \\
\hline PENK|5179 & $\mathrm{B} 2$ \\
\hline
\end{tabular}




\begin{tabular}{|c|c|}
\hline PEPD|5184 & B2 \\
\hline PER4|168741 & B2 \\
\hline PERP|64065 & $\mathrm{B} 2$ \\
\hline PES1|23481 & $\mathrm{B} 2$ \\
\hline PET112L|5188 & B2 \\
\hline PEX10|5192 & $\mathrm{B} 2$ \\
\hline PEX11A $\mid 8800$ & $\mathrm{~B} 2$ \\
\hline PEX11G|92960 & $\mathrm{B} 2$ \\
\hline PEX12|5193 & $\mathrm{B} 2$ \\
\hline PEX14|5195 & $\mathrm{B} 2$ \\
\hline PEX16|9409 & $\mathrm{B} 2$ \\
\hline PEX19|5824 & B2 \\
\hline PEX1|5189 & $\mathrm{B} 2$ \\
\hline PEX26|55670 & $\mathrm{B} 2$ \\
\hline PEX2|5828 & $\mathrm{B} 2$ \\
\hline PEX3|8504 & $\mathrm{B} 2$ \\
\hline PEX5L|51555 & $\mathrm{B} 2$ \\
\hline PEX6|5190 & $\mathrm{B} 2$ \\
\hline PEX7|5191 & $\mathrm{B} 2$ \\
\hline PF4V1|5197 & B2 \\
\hline PF4|5196 & $\mathrm{B} 2$ \\
\hline PFAS|5198 & $\mathrm{B} 2$ \\
\hline PFDN1|5201 & $\mathrm{B} 2$ \\
\hline PFDN4|5203 & $\mathrm{B} 2$ \\
\hline PFKFB2|5208 & $\mathrm{B} 2$ \\
\hline PFKL|5211 & $\mathrm{B} 2$ \\
\hline PFKM|5213 & B2 \\
\hline PFKP|5214 & $\mathrm{B} 2$ \\
\hline PFN3|345456 & $\mathrm{B} 2$ \\
\hline PFN4|375189 & $\mathrm{B} 2$ \\
\hline PGA4|643847 & $\mathrm{B} 2$ \\
\hline PGA5|5222 & $\mathrm{B} 2$ \\
\hline PGAM2|5224 & $\mathrm{B} 2$ \\
\hline PGAM4|441531 & $\mathrm{B} 2$ \\
\hline PGAP2|27315 & B2 \\
\hline $\begin{array}{l}\text { PGAP3|93210 } \\
\end{array}$ & $\mathrm{B} 2$ \\
\hline PGBD1|84547 & $\mathrm{B} 2$ \\
\hline PGBD2|267002 & $\mathrm{B} 2$ \\
\hline \begin{tabular}{l|l|} 
PGBD3|267004 &
\end{tabular} & $\mathrm{B} 2$ \\
\hline PGBD5|79605 & $\mathrm{B} 2$ \\
\hline PGC|5225 & $\mathrm{B} 2$ \\
\hline PGD|5226 & B2 \\
\hline PGF|5228 & $\mathrm{B} 2$ \\
\hline PGK2|5232 & $\mathrm{B} 2$ \\
\hline PGLS|25796 & $\mathrm{B} 2$ \\
\hline PGLYRP1|8993 & $\mathrm{B} 2$ \\
\hline PGLYRP2|114770 & $\mathrm{B} 2$ \\
\hline PGLYRP3|114771 & $\mathrm{B} 2$ \\
\hline PGLYRP4|57115 & $\mathrm{B} 2$ \\
\hline PGM2L1|283209 & B2 \\
\hline PGPEP1L|145814 & $\mathrm{B} 2$ \\
\hline
\end{tabular}




\begin{tabular}{|c|c|}
\hline PGRMC1|10857 & B2 \\
\hline PGRMC2|10424 & B2 \\
\hline PGR|5241 & B2 \\
\hline PGS1|9489 & $\mathrm{B} 2$ \\
\hline PHACTR1|221692 & $\mathrm{B} 2$ \\
\hline PHACTR3|116154 & $\mathrm{B} 2$ \\
\hline PHACTR4|65979 & $\mathrm{B} 2$ \\
\hline PHAX|51808 & $\mathrm{B} 2$ \\
\hline PHB2|11331 & $\mathrm{B} 2$ \\
\hline PHB|5245 & $\mathrm{B} 2$ \\
\hline PHC1|1911 & $\mathrm{B} 2$ \\
\hline PHC2|1912 & $\mathrm{B} 2$ \\
\hline PHC3|80012 & $\mathrm{B} 2$ \\
\hline PHEX|5251 & $\mathrm{B} 2$ \\
\hline PHF11|51131 & $\mathrm{B} 2$ \\
\hline PHF12|57649 & $\mathrm{B} 2$ \\
\hline PHF13|148479 & $\mathrm{B} 2$ \\
\hline \begin{tabular}{l|l|l|} 
PHF15338 & 2338
\end{tabular} & $\mathrm{~B} 2$ \\
\hline PHF19|26147 & $\mathrm{B} 2$ \\
\hline PHF20L1|51105 & $\mathrm{B} 2$ \\
\hline PHF20|51230 & $\mathrm{B} 2$ \\
\hline PHF21B|112885 & $\mathrm{B} 2$ \\
\hline PHF23|79142 & $\mathrm{B} 2$ \\
\hline PHF5A|84844 & $\mathrm{B} 2$ \\
\hline PHF8|23133 & $\mathrm{B} 2$ \\
\hline PHGDH|26227 & $\mathrm{B} 2$ \\
\hline PHGR1|644844 & $\mathrm{B} 2$ \\
\hline PHIP $\mid 55023$ & $\mathrm{~B} 2$ \\
\hline PHKA1|5255 & $\mathrm{B} 2$ \\
\hline PHKB $\mid 5257$ & $\mathrm{~B} 2$ \\
\hline PHKG1|5260 & $\mathrm{B} 2$ \\
\hline PHLDA2|7262 & $\mathrm{B} 2$ \\
\hline PHLPP2|23035 & $\mathrm{B} 2$ \\
\hline PHOSPHO1|162466 & $\mathrm{B} 2$ \\
\hline PHOSPHO2|493911 & $\mathrm{B} 2$ \\
\hline PHOX2A|401 & $\mathrm{B} 2$ \\
\hline PHOX2B|8929 & $\mathrm{B} 2$ \\
\hline PHPT1|29085 & $\mathrm{B} 2$ \\
\hline PHRF1|57661 & $\mathrm{B} 2$ \\
\hline PHTF2|57157 & $\mathrm{B} 2$ \\
\hline PHYH|5264 & $\mathrm{B} 2$ \\
\hline PI3|5266 & $\mathrm{B} 2$ \\
\hline PI4K2A|55361 & $\mathrm{B} 2$ \\
\hline $\begin{array}{l}\mathrm{PI} 4 \mathrm{~K} 2 \mathrm{~B} \mid 55300 \\
\end{array}$ & $\mathrm{~B} 2$ \\
\hline PI4KA|5297 & $\mathrm{B} 2$ \\
\hline PI4KB|5298 & $\mathrm{B} 2$ \\
\hline PIAS1|8554 & $\mathrm{B} 2$ \\
\hline PIAS2|9063 & $\mathrm{B} 2$ \\
\hline PIBF1|10464 & $\mathrm{B} 2$ \\
\hline PICK1|9463 & $\mathrm{B} 2$ \\
\hline PIGA|5277 & B2 \\
\hline
\end{tabular}




\begin{tabular}{|c|c|}
\hline PIGB|9488 & B2 \\
\hline PIGF|5281 & $\mathrm{B} 2$ \\
\hline PIGG|54872 & B2 \\
\hline PIGH|5283 & $\mathrm{B} 2$ \\
\hline PIGK|10026 & B2 \\
\hline PIGN|23556 & $\mathrm{B} 2$ \\
\hline PIGP|51227 & B2 \\
\hline PIGR|5284 & $\mathrm{B} 2$ \\
\hline PIGS|94005 & $\mathrm{B} 2$ \\
\hline PIGT|51604 & $\mathrm{B} 2$ \\
\hline PIGV|55650 & B2 \\
\hline PIGY|84992 & $\mathrm{B} 2$ \\
\hline PIH1D1|55011 & $\mathrm{B} 2$ \\
\hline PIH1D2|120379 & $\mathrm{B} 2$ \\
\hline PIK3AP1|118788 & $\mathrm{B} 2$ \\
\hline PIK3C2A|5286 & $\mathrm{B} 2$ \\
\hline PIK3C3|5289 & $\mathrm{B} 2$ \\
\hline $\begin{array}{l}\text { PIK3CB } \\
5291\end{array}$ & $\mathrm{~B} 2$ \\
\hline \begin{tabular}{l|l|} 
PIK3CD 5293 \\
\end{tabular} & $\mathrm{~B} 2$ \\
\hline PIK3CG|5294 & $\mathrm{B} 2$ \\
\hline PIK3R4|30849 & $\mathrm{B} 2$ \\
\hline PIK3R5|23533 & $\mathrm{B} 2$ \\
\hline PIK3R6|146850 & $\mathrm{B} 2$ \\
\hline PILRA|29992 & $\mathrm{B} 2$ \\
\hline PILRB|29990 & $\mathrm{B} 2$ \\
\hline PIM1|5292 & $\mathrm{B} 2$ \\
\hline PIM2|11040 & $\mathrm{B} 2$ \\
\hline PIM3|415116 & $\mathrm{B} 2$ \\
\hline PIN1L|5301 & $\mathrm{B} 2$ \\
\hline PIN1|5300 & $\mathrm{B} 2$ \\
\hline PIN4|5303 & $\mathrm{B} 2$ \\
\hline PION|54103 & $\mathrm{B} 2$ \\
\hline PIP4K2A|5305 & $\mathrm{B} 2$ \\
\hline PIP4K2B|8396 & $\mathrm{B} 2$ \\
\hline PIP5K1B|8395 & $\mathrm{B} 2$ \\
\hline PIP5K1C|23396 & $\mathrm{B} 2$ \\
\hline PIP5K1P1|206426 & $\mathrm{B} 2$ \\
\hline PIP5KL1|138429 & $\mathrm{B} 2$ \\
\hline PIPOX|51268 & $\mathrm{B} 2$ \\
\hline PIP|5304 & $\mathrm{B} 2$ \\
\hline PIRT|644139 & $\mathrm{B} 2$ \\
\hline PIR|8544 & $\mathrm{B} 2$ \\
\hline PITPNA|5306 & $\mathrm{B} 2$ \\
\hline PITPNB|23760 & $\mathrm{B} 2$ \\
\hline PITPNC1|26207 & $\mathrm{B} 2$ \\
\hline PITPNM1|9600 & $\mathrm{B} 2$ \\
\hline PITPNM3|83394 & $\mathrm{B} 2$ \\
\hline PITRM1|10531 & $\mathrm{B} 2$ \\
\hline PITX2|5308 & $\mathrm{B} 2$ \\
\hline \begin{tabular}{l|l} 
PITX3|5309 &
\end{tabular} & $\mathrm{B} 2$ \\
\hline PIWIL1|9271 & $\mathrm{B} 2$ \\
\hline
\end{tabular}




\begin{tabular}{|c|c|}
\hline PIWIL2|55124 & B2 \\
\hline PIWIL3|440822 & B2 \\
\hline PKD1L1|168507 & $\mathrm{B} 2$ \\
\hline PKD2L1|9033 & $\mathrm{B} 2$ \\
\hline PKD2L2|27039 & B2 \\
\hline PKHD1|5314 & $\mathrm{B} 2$ \\
\hline PKIA|5569 & $\mathrm{B} 2$ \\
\hline PKLR|5313 & B2 \\
\hline PKN1|5585 & B2 \\
\hline PKNOX1|5316 & $\mathrm{B} 2$ \\
\hline PKNOX2|63876 & $\mathrm{B} 2$ \\
\hline PKP1|5317 & $\mathrm{B} 2$ \\
\hline PKP2|5318 & $\mathrm{B} 2$ \\
\hline PKP4|8502 & $\mathrm{B} 2$ \\
\hline PLA1A|51365 & $\mathrm{B} 2$ \\
\hline PLA2G10|8399 & B2 \\
\hline PLA2G12A|81579 & $\mathrm{B} 2$ \\
\hline PLA2G12B|84647 & $\mathrm{B} 2$ \\
\hline PLA2G15|23659 & $\mathrm{B} 2$ \\
\hline PLA2G1B|5319 & B2 \\
\hline PLA2G2A|5320 & $\mathrm{B} 2$ \\
\hline PLA2G2C|391013 & $\mathrm{B} 2$ \\
\hline PLA2G2D|26279 & $\mathrm{B} 2$ \\
\hline PLA2G2F|64600 & $\mathrm{B} 2$ \\
\hline PLA2G3|50487 & $\mathrm{B} 2$ \\
\hline PLA2G4C|8605 & $\mathrm{B} 2$ \\
\hline PLA2G4D|283748 & $\mathrm{B} 2$ \\
\hline PLA2G4E|123745 & $\mathrm{B} 2$ \\
\hline PLA2G7|7941 & $\mathrm{B} 2$ \\
\hline PLAC4|191585 & $\mathrm{B} 2$ \\
\hline PLAC8L1|153770 & $\mathrm{B} 2$ \\
\hline PLAC8|51316 & $\mathrm{B} 2$ \\
\hline \begin{tabular}{l|l|} 
PLAG1|5324 &
\end{tabular} & $\mathrm{B} 2$ \\
\hline PLAGL2|5326 & $\mathrm{B} 2$ \\
\hline PLAT|5327 & $\mathrm{B} 2$ \\
\hline PLBD1|79887 & $\mathrm{B} 2$ \\
\hline PLBD2|196463 & $\mathrm{B} 2$ \\
\hline PLCB1|23236 & $\mathrm{B} 2$ \\
\hline PLCB2|5330 & $\mathrm{B} 2$ \\
\hline PLCB4|5332 & $\mathrm{B} 2$ \\
\hline PLCD4|84812 & $\mathrm{B} 2$ \\
\hline $\begin{array}{l}\text { PLCE1|51196 } \\
\end{array}$ & $\mathrm{B} 2$ \\
\hline PLCG1|5335 & $\mathrm{B} 2$ \\
\hline PLCG2|5336 & $\mathrm{B} 2$ \\
\hline \begin{tabular}{l|} 
PLCH1|23007 \\
\end{tabular} & $\mathrm{B} 2$ \\
\hline PLCL1|5334 & $\mathrm{B} 2$ \\
\hline PLCXD1|55344 & $\mathrm{B} 2$ \\
\hline PLCXD2|257068 & $\mathrm{B} 2$ \\
\hline PLD3|23646 & $\mathrm{B} 2$ \\
\hline PLD4|122618 & $\mathrm{B} 2$ \\
\hline \begin{tabular}{l|l|} 
PLD5|200150 &
\end{tabular} & $\mathrm{B} 2$ \\
\hline
\end{tabular}




\begin{tabular}{|c|c|}
\hline PLD6|201164 & $\mathrm{B} 2$ \\
\hline PLDN|26258 & B2 \\
\hline PLEC|5339 & $\mathrm{B} 2$ \\
\hline PLEKHA1|59338 & $\mathrm{B} 2$ \\
\hline PLEKHA2|59339 & $\mathrm{B} 2$ \\
\hline PLEKHA3|65977 & $\mathrm{B} 2$ \\
\hline PLEKHA5|54477 & $\mathrm{B} 2$ \\
\hline PLEKHA6|22874 & $\mathrm{B} 2$ \\
\hline PLEKHB1|58473 & B2 \\
\hline PLEKHG2|64857 & $\mathrm{B} 2$ \\
\hline PLEKHG3|26030 & $\mathrm{B} 2$ \\
\hline PLEKHG4B|153478 & $\mathrm{B} 2$ \\
\hline PLEKHG4|25894 & $\mathrm{B} 2$ \\
\hline PLEKHG6|55200 & $\mathrm{B} 2$ \\
\hline PLEKHG7|440107 & $\mathrm{B} 2$ \\
\hline PLEKHH1|57475 & $\mathrm{B} 2$ \\
\hline PLEKHH3|79990 & $\mathrm{B} 2$ \\
\hline PLEKHJ1|55111 & $\mathrm{B} 2$ \\
\hline PLEKHM2|23207 & $\mathrm{B} 2$ \\
\hline PLEKHO1|51177 & $\mathrm{B} 2$ \\
\hline PLEKHO2|80301 & $\mathrm{B} 2$ \\
\hline PLEK|5341 & $\mathrm{B} 2$ \\
\hline PLGLB2|5342 & $\mathrm{B} 2$ \\
\hline PLG|5340 & $\mathrm{B} 2$ \\
\hline $\begin{array}{l}\text { PLIN5|440503 } \\
\end{array}$ & $\mathrm{B} 2$ \\
\hline PLK2|10769 & $\mathrm{B} 2$ \\
\hline PLK5P|126520 & $\mathrm{B} 2$ \\
\hline PLN|5350 & $\mathrm{B} 2$ \\
\hline PLOD1|5351 & $\mathrm{B} 2$ \\
\hline PLOD2|5352 & $\mathrm{B} 2$ \\
\hline PLOD3|8985 & $\mathrm{B} 2$ \\
\hline PLP2|5355 & $\mathrm{B} 2$ \\
\hline PLRG1|5356 & $\mathrm{B} 2$ \\
\hline $\begin{array}{l}\text { PLSCR1|5359 } \\
\end{array}$ & $\mathrm{B} 2$ \\
\hline PLSCR2|57047 & $\mathrm{B} 2$ \\
\hline PLSCR3|57048 & $\mathrm{B} 2$ \\
\hline PLSCR5|389158 & $\mathrm{B} 2$ \\
\hline PLUNC|51297 & $\mathrm{B} 2$ \\
\hline \begin{tabular}{l|l|} 
PLVAP 83483 \\
\end{tabular} & $\mathrm{~B} 2$ \\
\hline PLXDC1|57125 & $\mathrm{B} 2$ \\
\hline \begin{tabular}{l|l} 
PLXDC2|84898 \\
\end{tabular} & $\mathrm{B} 2$ \\
\hline PLXNA1|5361 & $\mathrm{B} 2$ \\
\hline PLXNA2|5362 & $\mathrm{B} 2$ \\
\hline PLXNB1|5364 & $\mathrm{B} 2$ \\
\hline PLXNB2|23654 & $\mathrm{B} 2$ \\
\hline PLXNB3|5365 & $\mathrm{B} 2$ \\
\hline PLXND1|23129 & $\mathrm{B} 2$ \\
\hline PMCHL1|5369 & $\mathrm{B} 2$ \\
\hline PMCHL2|5370 & $\mathrm{B} 2$ \\
\hline PMEPA1|56937 & $\mathrm{B} 2$ \\
\hline PMFBP1|83449 & $\mathrm{B} 2$ \\
\hline
\end{tabular}




\begin{tabular}{|c|c|}
\hline PML|5371 & B2 \\
\hline PMM1|5372 & B2 \\
\hline PMP2|5375 & $\mathrm{B} 2$ \\
\hline PMPCA|23203 & $\mathrm{B} 2$ \\
\hline PMPCB $\mid 9512$ & B2 \\
\hline PMS1|5378 & B2 \\
\hline PMS2CL|441194 & $\mathrm{B} 2$ \\
\hline PMS2L11|441263 & $\mathrm{B} 2$ \\
\hline PMS2L2|5380 & B2 \\
\hline \begin{tabular}{l|l} 
PMS2L3|5387 \\
\end{tabular} & $\mathrm{B} 2$ \\
\hline PMS2|5395 & $\mathrm{B} 2$ \\
\hline PMVK|10654 & B2 \\
\hline PNCK|139728 & $\mathrm{B} 2$ \\
\hline PNLDC1|154197 & $\mathrm{B} 2$ \\
\hline PNLIPRP1|5407 & $\mathrm{B} 2$ \\
\hline PNLIPRP2|5408 & B2 \\
\hline PNLIPRP3|119548 & $\mathrm{B} 2$ \\
\hline PNLIP|5406 & $\mathrm{B} 2$ \\
\hline PNMA1|9240 & $\mathrm{B} 2$ \\
\hline PNMA2|10687 & $\mathrm{B} 2$ \\
\hline \begin{tabular}{|l|} 
PNMA3|29944 \\
\end{tabular} & $\mathrm{B} 2$ \\
\hline PNMA5|114824 & $\mathrm{B} 2$ \\
\hline PNMA6A|84968 & $\mathrm{B} 2$ \\
\hline PNMAL1|55228 & $\mathrm{B} 2$ \\
\hline PNMT|5409 & $\mathrm{B} 2$ \\
\hline PNN|5411 & $\mathrm{B} 2$ \\
\hline PNOC|5368 & $\mathrm{B} 2$ \\
\hline PNPLA1|285848 & $\mathrm{B} 2$ \\
\hline \begin{tabular}{|l|l|} 
PNPLA3|80339 \\
\end{tabular} & $\mathrm{B} 2$ \\
\hline PNPLA4|8228 & $\mathrm{B} 2$ \\
\hline PNPLA5|150379 & $\mathrm{B} 2$ \\
\hline PNPLA6|10908 & $\mathrm{B} 2$ \\
\hline PNPLA8|50640 & $\mathrm{B} 2$ \\
\hline PNPO|55163 & $\mathrm{B} 2$ \\
\hline PNPT1|87178 & $\mathrm{B} 2$ \\
\hline PNRC2|55629 & $\mathrm{B} 2$ \\
\hline POC1B|282809 & $\mathrm{B} 2$ \\
\hline POC5|134359 & $\mathrm{B} 2$ \\
\hline PODNL1|79883 & $\mathrm{B} 2$ \\
\hline PODXL|5420 & $\mathrm{B} 2$ \\
\hline POF1B|79983 & $\mathrm{B} 2$ \\
\hline POFUT1|23509 & $\mathrm{B} 2$ \\
\hline POFUT2|23275 & $\mathrm{B} 2$ \\
\hline POGZ|23126 & $\mathrm{B} 2$ \\
\hline POLA1|5422 & $\mathrm{B} 2$ \\
\hline POLB|5423 & $\mathrm{B} 2$ \\
\hline POLD3|10714 & $\mathrm{B} 2$ \\
\hline POLDIP2|26073 & $\mathrm{B} 2$ \\
\hline POLDIP3|84271 & $\mathrm{B} 2$ \\
\hline POLE4|56655 & $\mathrm{B} 2$ \\
\hline POLG2|11232 & $\mathrm{B} 2$ \\
\hline
\end{tabular}




\begin{tabular}{|c|c|}
\hline POLG|5428 & B2 \\
\hline POLH|5429 & $\mathrm{B} 2$ \\
\hline POLK|51426 & $\mathrm{B} 2$ \\
\hline POLM|27434 & $\mathrm{B} 2$ \\
\hline POLN|353497 & B2 \\
\hline POLR1A|25885 & $\mathrm{B} 2$ \\
\hline POLR1B|84172 & $\mathrm{B} 2$ \\
\hline POLR1C|9533 & $\mathrm{B} 2$ \\
\hline POLR1D|51082 & $\mathrm{B} 2$ \\
\hline POLR2A|5430 & $\mathrm{B} 2$ \\
\hline POLR2B|5431 & $\mathrm{B} 2$ \\
\hline POLR2C|5432 & $\mathrm{B} 2$ \\
\hline POLR2F|5435 & $\mathrm{B} 2$ \\
\hline POLR2I|5438 & $\mathrm{B} 2$ \\
\hline POLR2J2|246721 & $\mathrm{B} 2$ \\
\hline POLR2J3|548644 & $\mathrm{B} 2$ \\
\hline POLR2L|5441 & $\mathrm{B} 2$ \\
\hline POLR3A|11128 & $\mathrm{B} 2$ \\
\hline 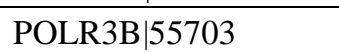 & $\mathrm{B} 2$ \\
\hline POLR3C|10623 & $\mathrm{B} 2$ \\
\hline POLR3D|661 & $\mathrm{B} 2$ \\
\hline POLR3E|55718 & $\mathrm{B} 2$ \\
\hline POLR3F|10621 & $\mathrm{B} 2$ \\
\hline POLR3G|10622 & $\mathrm{B} 2$ \\
\hline POLR3H|171568 & $\mathrm{B} 2$ \\
\hline POLRMT|5442 & $\mathrm{B} 2$ \\
\hline POM121C|100101267 & $\mathrm{B} 2$ \\
\hline POM121L10P|646074 & $\mathrm{B} 2$ \\
\hline POM121L1P|25812 & $\mathrm{B} 2$ \\
\hline POM121L2|94026 & $\mathrm{B} 2$ \\
\hline POM121L4P|266697 & $\mathrm{B} 2$ \\
\hline POM121L8P|29797 & $\mathrm{B} 2$ \\
\hline POM121|9883 & $\mathrm{B} 2$ \\
\hline POMC|5443 & $\mathrm{B} 2$ \\
\hline POMGNT1|55624 & B2 \\
\hline POMT1|10585 & $\mathrm{B} 2$ \\
\hline POMT2|29954 & $\mathrm{B} 2$ \\
\hline POMZP3|22932 & $\mathrm{B} 2$ \\
\hline PON1|5444 & $\mathrm{B} 2$ \\
\hline PON2|5445 & $\mathrm{B} 2$ \\
\hline \begin{tabular}{ll|l} 
PON3|5446 &
\end{tabular} & $\mathrm{B} 2$ \\
\hline POP5|51367 & $\mathrm{B} 2$ \\
\hline \begin{tabular}{l|l|} 
POPDC2|64091 & 1
\end{tabular} & $\mathrm{~B} 2$ \\
\hline POPDC3|64208 & $\mathrm{B} 2$ \\
\hline PORCN|64840 & $\mathrm{B} 2$ \\
\hline POR|5447 & $\mathrm{B} 2$ \\
\hline POT1|25913 & $\mathrm{B} 2$ \\
\hline POTEA|340441 & $\mathrm{B} 2$ \\
\hline POTEB|339010 & $\mathrm{B} 2$ \\
\hline POTEC|388468 & $\mathrm{B} 2$ \\
\hline POTED 317754 & $\mathrm{~B} 2$ \\
\hline
\end{tabular}




\begin{tabular}{|c|c|}
\hline POTEE|445582 & $\mathrm{B} 2$ \\
\hline POTEF|728378 & B2 \\
\hline POTEG|404785 & B2 \\
\hline POTEH|23784 & $\mathrm{B} 2$ \\
\hline POU1F1|5449 & $\mathrm{B} 2$ \\
\hline POU2AF1|5450 & $\mathrm{B} 2$ \\
\hline POU2F2|5452 & $\mathrm{B} 2$ \\
\hline POU2F3|25833 & $\mathrm{B} 2$ \\
\hline POU3F1|5453 & $\mathrm{B} 2$ \\
\hline POU3F2|5454 & $\mathrm{B} 2$ \\
\hline POU3F3|5455 & $\mathrm{B} 2$ \\
\hline POU3F4|5456 & $\mathrm{B} 2$ \\
\hline POU4F1|5457 & $\mathrm{B} 2$ \\
\hline POU4F2|5458 & $\mathrm{B} 2$ \\
\hline POU4F3|5459 & $\mathrm{B} 2$ \\
\hline POU5F1B|5462 & $\mathrm{B} 2$ \\
\hline POU5F1|5460 & B2 \\
\hline POU5F2|134187 & $\mathrm{B} 2$ \\
\hline POU6F2|11281 & $\mathrm{B} 2$ \\
\hline PPA1|5464 & $\mathrm{B} 2$ \\
\hline PPAN-P2RY11|692312 & $\mathrm{B} 2$ \\
\hline PPAN|56342 & $\mathrm{B} 2$ \\
\hline PPAP2C $\mid 8612$ & $\mathrm{~B} 2$ \\
\hline PPAPDC1B|84513 & $\mathrm{B} 2$ \\
\hline PPAPDC2 $\mid 403313$ & $\mathrm{~B} 2$ \\
\hline \begin{tabular}{l|l|} 
PPAPDC3|84814 &
\end{tabular} & $\mathrm{B} 2$ \\
\hline PPARD|5467 & $\mathrm{B} 2$ \\
\hline PPARGC1A|10891 & $\mathrm{B} 2$ \\
\hline PPBPL2|10895 & $\mathrm{B} 2$ \\
\hline PPBP|5473 & $\mathrm{B} 2$ \\
\hline PPCDC|60490 & $\mathrm{B} 2$ \\
\hline PPEF1|5475 & $\mathrm{B} 2$ \\
\hline PPEF2|5470 & $\mathrm{B} 2$ \\
\hline PPFIA1 $\mid 8500$ & $\mathrm{~B} 2$ \\
\hline PPFIA2|8499 & $\mathrm{B} 2$ \\
\hline PPFIA4|8497 & $\mathrm{B} 2$ \\
\hline PPFIBP1|8496 & $\mathrm{B} 2$ \\
\hline PPFIBP2|8495 & $\mathrm{B} 2$ \\
\hline PPHLN1|51535 & $\mathrm{B} 2$ \\
\hline PPIAL4D|645142 & $\mathrm{B} 2$ \\
\hline PPIAL4E|730262 & $\mathrm{B} 2$ \\
\hline PPIC|5480 & $\mathrm{B} 2$ \\
\hline PPID|5481 & $\mathrm{B} 2$ \\
\hline PPIE|10450 & $\mathrm{B} 2$ \\
\hline PPIF|10105 & $\mathrm{B} 2$ \\
\hline PPIG|9360 & $\mathrm{B} 2$ \\
\hline PPIL3|53938 & $\mathrm{B} 2$ \\
\hline PPIL4|85313 & $\mathrm{B} 2$ \\
\hline PPIL6|285755 & $\mathrm{B} 2$ \\
\hline PPIP5K1|9677 & $\mathrm{B} 2$ \\
\hline PPIP5K2|23262 & $\mathrm{B} 2$ \\
\hline
\end{tabular}




\begin{tabular}{|c|c|}
\hline PPM1A|5494 & B2 \\
\hline PPM1B|5495 & B2 \\
\hline PPM1D|8493 & $\mathrm{B} 2$ \\
\hline PPM1E|22843 & $\mathrm{B} 2$ \\
\hline PPM1K|152926 & B2 \\
\hline PPM1L|151742 & B2 \\
\hline PPM1N|147699 & $\mathrm{B} 2$ \\
\hline PPP1CB|5500 & $\mathrm{B} 2$ \\
\hline PPP1CC|5501 & B2 \\
\hline PPP1R13B|23368 & $\mathrm{B} 2$ \\
\hline PPP1R13L|10848 & $\mathrm{B} 2$ \\
\hline PPP1R14C|81706 & $\mathrm{B} 2$ \\
\hline PPP1R14D|54866 & $\mathrm{B} 2$ \\
\hline PPP1R15B|84919 & $\mathrm{B} 2$ \\
\hline PPP1R16A|84988 & $\mathrm{B} 2$ \\
\hline PPP1R1C|151242 & $\mathrm{B} 2$ \\
\hline PPP1R2P1|5505 & B2 \\
\hline PPP1R2P3|153743 & $\mathrm{B} 2$ \\
\hline PPP1R2P9|80316 & $\mathrm{B} 2$ \\
\hline PPP1R2|5504 & $\mathrm{B} 2$ \\
\hline PPP1R3A|5506 & $\mathrm{B} 2$ \\
\hline PPP1R3B|79660 & $\mathrm{B} 2$ \\
\hline PPP1R3C|5507 & $\mathrm{B} 2$ \\
\hline PPP1R3D|5509 & $\mathrm{B} 2$ \\
\hline PPP1R3F|89801 & $\mathrm{B} 2$ \\
\hline PPP1R7|5510 & $\mathrm{B} 2$ \\
\hline PPP1R8|5511 & $\mathrm{B} 2$ \\
\hline PPP1R9A|55607 & $\mathrm{B} 2$ \\
\hline PPP2R2A|5520 & $\mathrm{B} 2$ \\
\hline PPP2R2D|55844 & $\mathrm{B} 2$ \\
\hline PPP2R3A|5523 & $\mathrm{B} 2$ \\
\hline PPP2R3B|28227 & $\mathrm{B} 2$ \\
\hline PPP2R3C|55012 & $\mathrm{B} 2$ \\
\hline PPP2R5A|5525 & $\mathrm{B} 2$ \\
\hline PPP2R5B|5526 & $\mathrm{B} 2$ \\
\hline PPP2R5C|5527 & $\mathrm{B} 2$ \\
\hline PPP2R5D|5528 & $\mathrm{B} 2$ \\
\hline PPP3CA|5530 & $\mathrm{B} 2$ \\
\hline РPР3СВ 5532 & B2 \\
\hline PPP3R1|5534 & $\mathrm{B} 2$ \\
\hline PPP3R2|5535 & $\mathrm{B} 2$ \\
\hline PPP4R1|9989 & $\mathrm{B} 2$ \\
\hline PPP4R2|151987 & $\mathrm{B} 2$ \\
\hline PPP4R4|57718 & $\mathrm{B} 2$ \\
\hline PPP6C|5537 & $\mathrm{B} 2$ \\
\hline PPPDE2|27351 & $\mathrm{B} 2$ \\
\hline PPRC1|23082 & $\mathrm{B} 2$ \\
\hline PPT2|9374 & $\mathrm{B} 2$ \\
\hline PPTC7|160760 & $\mathrm{B} 2$ \\
\hline PPY2|23614 & $\mathrm{B} 2$ \\
\hline PPYR1|5540 & $\mathrm{B} 2$ \\
\hline
\end{tabular}




\begin{tabular}{|c|c|}
\hline PPY|5539 & B2 \\
\hline PQBP1|10084 & $\mathrm{B} 2$ \\
\hline PQLC3|130814 & $\mathrm{B} 2$ \\
\hline PRAM1|84106 & $\mathrm{B} 2$ \\
\hline PRAMEF11|440560 & B2 \\
\hline PRAMEF14|729528 & $\mathrm{B} 2$ \\
\hline PRAMEF16|654348 & $\mathrm{B} 2$ \\
\hline PRAMEF20|645425 & $\mathrm{B} 2$ \\
\hline PRAMEF22|653606 & B2 \\
\hline PRAMEF2|65122 & $\mathrm{B} 2$ \\
\hline PRAMEF4|400735 & $\mathrm{B} 2$ \\
\hline PRAMEF8|391002 & $\mathrm{B} 2$ \\
\hline PRAME|23532 & $\mathrm{B} 2$ \\
\hline PRAP1|118471 & $\mathrm{B} 2$ \\
\hline PRB1|5542 & $\mathrm{B} 2$ \\
\hline PRB2|653247 & $\mathrm{B} 2$ \\
\hline PRB3|5544 & B2 \\
\hline PRB4|5545 & $\mathrm{B} 2$ \\
\hline PRCP|5547 & $\mathrm{B} 2$ \\
\hline PRDM10|56980 & $\mathrm{B} 2$ \\
\hline PRDM12|59335 & $\mathrm{B} 2$ \\
\hline PRDM13|59336 & $\mathrm{B} 2$ \\
\hline PRDM14|63978 & $\mathrm{B} 2$ \\
\hline PRDM15|63977 & $\mathrm{B} 2$ \\
\hline PRDM1|639 & $\mathrm{B} 2$ \\
\hline PRDM4|11108 & $\mathrm{B} 2$ \\
\hline PRDM6|93166 & $\mathrm{B} 2$ \\
\hline PRDM7|11105 & $\mathrm{B} 2$ \\
\hline \begin{tabular}{l|l|} 
PRDM9|56979 \\
\end{tabular} & $\mathrm{B} 2$ \\
\hline PRDX3|10935 & $\mathrm{B} 2$ \\
\hline $\begin{array}{l}\text { PRDX5|25824 } \\
\end{array}$ & $\mathrm{B} 2$ \\
\hline PRDX6|9588 & $\mathrm{B} 2$ \\
\hline PREB|10113 & $\mathrm{B} 2$ \\
\hline PRELID2|153768 & $\mathrm{B} 2$ \\
\hline PREPL|9581 & $\mathrm{B} 2$ \\
\hline PREP|5550 & $\mathrm{B} 2$ \\
\hline PREX1|57580 & $\mathrm{B} 2$ \\
\hline PRF1|5551 & $\mathrm{B} 2$ \\
\hline PRG1|23574 & B2 \\
\hline PRG2|5553 & $\mathrm{B} 2$ \\
\hline PRG4|10216 & $\mathrm{B} 2$ \\
\hline PRH1|5554 & $\mathrm{B} 2$ \\
\hline PRH2|5555 & $\mathrm{B} 2$ \\
\hline PRHOXNB|646625 & $\mathrm{B} 2$ \\
\hline PRIC285|85441 & $\mathrm{B} 2$ \\
\hline PRICKLE1|144165 & $\mathrm{B} 2$ \\
\hline PRICKLE3|4007 & $\mathrm{B} 2$ \\
\hline PRICKLE4|29964 & $\mathrm{B} 2$ \\
\hline PRINS|100169750 & $\mathrm{B} 2$ \\
\hline PRKAA1|5562 & $\mathrm{B} 2$ \\
\hline PRKAA2|5563 & $\mathrm{B} 2$ \\
\hline
\end{tabular}




\begin{tabular}{|c|c|}
\hline PRKAB1|5564 & $\mathrm{B} 2$ \\
\hline PRKAB2|5565 & B2 \\
\hline PRKACA|5566 & $\mathrm{B} 2$ \\
\hline PRKACB|5567 & $\mathrm{B} 2$ \\
\hline PRKACG|5568 & B2 \\
\hline PRKAG1|5571 & $\mathrm{B} 2$ \\
\hline PRKAG3|53632 & $\mathrm{B} 2$ \\
\hline PRKAR1A|5573 & $\mathrm{B} 2$ \\
\hline PRKAR2A|5576 & B2 \\
\hline PRKCB|5579 & $\mathrm{B} 2$ \\
\hline PRKCDBP|112464 & $\mathrm{B} 2$ \\
\hline PRKCD 5580 & $\mathrm{~B} 2$ \\
\hline PRKCE|5581 & $\mathrm{B} 2$ \\
\hline PRKCG|5582 & $\mathrm{B} 2$ \\
\hline PRKCI|5584 & $\mathrm{B} 2$ \\
\hline PRKCQ|5588 & $\mathrm{B} 2$ \\
\hline PRKCSH|5589 & $\mathrm{B} 2$ \\
\hline PRKD2|25865 & $\mathrm{B} 2$ \\
\hline PRKG1|5592 & $\mathrm{B} 2$ \\
\hline PRKG2|5593 & $\mathrm{B} 2$ \\
\hline PRKRA|8575 & $\mathrm{B} 2$ \\
\hline PRKRIP1|79706 & $\mathrm{B} 2$ \\
\hline PRKRIR|5612 & $\mathrm{B} 2$ \\
\hline PRKX|5613 & $\mathrm{B} 2$ \\
\hline PRKY|5616 & $\mathrm{B} 2$ \\
\hline PRLHR|2834 & $\mathrm{B} 2$ \\
\hline PRLH|51052 & $\mathrm{B} 2$ \\
\hline PRL|5617 & $\mathrm{B} 2$ \\
\hline PRMT1|3276 & $\mathrm{B} 2$ \\
\hline PRMT3|10196 & $\mathrm{B} 2$ \\
\hline PRMT5|10419 & $\mathrm{B} 2$ \\
\hline PRMT6|55170 & $\mathrm{B} 2$ \\
\hline PRMT7|54496 & $\mathrm{B} 2$ \\
\hline PRMT8|56341 & $\mathrm{B} 2$ \\
\hline PRND|23627 & $\mathrm{B} 2$ \\
\hline PRO0611|28997 & $\mathrm{B} 2$ \\
\hline PRO0628|29053 & $\mathrm{B} 2$ \\
\hline PRO1768|29018 & $\mathrm{B} 2$ \\
\hline PROCA1|147011 & $\mathrm{B} 2$ \\
\hline PRODH|5625 & $\mathrm{B} 2$ \\
\hline PROK1|84432 & $\mathrm{B} 2$ \\
\hline PROK2|60675 & $\mathrm{B} 2$ \\
\hline PROKR2|128674 & $\mathrm{B} 2$ \\
\hline PROL1|58503 & $\mathrm{B} 2$ \\
\hline PROM1|8842 & $\mathrm{B} 2$ \\
\hline PROP1|5626 & $\mathrm{B} 2$ \\
\hline PROSC|11212 & $\mathrm{B} 2$ \\
\hline PROX2|283571 & $\mathrm{B} 2$ \\
\hline PROZ|8858 & $\mathrm{B} 2$ \\
\hline PRPF18|8559 & $\mathrm{B} 2$ \\
\hline PRPF31|26121 & $\mathrm{B} 2$ \\
\hline
\end{tabular}




\begin{tabular}{|c|c|}
\hline PRPF38A|84950 & $\mathrm{B} 2$ \\
\hline PRPF38B|55119 & B2 \\
\hline PRPF39|55015 & $\mathrm{B} 2$ \\
\hline PRPF3|9129 & $\mathrm{B} 2$ \\
\hline PRPF40B|25766 & $\mathrm{B} 2$ \\
\hline PRPF4B|8899 & $\mathrm{B} 2$ \\
\hline PRPF6|24148 & $\mathrm{B} 2$ \\
\hline PRPF8|10594 & $\mathrm{B} 2$ \\
\hline PRPH2|5961 & B2 \\
\hline PRPH $\mid 5630$ & $\mathrm{~B} 2$ \\
\hline PRPS1L1|221823 & $\mathrm{B} 2$ \\
\hline PRPSAP1|5635 & $\mathrm{B} 2$ \\
\hline PRPSAP2|5636 & $\mathrm{B} 2$ \\
\hline PRR11|55771 & $\mathrm{B} 2$ \\
\hline PRR12|57479 & $\mathrm{B} 2$ \\
\hline PRR15L|79170 & $\mathrm{B} 2$ \\
\hline PRR16|51334 & $\mathrm{B} 2$ \\
\hline PRR18|285800 & $\mathrm{B} 2$ \\
\hline PRR22|163154 & $\mathrm{B} 2$ \\
\hline PRR23A|729627 & $\mathrm{B} 2$ \\
\hline PRR24|255783 & $\mathrm{B} 2$ \\
\hline PRR25|388199 & $\mathrm{B} 2$ \\
\hline PRR3|80742 & $\mathrm{B} 2$ \\
\hline PRR4|11272 & $\mathrm{B} 2$ \\
\hline PRR5-ARHGAP8|553158 & $\mathrm{B} 2$ \\
\hline PRR5L|79899 & $\mathrm{B} 2$ \\
\hline PRRG2|5639 & $\mathrm{B} 2$ \\
\hline PRRG4|79056 & $\mathrm{B} 2$ \\
\hline PRRT1|80863 & $\mathrm{B} 2$ \\
\hline PRRT2|112476 & $\mathrm{B} 2$ \\
\hline PRRX1|5396 & $\mathrm{B} 2$ \\
\hline \begin{tabular}{l|l|} 
PRRX2|51450 \\
\end{tabular} & $\mathrm{B} 2$ \\
\hline PRSS12|8492 & $\mathrm{B} 2$ \\
\hline PRSS16|10279 & $\mathrm{B} 2$ \\
\hline PRSS1|5644 & $\mathrm{B} 2$ \\
\hline PRSS21|10942 & $\mathrm{B} 2$ \\
\hline PRSS22|64063 & $\mathrm{B} 2$ \\
\hline \begin{tabular}{l|l|l|} 
PRSS23|11098 \\
\end{tabular} & $\mathrm{B} 2$ \\
\hline PRSS27|83886 & $\mathrm{B} 2$ \\
\hline PRSS30P|124221 & $\mathrm{B} 2$ \\
\hline PRSS33|260429 & $\mathrm{B} 2$ \\
\hline PRSS35|167681 & $\mathrm{B} 2$ \\
\hline PRSS36|146547 & $\mathrm{B} 2$ \\
\hline PRSS37|136242 & $\mathrm{B} 2$ \\
\hline PRSS38|339501 & $\mathrm{B} 2$ \\
\hline $\begin{array}{l}\text { PRSS3|5646 } \\
\end{array}$ & $\mathrm{B} 2$ \\
\hline PRSS41|360226 & $\mathrm{B} 2$ \\
\hline PRSS42|339906 & $\mathrm{B} 2$ \\
\hline PRSS45|377047 & $\mathrm{B} 2$ \\
\hline PRSS48|345062 & $\mathrm{B} 2$ \\
\hline $\begin{array}{l}\text { PRSS53|339105 } \\
\end{array}$ & $\mathrm{B} 2$ \\
\hline
\end{tabular}




\begin{tabular}{|c|c|}
\hline PRSS54|221191 & B2 \\
\hline PRSSL1|400668 & $\mathrm{B} 2$ \\
\hline PRTFDC1|56952 & $\mathrm{B} 2$ \\
\hline PRTG|283659 & $\mathrm{B} 2$ \\
\hline PRTN3|5657 & $\mathrm{B} 2$ \\
\hline PSAPL1|768239 & $\mathrm{B} 2$ \\
\hline PSAP|5660 & $\mathrm{B} 2$ \\
\hline PSAT1|29968 & $\mathrm{B} 2$ \\
\hline PSCA $\mid 8000$ & $\mathrm{~B} 2$ \\
\hline PSD2|84249 & $\mathrm{B} 2$ \\
\hline PSD3|23362 & $\mathrm{B} 2$ \\
\hline PSD4|23550 & $\mathrm{B} 2$ \\
\hline PSD|5662 & $\mathrm{B} 2$ \\
\hline PSG10|653492 & $\mathrm{B} 2$ \\
\hline PSG11|5680 & $\mathrm{B} 2$ \\
\hline PSG1|5669 & $\mathrm{B} 2$ \\
\hline PSG2|5670 & $\mathrm{B} 2$ \\
\hline $\begin{array}{l}\text { PSG3|5671 } \\
\end{array}$ & $\mathrm{B} 2$ \\
\hline PSG4|5672 & $\mathrm{B} 2$ \\
\hline $\begin{array}{l}\text { PSG5|5673 } \\
\end{array}$ & $\mathrm{B} 2$ \\
\hline PSG6|5675 & $\mathrm{B} 2$ \\
\hline PSG7|5676 & $\mathrm{B} 2$ \\
\hline PSG8|440533 & $\mathrm{B} 2$ \\
\hline PSG9|5678 & $\mathrm{B} 2$ \\
\hline PSIMCT-1|100101490 & $\mathrm{B} 2$ \\
\hline PSIP1|11168 & $\mathrm{B} 2$ \\
\hline PSKH2|85481 & $\mathrm{B} 2$ \\
\hline PSMA1 5682 & $\mathrm{~B} 2$ \\
\hline PSMA8|143471 & $\mathrm{B} 2$ \\
\hline PSMB10|5699 & $\mathrm{B} 2$ \\
\hline PSMB11|122706 & $\mathrm{B} 2$ \\
\hline PSMB1|5689 & $\mathrm{B} 2$ \\
\hline PSMB6|5694 & $\mathrm{B} 2$ \\
\hline PSMB7|5695 & $\mathrm{B} 2$ \\
\hline PSMB8|5696 & $\mathrm{B} 2$ \\
\hline PSMB9|5698 & $\mathrm{B} 2$ \\
\hline PSMC1 15700 & $\mathrm{~B} 2$ \\
\hline PSMC3|5702 & $\mathrm{B} 2$ \\
\hline PSMC5|5705 & $\mathrm{B} 2$ \\
\hline PSMD13|5719 & $\mathrm{B} 2$ \\
\hline PSMD3|5709 & $\mathrm{B} 2$ \\
\hline PSMD6|9861 & $\mathrm{B} 2$ \\
\hline PSMD7|5713 & $\mathrm{B} 2$ \\
\hline PSMD8|5714 & $\mathrm{B} 2$ \\
\hline PSMD9|5715 & $\mathrm{B} 2$ \\
\hline PSMF1|9491 & $\mathrm{B} 2$ \\
\hline PSMG1|8624 & $\mathrm{B} 2$ \\
\hline \begin{tabular}{l|l|} 
PSMG2|56984 \\
\end{tabular} & $\mathrm{B} 2$ \\
\hline PSMG4|389362 & $\mathrm{B} 2$ \\
\hline PSORS1C1|170679 & $\mathrm{B} 2$ \\
\hline PSORS1C2|170680 & $\mathrm{B} 2$ \\
\hline
\end{tabular}




\begin{tabular}{|c|c|}
\hline PSORS1C3|100130889 & B2 \\
\hline PSPH $\mid 5723$ & $\mathrm{~B} 2$ \\
\hline PSPN|5623 & $\mathrm{B} 2$ \\
\hline PSTK|118672 & $\mathrm{B} 2$ \\
\hline PSTPIP1|9051 & $\mathrm{B} 2$ \\
\hline PSTPIP2|9050 & B2 \\
\hline PTAFR|5724 & $\mathrm{B} 2$ \\
\hline PTBP2|58155 & $\mathrm{B} 2$ \\
\hline PTCD2|79810 & $\mathrm{B} 2$ \\
\hline PTCD3|55037 & $\mathrm{B} 2$ \\
\hline PTCHD2|57540 & $\mathrm{B} 2$ \\
\hline PTCHD3|374308 & $\mathrm{B} 2$ \\
\hline PTCRA|171558 & $\mathrm{B} 2$ \\
\hline PTDSS1|9791 & $\mathrm{B} 2$ \\
\hline PTDSS2|81490 & $\mathrm{B} 2$ \\
\hline PTENP1|11191 & $\mathrm{B} 2$ \\
\hline PTER|9317 & $\mathrm{B} 2$ \\
\hline PTF1A|256297 & $\mathrm{B} 2$ \\
\hline PTGDR|5729 & $\mathrm{B} 2$ \\
\hline PTGER1|5731 & $\mathrm{B} 2$ \\
\hline PTGER2|5732 & $\mathrm{B} 2$ \\
\hline PTGES2|80142 & $\mathrm{B} 2$ \\
\hline PTGES|9536 & $\mathrm{B} 2$ \\
\hline PTGFRN|5738 & $\mathrm{B} 2$ \\
\hline PTGIR|5739 & $\mathrm{B} 2$ \\
\hline PTGR1|22949 & $\mathrm{B} 2$ \\
\hline PTGR2|145482 & $\mathrm{B} 2$ \\
\hline PTGS1|5742 & $\mathrm{B} 2$ \\
\hline PTGS2|5743 & $\mathrm{B} 2$ \\
\hline PTH2R|5746 & $\mathrm{B} 2$ \\
\hline PTH2|113091 & $\mathrm{B} 2$ \\
\hline PTHLH|5744 & $\mathrm{B} 2$ \\
\hline PTK2B|2185 & $\mathrm{B} 2$ \\
\hline PTOV1|53635 & $\mathrm{B} 2$ \\
\hline PTP4A1|7803 & $\mathrm{B} 2$ \\
\hline PTP4A2|8073 & $\mathrm{B} 2$ \\
\hline PTP4A3|11156 & $\mathrm{B} 2$ \\
\hline PTPDC1|138639 & $\mathrm{B} 2$ \\
\hline PTPLAD2|401494 & $\mathrm{B} 2$ \\
\hline PTPLB|201562 & $\mathrm{B} 2$ \\
\hline PTPMT1|114971 & $\mathrm{B} 2$ \\
\hline $\begin{array}{l}\text { PTPN12|5782 } \\
\end{array}$ & $\mathrm{B} 2$ \\
\hline PTPN13|5783 & $\mathrm{B} 2$ \\
\hline PTPN18|26469 & $\mathrm{B} 2$ \\
\hline PTPN1 15770 & $\mathrm{~B} 2$ \\
\hline PTPN20A|653129 & B2 \\
\hline \begin{tabular}{l|l|} 
PTPN20B|26095 \\
\end{tabular} & $\mathrm{B} 2$ \\
\hline PTPN22|26191 & $\mathrm{B} 2$ \\
\hline PTPN23|25930 & $\mathrm{B} 2$ \\
\hline PTPN2|5771 & $\mathrm{B} 2$ \\
\hline PTPN3|5774 & $\mathrm{B} 2$ \\
\hline
\end{tabular}




\begin{tabular}{|c|c|}
\hline PTPN5|84867 & B2 \\
\hline PTPN7|5778 & B2 \\
\hline PTPN9|5780 & $\mathrm{B} 2$ \\
\hline PTPRA|5786 & $\mathrm{B} 2$ \\
\hline PTPRCAP|5790 & B2 \\
\hline PTPRC|5788 & B2 \\
\hline PTPRD|5789 & $\mathrm{B} 2$ \\
\hline PTPRE|5791 & $\mathrm{B} 2$ \\
\hline PTPRF|5792 & $\mathrm{B} 2$ \\
\hline PTPRH|5794 & $\mathrm{B} 2$ \\
\hline PTPRJ|5795 & $\mathrm{B} 2$ \\
\hline PTPRK|5796 & B2 \\
\hline PTPRN2|5799 & $\mathrm{B} 2$ \\
\hline PTPRN|5798 & $\mathrm{B} 2$ \\
\hline PTPRO|5800 & $\mathrm{B} 2$ \\
\hline PTPRQ|374462 & B2 \\
\hline PTPRR|5801 & $\mathrm{B} 2$ \\
\hline PTPRT|11122 & $\mathrm{B} 2$ \\
\hline PTPRU|10076 & $\mathrm{B} 2$ \\
\hline PTPRVP|148713 & $\mathrm{B} 2$ \\
\hline PTRH2|51651 & $\mathrm{B} 2$ \\
\hline PTTG2|10744 & $\mathrm{B} 2$ \\
\hline PTX3|5806 & $\mathrm{B} 2$ \\
\hline PTX4|390667 & $\mathrm{B} 2$ \\
\hline PUM1|9698 & $\mathrm{B} 2$ \\
\hline PUM2|23369 & $\mathrm{B} 2$ \\
\hline PURA|5813 & B2 \\
\hline PURB|5814 & $\mathrm{B} 2$ \\
\hline PURG|29942 & $\mathrm{B} 2$ \\
\hline PUS10|150962 & $\mathrm{B} 2$ \\
\hline PUS1|80324 & $\mathrm{B} 2$ \\
\hline PUS3|83480 & $\mathrm{B} 2$ \\
\hline PVALB|5816 & $\mathrm{B} 2$ \\
\hline PVRIG|79037 & $\mathrm{B} 2$ \\
\hline PVRL1|5818 & $\mathrm{B} 2$ \\
\hline PVR|5817 & $\mathrm{B} 2$ \\
\hline $\begin{array}{l}\text { PVT1|5820 } \\
\end{array}$ & $\mathrm{B} 2$ \\
\hline PWP1|11137 & $\mathrm{B} 2$ \\
\hline PWP2|5822 & $\mathrm{B} 2$ \\
\hline PWRN1|791114 & $\mathrm{B} 2$ \\
\hline PWRN2|791115 & $\mathrm{B} 2$ \\
\hline PWWP2A|114825 & B2 \\
\hline PWWP2B|170394 & $\mathrm{B} 2$ \\
\hline PXDNL|137902 & $\mathrm{B} 2$ \\
\hline PXDN|7837 & $\mathrm{B} 2$ \\
\hline PXK|54899 & $\mathrm{B} 2$ \\
\hline PXMP2|5827 & $\mathrm{B} 2$ \\
\hline PXMP4|11264 & $\mathrm{B} 2$ \\
\hline PXT1|222659 & $\mathrm{B} 2$ \\
\hline PYDC1|260434 & B2 \\
\hline PYGB|5834 & $\mathrm{B} 2$ \\
\hline
\end{tabular}




\begin{tabular}{|c|c|}
\hline PYGL|5836 & B2 \\
\hline PYGM|5837 & $\mathrm{B} 2$ \\
\hline PYGO1|26108 & B2 \\
\hline PYHIN1|149628 & $\mathrm{B} 2$ \\
\hline PYROXD1|79912 & B2 \\
\hline PYY2|23615 & B2 \\
\hline PYY|5697 & B2 \\
\hline QARS|5859 & $\mathrm{B} 2$ \\
\hline QDPR|5860 & $\mathrm{B} 2$ \\
\hline QPCTL|54814 & $\mathrm{B} 2$ \\
\hline QPCT|25797 & B2 \\
\hline QPRT|23475 & $\mathrm{B} 2$ \\
\hline QRFPR|84109 & $\mathrm{B} 2$ \\
\hline QRFP|347148 & $\mathrm{B} 2$ \\
\hline QRICH1|54870 & $\mathrm{B} 2$ \\
\hline QRICH2|84074 & $\mathrm{B} 2$ \\
\hline QRSL1|55278 & $\mathrm{B} 2$ \\
\hline QSER1|79832 & $\mathrm{B} 2$ \\
\hline QSOX1|5768 & $\mathrm{B} 2$ \\
\hline QSOX2|169714 & $\mathrm{B} 2$ \\
\hline QTRT1|81890 & $\mathrm{B} 2$ \\
\hline R3HDM2|22864 & $\mathrm{B} 2$ \\
\hline R3HDML|140902 & $\mathrm{B} 2$ \\
\hline RAB11A|8766 & $\mathrm{B} 2$ \\
\hline RAB11B|9230 & $\mathrm{B} 2$ \\
\hline RAB11FIP1|80223 & $\mathrm{B} 2$ \\
\hline RAB11FIP5|26056 & $\mathrm{B} 2$ \\
\hline RAB12|201475 & $\mathrm{B} 2$ \\
\hline RAB14|51552 & $\mathrm{B} 2$ \\
\hline RAB15|376267 & $\mathrm{B} 2$ \\
\hline RAB18|22931 & $\mathrm{B} 2$ \\
\hline RAB1B|81876 & $\mathrm{B} 2$ \\
\hline \begin{tabular}{l|l|l|} 
RAB20|55647 &
\end{tabular} & $\mathrm{B} 2$ \\
\hline RAB21|23011 & $\mathrm{B} 2$ \\
\hline RAB22A|57403 & $\mathrm{B} 2$ \\
\hline RAB23|51715 & $\mathrm{B} 2$ \\
\hline RAB24|53917 & $\mathrm{B} 2$ \\
\hline RAB27A|5873 & $\mathrm{B} 2$ \\
\hline RAB27B|5874 & $\mathrm{B} 2$ \\
\hline RAB2B|84932 & $\mathrm{B} 2$ \\
\hline RAB30|27314 & $\mathrm{B} 2$ \\
\hline RAB32|10981 & $\mathrm{B} 2$ \\
\hline RAB33B|83452 & $\mathrm{B} 2$ \\
\hline RAB34|83871 & $\mathrm{B} 2$ \\
\hline RAB35|11021 & B2 \\
\hline RAB36|9609 & $\mathrm{B} 2$ \\
\hline RAB37|326624 & $\mathrm{B} 2$ \\
\hline RAB38|23682 & $\mathrm{B} 2$ \\
\hline RAB39B|116442 & $\mathrm{B} 2$ \\
\hline 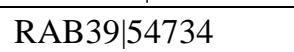 & $\mathrm{B} 2$ \\
\hline RAB3B|5865 & B2 \\
\hline
\end{tabular}




\begin{tabular}{|c|c|}
\hline RAB3C|115827 & B2 \\
\hline RAB3GAP1|22930 & B2 \\
\hline RAB3GAP2|25782 & $\mathrm{B} 2$ \\
\hline RAB40AL|282808 & $\mathrm{B} 2$ \\
\hline RAB41|347517 & B2 \\
\hline RAB42|115273 & $\mathrm{B} 2$ \\
\hline RAB43|339122 & B2 \\
\hline RAB4A|5867 & $\mathrm{B} 2$ \\
\hline RAB5A|5868 & $\mathrm{B} 2$ \\
\hline RAB5B|5869 & $\mathrm{B} 2$ \\
\hline RAB5C|5878 & $\mathrm{B} 2$ \\
\hline RAB6C|84084 & $\mathrm{B} 2$ \\
\hline RAB7A|7879 & $\mathrm{B} 2$ \\
\hline RAB7L1|8934 & $\mathrm{B} 2$ \\
\hline RAB8B|51762 & B2 \\
\hline RAB9A|9367 & B2 \\
\hline RABAC1|10567 & B2 \\
\hline RABEP1|9135 & B2 \\
\hline RABGAP1L $\mid 9910$ & B2 \\
\hline RABGAP1|23637 & $\mathrm{B} 2$ \\
\hline RABGEF1|27342 & $\mathrm{B} 2$ \\
\hline RABGGTA|5875 & $\mathrm{B} 2$ \\
\hline RABGGTB|5876 & $\mathrm{B} 2$ \\
\hline RABL2A|11159 & $\mathrm{B} 2$ \\
\hline RABL2B|11158 & $\mathrm{B} 2$ \\
\hline RABL3|285282 & B2 \\
\hline RAC1|5879 & B2 \\
\hline RAC2|5880 & $\mathrm{B} 2$ \\
\hline RAD17|5884 & $\mathrm{B} 2$ \\
\hline \begin{tabular}{l|l|l|} 
RAD186852 &
\end{tabular} & $\mathrm{B} 2$ \\
\hline RAD21L1|642636 & $\mathrm{B} 2$ \\
\hline RAD23A|5886 & $\mathrm{B} 2$ \\
\hline RAD23B|5887 & $\mathrm{B} 2$ \\
\hline RAD50|10111 & \begin{tabular}{|l}
$\mathrm{B} 2$ \\
\end{tabular} \\
\hline RAD51AP2|729475 & $\mathrm{B} 2$ \\
\hline RAD51C|5889 & $\mathrm{B} 2$ \\
\hline RAD51L1|5890 & B2 \\
\hline RAD51L3|5892 & B2 \\
\hline RAD52|5893 & $\mathrm{B} 2$ \\
\hline RAD9A|5883 & B2 \\
\hline RAD9B|144715 & B2 \\
\hline RAET1E|135250 & B2 \\
\hline RAET1G|353091 & $\mathrm{B} 2$ \\
\hline RAET1L|154064 & $\mathrm{B} 2$ \\
\hline RAF1|5894 & $\mathrm{B} 2$ \\
\hline RAG2|5897 & $\mathrm{B} 2$ \\
\hline RAGE|5891 & $\mathrm{B} 2$ \\
\hline RAI1|10743 & B2 \\
\hline RALBP1|10928 & B2 \\
\hline RALB|5899 & $\mathrm{B} 2$ \\
\hline RALGAPA1|253959 & B2 \\
\hline
\end{tabular}




\begin{tabular}{|c|c|}
\hline RALGAPA2|57186 & B2 \\
\hline RALGAPB|57148 & $\mathrm{B} 2$ \\
\hline RALGPS1|9649 & B2 \\
\hline RALYL|138046 & $\mathrm{B} 2$ \\
\hline RAMP1|10267 & $\mathrm{B} 2$ \\
\hline RAMP3|10268 & B2 \\
\hline RANBP2|5903 & B2 \\
\hline RANBP3L|202151 & $\mathrm{B} 2$ \\
\hline RANBP3|8498 & $\mathrm{B} 2$ \\
\hline RANBP6|26953 & $\mathrm{B} 2$ \\
\hline RANGRF|29098 & B2 \\
\hline RAP1A|5906 & $\mathrm{B} 2$ \\
\hline $\begin{array}{l}\text { RAP1B|5908 } \\
\end{array}$ & $\mathrm{B} 2$ \\
\hline RAP1GAP2|23108 & $\mathrm{B} 2$ \\
\hline RAP1GAP|5909 & $\mathrm{B} 2$ \\
\hline RAP1GDS1|5910 & $\mathrm{B} 2$ \\
\hline RAP2A|5911 & $\mathrm{B} 2$ \\
\hline RAP2B|5912 & $\mathrm{B} 2$ \\
\hline RAPGEF4|11069 & $\mathrm{B} 2$ \\
\hline RAPGEF5|9771 & $\mathrm{B} 2$ \\
\hline RAPGEF6|51735 & $\mathrm{B} 2$ \\
\hline RAPGEFL1|51195 & $\mathrm{B} 2$ \\
\hline RAPH1 65059 & $\mathrm{~B} 2$ \\
\hline RAPSN|5913 & $\mathrm{B} 2$ \\
\hline RARA|5914 & $\mathrm{B} 2$ \\
\hline RARG|5916 & $\mathrm{B} 2$ \\
\hline RARRES1|5918 & $\mathrm{B} 2$ \\
\hline RARS2|57038 & $\mathrm{B} 2$ \\
\hline RASA1|5921 & $\mathrm{B} 2$ \\
\hline RASA4P|401331 & $\mathrm{B} 2$ \\
\hline RASAL1|8437 & $\mathrm{B} 2$ \\
\hline RASAL2|9462 & $\mathrm{B} 2$ \\
\hline RASAL3|64926 & $\mathrm{B} 2$ \\
\hline \begin{tabular}{l|l} 
RASD2|23551 &
\end{tabular} & B2 \\
\hline RASEF|158158 & $\mathrm{B} 2$ \\
\hline RASGEF1A|221002 & $\mathrm{B} 2$ \\
\hline RASGEF1C|255426 & $\mathrm{B} 2$ \\
\hline RASGRF1|5923 & $\mathrm{B} 2$ \\
\hline RASGRF2|5924 & $\mathrm{B} 2$ \\
\hline RASGRP1|10125 & $\mathrm{B} 2$ \\
\hline RASGRP3|25780 & $\mathrm{B} 2$ \\
\hline RASGRP4|115727 & $\mathrm{B} 2$ \\
\hline \begin{tabular}{l|l} 
RASL10B $\mid 91608$ \\
\end{tabular} & $\mathrm{~B} 2$ \\
\hline RASSF10|644943 & $\mathrm{B} 2$ \\
\hline $\begin{array}{l}\text { RASSF2|9770 } \\
\end{array}$ & B2 \\
\hline RASSF4|83937 & $\mathrm{B} 2$ \\
\hline RASSF5|83593 & $\mathrm{B} 2$ \\
\hline RASSF8|11228 & $\mathrm{B} 2$ \\
\hline RAX|30062 & $\mathrm{B} 2$ \\
\hline RB1CC1|9821 & B2 \\
\hline RB1|5925 & B2 \\
\hline
\end{tabular}




\begin{tabular}{|c|c|}
\hline RBAK|57786 & B2 \\
\hline RBBP6|5930 & $\mathrm{B} 2$ \\
\hline RBBP7|5931 & $\mathrm{B} 2$ \\
\hline RBBP8|5932 & $\mathrm{B} 2$ \\
\hline RBBP9|10741 & $\mathrm{B} 2$ \\
\hline RBCK1|10616 & $\mathrm{B} 2$ \\
\hline RBM10|8241 & $\mathrm{B} 2$ \\
\hline RBM11|54033 & $\mathrm{B} 2$ \\
\hline RBM12|10137 & $\mathrm{B} 2$ \\
\hline RBM14|10432 & $\mathrm{B} 2$ \\
\hline RBM15B|29890 & $\mathrm{B} 2$ \\
\hline RBM15|64783 & $\mathrm{B} 2$ \\
\hline RBM16|22828 & $\mathrm{B} 2$ \\
\hline RBM17|84991 & $\mathrm{B} 2$ \\
\hline RBM18|92400 & $\mathrm{B} 2$ \\
\hline RBM19|9904 & $\mathrm{B} 2$ \\
\hline RBM20|282996 & $\mathrm{B} 2$ \\
\hline RBM22|55696 & $\mathrm{B} 2$ \\
\hline RBM23|55147 & $\mathrm{B} 2$ \\
\hline RBM24|221662 & $\mathrm{B} 2$ \\
\hline RBM25|58517 & $\mathrm{B} 2$ \\
\hline RBM26|64062 & $\mathrm{B} 2$ \\
\hline RBM27|54439 & $\mathrm{B} 2$ \\
\hline RBM28|55131 & $\mathrm{B} 2$ \\
\hline RBM38|55544 & $\mathrm{B} 2$ \\
\hline RBM39|9584 & $\mathrm{B} 2$ \\
\hline RBM3|5935 & $\mathrm{B} 2$ \\
\hline RBM41|55285 & $\mathrm{B} 2$ \\
\hline RBM42|79171 & $\mathrm{B} 2$ \\
\hline RBM43|375287 & $\mathrm{B} 2$ \\
\hline RBM44|375316 & $\mathrm{B} 2$ \\
\hline RBM46|166863 & $\mathrm{B} 2$ \\
\hline RBM4B|83759 & $\mathrm{B} 2$ \\
\hline RBMX2|51634 & $\mathrm{B} 2$ \\
\hline RBMXL2|27288 & $\mathrm{B} 2$ \\
\hline RBMX|27316 & $\mathrm{B} 2$ \\
\hline RBP1|5947 & $\mathrm{B} 2$ \\
\hline RBP2|5948 & $\mathrm{B} 2$ \\
\hline RBP3|5949 & $\mathrm{B} 2$ \\
\hline RBP5|83758 & $\mathrm{B} 2$ \\
\hline RBPJL|11317 & $\mathrm{B} 2$ \\
\hline RC3H1|149041 & $\mathrm{B} 2$ \\
\hline RC3H2|54542 & $\mathrm{B} 2$ \\
\hline RCAN3|11123 & $\mathrm{B} 2$ \\
\hline RCBTB1|55213 & $\mathrm{B} 2$ \\
\hline RCCD1|91433 & $\mathrm{B} 2$ \\
\hline RCE1|9986 & $\mathrm{B} 2$ \\
\hline RCHY1|25898 & $\mathrm{B} 2$ \\
\hline RCL1|10171 & $\mathrm{B} 2$ \\
\hline \begin{tabular}{l|l|l|} 
RCN1|5954 &
\end{tabular} & $\mathrm{B} 2$ \\
\hline RCN2|5955 & $\mathrm{B} 2$ \\
\hline
\end{tabular}




\begin{tabular}{|c|c|}
\hline RCN3|57333 & B2 \\
\hline RCOR1|23186 & B2 \\
\hline RCOR2|283248 & $\mathrm{B} 2$ \\
\hline RCOR3|55758 & $\mathrm{B} 2$ \\
\hline RD3|343035 & $\mathrm{B} 2$ \\
\hline RDBP|7936 & $\mathrm{B} 2$ \\
\hline RDH10|157506 & $\mathrm{B} 2$ \\
\hline RDH12|145226 & $\mathrm{B} 2$ \\
\hline RDH14|57665 & $\mathrm{B} 2$ \\
\hline RDH16|8608 & $\mathrm{B} 2$ \\
\hline RDH8|50700 & $\mathrm{B} 2$ \\
\hline RDX|5962 & $\mathrm{B} 2$ \\
\hline REC8|9985 & $\mathrm{B} 2$ \\
\hline RECQL5|9400 & $\mathrm{B} 2$ \\
\hline RECQL|5965 & B2 \\
\hline REEP1|65055 & $\mathrm{B} 2$ \\
\hline REEP2|51308 & $\mathrm{B} 2$ \\
\hline REEP3|221035 & $\mathrm{B} 2$ \\
\hline REEP5|7905 & $\mathrm{B} 2$ \\
\hline \begin{tabular}{l|l|} 
REEP6|92840 \\
\end{tabular} & $\mathrm{B} 2$ \\
\hline REG1A|5967 & $\mathrm{B} 2$ \\
\hline REG3G|130120 & B2 \\
\hline REG4|83998 & $\mathrm{B} 2$ \\
\hline RELA|5970 & $\mathrm{B} 2$ \\
\hline RELB|5971 & $\mathrm{B} 2$ \\
\hline RELL1|768211 & $\mathrm{B} 2$ \\
\hline REL|5966 & $\mathrm{B} 2$ \\
\hline RENBP|5973 & $\mathrm{B} 2$ \\
\hline REN|5972 & $\mathrm{B} 2$ \\
\hline REP15|387849 & $\mathrm{B} 2$ \\
\hline REPS1|85021 & $\mathrm{B} 2$ \\
\hline RER1|11079 & $\mathrm{B} 2$ \\
\hline RERG|85004 & $\mathrm{B} 2$ \\
\hline RESP18|389075 & $\mathrm{B} 2$ \\
\hline REST|5978 & $\mathrm{B} 2$ \\
\hline RETNLB|84666 & $\mathrm{B} 2$ \\
\hline RETN|56729 & $\mathrm{B} 2$ \\
\hline RET|5979 & $\mathrm{B} 2$ \\
\hline REXO1L1|254958 & $\mathrm{B} 2$ \\
\hline REXO1|57455 & $\mathrm{B} 2$ \\
\hline REXO2|25996 & $\mathrm{B} 2$ \\
\hline REXO4|57109 & $\mathrm{B} 2$ \\
\hline RFC1|5981 & $\mathrm{B} 2$ \\
\hline RFESD|317671 & $\mathrm{B} 2$ \\
\hline RFK|55312 & $\mathrm{B} 2$ \\
\hline RFNG|5986 & $\mathrm{B} 2$ \\
\hline RFPL1S|10740 & $\mathrm{B} 2$ \\
\hline RFPL1|5988 & $\mathrm{B} 2$ \\
\hline RFPL2|10739 & $\mathrm{B} 2$ \\
\hline RFPL3S|10737 & $\mathrm{B} 2$ \\
\hline RFPL3|10738 & $\mathrm{B} 2$ \\
\hline
\end{tabular}




\begin{tabular}{|c|c|}
\hline RFPL4A|342931 & B2 \\
\hline RFPL4B|442247 & B2 \\
\hline RFT1|91869 & $\mathrm{B} 2$ \\
\hline RFTN1|23180 & $\mathrm{B} 2$ \\
\hline RFX1|5989 & B2 \\
\hline RFX3|5991 & $\mathrm{B} 2$ \\
\hline RFX4|5992 & $\mathrm{B} 2$ \\
\hline RFX6|222546 & $\mathrm{B} 2$ \\
\hline RFX7|64864 & $\mathrm{B} 2$ \\
\hline RFX8|731220 & $\mathrm{B} 2$ \\
\hline RG9MTD1|54931 & $\mathrm{B} 2$ \\
\hline RGAG1|57529 & $\mathrm{B} 2$ \\
\hline RGL2|5863 & $\mathrm{B} 2$ \\
\hline RGL3|57139 & $\mathrm{B} 2$ \\
\hline RGL4|266747 & $\mathrm{B} 2$ \\
\hline RGP1|9827 & $\mathrm{B} 2$ \\
\hline RGPD1|400966 & $\mathrm{B} 2$ \\
\hline RGPD8|727851 & $\mathrm{B} 2$ \\
\hline RGR|5995 & $\mathrm{B} 2$ \\
\hline RGS11|8786 & $\mathrm{B} 2$ \\
\hline RGS13|6003 & $\mathrm{B} 2$ \\
\hline RGS16|6004 & $\mathrm{B} 2$ \\
\hline RGS17|26575 & $\mathrm{B} 2$ \\
\hline RGS18|64407 & $\mathrm{B} 2$ \\
\hline RGS1|5996 & $\mathrm{B} 2$ \\
\hline RGS20|8601 & $\mathrm{B} 2$ \\
\hline RGS21|431704 & $\mathrm{B} 2$ \\
\hline RGS22|26166 & $\mathrm{B} 2$ \\
\hline RGS3|5998 & $\mathrm{B} 2$ \\
\hline RGS7|6000 & $\mathrm{B} 2$ \\
\hline RGS8|85397 & $\mathrm{B} 2$ \\
\hline RGS9BP|388531 & $\mathrm{B} 2$ \\
\hline RGS9|8787 & $\mathrm{B} 2$ \\
\hline RGSL1|353299 & $\mathrm{B} 2$ \\
\hline RHAG|6005 & $\mathrm{B} 2$ \\
\hline RHBDD1|84236 & $\mathrm{B} 2$ \\
\hline RHBDL3|162494 & $\mathrm{B} 2$ \\
\hline RHBG|57127 & $\mathrm{B} 2$ \\
\hline RHCG|51458 & $\mathrm{B} 2$ \\
\hline RHD|6007 & $\mathrm{B} 2$ \\
\hline RHEBL1|121268 & $\mathrm{B} 2$ \\
\hline RHEB|6009 & $\mathrm{B} 2$ \\
\hline RHOA|387 & $\mathrm{B} 2$ \\
\hline RHOBTB1|9886 & $\mathrm{B} 2$ \\
\hline RHOBTB2|23221 & $\mathrm{B} 2$ \\
\hline RHOBTB3|22836 & $\mathrm{B} 2$ \\
\hline RHOB|388 & $\mathrm{B} 2$ \\
\hline RHOC|389 & $\mathrm{B} 2$ \\
\hline RHOG|391 & $\mathrm{B} 2$ \\
\hline RHOH|399 & $\mathrm{B} 2$ \\
\hline RHOT1|55288 & $\mathrm{B} 2$ \\
\hline
\end{tabular}




\begin{tabular}{|c|c|}
\hline RHOV|171177 & $\mathrm{B} 2$ \\
\hline RHOXF2B|727940 & B2 \\
\hline RHO 6010 & $\mathrm{~B} 2$ \\
\hline RHPN2|85415 & $\mathrm{B} 2$ \\
\hline RIBC1|158787 & $\mathrm{B} 2$ \\
\hline RIC8A|60626 & $\mathrm{B} 2$ \\
\hline RIC8B|55188 & $\mathrm{B} 2$ \\
\hline RICH2|9912 & $\mathrm{B} 2$ \\
\hline RICTOR/253260 & $\mathrm{B} 2$ \\
\hline RIF1|55183 & $\mathrm{B} 2$ \\
\hline RIMBP2|23504 & $\mathrm{B} 2$ \\
\hline RIMBP3C|150221 & $\mathrm{B} 2$ \\
\hline RIMBP3|85376 & $\mathrm{B} 2$ \\
\hline RIMKLA|284716 & $\mathrm{B} 2$ \\
\hline RIMS1|22999 & $\mathrm{B} 2$ \\
\hline RIMS2|9699 & $\mathrm{B} 2$ \\
\hline RIN1|9610 & $\mathrm{B} 2$ \\
\hline RIN2|54453 & $\mathrm{B} 2$ \\
\hline RIN3|79890 & $\mathrm{B} 2$ \\
\hline RING1|6015 & $\mathrm{B} 2$ \\
\hline RINL|126432 & $\mathrm{B} 2$ \\
\hline RIOK1|83732 & $\mathrm{B} 2$ \\
\hline RIOK2|55781 & $\mathrm{B} 2$ \\
\hline RIOK3|8780 & $\mathrm{B} 2$ \\
\hline RIPK1|8737 & $\mathrm{B} 2$ \\
\hline RIPK3|11035 & $\mathrm{B} 2$ \\
\hline RIPPLY1|92129 & $\mathrm{B} 2$ \\
\hline RIPPLY2|134701 & $\mathrm{B} 2$ \\
\hline RIT2|6014 & $\mathrm{B} 2$ \\
\hline RLBP1 6017 & $\mathrm{~B} 2$ \\
\hline RLF|6018 & $\mathrm{B} 2$ \\
\hline RLIM|51132 & $\mathrm{B} 2$ \\
\hline RLN1|6013 & $\mathrm{B} 2$ \\
\hline RLN2|6019 & $\mathrm{B} 2$ \\
\hline RLN3|117579 & $\mathrm{B} 2$ \\
\hline RLTPR|146206 & $\mathrm{B} 2$ \\
\hline RMND1|55005 & $\mathrm{B} 2$ \\
\hline RMND5A|64795 & $\mathrm{B} 2$ \\
\hline RMRP|6023 & $\mathrm{B} 2$ \\
\hline RMST|196475 & $\mathrm{B} 2$ \\
\hline RNASE10|338879 & $\mathrm{B} 2$ \\
\hline RNASE11|122651 & $\mathrm{B} 2$ \\
\hline RNASE12|493901 & $\mathrm{B} 2$ \\
\hline RNASE2|6036 & $\mathrm{B} 2$ \\
\hline RNASE3|6037 & $\mathrm{B} 2$ \\
\hline RNASE6|6039 & $\mathrm{B} 2$ \\
\hline RNASE8|122665 & $\mathrm{B} 2$ \\
\hline RNASEH1|246243 & $\mathrm{B} 2$ \\
\hline RNASEH2B|79621 & $\mathrm{B} 2$ \\
\hline RNASEH2C|84153 & $\mathrm{B} 2$ \\
\hline RNASEK|440400 & B2 \\
\hline
\end{tabular}




\begin{tabular}{|c|c|}
\hline RNASEL|6041 & $\mathrm{B} 2$ \\
\hline RNASEN|29102 & B2 \\
\hline RNASET2|8635 & $\mathrm{B} 2$ \\
\hline RND2|8153 & $\mathrm{B} 2$ \\
\hline RNF10|9921 & $\mathrm{B} 2$ \\
\hline RNF111|54778 & $\mathrm{B} 2$ \\
\hline RNF113A|7737 & $\mathrm{B} 2$ \\
\hline RNF113B|140432 & $\mathrm{B} 2$ \\
\hline RNF114|55905 & $\mathrm{B} 2$ \\
\hline RNF121|55298 & $\mathrm{B} 2$ \\
\hline RNF122|79845 & $\mathrm{B} 2$ \\
\hline RNF123|63891 & $\mathrm{B} 2$ \\
\hline RNF126P1|376412 & $\mathrm{B} 2$ \\
\hline RNF126|55658 & $\mathrm{B} 2$ \\
\hline RNF128|79589 & $\mathrm{B} 2$ \\
\hline RNF133|168433 & $\mathrm{B} 2$ \\
\hline RNF135|84282 & B2 \\
\hline RNF138|51444 & $\mathrm{B} 2$ \\
\hline RNF13|11342 & $\mathrm{B} 2$ \\
\hline RNF141|50862 & $\mathrm{B} 2$ \\
\hline RNF144A|9781 & $\mathrm{B} 2$ \\
\hline RNF144B|255488 & $\mathrm{B} 2$ \\
\hline RNF148|378925 & $\mathrm{B} 2$ \\
\hline RNF14|9604 & $\mathrm{B} 2$ \\
\hline RNF151|146310 & $\mathrm{B} 2$ \\
\hline RNF152|220441 & $\mathrm{B} 2$ \\
\hline RNF160|26046 & $\mathrm{B} 2$ \\
\hline RNF166|115992 & $\mathrm{B} 2$ \\
\hline RNF167|26001 & $\mathrm{B} 2$ \\
\hline RNF168|165918 & $\mathrm{B} 2$ \\
\hline RNF169|254225 & $\mathrm{B} 2$ \\
\hline RNF170|81790 & B2 \\
\hline RNF175|285533 & $\mathrm{B} 2$ \\
\hline RNF17|56163 & $\mathrm{B} 2$ \\
\hline RNF182|221687 & $\mathrm{B} 2$ \\
\hline RNF183|138065 & $\mathrm{B} 2$ \\
\hline RNF19A|25897 & $\mathrm{B} 2$ \\
\hline \begin{tabular}{l|l|} 
RNF19B|127544 \\
\end{tabular} & $\mathrm{B} 2$ \\
\hline RNF207|388591 & $\mathrm{B} 2$ \\
\hline $\begin{array}{l}\text { RNF20|56254 } \\
\end{array}$ & $\mathrm{B} 2$ \\
\hline RNF212|285498 & $\mathrm{B} 2$ \\
\hline RNF215|200312 & $\mathrm{B} 2$ \\
\hline RNF216L|441191 & $\mathrm{B} 2$ \\
\hline RNF216|54476 & $\mathrm{B} 2$ \\
\hline RNF219|79596 & $\mathrm{B} 2$ \\
\hline RNF220|55182 & $\mathrm{B} 2$ \\
\hline \begin{tabular}{l|l|} 
RNF222|643904 \\
\end{tabular} & $\mathrm{B} 2$ \\
\hline RNF24|11237 & $\mathrm{B} 2$ \\
\hline RNF25|64320 & $\mathrm{B} 2$ \\
\hline RNF26|79102 & $\mathrm{B} 2$ \\
\hline RNF31|55072 & $\mathrm{B} 2$ \\
\hline
\end{tabular}




\begin{tabular}{|c|c|}
\hline RNF32|140545 & B2 \\
\hline RNF39|80352 & B2 \\
\hline RNF43|54894 & B2 \\
\hline RNF44|22838 & B2 \\
\hline RNF5P1|286140 & B2 \\
\hline RNF5|6048 & B2 \\
\hline RNF6|6049 & B2 \\
\hline RNF8|9025 & B2 \\
\hline RNFT1|51136 & B2 \\
\hline RNGTT|8732 & B2 \\
\hline RNH1|6050 & $\mathrm{B} 2$ \\
\hline RNMTL1|55178 & B2 \\
\hline RNMT|8731 & $\mathrm{B} 2$ \\
\hline RNPEPL1|57140 & $\mathrm{B} 2$ \\
\hline RNU11|26824 & $\mathrm{B} 2$ \\
\hline RNU4ATAC|100151683 & B2 \\
\hline RNU6ATAC|100151684 & $\mathrm{B} 2$ \\
\hline ROBO1|6091 & B2 \\
\hline ROBO2|6092 & $\mathrm{B} 2$ \\
\hline ROCK1|6093 & $\mathrm{B} 2$ \\
\hline ROM1|6094 & $\mathrm{B} 2$ \\
\hline ROMO1|140823 & $\mathrm{B} 2$ \\
\hline ROPN1B|152015 & $\mathrm{B} 2$ \\
\hline ROPN1L|83853 & B2 \\
\hline ROPN1|54763 & $\mathrm{B} 2$ \\
\hline RORA|6095 & $\mathrm{B} 2$ \\
\hline RORB|6096 & $\mathrm{B} 2$ \\
\hline RORC|6097 & B2 \\
\hline ROS1|6098 & B2 \\
\hline RP1-177G6.2|286411 & $\mathrm{B} 2$ \\
\hline RP1|6101 & $\mathrm{B} 2$ \\
\hline RP2|6102 & $\mathrm{B} 2$ \\
\hline RP9P|441212 & $\mathrm{B} 2$ \\
\hline RP9|6100 & $\mathrm{B} 2$ \\
\hline RPA1|6117 & $\mathrm{B} 2$ \\
\hline RPA2|6118 & B2 \\
\hline RPA4|29935 & $\mathrm{B} 2$ \\
\hline RPAIN|84268 & $\mathrm{B} 2$ \\
\hline RPAP1|26015 & $\mathrm{B} 2$ \\
\hline RPAP2|79871 & $\mathrm{B} 2$ \\
\hline RPF1|80135 & $\mathrm{B} 2$ \\
\hline RPF2|84154 & $\mathrm{B} 2$ \\
\hline RPGRIP1|57096 & $\mathrm{B} 2$ \\
\hline RPH3AL|9501 & B2 \\
\hline RPH3A|22895 & $\mathrm{B} 2$ \\
\hline RPIA|22934 & $\mathrm{B} 2$ \\
\hline RPL10L|140801 & $\mathrm{B} 2$ \\
\hline RPL10|6134 & B2 \\
\hline RPL12|6136 & $\mathrm{B} 2$ \\
\hline RPL13AP17|399670 & $\mathrm{B} 2$ \\
\hline RPL13AP20|387841 & B2 \\
\hline
\end{tabular}




\begin{tabular}{|c|c|}
\hline RPL13AP3|645683 & B2 \\
\hline RPL13AP6|644511 & B2 \\
\hline RPL13A|23521 & $\mathrm{B} 2$ \\
\hline RPL13P5|283345 & $\mathrm{B} 2$ \\
\hline RPL13|6137 & B2 \\
\hline RPL14|9045 & $\mathrm{B} 2$ \\
\hline RPL17|6139 & $\mathrm{B} 2$ \\
\hline RPL18A|6142 & $\mathrm{B} 2$ \\
\hline RPL18|6141 & $\mathrm{B} 2$ \\
\hline RPL19P12|100129424 & $\mathrm{B} 2$ \\
\hline RPL19|6143 & $\mathrm{B} 2$ \\
\hline RPL22L1|200916 & $\mathrm{B} 2$ \\
\hline RPL23AP7|118433 & $\mathrm{B} 2$ \\
\hline RPL23AP82|284942 & $\mathrm{B} 2$ \\
\hline RPL23A|6147 & $\mathrm{B} 2$ \\
\hline RPL23P8|222901 & $\mathrm{B} 2$ \\
\hline RPL23|9349 & $\mathrm{B} 2$ \\
\hline RPL24|6152 & $\mathrm{B} 2$ \\
\hline RPL27A|6157 & $\mathrm{B} 2$ \\
\hline RPL27|6155 & $\mathrm{B} 2$ \\
\hline RPL28|6158 & $\mathrm{B} 2$ \\
\hline RPL29P2|118432 & $\mathrm{B} 2$ \\
\hline RPL29|6159 & $\mathrm{B} 2$ \\
\hline RPL30|6156 & $\mathrm{B} 2$ \\
\hline RPL31|6160 & B2 \\
\hline RPL32P3|132241 & $\mathrm{B} 2$ \\
\hline RPL35A|6165 & $\mathrm{B} 2$ \\
\hline RPL35|11224 & $\mathrm{B} 2$ \\
\hline RPL36AL|6166 & $\mathrm{B} 2$ \\
\hline RPL36A|6173 & $\mathrm{B} 2$ \\
\hline RPL36|25873 & $\mathrm{B} 2$ \\
\hline RPL37A|6168 & $\mathrm{B} 2$ \\
\hline RPL37|6167 & $\mathrm{B} 2$ \\
\hline RPL38|6169 & $\mathrm{B} 2$ \\
\hline RPL39|6170 & $\mathrm{B} 2$ \\
\hline RPL3L|6123 & $\mathrm{B} 2$ \\
\hline RPL41|6171 & $\mathrm{B} 2$ \\
\hline RPL6|6128 & $\mathrm{B} 2$ \\
\hline RPL7A|6130 & $\mathrm{B} 2$ \\
\hline RPL7L1|285855 & $\mathrm{B} 2$ \\
\hline RPL7|6129 & $\mathrm{B} 2$ \\
\hline RPL8|6132 & $\mathrm{B} 2$ \\
\hline RPL9|6133 & $\mathrm{B} 2$ \\
\hline RPLP0|6175 & $\mathrm{B} 2$ \\
\hline RPLP1|6176 & $\mathrm{B} 2$ \\
\hline RPLP2|6181 & $\mathrm{B} 2$ \\
\hline RPP14|11102 & $\mathrm{B} 2$ \\
\hline RPP25|54913 & $\mathrm{B} 2$ \\
\hline RPP38|10557 & $\mathrm{B} 2$ \\
\hline RPPH1|85495 & $\mathrm{B} 2$ \\
\hline RPRD1A|55197 & $\mathrm{B} 2$ \\
\hline
\end{tabular}




\begin{tabular}{|c|c|}
\hline RPRD1B|58490 & B2 \\
\hline RPRML/388394 & $\mathrm{B} 2$ \\
\hline RPRM|56475 & $\mathrm{B} 2$ \\
\hline RPS10P7|376693 & $\mathrm{B} 2$ \\
\hline RPS10|6204 & $\mathrm{B} 2$ \\
\hline RPS11|6205 & $\mathrm{B} 2$ \\
\hline RPS12|6206 & $\mathrm{B} 2$ \\
\hline RPS15AP10|728963 & $\mathrm{B} 2$ \\
\hline RPS15A|6210 & $\mathrm{B} 2$ \\
\hline RPS15|6209 & $\mathrm{B} 2$ \\
\hline RPS16|6217 & $\mathrm{B} 2$ \\
\hline RPS17|6218 & $\mathrm{B} 2$ \\
\hline RPS18|6222 & $\mathrm{B} 2$ \\
\hline RPS19BP1|91582 & $\mathrm{B} 2$ \\
\hline RPS19|6223 & $\mathrm{B} 2$ \\
\hline RPS21|6227 & $\mathrm{B} 2$ \\
\hline RPS24|6229 & B2 \\
\hline RPS26P11|441502 & $\mathrm{B} 2$ \\
\hline RPS26|6231 & $\mathrm{B} 2$ \\
\hline RPS27L|51065 & $\mathrm{B} 2$ \\
\hline RPS27|6232 & $\mathrm{B} 2$ \\
\hline RPS28|6234 & $\mathrm{B} 2$ \\
\hline RPS29|6235 & $\mathrm{B} 2$ \\
\hline RPS2P32|256355 & $\mathrm{B} 2$ \\
\hline RPS2|6187 & $\mathrm{B} 2$ \\
\hline RPS3|6188 & $\mathrm{B} 2$ \\
\hline RPS4X|6191 & $\mathrm{B} 2$ \\
\hline RPS4Y1|6192 & $\mathrm{B} 2$ \\
\hline RPS5|6193 & $\mathrm{B} 2$ \\
\hline RPS6KA4|8986 & $\mathrm{B} 2$ \\
\hline RPS6KA6|27330 & $\mathrm{B} 2$ \\
\hline RPS6KB1|6198 & $\mathrm{B} 2$ \\
\hline RPS6KL1|83694 & $\mathrm{B} 2$ \\
\hline RPS6|6194 & $\mathrm{B} 2$ \\
\hline RPS7|6201 & $\mathrm{B} 2$ \\
\hline RPS8|6202 & $\mathrm{B} 2$ \\
\hline RPSAP52|204010 & $\mathrm{B} 2$ \\
\hline RPSAP58|388524 & $\mathrm{B} 2$ \\
\hline RPSAP9|653162 & $\mathrm{B} 2$ \\
\hline RPSA|3921 & $\mathrm{B} 2$ \\
\hline RPTN|126638 & $\mathrm{B} 2$ \\
\hline RPTOR|57521 & $\mathrm{B} 2$ \\
\hline RPUSD2|27079 & $\mathrm{B} 2$ \\
\hline RPUSD3|285367 & $\mathrm{B} 2$ \\
\hline RRAD|6236 & $\mathrm{B} 2$ \\
\hline RRAGA|10670 & $\mathrm{B} 2$ \\
\hline RRAGB|10325 & $\mathrm{B} 2$ \\
\hline RRAGC|64121 & $\mathrm{B} 2$ \\
\hline RRBP1|6238 & $\mathrm{B} 2$ \\
\hline RREB1|6239 & $\mathrm{B} 2$ \\
\hline RRH|10692 & B2 \\
\hline
\end{tabular}




\begin{tabular}{|c|c|}
\hline RRM2B|50484 & B2 \\
\hline RRN3P2|653390 & $\mathrm{B} 2$ \\
\hline RRN3P3|100131998 & $\mathrm{B} 2$ \\
\hline RRP15|51018 & $\mathrm{B} 2$ \\
\hline RRP1B|23076 & $\mathrm{B} 2$ \\
\hline RRP1|8568 & $\mathrm{B} 2$ \\
\hline RRP7A|27341 & $\mathrm{B} 2$ \\
\hline RRP7B|91695 & $\mathrm{B} 2$ \\
\hline RRP8|23378 & $\mathrm{B} 2$ \\
\hline RRP9|9136 & B2 \\
\hline RS1|6247 & $\mathrm{B} 2$ \\
\hline RSAD1|55316 & B2 \\
\hline RSBN1L|222194 & $\mathrm{B} 2$ \\
\hline RSBN1|54665 & $\mathrm{B} 2$ \\
\hline RSC1A1|6248 & $\mathrm{B} 2$ \\
\hline RSF1|51773 & $\mathrm{B} 2$ \\
\hline RSL1D1|26156 & B2 \\
\hline RSL24D1|51187 & $\mathrm{B} 2$ \\
\hline RSPH10B2|728194 & B2 \\
\hline RSPH3|83861 & $\mathrm{B} 2$ \\
\hline RSPH4A|345895 & $\mathrm{B} 2$ \\
\hline RSPH6A|81492 & $\mathrm{B} 2$ \\
\hline \begin{tabular}{l|l|} 
RSPH9|221421 \\
\end{tabular} & $\mathrm{B} 2$ \\
\hline RSPO1|284654 & $\mathrm{B} 2$ \\
\hline RSPO2|340419 & $\mathrm{B} 2$ \\
\hline RSPO4|343637 & $\mathrm{B} 2$ \\
\hline RSPRY1|89970 & $\mathrm{B} 2$ \\
\hline RSRC1|51319 & B2 \\
\hline RSRC2|65117 & $\mathrm{B} 2$ \\
\hline RSU1|6251 & $\mathrm{B} 2$ \\
\hline RTBDN|83546 & $\mathrm{B} 2$ \\
\hline RTDR1|27156 & $\mathrm{B} 2$ \\
\hline RTEL1|51750 & $\mathrm{B} 2$ \\
\hline RTF1|23168 & $\mathrm{B} 2$ \\
\hline RTL1|388015 & $\mathrm{B} 2$ \\
\hline RTN1|6252 & $\mathrm{B} 2$ \\
\hline RTN2|6253 & $\mathrm{B} 2$ \\
\hline RTN3|10313 & $\mathrm{B} 2$ \\
\hline RTN4IP1|84816 & B2 \\
\hline RTN4RL1|146760 & $\mathrm{B} 2$ \\
\hline RTN4R|65078 & B2 \\
\hline RTP1|132112 & $\mathrm{B} 2$ \\
\hline RTP2|344892 & B2 \\
\hline RTP3|83597 & $\mathrm{B} 2$ \\
\hline RTP4|64108 & $\mathrm{B} 2$ \\
\hline RTTN|25914 & $\mathrm{B} 2$ \\
\hline $\begin{array}{l}\text { RUFY1|80230 } \\
\end{array}$ & $\mathrm{B} 2$ \\
\hline RUFY2|55680 & $\mathrm{B} 2$ \\
\hline RUFY3|22902 & $\mathrm{B} 2$ \\
\hline RUFY4|285180 & $\mathrm{B} 2$ \\
\hline RUNDC1|146923 & $\mathrm{B} 2$ \\
\hline
\end{tabular}




\begin{tabular}{|c|c|}
\hline RUNDC2A|84127 & B2 \\
\hline RUNDC2C|440352 & B2 \\
\hline RUNDC3A|10900 & $\mathrm{B} 2$ \\
\hline RUNX1|861 & $\mathrm{B} 2$ \\
\hline RUNX3|864 & $\mathrm{B} 2$ \\
\hline RWDD1|51389 & $\mathrm{B} 2$ \\
\hline RWDD2A|112611 & $\mathrm{B} 2$ \\
\hline RWDD2B|10069 & $\mathrm{B} 2$ \\
\hline RWDD3|25950 & $\mathrm{B} 2$ \\
\hline RWDD4A|201965 & $\mathrm{B} 2$ \\
\hline RXFP1|59350 & $\mathrm{B} 2$ \\
\hline RXFP2|122042 & $\mathrm{B} 2$ \\
\hline RXFP4|339403 & $\mathrm{B} 2$ \\
\hline RXRA|6256 & $\mathrm{B} 2$ \\
\hline RXRB|6257 & $\mathrm{B} 2$ \\
\hline RXRG|6258 & $\mathrm{B} 2$ \\
\hline RYBP|23429 & $\mathrm{B} 2$ \\
\hline RYR1|6261 & $\mathrm{B} 2$ \\
\hline RYR2|6262 & $\mathrm{B} 2$ \\
\hline S100A10|6281 & $\mathrm{B} 2$ \\
\hline \begin{tabular}{l|l|l|} 
S100A12 6283 \\
\end{tabular} & $\mathrm{~B} 2$ \\
\hline \begin{tabular}{l|l|l|} 
S100A13|6284 \\
\end{tabular} & $\mathrm{B} 2$ \\
\hline S100A1|6271 & $\mathrm{B} 2$ \\
\hline S100A2|6273 & $\mathrm{B} 2$ \\
\hline S100A3|6274 & $\mathrm{B} 2$ \\
\hline S100A5|6276 & $\mathrm{B} 2$ \\
\hline S100A6|6277 & $\mathrm{B} 2$ \\
\hline S100A7A|338324 & $\mathrm{B} 2$ \\
\hline S100A7|6278 & $\mathrm{B} 2$ \\
\hline S100A8|6279 & $\mathrm{B} 2$ \\
\hline S100A9|6280 & $\mathrm{B} 2$ \\
\hline S100G|795 & $\mathrm{B} 2$ \\
\hline S100PBP|64766 & $\mathrm{B} 2$ \\
\hline S100P|6286 & $\mathrm{B} 2$ \\
\hline S100Z|170591 & $\mathrm{B} 2$ \\
\hline S1PR2|9294 & $\mathrm{B} 2$ \\
\hline S1PR3|1903 & $\mathrm{B} 2$ \\
\hline S1PR4|8698 & $\mathrm{B} 2$ \\
\hline S1PR5|53637 & $\mathrm{B} 2$ \\
\hline SACM1L|22908 & $\mathrm{B} 2$ \\
\hline SAFB2|9667 & $\mathrm{B} 2$ \\
\hline SAFB|6294 & $\mathrm{B} 2$ \\
\hline SAGE1|55511 & $\mathrm{B} 2$ \\
\hline SAG|6295 & B2 \\
\hline SALL1|6299 & $\mathrm{B} 2$ \\
\hline $\begin{array}{l}\text { SALL2|6297 } \\
\end{array}$ & $\mathrm{B} 2$ \\
\hline $\begin{array}{l}\text { SALL3|27164 } \\
\end{array}$ & $\mathrm{B} 2$ \\
\hline SAMD13|148418 & $\mathrm{B} 2$ \\
\hline SAMD3|154075 & $\mathrm{B} 2$ \\
\hline SAMD4B|55095 & $\mathrm{B} 2$ \\
\hline SAMD7|344658 & $\mathrm{B} 2$ \\
\hline
\end{tabular}




\begin{tabular}{|c|c|}
\hline SAMD8|142891 & B2 \\
\hline SAMD9L|219285 & $\mathrm{B} 2$ \\
\hline SAMD9|54809 & $\mathrm{B} 2$ \\
\hline SAMHD1|25939 & $\mathrm{B} 2$ \\
\hline SAMM50|25813 & $\mathrm{B} 2$ \\
\hline SAP18|10284 & $\mathrm{B} 2$ \\
\hline SAP30BP|29115 & $\mathrm{B} 2$ \\
\hline SAPS2|9701 & $\mathrm{B} 2$ \\
\hline SAPS3|55291 & $\mathrm{B} 2$ \\
\hline SAR1A|56681 & $\mathrm{B} 2$ \\
\hline SARDH|1757 & $\mathrm{B} 2$ \\
\hline SARM1|23098 & $\mathrm{B} 2$ \\
\hline \begin{tabular}{l|l|l|} 
SARS2|54938 \\
\end{tabular} & $\mathrm{B} 2$ \\
\hline SARS|6301 & $\mathrm{B} 2$ \\
\hline SART1|9092 & $\mathrm{B} 2$ \\
\hline SART3|9733 & $\mathrm{B} 2$ \\
\hline \begin{tabular}{l|l|} 
SASH3 54440 \\
\end{tabular} & $\mathrm{~B} 2$ \\
\hline SAT1|6303 & $\mathrm{B} 2$ \\
\hline SATB2|23314 & $\mathrm{B} 2$ \\
\hline SATL1|340562 & $\mathrm{B} 2$ \\
\hline SBDSP1|155370 & $\mathrm{B} 2$ \\
\hline SBF1P1|100133234 & $\mathrm{B} 2$ \\
\hline SBK2|646643 & $\mathrm{B} 2$ \\
\hline \begin{tabular}{l|l|} 
SBNO15206 &
\end{tabular} & B2 \\
\hline \begin{tabular}{l|l|l|} 
SBNO22904 \\
\end{tabular} & $\mathrm{B} 2$ \\
\hline SBSN|374897 & $\mathrm{B} 2$ \\
\hline SC4MOL|6307 & $\mathrm{B} 2$ \\
\hline SC5DL|6309 & $\mathrm{B} 2$ \\
\hline SCAF1|58506 & $\mathrm{B} 2$ \\
\hline SCAI|286205 & $\mathrm{B} 2$ \\
\hline SCAMP1|9522 & $\mathrm{B} 2$ \\
\hline SCAMP2|10066 & $\mathrm{B} 2$ \\
\hline SCAMP4|113178 & $\mathrm{B} 2$ \\
\hline SCAMP5|192683 & $\mathrm{B} 2$ \\
\hline SCAND1|51282 & $\mathrm{B} 2$ \\
\hline SCAND2|54581 & $\mathrm{B} 2$ \\
\hline SCAND3|114821 & $\mathrm{B} 2$ \\
\hline SCAP|22937 & $\mathrm{B} 2$ \\
\hline SCARA3|51435 & $\mathrm{B} 2$ \\
\hline SCARB1|949 & $\mathrm{B} 2$ \\
\hline SCARB2|950 & $\mathrm{B} 2$ \\
\hline SCARF2|91179 & $\mathrm{B} 2$ \\
\hline SCARNA10|692148 & $\mathrm{B} 2$ \\
\hline SCARNA12|677777 & $\mathrm{B} 2$ \\
\hline SCARNA16|677781 & $\mathrm{B} 2$ \\
\hline $\begin{array}{l}\text { SCARNA1|677774 } \\
\end{array}$ & $\mathrm{B} 2$ \\
\hline SCARNA21|677763 & $\mathrm{B} 2$ \\
\hline SCARNA2|677766 & $\mathrm{B} 2$ \\
\hline SCARNA4|677771 & $\mathrm{B} 2$ \\
\hline SCARNA5|677775 & $\mathrm{B} 2$ \\
\hline SCARNA6|677772 & B2 \\
\hline
\end{tabular}




\begin{tabular}{|c|c|}
\hline SCARNA7|677767 & B2 \\
\hline SCARNA8|677776 & $\mathrm{B} 2$ \\
\hline SCARNA9L|100158262 & $\mathrm{B} 2$ \\
\hline SCARNA9|619383 & $\mathrm{B} 2$ \\
\hline SCD5|79966 & B2 \\
\hline $\operatorname{SCD} \mid 6319$ & $\mathrm{~B} 2$ \\
\hline SCEL|8796 & $\mathrm{B} 2$ \\
\hline SCFD1|23256 & $\mathrm{B} 2$ \\
\hline SCFD2|152579 & $\mathrm{B} 2$ \\
\hline SCG2|7857 & $\mathrm{B} 2$ \\
\hline SCG3|29106 & $\mathrm{B} 2$ \\
\hline SCG5|6447 & $\mathrm{B} 2$ \\
\hline SCGB1A1|7356 & $\mathrm{B} 2$ \\
\hline SCGB1C1|147199 & $\mathrm{B} 2$ \\
\hline SCGB1D1|10648 & $\mathrm{B} 2$ \\
\hline SCGB1D2|10647 & $\mathrm{B} 2$ \\
\hline SCGB1D4|404552 & $\mathrm{B} 2$ \\
\hline SCGB2A1|4246 & $\mathrm{B} 2$ \\
\hline SCGB2A2|4250 & $\mathrm{B} 2$ \\
\hline SCGB3A1|92304 & $\mathrm{B} 2$ \\
\hline SCGB3A2|117156 & $\mathrm{B} 2$ \\
\hline SCGBL|284402 & $\mathrm{B} 2$ \\
\hline SCGN|10590 & $\mathrm{B} 2$ \\
\hline SCHIP1|29970 & $\mathrm{B} 2$ \\
\hline SCIN|85477 & $\mathrm{B} 2$ \\
\hline SCLT1|132320 & $\mathrm{B} 2$ \\
\hline SCML2|10389 & $\mathrm{B} 2$ \\
\hline SCML4|256380 & $\mathrm{B} 2$ \\
\hline SCN10A|6336 & $\mathrm{B} 2$ \\
\hline SCN1A|6323 & $\mathrm{B} 2$ \\
\hline SCN1B|6324 & $\mathrm{B} 2$ \\
\hline SCN4A|6329 & $\mathrm{B} 2$ \\
\hline SCN7A|6332 & $\mathrm{B} 2$ \\
\hline SCN8A|6334 & $\mathrm{B} 2$ \\
\hline SCNN1A|6337 & $\mathrm{B} 2$ \\
\hline SCNN1B|6338 & $\mathrm{B} 2$ \\
\hline SCNN1D|6339 & $\mathrm{B} 2$ \\
\hline SCNN1G|6340 & $\mathrm{B} 2$ \\
\hline SCO1|6341 & $\mathrm{B} 2$ \\
\hline SCOC $\mid 60592$ & $\mathrm{~B} 2$ \\
\hline SCP2|6342 & $\mathrm{B} 2$ \\
\hline SCPEP1|59342 & $\mathrm{B} 2$ \\
\hline SCRG1|11341 & $\mathrm{B} 2$ \\
\hline SCRIB|23513 & $\mathrm{B} 2$ \\
\hline SCRN1|9805 & $\mathrm{B} 2$ \\
\hline \begin{tabular}{l|l|l|} 
SCRN3|79634
\end{tabular} & $\mathrm{B} 2$ \\
\hline SCRT1|83482 & $\mathrm{B} 2$ \\
\hline \begin{tabular}{l|l|} 
SCRT2|8508 &
\end{tabular} & $\mathrm{B} 2$ \\
\hline SCUBE1|80274 & $\mathrm{B} 2$ \\
\hline SCUBE2|57758 & $\mathrm{B} 2$ \\
\hline SCUBE3|222663 & $\mathrm{B} 2$ \\
\hline
\end{tabular}




\begin{tabular}{|c|c|}
\hline SCXB $\mid 642658$ & B2 \\
\hline SCYL1|57410 & $\mathrm{B} 2$ \\
\hline SDAD1|55153 & $\mathrm{B} 2$ \\
\hline SDC2|6383 & $\mathrm{B} 2$ \\
\hline SDC4|6385 & B2 \\
\hline SDCBP2|27111 & $\mathrm{B} 2$ \\
\hline SDCBP|6386 & $\mathrm{B} 2$ \\
\hline SDCCAG1|9147 & $\mathrm{B} 2$ \\
\hline SDCCAG3|10807 & $\mathrm{B} 2$ \\
\hline SDCCAG8|10806 & $\mathrm{B} 2$ \\
\hline SDF2|6388 & $\mathrm{B} 2$ \\
\hline SDF4|51150 & $\mathrm{B} 2$ \\
\hline SDHAF1|644096 & $\mathrm{B} 2$ \\
\hline SDHAF2|54949 & $\mathrm{B} 2$ \\
\hline SDHA|6389 & $\mathrm{B} 2$ \\
\hline SDHB|6390 & $\mathrm{B} 2$ \\
\hline SDK2|54549 & $\mathrm{B} 2$ \\
\hline SDR16C5|195814 & $\mathrm{B} 2$ \\
\hline \begin{tabular}{l|l|} 
SDR39U1|56948 &
\end{tabular} & $\mathrm{B} 2$ \\
\hline SDR42E1|93517 & $\mathrm{B} 2$ \\
\hline SDR9C7|121214 & $\mathrm{B} 2$ \\
\hline SEBOX|645832 & $\mathrm{B} 2$ \\
\hline SEC11A|23478 & $\mathrm{B} 2$ \\
\hline SEC14L2|23541 & $\mathrm{B} 2$ \\
\hline SEC14L3|266629 & $\mathrm{B} 2$ \\
\hline SEC14L4|284904 & $\mathrm{B} 2$ \\
\hline SEC14L5|9717 & $\mathrm{B} 2$ \\
\hline SEC22A|26984 & $\mathrm{B} 2$ \\
\hline SEC22B|9554 & $\mathrm{B} 2$ \\
\hline SEC23A|10484 & $\mathrm{B} 2$ \\
\hline SEC23IP|11196 & $\mathrm{B} 2$ \\
\hline SEC24A|10802 & $\mathrm{B} 2$ \\
\hline SEC24B|10427 & $\mathrm{B} 2$ \\
\hline $\begin{array}{l}\text { SEC24C|9632 } \\
\end{array}$ & $\mathrm{B} 2$ \\
\hline SEC62|7095 & $\mathrm{B} 2$ \\
\hline SEC63|11231 & $\mathrm{B} 2$ \\
\hline SECISBP2|79048 & $\mathrm{B} 2$ \\
\hline SEH1L|81929 & $\mathrm{B} 2$ \\
\hline \begin{tabular}{l|l|} 
SEL1L2|80343 \\
\end{tabular} & $\mathrm{B} 2$ \\
\hline SEL1L3|23231 & $\mathrm{B} 2$ \\
\hline SEL1L|6400 & $\mathrm{B} 2$ \\
\hline SELENBP1|8991 & $\mathrm{B} 2$ \\
\hline SELE|6401 & $\mathrm{B} 2$ \\
\hline SELK|58515 & $\mathrm{B} 2$ \\
\hline SELL|6402 & $\mathrm{B} 2$ \\
\hline SELM|140606 & $\mathrm{B} 2$ \\
\hline SELO|83642 & $\mathrm{B} 2$ \\
\hline SELPLG|6404 & $\mathrm{B} 2$ \\
\hline SELV|348303 & $\mathrm{B} 2$ \\
\hline SEMA3B|7869 & $\mathrm{B} 2$ \\
\hline SEMA3E|9723 & $\mathrm{B} 2$ \\
\hline
\end{tabular}




\begin{tabular}{|c|c|}
\hline SEMA4A|64218 & B2 \\
\hline SEMA4B|10509 & B2 \\
\hline SEMA4C|54910 & $\mathrm{B} 2$ \\
\hline SEMA4D|10507 & $\mathrm{B} 2$ \\
\hline SEMA4F|10505 & B2 \\
\hline SEMA4G|57715 & $\mathrm{B} 2$ \\
\hline SEMA5B|54437 & $\mathrm{B} 2$ \\
\hline SEMA6B|10501 & $\mathrm{B} 2$ \\
\hline SEMG1|6406 & $\mathrm{B} 2$ \\
\hline SEMG2|6407 & $\mathrm{B} 2$ \\
\hline SENP2|59343 & $\mathrm{B} 2$ \\
\hline SENP3|26168 & $\mathrm{B} 2$ \\
\hline SENP5|205564 & $\mathrm{B} 2$ \\
\hline SENP6|26054 & $\mathrm{B} 2$ \\
\hline SENP7|57337 & $\mathrm{B} 2$ \\
\hline SENP8|123228 & $\mathrm{B} 2$ \\
\hline SEP15|9403 & $\mathrm{B} 2$ \\
\hline SEPHS1|22929 & $\mathrm{B} 2$ \\
\hline SEPN1|57190 & $\mathrm{B} 2$ \\
\hline SEPSECS|51091 & $\mathrm{B} 2$ \\
\hline SEPT11|55752 & $\mathrm{B} 2$ \\
\hline SEPT12|124404 & $\mathrm{B} 2$ \\
\hline SEPT14|346288 & $\mathrm{B} 2$ \\
\hline SEPT1|1731 & $\mathrm{B} 2$ \\
\hline SEPT2|4735 & $\mathrm{B} 2$ \\
\hline SEPT3|55964 & $\mathrm{B} 2$ \\
\hline SEPT5|5413 & $\mathrm{B} 2$ \\
\hline SEPT6|23157 & $\mathrm{B} 2$ \\
\hline SEPT7L|285961 & $\mathrm{B} 2$ \\
\hline SEPT7P2|641977 & $\mathrm{B} 2$ \\
\hline SEPT7|989 & $\mathrm{B} 2$ \\
\hline SEPW1|6415 & $\mathrm{B} 2$ \\
\hline SERAC1|84947 & $\mathrm{B} 2$ \\
\hline SERBP1|26135 & $\mathrm{B} 2$ \\
\hline SERF1A|8293 & $\mathrm{B} 2$ \\
\hline SERF2|10169 & $\mathrm{B} 2$ \\
\hline SERGEF|26297 & $\mathrm{B} 2$ \\
\hline SERHL2|253190 & $\mathrm{B} 2$ \\
\hline SERHL|94009 & $\mathrm{B} 2$ \\
\hline SERINC3|10955 & $\mathrm{B} 2$ \\
\hline SERINC4|619189 & $\mathrm{B} 2$ \\
\hline SERINC5|256987 & $\mathrm{B} 2$ \\
\hline SERP2|387923 & $\mathrm{B} 2$ \\
\hline SERPINA10|51156 & $\mathrm{B} 2$ \\
\hline SERPINA11|256394 & $\mathrm{B} 2$ \\
\hline SERPINA12|145264 & $\mathrm{B} 2$ \\
\hline SERPINA13|388007 & $\mathrm{B} 2$ \\
\hline SERPINA1|5265 & $\mathrm{B} 2$ \\
\hline SERPINA3|12 & $\mathrm{B} 2$ \\
\hline SERPINA4|5267 & $\mathrm{B} 2$ \\
\hline $\begin{array}{l}\text { SERPINA5|5104 } \\
\text { S }\end{array}$ & $\mathrm{B} 2$ \\
\hline
\end{tabular}




\begin{tabular}{|c|c|}
\hline SERPINA6|866 & B2 \\
\hline SERPINA9|327657 & B2 \\
\hline SERPINB10|5273 & $\mathrm{B} 2$ \\
\hline SERPINB11|89778 & $\mathrm{B} 2$ \\
\hline SERPINB12|89777 & B2 \\
\hline SERPINB13|5275 & $\mathrm{B} 2$ \\
\hline SERPINB1|1992 & $\mathrm{B} 2$ \\
\hline SERPINB2|5055 & $\mathrm{B} 2$ \\
\hline SERPINB3|6317 & $\mathrm{B} 2$ \\
\hline SERPINB4|6318 & $\mathrm{B} 2$ \\
\hline SERPINB5|5268 & $\mathrm{B} 2$ \\
\hline SERPINB6|5269 & $\mathrm{B} 2$ \\
\hline SERPINB7|8710 & $\mathrm{B} 2$ \\
\hline SERPINB8|5271 & $\mathrm{B} 2$ \\
\hline SERPINB9|5272 & $\mathrm{B} 2$ \\
\hline SERPINC1|462 & $\mathrm{B} 2$ \\
\hline SERPIND1|3053 & B2 \\
\hline SERPINE1|5054 & $\mathrm{B} 2$ \\
\hline SERPINE2|5270 & $\mathrm{B} 2$ \\
\hline SERPINE3|647174 & $\mathrm{B} 2$ \\
\hline SERPINI1|5274 & $\mathrm{B} 2$ \\
\hline SERPINI2|5276 & $\mathrm{B} 2$ \\
\hline SERTAD1|29950 & $\mathrm{B} 2$ \\
\hline SERTAD2|9792 & $\mathrm{B} 2$ \\
\hline SERTAD3|29946 & $\mathrm{B} 2$ \\
\hline SERTAD4|56256 & $\mathrm{B} 2$ \\
\hline SESN2|83667 & $\mathrm{B} 2$ \\
\hline SESN3|143686 & $\mathrm{B} 2$ \\
\hline SESTD1|91404 & $\mathrm{B} 2$ \\
\hline SETD1A|9739 & $\mathrm{B} 2$ \\
\hline SETD1B|23067 & $\mathrm{B} 2$ \\
\hline SETD2|29072 & $\mathrm{B} 2$ \\
\hline SETD3|84193 & $\mathrm{B} 2$ \\
\hline $\begin{array}{l}\text { SETD4|54093 } \\
\text { SET }\end{array}$ & $\mathrm{B} 2$ \\
\hline SETD5|55209 & $\mathrm{B} 2$ \\
\hline SETD6|79918 & $\mathrm{B} 2$ \\
\hline SETD8|387893 & $\mathrm{B} 2$ \\
\hline SEZ6L|23544 & $\mathrm{B} 2$ \\
\hline SEZ6|124925 & $\mathrm{B} 2$ \\
\hline SF3A2|8175 & $\mathrm{B} 2$ \\
\hline SF3A3|10946 & $\mathrm{B} 2$ \\
\hline SF3B1|23451 & $\mathrm{B} 2$ \\
\hline SF3B2|10992 & $\mathrm{B} 2$ \\
\hline SF3B3|23450 & $\mathrm{B} 2$ \\
\hline SF3B5|83443 & $\mathrm{B} 2$ \\
\hline SF4|57794 & $\mathrm{B} 2$ \\
\hline SFI1|9814 & $\mathrm{B} 2$ \\
\hline SFMBT1|51460 & $\mathrm{B} 2$ \\
\hline SFMBT2|57713 & $\mathrm{B} 2$ \\
\hline SFN|2810 & $\mathrm{B} 2$ \\
\hline SFRP4|6424 & $\mathrm{B} 2$ \\
\hline
\end{tabular}




\begin{tabular}{|c|c|}
\hline SFRP5|6425 & B2 \\
\hline SFRS11|9295 & B2 \\
\hline SFRS12IP1|285672 & $\mathrm{B} 2$ \\
\hline SFRS12|140890 & $\mathrm{B} 2$ \\
\hline SFRS13A|10772 & B2 \\
\hline SFRS13B|135295 & $\mathrm{B} 2$ \\
\hline SFRS14|10147 & $\mathrm{B} 2$ \\
\hline SFRS15|57466 & $\mathrm{B} 2$ \\
\hline SFRS16|11129 & B2 \\
\hline SFRS17A|8227 & $\mathrm{B} 2$ \\
\hline SFRS2IP|9169 & $\mathrm{B} 2$ \\
\hline SFRS3|6428 & $\mathrm{B} 2$ \\
\hline SFRS6|6431 & $\mathrm{B} 2$ \\
\hline SFRS7|6432 & $\mathrm{B} 2$ \\
\hline SFRS8|6433 & $\mathrm{B} 2$ \\
\hline SFT2D1|113402 & $\mathrm{B} 2$ \\
\hline SFT2D2|375035 & $\mathrm{B} 2$ \\
\hline SFT2D3|84826 & $\mathrm{B} 2$ \\
\hline SFTA1P|207107 & $\mathrm{B} 2$ \\
\hline SFTA2|389376 & $\mathrm{B} 2$ \\
\hline $\begin{array}{l}\text { SFTA3|253970 } \\
\end{array}$ & $\mathrm{B} 2$ \\
\hline SFTPA1|653509 & $\mathrm{B} 2$ \\
\hline SFTPA2|729238 & $\mathrm{B} 2$ \\
\hline SFTPB|6439 & $\mathrm{B} 2$ \\
\hline SFTPC|6440 & $\mathrm{B} 2$ \\
\hline SFTPD|6441 & $\mathrm{B} 2$ \\
\hline SFXN3|81855 & $\mathrm{B} 2$ \\
\hline SFXN4|119559 & $\mathrm{B} 2$ \\
\hline SGCA|6442 & $\mathrm{B} 2$ \\
\hline SGCD|6444 & $\mathrm{B} 2$ \\
\hline SGCG|6445 & $\mathrm{B} 2$ \\
\hline SGEF|26084 & $\mathrm{B} 2$ \\
\hline SGIP1|84251 & $\mathrm{B} 2$ \\
\hline SGK196|84197 & $\mathrm{B} 2$ \\
\hline SGK223|157285 & $\mathrm{B} 2$ \\
\hline SGK3|23678 & $\mathrm{B} 2$ \\
\hline SGK494|124923 & $\mathrm{B} 2$ \\
\hline SGMS1|259230 & $\mathrm{B} 2$ \\
\hline \begin{tabular}{l|l|l|} 
SGMS2|16929 \\
\end{tabular} & $\mathrm{B} 2$ \\
\hline SGPP1|81537 & $\mathrm{B} 2$ \\
\hline SGPP2|130367 & $\mathrm{B} 2$ \\
\hline SGSH|6448 & $\mathrm{B} 2$ \\
\hline \begin{tabular}{ll|l} 
SGSM3|27352 &
\end{tabular} & $\mathrm{B} 2$ \\
\hline SGTA|6449 & $\mathrm{B} 2$ \\
\hline SH2B1|25970 & $\mathrm{B} 2$ \\
\hline SH2B2|10603 & $\mathrm{B} 2$ \\
\hline SH2B3|10019 & $\mathrm{B} 2$ \\
\hline SH2D1A|4068 & $\mathrm{B} 2$ \\
\hline SH2D1B|117157 & $\mathrm{B} 2$ \\
\hline SH2D3A|10045 & $\mathrm{B} 2$ \\
\hline \begin{tabular}{l|l|l|} 
SH2D4B 387694 \\
\end{tabular} & $\mathrm{~B} 2$ \\
\hline
\end{tabular}




\begin{tabular}{|c|c|}
\hline SH2D5|400745 & B2 \\
\hline SH2D6|284948 & B2 \\
\hline SH2D7|646892 & $\mathrm{B} 2$ \\
\hline SH3BGRL3|83442 & $\mathrm{B} 2$ \\
\hline SH3BGR|6450 & $\mathrm{B} 2$ \\
\hline SH3BP1|23616 & $\mathrm{B} 2$ \\
\hline SH3BP4|23677 & $\mathrm{B} 2$ \\
\hline SH3D20|201175 & $\mathrm{B} 2$ \\
\hline SH3GL2|6456 & $\mathrm{B} 2$ \\
\hline SH3GL3|6457 & $\mathrm{B} 2$ \\
\hline SH3GLB2|56904 & $\mathrm{B} 2$ \\
\hline SH3PXD2A|9644 & $\mathrm{B} 2$ \\
\hline SH3PXD2B|285590 & $\mathrm{B} 2$ \\
\hline SH3TC1|54436 & $\mathrm{B} 2$ \\
\hline SH3YL1|26751 & B2 \\
\hline SHANK1|50944 & $\mathrm{B} 2$ \\
\hline SHANK2|22941 & $\mathrm{B} 2$ \\
\hline SHB|6461 & $\mathrm{B} 2$ \\
\hline SHC1|6464 & $\mathrm{B} 2$ \\
\hline $\begin{array}{l}\text { SHC2|25759 } \\
\end{array}$ & $\mathrm{B} 2$ \\
\hline SHC3|53358 & $\mathrm{B} 2$ \\
\hline SHC4|399694 & $\mathrm{B} 2$ \\
\hline $\begin{array}{l}\text { SHD|56961 } \\
\end{array}$ & $\mathrm{B} 2$ \\
\hline SHF|90525 & $\mathrm{B} 2$ \\
\hline SHH|6469 & $\mathrm{B} 2$ \\
\hline $\begin{array}{l}\text { SHISA2|387914 } \\
\end{array}$ & $\mathrm{B} 2$ \\
\hline SHISA4|149345 & $\mathrm{B} 2$ \\
\hline SHISA6|388336 & $\mathrm{B} 2$ \\
\hline SHISA7|729956 & $\mathrm{B} 2$ \\
\hline \begin{tabular}{l|l|l|} 
SHOC2|8036 \\
\end{tabular} & $\mathrm{B} 2$ \\
\hline SHPK|23729 & $\mathrm{B} 2$ \\
\hline SHROOM1|134549 & $\mathrm{B} 2$ \\
\hline SHROOM3|57619 & $\mathrm{B} 2$ \\
\hline SIAE|54414 & $\mathrm{B} 2$ \\
\hline SIAH1|6477 & $\mathrm{B} 2$ \\
\hline \begin{tabular}{l|l|l|} 
SIAH3 283514 \\
\end{tabular} & $\mathrm{~B} 2$ \\
\hline SIGLEC10|89790 & $\mathrm{B} 2$ \\
\hline SIGLEC11|114132 & $\mathrm{B} 2$ \\
\hline SIGLEC12|89858 & $\mathrm{B} 2$ \\
\hline SIGLEC14|100049587 & $\mathrm{B} 2$ \\
\hline SIGLEC15|284266 & $\mathrm{B} 2$ \\
\hline SIGLEC16|400709 & $\mathrm{B} 2$ \\
\hline SIGLEC1|6614 & $\mathrm{B} 2$ \\
\hline SIGLEC5|8778 & $\mathrm{B} 2$ \\
\hline SIGLEC6|946 & $\mathrm{B} 2$ \\
\hline SIGLEC7|27036 & $\mathrm{B} 2$ \\
\hline SIGLEC8|27181 & $\mathrm{B} 2$ \\
\hline $\begin{array}{l}\text { SIGLEC9|27180 } \\
\end{array}$ & $\mathrm{B} 2$ \\
\hline SIGLECP3|284367 & $\mathrm{B} 2$ \\
\hline SIGMAR1|10280 & $\mathrm{B} 2$ \\
\hline SIK1|150094 & $\mathrm{B} 2$ \\
\hline
\end{tabular}




\begin{tabular}{|c|c|}
\hline SIKE1|80143 & B2 \\
\hline SIL1 $\mid 64374$ & B2 \\
\hline SILV|6490 & $\mathrm{B} 2$ \\
\hline SIM1|6492 & $\mathrm{B} 2$ \\
\hline SIN3A|25942 & B2 \\
\hline SIN3B|23309 & $\mathrm{B} 2$ \\
\hline SIP1|8487 & B2 \\
\hline SIPA1L1|26037 & $\mathrm{B} 2$ \\
\hline SIPA1L2|57568 & $\mathrm{B} 2$ \\
\hline SIPA1|6494 & $\mathrm{B} 2$ \\
\hline SIRPB2|284759 & B2 \\
\hline SIRPD|128646 & B2 \\
\hline SIRPG|55423 & B2 \\
\hline SIRT2|22933 & $\mathrm{B} 2$ \\
\hline SIRT3|23410 & B2 \\
\hline SIRT4|23409 & $\mathrm{B} 2$ \\
\hline SIRT5|23408 & B2 \\
\hline SIT1|27240 & B2 \\
\hline SIVA1|10572 & B2 \\
\hline SIX1|6495 & $\mathrm{B} 2$ \\
\hline SIX2|10736 & B2 \\
\hline SIX3|6496 & $\mathrm{B} 2$ \\
\hline SIX5|147912 & $\mathrm{B} 2$ \\
\hline SIX6|4990 & B2 \\
\hline SKAP1|8631 & $\mathrm{B} 2$ \\
\hline SKIV2L2|23517 & B2 \\
\hline SKP1|6500 & $\mathrm{B} 2$ \\
\hline SKP2|6502 & B2 \\
\hline SLA2|84174 & $\mathrm{B} 2$ \\
\hline SLAIN1|122060 & B2 \\
\hline SLAIN2|57606 & $\mathrm{B} 2$ \\
\hline SLAMF1|6504 & B2 \\
\hline SLAMF6|114836 & B2 \\
\hline SLAMF7|57823 & B2 \\
\hline SLAMF9|89886 & $\mathrm{B} 2$ \\
\hline SLA 6503 & $\mathrm{~B} 2$ \\
\hline SLC10A1|6554 & B2 \\
\hline SLC10A2|6555 & $\mathrm{B} 2$ \\
\hline SLC10A3|8273 & B2 \\
\hline SLC10A4|201780 & $\mathrm{B} 2$ \\
\hline SLC10A5|347051 & B2 \\
\hline SLC10A7|84068 & $\mathrm{B} 2$ \\
\hline SLC11A2|4891 & B2 \\
\hline SLC12A1|6557 & B2 \\
\hline SLC12A2|6558 & $\mathrm{B} 2$ \\
\hline SLC12A3|6559 & B2 \\
\hline SLC12A5|57468 & $\mathrm{B} 2$ \\
\hline SLC12A9|56996 & B2 \\
\hline SLC13A1|6561 & $\mathrm{B} 2$ \\
\hline SLC13A2|9058 & B2 \\
\hline SLC13A3|64849 & $\mathrm{B} 2$ \\
\hline
\end{tabular}




\begin{tabular}{|c|c|}
\hline SLC13A4|26266 & B2 \\
\hline SLC13A5|284111 & B2 \\
\hline SLC14A1|6563 & $\mathrm{B} 2$ \\
\hline SLC14A2|8170 & $\mathrm{B} 2$ \\
\hline SLC15A1|6564 & $\mathrm{B} 2$ \\
\hline SLC15A2|6565 & $\mathrm{B} 2$ \\
\hline SLC15A3|51296 & $\mathrm{B} 2$ \\
\hline SLC15A4|121260 & $\mathrm{B} 2$ \\
\hline SLC16A10|117247 & $\mathrm{B} 2$ \\
\hline SLC16A13|201232 & $\mathrm{B} 2$ \\
\hline SLC16A14|151473 & $\mathrm{B} 2$ \\
\hline SLC16A1|6566 & $\mathrm{B} 2$ \\
\hline SLC16A4|9122 & $\mathrm{B} 2$ \\
\hline SLC16A5|9121 & $\mathrm{B} 2$ \\
\hline SLC16A6|9120 & B2 \\
\hline SLC16A8|23539 & $\mathrm{B} 2$ \\
\hline SLC16A9|220963 & $\mathrm{B} 2$ \\
\hline SLC17A1|6568 & $\mathrm{B} 2$ \\
\hline SLC17A3|10786 & $\mathrm{B} 2$ \\
\hline SLC17A4|10050 & $\mathrm{B} 2$ \\
\hline SLC17A5|26503 & $\mathrm{B} 2$ \\
\hline SLC17A6|57084 & $\mathrm{B} 2$ \\
\hline SLC17A8|246213 & $\mathrm{B} 2$ \\
\hline $\begin{array}{l}\text { SLC17A9|63910 } \\
\end{array}$ & $\mathrm{B} 2$ \\
\hline SLC18A1|6570 & $\mathrm{B} 2$ \\
\hline SLC18A2|6571 & $\mathrm{B} 2$ \\
\hline SLC18A3|6572 & $\mathrm{B} 2$ \\
\hline SLC1A1|6505 & $\mathrm{B} 2$ \\
\hline SLC1A2|6506 & $\mathrm{B} 2$ \\
\hline SLC1A5|6510 & $\mathrm{B} 2$ \\
\hline SLC1A6|6511 & $\mathrm{B} 2$ \\
\hline SLC20A2|6575 & $\mathrm{B} 2$ \\
\hline SLC22A10|387775 & $\mathrm{B} 2$ \\
\hline SLC22A13|9390 & $\mathrm{B} 2$ \\
\hline SLC22A14|9389 & $\mathrm{B} 2$ \\
\hline SLC22A15|55356 & $\mathrm{B} 2$ \\
\hline SLC22A16|85413 & $\mathrm{B} 2$ \\
\hline SLC22A17|51310 & $\mathrm{B} 2$ \\
\hline SLC22A18AS|5003 & $\mathrm{B} 2$ \\
\hline SLC22A1 6580 & $\mathrm{~B} 2$ \\
\hline SLC22A20|440044 & $\mathrm{B} 2$ \\
\hline SLC22A23|63027 & $\mathrm{B} 2$ \\
\hline SLC22A24|283238 & $\mathrm{B} 2$ \\
\hline SLC22A25|387601 & $\mathrm{B} 2$ \\
\hline SLC22A2|6582 & $\mathrm{B} 2$ \\
\hline SLC22A4|6583 & $\mathrm{B} 2$ \\
\hline $\begin{array}{l}\text { SLC22A5|6584 } \\
\end{array}$ & $\mathrm{B} 2$ \\
\hline SLC22A6|9356 & $\mathrm{B} 2$ \\
\hline SLC22A7|10864 & $\mathrm{B} 2$ \\
\hline SLC22A8|9376 & $\mathrm{B} 2$ \\
\hline SLC22A9|114571 & $\mathrm{B} 2$ \\
\hline
\end{tabular}




\begin{tabular}{|c|c|}
\hline SLC23A3|151295 & B2 \\
\hline SLC24A1|9187 & B2 \\
\hline SLC24A3|57419 & $\mathrm{B} 2$ \\
\hline SLC24A4|123041 & $\mathrm{B} 2$ \\
\hline SLC24A5|283652 & $\mathrm{B} 2$ \\
\hline SLC24A6|80024 & $\mathrm{B} 2$ \\
\hline SLC25A10|1468 & $\mathrm{B} 2$ \\
\hline SLC25A11|8402 & $\mathrm{B} 2$ \\
\hline SLC25A12|8604 & $\mathrm{B} 2$ \\
\hline SLC25A13|10165 & $\mathrm{B} 2$ \\
\hline SLC25A16|8034 & $\mathrm{B} 2$ \\
\hline SLC25A18|83733 & $\mathrm{B} 2$ \\
\hline SLC25A1|6576 & $\mathrm{B} 2$ \\
\hline SLC25A21|89874 & $\mathrm{B} 2$ \\
\hline SLC25A23|79085 & $\mathrm{B} 2$ \\
\hline SLC25A29|123096 & $\mathrm{B} 2$ \\
\hline 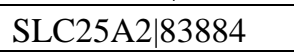 & $\mathrm{B} 2$ \\
\hline SLC25A31|83447 & $\mathrm{B} 2$ \\
\hline SLC25A32|81034 & $\mathrm{B} 2$ \\
\hline SLC25A33|84275 & $\mathrm{B} 2$ \\
\hline SLC25A34|284723 & $\mathrm{B} 2$ \\
\hline SLC25A35|399512 & $\mathrm{B} 2$ \\
\hline SLC25A37|51312 & $\mathrm{B} 2$ \\
\hline SLC25A38|54977 & $\mathrm{B} 2$ \\
\hline SLC25A3|5250 & $\mathrm{B} 2$ \\
\hline SLC25A40|55972 & $\mathrm{B} 2$ \\
\hline SLC25A41|284427 & $\mathrm{B} 2$ \\
\hline SLC25A43|203427 & $\mathrm{B} 2$ \\
\hline SLC25A45|283130 & $\mathrm{B} 2$ \\
\hline SLC25A46|91137 & $\mathrm{B} 2$ \\
\hline SLC25A4|291 & $\mathrm{B} 2$ \\
\hline SLC25A6|293 & $\mathrm{B} 2$ \\
\hline SLC26A11|284129 & $\mathrm{B} 2$ \\
\hline SLC26A1|10861 & $\mathrm{B} 2$ \\
\hline SLC26A2|1836 & $\mathrm{B} 2$ \\
\hline SLC26A3|1811 & $\mathrm{B} 2$ \\
\hline SLC26A5|375611 & $\mathrm{B} 2$ \\
\hline SLC26A6|65010 & $\mathrm{B} 2$ \\
\hline SLC26A7|115111 & $\mathrm{B} 2$ \\
\hline SLC26A8|116369 & $\mathrm{B} 2$ \\
\hline SLC26A9|115019 & $\mathrm{B} 2$ \\
\hline SLC27A2|11001 & $\mathrm{B} 2$ \\
\hline SLC27A3|11000 & $\mathrm{B} 2$ \\
\hline $\begin{array}{l}\text { SLC27A5|10998 } \\
\end{array}$ & $\mathrm{B} 2$ \\
\hline $\begin{array}{l}\text { SLC28A1|9154 } \\
\end{array}$ & $\mathrm{B} 2$ \\
\hline SLC28A2|9153 & $\mathrm{B} 2$ \\
\hline \begin{tabular}{l|l|l|} 
SLC28A3|64078 \\
\end{tabular} & $\mathrm{B} 2$ \\
\hline SLC29A2|3177 & $\mathrm{B} 2$ \\
\hline SLC2A11|66035 & $\mathrm{B} 2$ \\
\hline SLC2A13|114134 & $\mathrm{B} 2$ \\
\hline SLC2A14|144195 & $\mathrm{B} 2$ \\
\hline
\end{tabular}




\begin{tabular}{|c|c|}
\hline SLC2A2|6514 & B2 \\
\hline SLC2A3|6515 & $\mathrm{B} 2$ \\
\hline SLC2A4RG|56731 & $\mathrm{B} 2$ \\
\hline SLC2A5|6518 & $\mathrm{B} 2$ \\
\hline SLC2A7|155184 & B2 \\
\hline SLC2A8|29988 & $\mathrm{B} 2$ \\
\hline SLC2A9|56606 & $\mathrm{B} 2$ \\
\hline SLC30A10|55532 & $\mathrm{B} 2$ \\
\hline SLC30A2|7780 & $\mathrm{B} 2$ \\
\hline SLC30A3|7781 & $\mathrm{B} 2$ \\
\hline SLC30A4|7782 & $\mathrm{B} 2$ \\
\hline SLC30A5|64924 & $\mathrm{B} 2$ \\
\hline SLC30A6|55676 & $\mathrm{B} 2$ \\
\hline SLC30A8|169026 & $\mathrm{B} 2$ \\
\hline SLC30A9|10463 & $\mathrm{B} 2$ \\
\hline SLC31A1|1317 & $\mathrm{B} 2$ \\
\hline SLC31A2|1318 & B2 \\
\hline SLC32A1|140679 & $\mathrm{B} 2$ \\
\hline SLC33A1|9197 & $\mathrm{B} 2$ \\
\hline SLC34A1|6569 & $\mathrm{B} 2$ \\
\hline $\begin{array}{l}\text { SLC34A2|10568 } \\
\end{array}$ & $\mathrm{B} 2$ \\
\hline SLC34A3|142680 & $\mathrm{B} 2$ \\
\hline SLC35A1|10559 & $\mathrm{B} 2$ \\
\hline SLC35A4|113829 & $\mathrm{B} 2$ \\
\hline SLC35A5|55032 & $\mathrm{B} 2$ \\
\hline SLC35B3|51000 & $\mathrm{B} 2$ \\
\hline SLC35C1|55343 & $\mathrm{B} 2$ \\
\hline SLC35C2|51006 & $\mathrm{B} 2$ \\
\hline SLC35D2|11046 & $\mathrm{B} 2$ \\
\hline SLC35D3|340146 & $\mathrm{B} 2$ \\
\hline SLC35E1|79939 & $\mathrm{B} 2$ \\
\hline SLC35E2|728661 & $\mathrm{B} 2$ \\
\hline SLC35E2|9906 & $\mathrm{B} 2$ \\
\hline SLC35E4|339665 & $\mathrm{B} 2$ \\
\hline SLC35F1|222553 & $\mathrm{B} 2$ \\
\hline SLC35F2|54733 & $\mathrm{B} 2$ \\
\hline SLC35F3|148641 & $\mathrm{B} 2$ \\
\hline SLC35F4|341880 & $\mathrm{B} 2$ \\
\hline SLC36A1|206358 & $\mathrm{B} 2$ \\
\hline SLC36A2|153201 & $\mathrm{B} 2$ \\
\hline SLC36A3|285641 & $\mathrm{B} 2$ \\
\hline SLC36A4|120103 & $\mathrm{B} 2$ \\
\hline SLC37A2|219855 & $\mathrm{B} 2$ \\
\hline SLC37A3|84255 & $\mathrm{B} 2$ \\
\hline SLC37A4|2542 & $\mathrm{B} 2$ \\
\hline SLC38A10|124565 & $\mathrm{B} 2$ \\
\hline SLC38A11|151258 & $\mathrm{B} 2$ \\
\hline $\begin{array}{l}\text { SLC38A1|81539 } \\
\end{array}$ & $\mathrm{B} 2$ \\
\hline SLC38A2|54407 & $\mathrm{B} 2$ \\
\hline SLC38A3|10991 & $\mathrm{B} 2$ \\
\hline SLC38A4|55089 & $\mathrm{B} 2$ \\
\hline
\end{tabular}




\begin{tabular}{|c|c|}
\hline SLC38A6|145389 & B2 \\
\hline SLC38A8|146167 & B2 \\
\hline SLC38A9|153129 & $\mathrm{B} 2$ \\
\hline SLC39A12|221074 & $\mathrm{B} 2$ \\
\hline SLC39A13|91252 & $\mathrm{B} 2$ \\
\hline SLC39A14|23516 & $\mathrm{B} 2$ \\
\hline SLC39A2|29986 & $\mathrm{B} 2$ \\
\hline SLC39A5|283375 & $\mathrm{B} 2$ \\
\hline SLC39A6|25800 & $\mathrm{B} 2$ \\
\hline SLC39A8|64116 & $\mathrm{B} 2$ \\
\hline SLC3A1|6519 & $\mathrm{B} 2$ \\
\hline SLC3A2|6520 & $\mathrm{B} 2$ \\
\hline SLC40A1|30061 & $\mathrm{B} 2$ \\
\hline SLC41A1|254428 & $\mathrm{B} 2$ \\
\hline SLC41A2|84102 & B2 \\
\hline SLC43A1|8501 & $\mathrm{B} 2$ \\
\hline SLC43A2|124935 & $\mathrm{B} 2$ \\
\hline SLC43A3|29015 & $\mathrm{B} 2$ \\
\hline SLC44A2|57153 & $\mathrm{B} 2$ \\
\hline SLC44A3|126969 & $\mathrm{B} 2$ \\
\hline SLC44A5|204962 & $\mathrm{B} 2$ \\
\hline SLC45A1|50651 & $\mathrm{B} 2$ \\
\hline $\begin{array}{l}\text { SLC45A2|51151 } \\
\end{array}$ & $\mathrm{B} 2$ \\
\hline $\begin{array}{l}\text { SLC45A3|85414 } \\
\end{array}$ & $\mathrm{B} 2$ \\
\hline SLC45A4|57210 & $\mathrm{B} 2$ \\
\hline SLC46A1|113235 & $\mathrm{B} 2$ \\
\hline SLC46A2|57864 & $\mathrm{B} 2$ \\
\hline SLC46A3|283537 & $\mathrm{B} 2$ \\
\hline \begin{tabular}{l|l|l|} 
SLC47A244 \\
\end{tabular} & $\mathrm{B} 2$ \\
\hline SLC4A10|57282 & $\mathrm{B} 2$ \\
\hline SLC4A11|83959 & $\mathrm{B} 2$ \\
\hline SLC4A1AP|22950 & $\mathrm{B} 2$ \\
\hline SLC4A1|6521 & $\mathrm{B} 2$ \\
\hline SLC4A3|6508 & $\mathrm{B} 2$ \\
\hline SLC4A4|8671 & $\mathrm{B} 2$ \\
\hline SLC4A7|9497 & $\mathrm{B} 2$ \\
\hline $\begin{array}{l}\text { SLC4A9|83697 } \\
\end{array}$ & $\mathrm{B} 2$ \\
\hline SLC5A10|125206 & $\mathrm{B} 2$ \\
\hline SLC5A11|115584 & $\mathrm{B} 2$ \\
\hline SLC5A12|159963 & $\mathrm{B} 2$ \\
\hline SLC5A1|6523 & $\mathrm{B} 2$ \\
\hline \begin{tabular}{l|l|l|} 
SLC5A2|6524 \\
\end{tabular} & $\mathrm{B} 2$ \\
\hline SLC5A3|6526 & $\mathrm{B} 2$ \\
\hline \begin{tabular}{l|l|l|} 
SLC5A5 6528 \\
\end{tabular} & $\mathrm{~B} 2$ \\
\hline $\begin{array}{l}\text { SLC5A6|8884 } \\
\end{array}$ & $\mathrm{B} 2$ \\
\hline SLC5A7|60482 & $\mathrm{B} 2$ \\
\hline \begin{tabular}{l|l|} 
SLC5A8|160728 \\
\end{tabular} & $\mathrm{B} 2$ \\
\hline SLC6A10P|386757 & $\mathrm{B} 2$ \\
\hline SLC6A11|6538 & $\mathrm{B} 2$ \\
\hline SLC6A12|6539 & $\mathrm{B} 2$ \\
\hline SLC6A13|6540 & $\mathrm{B} 2$ \\
\hline
\end{tabular}




\begin{tabular}{|c|c|}
\hline SLC6A14|11254 & B2 \\
\hline SLC6A15|55117 & B2 \\
\hline SLC6A17|388662 & $\mathrm{B} 2$ \\
\hline SLC6A18|348932 & $\mathrm{B} 2$ \\
\hline SLC6A19|340024 & B2 \\
\hline SLC6A20|54716 & $\mathrm{B} 2$ \\
\hline SLC6A2|6530 & $\mathrm{B} 2$ \\
\hline SLC6A3|6531 & $\mathrm{B} 2$ \\
\hline SLC6A4|6532 & $\mathrm{B} 2$ \\
\hline SLC6A6 6533 & $\mathrm{~B} 2$ \\
\hline SLC6A7|6534 & $\mathrm{B} 2$ \\
\hline SLC6A8|6535 & $\mathrm{B} 2$ \\
\hline SLC7A13|157724 & $\mathrm{B} 2$ \\
\hline SLC7A1|6541 & $\mathrm{B} 2$ \\
\hline SLC7A2|6542 & $\mathrm{B} 2$ \\
\hline SLC7A4|6545 & $\mathrm{B} 2$ \\
\hline SLC7A5P1|81893 & $\mathrm{B} 2$ \\
\hline SLC7A5P2|387254 & $\mathrm{B} 2$ \\
\hline SLC7A5|8140 & $\mathrm{B} 2$ \\
\hline SLC7A6OS|84138 & $\mathrm{B} 2$ \\
\hline SLC7A6|9057 & $\mathrm{B} 2$ \\
\hline SLC7A7|9056 & $\mathrm{B} 2$ \\
\hline SLC7A9|11136 & $\mathrm{B} 2$ \\
\hline SLC8A1|6546 & $\mathrm{B} 2$ \\
\hline SLC8A2|6543 & $\mathrm{B} 2$ \\
\hline \begin{tabular}{l|l|} 
SLC8A3|6547 &
\end{tabular} & $\mathrm{B} 2$ \\
\hline SLC9A10|285335 & $\mathrm{B} 2$ \\
\hline SLC9A11|284525 & $\mathrm{B} 2$ \\
\hline $\begin{array}{l}\text { SLC9A1|6548 } \\
\end{array}$ & $\mathrm{B} 2$ \\
\hline $\begin{array}{l}\text { SLC9A2|6549 } \\
\end{array}$ & $\mathrm{B} 2$ \\
\hline SLC9A3R2|9351 & $\mathrm{B} 2$ \\
\hline \begin{tabular}{ll|l|} 
SLC9A3 6550 \\
\end{tabular} & $\mathrm{~B} 2$ \\
\hline SLC9A4|389015 & $\mathrm{B} 2$ \\
\hline SLC9A5|6553 & $\mathrm{B} 2$ \\
\hline SLC9A7|84679 & $\mathrm{B} 2$ \\
\hline SLC9A8|23315 & $\mathrm{B} 2$ \\
\hline SLCO1B1|10599 & $\mathrm{B} 2$ \\
\hline SLCO1B3|28234 & $\mathrm{B} 2$ \\
\hline SLCO2A1|6578 & $\mathrm{B} 2$ \\
\hline SLCO2B1|11309 & $\mathrm{B} 2$ \\
\hline SLCO3A1|28232 & $\mathrm{B} 2$ \\
\hline SLCO4A1|28231 & $\mathrm{B} 2$ \\
\hline SLCO4C1|353189 & $\mathrm{B} 2$ \\
\hline SLCO5A1|81796 & $\mathrm{B} 2$ \\
\hline SLCO6A1|133482 & $\mathrm{B} 2$ \\
\hline SLED1|643036 & $\mathrm{B} 2$ \\
\hline SLFN11|91607 & $\mathrm{B} 2$ \\
\hline SLFN12L|342615 & $\mathrm{B} 2$ \\
\hline SLFN12|55106 & $\mathrm{B} 2$ \\
\hline SLFN13|146857 & $\mathrm{B} 2$ \\
\hline SLFN14|342618 & $\mathrm{B} 2$ \\
\hline
\end{tabular}




\begin{tabular}{|c|c|}
\hline SLFN5|162394 & B2 \\
\hline SLFNL1|200172 & $\mathrm{B} 2$ \\
\hline SLIT1|6585 & $\mathrm{B} 2$ \\
\hline SLITRK1|114798 & $\mathrm{B} 2$ \\
\hline SLITRK3|22865 & $\mathrm{B} 2$ \\
\hline SLITRK4|139065 & $\mathrm{B} 2$ \\
\hline SLITRK5|26050 & $\mathrm{B} 2$ \\
\hline SLITRK6|84189 & $\mathrm{B} 2$ \\
\hline SLK|9748 & $\mathrm{B} 2$ \\
\hline SLMAP|7871 & $\mathrm{B} 2$ \\
\hline SLMO1|10650 & $\mathrm{B} 2$ \\
\hline SLN|6588 & $\mathrm{B} 2$ \\
\hline SLPI 6590 & $\mathrm{~B} 2$ \\
\hline SLTM|79811 & $\mathrm{B} 2$ \\
\hline SLURP1|57152 & $\mathrm{B} 2$ \\
\hline SMAD1|4086 & $\mathrm{B} 2$ \\
\hline SMAD2|4087 & $\mathrm{B} 2$ \\
\hline $\begin{array}{l}\text { SMAD5|4090 } \\
\end{array}$ & $\mathrm{B} 2$ \\
\hline SMAD7|4092 & $\mathrm{B} 2$ \\
\hline SMAGP|57228 & $\mathrm{B} 2$ \\
\hline SMAP1|60682 & $\mathrm{B} 2$ \\
\hline SMAP2|64744 & $\mathrm{B} 2$ \\
\hline SMARCA1|6594 & $\mathrm{B} 2$ \\
\hline \begin{tabular}{|l|} 
SMARCA5|8467 \\
\end{tabular} & $\mathrm{B} 2$ \\
\hline SMARCAD1|56916 & $\mathrm{B} 2$ \\
\hline SMARCAL1|50485 & $\mathrm{B} 2$ \\
\hline SMARCD1|6602 & $\mathrm{B} 2$ \\
\hline SMARCD2|6603 & $\mathrm{B} 2$ \\
\hline $\begin{array}{l}\text { SMARCD3|6604 } \\
\end{array}$ & $\mathrm{B} 2$ \\
\hline SMARCE1|6605 & $\mathrm{B} 2$ \\
\hline SMC1B|27127 & $\mathrm{B} 2$ \\
\hline SMC3|9126 & $\mathrm{B} 2$ \\
\hline SMC5|23137 & $\mathrm{B} 2$ \\
\hline SMCHD1|23347 & $\mathrm{B} 2$ \\
\hline SMCR5|140771 & $\mathrm{B} 2$ \\
\hline SMCR7L|54471 & $\mathrm{B} 2$ \\
\hline SMCR7|125170 & $\mathrm{B} 2$ \\
\hline SMCR8|140775 & $\mathrm{B} 2$ \\
\hline SMEK1|55671 & $\mathrm{B} 2$ \\
\hline SMEK2|57223 & $\mathrm{B} 2$ \\
\hline SMEK3P|139420 & $\mathrm{B} 2$ \\
\hline SMG1|23049 & $\mathrm{B} 2$ \\
\hline SMN1|6606 & $\mathrm{B} 2$ \\
\hline SMN2|6607 & $\mathrm{B} 2$ \\
\hline SMNDC1|10285 & $\mathrm{B} 2$ \\
\hline SMOC2|64094 & B2 \\
\hline SMOX|54498 & $\mathrm{B} 2$ \\
\hline SMO|6608 & $\mathrm{B} 2$ \\
\hline SMPD1|6609 & $\mathrm{B} 2$ \\
\hline SMPD3|55512 & $\mathrm{B} 2$ \\
\hline SMPDL3A|10924 & $\mathrm{B} 2$ \\
\hline
\end{tabular}




\begin{tabular}{|c|c|}
\hline SMPDL3B|27293 & B2 \\
\hline SMPX|23676 & $\mathrm{B} 2$ \\
\hline SMR3A|26952 & $\mathrm{B} 2$ \\
\hline SMR3B|10879 & $\mathrm{B} 2$ \\
\hline SMTNL1|219537 & B2 \\
\hline SMTNL2|342527 & $\mathrm{B} 2$ \\
\hline SMU1|55234 & $\mathrm{B} 2$ \\
\hline SMURF1|57154 & $\mathrm{B} 2$ \\
\hline SMURF2|64750 & $\mathrm{B} 2$ \\
\hline SMYD1|150572 & $\mathrm{B} 2$ \\
\hline SMYD2|56950 & $\mathrm{B} 2$ \\
\hline SMYD5|10322 & B2 \\
\hline SNAI1|6615 & $\mathrm{B} 2$ \\
\hline \begin{tabular}{l|l|} 
SNAI3|333929 \\
\end{tabular} & $\mathrm{B} 2$ \\
\hline SNAP23|8773 & $\mathrm{B} 2$ \\
\hline SNAP25|6616 & $\mathrm{B} 2$ \\
\hline SNAP29|9342 & B2 \\
\hline SNAP91|9892 & $\mathrm{B} 2$ \\
\hline \begin{tabular}{l|l|l|} 
SNAPC1|6617 &
\end{tabular} & B2 \\
\hline SNAPC3|6619 & $\mathrm{B} 2$ \\
\hline SNAPC4|6621 & $\mathrm{B} 2$ \\
\hline SNAPC5|10302 & $\mathrm{B} 2$ \\
\hline SNAPIN|23557 & $\mathrm{B} 2$ \\
\hline SNCAIP|9627 & $\mathrm{B} 2$ \\
\hline SNCB 6620 & $\mathrm{~B} 2$ \\
\hline SND1|27044 & $\mathrm{B} 2$ \\
\hline SNF8|11267 & $\mathrm{B} 2$ \\
\hline SNHG10|283596 & B2 \\
\hline SNHG11|128439 & $\mathrm{B} 2$ \\
\hline \begin{tabular}{l|l|} 
SNHG12|85028 \\
\end{tabular} & $\mathrm{B} 2$ \\
\hline \begin{tabular}{l|l|l|} 
SNHG1 23642 \\
\end{tabular} & $\mathrm{~B} 2$ \\
\hline SNHG3-RCC1|751867 & $\mathrm{B} 2$ \\
\hline SNHG3|8420 & $\mathrm{B} 2$ \\
\hline SNHG4|724102 & $\mathrm{B} 2$ \\
\hline SNHG6|641638 & $\mathrm{B} 2$ \\
\hline SNHG7|84973 & $\mathrm{B} 2$ \\
\hline \begin{tabular}{l|l|} 
SNHG9|735301 \\
\end{tabular} & B2 \\
\hline SNIP1|79753 & $\mathrm{B} 2$ \\
\hline SNORA10|574042 & B2 \\
\hline SNORA11|677799 & $\mathrm{B} 2$ \\
\hline SNORA12|677800 & B2 \\
\hline SNORA14A|677801 & $\mathrm{B} 2$ \\
\hline SNORA14B|677802 & B2 \\
\hline SNORA15|677803 & $\mathrm{B} 2$ \\
\hline SNORA16A|692073 & $\mathrm{B} 2$ \\
\hline SNORA16B|692157 & $\mathrm{B} 2$ \\
\hline SNORA18|677805 & $\mathrm{B} 2$ \\
\hline SNORA1|677792 & $\mathrm{B} 2$ \\
\hline SNORA20|677806 & $\mathrm{B} 2$ \\
\hline SNORA21|619505 & $\mathrm{B} 2$ \\
\hline SNORA22|677807 & $\mathrm{B} 2$ \\
\hline
\end{tabular}




\begin{tabular}{|c|c|}
\hline SNORA23|677808 & B2 \\
\hline SNORA24|677809 & $\mathrm{B} 2$ \\
\hline SNORA25|684959 & $\mathrm{B} 2$ \\
\hline SNORA26|677810 & $\mathrm{B} 2$ \\
\hline SNORA27|619499 & B2 \\
\hline SNORA29|677812 & $\mathrm{B} 2$ \\
\hline SNORA2A|677793 & $\mathrm{B} 2$ \\
\hline SNORA2B|677794 & $\mathrm{B} 2$ \\
\hline SNORA31|677814 & $\mathrm{B} 2$ \\
\hline SNORA34|677815 & $\mathrm{B} 2$ \\
\hline SNORA37|677819 & $\mathrm{B} 2$ \\
\hline SNORA38B|100124536 & $\mathrm{B} 2$ \\
\hline SNORA38|677820 & $\mathrm{B} 2$ \\
\hline SNORA39|677821 & $\mathrm{B} 2$ \\
\hline SNORA3|619562 & $\mathrm{B} 2$ \\
\hline SNORA40|677822 & $\mathrm{B} 2$ \\
\hline SNORA41|619569 & $\mathrm{B} 2$ \\
\hline SNORA44|677825 & $\mathrm{B} 2$ \\
\hline SNORA45|677826 & $\mathrm{B} 2$ \\
\hline SNORA46|677827 & $\mathrm{B} 2$ \\
\hline SNORA47|677828 & $\mathrm{B} 2$ \\
\hline SNORA48|652965 & $\mathrm{B} 2$ \\
\hline SNORA49|677829 & $\mathrm{B} 2$ \\
\hline SNORA4|619568 & $\mathrm{B} 2$ \\
\hline SNORA50|677830 & $\mathrm{B} 2$ \\
\hline SNORA52|619565 & $\mathrm{B} 2$ \\
\hline SNORA53|677832 & $\mathrm{B} 2$ \\
\hline SNORA54|677833 & $\mathrm{B} 2$ \\
\hline SNORA55|677834 & $\mathrm{B} 2$ \\
\hline SNORA56|677835 & $\mathrm{B} 2$ \\
\hline SNORA57|692158 & $\mathrm{B} 2$ \\
\hline SNORA59B|677882 & $\mathrm{B} 2$ \\
\hline SNORA5A|654319 & $\mathrm{B} 2$ \\
\hline SNORA5B|677795 & $\mathrm{B} 2$ \\
\hline SNORA5C|677796 & $\mathrm{B} 2$ \\
\hline SNORA61|677838 & $\mathrm{B} 2$ \\
\hline SNORA62|6044 & $\mathrm{B} 2$ \\
\hline SNORA63|6043 & $\mathrm{B} 2$ \\
\hline SNORA64|26784 & $\mathrm{B} 2$ \\
\hline SNORA65|26783 & $\mathrm{B} 2$ \\
\hline SNORA67|26781 & $\mathrm{B} 2$ \\
\hline SNORA68|26780 & $\mathrm{B} 2$ \\
\hline SNORA6|574040 & $\mathrm{B} 2$ \\
\hline SNORA70|26778 & $\mathrm{B} 2$ \\
\hline SNORA71A|26777 & $\mathrm{B} 2$ \\
\hline SNORA71B|26776 & $\mathrm{B} 2$ \\
\hline SNORA71D|677840 & $\mathrm{B} 2$ \\
\hline SNORA72|26775 & $\mathrm{B} 2$ \\
\hline SNORA74A|26821 & $\mathrm{B} 2$ \\
\hline SNORA74B|677841 & $\mathrm{B} 2$ \\
\hline SNORA75|654321 & $\mathrm{B} 2$ \\
\hline
\end{tabular}




\begin{tabular}{|c|c|}
\hline SNORA76|677842 & B2 \\
\hline SNORA7B|677797 & $\mathrm{B} 2$ \\
\hline SNORA80|677846 & $\mathrm{B} 2$ \\
\hline SNORA81|677847 & $\mathrm{B} 2$ \\
\hline SNORA84|100124534 & B2 \\
\hline SNORA8|654320 & $\mathrm{B} 2$ \\
\hline SNORA9|677798 & $\mathrm{B} 2$ \\
\hline SNORD10|652966 & $\mathrm{B} 2$ \\
\hline SNORD115-26|100033802 & $\mathrm{B} 2$ \\
\hline SNORD116-28|100033820 & $\mathrm{B} 2$ \\
\hline SNORD15A|6079 & $\mathrm{B} 2$ \\
\hline SNORD15B|114599 & B2 \\
\hline SNORD17|692086 & $\mathrm{B} 2$ \\
\hline SNORD1C|677850 & B2 \\
\hline SNORD22|9304 & $\mathrm{B} 2$ \\
\hline SNORD94|692225 & $\mathrm{B} 2$ \\
\hline SNORD97|692223 & $\mathrm{B} 2$ \\
\hline SNPH|9751 & $\mathrm{B} 2$ \\
\hline SNRNP200|23020 & B2 \\
\hline SNRNP27|11017 & $\mathrm{B} 2$ \\
\hline SNRNP35|11066 & B2 \\
\hline SNRNP48|154007 & $\mathrm{B} 2$ \\
\hline SNRNP70|6625 & $\mathrm{B} 2$ \\
\hline SNRPA|6626 & $\mathrm{B} 2$ \\
\hline $\begin{array}{l}\text { SNRPD2|6633 } \\
\end{array}$ & $\mathrm{B} 2$ \\
\hline SNRPN|6638 & $\mathrm{B} 2$ \\
\hline SNTA1|6640 & $\mathrm{B} 2$ \\
\hline SNTG1|54212 & B2 \\
\hline SNTN|132203 & $\mathrm{B} 2$ \\
\hline SNUPN|10073 & $\mathrm{B} 2$ \\
\hline SNURF|8926 & $\mathrm{B} 2$ \\
\hline SNW1|22938 & B2 \\
\hline \begin{tabular}{ll|l|} 
SNX10|29887 \\
\end{tabular} & $\mathrm{B} 2$ \\
\hline SNX11|29916 & $\mathrm{B} 2$ \\
\hline \begin{tabular}{l|l|l|} 
SNX129934 & \\
\end{tabular} & $\mathrm{B} 2$ \\
\hline SNX13|23161 & $\mathrm{B} 2$ \\
\hline SNX14|57231 & $\mathrm{B} 2$ \\
\hline $\begin{array}{l}\text { SNX16|64089 } \\
\end{array}$ & $\mathrm{B} 2$ \\
\hline SNX17|9784 & $\mathrm{B} 2$ \\
\hline SNX19|399979 & $\mathrm{B} 2$ \\
\hline SNX20|124460 & B2 \\
\hline SNX22|79856 & $\mathrm{B} 2$ \\
\hline \begin{tabular}{l|l|} 
SNX25|83891 &
\end{tabular} & B2 \\
\hline SNX2|6643 & $\mathrm{B} 2$ \\
\hline SNX30|401548 & $\mathrm{B} 2$ \\
\hline SNX31|169166 & $\mathrm{B} 2$ \\
\hline SNX32|254122 & $\mathrm{B} 2$ \\
\hline $\begin{array}{l}\text { SNX3|8724 } \\
\end{array}$ & $\mathrm{B} 2$ \\
\hline SNX4|8723 & $\mathrm{B} 2$ \\
\hline SNX5|27131 & B2 \\
\hline SNX6|58533 & $\mathrm{B} 2$ \\
\hline
\end{tabular}




\begin{tabular}{|c|c|}
\hline SNX7|51375 & B2 \\
\hline SNX8|29886 & $\mathrm{B} 2$ \\
\hline SNX9|51429 & $\mathrm{B} 2$ \\
\hline SOAT1|6646 & $\mathrm{B} 2$ \\
\hline SOAT2|8435 & $\mathrm{B} 2$ \\
\hline SOCS1|8651 & $\mathrm{B} 2$ \\
\hline SOCS4|122809 & $\mathrm{B} 2$ \\
\hline SOCS6|9306 & $\mathrm{B} 2$ \\
\hline SOCS7|30837 & $\mathrm{B} 2$ \\
\hline SOD1|6647 & $\mathrm{B} 2$ \\
\hline SOHLH1|402381 & $\mathrm{B} 2$ \\
\hline SOHLH2|54937 & $\mathrm{B} 2$ \\
\hline SON|6651 & $\mathrm{B} 2$ \\
\hline SORCS1|114815 & $\mathrm{B} 2$ \\
\hline SORCS2|57537 & $\mathrm{B} 2$ \\
\hline SORCS3|22986 & $\mathrm{B} 2$ \\
\hline SORL1|6653 & $\mathrm{B} 2$ \\
\hline SORT1|6272 & $\mathrm{B} 2$ \\
\hline \begin{tabular}{l|l} 
SOST|50964 \\
\end{tabular} & $\mathrm{B} 2$ \\
\hline SOX11|6664 & $\mathrm{B} 2$ \\
\hline SOX13|9580 & $\mathrm{B} 2$ \\
\hline SOX14|8403 & $\mathrm{B} 2$ \\
\hline SOX15|6665 & $\mathrm{B} 2$ \\
\hline SOX18|54345 & $\mathrm{B} 2$ \\
\hline SOX1|6656 & $\mathrm{B} 2$ \\
\hline SOX21|11166 & $\mathrm{B} 2$ \\
\hline SOX2OT|347689 & $\mathrm{B} 2$ \\
\hline SOX2|6657 & $\mathrm{B} 2$ \\
\hline SOX30|11063 & $\mathrm{B} 2$ \\
\hline SOX3|6658 & $\mathrm{B} 2$ \\
\hline SOX6|55553 & $\mathrm{B} 2$ \\
\hline SOX8|30812 & $\mathrm{B} 2$ \\
\hline SOX9|6662 & $\mathrm{B} 2$ \\
\hline SP100|6672 & $\mathrm{B} 2$ \\
\hline SP140L|93349 & $\mathrm{B} 2$ \\
\hline SP140|11262 & $\mathrm{B} 2$ \\
\hline SP1|6667 & $\mathrm{B} 2$ \\
\hline SP2|6668 & $\mathrm{B} 2$ \\
\hline SP3|6670 & $\mathrm{B} 2$ \\
\hline SP4|6671 & $\mathrm{B} 2$ \\
\hline SP5|389058 & $\mathrm{B} 2$ \\
\hline SP6|80320 & $\mathrm{B} 2$ \\
\hline SP7|121340 & $\mathrm{B} 2$ \\
\hline SP8|221833 & $\mathrm{B} 2$ \\
\hline SP9|100131390 & $\mathrm{B} 2$ \\
\hline SPACA3|124912 & $\mathrm{B} 2$ \\
\hline SPACA4|171169 & $\mathrm{B} 2$ \\
\hline SPACA5|389852 & $\mathrm{B} 2$ \\
\hline SPAG11A|653423 & $\mathrm{B} 2$ \\
\hline SPAG16|79582 & $\mathrm{B} 2$ \\
\hline SPAG17|200162 & $\mathrm{B} 2$ \\
\hline
\end{tabular}




\begin{tabular}{|c|c|}
\hline SPAG4|6676 & $\mathrm{B} 2$ \\
\hline SPAG6|9576 & $\mathrm{B} 2$ \\
\hline SPAG7|9552 & $\mathrm{B} 2$ \\
\hline SPAG8|26206 & $\mathrm{B} 2$ \\
\hline SPAG9|9043 & B2 \\
\hline SPAM1|6677 & $\mathrm{B} 2$ \\
\hline SPANXA2|728712 & $\mathrm{B} 2$ \\
\hline SPANXB2|100133171 & $\mathrm{B} 2$ \\
\hline SPANXC|64663 & B2 \\
\hline SPANXE|171489 & $\mathrm{B} 2$ \\
\hline SPANXN3|139067 & $\mathrm{B} 2$ \\
\hline SPARC|6678 & $\mathrm{B} 2$ \\
\hline \begin{tabular}{l|l} 
SPAST 6683 \\
\end{tabular} & $\mathrm{~B} 2$ \\
\hline SPATA12|353324 & $\mathrm{B} 2$ \\
\hline SPATA13|221178 & $\mathrm{B} 2$ \\
\hline SPATA16|83893 & $\mathrm{B} 2$ \\
\hline SPATA1|64173 & $\mathrm{B} 2$ \\
\hline SPATA20|64847 & $\mathrm{B} 2$ \\
\hline SPATA21|374955 & $\mathrm{B} 2$ \\
\hline SPATA22|84690 & $\mathrm{B} 2$ \\
\hline SPATA24|202051 & $\mathrm{B} 2$ \\
\hline SPATA2L|124044 & $\mathrm{B} 2$ \\
\hline SPATA2|9825 & $\mathrm{B} 2$ \\
\hline SPATA3|130560 & $\mathrm{B} 2$ \\
\hline SPATA4|132851 & $\mathrm{B} 2$ \\
\hline SPATA5L1|79029 & $\mathrm{B} 2$ \\
\hline SPATA5|166378 & $\mathrm{B} 2$ \\
\hline SPATA6|54558 & $\mathrm{B} 2$ \\
\hline SPATA8|145946 & $\mathrm{B} 2$ \\
\hline SPATC1|375686 & $\mathrm{B} 2$ \\
\hline SPATS1|221409 & $\mathrm{B} 2$ \\
\hline SPATS2L|26010 & $\mathrm{B} 2$ \\
\hline SPCS3|60559 & $\mathrm{B} 2$ \\
\hline SPDYA|245711 & $\mathrm{B} 2$ \\
\hline SPDYC|387778 & $\mathrm{B} 2$ \\
\hline SPDYE1|285955 & $\mathrm{B} 2$ \\
\hline SPDYE2|441273 & $\mathrm{B} 2$ \\
\hline SPDYE3|441272 & $\mathrm{B} 2$ \\
\hline SPDYE4|388333 & $\mathrm{B} 2$ \\
\hline SPDYE5|442590 & $\mathrm{B} 2$ \\
\hline SPDYE6|729597 & $\mathrm{B} 2$ \\
\hline SPDYE7P|441251 & $\mathrm{B} 2$ \\
\hline SPDYE8P|389517 & $\mathrm{B} 2$ \\
\hline SPEF2|79925 & $\mathrm{B} 2$ \\
\hline SPEG|10290 & $\mathrm{B} 2$ \\
\hline SPEM1|374768 & $\mathrm{B} 2$ \\
\hline SPERT|220082 & $\mathrm{B} 2$ \\
\hline SPESP1|246777 & $\mathrm{B} 2$ \\
\hline SPG11|80208 & $\mathrm{B} 2$ \\
\hline SPG21|51324 & $\mathrm{B} 2$ \\
\hline SPG7|6687 & $\mathrm{B} 2$ \\
\hline
\end{tabular}




\begin{tabular}{|c|c|}
\hline SPHAR|10638 & $\mathrm{B} 2$ \\
\hline SPHK1|8877 & B2 \\
\hline SPHK2|56848 & $\mathrm{B} 2$ \\
\hline SPI1|6688 & $\mathrm{B} 2$ \\
\hline SPIB|6689 & $\mathrm{B} 2$ \\
\hline SPIC|121599 & $\mathrm{B} 2$ \\
\hline SPIN1|10927 & $\mathrm{B} 2$ \\
\hline SPIN2B|474343 & $\mathrm{B} 2$ \\
\hline SPIN3|169981 & $\mathrm{B} 2$ \\
\hline SPIN4|139886 & $\mathrm{B} 2$ \\
\hline SPINK13|153218 & $\mathrm{B} 2$ \\
\hline SPINK1|6690 & $\mathrm{B} 2$ \\
\hline SPINK2|6691 & $\mathrm{B} 2$ \\
\hline SPINK4|27290 & $\mathrm{B} 2$ \\
\hline SPINK5|11005 & $\mathrm{B} 2$ \\
\hline SPINK6|404203 & $\mathrm{B} 2$ \\
\hline SPINK7|84651 & $\mathrm{B} 2$ \\
\hline SPINK8|646424 & $\mathrm{B} 2$ \\
\hline \begin{tabular}{l|l|} 
SPINK9|643394 &
\end{tabular} & $\mathrm{B} 2$ \\
\hline SPINLW1|57119 & $\mathrm{B} 2$ \\
\hline SPINT3|10816 & $\mathrm{B} 2$ \\
\hline SPIRE1|56907 & $\mathrm{B} 2$ \\
\hline SPNS3|201305 & $\mathrm{B} 2$ \\
\hline SPN|6693 & $\mathrm{B} 2$ \\
\hline SPOCK1|6695 & $\mathrm{B} 2$ \\
\hline SPOCK2|9806 & $\mathrm{B} 2$ \\
\hline SPOCK3|50859 & $\mathrm{B} 2$ \\
\hline SPON1|10418 & $\mathrm{B} 2$ \\
\hline SPON2|10417 & $\mathrm{B} 2$ \\
\hline SPOP $\mid 8405$ & $\mathrm{~B} 2$ \\
\hline \begin{tabular}{l|l|l|} 
SPP2|6694 &
\end{tabular} & $\mathrm{B} 2$ \\
\hline $\begin{array}{l}\text { SPPL2A|84888 } \\
\end{array}$ & B2 \\
\hline \begin{tabular}{l|l|} 
SPPL2B 56928 \\
\end{tabular} & $\mathrm{~B} 2$ \\
\hline SPPL3|121665 & $\mathrm{B} 2$ \\
\hline SPRED1|161742 & $\mathrm{B} 2$ \\
\hline SPRED2|200734 & $\mathrm{B} 2$ \\
\hline SPRED3|399473 & $\mathrm{B} 2$ \\
\hline SPRN|503542 & $\mathrm{B} 2$ \\
\hline SPRR1A|6698 & $\mathrm{B} 2$ \\
\hline \begin{tabular}{l|l|} 
SPRR1B|6699 \\
\end{tabular} & $\mathrm{B} 2$ \\
\hline SPRR2A|6700 & $\mathrm{B} 2$ \\
\hline SPRR2B|6701 & $\mathrm{B} 2$ \\
\hline SPRR2C|6702 & $\mathrm{B} 2$ \\
\hline \begin{tabular}{l|l|l|} 
SPRR2D & 6703 \\
\end{tabular} & $\mathrm{~B} 2$ \\
\hline SPRR2E|6704 & $\mathrm{B} 2$ \\
\hline $\begin{array}{l}\text { SPRR2F|6705 } \\
\end{array}$ & $\mathrm{B} 2$ \\
\hline SPRR2G|6706 & $\mathrm{B} 2$ \\
\hline $\begin{array}{l}\text { SPRR3|6707 } \\
\end{array}$ & $\mathrm{B} 2$ \\
\hline SPRR4|163778 & $\mathrm{B} 2$ \\
\hline SPRY3|10251 & $\mathrm{B} 2$ \\
\hline SPRY4|81848 & $\mathrm{B} 2$ \\
\hline
\end{tabular}




\begin{tabular}{|c|c|}
\hline SPRYD3|84926 & B2 \\
\hline SPRYD4|283377 & B2 \\
\hline SPRYD5|84767 & $\mathrm{B} 2$ \\
\hline SPSB1|80176 & $\mathrm{B} 2$ \\
\hline SPSB2|84727 & $\mathrm{B} 2$ \\
\hline SPSB3|90864 & $\mathrm{B} 2$ \\
\hline SPSB4|92369 & $\mathrm{B} 2$ \\
\hline SPTA1|6708 & $\mathrm{B} 2$ \\
\hline SPTAN1|6709 & $\mathrm{B} 2$ \\
\hline SPTBN2|6712 & $\mathrm{B} 2$ \\
\hline SPTBN4|57731 & $\mathrm{B} 2$ \\
\hline SPTBN5|51332 & $\mathrm{B} 2$ \\
\hline SPTB $\mid 6710$ & $\mathrm{~B} 2$ \\
\hline SPTLC1|10558 & $\mathrm{B} 2$ \\
\hline SPTLC2|9517 & $\mathrm{B} 2$ \\
\hline \begin{tabular}{l|l|l|} 
SPTLC3 5304 \\
\end{tabular} & $\mathrm{~B} 2$ \\
\hline SPTY2D1|144108 & $\mathrm{B} 2$ \\
\hline SPZ1|84654 & $\mathrm{B} 2$ \\
\hline SQSTM1|8878 & $\mathrm{B} 2$ \\
\hline SR140|23350 & $\mathrm{B} 2$ \\
\hline SRBD1|55133 & $\mathrm{B} 2$ \\
\hline SRCIN1|80725 & $\mathrm{B} 2$ \\
\hline SRCRB4D|136853 & $\mathrm{B} 2$ \\
\hline $\begin{array}{l}\text { SRD5A1|6715 } \\
\end{array}$ & $\mathrm{B} 2$ \\
\hline SRD5A2|6716 & $\mathrm{B} 2$ \\
\hline SREBF2|6721 & $\mathrm{B} 2$ \\
\hline SRFBP1|153443 & $\mathrm{B} 2$ \\
\hline SRGAP1|57522 & $\mathrm{B} 2$ \\
\hline SRGAP3|9901 & $\mathrm{B} 2$ \\
\hline SRGN|5552 & $\mathrm{B} 2$ \\
\hline SRI|6717 & $\mathrm{B} 2$ \\
\hline SRL|6345 & B2 \\
\hline SRM|6723 & $\mathrm{B} 2$ \\
\hline SRP14|6727 & $\mathrm{B} 2$ \\
\hline SRP68|6730 & $\mathrm{B} 2$ \\
\hline SRPK3|26576 & $\mathrm{B} 2$ \\
\hline SRPR|6734 & $\mathrm{B} 2$ \\
\hline SRPX2|27286 & $\mathrm{B} 2$ \\
\hline SRRD|402055 & $\mathrm{B} 2$ \\
\hline SRRM1|10250 & $\mathrm{B} 2$ \\
\hline SRRM2|23524 & $\mathrm{B} 2$ \\
\hline SRRM3|222183 & $\mathrm{B} 2$ \\
\hline SRRM4|84530 & $\mathrm{B} 2$ \\
\hline SRRM5|100170229 & $\mathrm{B} 2$ \\
\hline SRR|63826 & $\mathrm{B} 2$ \\
\hline SS18L1|26039 & $\mathrm{B} 2$ \\
\hline \begin{tabular}{l|l|l|l|l|} 
SS18L2|51 \\
\end{tabular} & $\mathrm{B} 2$ \\
\hline SS18|6760 & $\mathrm{B} 2$ \\
\hline SSBP3|23648 & $\mathrm{B} 2$ \\
\hline SSBP4|170463 & $\mathrm{B} 2$ \\
\hline SSB|6741 & $\mathrm{B} 2$ \\
\hline
\end{tabular}




\begin{tabular}{|c|c|}
\hline SSC5D|284297 & B2 \\
\hline SSFA2|6744 & $\mathrm{B} 2$ \\
\hline SSH1|54434 & $\mathrm{B} 2$ \\
\hline SSH2|85464 & $\mathrm{B} 2$ \\
\hline SSH3|54961 & $\mathrm{B} 2$ \\
\hline SSPO|23145 & $\mathrm{B} 2$ \\
\hline SSR3|6747 & $\mathrm{B} 2$ \\
\hline SSRP1|6749 & $\mathrm{B} 2$ \\
\hline SSSCA1|10534 & $\mathrm{B} 2$ \\
\hline SSTR2|6752 & $\mathrm{B} 2$ \\
\hline SSTR3|6753 & $\mathrm{B} 2$ \\
\hline SSTR5|6755 & $\mathrm{B} 2$ \\
\hline SST 6750 & $\mathrm{~B} 2$ \\
\hline SSU72|29101 & $\mathrm{B} 2$ \\
\hline SSX1|6756 & $\mathrm{B} 2$ \\
\hline SSX2|6757 & $\mathrm{B} 2$ \\
\hline SSX3|10214 & $\mathrm{B} 2$ \\
\hline SSX4|6759 & $\mathrm{B} 2$ \\
\hline SSX5|6758 & $\mathrm{B} 2$ \\
\hline SSX6|280657 & $\mathrm{B} 2$ \\
\hline ST18|9705 & $\mathrm{B} 2$ \\
\hline ST3GAL1|6482 & $\mathrm{B} 2$ \\
\hline ST3GAL2|6483 & $\mathrm{B} 2$ \\
\hline ST3GAL4|6484 & B2 \\
\hline $\begin{array}{l}\text { ST3GAL5|8869 } \\
\end{array}$ & $\mathrm{B} 2$ \\
\hline ST3GAL6|10402 & $\mathrm{B} 2$ \\
\hline ST6GAL1|6480 & $\mathrm{B} 2$ \\
\hline $\begin{array}{l}\text { ST6GALNAC1|55808 } \\
\end{array}$ & $\mathrm{B} 2$ \\
\hline \begin{tabular}{l|l|l|} 
ST6GALNAC2|10610 \\
\end{tabular} & $\mathrm{B} 2$ \\
\hline ST6GALNAC4|27090 & $\mathrm{B} 2$ \\
\hline \begin{tabular}{l|l|} 
ST6GALNAC5|81849 \\
\end{tabular} & $\mathrm{B} 2$ \\
\hline ST7L|54879 & $\mathrm{B} 2$ \\
\hline ST7OT2|93654 & $\mathrm{B} 2$ \\
\hline ST7OT3|93655 & $\mathrm{B} 2$ \\
\hline ST7OT4|338069 & $\mathrm{B} 2$ \\
\hline ST7|7982 & $\mathrm{B} 2$ \\
\hline ST8SIA1|6489 & $\mathrm{B} 2$ \\
\hline ST8SIA3|51046 & $\mathrm{B} 2$ \\
\hline ST8SIA4|7903 & $\mathrm{B} 2$ \\
\hline ST8SIA5|29906 & $\mathrm{B} 2$ \\
\hline ST8SIA6|338596 & $\mathrm{B} 2$ \\
\hline STAC3|246329 & $\mathrm{B} 2$ \\
\hline STAC|6769 & $\mathrm{B} 2$ \\
\hline STAG1|10274 & $\mathrm{B} 2$ \\
\hline STAG2|10735 & $\mathrm{B} 2$ \\
\hline STAG3L1|54441 & $\mathrm{B} 2$ \\
\hline STAG3L2|442582 & $\mathrm{B} 2$ \\
\hline STAG3L4|64940 & $\mathrm{B} 2$ \\
\hline \begin{tabular}{l|l|l|} 
STAG3 & 10734 \\
\end{tabular} & $\mathrm{~B} 2$ \\
\hline STAM2|10254 & $\mathrm{B} 2$ \\
\hline STAMBPL1|57559 & B2 \\
\hline
\end{tabular}




\begin{tabular}{|c|c|}
\hline STAP1|26228 & B2 \\
\hline STAP2|55620 & $\mathrm{B} 2$ \\
\hline STARD3NL|83930 & $\mathrm{B} 2$ \\
\hline STARD3|10948 & $\mathrm{B} 2$ \\
\hline STARD4|134429 & $\mathrm{B} 2$ \\
\hline STARD5|80765 & $\mathrm{B} 2$ \\
\hline STARD6|147323 & $\mathrm{B} 2$ \\
\hline STARD7|56910 & $\mathrm{B} 2$ \\
\hline STAR|6770 & B2 \\
\hline STAT2|6773 & $\mathrm{B} 2$ \\
\hline STAT3|6774 & $\mathrm{B} 2$ \\
\hline STAT4|6775 & $\mathrm{B} 2$ \\
\hline STATH|6779 & $\mathrm{B} 2$ \\
\hline STAU2|27067 & $\mathrm{B} 2$ \\
\hline STC1|6781 & $\mathrm{B} 2$ \\
\hline STC2|8614 & $\mathrm{B} 2$ \\
\hline STEAP1|26872 & $\mathrm{B} 2$ \\
\hline STEAP2|261729 & $\mathrm{B} 2$ \\
\hline STEAP3|55240 & $\mathrm{B} 2$ \\
\hline STEAP4|79689 & $\mathrm{B} 2$ \\
\hline STH|246744 & $\mathrm{B} 2$ \\
\hline STK10|6793 & $\mathrm{B} 2$ \\
\hline STK11|6794 & $\mathrm{B} 2$ \\
\hline STK16|8576 & $\mathrm{B} 2$ \\
\hline STK17A|9263 & $\mathrm{B} 2$ \\
\hline STK17B|9262 & $\mathrm{B} 2$ \\
\hline STK19|8859 & $\mathrm{B} 2$ \\
\hline STK24|8428 & $\mathrm{B} 2$ \\
\hline STK25|10494 & $\mathrm{B} 2$ \\
\hline STK31|56164 & $\mathrm{B} 2$ \\
\hline STK32B|55351 & $\mathrm{B} 2$ \\
\hline STK32C|282974 & $\mathrm{B} 2$ \\
\hline $\begin{array}{l}\text { STK33|65975 } \\
\end{array}$ & $\mathrm{B} 2$ \\
\hline STK35|140901 & $\mathrm{B} 2$ \\
\hline \begin{tabular}{l|l|l|} 
STK36 27148 \\
\end{tabular} & $\mathrm{~B} 2$ \\
\hline STK38L|23012 & $\mathrm{B} 2$ \\
\hline STK38|11329 & $\mathrm{B} 2$ \\
\hline \begin{tabular}{l|l|l|} 
STK39|27347 \\
\end{tabular} & $\mathrm{B} 2$ \\
\hline $\begin{array}{l}\text { STK3|6788 } \\
\end{array}$ & $\mathrm{B} 2$ \\
\hline STK4|6789 & $\mathrm{B} 2$ \\
\hline STMN2|11075 & $\mathrm{B} 2$ \\
\hline STMN3|50861 & $\mathrm{B} 2$ \\
\hline STMN4|81551 & $\mathrm{B} 2$ \\
\hline STOML1|9399 & $\mathrm{B} 2$ \\
\hline STOML2|30968 & $\mathrm{B} 2$ \\
\hline STOML3|161003 & $\mathrm{B} 2$ \\
\hline STOM|2040 & $\mathrm{B} 2$ \\
\hline STON1-GTF2A1L|286749 & $\mathrm{B} 2$ \\
\hline STON2|85439 & $\mathrm{B} 2$ \\
\hline STOX1|219736 & $\mathrm{B} 2$ \\
\hline STRA6|64220 & $\mathrm{B} 2$ \\
\hline
\end{tabular}




\begin{tabular}{|c|c|}
\hline STRA8|346673 & B2 \\
\hline STRADA|92335 & $\mathrm{B} 2$ \\
\hline STRADB|55437 & $\mathrm{B} 2$ \\
\hline STRAP|11171 & $\mathrm{B} 2$ \\
\hline STRC|161497 & $\mathrm{B} 2$ \\
\hline STRN3|29966 & B2 \\
\hline STRN4|29888 & $\mathrm{B} 2$ \\
\hline STRN|6801 & $\mathrm{B} 2$ \\
\hline STS $\mid 412$ & B2 \\
\hline STT3B|201595 & $\mathrm{B} 2$ \\
\hline STUB1|10273 & $\mathrm{B} 2$ \\
\hline STX10|8677 & $\mathrm{B} 2$ \\
\hline STX16|8675 & $\mathrm{B} 2$ \\
\hline STX17|55014 & $\mathrm{B} 2$ \\
\hline STX18|53407 & B2 \\
\hline STX19|415117 & $\mathrm{B} 2$ \\
\hline STX1B|112755 & B2 \\
\hline STX4|6810 & $\mathrm{B} 2$ \\
\hline $\begin{array}{l}\text { STX5|6811 } \\
\text { ST }\end{array}$ & B2 \\
\hline STXBP3|6814 & $\mathrm{B} 2$ \\
\hline STXBP4|252983 & $\mathrm{B} 2$ \\
\hline STXBP5L|9515 & $\mathrm{B} 2$ \\
\hline STXBP5|134957 & $\mathrm{B} 2$ \\
\hline STYK1|55359 & $\mathrm{B} 2$ \\
\hline STYX|6815 & $\mathrm{B} 2$ \\
\hline SUB1|10923 & $\mathrm{B} 2$ \\
\hline SUCLG2|8801 & B2 \\
\hline $\begin{array}{l}\text { SUCNR1|56670 } \\
\end{array}$ & B2 \\
\hline SUDS3|64426 & $\mathrm{B} 2$ \\
\hline SUFU|51684 & $\mathrm{B} 2$ \\
\hline $\begin{array}{l}\text { SUGT1|10910 } \\
\end{array}$ & $\mathrm{B} 2$ \\
\hline SULT1A1|6817 & $\mathrm{B} 2$ \\
\hline SULT1A2|6799 & $\mathrm{B} 2$ \\
\hline SULT1A3|6818 & B2 \\
\hline SULT1B1|27284 & $\mathrm{B} 2$ \\
\hline SULT1C2|6819 & $\mathrm{B} 2$ \\
\hline SULT1C3|442038 & $\mathrm{B} 2$ \\
\hline SULT1E1|6783 & $\mathrm{B} 2$ \\
\hline SULT2A1|6822 & $\mathrm{B} 2$ \\
\hline SULT2B1|6820 & $\mathrm{B} 2$ \\
\hline SULT4A1|25830 & B2 \\
\hline SULT6B1|391365 & $\mathrm{B} 2$ \\
\hline SUMF1|285362 & B2 \\
\hline SUMF2|25870 & $\mathrm{B} 2$ \\
\hline SUMO1P1|391257 & $\mathrm{B} 2$ \\
\hline SUMO4|387082 & $\mathrm{B} 2$ \\
\hline $\begin{array}{l}\text { SUN3|256979 } \\
\end{array}$ & $\mathrm{B} 2$ \\
\hline SUOX|6821 & $\mathrm{B} 2$ \\
\hline SUPT4H1|6827 & $\mathrm{B} 2$ \\
\hline SUPT5H|6829 & $\mathrm{B} 2$ \\
\hline SUPT6H|6830 & $\mathrm{B} 2$ \\
\hline
\end{tabular}




\begin{tabular}{|c|c|}
\hline SUPT7L|9913 & B2 \\
\hline SUPV3L1|6832 & B2 \\
\hline SURF1|6834 & $\mathrm{B} 2$ \\
\hline SURF2|6835 & $\mathrm{B} 2$ \\
\hline SURF4|6836 & B2 \\
\hline SURF6|6838 & $\mathrm{B} 2$ \\
\hline SUSD1|64420 & $\mathrm{B} 2$ \\
\hline $\begin{array}{l}\text { SUSD2|56241 } \\
\end{array}$ & $\mathrm{B} 2$ \\
\hline SUSD4|55061 & $\mathrm{B} 2$ \\
\hline \begin{tabular}{l|l|} 
SUSD5|26032 &
\end{tabular} & $\mathrm{B} 2$ \\
\hline SUV420H1|51111 & $\mathrm{B} 2$ \\
\hline SUZ12P|440423 & $\mathrm{B} 2$ \\
\hline \begin{tabular}{l|l|l|} 
SUZ12 & 23512
\end{tabular} & $\mathrm{~B} 2$ \\
\hline SV2A|9900 & $\mathrm{B} 2$ \\
\hline SV2C|22987 & $\mathrm{B} 2$ \\
\hline SVIL|6840 & $\mathrm{B} 2$ \\
\hline SVOPL|136306 & $\mathrm{B} 2$ \\
\hline SVOP|55530 & $\mathrm{B} 2$ \\
\hline SYBU|55638 & $\mathrm{B} 2$ \\
\hline SYCE1L|100130958 & $\mathrm{B} 2$ \\
\hline SYCE1|93426 & $\mathrm{B} 2$ \\
\hline SYCN|342898 & $\mathrm{B} 2$ \\
\hline SYCP1|6847 & $\mathrm{B} 2$ \\
\hline SYCP2L|221711 & $\mathrm{B} 2$ \\
\hline SYCP2|10388 & $\mathrm{B} 2$ \\
\hline SYCP3|50511 & $\mathrm{B} 2$ \\
\hline SYDE2|84144 & $\mathrm{B} 2$ \\
\hline SYK $\mid 6850$ & $\mathrm{~B} 2$ \\
\hline SYMPK|8189 & $\mathrm{B} 2$ \\
\hline SYN1|6853 & $\mathrm{B} 2$ \\
\hline SYN3|8224 & $\mathrm{B} 2$ \\
\hline SYNCRIP|10492 & $\mathrm{B} 2$ \\
\hline SYNC|81493 & $\mathrm{B} 2$ \\
\hline SYNGAP1|8831 & $\mathrm{B} 2$ \\
\hline SYNGR1|9145 & $\mathrm{B} 2$ \\
\hline SYNJ1|8867 & $\mathrm{B} 2$ \\
\hline SYNJ2BP|55333 & $\mathrm{B} 2$ \\
\hline SYNJ2|8871 & $\mathrm{B} 2$ \\
\hline SYNPO2L|79933 & $\mathrm{B} 2$ \\
\hline SYNPR|132204 & $\mathrm{B} 2$ \\
\hline SYNRG|11276 & $\mathrm{B} 2$ \\
\hline SYPL1|6856 & $\mathrm{B} 2$ \\
\hline SYPL2|284612 & $\mathrm{B} 2$ \\
\hline SYP|6855 & $\mathrm{B} 2$ \\
\hline SYS1-DBNDD2|767557 & $\mathrm{B} 2$ \\
\hline SYS1|90196 & $\mathrm{B} 2$ \\
\hline SYT10|341359 & $\mathrm{B} 2$ \\
\hline SYT11|23208 & $\mathrm{B} 2$ \\
\hline $\begin{array}{l}\text { SYT12|91683 } \\
\end{array}$ & $\mathrm{B} 2$ \\
\hline SYT13|57586 & $\mathrm{B} 2$ \\
\hline SYT14L|401135 & $\mathrm{B} 2$ \\
\hline
\end{tabular}




\begin{tabular}{|c|c|}
\hline SYT14|255928 & B2 \\
\hline SYT16|83851 & $\mathrm{B} 2$ \\
\hline SYT17|51760 & $\mathrm{B} 2$ \\
\hline SYT1|6857 & $\mathrm{B} 2$ \\
\hline SYT3|84258 & $\mathrm{B} 2$ \\
\hline SYT4|6860 & B2 \\
\hline SYT5|6861 & $\mathrm{B} 2$ \\
\hline SYT6|148281 & $\mathrm{B} 2$ \\
\hline SYT7|9066 & $\mathrm{B} 2$ \\
\hline SYT9|143425 & $\mathrm{B} 2$ \\
\hline SYTL1|84958 & $\mathrm{B} 2$ \\
\hline $\begin{array}{l}\text { SYTL2|54843 } \\
\end{array}$ & $\mathrm{B} 2$ \\
\hline SYTL4|94121 & $\mathrm{B} 2$ \\
\hline SYTL5|94122 & $\mathrm{B} 2$ \\
\hline SYVN1|84447 & B2 \\
\hline TAAR1|134864 & $\mathrm{B} 2$ \\
\hline TAB1|10454 & B2 \\
\hline TAB2|23118 & $\mathrm{B} 2$ \\
\hline \begin{tabular}{l|l|} 
TAB3|257397
\end{tabular} & B2 \\
\hline TAC3|6866 & $\mathrm{B} 2$ \\
\hline TAC4|255061 & $\mathrm{B} 2$ \\
\hline TACC2|10579 & $\mathrm{B} 2$ \\
\hline TACR2|6865 & $\mathrm{B} 2$ \\
\hline TACR3|6870 & $\mathrm{B} 2$ \\
\hline TACSTD2|4070 & $\mathrm{B} 2$ \\
\hline TADA2A|6871 & $\mathrm{B} 2$ \\
\hline TADA2B|93624 & B2 \\
\hline TADA3|10474 & B2 \\
\hline TAF10|6881 & $\mathrm{B} 2$ \\
\hline TAF13|6884 & $\mathrm{B} 2$ \\
\hline TAF15|8148 & $\mathrm{B} 2$ \\
\hline TAF1A|9015 & $\mathrm{B} 2$ \\
\hline TAF1B|9014 & $\mathrm{B} 2$ \\
\hline TAF1D|79101 & B2 \\
\hline TAF1L|138474 & $\mathrm{B} 2$ \\
\hline TAF1|6872 & $\mathrm{B} 2$ \\
\hline TAF3|83860 & B2 \\
\hline TAF4B|6875 & $\mathrm{B} 2$ \\
\hline TAF5|6877 & $\mathrm{B} 2$ \\
\hline TAF6L|10629 & $\mathrm{B} 2$ \\
\hline TAF7L|54457 & B2 \\
\hline TAF7|6879 & $\mathrm{B} 2$ \\
\hline TAF9B|51616 & B2 \\
\hline TAF9|6880 & $\mathrm{B} 2$ \\
\hline TAGAP|117289 & $\mathrm{B} 2$ \\
\hline TAGLN3|29114 & $\mathrm{B} 2$ \\
\hline TAL2|6887 & $\mathrm{B} 2$ \\
\hline TALDO1|6888 & $\mathrm{B} 2$ \\
\hline TANC1|85461 & $\mathrm{B} 2$ \\
\hline TAOK1|57551 & $\mathrm{B} 2$ \\
\hline TAOK2|9344 & $\mathrm{B} 2$ \\
\hline
\end{tabular}




\begin{tabular}{|c|c|}
\hline TAOK3|51347 & B2 \\
\hline TAP2|6891 & $\mathrm{B} 2$ \\
\hline TAPBPL|55080 & $\mathrm{B} 2$ \\
\hline TAPT1|202018 & $\mathrm{B} 2$ \\
\hline TARBP1|6894 & B2 \\
\hline TARP|445347 & $\mathrm{B} 2$ \\
\hline TARSL2|123283 & $\mathrm{B} 2$ \\
\hline TAS1R1|80835 & $\mathrm{B} 2$ \\
\hline TAS1R2|80834 & $\mathrm{B} 2$ \\
\hline TAS1R3|83756 & $\mathrm{B} 2$ \\
\hline TAS2R10|50839 & $\mathrm{B} 2$ \\
\hline TAS2R13|50838 & B2 \\
\hline TAS2R14|50840 & $\mathrm{B} 2$ \\
\hline TAS2R19|259294 & $\mathrm{B} 2$ \\
\hline TAS2R1|50834 & $\mathrm{B} 2$ \\
\hline TAS2R20|259295 & $\mathrm{B} 2$ \\
\hline TAS2R30|259293 & $\mathrm{B} 2$ \\
\hline TAS2R31|259290 & $\mathrm{B} 2$ \\
\hline TAS2R38|5726 & B2 \\
\hline TAS2R39|259285 & $\mathrm{B} 2$ \\
\hline TAS2R3|50831 & $\mathrm{B} 2$ \\
\hline TAS2R40|259286 & $\mathrm{B} 2$ \\
\hline TAS2R42|353164 & $\mathrm{B} 2$ \\
\hline $\begin{array}{l}\text { TAS2R43|259289 } \\
\end{array}$ & $\mathrm{B} 2$ \\
\hline TAS2R46|259292 & $\mathrm{B} 2$ \\
\hline TAS2R4|50832 & $\mathrm{B} 2$ \\
\hline TAS2R50|259296 & $\mathrm{B} 2$ \\
\hline TAS2R60|338398 & B2 \\
\hline TAS2R7|50837 & $\mathrm{B} 2$ \\
\hline TAS2R8|50836 & $\mathrm{B} 2$ \\
\hline TAS2R9|50835 & $\mathrm{B} 2$ \\
\hline TASP1|55617 & $\mathrm{B} 2$ \\
\hline TATDN1|83940 & $\mathrm{B} 2$ \\
\hline TATDN2|9797 & B2 \\
\hline TATDN3|128387 & $\mathrm{B} 2$ \\
\hline TAT|6898 & $\mathrm{B} 2$ \\
\hline TAX1BP3|30851 & $\mathrm{B} 2$ \\
\hline TAZ|6901 & $\mathrm{B} 2$ \\
\hline TBC1D10A|83874 & $\mathrm{B} 2$ \\
\hline TBC1D10C|374403 & $\mathrm{B} 2$ \\
\hline TBC1D12|23232 & B2 \\
\hline TBC1D16|125058 & $\mathrm{B} 2$ \\
\hline TBC1D19|55296 & B2 \\
\hline TBC1D1|23216 & $\mathrm{B} 2$ \\
\hline TBC1D20|128637 & $\mathrm{B} 2$ \\
\hline TBC1D22A|25771 & $\mathrm{B} 2$ \\
\hline TBC1D22B|55633 & $\mathrm{B} 2$ \\
\hline TBC1D23|55773 & $\mathrm{B} 2$ \\
\hline TBC1D24|57465 & $\mathrm{B} 2$ \\
\hline TBC1D26|353149 & $\mathrm{B} 2$ \\
\hline TBC1D28|254272 & B2 \\
\hline
\end{tabular}




\begin{tabular}{|c|c|}
\hline TBC1D29|26083 & B2 \\
\hline TBC1D2|55357 & B2 \\
\hline TBC1D3B|414059 & B2 \\
\hline TBC1D3C|414060 & $\mathrm{B} 2$ \\
\hline TBC1D3G|654341 & $\mathrm{B} 2$ \\
\hline TBC1D3H|729877 & $\mathrm{B} 2$ \\
\hline TBC1D3P2|440452 & $\mathrm{B} 2$ \\
\hline TBC1D3|729873 & $\mathrm{B} 2$ \\
\hline TBC1D8B|54885 & $\mathrm{B} 2$ \\
\hline TBC1D8|11138 & B2 \\
\hline TBC1D9B|23061 & $\mathrm{B} 2$ \\
\hline TBCCD1|55171 & $\mathrm{B} 2$ \\
\hline ТВСС $\mid 6903$ & $\mathrm{~B} 2$ \\
\hline TBCD|6904 & $\mathrm{B} 2$ \\
\hline TBCEL|219899 & $\mathrm{B} 2$ \\
\hline TBCK|93627 & $\mathrm{B} 2$ \\
\hline TBK1|29110 & $\mathrm{B} 2$ \\
\hline TBL1X|6907 & $\mathrm{B} 2$ \\
\hline TBL1Y|90665 & $\mathrm{B} 2$ \\
\hline TBPL1|9519 & $\mathrm{B} 2$ \\
\hline TBPL2|387332 & $\mathrm{B} 2$ \\
\hline TBP|6908 & $\mathrm{B} 2$ \\
\hline TBR1|10716 & $\mathrm{B} 2$ \\
\hline TBX10|347853 & $\mathrm{B} 2$ \\
\hline TBX19|9095 & $\mathrm{B} 2$ \\
\hline TBX1|6899 & $\mathrm{B} 2$ \\
\hline TBX20|57057 & $\mathrm{B} 2$ \\
\hline TBX21|30009 & $\mathrm{B} 2$ \\
\hline TBX22|50945 & $\mathrm{B} 2$ \\
\hline TBX2|6909 & $\mathrm{B} 2$ \\
\hline TBX3|6926 & $\mathrm{B} 2$ \\
\hline TBX4|9496 & $\mathrm{B} 2$ \\
\hline TBX5|6910 & $\mathrm{B} 2$ \\
\hline TBX6|6911 & $\mathrm{B} 2$ \\
\hline TBXA2R|6915 & $\mathrm{B} 2$ \\
\hline TBXAS1|6916 & $\mathrm{B} 2$ \\
\hline TC2N|123036 & $\mathrm{B} 2$ \\
\hline TCAM1P|146771 & $\mathrm{B} 2$ \\
\hline TCAP $\mid 8557$ & $\mathrm{~B} 2$ \\
\hline TCEA2|6919 & $\mathrm{B} 2$ \\
\hline TCEA3|6920 & $\mathrm{B} 2$ \\
\hline TCEAL1|9338 & $\mathrm{B} 2$ \\
\hline TCEAL3|85012 & $\mathrm{B} 2$ \\
\hline TCEAL4|79921 & $\mathrm{B} 2$ \\
\hline TCEAL6|158931 & $\mathrm{B} 2$ \\
\hline TCEAL8|90843 & $\mathrm{B} 2$ \\
\hline TCEANC|170082 & $\mathrm{B} 2$ \\
\hline TCEB3B|51224 & $\mathrm{B} 2$ \\
\hline TCEB3C|162699 & $\mathrm{B} 2$ \\
\hline TCEB3|6924 & $\mathrm{B} 2$ \\
\hline TCERG1L|256536 & $\mathrm{B} 2$ \\
\hline
\end{tabular}




\begin{tabular}{|c|c|}
\hline TCERG1|10915 & B2 \\
\hline TCF12|6938 & B2 \\
\hline TCF20|6942 & B2 \\
\hline TCF23|150921 & $\mathrm{B} 2$ \\
\hline TCFL5|10732 & $\mathrm{B} 2$ \\
\hline TCHHL1|126637 & $\mathrm{B} 2$ \\
\hline TCHH|7062 & $\mathrm{B} 2$ \\
\hline TCHP $\mid 84260$ & $\mathrm{~B} 2$ \\
\hline TCIRG1|10312 & $\mathrm{B} 2$ \\
\hline TCL1A|8115 & $\mathrm{B} 2$ \\
\hline TCL1B $\mid 9623$ & $\mathrm{~B} 2$ \\
\hline TCL6|27004 & $\mathrm{B} 2$ \\
\hline TCN1|6947 & $\mathrm{B} 2$ \\
\hline TCOF1|6949 & $\mathrm{B} 2$ \\
\hline TCP10L2|401285 & $\mathrm{B} 2$ \\
\hline TCP10L $\mid 140290$ & $\mathrm{~B} 2$ \\
\hline TCP11L1|55346 & $\mathrm{B} 2$ \\
\hline TCP11|6954 & $\mathrm{B} 2$ \\
\hline TCP1|6950 & $\mathrm{B} 2$ \\
\hline TCTA|6988 & $\mathrm{B} 2$ \\
\hline TCTE1|202500 & $\mathrm{B} 2$ \\
\hline TCTE3|6991 & $\mathrm{B} 2$ \\
\hline TCTEX1D1|200132 & $\mathrm{B} 2$ \\
\hline TCTEX1D4|343521 & $\mathrm{B} 2$ \\
\hline TCTN1|79600 & $\mathrm{B} 2$ \\
\hline TCTN2|79867 & $\mathrm{B} 2$ \\
\hline TCTN3|26123 & $\mathrm{B} 2$ \\
\hline TDGF1|6997 & $\mathrm{B} 2$ \\
\hline TDGF3|6998 & $\mathrm{B} 2$ \\
\hline TDH|157739 & $\mathrm{B} 2$ \\
\hline TDO2|6999 & $\mathrm{B} 2$ \\
\hline TDP2|51567 & $\mathrm{B} 2$ \\
\hline TDRD12|91646 & $\mathrm{B} 2$ \\
\hline TDRD1|56165 & $\mathrm{B} 2$ \\
\hline TDRD3|81550 & $\mathrm{B} 2$ \\
\hline TDRD5|163589 & $\mathrm{B} 2$ \\
\hline TDRD7|23424 & $\mathrm{B} 2$ \\
\hline TDRD9|122402 & $\mathrm{B} 2$ \\
\hline TDRG1|732253 & $\mathrm{B} 2$ \\
\hline TEAD2|8463 & $\mathrm{B} 2$ \\
\hline TEAD3|7005 & $\mathrm{B} 2$ \\
\hline TECPR1|25851 & $\mathrm{B} 2$ \\
\hline TECRL|253017 & $\mathrm{B} 2$ \\
\hline TECR $\mid 9524$ & $\mathrm{~B} 2$ \\
\hline TECTA|7007 & $\mathrm{B} 2$ \\
\hline TECTB|6975 & $\mathrm{B} 2$ \\
\hline TEC|7006 & $\mathrm{B} 2$ \\
\hline TEDDM1|127670 & $\mathrm{B} 2$ \\
\hline TEKT1|83659 & $\mathrm{B} 2$ \\
\hline TEKT2|27285 & $\mathrm{B} 2$ \\
\hline \begin{tabular}{l|l|l|} 
TEKT3|64518 \\
\end{tabular} & B2 \\
\hline
\end{tabular}




\begin{tabular}{|c|c|}
\hline TEKT4|150483 & B2 \\
\hline TEKT5|146279 & B2 \\
\hline TELO2|9894 & B2 \\
\hline TEP1|7011 & $\mathrm{B} 2$ \\
\hline TERC|7012 & $\mathrm{B} 2$ \\
\hline TERF1|7013 & $\mathrm{B} 2$ \\
\hline TERF2IP|54386 & $\mathrm{B} 2$ \\
\hline TERF2|7014 & $\mathrm{B} 2$ \\
\hline TERT|7015 & $\mathrm{B} 2$ \\
\hline TESK1|7016 & B2 \\
\hline TESK2|10420 & $\mathrm{B} 2$ \\
\hline TES|26136 & $\mathrm{B} 2$ \\
\hline TET1|80312 & $\mathrm{B} 2$ \\
\hline TET2|54790 & $\mathrm{B} 2$ \\
\hline TEX101|83639 & $\mathrm{B} 2$ \\
\hline TEX10|54881 & $\mathrm{B} 2$ \\
\hline TEX11|56159 & $\mathrm{B} 2$ \\
\hline TEX12|56158 & $\mathrm{B} 2$ \\
\hline TEX13B|56156 & $\mathrm{B} 2$ \\
\hline TEX14|56155 & $\mathrm{B} 2$ \\
\hline TEX15|56154 & $\mathrm{B} 2$ \\
\hline TEX19|400629 & $\mathrm{B} 2$ \\
\hline TEX261|113419 & $\mathrm{B} 2$ \\
\hline TEX264|51368 & $\mathrm{B} 2$ \\
\hline TEX2|55852 & $\mathrm{B} 2$ \\
\hline TEX9|374618 & $\mathrm{B} 2$ \\
\hline TFAMP1|260341 & $\mathrm{B} 2$ \\
\hline TFAM|7019 & $\mathrm{B} 2$ \\
\hline TFAP2B|7021 & $\mathrm{B} 2$ \\
\hline TFAP2C|7022 & $\mathrm{B} 2$ \\
\hline TFAP2D|83741 & $\mathrm{B} 2$ \\
\hline TFAP2E|339488 & $\mathrm{B} 2$ \\
\hline TFAP4|7023 & $\mathrm{B} 2$ \\
\hline TFB1M|51106 & $\mathrm{B} 2$ \\
\hline TFCP2L1|29842 & $\mathrm{B} 2$ \\
\hline TFCP2|7024 & $\mathrm{B} 2$ \\
\hline TFDP2|7029 & $\mathrm{B} 2$ \\
\hline \begin{tabular}{l|l|l|} 
TFDP3 & 51270 \\
\end{tabular} & $\mathrm{~B} 2$ \\
\hline TFEB|7942 & $\mathrm{B} 2$ \\
\hline TFEC|22797 & $\mathrm{B} 2$ \\
\hline TFF1|7031 & $\mathrm{B} 2$ \\
\hline TFF2|7032 & $\mathrm{B} 2$ \\
\hline TFF3|7033 & $\mathrm{B} 2$ \\
\hline TFIP11|24144 & $\mathrm{B} 2$ \\
\hline TFPI2|7980 & $\mathrm{B} 2$ \\
\hline TGDS|23483 & $\mathrm{B} 2$ \\
\hline TGFA|7039 & $\mathrm{B} 2$ \\
\hline TGFB2|7042 & $\mathrm{B} 2$ \\
\hline TGFBI|7045 & $\mathrm{B} 2$ \\
\hline TGFBR1|7046 & $\mathrm{B} 2$ \\
\hline TGFBRAP1|9392 & $\mathrm{B} 2$ \\
\hline
\end{tabular}




\begin{tabular}{|c|c|}
\hline TGIF2LX|90316 & B2 \\
\hline TGIF2LY|90655 & B2 \\
\hline TGIF2|60436 & $\mathrm{B} 2$ \\
\hline TGM1|7051 & $\mathrm{B} 2$ \\
\hline TGM2|7052 & $\mathrm{B} 2$ \\
\hline TGM3|7053 & $\mathrm{B} 2$ \\
\hline TGM4|7047 & $\mathrm{B} 2$ \\
\hline TGM5|9333 & $\mathrm{B} 2$ \\
\hline TGM7|116179 & B2 \\
\hline TGOLN2|10618 & B2 \\
\hline TGS1|96764 & B2 \\
\hline TG|7038 & B2 \\
\hline TH1L|51497 & $\mathrm{B} 2$ \\
\hline THADA|63892 & $\mathrm{B} 2$ \\
\hline THAP10|56906 & $\mathrm{B} 2$ \\
\hline THAP11|57215 & B2 \\
\hline THAP1|55145 & B2 \\
\hline THAP2|83591 & $\mathrm{B} 2$ \\
\hline THAP3|90326 & B2 \\
\hline THAP4|51078 & B2 \\
\hline THAP5|168451 & B2 \\
\hline THAP6|152815 & $\mathrm{B} 2$ \\
\hline THAP7|80764 & $\mathrm{B} 2$ \\
\hline THAP8|199745 & $\mathrm{B} 2$ \\
\hline $\begin{array}{l}\text { THAP9|79725 } \\
\end{array}$ & $\mathrm{B} 2$ \\
\hline THBS1|7057 & $\mathrm{B} 2$ \\
\hline THBS3|7059 & $\mathrm{B} 2$ \\
\hline THBS4|7060 & B2 \\
\hline THEG|51298 & $\mathrm{B} 2$ \\
\hline THEM4|117145 & B2 \\
\hline THEM5|284486 & $\mathrm{B} 2$ \\
\hline THEMIS|387357 & B2 \\
\hline THG1L|54974 & $\mathrm{B} 2$ \\
\hline THNSL1|79896 & $\mathrm{B} 2$ \\
\hline THNSL2|55258 & $\mathrm{B} 2$ \\
\hline THOC1|9984 & $\mathrm{B} 2$ \\
\hline THOC5|8563 & $\mathrm{B} 2$ \\
\hline THOC7|80145 & $\mathrm{B} 2$ \\
\hline THOP1|7064 & B2 \\
\hline THPO|7066 & $\mathrm{B} 2$ \\
\hline THRAP3|9967 & B2 \\
\hline THSD4|79875 & B2 \\
\hline THTPA|79178 & B2 \\
\hline THUMPD1|55623 & $\mathrm{B} 2$ \\
\hline THUMPD $2 \mid 80745$ & $\mathrm{~B} 2$ \\
\hline TH|7054 & $\mathrm{B} 2$ \\
\hline TIA1|7072 & B2 \\
\hline TIAF1|9220 & $\mathrm{B} 2$ \\
\hline TIAL1|7073 & $\mathrm{B} 2$ \\
\hline TIAM1|7074 & B2 \\
\hline TIAM2|26230 & $\mathrm{B} 2$ \\
\hline
\end{tabular}




\begin{tabular}{|c|c|}
\hline TICAM1|148022 & B2 \\
\hline TICAM2|353376 & B2 \\
\hline TIFAB|497189 & $\mathrm{B} 2$ \\
\hline TIFA|92610 & $\mathrm{B} 2$ \\
\hline TIGD1|200765 & B2 \\
\hline TIGD2|166815 & $\mathrm{B} 2$ \\
\hline TIGD4|201798 & B2 \\
\hline TIGD6|81789 & $\mathrm{B} 2$ \\
\hline TIGD7|91151 & B2 \\
\hline TIMD4|91937 & $\mathrm{B} 2$ \\
\hline TIMM10|26519 & $\mathrm{B} 2$ \\
\hline TIMM13|26517 & $\mathrm{B} 2$ \\
\hline TIMM22|29928 & $\mathrm{B} 2$ \\
\hline TIMM44|10469 & $\mathrm{B} 2$ \\
\hline TIMM50|92609 & $\mathrm{B} 2$ \\
\hline TIMM8B|26521 & $\mathrm{B} 2$ \\
\hline TIMM9|26520 & $\mathrm{B} 2$ \\
\hline TIMP2|7077 & $\mathrm{B} 2$ \\
\hline TIMP3|7078 & $\mathrm{B} 2$ \\
\hline TINF2|26277 & $\mathrm{B} 2$ \\
\hline TIRAP|114609 & $\mathrm{B} 2$ \\
\hline TJAP1|93643 & $\mathrm{B} 2$ \\
\hline TKTL2|84076 & $\mathrm{B} 2$ \\
\hline TKT|7086 & $\mathrm{B} 2$ \\
\hline TLE1|7088 & $\mathrm{B} 2$ \\
\hline TLK1|9874 & $\mathrm{B} 2$ \\
\hline TLK2|11011 & $\mathrm{B} 2$ \\
\hline TLR10|81793 & $\mathrm{B} 2$ \\
\hline TLR1|7096 & $\mathrm{B} 2$ \\
\hline TLR2|7097 & $\mathrm{B} 2$ \\
\hline TLR5|7100 & $\mathrm{B} 2$ \\
\hline TLR6|10333 & $\mathrm{B} 2$ \\
\hline TLR7|51284 & $\mathrm{B} 2$ \\
\hline TLR8|51311 & $\mathrm{B} 2$ \\
\hline TLR9|54106 & $\mathrm{B} 2$ \\
\hline TLX1NB|100038246 & $\mathrm{B} 2$ \\
\hline TLX1|3195 & $\mathrm{B} 2$ \\
\hline TLX2|3196 & $\mathrm{B} 2$ \\
\hline TLX3|30012 & $\mathrm{B} 2$ \\
\hline TM2D1|83941 & $\mathrm{B} 2$ \\
\hline \begin{tabular}{l|l|} 
TM2D2|83877 &
\end{tabular} & $\mathrm{B} 2$ \\
\hline TM2D3|80213 & $\mathrm{B} 2$ \\
\hline TM4SF19|116211 & $\mathrm{B} 2$ \\
\hline TM4SF1|4071 & $\mathrm{B} 2$ \\
\hline TM4SF20|79853 & $\mathrm{B} 2$ \\
\hline TM4SF4|7104 & $\mathrm{B} 2$ \\
\hline TM4SF5|9032 & $\mathrm{B} 2$ \\
\hline TM6SF1|53346 & $\mathrm{B} 2$ \\
\hline TM7SF2|7108 & $\mathrm{B} 2$ \\
\hline TM7SF3|51768 & $\mathrm{B} 2$ \\
\hline TM7SF4|81501 & $\mathrm{B} 2$ \\
\hline
\end{tabular}




\begin{tabular}{|c|c|}
\hline TM9SF3|56889 & B2 \\
\hline TMBIM4|51643 & B2 \\
\hline TMC1|117531 & $\mathrm{B} 2$ \\
\hline TMC3|342125 & $\mathrm{B} 2$ \\
\hline TMC5|79838 & B2 \\
\hline TMC7|79905 & $\mathrm{B} 2$ \\
\hline TMC8|147138 & $\mathrm{B} 2$ \\
\hline TMCC1|23023 & $\mathrm{B} 2$ \\
\hline TMCC2|9911 & $\mathrm{B} 2$ \\
\hline TMCO2|127391 & $\mathrm{B} 2$ \\
\hline TMCO5A|145942 & $\mathrm{B} 2$ \\
\hline TMED10|10972 & B2 \\
\hline TMED1|11018 & $\mathrm{B} 2$ \\
\hline TMED5|50999 & $\mathrm{B} 2$ \\
\hline TMED6|146456 & $\mathrm{B} 2$ \\
\hline TMED7-TICAM2|100302736 & $\mathrm{B} 2$ \\
\hline TMED8|283578 & B2 \\
\hline TMEFF1|8577 & $\mathrm{B} 2$ \\
\hline TMEFF2|23671 & B2 \\
\hline TMEM101|84336 & $\mathrm{B} 2$ \\
\hline TMEM102|284114 & $\mathrm{B} 2$ \\
\hline TMEM105|284186 & $\mathrm{B} 2$ \\
\hline TMEM106A|113277 & $\mathrm{B} 2$ \\
\hline TMEM106B|54664 & $\mathrm{B} 2$ \\
\hline TMEM107|84314 & $\mathrm{B} 2$ \\
\hline TMEM108|66000 & $\mathrm{B} 2$ \\
\hline TMEM110|375346 & $\mathrm{B} 2$ \\
\hline TMEM111|55831 & B2 \\
\hline TMEM114|283953 & $\mathrm{B} 2$ \\
\hline TMEM115|11070 & $\mathrm{B} 2$ \\
\hline TMEM116|89894 & $\mathrm{B} 2$ \\
\hline TMEM119|338773 & $\mathrm{B} 2$ \\
\hline TMEM11|8834 & $\mathrm{B} 2$ \\
\hline TMEM120A|83862 & B2 \\
\hline TMEM121|80757 & $\mathrm{B} 2$ \\
\hline TMEM123|114908 & $\mathrm{B} 2$ \\
\hline TMEM126A|84233 & $\mathrm{B} 2$ \\
\hline TMEM126B|55863 & $\mathrm{B} 2$ \\
\hline TMEM128|85013 & $\mathrm{B} 2$ \\
\hline TMEM129|92305 & $\mathrm{B} 2$ \\
\hline TMEM130|222865 & B2 \\
\hline TMEM131|23505 & $\mathrm{B} 2$ \\
\hline TMEM132B|114795 & B2 \\
\hline TMEM132D|121256 & $\mathrm{B} 2$ \\
\hline TMEM132E|124842 & $\mathrm{B} 2$ \\
\hline TMEM135|65084 & $\mathrm{B} 2$ \\
\hline TMEM136|219902 & $\mathrm{B} 2$ \\
\hline TMEM138|51524 & $\mathrm{B} 2$ \\
\hline TMEM139|135932 & $\mathrm{B} 2$ \\
\hline TMEM143|55260 & $\mathrm{B} 2$ \\
\hline TMEM144|55314 & $\mathrm{B} 2$ \\
\hline
\end{tabular}




\begin{tabular}{|c|c|}
\hline TMEM145|284339 & B2 \\
\hline TMEM146|257062 & B2 \\
\hline TMEM149|79713 & $\mathrm{B} 2$ \\
\hline TMEM14B $\mid 81853$ & $\mathrm{~B} 2$ \\
\hline TMEM14C|51522 & B2 \\
\hline TMEM14E|645843 & $\mathrm{B} 2$ \\
\hline TMEM150A|129303 & $\mathrm{B} 2$ \\
\hline TMEM150B|284417 & $\mathrm{B} 2$ \\
\hline TMEM150C|441027 & $\mathrm{B} 2$ \\
\hline TMEM151A|256472 & $\mathrm{B} 2$ \\
\hline TMEM151B|441151 & $\mathrm{B} 2$ \\
\hline TMEM154|201799 & B2 \\
\hline TMEM155|132332 & $\mathrm{B} 2$ \\
\hline TMEM156|80008 & $\mathrm{B} 2$ \\
\hline TMEM158|25907 & B2 \\
\hline TMEM159|57146 & $\mathrm{B} 2$ \\
\hline TMEM160|54958 & $\mathrm{B} 2$ \\
\hline TMEM161A|54929 & $\mathrm{B} 2$ \\
\hline TMEM161B|153396 & B2 \\
\hline TMEM163|81615 & $\mathrm{B} 2$ \\
\hline TMEM164|84187 & $\mathrm{B} 2$ \\
\hline TMEM167A|153339 & $\mathrm{B} 2$ \\
\hline TMEM167B|56900 & $\mathrm{B} 2$ \\
\hline TMEM168|64418 & $\mathrm{B} 2$ \\
\hline TMEM169|92691 & $\mathrm{B} 2$ \\
\hline TMEM171|134285 & $\mathrm{B} 2$ \\
\hline TMEM173|340061 & $\mathrm{B} 2$ \\
\hline TMEM174|134288 & B2 \\
\hline TMEM175|84286 & $\mathrm{B} 2$ \\
\hline TMEM176A|55365 & $\mathrm{B} 2$ \\
\hline TMEM176B|28959 & $\mathrm{B} 2$ \\
\hline TMEM179|388021 & $\mathrm{B} 2$ \\
\hline TMEM17|200728 & $\mathrm{B} 2$ \\
\hline TMEM180|79847 & B2 \\
\hline TMEM181|57583 & $\mathrm{B} 2$ \\
\hline TMEM182|130827 & $\mathrm{B} 2$ \\
\hline TMEM184B|25829 & $\mathrm{B} 2$ \\
\hline TMEM184C|55751 & $\mathrm{B} 2$ \\
\hline TMEM185A|84548 & $\mathrm{B} 2$ \\
\hline TMEM185B|79134 & $\mathrm{B} 2$ \\
\hline TMEM186|25880 & B2 \\
\hline TMEM187|8269 & $\mathrm{B} 2$ \\
\hline TMEM188|255919 & B2 \\
\hline TMEM189-UBE2V1|387522 & $\mathrm{B} 2$ \\
\hline TMEM189|387521 & $\mathrm{B} 2$ \\
\hline TMEM190|147744 & $\mathrm{B} 2$ \\
\hline TMEM192|201931 & $\mathrm{B} 2$ \\
\hline TMEM194B|100131211 & $\mathrm{B} 2$ \\
\hline TMEM196|256130 & $\mathrm{B} 2$ \\
\hline TMEM198|130612 & $\mathrm{B} 2$ \\
\hline TMEM19|55266 & $\mathrm{B} 2$ \\
\hline
\end{tabular}




\begin{tabular}{|c|c|}
\hline TMEM200A|114801 & B2 \\
\hline TMEM200C|645369 & $\mathrm{B} 2$ \\
\hline TMEM201|199953 & B2 \\
\hline TMEM203|94107 & B2 \\
\hline TMEM204|79652 & B2 \\
\hline TMEM211|255349 & B2 \\
\hline TMEM212|389177 & B2 \\
\hline TMEM213|155006 & B2 \\
\hline TMEM214|54867 & B2 \\
\hline TMEM215|401498 & B2 \\
\hline TMEM216|51259 & B2 \\
\hline TMEM217|221468 & B2 \\
\hline TMEM219|124446 & $\mathrm{B} 2$ \\
\hline TMEM222|84065 & B2 \\
\hline TMEM229A|730130 & B2 \\
\hline TMEM229B|161145 & B2 \\
\hline TMEM231|79583 & B2 \\
\hline TMEM232|642987 & B2 \\
\hline TMEM233|387890 & $\mathrm{B} 2$ \\
\hline TMEM25|84866 & B2 \\
\hline TMEM26|219623 & B2 \\
\hline TMEM27|57393 & B2 \\
\hline TMEM2|23670 & $\mathrm{B} 2$ \\
\hline TMEM30A|55754 & B2 \\
\hline TMEM30B|161291 & $\mathrm{B} 2$ \\
\hline TMEM31|203562 & $\mathrm{B} 2$ \\
\hline TMEM38A|79041 & $\mathrm{B} 2$ \\
\hline TMEM38B|55151 & $\mathrm{B} 2$ \\
\hline TMEM39A|55254 & B2 \\
\hline TMEM39B|55116 & $\mathrm{B} 2$ \\
\hline TMEM40|55287 & $\mathrm{B} 2$ \\
\hline TMEM44|93109 & $\mathrm{B} 2$ \\
\hline TMEM45A|55076 & B2 \\
\hline TMEM45B|120224 & $\mathrm{B} 2$ \\
\hline TMEM49|81671 & $\mathrm{B} 2$ \\
\hline TMEM50A|23585 & B2 \\
\hline TMEM50B|757 & $\mathrm{B} 2$ \\
\hline TMEM51|55092 & $\mathrm{B} 2$ \\
\hline TMEM52|339456 & $\mathrm{B} 2$ \\
\hline TMEM53|79639 & $\mathrm{B} 2$ \\
\hline TMEM55B|90809 & $\mathrm{B} 2$ \\
\hline TMEM56|148534 & $\mathrm{B} 2$ \\
\hline TMEM59L|25789 & B2 \\
\hline TMEM59|9528 & $\mathrm{B} 2$ \\
\hline TMEM5|10329 & $\mathrm{B} 2$ \\
\hline TMEM60|85025 & B2 \\
\hline TMEM61|199964 & $\mathrm{B} 2$ \\
\hline TMEM62|80021 & $\mathrm{B} 2$ \\
\hline TMEM63A|9725 & $\mathrm{B} 2$ \\
\hline TMEM64|169200 & $\mathrm{B} 2$ \\
\hline TMEM65|157378 & $\mathrm{B} 2$ \\
\hline
\end{tabular}




\begin{tabular}{|c|c|}
\hline TMEM66|51669 & B2 \\
\hline TMEM67|91147 & $\mathrm{B} 2$ \\
\hline TMEM68|137695 & $\mathrm{B} 2$ \\
\hline TMEM70|54968 & $\mathrm{B} 2$ \\
\hline TMEM72|643236 & B2 \\
\hline TMEM74|157753 & $\mathrm{B} 2$ \\
\hline TMEM81|388730 & $\mathrm{B} 2$ \\
\hline TMEM82|388595 & $\mathrm{B} 2$ \\
\hline TMEM84|283673 & $\mathrm{B} 2$ \\
\hline TMEM85|51234 & $\mathrm{B} 2$ \\
\hline TMEM86A|144110 & $\mathrm{B} 2$ \\
\hline TMEM86B|255043 & B2 \\
\hline TMEM87A|25963 & $\mathrm{B} 2$ \\
\hline TMEM88B|643965 & $\mathrm{B} 2$ \\
\hline TMEM89|440955 & $\mathrm{B} 2$ \\
\hline TMEM8B|51754 & $\mathrm{B} 2$ \\
\hline TMEM8C|389827 & B2 \\
\hline TMEM90A|646658 & $\mathrm{B} 2$ \\
\hline TMEM91|641649 & B2 \\
\hline TMEM92|162461 & $\mathrm{B} 2$ \\
\hline TMEM93|83460 & $\mathrm{B} 2$ \\
\hline TMEM95|339168 & $\mathrm{B} 2$ \\
\hline TMEM98|26022 & $\mathrm{B} 2$ \\
\hline TMEM99|147184 & $\mathrm{B} 2$ \\
\hline TMF1|7110 & $\mathrm{B} 2$ \\
\hline TMIGD1|388364 & $\mathrm{B} 2$ \\
\hline TMIGD2|126259 & $\mathrm{B} 2$ \\
\hline TMOD1|7111 & B2 \\
\hline TMOD3|29766 & $\mathrm{B} 2$ \\
\hline TMOD4|29765 & $\mathrm{B} 2$ \\
\hline TMPPE|643853 & $\mathrm{B} 2$ \\
\hline TMPRSS11A|339967 & $\mathrm{B} 2$ \\
\hline TMPRSS11BNL|401136 & $\mathrm{B} 2$ \\
\hline TMPRSS11B|132724 & $\mathrm{B} 2$ \\
\hline TMPRSS11D|9407 & $\mathrm{B} 2$ \\
\hline TMPRSS11F|389208 & $\mathrm{B} 2$ \\
\hline TMPRSS12|283471 & $\mathrm{B} 2$ \\
\hline TMPRSS13|84000 & $\mathrm{B} 2$ \\
\hline TMPRSS15|5651 & $\mathrm{B} 2$ \\
\hline TMPRSS2|7113 & $\mathrm{B} 2$ \\
\hline TMPRSS3|64699 & B2 \\
\hline TMPRSS4|56649 & $\mathrm{B} 2$ \\
\hline TMPRSS5|80975 & B2 \\
\hline TMPRSS6|164656 & $\mathrm{B} 2$ \\
\hline TMPRSS7|344805 & $\mathrm{B} 2$ \\
\hline TMPRSS9|360200 & $\mathrm{B} 2$ \\
\hline TMSB15A|11013 & $\mathrm{B} 2$ \\
\hline TMSB15B|286527 & $\mathrm{B} 2$ \\
\hline TMSL3|7117 & B2 \\
\hline TMTC2|160335 & $\mathrm{B} 2$ \\
\hline TMUB1|83590 & $\mathrm{B} 2$ \\
\hline
\end{tabular}




\begin{tabular}{|c|c|}
\hline TMUB2|79089 & B2 \\
\hline TMX2|51075 & B2 \\
\hline TMX3|54495 & B2 \\
\hline TMX4|56255 & B2 \\
\hline TNC|3371 & B2 \\
\hline TNFAIP1|7126 & B2 \\
\hline TNFAIP2|7127 & B2 \\
\hline TNFAIP3|7128 & B2 \\
\hline TNFAIP6|7130 & B2 \\
\hline TNFAIP8L2|79626 & B2 \\
\hline TNFAIP8L3|388121 & B2 \\
\hline TNFAIP8|25816 & B2 \\
\hline TNFRSF10A|8797 & $\mathrm{B} 2$ \\
\hline TNFRSF10B|8795 & B2 \\
\hline TNFRSF10C|8794 & B2 \\
\hline TNFRSF11A|8792 & B2 \\
\hline TNFRSF11B|4982 & $\mathrm{B} 2$ \\
\hline TNFRSF12A|51330 & B2 \\
\hline TNFRSF13B|23495 & $\mathrm{B} 2$ \\
\hline TNFRSF13C|115650 & B2 \\
\hline TNFRSF14|8764 & B2 \\
\hline TNFRSF17|608 & $\mathrm{B} 2$ \\
\hline TNFRSF19|55504 & $\mathrm{B} 2$ \\
\hline TNFRSF1A|7132 & B2 \\
\hline TNFRSF25|8718 & $\mathrm{B} 2$ \\
\hline TNFRSF6B|8771 & $\mathrm{B} 2$ \\
\hline TNFRSF9|3604 & $\mathrm{B} 2$ \\
\hline TNFSF10|8743 & $\mathrm{B} 2$ \\
\hline TNFSF11|8600 & B2 \\
\hline TNFSF12-TNFSF13|407977 & $\mathrm{B} 2$ \\
\hline TNFSF13B|10673 & $\mathrm{B} 2$ \\
\hline TNFSF13|8741 & $\mathrm{B} 2$ \\
\hline TNFSF14|8740 & B2 \\
\hline TNFSF15|9966 & $\mathrm{B} 2$ \\
\hline TNFSF18|8995 & $\mathrm{B} 2$ \\
\hline TNFSF8|944 & B2 \\
\hline TNFSF9|8744 & $\mathrm{B} 2$ \\
\hline TNF|7124 & $\mathrm{B} 2$ \\
\hline TNIK|23043 & $\mathrm{B} 2$ \\
\hline TNIP2|79155 & $\mathrm{B} 2$ \\
\hline TNIP3|79931 & $\mathrm{B} 2$ \\
\hline TNK1|8711 & $\mathrm{B} 2$ \\
\hline TNK2|10188 & $\mathrm{B} 2$ \\
\hline TNKS1BP1|85456 & $\mathrm{B} 2$ \\
\hline TNKS2|80351 & $\mathrm{B} 2$ \\
\hline TNKS|8658 & $\mathrm{B} 2$ \\
\hline TNNC1|7134 & $\mathrm{B} 2$ \\
\hline TNNC2|7125 & $\mathrm{B} 2$ \\
\hline TNNI1|7135 & $\mathrm{B} 2$ \\
\hline TNNI2|7136 & $\mathrm{B} 2$ \\
\hline TNNI3K|51086 & B2 \\
\hline
\end{tabular}




\begin{tabular}{|c|c|}
\hline TNNI3|7137 & B2 \\
\hline TNNT1|7138 & B2 \\
\hline TNNT2|7139 & $\mathrm{B} 2$ \\
\hline TNNT3|7140 & $\mathrm{B} 2$ \\
\hline TNN|63923 & B2 \\
\hline TNP1|7141 & $\mathrm{B} 2$ \\
\hline TNP2|7142 & $\mathrm{B} 2$ \\
\hline TNPO1|3842 & $\mathrm{B} 2$ \\
\hline TNPO2|30000 & $\mathrm{B} 2$ \\
\hline TNPO3|23534 & $\mathrm{B} 2$ \\
\hline TNRC18|84629 & $\mathrm{B} 2$ \\
\hline TNRC6A|27327 & $\mathrm{B} 2$ \\
\hline TNRC6C|57690 & $\mathrm{B} 2$ \\
\hline TNR/7143 & $\mathrm{B} 2$ \\
\hline TNS3|64759 & $\mathrm{B} 2$ \\
\hline TOB1|10140 & $\mathrm{B} 2$ \\
\hline TOB2|10766 & $\mathrm{B} 2$ \\
\hline TOE1|114034 & $\mathrm{B} 2$ \\
\hline TOLLIP|54472 & $\mathrm{B} 2$ \\
\hline TOM1L1|10040 & $\mathrm{B} 2$ \\
\hline TOM1|10043 & $\mathrm{B} 2$ \\
\hline TOMM20L|387990 & $\mathrm{B} 2$ \\
\hline TOMM20|9804 & $\mathrm{B} 2$ \\
\hline TOMM22|56993 & $\mathrm{B} 2$ \\
\hline TOMM5|401505 & $\mathrm{B} 2$ \\
\hline TOMM70A|9868 & $\mathrm{B} 2$ \\
\hline TOP1MT|116447 & $\mathrm{B} 2$ \\
\hline TOP2B|7155 & $\mathrm{B} 2$ \\
\hline TOP3A|7156 & $\mathrm{B} 2$ \\
\hline TOP3B $\mid 8940$ & $\mathrm{~B} 2$ \\
\hline TOPORS|10210 & $\mathrm{B} 2$ \\
\hline TOR1AIP1|26092 & $\mathrm{B} 2$ \\
\hline TOX2|84969 & $\mathrm{B} 2$ \\
\hline TOX4|9878 & $\mathrm{B} 2$ \\
\hline TOX|9760 & $\mathrm{B} 2$ \\
\hline TP53BP1|7158 & $\mathrm{B} 2$ \\
\hline TP53I13|90313 & $\mathrm{B} 2$ \\
\hline TP53I3|9540 & $\mathrm{B} 2$ \\
\hline TP53INP2|58476 & $\mathrm{B} 2$ \\
\hline TP53TG1|11257 & $\mathrm{B} 2$ \\
\hline TP53TG5|27296 & $\mathrm{B} 2$ \\
\hline TP53|7157 & $\mathrm{B} 2$ \\
\hline TP73|7161 & $\mathrm{B} 2$ \\
\hline TPCN2|219931 & $\mathrm{B} 2$ \\
\hline TPD52L1|7164 & $\mathrm{B} 2$ \\
\hline TPD52L2|7165 & $\mathrm{B} 2$ \\
\hline TPD52L3|89882 & $\mathrm{B} 2$ \\
\hline TPH1|7166 & $\mathrm{B} 2$ \\
\hline TPH2|121278 & $\mathrm{B} 2$ \\
\hline TPI1P3|728402 & $\mathrm{B} 2$ \\
\hline TPK1|27010 & $\mathrm{B} 2$ \\
\hline
\end{tabular}




\begin{tabular}{|c|c|}
\hline TPM1|7168 & B2 \\
\hline TPM2|7169 & $\mathrm{B} 2$ \\
\hline TPP1|1200 & $\mathrm{B} 2$ \\
\hline TPP2|7174 & $\mathrm{B} 2$ \\
\hline TPRA1|131601 & B2 \\
\hline TPRG1|285386 & $\mathrm{B} 2$ \\
\hline TPRX1|284355 & $\mathrm{B} 2$ \\
\hline TPRXL|348825 & $\mathrm{B} 2$ \\
\hline TPR|7175 & $\mathrm{B} 2$ \\
\hline TPSAB1|7177 & $\mathrm{B} 2$ \\
\hline TPSB2|64499 & $\mathrm{B} 2$ \\
\hline TPSD1|23430 & B2 \\
\hline TPSG1|25823 & $\mathrm{B} 2$ \\
\hline TPST1|8460 & $\mathrm{B} 2$ \\
\hline TPST2|8459 & B2 \\
\hline TPTE2|93492 & $\mathrm{B} 2$ \\
\hline TPTE|7179 & $\mathrm{B} 2$ \\
\hline TRA2A|29896 & $\mathrm{B} 2$ \\
\hline TRA2B|6434 & B2 \\
\hline TRABD|80305 & $\mathrm{B} 2$ \\
\hline TRADD|8717 & $\mathrm{B} 2$ \\
\hline TRAF1|7185 & $\mathrm{B} 2$ \\
\hline TRAF3IP1|26146 & $\mathrm{B} 2$ \\
\hline TRAF3IP2|10758 & $\mathrm{B} 2$ \\
\hline TRAF3IP3|80342 & $\mathrm{B} 2$ \\
\hline TRAF3|7187 & $\mathrm{B} 2$ \\
\hline TRAF4|9618 & $\mathrm{B} 2$ \\
\hline TRAK1|22906 & B2 \\
\hline TRAK2|66008 & $\mathrm{B} 2$ \\
\hline TRAM1L1|133022 & $\mathrm{B} 2$ \\
\hline TRAM1|23471 & $\mathrm{B} 2$ \\
\hline TRAM2|9697 & B2 \\
\hline TRANK1|9881 & $\mathrm{B} 2$ \\
\hline TRAP1|10131 & B2 \\
\hline TRAPPC10|7109 & $\mathrm{B} 2$ \\
\hline TRAPPC1|58485 & $\mathrm{B} 2$ \\
\hline TRAPPC2L|51693 & $\mathrm{B} 2$ \\
\hline TRAPPC2P1|10597 & $\mathrm{B} 2$ \\
\hline TRAPPC2|6399 & $\mathrm{B} 2$ \\
\hline TRAPPC3|27095 & $\mathrm{B} 2$ \\
\hline TRAPPC4|51399 & B2 \\
\hline TRAPPC5|126003 & $\mathrm{B} 2$ \\
\hline TRAPPC6A|79090 & B2 \\
\hline TRAPPC6B|122553 & $\mathrm{B} 2$ \\
\hline TRAT1|50852 & $\mathrm{B} 2$ \\
\hline TRDN|10345 & $\mathrm{B} 2$ \\
\hline TREM2|54209 & $\mathrm{B} 2$ \\
\hline TREML1|340205 & $\mathrm{B} 2$ \\
\hline TREML2|79865 & $\mathrm{B} 2$ \\
\hline TREML3|340206 & $\mathrm{B} 2$ \\
\hline TREML4|285852 & $\mathrm{B} 2$ \\
\hline
\end{tabular}




\begin{tabular}{|c|c|}
\hline TREX1|11277 & B2 \\
\hline TREX2|11219 & B2 \\
\hline TRHR|7201 & $\mathrm{B} 2$ \\
\hline TRH|7200 & $\mathrm{B} 2$ \\
\hline TRIAP1|51499 & B2 \\
\hline TRIB1|10221 & $\mathrm{B} 2$ \\
\hline TRIB2|28951 & $\mathrm{B} 2$ \\
\hline TRIL|9865 & $\mathrm{B} 2$ \\
\hline TRIM10|10107 & $\mathrm{B} 2$ \\
\hline TRIM13|10206 & $\mathrm{B} 2$ \\
\hline TRIM15|89870 & $\mathrm{B} 2$ \\
\hline TRIM16L|147166 & B2 \\
\hline TRIM16|10626 & $\mathrm{B} 2$ \\
\hline TRIM17|51127 & $\mathrm{B} 2$ \\
\hline TRIM21|6737 & $\mathrm{B} 2$ \\
\hline TRIM22|10346 & $\mathrm{B} 2$ \\
\hline TRIM25|7706 & B2 \\
\hline TRIM31|11074 & $\mathrm{B} 2$ \\
\hline TRIM32|22954 & B2 \\
\hline TRIM33|51592 & $\mathrm{B} 2$ \\
\hline TRIM34|53840 & B2 \\
\hline TRIM36|55521 & $\mathrm{B} 2$ \\
\hline TRIM37|4591 & $\mathrm{B} 2$ \\
\hline TRIM38|10475 & $\mathrm{B} 2$ \\
\hline TRIM39|56658 & B2 \\
\hline TRIM3|10612 & $\mathrm{B} 2$ \\
\hline TRIM40|135644 & $\mathrm{B} 2$ \\
\hline TRIM41|90933 & B2 \\
\hline TRIM42|287015 & $\mathrm{B} 2$ \\
\hline TRIM43|129868 & $\mathrm{B} 2$ \\
\hline TRIM44|54765 & $\mathrm{B} 2$ \\
\hline TRIM45|80263 & $\mathrm{B} 2$ \\
\hline TRIM47|91107 & $\mathrm{B} 2$ \\
\hline TRIM48|79097 & B2 \\
\hline TRIM49L|729384 & $\mathrm{B} 2$ \\
\hline TRIM49|57093 & $\mathrm{B} 2$ \\
\hline TRIM4|89122 & $\mathrm{B} 2$ \\
\hline TRIM52|84851 & $\mathrm{B} 2$ \\
\hline TRIM54|57159 & B2 \\
\hline TRIM55|84675 & $\mathrm{B} 2$ \\
\hline TRIM58|25893 & B2 \\
\hline TRIM5|85363 & $\mathrm{B} 2$ \\
\hline TRIM6-TRIM34|445372 & B2 \\
\hline TRIM60|166655 & $\mathrm{B} 2$ \\
\hline TRIM63|84676 & $\mathrm{B} 2$ \\
\hline TRIM65|201292 & $\mathrm{B} 2$ \\
\hline TRIM67|440730 & $\mathrm{B} 2$ \\
\hline TRIM69|140691 & $\mathrm{B} 2$ \\
\hline TRIM6|117854 & $\mathrm{B} 2$ \\
\hline TRIM71|131405 & $\mathrm{B} 2$ \\
\hline TRIM72|493829 & $\mathrm{B} 2$ \\
\hline
\end{tabular}




\begin{tabular}{|c|c|}
\hline TRIM74|378108 & B2 \\
\hline TRIM78P|117852 & B2 \\
\hline TRIM7|81786 & $\mathrm{B} 2$ \\
\hline TRIM8|81603 & $\mathrm{B} 2$ \\
\hline TRIM9|114088 & B2 \\
\hline TRIML1|339976 & $\mathrm{B} 2$ \\
\hline TRIML2|205860 & B2 \\
\hline TRIO|7204 & $\mathrm{B} 2$ \\
\hline TRIP11|9321 & B2 \\
\hline TRIP12|9320 & $\mathrm{B} 2$ \\
\hline TRIP4|9325 & $\mathrm{B} 2$ \\
\hline TRIP6|7205 & $\mathrm{B} 2$ \\
\hline TRIT1|54802 & $\mathrm{B} 2$ \\
\hline TRMT1|55621 & $\mathrm{B} 2$ \\
\hline TRMT2A|27037 & $\mathrm{B} 2$ \\
\hline TRMT2B|79979 & $\mathrm{B} 2$ \\
\hline TRMT61A|115708 & $\mathrm{B} 2$ \\
\hline TRMT61B|55006 & $\mathrm{B} 2$ \\
\hline TRMU|55687 & $\mathrm{B} 2$ \\
\hline TRNAU1AP|54952 & $\mathrm{B} 2$ \\
\hline TRNT1|51095 & $\mathrm{B} 2$ \\
\hline TRPA1|8989 & $\mathrm{B} 2$ \\
\hline TRPC2|7221 & $\mathrm{B} 2$ \\
\hline TRPC3|7222 & $\mathrm{B} 2$ \\
\hline TRPC4AP|26133 & $\mathrm{B} 2$ \\
\hline TRPC4|7223 & $\mathrm{B} 2$ \\
\hline TRPC5|7224 & $\mathrm{B} 2$ \\
\hline TRPC7|57113 & $\mathrm{B} 2$ \\
\hline TRPM1 $\mid 4308$ & $\mathrm{~B} 2$ \\
\hline TRPM4|54795 & $\mathrm{B} 2$ \\
\hline TRPM5|29850 & $\mathrm{B} 2$ \\
\hline TRPM7|54822 & $\mathrm{B} 2$ \\
\hline TRPM8|79054 & $\mathrm{B} 2$ \\
\hline TRPT1|83707 & $\mathrm{B} 2$ \\
\hline TRPV1|7442 & $\mathrm{B} 2$ \\
\hline TRPV2|51393 & $\mathrm{B} 2$ \\
\hline TRPV3|162514 & $\mathrm{B} 2$ \\
\hline TRPV4|59341 & $\mathrm{B} 2$ \\
\hline TRPV5|56302 & $\mathrm{B} 2$ \\
\hline TRPV6|55503 & $\mathrm{B} 2$ \\
\hline TRRAP|8295 & $\mathrm{B} 2$ \\
\hline TRY6|154754 & $\mathrm{B} 2$ \\
\hline TRYX3|136541 & $\mathrm{B} 2$ \\
\hline TSC22D2|9819 & $\mathrm{B} 2$ \\
\hline TSC22D4|81628 & $\mathrm{B} 2$ \\
\hline TSC2|7249 & $\mathrm{B} 2$ \\
\hline TSEN2|80746 & $\mathrm{B} 2$ \\
\hline TSG1|643432 & $\mathrm{B} 2$ \\
\hline TSGA10IP|254187 & $\mathrm{B} 2$ \\
\hline TSGA10|80705 & $\mathrm{B} 2$ \\
\hline TSGA13|114960 & $\mathrm{B} 2$ \\
\hline
\end{tabular}




\begin{tabular}{|c|c|}
\hline TSGA14|95681 & B2 \\
\hline TSHB|7252 & B2 \\
\hline TSHR|7253 & B2 \\
\hline TSHZ3|57616 & $\mathrm{B} 2$ \\
\hline TSIX $\mid 9383$ & B2 \\
\hline TSKU|25987 & B2 \\
\hline TSNARE1|203062 & $\mathrm{B} 2$ \\
\hline TSNAX-DISC1|100303453 & B2 \\
\hline TSNAXIP1|55815 & B2 \\
\hline TSN|7247 & B2 \\
\hline TSPAN10|83882 & B2 \\
\hline TSPAN12|23554 & B2 \\
\hline TSPAN16|26526 & B2 \\
\hline TSPAN19|144448 & B2 \\
\hline TSPAN31|6302 & B2 \\
\hline TSPAN32|10077 & B2 \\
\hline TSPAN33|340348 & $\mathrm{B} 2$ \\
\hline TSPAN5|10098 & B2 \\
\hline TSPAN8|7103 & B2 \\
\hline TSPAN9|10867 & B2 \\
\hline TSPO2|222642 & B2 \\
\hline TSPO|706 & $\mathrm{B} 2$ \\
\hline TSPYL1|7259 & B2 \\
\hline TSPYL3|128854 & B2 \\
\hline TSPYL4|23270 & $\mathrm{B} 2$ \\
\hline TSPYL5|85453 & B2 \\
\hline TSPYL6|388951 & B2 \\
\hline TSR1|55720 & $\mathrm{B} 2$ \\
\hline TSR2|90121 & $\mathrm{B} 2$ \\
\hline TSSC4|10078 & B2 \\
\hline TSSK1B $\mid 83942$ & B2 \\
\hline TSSK6|83983 & $\mathrm{B} 2$ \\
\hline TSTD2|158427 & $\mathrm{B} 2$ \\
\hline TST|7263 & B2 \\
\hline TTBK1|84630 & B2 \\
\hline TTC12|54970 & B2 \\
\hline TTC14|151613 & B2 \\
\hline TTC15|51112 & B2 \\
\hline TTC16|158248 & $\mathrm{B} 2$ \\
\hline TTC17|55761 & B2 \\
\hline TTC18|118491 & B2 \\
\hline TTC19|54902 & $\mathrm{B} 2$ \\
\hline TTC1|7265 & $\mathrm{B} 2$ \\
\hline TTC21A|199223 & $\mathrm{B} 2$ \\
\hline TTC22|55001 & B2 \\
\hline TTC23L|153657 & B2 \\
\hline TTC24|164118 & $\mathrm{B} 2$ \\
\hline TTC25|83538 & B2 \\
\hline TTC27|55622 & B2 \\
\hline TTC29|83894 & $\mathrm{B} 2$ \\
\hline TTC30A|92104 & B2 \\
\hline
\end{tabular}




\begin{tabular}{|c|c|}
\hline ТТС30В|150737 & B2 \\
\hline TTC31|64427 & B2 \\
\hline TTC32|130502 & $\mathrm{B} 2$ \\
\hline TTC35|9694 & $\mathrm{B} 2$ \\
\hline TTC36|143941 & B2 \\
\hline TTC37|9652 & $\mathrm{B} 2$ \\
\hline ТTC39B|158219 & B2 \\
\hline TTC39C|125488 & B2 \\
\hline TTC3|7267 & B2 \\
\hline TTC4|7268 & $\mathrm{B} 2$ \\
\hline TTC5|91875 & $\mathrm{B} 2$ \\
\hline TTC7A|57217 & $\mathrm{B} 2$ \\
\hline TTC8|123016 & $\mathrm{B} 2$ \\
\hline TTF1|7270 & $\mathrm{B} 2$ \\
\hline TTF2|8458 & $\mathrm{B} 2$ \\
\hline TTLL13|440307 & B2 \\
\hline TTLL1|25809 & $\mathrm{B} 2$ \\
\hline TTLL2|83887 & $\mathrm{B} 2$ \\
\hline TTLL4|9654 & $\mathrm{B} 2$ \\
\hline TTLL5|23093 & $\mathrm{B} 2$ \\
\hline TTLL6|284076 & $\mathrm{B} 2$ \\
\hline TTLL7|79739 & $\mathrm{B} 2$ \\
\hline TTLL8|164714 & $\mathrm{B} 2$ \\
\hline TTLL9|164395 & $\mathrm{B} 2$ \\
\hline TTL|150465 & $\mathrm{B} 2$ \\
\hline TTN|7273 & $\mathrm{B} 2$ \\
\hline TTPAL|79183 & $\mathrm{B} 2$ \\
\hline TTPA|7274 & $\mathrm{B} 2$ \\
\hline TTR/7276 & $\mathrm{B} 2$ \\
\hline TTTY15|64595 & $\mathrm{B} 2$ \\
\hline TTYH1|57348 & $\mathrm{B} 2$ \\
\hline TTYH2|94015 & $\mathrm{B} 2$ \\
\hline TUBA1A|7846 & $\mathrm{B} 2$ \\
\hline TUBA3C|7278 & $\mathrm{B} 2$ \\
\hline TUBA3D|113457 & $\mathrm{B} 2$ \\
\hline TUBA3E|112714 & $\mathrm{B} 2$ \\
\hline TUBA4A|7277 & $\mathrm{B} 2$ \\
\hline TUBA4B|80086 & $\mathrm{B} 2$ \\
\hline TUBA8|51807 & $\mathrm{B} 2$ \\
\hline TUBAL3|79861 & $\mathrm{B} 2$ \\
\hline TUBB1|81027 & $\mathrm{B} 2$ \\
\hline TUBB4Q|56604 & $\mathrm{B} 2$ \\
\hline TUBB4|10382 & $\mathrm{B} 2$ \\
\hline TUBBP5|643224 & $\mathrm{B} 2$ \\
\hline TUBD1|51174 & $\mathrm{B} 2$ \\
\hline TUBGCP2|10844 & $\mathrm{B} 2$ \\
\hline TUBGCP3|10426 & $\mathrm{B} 2$ \\
\hline TUBGCP4|27229 & $\mathrm{B} 2$ \\
\hline TUBGCP5|114791 & $\mathrm{B} 2$ \\
\hline TUG1|55000 & $\mathrm{B} 2$ \\
\hline TULP1|7287 & $\mathrm{B} 2$ \\
\hline
\end{tabular}




\begin{tabular}{|c|c|}
\hline TULP3|7289 & B2 \\
\hline TUSC1|286319 & B2 \\
\hline TUSC2|11334 & B2 \\
\hline TUSC3|7991 & $\mathrm{B} 2$ \\
\hline TUT1|64852 & $\mathrm{B} 2$ \\
\hline TWF2|11344 & $\mathrm{B} 2$ \\
\hline TWISTNB|221830 & $\mathrm{B} 2$ \\
\hline TXK|7294 & $\mathrm{B} 2$ \\
\hline TXLNA|200081 & $\mathrm{B} 2$ \\
\hline TXLNB|167838 & $\mathrm{B} 2$ \\
\hline TXLNG|55787 & $\mathrm{B} 2$ \\
\hline TXN2|25828 & $\mathrm{B} 2$ \\
\hline TXNDC15|79770 & $\mathrm{B} 2$ \\
\hline TXNDC16|57544 & $\mathrm{B} 2$ \\
\hline TXNDC3|51314 & $\mathrm{B} 2$ \\
\hline TXNDC5|81567 & $\mathrm{B} 2$ \\
\hline TXNL4B|54957 & $\mathrm{B} 2$ \\
\hline TXNRD1|7296 & $\mathrm{B} 2$ \\
\hline TXNRD2|10587 & $\mathrm{B} 2$ \\
\hline TXNRD3IT1|645840 & $\mathrm{B} 2$ \\
\hline TYK2|7297 & $\mathrm{B} 2$ \\
\hline TYROBP|7305 & $\mathrm{B} 2$ \\
\hline TYRP1|7306 & $\mathrm{B} 2$ \\
\hline TYR|7299 & $\mathrm{B} 2$ \\
\hline TYSND1|219743 & $\mathrm{B} 2$ \\
\hline TYW1B|441250 & $\mathrm{B} 2$ \\
\hline TYW1|55253 & $\mathrm{B} 2$ \\
\hline $\mathrm{T} \mid 6862$ & $\mathrm{~B} 2$ \\
\hline U2AF1L4|199746 & $\mathrm{B} 2$ \\
\hline U2AF1|7307 & $\mathrm{B} 2$ \\
\hline UAP1L1|91373 & $\mathrm{B} 2$ \\
\hline UAP1|6675 & $\mathrm{B} 2$ \\
\hline UBA2|10054 & $\mathrm{B} 2$ \\
\hline UBA3|9039 & $\mathrm{B} 2$ \\
\hline UBA52|7311 & $\mathrm{B} 2$ \\
\hline UBA5|79876 & $\mathrm{B} 2$ \\
\hline UBA6|55236 & $\mathrm{B} 2$ \\
\hline UBA7|7318 & $\mathrm{B} 2$ \\
\hline UBAC1|10422 & $\mathrm{B} 2$ \\
\hline UBAC2|337867 & $\mathrm{B} 2$ \\
\hline UBAP2|55833 & $\mathrm{B} 2$ \\
\hline UBASH3A|53347 & $\mathrm{B} 2$ \\
\hline UBASH3B|84959 & $\mathrm{B} 2$ \\
\hline UBB|7314 & $\mathrm{B} 2$ \\
\hline UBC|7316 & $\mathrm{B} 2$ \\
\hline UBD|10537 & $\mathrm{B} 2$ \\
\hline UBE2B|7320 & $\mathrm{B} 2$ \\
\hline UBE2CBP $\mid 90025$ & $\mathrm{~B} 2$ \\
\hline UBE2D1|7321 & $\mathrm{B} 2$ \\
\hline UBE2D3|7323 & $\mathrm{B} 2$ \\
\hline UBE2D4|51619 & B2 \\
\hline
\end{tabular}




\begin{tabular}{|c|c|}
\hline UBE2DNL|100131816 & B2 \\
\hline UBE2E2|7325 & B2 \\
\hline UBE2G1|7326 & $\mathrm{B} 2$ \\
\hline UBE2J1|51465 & $\mathrm{B} 2$ \\
\hline UBE2J2|118424 & $\mathrm{B} 2$ \\
\hline UBE2L3|7332 & $\mathrm{B} 2$ \\
\hline UBE2NL|389898 & $\mathrm{B} 2$ \\
\hline UBE2Q2|92912 & $\mathrm{B} 2$ \\
\hline UBE2QL1|134111 & B2 \\
\hline UBE2R2|54926 & $\mathrm{B} 2$ \\
\hline UBE2U|148581 & $\mathrm{B} 2$ \\
\hline UBE2V1|7335 & B2 \\
\hline UBE2Z|65264 & $\mathrm{B} 2$ \\
\hline UBE3A|7337 & $\mathrm{B} 2$ \\
\hline UBE3B|89910 & B2 \\
\hline UBE3C|9690 & $\mathrm{B} 2$ \\
\hline UBE4A|9354 & $\mathrm{B} 2$ \\
\hline UBE4B|10277 & $\mathrm{B} 2$ \\
\hline UBIAD1|29914 & B2 \\
\hline UBL3|5412 & $\mathrm{B} 2$ \\
\hline UBL4B|164153 & $\mathrm{B} 2$ \\
\hline UBL7|84993 & $\mathrm{B} 2$ \\
\hline UBLCP1|134510 & $\mathrm{B} 2$ \\
\hline \begin{tabular}{l|l|l|} 
UBN2|254048 \\
\end{tabular} & $\mathrm{B} 2$ \\
\hline $\begin{array}{l}\text { UBOX5|22888 } \\
\end{array}$ & B2 \\
\hline UBP1|7342 & $\mathrm{B} 2$ \\
\hline UBQLN1|29979 & $\mathrm{B} 2$ \\
\hline UBQLN2|29978 & B2 \\
\hline UBQLN3|50613 & $\mathrm{B} 2$ \\
\hline UBQLNL|143630 & $\mathrm{B} 2$ \\
\hline UBR1|197131 & $\mathrm{B} 2$ \\
\hline UBR2|23304 & $\mathrm{B} 2$ \\
\hline UBR3|130507 & $\mathrm{B} 2$ \\
\hline UBR4|23352 & $\mathrm{B} 2$ \\
\hline UBR5|51366 & $\mathrm{B} 2$ \\
\hline UBTD1|80019 & $\mathrm{B} 2$ \\
\hline UBTF|7343 & $\mathrm{B} 2$ \\
\hline UBXN10|127733 & $\mathrm{B} 2$ \\
\hline UBXN11|91544 & $\mathrm{B} 2$ \\
\hline UBXN1|51035 & $\mathrm{B} 2$ \\
\hline UBXN2A|165324 & B2 \\
\hline UBXN6|80700 & $\mathrm{B} 2$ \\
\hline UBXN7|26043 & B2 \\
\hline UBXN8|7993 & $\mathrm{B} 2$ \\
\hline UCA1|652995 & B2 \\
\hline UCHL1|7345 & $\mathrm{B} 2$ \\
\hline UCHL3|7347 & $\mathrm{B} 2$ \\
\hline UCK1|83549 & $\mathrm{B} 2$ \\
\hline UCKL1AS|100113386 & $\mathrm{B} 2$ \\
\hline UCKL1|54963 & $\mathrm{B} 2$ \\
\hline UCMA|221044 & $\mathrm{B} 2$ \\
\hline
\end{tabular}




\begin{tabular}{|c|c|}
\hline UCN2|90226 & B2 \\
\hline \begin{tabular}{l|l|l|} 
UCN3 & 114131 \\
\end{tabular} & $\mathrm{~B} 2$ \\
\hline UCN|7349 & $\mathrm{B} 2$ \\
\hline UCP1|7350 & $\mathrm{B} 2$ \\
\hline UCP2|7351 & $\mathrm{B} 2$ \\
\hline UCP3|7352 & $\mathrm{B} 2$ \\
\hline UEVLD|55293 & $\mathrm{B} 2$ \\
\hline UFM1|51569 & $\mathrm{B} 2$ \\
\hline UFSP1|402682 & $\mathrm{B} 2$ \\
\hline UGCG|7357 & $\mathrm{B} 2$ \\
\hline UGDH|7358 & $\mathrm{B} 2$ \\
\hline UGGT2|55757 & $\mathrm{B} 2$ \\
\hline UGT1A10|54575 & $\mathrm{B} 2$ \\
\hline UGT1A1|54658 & $\mathrm{B} 2$ \\
\hline UGT1A3|54659 & $\mathrm{B} 2$ \\
\hline UGT1A4|54657 & $\mathrm{B} 2$ \\
\hline UGT1A6|54578 & $\mathrm{B} 2$ \\
\hline UGT1A7|54577 & $\mathrm{B} 2$ \\
\hline UGT1A8|54576 & $\mathrm{B} 2$ \\
\hline UGT1A9|54600 & $\mathrm{B} 2$ \\
\hline UGT2A1|10941 & $\mathrm{B} 2$ \\
\hline UGT2A3|79799 & $\mathrm{B} 2$ \\
\hline UGT2B10|7365 & $\mathrm{B} 2$ \\
\hline UGT2B11|10720 & $\mathrm{B} 2$ \\
\hline UGT2B15|7366 & $\mathrm{B} 2$ \\
\hline UGT2B28|54490 & $\mathrm{B} 2$ \\
\hline UGT2B4|7363 & $\mathrm{B} 2$ \\
\hline UGT2B7|7364 & $\mathrm{B} 2$ \\
\hline UGT3A1|133688 & $\mathrm{B} 2$ \\
\hline UGT3A2|167127 & $\mathrm{B} 2$ \\
\hline UGT8|7368 & $\mathrm{B} 2$ \\
\hline UHMK1|127933 & $\mathrm{B} 2$ \\
\hline UHRF1BP1L|23074 & $\mathrm{B} 2$ \\
\hline UHRF1BP1|54887 & $\mathrm{B} 2$ \\
\hline UHRF2|115426 & $\mathrm{B} 2$ \\
\hline ULBP1|80329 & $\mathrm{B} 2$ \\
\hline ULBP2|80328 & $\mathrm{B} 2$ \\
\hline $\begin{array}{l}\text { ULBP3|79465 } \\
\end{array}$ & $\mathrm{B} 2$ \\
\hline ULK1|8408 & $\mathrm{B} 2$ \\
\hline ULK2|9706 & $\mathrm{B} 2$ \\
\hline ULK3|25989 & $\mathrm{B} 2$ \\
\hline ULK4|54986 & $\mathrm{B} 2$ \\
\hline UMODL1|89766 & $\mathrm{B} 2$ \\
\hline UMOD|7369 & $\mathrm{B} 2$ \\
\hline UNC119|9094 & $\mathrm{B} 2$ \\
\hline UNC13A|23025 & $\mathrm{B} 2$ \\
\hline UNC13B|10497 & $\mathrm{B} 2$ \\
\hline UNC13C|440279 & $\mathrm{B} 2$ \\
\hline UNC13D|201294 & $\mathrm{B} 2$ \\
\hline UNC45A|55898 & $\mathrm{B} 2$ \\
\hline \begin{tabular}{l|l} 
UNC45B|146862 \\
\end{tabular} & $\mathrm{B} 2$ \\
\hline
\end{tabular}




\begin{tabular}{|c|c|}
\hline UNC50|25972 & B2 \\
\hline UNC5A|90249 & B2 \\
\hline UNC5CL|222643 & B2 \\
\hline UNC5C $\mid 8633$ & $\mathrm{~B} 2$ \\
\hline UNC5D|137970 & $\mathrm{B} 2$ \\
\hline UNC80|285175 & $\mathrm{B} 2$ \\
\hline UNC93A|54346 & $\mathrm{B} 2$ \\
\hline UNG|7374 & $\mathrm{B} 2$ \\
\hline UNK|85451 & $\mathrm{B} 2$ \\
\hline UOX|391051 & $\mathrm{B} 2$ \\
\hline UPB $1 \mid 51733$ & $\mathrm{~B} 2$ \\
\hline UPF0639|400224 & $\mathrm{B} 2$ \\
\hline UPF1|5976 & $\mathrm{B} 2$ \\
\hline UPF2|26019 & $\mathrm{B} 2$ \\
\hline UPF3A|65110 & $\mathrm{B} 2$ \\
\hline UPF3B|65109 & $\mathrm{B} 2$ \\
\hline UPK1A|11045 & $\mathrm{B} 2$ \\
\hline UPK1B|7348 & $\mathrm{B} 2$ \\
\hline UPK2|7379 & $\mathrm{B} 2$ \\
\hline UPK3A|7380 & $\mathrm{B} 2$ \\
\hline UPK3BL|100134938 & $\mathrm{B} 2$ \\
\hline UPK3B $\mid 80761$ & $\mathrm{~B} 2$ \\
\hline UPP1|7378 & $\mathrm{B} 2$ \\
\hline UPP2|151531 & $\mathrm{B} 2$ \\
\hline UQCC|55245 & $\mathrm{B} 2$ \\
\hline UQCR10|29796 & $\mathrm{B} 2$ \\
\hline UQCR11|10975 & $\mathrm{B} 2$ \\
\hline UQCRB|7381 & $\mathrm{B} 2$ \\
\hline UQCRC1|7384 & $\mathrm{B} 2$ \\
\hline UQCRC2|7385 & $\mathrm{B} 2$ \\
\hline UQCRFS1|7386 & $\mathrm{B} 2$ \\
\hline UQCRHL|440567 & $\mathrm{B} 2$ \\
\hline UQCRH|7388 & $\mathrm{B} 2$ \\
\hline URB1|9875 & $\mathrm{B} 2$ \\
\hline URGCP|55665 & $\mathrm{B} 2$ \\
\hline URM1|81605 & $\mathrm{B} 2$ \\
\hline UROC1|131669 & $\mathrm{B} 2$ \\
\hline UROS|7390 & $\mathrm{B} 2$ \\
\hline USE1|55850 & $\mathrm{B} 2$ \\
\hline USF2|7392 & $\mathrm{B} 2$ \\
\hline USH1C|10083 & $\mathrm{B} 2$ \\
\hline USH1G|124590 & $\mathrm{B} 2$ \\
\hline USH2A|7399 & $\mathrm{B} 2$ \\
\hline USMG5|84833 & $\mathrm{B} 2$ \\
\hline USO1|8615 & $\mathrm{B} 2$ \\
\hline USP10|9100 & $\mathrm{B} 2$ \\
\hline USP12|219333 & $\mathrm{B} 2$ \\
\hline USP13|8975 & $\mathrm{B} 2$ \\
\hline USP15|9958 & $\mathrm{B} 2$ \\
\hline USP16|10600 & $\mathrm{B} 2$ \\
\hline USP17L2|377630 & B2 \\
\hline
\end{tabular}




\begin{tabular}{|c|c|}
\hline USP17L6P|391622 & B2 \\
\hline USP17|391627 & B2 \\
\hline USP1|7398 & $\mathrm{B} 2$ \\
\hline USP20|10868 & $\mathrm{B} 2$ \\
\hline USP22|23326 & B2 \\
\hline USP24|23358 & $\mathrm{B} 2$ \\
\hline USP26|83844 & $\mathrm{B} 2$ \\
\hline USP27X|389856 & $\mathrm{B} 2$ \\
\hline USP28|57646 & $\mathrm{B} 2$ \\
\hline USP29|57663 & $\mathrm{B} 2$ \\
\hline USP2|9099 & $\mathrm{B} 2$ \\
\hline USP32|84669 & $\mathrm{B} 2$ \\
\hline USP33|23032 & $\mathrm{B} 2$ \\
\hline USP34|9736 & $\mathrm{B} 2$ \\
\hline USP35|57558 & $\mathrm{B} 2$ \\
\hline USP36|57602 & $\mathrm{B} 2$ \\
\hline USP38|84640 & $\mathrm{B} 2$ \\
\hline USP3|9960 & $\mathrm{B} 2$ \\
\hline USP40|55230 & $\mathrm{B} 2$ \\
\hline USP42|84132 & $\mathrm{B} 2$ \\
\hline USP43|124739 & $\mathrm{B} 2$ \\
\hline USP45|85015 & $\mathrm{B} 2$ \\
\hline USP46|64854 & $\mathrm{B} 2$ \\
\hline USP47|55031 & $\mathrm{B} 2$ \\
\hline USP48|84196 & $\mathrm{B} 2$ \\
\hline USP49|25862 & $\mathrm{B} 2$ \\
\hline USP4|7375 & $\mathrm{B} 2$ \\
\hline USP50|373509 & $\mathrm{B} 2$ \\
\hline USP51|158880 & $\mathrm{B} 2$ \\
\hline USP54|159195 & $\mathrm{B} 2$ \\
\hline USP6NL|9712 & $\mathrm{B} 2$ \\
\hline USP6|9098 & $\mathrm{B} 2$ \\
\hline USP8|9101 & $\mathrm{B} 2$ \\
\hline USP9X|8239 & $\mathrm{B} 2$ \\
\hline USP9Y|8287 & $\mathrm{B} 2$ \\
\hline USPL1|10208 & $\mathrm{B} 2$ \\
\hline UTF1|8433 & $\mathrm{B} 2$ \\
\hline UTP11L|51118 & $\mathrm{B} 2$ \\
\hline UTP14A|10813 & $\mathrm{B} 2$ \\
\hline UTP14C|9724 & $\mathrm{B} 2$ \\
\hline UTP15|84135 & $\mathrm{B} 2$ \\
\hline UTP18|51096 & $\mathrm{B} 2$ \\
\hline UTP20|27340 & $\mathrm{B} 2$ \\
\hline UTP23|84294 & $\mathrm{B} 2$ \\
\hline UTP3|57050 & $\mathrm{B} 2$ \\
\hline UTP6|55813 & $\mathrm{B} 2$ \\
\hline UTS2R/2837 & $\mathrm{B} 2$ \\
\hline UTS2|10911 & $\mathrm{B} 2$ \\
\hline UTY|7404 & $\mathrm{B} 2$ \\
\hline UXT|8409 & $\mathrm{B} 2$ \\
\hline VAC14|55697 & $\mathrm{B} 2$ \\
\hline
\end{tabular}




\begin{tabular}{|c|c|}
\hline VAMP1|6843 & B2 \\
\hline VAMP4|8674 & $\mathrm{B} 2$ \\
\hline VAMP5|10791 & $\mathrm{B} 2$ \\
\hline VAMP7|6845 & $\mathrm{B} 2$ \\
\hline VANGL2|57216 & $\mathrm{B} 2$ \\
\hline VAPA|9218 & $\mathrm{B} 2$ \\
\hline VAPB|9217 & $\mathrm{B} 2$ \\
\hline VASH1|22846 & $\mathrm{B} 2$ \\
\hline VASH2|79805 & B2 \\
\hline VASN|114990 & $\mathrm{B} 2$ \\
\hline VAT1L|57687 & $\mathrm{B} 2$ \\
\hline VAT1|10493 & B2 \\
\hline VAV1|7409 & $\mathrm{B} 2$ \\
\hline VAX1|11023 & $\mathrm{B} 2$ \\
\hline VAX2|25806 & $\mathrm{B} 2$ \\
\hline VCAM1|7412 & $\mathrm{B} 2$ \\
\hline VCL|7414 & B2 \\
\hline VCPIP1|80124 & $\mathrm{B} 2$ \\
\hline 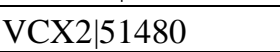 & B2 \\
\hline VCX3A|51481 & B2 \\
\hline VCX3B|425054 & B2 \\
\hline VCX 26609 & $\mathrm{~B} 2$ \\
\hline VDAC2|7417 & B2 \\
\hline VDAC3|7419 & $\mathrm{B} 2$ \\
\hline VDR|7421 & $\mathrm{B} 2$ \\
\hline VEGFA|7422 & $\mathrm{B} 2$ \\
\hline VENTXP1|139538 & $\mathrm{B} 2$ \\
\hline VENTXP7|391518 & B2 \\
\hline VEPH1|79674 & $\mathrm{B} 2$ \\
\hline VEZF1|7716 & B2 \\
\hline $\begin{array}{l}\text { VGF|7425 } \\
\end{array}$ & $\mathrm{B} 2$ \\
\hline VGLL1|51442 & B2 \\
\hline VGLL2|245806 & $\mathrm{B} 2$ \\
\hline VGLL4|9686 & B2 \\
\hline VHLL|391104 & B2 \\
\hline VHL|7428 & $\mathrm{B} 2$ \\
\hline VIL1|7429 & $\mathrm{B} 2$ \\
\hline VIPAR|63894 & $\mathrm{B} 2$ \\
\hline VIPR2|7434 & $\mathrm{B} 2$ \\
\hline VMA21|203547 & $\mathrm{B} 2$ \\
\hline VMAC|400673 & B2 \\
\hline VMO1|284013 & B2 \\
\hline VN1R1|57191 & B2 \\
\hline VN1R2|317701 & $\mathrm{B} 2$ \\
\hline VN1R5|317705 & $\mathrm{B} 2$ \\
\hline VNN1|8876 & $\mathrm{B} 2$ \\
\hline VNN2|8875 & $\mathrm{B} 2$ \\
\hline \begin{tabular}{l|l|l|} 
VNN35350 \\
\end{tabular} & $\mathrm{B} 2$ \\
\hline VPRBP|9730 & $\mathrm{B} 2$ \\
\hline VPREB1|7441 & B2 \\
\hline VPREB3|29802 & $\mathrm{B} 2$ \\
\hline
\end{tabular}




\begin{tabular}{|c|c|}
\hline VPS13B|157680 & B2 \\
\hline VPS13C|54832 & B2 \\
\hline VPS16|64601 & $\mathrm{B} 2$ \\
\hline VPS18|57617 & $\mathrm{B} 2$ \\
\hline VPS26A|9559 & B2 \\
\hline VPS26B|112936 & B2 \\
\hline VPS29|51699 & $\mathrm{B} 2$ \\
\hline VPS33B|26276 & $\mathrm{B} 2$ \\
\hline VPS35|55737 & $\mathrm{B} 2$ \\
\hline VPS37A|137492 & $\mathrm{B} 2$ \\
\hline VPS37B|79720 & $\mathrm{B} 2$ \\
\hline VPS37D|155382 & B2 \\
\hline VPS41|27072 & $\mathrm{B} 2$ \\
\hline VPS4A|27183 & $\mathrm{B} 2$ \\
\hline VPS4B|9525 & $\mathrm{B} 2$ \\
\hline VPS53|55275 & $\mathrm{B} 2$ \\
\hline VPS54|51542 & $\mathrm{B} 2$ \\
\hline VPS8|23355 & $\mathrm{B} 2$ \\
\hline VRK2|7444 & B2 \\
\hline VRK3|51231 & $\mathrm{B} 2$ \\
\hline VSIG10L|147645 & $\mathrm{B} 2$ \\
\hline VSIG10|54621 & $\mathrm{B} 2$ \\
\hline VSIG1|340547 & $\mathrm{B} 2$ \\
\hline VSIG2|23584 & $\mathrm{B} 2$ \\
\hline VSIG4|11326 & $\mathrm{B} 2$ \\
\hline VSIG8|391123 & $\mathrm{B} 2$ \\
\hline VSTM1|284415 & B2 \\
\hline VSTM2A|222008 & B2 \\
\hline VSTM2B|342865 & $\mathrm{B} 2$ \\
\hline VSTM2L|128434 & $\mathrm{B} 2$ \\
\hline VSX1|30813 & $\mathrm{B} 2$ \\
\hline VSX2|338917 & $\mathrm{B} 2$ \\
\hline \begin{tabular}{|l||r|} 
VTA1 51534 \\
\end{tabular} & $\mathrm{~B} 2$ \\
\hline VTCN1|79679 & B2 \\
\hline VTI1A|143187 & $\mathrm{B} 2$ \\
\hline VTI1B|10490 & $\mathrm{B} 2$ \\
\hline VTN|7448 & $\mathrm{B} 2$ \\
\hline VWA2|340706 & $\mathrm{B} 2$ \\
\hline VWA3A|146177 & $\mathrm{B} 2$ \\
\hline VWA3B|200403 & $\mathrm{B} 2$ \\
\hline VWA5B1|127731 & B2 \\
\hline VWA5B2|90113 & $\mathrm{B} 2$ \\
\hline VWC2L|402117 & B2 \\
\hline VWC2|375567 & $\mathrm{B} 2$ \\
\hline VWDE|221806 & B2 \\
\hline WAC|51322 & $\mathrm{B} 2$ \\
\hline WAPAL|23063 & $\mathrm{B} 2$ \\
\hline WARS2|10352 & $\mathrm{B} 2$ \\
\hline WARS|7453 & $\mathrm{B} 2$ \\
\hline WASF1|8936 & $\mathrm{B} 2$ \\
\hline WASH2P 375260 & $\mathrm{~B} 2$ \\
\hline
\end{tabular}




\begin{tabular}{|c|c|}
\hline WASH3P|374666 & B2 \\
\hline WASH5P 375690 & $\mathrm{~B} 2$ \\
\hline WASH7P|653635 & $\mathrm{B} 2$ \\
\hline WASL|8976 & $\mathrm{B} 2$ \\
\hline WAS|7454 & $\mathrm{B} 2$ \\
\hline WBP11P1|441818 & B2 \\
\hline WBP11|51729 & $\mathrm{B} 2$ \\
\hline WBP2|23558 & B2 \\
\hline WBP5|51186 & $\mathrm{B} 2$ \\
\hline WBSCR16|81554 & $\mathrm{B} 2$ \\
\hline WBSCR17|64409 & $\mathrm{B} 2$ \\
\hline WBSCR26|171022 & B2 \\
\hline WBSCR27|155368 & $\mathrm{B} 2$ \\
\hline WDFY1|57590 & $\mathrm{B} 2$ \\
\hline WDFY3|23001 & $\mathrm{B} 2$ \\
\hline WDFY4|57705 & $\mathrm{B} 2$ \\
\hline WDR16|146845 & B2 \\
\hline WDR17|116966 & $\mathrm{B} 2$ \\
\hline WDR18|57418 & B2 \\
\hline WDR20|91833 & $\mathrm{B} 2$ \\
\hline WDR25|79446 & $\mathrm{B} 2$ \\
\hline WDR31|114987 & $\mathrm{B} 2$ \\
\hline WDR33|55339 & $\mathrm{B} 2$ \\
\hline \begin{tabular}{l|l|} 
WDR35 & 57539 \\
\end{tabular} & $\mathrm{~B} 2$ \\
\hline WDR36|134430 & $\mathrm{B} 2$ \\
\hline WDR38|401551 & $\mathrm{B} 2$ \\
\hline WDR3|10885 & B2 \\
\hline WDR41|55255 & B2 \\
\hline WDR43|23160 & $\mathrm{B} 2$ \\
\hline WDR44|54521 & $\mathrm{B} 2$ \\
\hline WDR45L|56270 & $\mathrm{B} 2$ \\
\hline WDR45|11152 & B2 \\
\hline WDR46|9277 & $\mathrm{B} 2$ \\
\hline WDR47|22911 & B2 \\
\hline WDR49|151790 & $\mathrm{B} 2$ \\
\hline WDR4|10785 & $\mathrm{B} 2$ \\
\hline \begin{tabular}{l|l|} 
WDR52|55779 \\
\end{tabular} & $\mathrm{B} 2$ \\
\hline WDR53|348793 & $\mathrm{B} 2$ \\
\hline WDR5B|54554 & $\mathrm{B} 2$ \\
\hline WDR5|11091 & $\mathrm{B} 2$ \\
\hline WDR60|55112 & B2 \\
\hline WDR61|80349 & $\mathrm{B} 2$ \\
\hline WDR62|284403 & B2 \\
\hline WDR63|126820 & $\mathrm{B} 2$ \\
\hline WDR64|128025 & B2 \\
\hline WDR65|149465 & $\mathrm{B} 2$ \\
\hline WDR66|144406 & $\mathrm{B} 2$ \\
\hline WDR69|164781 & $\mathrm{B} 2$ \\
\hline WDR6|11180 & $\mathrm{B} 2$ \\
\hline WDR70|55100 & $\mathrm{B} 2$ \\
\hline WDR72|256764 & $\mathrm{B} 2$ \\
\hline
\end{tabular}




\begin{tabular}{|c|c|}
\hline WDR73|84942 & B2 \\
\hline WDR74|54663 & B2 \\
\hline WDR77|79084 & $\mathrm{B} 2$ \\
\hline WDR78|79819 & $\mathrm{B} 2$ \\
\hline WDR7|23335 & B2 \\
\hline WDR82|80335 & B2 \\
\hline WDR83|84292 & $\mathrm{B} 2$ \\
\hline WDR85|92715 & B2 \\
\hline WDR87|83889 & $\mathrm{B} 2$ \\
\hline WDR88|126248 & $\mathrm{B} 2$ \\
\hline WDR89|112840 & $\mathrm{B} 2$ \\
\hline WDR8|49856 & B2 \\
\hline WDR91|29062 & $\mathrm{B} 2$ \\
\hline WDR92|116143 & $\mathrm{B} 2$ \\
\hline WDR93|56964 & B2 \\
\hline WDSUB1|151525 & $\mathrm{B} 2$ \\
\hline WEE1|7465 & $\mathrm{B} 2$ \\
\hline WEE2|494551 & $\mathrm{B} 2$ \\
\hline WFDC10A|140832 & B2 \\
\hline WFDC10B|280664 & $\mathrm{B} 2$ \\
\hline WFDC11|259239 & $\mathrm{B} 2$ \\
\hline WFDC12|128488 & $\mathrm{B} 2$ \\
\hline WFDC13|164237 & $\mathrm{B} 2$ \\
\hline WFDC1|58189 & $\mathrm{B} 2$ \\
\hline WFDC2|10406 & $\mathrm{B} 2$ \\
\hline WFDC3|140686 & $\mathrm{B} 2$ \\
\hline WFDC5|149708 & B2 \\
\hline WFDC6|140870 & B2 \\
\hline $\begin{array}{l}\text { WFDC8|90199 } \\
\end{array}$ & $\mathrm{B} 2$ \\
\hline WFIKKN1|117166 & $\mathrm{B} 2$ \\
\hline WFIKKN2|124857 & $\mathrm{B} 2$ \\
\hline WFS1|7466 & B2 \\
\hline WHSC1L1|54904 & $\mathrm{B} 2$ \\
\hline WHSC2|7469 & B2 \\
\hline WIF1|11197 & $\mathrm{B} 2$ \\
\hline WIPF1|7456 & $\mathrm{B} 2$ \\
\hline WIPF2|147179 & $\mathrm{B} 2$ \\
\hline WIPF3|644150 & $\mathrm{B} 2$ \\
\hline WIPI1|55062 & $\mathrm{B} 2$ \\
\hline WIPI2|26100 & $\mathrm{B} 2$ \\
\hline WISP3|8838 & B2 \\
\hline WIT1|51352 & $\mathrm{B} 2$ \\
\hline WIZ|58525 & B2 \\
\hline WNK1|65125 & $\mathrm{B} 2$ \\
\hline WNK3|65267 & $\mathrm{B} 2$ \\
\hline WNK4|65266 & $\mathrm{B} 2$ \\
\hline WNT10A|80326 & $\mathrm{B} 2$ \\
\hline WNT10B|7480 & $\mathrm{B} 2$ \\
\hline WNT11|7481 & $\mathrm{B} 2$ \\
\hline \begin{tabular}{l|l|} 
WNT16 &
\end{tabular} & $\mathrm{B} 2$ \\
\hline WNT1|7471 & $\mathrm{B} 2$ \\
\hline
\end{tabular}




\begin{tabular}{|c|c|}
\hline WNT2B|7482 & B2 \\
\hline WNT3A|89780 & B2 \\
\hline WNT3|7473 & B2 \\
\hline WNT4|54361 & B2 \\
\hline WNT5A|7474 & B2 \\
\hline WNT5B|81029 & $\mathrm{B} 2$ \\
\hline WNT6|7475 & B2 \\
\hline WNT7A|7476 & $\mathrm{B} 2$ \\
\hline WNT8A|7478 & B2 \\
\hline WNT8B|7479 & B2 \\
\hline WNT9A|7483 & B2 \\
\hline WRAP53|55135 & $\mathrm{B} 2$ \\
\hline WRB|7485 & $\mathrm{B} 2$ \\
\hline WRN|7486 & B2 \\
\hline WSB2|55884 & $\mathrm{B} 2$ \\
\hline WT1|7490 & B2 \\
\hline WWC1|23286 & B2 \\
\hline \begin{tabular}{l|l|} 
WWC2|80014 \\
\end{tabular} & $\mathrm{B} 2$ \\
\hline WWC3|55841 & $\mathrm{B} 2$ \\
\hline WWOX|51741 & $\mathrm{B} 2$ \\
\hline WWP1|11059 & $\mathrm{B} 2$ \\
\hline WWP2|11060 & $\mathrm{B} 2$ \\
\hline WWTR1|25937 & $\mathrm{B} 2$ \\
\hline $\begin{array}{l}\text { XAB2|56949 } \\
\end{array}$ & $\mathrm{B} 2$ \\
\hline XAF1|54739 & $\mathrm{B} 2$ \\
\hline XAGE1D|9503 & $\mathrm{B} 2$ \\
\hline XAGE2|9502 & B2 \\
\hline XAGE3|170626 & $\mathrm{B} 2$ \\
\hline XAGE5|170627 & B2 \\
\hline XCL1|6375 & $\mathrm{B} 2$ \\
\hline XCL2|6846 & $\mathrm{B} 2$ \\
\hline XCR1|2829 & $\mathrm{B} 2$ \\
\hline XDH|7498 & $\mathrm{B} 2$ \\
\hline XG|7499 & $\mathrm{B} 2$ \\
\hline \begin{tabular}{l|l|l|} 
XIAP & 331 \\
\end{tabular} & $\mathrm{~B} 2$ \\
\hline XIRP1|165904 & $\mathrm{B} 2$ \\
\hline XIRP2|129446 & $\mathrm{B} 2$ \\
\hline XKR3|150165 & $\mathrm{B} 2$ \\
\hline XKR4|114786 & B2 \\
\hline \begin{tabular}{l|l|} 
XKR5|389610 \\
\end{tabular} & $\mathrm{B} 2$ \\
\hline XKR7|343702 & $\mathrm{B} 2$ \\
\hline XKR8|55113 & $\mathrm{B} 2$ \\
\hline XKR9|389668 & $\mathrm{B} 2$ \\
\hline XKRX|402415 & B2 \\
\hline XK|7504 & $\mathrm{B} 2$ \\
\hline XPNPEP1|7511 & $\mathrm{B} 2$ \\
\hline $\begin{array}{l}\text { XPNPEP3|63929 } \\
\end{array}$ & B2 \\
\hline XPO4|64328 & B2 \\
\hline XPO7|23039 & B2 \\
\hline XRCC1|7515 & B2 \\
\hline XRCC3|7517 & B2 \\
\hline
\end{tabular}




\begin{tabular}{|c|c|}
\hline XRCC6BP1|91419 & B2 \\
\hline XRCC6|2547 & $\mathrm{B} 2$ \\
\hline XRN1|54464 & $\mathrm{B} 2$ \\
\hline XRN2|22803 & B2 \\
\hline XRRA1|143570 & $\mathrm{B} 2$ \\
\hline XYLT1|64131 & B2 \\
\hline XYLT2|64132 & $\mathrm{B} 2$ \\
\hline YAF2|10138 & $\mathrm{B} 2$ \\
\hline YBX1|4904 & $\mathrm{B} 2$ \\
\hline YBX2|51087 & $\mathrm{B} 2$ \\
\hline YEATS2|55689 & B2 \\
\hline YES1|7525 & B2 \\
\hline YIPF4|84272 & B2 \\
\hline YIPF5|81555 & $\mathrm{B} 2$ \\
\hline YIPF7|285525 & $\mathrm{B} 2$ \\
\hline YJEFN3|374887 & $\mathrm{B} 2$ \\
\hline YOD1|55432 & $\mathrm{B} 2$ \\
\hline YPEL1|29799 & $\mathrm{B} 2$ \\
\hline YPEL2|388403 & B2 \\
\hline YPEL3|83719 & B2 \\
\hline YPEL5|51646 & $\mathrm{B} 2$ \\
\hline YRDC|79693 & B2 \\
\hline YSK4|80122 & $\mathrm{B} 2$ \\
\hline YTHDC1|91746 & B2 \\
\hline \begin{tabular}{l|l|} 
YTHDC2|64848 \\
\end{tabular} & $\mathrm{B} 2$ \\
\hline YTHDF2|51441 & B2 \\
\hline YTHDF3|253943 & $\mathrm{B} 2$ \\
\hline YWHAE|7531 & B2 \\
\hline YWHAG|7532 & $\mathrm{B} 2$ \\
\hline YWHAQ|10971 & $\mathrm{B} 2$ \\
\hline ZACN|353174 & B2 \\
\hline ZADH2|284273 & $\mathrm{B} 2$ \\
\hline ZAN|7455 & B2 \\
\hline ZAP70|7535 & $\mathrm{B} 2$ \\
\hline ZAR1L|646799 & B2 \\
\hline ZAR1|326340 & $\mathrm{B} 2$ \\
\hline ZBBX|79740 & B2 \\
\hline ZBED1|9189 & $\mathrm{B} 2$ \\
\hline ZBED2|79413 & $\mathrm{B} 2$ \\
\hline \begin{tabular}{l|l|} 
ZBED5|58486 &
\end{tabular} & $\mathrm{B} 2$ \\
\hline ZBP1|81030 & B2 \\
\hline ZBTB10|65986 & B2 \\
\hline ZBTB11|27107 & B2 \\
\hline ZBTB17|7709 & B2 \\
\hline \begin{tabular}{l|l|} 
ZBTB22|9278 \\
\end{tabular} & $\mathrm{B} 2$ \\
\hline ZBTB24|9841 & B2 \\
\hline $\begin{array}{l}\text { ZBTB25|7597 } \\
\end{array}$ & $\mathrm{B} 2$ \\
\hline ZBTB26|57684 & B2 \\
\hline $\begin{array}{l}\text { ZBTB2|57621 } \\
\end{array}$ & $\mathrm{B} 2$ \\
\hline ZBTB32|27033 & B2 \\
\hline ZBTB33|10009 & $\mathrm{B} 2$ \\
\hline
\end{tabular}




\begin{tabular}{|c|c|}
\hline ZBTB37|84614 & B2 \\
\hline ZBTB39|9880 & B2 \\
\hline ZBTB3|79842 & $\mathrm{B} 2$ \\
\hline ZBTB43|23099 & $\mathrm{B} 2$ \\
\hline ZBTB45|84878 & $\mathrm{B} 2$ \\
\hline ZBTB46|140685 & B2 \\
\hline ZBTB48|3104 & $\mathrm{B} 2$ \\
\hline ZBTB5|9925 & $\mathrm{B} 2$ \\
\hline ZBTB6|10773 & B2 \\
\hline ZBTB7A|51341 & $\mathrm{B} 2$ \\
\hline ZBTB7C|201501 & $\mathrm{B} 2$ \\
\hline ZBTB8A|653121 & $\mathrm{B} 2$ \\
\hline ZBTB8B|728116 & $\mathrm{B} 2$ \\
\hline ZC3H10|84872 & $\mathrm{B} 2$ \\
\hline ZC3H11A|9877 & $\mathrm{B} 2$ \\
\hline ZC3H12A|80149 & $\mathrm{B} 2$ \\
\hline ZC3H12D|340152 & $\mathrm{B} 2$ \\
\hline ZC3H14|79882 & $\mathrm{B} 2$ \\
\hline ZC3H18|124245 & B2 \\
\hline ZC3H4|23211 & $\mathrm{B} 2$ \\
\hline ZC3H7A|29066 & B2 \\
\hline ZC3H8|84524 & $\mathrm{B} 2$ \\
\hline ZC3HAV1L|92092 & $\mathrm{B} 2$ \\
\hline ZC3HAV1|56829 & $\mathrm{B} 2$ \\
\hline ZC3HC1|51530 & $\mathrm{B} 2$ \\
\hline ZC4H2|55906 & $\mathrm{B} 2$ \\
\hline ZCCHC14|23174 & $\mathrm{B} 2$ \\
\hline ZCCHC16|340595 & B2 \\
\hline ZCCHC17|51538 & $\mathrm{B} 2$ \\
\hline ZCCHC18|644353 & $\mathrm{B} 2$ \\
\hline ZCCHC2|54877 & $\mathrm{B} 2$ \\
\hline \begin{tabular}{ll|} 
ZCCHC3|85364 \\
\end{tabular} & $\mathrm{B} 2$ \\
\hline ZCCHC5|203430 & $\mathrm{B} 2$ \\
\hline ZCCHC6|79670 & B2 \\
\hline ZCCHC7|84186 & $\mathrm{B} 2$ \\
\hline ZCCHC8|55596 & $\mathrm{B} 2$ \\
\hline \begin{tabular}{l|l} 
ZCCHC9|84240 \\
\end{tabular} & $\mathrm{B} 2$ \\
\hline ZCRB1|85437 & $\mathrm{B} 2$ \\
\hline ZDHHC11|79844 & $\mathrm{B} 2$ \\
\hline ZDHHC14|79683 & $\mathrm{B} 2$ \\
\hline ZDHHC18|84243 & B2 \\
\hline ZDHHC19|131540 & $\mathrm{B} 2$ \\
\hline ZDHHC20|253832 & B2 \\
\hline ZDHHC21|340481 & $\mathrm{B} 2$ \\
\hline ZDHHC22|283576 & $\mathrm{B} 2$ \\
\hline ZDHHC23|254887 & $\mathrm{B} 2$ \\
\hline $\begin{array}{l}\text { ZDHHC3|51304 } \\
\end{array}$ & $\mathrm{B} 2$ \\
\hline ZDHHC4|55146 & $\mathrm{B} 2$ \\
\hline $\begin{array}{l}\text { ZDHHC5|25921 } \\
\end{array}$ & $\mathrm{B} 2$ \\
\hline ZDHHC7|55625 & B2 \\
\hline ZDHHC8P1|150244 & $\mathrm{B} 2$ \\
\hline
\end{tabular}




\begin{tabular}{|c|c|}
\hline ZEB1|6935 & B2 \\
\hline ZFAND1|79752 & $\mathrm{B} 2$ \\
\hline ZFAND2A|90637 & B2 \\
\hline ZFAND2B|130617 & $\mathrm{B} 2$ \\
\hline ZFAND3|60685 & $\mathrm{B} 2$ \\
\hline ZFAND6|54469 & B2 \\
\hline ZFATAS|594840 & $\mathrm{B} 2$ \\
\hline ZFAT|57623 & $\mathrm{B} 2$ \\
\hline ZFC3H1|196441 & B2 \\
\hline ZFHX3|463 & $\mathrm{B} 2$ \\
\hline ZFP106|64397 & $\mathrm{B} 2$ \\
\hline ZFP112|7771 & $\mathrm{B} 2$ \\
\hline ZFP14|57677 & $\mathrm{B} 2$ \\
\hline ZFP1|162239 & $\mathrm{B} 2$ \\
\hline ZFP30|22835 & B2 \\
\hline ZFP3|124961 & $\mathrm{B} 2$ \\
\hline ZFP41|286128 & $\mathrm{B} 2$ \\
\hline ZFP42|132625 & $\mathrm{B} 2$ \\
\hline ZFP57|346171 & B2 \\
\hline ZFP82|284406 & $\mathrm{B} 2$ \\
\hline ZFP91-CNTF|386607 & $\mathrm{B} 2$ \\
\hline ZFP91|80829 & $\mathrm{B} 2$ \\
\hline ZFP92|139735 & $\mathrm{B} 2$ \\
\hline ZFPL1|7542 & $\mathrm{B} 2$ \\
\hline ZFR2|23217 & $\mathrm{B} 2$ \\
\hline ZFR|51663 & $\mathrm{B} 2$ \\
\hline ZFX|7543 & $\mathrm{B} 2$ \\
\hline ZFYVE16|9765 & B2 \\
\hline ZFYVE19|84936 & $\mathrm{B} 2$ \\
\hline ZFYVE26|23503 & $\mathrm{B} 2$ \\
\hline ZFYVE27|118813 & $\mathrm{B} 2$ \\
\hline ZFY|7544 & $\mathrm{B} 2$ \\
\hline ZG16|653808 & $\mathrm{B} 2$ \\
\hline ZGLP1|100125288 & B2 \\
\hline ZGPAT|84619 & $\mathrm{B} 2$ \\
\hline ZHX1|11244 & $\mathrm{B} 2$ \\
\hline ZHX2|22882 & $\mathrm{B} 2$ \\
\hline ZIC1|7545 & $\mathrm{B} 2$ \\
\hline ZIC3|7547 & $\mathrm{B} 2$ \\
\hline ZIC4|84107 & $\mathrm{B} 2$ \\
\hline ZIC5|85416 & B2 \\
\hline ZIK1|284307 & $\mathrm{B} 2$ \\
\hline ZIM2|23619 & B2 \\
\hline ZKSCAN1|7586 & $\mathrm{B} 2$ \\
\hline ZKSCAN2|342357 & B2 \\
\hline \begin{tabular}{l|l|} 
ZKSCAN3|80317 &
\end{tabular} & $\mathrm{B} 2$ \\
\hline ZKSCAN4|387032 & $\mathrm{B} 2$ \\
\hline 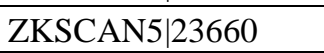 & $\mathrm{B} 2$ \\
\hline ZMAT2|153527 & $\mathrm{B} 2$ \\
\hline ZMAT4|79698 & $\mathrm{B} 2$ \\
\hline ZMAT5|55954 & $\mathrm{B} 2$ \\
\hline
\end{tabular}




\begin{tabular}{|c|c|}
\hline ZMIZ1|57178 & B2 \\
\hline ZMIZ2|83637 & B2 \\
\hline ZMYM1|79830 & $\mathrm{B} 2$ \\
\hline ZMYM2|7750 & $\mathrm{B} 2$ \\
\hline ZMYM3|9203 & $\mathrm{B} 2$ \\
\hline ZMYM4|9202 & $\mathrm{B} 2$ \\
\hline ZMYM5|9205 & $\mathrm{B} 2$ \\
\hline ZMYM6|9204 & $\mathrm{B} 2$ \\
\hline ZMYND11|10771 & $\mathrm{B} 2$ \\
\hline ZMYND12|84217 & $\mathrm{B} 2$ \\
\hline ZMYND15|84225 & $\mathrm{B} 2$ \\
\hline ZMYND17|118490 & $\mathrm{B} 2$ \\
\hline ZNF100|163227 & $\mathrm{B} 2$ \\
\hline ZNF101|94039 & $\mathrm{B} 2$ \\
\hline ZNF107|51427 & B2 \\
\hline ZNF114|163071 & $\mathrm{B} 2$ \\
\hline ZNF117|51351 & $\mathrm{B} 2$ \\
\hline ZNF121|7675 & $\mathrm{B} 2$ \\
\hline ZNF12|7559 & B2 \\
\hline ZNF131|7690 & $\mathrm{B} 2$ \\
\hline ZNF134|7693 & B2 \\
\hline ZNF135|7694 & $\mathrm{B} 2$ \\
\hline ZNF136|7695 & $\mathrm{B} 2$ \\
\hline ZNF137|7696 & $\mathrm{B} 2$ \\
\hline ZNF140|7699 & $\mathrm{B} 2$ \\
\hline ZNF141|7700 & $\mathrm{B} 2$ \\
\hline ZNF142|7701 & $\mathrm{B} 2$ \\
\hline ZNF143|7702 & B2 \\
\hline ZNF148|7707 & $\mathrm{B} 2$ \\
\hline ZNF14|7561 & $\mathrm{B} 2$ \\
\hline ZNF155|7711 & $\mathrm{B} 2$ \\
\hline ZNF165|7718 & $\mathrm{B} 2$ \\
\hline ZNF169|169841 & $\mathrm{B} 2$ \\
\hline ZNF16|7564 & B2 \\
\hline ZNF175|7728 & $\mathrm{B} 2$ \\
\hline ZNF177|7730 & $\mathrm{B} 2$ \\
\hline ZNF17|7565 & $\mathrm{B} 2$ \\
\hline ZNF180|7733 & $\mathrm{B} 2$ \\
\hline ZNF181|339318 & B2 \\
\hline ZNF182|7569 & $\mathrm{B} 2$ \\
\hline ZNF185|7739 & B2 \\
\hline ZNF18|7566 & $\mathrm{B} 2$ \\
\hline ZNF192|7745 & B2 \\
\hline ZNF193|7746 & $\mathrm{B} 2$ \\
\hline ZNF195|7748 & B2 \\
\hline ZNF197|10168 & $\mathrm{B} 2$ \\
\hline ZNF19|7567 & $\mathrm{B} 2$ \\
\hline ZNF205|7755 & $\mathrm{B} 2$ \\
\hline ZNF20|7568 & $\mathrm{B} 2$ \\
\hline ZNF211|10520 & $\mathrm{B} 2$ \\
\hline ZNF212|7988 & $\mathrm{B} 2$ \\
\hline
\end{tabular}




\begin{tabular}{|c|c|}
\hline ZNF214|7761 & B2 \\
\hline ZNF215|7762 & B2 \\
\hline ZNF217|7764 & $\mathrm{B} 2$ \\
\hline ZNF221|7638 & $\mathrm{B} 2$ \\
\hline ZNF222|7673 & $\mathrm{B} 2$ \\
\hline ZNF223|7766 & B2 \\
\hline ZNF224|7767 & $\mathrm{B} 2$ \\
\hline ZNF225|7768 & B2 \\
\hline ZNF226|7769 & B2 \\
\hline ZNF227|7770 & $\mathrm{B} 2$ \\
\hline ZNF229|7772 & $\mathrm{B} 2$ \\
\hline ZNF230|7773 & B2 \\
\hline ZNF232|7775 & $\mathrm{B} 2$ \\
\hline ZNF233|353355 & $\mathrm{B} 2$ \\
\hline ZNF234|10780 & B2 \\
\hline ZNF235|9310 & $\mathrm{B} 2$ \\
\hline ZNF236|7776 & $\mathrm{B} 2$ \\
\hline ZNF238|10472 & $\mathrm{B} 2$ \\
\hline ZNF24|7572 & B2 \\
\hline ZNF250|58500 & $\mathrm{B} 2$ \\
\hline ZNF251|90987 & $\mathrm{B} 2$ \\
\hline ZNF252|286101 & $\mathrm{B} 2$ \\
\hline ZNF253|56242 & $\mathrm{B} 2$ \\
\hline ZNF254|9534 & $\mathrm{B} 2$ \\
\hline \begin{tabular}{l|l|} 
ZNF256|10172 \\
\end{tabular} & $\mathrm{B} 2$ \\
\hline ZNF257|113835 & $\mathrm{B} 2$ \\
\hline ZNF259|8882 & B2 \\
\hline ZNF263|10127 & B2 \\
\hline ZNF264|9422 & $\mathrm{B} 2$ \\
\hline ZNF266|10781 & $\mathrm{B} 2$ \\
\hline ZNF268|10795 & $\mathrm{B} 2$ \\
\hline ZNF26|7574 & B2 \\
\hline ZNF271|10778 & $\mathrm{B} 2$ \\
\hline ZNF274|10782 & B2 \\
\hline \begin{tabular}{l|l|l|} 
ZNF275|10838 & \\
\end{tabular} & $\mathrm{B} 2$ \\
\hline ZNF277|11179 & $\mathrm{B} 2$ \\
\hline ZNF280A|129025 & $\mathrm{B} 2$ \\
\hline \begin{tabular}{l|l|l|} 
ZNF280B|140883 \\
\end{tabular} & $\mathrm{B} 2$ \\
\hline ZNF280C|55609 & B2 \\
\hline ZNF282|8427 & $\mathrm{B} 2$ \\
\hline \begin{tabular}{l|l|} 
ZNF283|284349 \\
\end{tabular} & B2 \\
\hline ZNF284|342909 & $\mathrm{B} 2$ \\
\hline \begin{tabular}{l|l|} 
ZNF285|26974 & \\
\end{tabular} & B2 \\
\hline ZNF286A|57335 & $\mathrm{B} 2$ \\
\hline $\begin{array}{l}\text { ZNF286B|729288 } \\
\end{array}$ & B2 \\
\hline ZNF287|57336 & $\mathrm{B} 2$ \\
\hline ZNF292|23036 & $\mathrm{B} 2$ \\
\hline \begin{tabular}{l|l|} 
ZNF295|49854 \\
\end{tabular} & $\mathrm{B} 2$ \\
\hline ZNF2|7549 & $\mathrm{B} 2$ \\
\hline ZNF300|91975 & $\mathrm{B} 2$ \\
\hline \begin{tabular}{l|l|} 
ZNF302|55900 \\
\end{tabular} & $\mathrm{B} 2$ \\
\hline
\end{tabular}




\begin{tabular}{|c|c|}
\hline ZNF304|57343 & B2 \\
\hline ZNF30|90075 & B2 \\
\hline ZNF311|282890 & $\mathrm{B} 2$ \\
\hline ZNF317|57693 & $\mathrm{B} 2$ \\
\hline ZNF318|24149 & $\mathrm{B} 2$ \\
\hline ZNF320|162967 & B2 \\
\hline ZNF321|399669 & $\mathrm{B} 2$ \\
\hline ZNF322A|79692 & $\mathrm{B} 2$ \\
\hline ZNF322B|387328 & B2 \\
\hline ZNF323|64288 & $\mathrm{B} 2$ \\
\hline ZNF324B|388569 & $\mathrm{B} 2$ \\
\hline ZNF324|25799 & B2 \\
\hline ZNF326|284695 & $\mathrm{B} 2$ \\
\hline $\begin{array}{l}\text { ZNF329|79673 } \\
\end{array}$ & $\mathrm{B} 2$ \\
\hline ZNF32|7580 & $\mathrm{B} 2$ \\
\hline 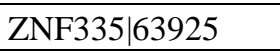 & $\mathrm{B} 2$ \\
\hline ZNF33A|7581 & $\mathrm{B} 2$ \\
\hline \begin{tabular}{l|l|} 
ZNF33B & 7582 \\
\end{tabular} & $\mathrm{~B} 2$ \\
\hline \begin{tabular}{l|l|l|l|} 
ZNF343|79175
\end{tabular} & B2 \\
\hline ZNF346|23567 & $\mathrm{B} 2$ \\
\hline ZNF347|84671 & B2 \\
\hline ZNF34|80778 & $\mathrm{B} 2$ \\
\hline $\begin{array}{l}\text { ZNF350|59348 } \\
\end{array}$ & $\mathrm{B} 2$ \\
\hline ZNF354A|6940 & $\mathrm{B} 2$ \\
\hline ZNF358|140467 & $\mathrm{B} 2$ \\
\hline ZNF35|7584 & $\mathrm{B} 2$ \\
\hline ZNF362|149076 & $\mathrm{B} 2$ \\
\hline \begin{tabular}{l|l|} 
ZNF365|22891 & \\
\end{tabular} & B2 \\
\hline ZNF37A|7587 & $\mathrm{B} 2$ \\
\hline ZNF382|84911 & $\mathrm{B} 2$ \\
\hline ZNF384|171017 & $\mathrm{B} 2$ \\
\hline ZNF385A|25946 & $\mathrm{B} 2$ \\
\hline \begin{tabular}{l|l|l|} 
ZNF385B|151126 \\
\end{tabular} & $\mathrm{B} 2$ \\
\hline ZNF385D|79750 & B2 \\
\hline ZNF394|84124 & $\mathrm{B} 2$ \\
\hline ZNF396|252884 & $\mathrm{B} 2$ \\
\hline \begin{tabular}{l|l|} 
ZNF398|57541 \\
\end{tabular} & $\mathrm{B} 2$ \\
\hline ZNF3|7551 & $\mathrm{B} 2$ \\
\hline ZNF404|342908 & B2 \\
\hline 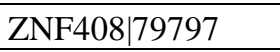 & $\mathrm{B} 2$ \\
\hline ZNF414|84330 & B2 \\
\hline \begin{tabular}{l|l|} 
ZNF415|55786 \\
\end{tabular} & $\mathrm{B} 2$ \\
\hline \begin{tabular}{l|l|} 
ZNF416|55659 &
\end{tabular} & B2 \\
\hline ZNF417|147687 & $\mathrm{B} 2$ \\
\hline ZNF418|147686 & $\mathrm{B} 2$ \\
\hline \begin{tabular}{l|l|l|} 
ZNF419|7974 \\
\end{tabular} & $\mathrm{B} 2$ \\
\hline ZNF41|7592 & $\mathrm{B} 2$ \\
\hline ZNF420|147923 & $\mathrm{B} 2$ \\
\hline ZNF425|155054 & $\mathrm{B} 2$ \\
\hline ZNF428|126299 & B2 \\
\hline ZNF429|353088 & $\mathrm{B} 2$ \\
\hline
\end{tabular}




\begin{tabular}{|c|c|}
\hline ZNF430|80264 & B2 \\
\hline ZNF431|170959 & B2 \\
\hline ZNF432|9668 & $\mathrm{B} 2$ \\
\hline ZNF433|163059 & $\mathrm{B} 2$ \\
\hline ZNF436|80818 & $\mathrm{B} 2$ \\
\hline ZNF439|90594 & B2 \\
\hline ZNF440|126070 & $\mathrm{B} 2$ \\
\hline ZNF441|126068 & B2 \\
\hline ZNF442|79973 & B2 \\
\hline ZNF445|353274 & $\mathrm{B} 2$ \\
\hline ZNF446|55663 & $\mathrm{B} 2$ \\
\hline ZNF44|51710 & $\mathrm{B} 2$ \\
\hline ZNF451|26036 & $\mathrm{B} 2$ \\
\hline \begin{tabular}{l|l|l|} 
ZNF4596 &
\end{tabular} & $\mathrm{B} 2$ \\
\hline ZNF460|10794 & $\mathrm{B} 2$ \\
\hline ZNF461|92283 & $\mathrm{B} 2$ \\
\hline \begin{tabular}{l|l|l|} 
ZNF473 & 25888 \\
\end{tabular} & $\mathrm{~B} 2$ \\
\hline ZNF474|133923 & $\mathrm{B} 2$ \\
\hline $\begin{array}{l}\text { ZNF479|90827 } \\
\end{array}$ & B2 \\
\hline 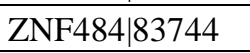 & $\mathrm{B} 2$ \\
\hline ZNF485|220992 & B2 \\
\hline ZNF486|90649 & $\mathrm{B} 2$ \\
\hline ZNF491|126069 & B2 \\
\hline \begin{tabular}{l|l|l|} 
ZNF492|57615 \\
\end{tabular} & $\mathrm{B} 2$ \\
\hline ZNF493|284443 & $\mathrm{B} 2$ \\
\hline \begin{tabular}{l|l} 
ZNF496|84838 \\
\end{tabular} & $\mathrm{B} 2$ \\
\hline ZNF497|162968 & $\mathrm{B} 2$ \\
\hline ZNF500|26048 & B2 \\
\hline \begin{tabular}{l|l|} 
ZNF503|84858 \\
\end{tabular} & $\mathrm{B} 2$ \\
\hline ZNF506|440515 & $\mathrm{B} 2$ \\
\hline ZNF511|118472 & $\mathrm{B} 2$ \\
\hline ZNF512B|57473 & B2 \\
\hline $\begin{array}{l}\text { ZNF512|84450 } \\
\end{array}$ & $\mathrm{B} 2$ \\
\hline ZNF513|130557 & B2 \\
\hline ZNF514|84874 & $\mathrm{B} 2$ \\
\hline ZNF516|9658 & $\mathrm{B} 2$ \\
\hline ZNF517|340385 & $\mathrm{B} 2$ \\
\hline ZNF518A|9849 & $\mathrm{B} 2$ \\
\hline ZNF518B $\mid 85460$ & B2 \\
\hline ZNF519|162655 & $\mathrm{B} 2$ \\
\hline ZNF524|147807 & B2 \\
\hline ZNF526|116115 & $\mathrm{B} 2$ \\
\hline ZNF528|84436 & B2 \\
\hline \begin{tabular}{l|l|} 
ZNF529|57711 \\
\end{tabular} & $\mathrm{B} 2$ \\
\hline ZNF530|348327 & $\mathrm{B} 2$ \\
\hline \begin{tabular}{l|l|} 
ZNF532|55205 \\
\end{tabular} & $\mathrm{B} 2$ \\
\hline ZNF534|147658 & $\mathrm{B} 2$ \\
\hline ZNF536|9745 & $\mathrm{B} 2$ \\
\hline \begin{tabular}{l|l|} 
ZNF541|84215 \\
\end{tabular} & $\mathrm{B} 2$ \\
\hline ZNF542|147947 & B2 \\
\hline ZNF543|125919 & $\mathrm{B} 2$ \\
\hline
\end{tabular}




\begin{tabular}{|c|c|}
\hline ZNF547|284306 & B2 \\
\hline ZNF548|147694 & B2 \\
\hline ZNF549|256051 & $\mathrm{B} 2$ \\
\hline ZNF550|162972 & $\mathrm{B} 2$ \\
\hline ZNF551|90233 & B2 \\
\hline ZNF555|148254 & B2 \\
\hline ZNF556|80032 & $\mathrm{B} 2$ \\
\hline ZNF557|79230 & $\mathrm{B} 2$ \\
\hline ZNF558|148156 & B2 \\
\hline ZNF560|147741 & $\mathrm{B} 2$ \\
\hline ZNF562|54811 & $\mathrm{B} 2$ \\
\hline ZNF563|147837 & $\mathrm{B} 2$ \\
\hline ZNF564|163050 & $\mathrm{B} 2$ \\
\hline ZNF565|147929 & $\mathrm{B} 2$ \\
\hline ZNF566|84924 & $\mathrm{B} 2$ \\
\hline ZNF567|163081 & $\mathrm{B} 2$ \\
\hline ZNF568|374900 & $\mathrm{B} 2$ \\
\hline ZNF569|148266 & $\mathrm{B} 2$ \\
\hline ZNF570|148268 & B2 \\
\hline ZNF571|51276 & $\mathrm{B} 2$ \\
\hline ZNF572|137209 & B2 \\
\hline ZNF573|126231 & $\mathrm{B} 2$ \\
\hline ZNF574|64763 & $\mathrm{B} 2$ \\
\hline ZNF575|284346 & $\mathrm{B} 2$ \\
\hline $\begin{array}{l}\text { ZNF576|79177 } \\
\end{array}$ & $\mathrm{B} 2$ \\
\hline ZNF577|84765 & $\mathrm{B} 2$ \\
\hline ZNF578|147660 & $\mathrm{B} 2$ \\
\hline $\begin{array}{l}\text { ZNF579|163033 } \\
\end{array}$ & $\mathrm{B} 2$ \\
\hline ZNF57|126295 & $\mathrm{B} 2$ \\
\hline ZNF580|51157 & $\mathrm{B} 2$ \\
\hline ZNF581|51545 & $\mathrm{B} 2$ \\
\hline ZNF582|147948 & $\mathrm{B} 2$ \\
\hline ZNF583|147949 & $\mathrm{B} 2$ \\
\hline ZNF584|201514 & $\mathrm{B} 2$ \\
\hline ZNF585A|199704 & $\mathrm{B} 2$ \\
\hline $\begin{array}{l}\text { ZNF585B|92285 } \\
\end{array}$ & $\mathrm{B} 2$ \\
\hline ZNF586|54807 & $\mathrm{B} 2$ \\
\hline \begin{tabular}{l|l|l|} 
ZNF593|51042 \\
\end{tabular} & $\mathrm{B} 2$ \\
\hline ZNF594|84622 & $\mathrm{B} 2$ \\
\hline ZNF595|152687 & $\mathrm{B} 2$ \\
\hline ZNF597|146434 & B2 \\
\hline \begin{tabular}{l|l|} 
ZNF599|148103 \\
\end{tabular} & $\mathrm{B} 2$ \\
\hline ZNF600|162966 & $\mathrm{B} 2$ \\
\hline ZNF605|100289635 & $\mathrm{B} 2$ \\
\hline ZNF606|80095 & $\mathrm{B} 2$ \\
\hline ZNF607|84775 & $\mathrm{B} 2$ \\
\hline \begin{tabular}{l|l|} 
ZNF609|23060 \\
\end{tabular} & $\mathrm{B} 2$ \\
\hline ZNF610|162963 & $\mathrm{B} 2$ \\
\hline ZNF611|81856 & $\mathrm{B} 2$ \\
\hline \begin{tabular}{l|l|l|} 
ZNF613|79898 \\
\end{tabular} & $\mathrm{B} 2$ \\
\hline ZNF614|80110 & $\mathrm{B} 2$ \\
\hline
\end{tabular}




\begin{tabular}{|c|c|}
\hline ZNF615|284370 & B2 \\
\hline ZNF616|90317 & $\mathrm{B} 2$ \\
\hline ZNF618|114991 & $\mathrm{B} 2$ \\
\hline ZNF619|285267 & $\mathrm{B} 2$ \\
\hline ZNF620|253639 & $\mathrm{B} 2$ \\
\hline ZNF621|285268 & B2 \\
\hline ZNF622|90441 & $\mathrm{B} 2$ \\
\hline ZNF624|57547 & B2 \\
\hline ZNF625|90589 & B2 \\
\hline ZNF626|199777 & $\mathrm{B} 2$ \\
\hline ZNF627|199692 & $\mathrm{B} 2$ \\
\hline ZNF628|89887 & B2 \\
\hline \begin{tabular}{l|l|} 
ZNF629|23361 \\
\end{tabular} & $\mathrm{B} 2$ \\
\hline ZNF630|57232 & $\mathrm{B} 2$ \\
\hline ZNF638|27332 & B2 \\
\hline \begin{tabular}{l|l|} 
ZNF639|51193 \\
\end{tabular} & $\mathrm{B} 2$ \\
\hline ZNF641|121274 & $\mathrm{B} 2$ \\
\hline ZNF644|84146 & $\mathrm{B} 2$ \\
\hline ZNF645|158506 & B2 \\
\hline ZNF648|127665 & $\mathrm{B} 2$ \\
\hline \begin{tabular}{l|l|} 
ZNF649|65251 & \\
\end{tabular} & B2 \\
\hline ZNF652|22834 & $\mathrm{B} 2$ \\
\hline ZNF653|115950 & $\mathrm{B} 2$ \\
\hline ZNF654|55279 & $\mathrm{B} 2$ \\
\hline ZNF664|144348 & $\mathrm{B} 2$ \\
\hline \begin{tabular}{l|l|l|} 
ZNF665 & \\
\end{tabular} & $\mathrm{B} 2$ \\
\hline ZNF669|79862 & B2 \\
\hline ZNF670|93474 & B2 \\
\hline \begin{tabular}{l|l|} 
ZNF671|79891 \\
\end{tabular} & $\mathrm{B} 2$ \\
\hline ZNF674|641339 & $\mathrm{B} 2$ \\
\hline ZNF676|163223 & $\mathrm{B} 2$ \\
\hline $\begin{array}{l}\text { ZNF679|168417 } \\
\end{array}$ & $\mathrm{B} 2$ \\
\hline ZNF680|340252 & $\mathrm{B} 2$ \\
\hline \begin{tabular}{l|l|} 
ZNF683|257101 \\
\end{tabular} & B2 \\
\hline ZNF684|127396 & $\mathrm{B} 2$ \\
\hline ZNF688|146542 & $\mathrm{B} 2$ \\
\hline ZNF689|115509 & $\mathrm{B} 2$ \\
\hline ZNF691|51058 & $\mathrm{B} 2$ \\
\hline \begin{tabular}{l|l|l|} 
ZNF692|55657 &
\end{tabular} & B2 \\
\hline 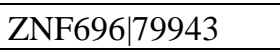 & $\mathrm{B} 2$ \\
\hline ZNF697|90874 & B2 \\
\hline $\begin{array}{l}\text { ZNF69|7620 } \\
\end{array}$ & $\mathrm{B} 2$ \\
\hline ZNF700|90592 & B2 \\
\hline \begin{tabular}{l|l|} 
ZNF701|55762 \\
\end{tabular} & $\mathrm{B} 2$ \\
\hline ZNF702P|79986 & B2 \\
\hline $\begin{array}{l}\text { ZNF703|80139 } \\
\end{array}$ & $\mathrm{B} 2$ \\
\hline ZNF705A|440077 & $\mathrm{B} 2$ \\
\hline $\begin{array}{l}\text { ZNF705D|728957 } \\
\end{array}$ & $\mathrm{B} 2$ \\
\hline ZNF706|51123 & $\mathrm{B} 2$ \\
\hline ZNF708|7562 & $\mathrm{B} 2$ \\
\hline ZNF709|163051 & $\mathrm{B} 2$ \\
\hline
\end{tabular}




\begin{tabular}{|c|c|}
\hline ZNF70|7621 & B2 \\
\hline ZNF710|374655 & B2 \\
\hline ZNF711|7552 & $\mathrm{B} 2$ \\
\hline ZNF713|349075 & $\mathrm{B} 2$ \\
\hline ZNF714|148206 & $\mathrm{B} 2$ \\
\hline ZNF716|441234 & B2 \\
\hline ZNF717|100131827 & $\mathrm{B} 2$ \\
\hline ZNF718|255403 & $\mathrm{B} 2$ \\
\hline ZNF71|58491 & B2 \\
\hline ZNF720|124411 & $\mathrm{B} 2$ \\
\hline ZNF721|170960 & $\mathrm{B} 2$ \\
\hline ZNF732|654254 & B2 \\
\hline ZNF737|100129842 & $\mathrm{B} 2$ \\
\hline ZNF738|148203 & $\mathrm{B} 2$ \\
\hline ZNF740|283337 & B2 \\
\hline ZNF746|155061 & $\mathrm{B} 2$ \\
\hline ZNF747|65988 & $\mathrm{B} 2$ \\
\hline ZNF749|388567 & $\mathrm{B} 2$ \\
\hline ZNF74|7625 & B2 \\
\hline ZNF750|79755 & $\mathrm{B} 2$ \\
\hline ZNF75D|7626 & $\mathrm{B} 2$ \\
\hline ZNF761|388561 & $\mathrm{B} 2$ \\
\hline $\begin{array}{l}\text { ZNF763|284390 } \\
\end{array}$ & B2 \\
\hline ZNF766|90321 & $\mathrm{B} 2$ \\
\hline ZNF76|7629 & $\mathrm{B} 2$ \\
\hline \begin{tabular}{l|l} 
ZNF770|54989 \\
\end{tabular} & $\mathrm{B} 2$ \\
\hline ZNF772|400720 & $\mathrm{B} 2$ \\
\hline ZNF773|374928 & B2 \\
\hline ZNF774|342132 & $\mathrm{B} 2$ \\
\hline ZNF775|285971 & $\mathrm{B} 2$ \\
\hline ZNF776|284309 & $\mathrm{B} 2$ \\
\hline ZNF777|27153 & $\mathrm{B} 2$ \\
\hline ZNF77|58492 & $\mathrm{B} 2$ \\
\hline ZNF780A|284323 & B2 \\
\hline \begin{tabular}{l|l} 
ZNF780B|163131 \\
\end{tabular} & $\mathrm{B} 2$ \\
\hline ZNF782|158431 & $\mathrm{B} 2$ \\
\hline ZNF784|147808 & $\mathrm{B} 2$ \\
\hline ZNF785|146540 & $\mathrm{B} 2$ \\
\hline ZNF786|136051 & $\mathrm{B} 2$ \\
\hline ZNF787|126208 & $\mathrm{B} 2$ \\
\hline ZNF788|388507 & B2 \\
\hline $\begin{array}{l}\text { ZNF789|285989 } \\
\end{array}$ & $\mathrm{B} 2$ \\
\hline ZNF790|388536 & B2 \\
\hline ZNF791|163049 & $\mathrm{B} 2$ \\
\hline ZNF792|126375 & $\mathrm{B} 2$ \\
\hline ZNF793|390927 & $\mathrm{B} 2$ \\
\hline $\begin{array}{l}\text { ZNF799|90576 } \\
\end{array}$ & $\mathrm{B} 2$ \\
\hline ZNF79|7633 & $\mathrm{B} 2$ \\
\hline ZNF7|7553 & $\mathrm{B} 2$ \\
\hline ZNF800|168850 & B2 \\
\hline ZNF804A|91752 & $\mathrm{B} 2$ \\
\hline
\end{tabular}




\begin{tabular}{|c|c|}
\hline ZNF804B|219578 & B2 \\
\hline ZNF805|390980 & B2 \\
\hline ZNF808|388558 & $\mathrm{B} 2$ \\
\hline ZNF80|7634 & $\mathrm{B} 2$ \\
\hline ZNF813|126017 & $\mathrm{B} 2$ \\
\hline ZNF814|730051 & B2 \\
\hline ZNF815|401303 & $\mathrm{B} 2$ \\
\hline ZNF816A|125893 & B2 \\
\hline ZNF81|347344 & B2 \\
\hline ZNF821|55565 & $\mathrm{B} 2$ \\
\hline ZNF823|55552 & $\mathrm{B} 2$ \\
\hline ZNF826|664701 & $\mathrm{B} 2$ \\
\hline ZNF827|152485 & $\mathrm{B} 2$ \\
\hline ZNF828|283489 & $\mathrm{B} 2$ \\
\hline ZNF829|374899 & $\mathrm{B} 2$ \\
\hline ZNF830|91603 & $\mathrm{B} 2$ \\
\hline ZNF831|128611 & $\mathrm{B} 2$ \\
\hline ZNF835|90485 & $\mathrm{B} 2$ \\
\hline ZNF836|162962 & B2 \\
\hline ZNF837|116412 & $\mathrm{B} 2$ \\
\hline \begin{tabular}{l|l|} 
ZNF839|55778 \\
\end{tabular} & $\mathrm{B} 2$ \\
\hline ZNF83|55769 & $\mathrm{B} 2$ \\
\hline ZNF841|284371 & B2 \\
\hline ZNF844|284391 & $\mathrm{B} 2$ \\
\hline \begin{tabular}{l|l|} 
ZNF845|91664 \\
\end{tabular} & $\mathrm{B} 2$ \\
\hline 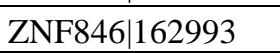 & $\mathrm{B} 2$ \\
\hline ZNF84|7637 & $\mathrm{B} 2$ \\
\hline ZNF860|344787 & B2 \\
\hline ZNF862|643641 & $\mathrm{B} 2$ \\
\hline $\begin{array}{l}\text { ZNF876P|642280 } \\
\end{array}$ & B2 \\
\hline ZNF878|729747 & $\mathrm{B} 2$ \\
\hline ZNF879|345462 & B2 \\
\hline ZNF880|400713 & $\mathrm{B} 2$ \\
\hline ZNF883|169834 & B2 \\
\hline ZNF8|7554 & $\mathrm{B} 2$ \\
\hline ZNF90|7643 & $\mathrm{B} 2$ \\
\hline ZNF91|7644 & $\mathrm{B} 2$ \\
\hline \begin{tabular}{l|l|l|} 
ZNF92|168374 \\
\end{tabular} & $\mathrm{B} 2$ \\
\hline 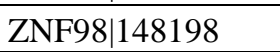 & B2 \\
\hline $\begin{array}{l}\text { ZNF99|7652 } \\
\end{array}$ & $\mathrm{B} 2$ \\
\hline ZNFX1|57169 & B2 \\
\hline ZNHIT1|10467 & $\mathrm{B} 2$ \\
\hline ZNHIT3|9326 & B2 \\
\hline ZNHIT6|54680 & $\mathrm{B} 2$ \\
\hline ZNRD1|30834 & $\mathrm{B} 2$ \\
\hline $\begin{array}{l}\text { ZNRF1|84937 } \\
\end{array}$ & $\mathrm{B} 2$ \\
\hline ZNRF3|84133 & $\mathrm{B} 2$ \\
\hline ZP1|22917 & $\mathrm{B} 2$ \\
\hline ZP2|7783 & $\mathrm{B} 2$ \\
\hline ZP3|7784 & B2 \\
\hline ZP4|57829 & B2 \\
\hline
\end{tabular}




\begin{tabular}{|c|c|}
\hline ZPBP2|124626 & B2 \\
\hline ZPBP|11055 & $\mathrm{B} 2$ \\
\hline ZPLD1|131368 & $\mathrm{B} 2$ \\
\hline ZRANB3|84083 & $\mathrm{B} 2$ \\
\hline ZRSR2|8233 & $\mathrm{B} 2$ \\
\hline ZSCAN10|84891 & B2 \\
\hline ZSCAN18|65982 & $\mathrm{B} 2$ \\
\hline ZSCAN1|284312 & B2 \\
\hline ZSCAN20|7579 & B2 \\
\hline ZSCAN21|7589 & $\mathrm{B} 2$ \\
\hline ZSCAN22|342945 & $\mathrm{B} 2$ \\
\hline ZSCAN29|146050 & $\mathrm{B} 2$ \\
\hline ZSCAN2|54993 & $\mathrm{B} 2$ \\
\hline ZSCAN4|201516 & $\mathrm{B} 2$ \\
\hline ZSCAN5A|79149 & $\mathrm{B} 2$ \\
\hline ZSCAN5B|342933 & $\mathrm{B} 2$ \\
\hline ZSWIM1|90204 & $\mathrm{B} 2$ \\
\hline ZSWIM2|151112 & $\mathrm{B} 2$ \\
\hline ZSWIM3|140831 & B2 \\
\hline ZSWIM4|65249 & $\mathrm{B} 2$ \\
\hline ZSWIM5|57643 & $\mathrm{B} 2$ \\
\hline ZSWIM6|57688 & $\mathrm{B} 2$ \\
\hline ZUFSP|221302 & $\mathrm{B} 2$ \\
\hline ZW10|9183 & $\mathrm{B} 2$ \\
\hline ZXDA|7789 & $\mathrm{B} 2$ \\
\hline ZXDB|158586 & $\mathrm{B} 2$ \\
\hline ZXDC|79364 & $\mathrm{B} 2$ \\
\hline ZYG11B|79699 & B2 \\
\hline ZYX|7791 & $\mathrm{B} 2$ \\
\hline $\begin{array}{l}\text { ZZZ3|26009 } \\
\end{array}$ & $\mathrm{B} 2$ \\
\hline tAKR|389932 & $\mathrm{B} 2$ \\
\hline $\begin{array}{l}\text { ACOT9|23597 } \\
\end{array}$ & B3 \\
\hline ACR/49 & B3 \\
\hline ADH6|130 & B3 \\
\hline AXL|558 & B3 \\
\hline BCHE|590 & B3 \\
\hline BRWD3|254065 & B3 \\
\hline C10orf108|414235 & B3 \\
\hline C11orf53|341032 & B3 \\
\hline C1QTNF2|114898 & B3 \\
\hline $\mathrm{C} 1 \mathrm{~S} \mid 716$ & B3 \\
\hline C21 orf62|56245 & B3 \\
\hline $\begin{array}{l}\text { CCDC92|80212 } \\
\end{array}$ & B3 \\
\hline CLEC2L|154790 & B3 \\
\hline CLEC4F|165530 & B3 \\
\hline COL15A1|1306 & B3 \\
\hline CPS1|1373 & B3 \\
\hline CRTAC1|55118 & B3 \\
\hline DPP4|1803 & B3 \\
\hline DZIP1|22873 & B3 \\
\hline EIF1|10209 & B3 \\
\hline
\end{tabular}




\begin{tabular}{|c|c|}
\hline ELMO1|9844 & B3 \\
\hline FAM127A|8933 & B3 \\
\hline FGD5|152273 & B3 \\
\hline FGF10|2255 & B3 \\
\hline FKBP1B|2281 & B3 \\
\hline FXYD2|486 & B3 \\
\hline GABRA2|2555 & B3 \\
\hline GLI1|2735 & B3 \\
\hline GPER|2852 & B3 \\
\hline HIBADH|11112 & B3 \\
\hline HMGCR|3156 & B3 \\
\hline HSPB11|51668 & B3 \\
\hline IGF2|3481 & B3 \\
\hline ILK|3611 & B3 \\
\hline ITGB1|3688 & B3 \\
\hline JDP2|122953 & B3 \\
\hline LOC158696|158696 & B3 \\
\hline LOC348926|348926 & B3 \\
\hline LOC400804|400804 & B3 \\
\hline MAGEE2|139599 & B3 \\
\hline MAP1A|4130 & B3 \\
\hline METTL6|131965 & B3 \\
\hline MFAP5|8076 & B3 \\
\hline MPZ|4359 & B3 \\
\hline NAA35|60560 & B3 \\
\hline NNMT|4837 & B3 \\
\hline NTRK3|4916 & B3 \\
\hline PKIG|11142 & B3 \\
\hline PLS1|5357 & B3 \\
\hline PPP1R1B|84152 & B3 \\
\hline PRMT2|3275 & B3 \\
\hline RAB3IL1|5866 & B3 \\
\hline RFFL|117584 & B3 \\
\hline RGS5|8490 & B3 \\
\hline RPP30|10556 & B3 \\
\hline S100B $\mid 6285$ & B3 \\
\hline SAMD10|140700 & B3 \\
\hline SEPT4|5414 & B3 \\
\hline SGTB|54557 & B3 \\
\hline SHOX2|6474 & B3 \\
\hline SNHG5|387066 & B3 \\
\hline STX2|2054 & B3 \\
\hline SULT1C4|27233 & B3 \\
\hline THOC2|57187 & B3 \\
\hline TSPAN18|90139 & B3 \\
\hline UVRAG|7405 & B3 \\
\hline $\begin{array}{l}\text { XKR6|286046 } \\
\end{array}$ & B3 \\
\hline ZNF208|7757 & B3 \\
\hline psiTPTE22|387590 & B3 \\
\hline AJAP1|55966 & B4 \\
\hline AKAP6|9472 & $\mathrm{B} 4$ \\
\hline
\end{tabular}




\begin{tabular}{|c|c|}
\hline C6orf105|84830 & B4 \\
\hline CASP1|834 & B4 \\
\hline CCDC68|80323 & B4 \\
\hline CEP164|22897 & B4 \\
\hline DIRC2|84925 & B4 \\
\hline FLJ39582|439931 & B4 \\
\hline GPR62|118442 & B4 \\
\hline GRIN2D|2906 & B4 \\
\hline KIAA0913|23053 & B4 \\
\hline LOC284276|284276 & B4 \\
\hline LOC401127|401127 & B4 \\
\hline MARCH4|57574 & B4 \\
\hline MLLT6|4302 & B4 \\
\hline NEUROG2|63973 & B4 \\
\hline NKPD1|284353 & B4 \\
\hline NMNAT3|349565 & B4 \\
\hline NPAS3|64067 & B4 \\
\hline SEMA6C|10500 & B4 \\
\hline SF1|7536 & B4 \\
\hline STAU1|6780 & B4 \\
\hline SYNPO2|171024 & B4 \\
\hline TMEM79|84283 & B4 \\
\hline TPTE2P3|220115 & B4 \\
\hline WSCD2|9671 & B4 \\
\hline ZFYVE28|57732 & B4 \\
\hline ZNF487|642819 & B4 \\
\hline ZNF589|51385 & B4 \\
\hline AMIGO2|347902 & B5 \\
\hline BRMS1L|84312 & B5 \\
\hline C11orf31|280636 & B5 \\
\hline C11orf93|120376 & B5 \\
\hline CA11|770 & B5 \\
\hline CDK6|1021 & B5 \\
\hline CSDAP1|440359 & B5 \\
\hline CSDA $\mid 8531$ & B5 \\
\hline CYP20A1|57404 & B5 \\
\hline CYP39A1|51302 & B5 \\
\hline $\begin{array}{l}\text { DCHS2|54798 } \\
\end{array}$ & B5 \\
\hline DISC1|27185 & B5 \\
\hline GNAQ|2776 & B5 \\
\hline IGF2BP2|10644 & B5 \\
\hline LPPR5|163404 & B5 \\
\hline MAP2|4133 & B5 \\
\hline OCA2|4948 & B5 \\
\hline SMOC1|64093 & B5 \\
\hline CAPN3|825 & B6 \\
\hline CLCA4|22802 & B6 \\
\hline GLT25D2|23127 & $\mathrm{B} 6$ \\
\hline JPH2|57158 & B6 \\
\hline LOC285419|285419 & $\mathrm{B} 6$ \\
\hline MYH3|4621 & B6 \\
\hline
\end{tabular}




\begin{tabular}{|l|l|} 
MYH7B|57644 & B6 \\
\hline PLP1|5354 & B6 \\
\hline TPM3|7170 & B6 \\
\hline ALPL|249 & B7 \\
\hline CHST2|9435 & B7 \\
\hline KCTD14|65987 & B7 \\
\hline KSR1|8844 & B7 \\
\hline MRAP2|112609 & B7 \\
\hline NAV3|89795 & B7 \\
\hline PLA2G4A|5321 & B7 \\
\hline TUBB2B|347733 & B7 \\
\hline ANKRD35|148741 & B8 \\
\hline ASXL3|80816 & B8 \\
\hline SEC1|653677 & B8 \\
\hline
\end{tabular}


Supplementary Table 2. Functional enrichment analysis for each class by ToppGene

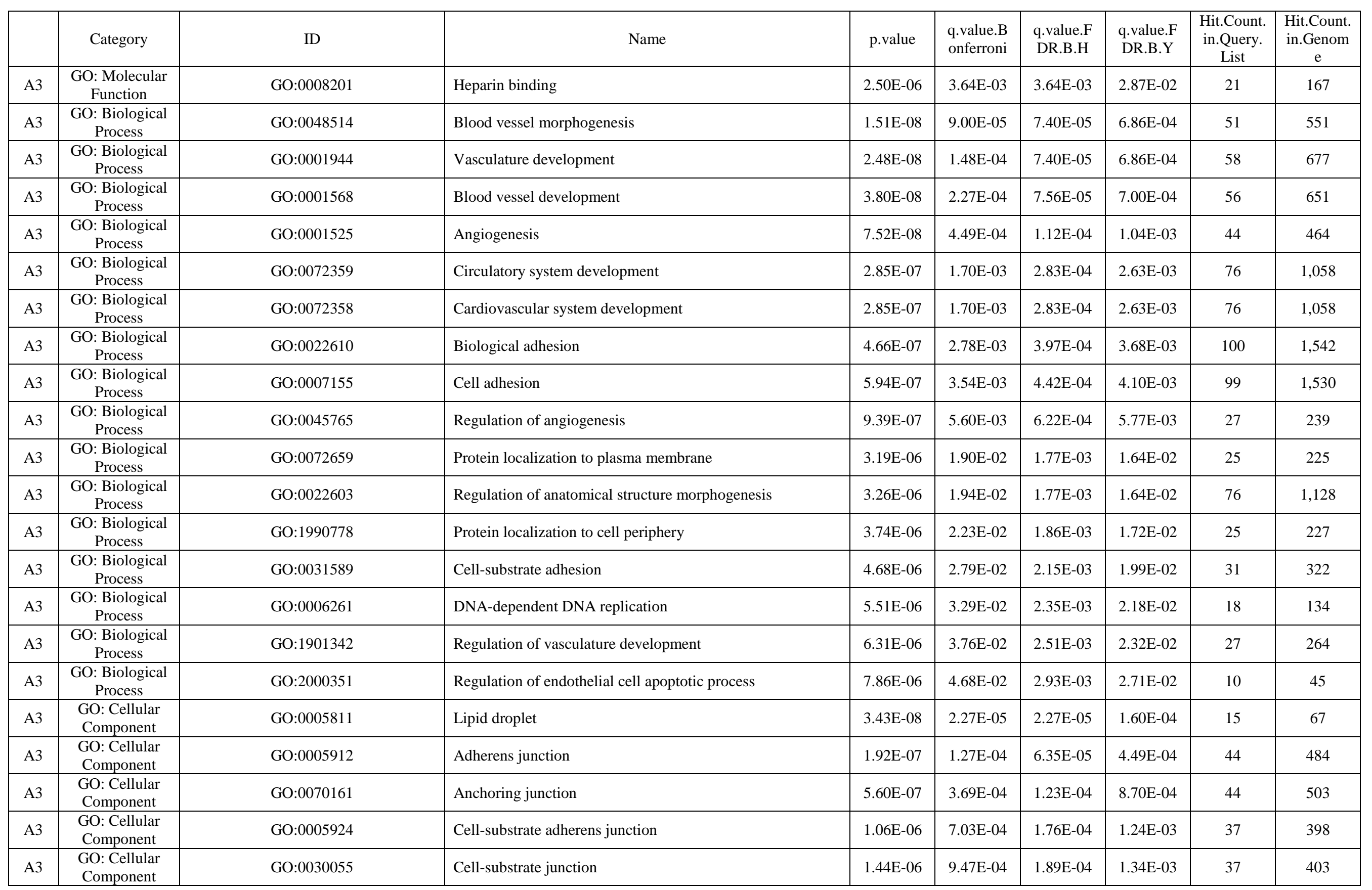




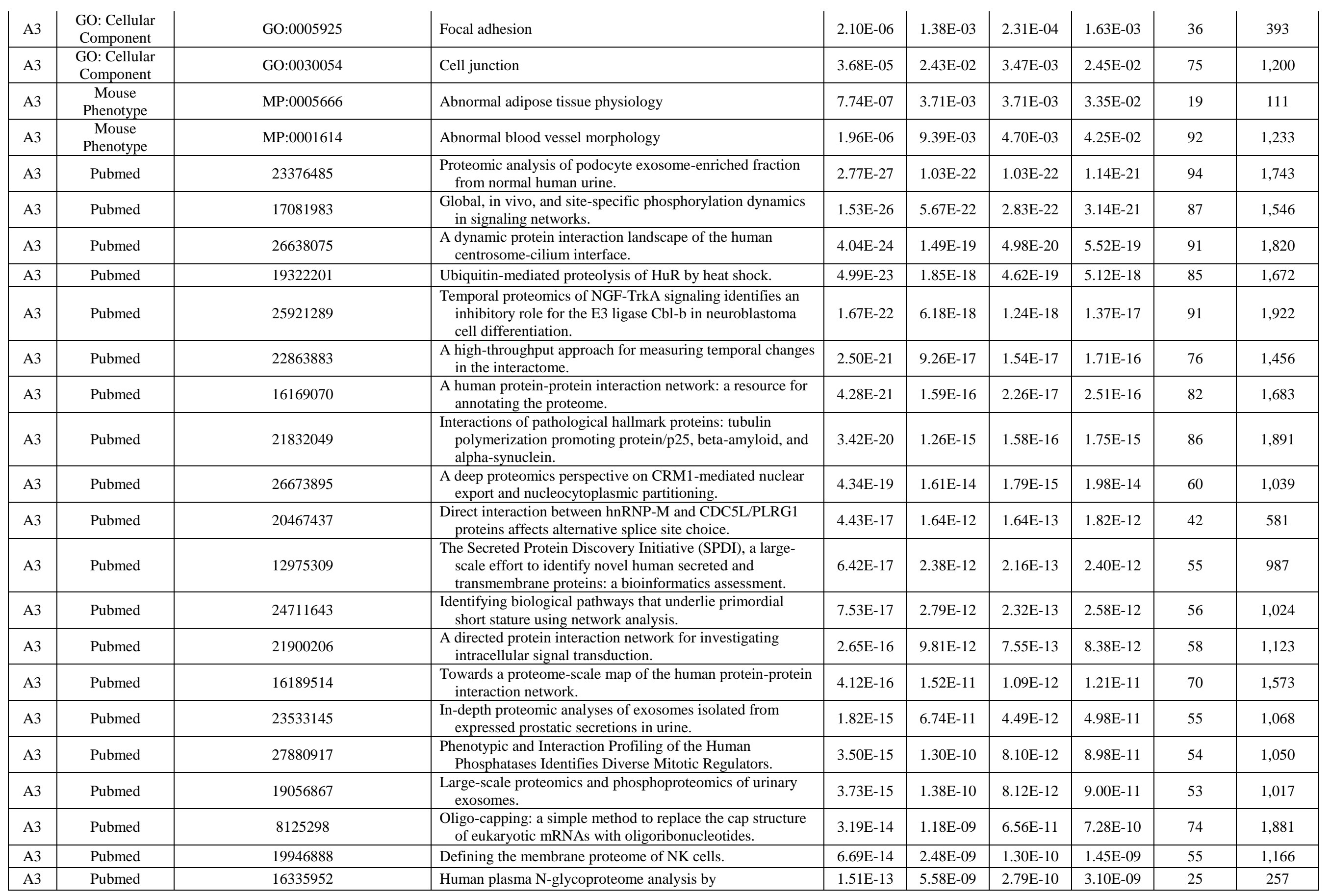


immunoaffinity subtraction, hydrazide chemistry, and mass spectrometry.

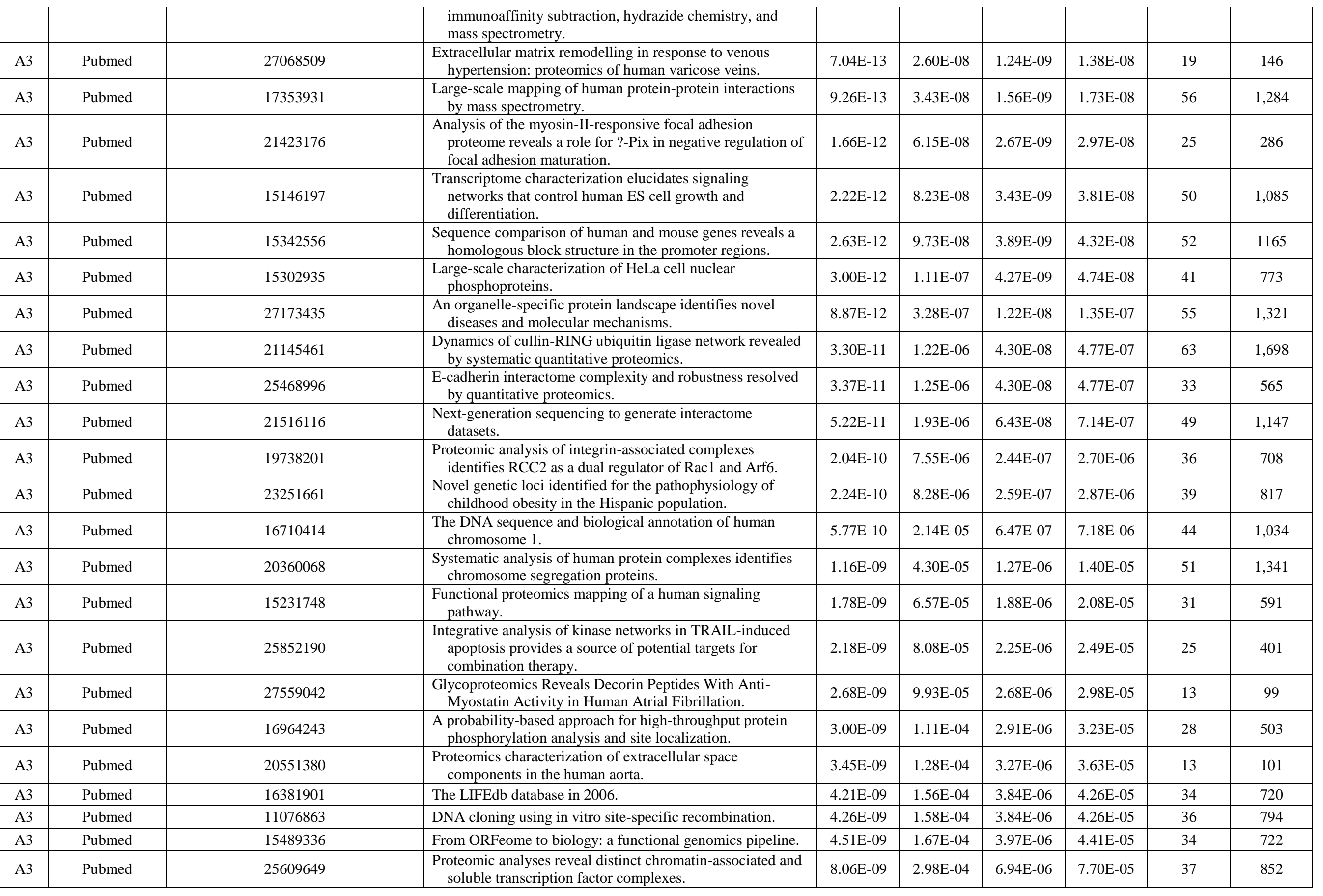




\begin{tabular}{|c|c|c|c|c|c|c|c|c|c|}
\hline A3 & Pubmed & 19615732 & $\begin{array}{l}\text { Defining the human deubiquitinating enzyme interaction } \\
\text { landscape. }\end{array}$ & $2.20 \mathrm{E}-08$ & 8.14E-04 & $1.85 \mathrm{E}-05$ & $2.05 \mathrm{E}-04$ & 40 & 1,005 \\
\hline A3 & Pubmed & 15242332 & $\begin{array}{l}\text { Vectorial proteomics reveal targeting, phosphorylation and } \\
\text { specific fragmentation of polymerase I and transcript } \\
\text { release factor (PTRF) at the surface of caveolae in human } \\
\text { adipocytes. }\end{array}$ & 2.92E-08 & $1.08 \mathrm{E}-03$ & $2.40 \mathrm{E}-05$ & $2.66 \mathrm{E}-04$ & 7 & 23 \\
\hline A3 & Pubmed & 20734064 & $\begin{array}{l}\text { A large-scale candidate gene association study of age at } \\
\text { menarche and age at natural menopause. }\end{array}$ & $3.63 \mathrm{E}-08$ & $1.34 \mathrm{E}-03$ & 2.92E-05 & $3.24 \mathrm{E}-04$ & 19 & 275 \\
\hline A3 & Pubmed & 20589320 & $\begin{array}{l}\text { mRNA levels of CD31, CD144, CD146 and von Willebrand } \\
\text { factor do not serve as surrogate markers for circulating } \\
\text { endothelial cells. }\end{array}$ & 4.74E-08 & $1.75 \mathrm{E}-03$ & $3.68 \mathrm{E}-05$ & $4.08 \mathrm{E}-04$ & 4 & 4 \\
\hline A3 & Pubmed & 20628624 & $\begin{array}{l}\text { Evaluation of candidate stromal epithelial cross-talk genes } \\
\text { identifies association between risk of serous ovarian } \\
\text { cancer and TERT, a cancer susceptibility "hot-spot". }\end{array}$ & $4.78 \mathrm{E}-08$ & $1.77 \mathrm{E}-03$ & $3.68 \mathrm{E}-05$ & $4.08 \mathrm{E}-04$ & 15 & 172 \\
\hline A3 & Pubmed & 24332808 & $\begin{array}{l}\text { PRP19 transforms into a sensor of RPA-ssDNA after DNA } \\
\text { damage and drives ATR activation via a ubiquitin- } \\
\text { mediated circuitry. }\end{array}$ & 4.87E-08 & $1.80 \mathrm{E}-03$ & $3.68 \mathrm{E}-05$ & $4.08 \mathrm{E}-04$ & 21 & 340 \\
\hline A3 & Pubmed & 22681889 & $\begin{array}{l}\text { The mRNA-bound proteome and its global occupancy } \\
\text { profile on protein-coding transcripts. }\end{array}$ & $6.29 \mathrm{E}-08$ & $2.33 \mathrm{E}-03$ & $4.58 \mathrm{E}-05$ & $5.09 \mathrm{E}-04$ & 34 & 805 \\
\hline A3 & Pubmed & 15815621 & $\begin{array}{l}\text { Generation and annotation of the DNA sequences of human } \\
\text { chromosomes } 2 \text { and } 4 \text {. }\end{array}$ & 6.32E-08 & $2.34 \mathrm{E}-03$ & 4.58E-05 & $5.09 \mathrm{E}-04$ & 24 & 442 \\
\hline A3 & Pubmed & 23383273 & $\begin{array}{l}\text { VCP phosphorylation-dependent interaction partners prevent } \\
\text { apoptosis in Helicobacter pylori-infected gastric epithelial } \\
\text { cells. }\end{array}$ & 7.54E-08 & 2.79E-03 & $5.26 \mathrm{E}-05$ & $5.84 \mathrm{E}-04$ & 19 & 288 \\
\hline A3 & Pubmed & 22446626 & $\begin{array}{l}\text { DBIRD complex integrates alternative mRNA splicing with } \\
\text { RNA polymerase II transcript elongation. }\end{array}$ & $8.48 \mathrm{E}-08$ & $3.14 \mathrm{E}-03$ & $5.81 \mathrm{E}-05$ & $6.45 \mathrm{E}-04$ & 11 & 89 \\
\hline A3 & Pubmed & 17207965 & $\begin{array}{l}\text { hORFeome v3.1: a resource of human open reading frames } \\
\text { representing over } 10,000 \text { human genes. }\end{array}$ & 9.49E-08 & $3.51 \mathrm{E}-03$ & $6.39 \mathrm{E}-05$ & 7.09E-04 & 54 & 1,674 \\
\hline A3 & Pubmed & 28065597 & $\begin{array}{l}\text { A Global Analysis of the Receptor Tyrosine Kinase-Protein } \\
\text { Phosphatase Interactome. }\end{array}$ & $9.75 \mathrm{E}-08$ & $3.61 \mathrm{E}-03$ & $6.44 \mathrm{E}-05$ & $7.15 \mathrm{E}-04$ & 23 & 419 \\
\hline A3 & Pubmed & 12107410 & $\begin{array}{l}\text { Expressed sequence tag analysis of human RPE/choroid for } \\
\text { the NEIBank Project: over } 6000 \text { non-redundant } \\
\text { transcripts, novel genes, and splice variants. }\end{array}$ & 9.97E-08 & $3.69 \mathrm{E}-03$ & $6.48 \mathrm{E}-05$ & 7.19E-04 & 12 & 111 \\
\hline A3 & Pubmed & 26167880 & $\begin{array}{l}\text { SR protein kinases promote splicing of nonconsensus } \\
\text { introns. }\end{array}$ & $1.34 \mathrm{E}-07$ & $4.94 \mathrm{E}-03$ & $8.52 \mathrm{E}-05$ & $9.46 \mathrm{E}-04$ & 21 & 361 \\
\hline A3 & Pubmed & 17474147 & $\begin{array}{l}\text { Systematic identification of SH3 domain-mediated human } \\
\text { protein-protein interactions by peptide array target } \\
\text { screening. }\end{array}$ & $1.37 \mathrm{E}-07$ & $5.08 \mathrm{E}-03$ & $8.61 \mathrm{E}-05$ & $9.55 \mathrm{E}-04$ & 20 & 330 \\
\hline A3 & Pubmed & 22113938 & $\begin{array}{l}\text { A bead-based approach for large-scale identification of in } \\
\text { vitro kinase substrates. }\end{array}$ & $1.61 \mathrm{E}-07$ & $5.96 \mathrm{E}-03$ & $9.69 \mathrm{E}-05$ & $1.08 \mathrm{E}-03$ & 14 & 163 \\
\hline A3 & Pubmed & 16713569 & $\begin{array}{l}\text { A protein-protein interaction network for human inherited } \\
\text { ataxias and disorders of Purkinje cell degeneration. }\end{array}$ & $1.62 \mathrm{E}-07$ & $6.01 \mathrm{E}-03$ & $9.69 \mathrm{E}-05$ & $1.08 \mathrm{E}-03$ & 28 & 608 \\
\hline A3 & Pubmed & 22268729 & Proteomic identification of common SCF ubiquitin ligase & $1.92 \mathrm{E}-07$ & 7.09E-03 & $1.13 \mathrm{E}-04$ & $1.25 \mathrm{E}-03$ & 28 & 613 \\
\hline
\end{tabular}




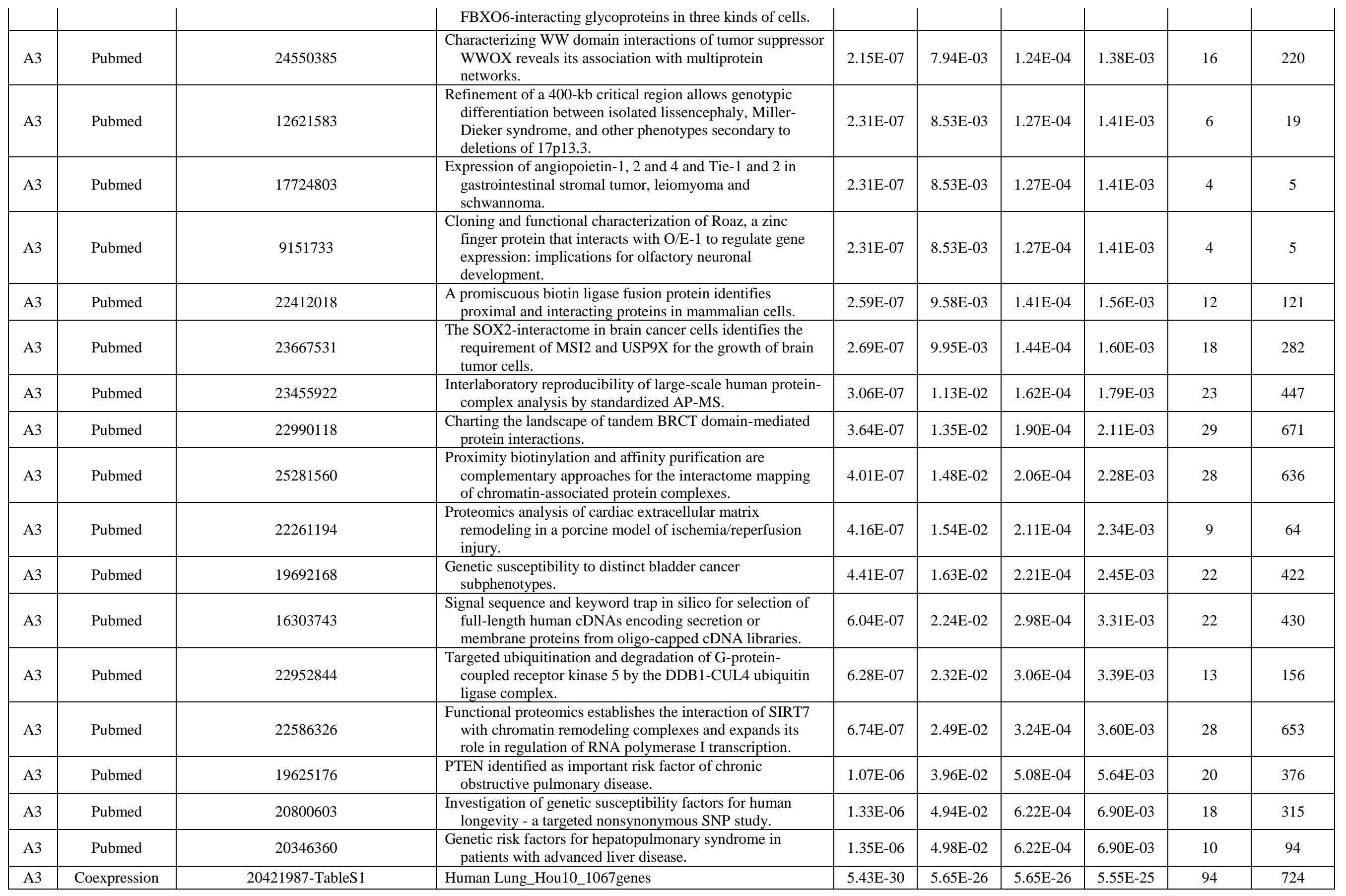




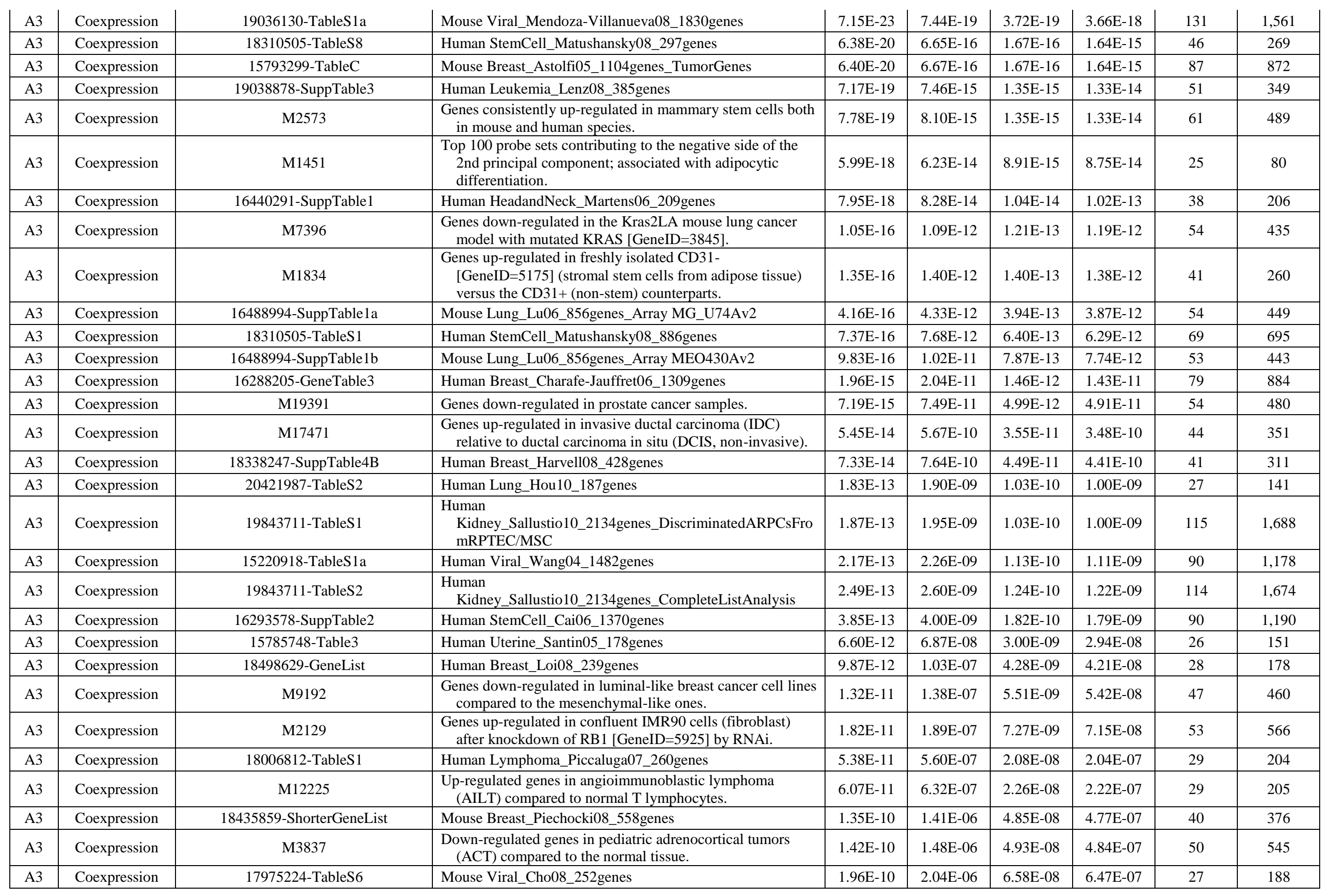




\begin{tabular}{|c|c|c|c|c|c|c|c|c|c|}
\hline $\mathrm{A} 3$ & Coexpression & 18310505-Table1 & Human StemCell_Matushansky08_69genes & $2.28 \mathrm{E}-10$ & $2.38 \mathrm{E}-06$ & 7.43E-08 & $7.30 \mathrm{E}-07$ & 15 & 55 \\
\hline A3 & Coexpression & 17683608-TableS1 & Mouse StemCell_Ulloa-Montoya07_546genes & $2.68 \mathrm{E}-10$ & $2.80 \mathrm{E}-06$ & $8.47 \mathrm{E}-08$ & $8.33 \mathrm{E}-07$ & 44 & 451 \\
\hline A3 & Coexpression & M4995 & $\begin{array}{l}\text { Genes specifically up-regulated in Cluster IIb of urothelial } \\
\text { cell carcinoma (UCC) tumors. }\end{array}$ & $4.05 \mathrm{E}-10$ & 4.22E-06 & $1.24 \mathrm{E}-07$ & $1.22 \mathrm{E}-06$ & 40 & 390 \\
\hline A3 & Coexpression & M97 & $\begin{array}{l}\text { Genes up-regulated in glomeruli of kidneys from patients } \\
\text { with diabetic nephropathy (type } 2 \text { diabetes mellitus). }\end{array}$ & 4.27E-10 & 4.44E-06 & $1.27 \mathrm{E}-07$ & $1.25 \mathrm{E}-06$ & 18 & 86 \\
\hline $\mathrm{A} 3$ & Coexpression & 19286929-SuppTable1 & Mouse Lung_Rangasamy09_2003genes & $4.92 \mathrm{E}-10$ & 5.13E-06 & $1.42 \mathrm{E}-07$ & $1.40 \mathrm{E}-06$ & 102 & 1,621 \\
\hline A3 & Coexpression & 17234769-TableS2b & Human StemCell_West07_669genes & $5.16 \mathrm{E}-10$ & $5.37 \mathrm{E}-06$ & $1.45 \mathrm{E}-07$ & $1.43 \mathrm{E}-06$ & 48 & 530 \\
\hline $\mathrm{A} 3$ & Coexpression & M12921 & $\begin{array}{l}\text { Genes up-regulated in brain from patients with Alzheimer's } \\
\text { disease. }\end{array}$ & $5.36 \mathrm{E}-10$ & $5.58 \mathrm{E}-06$ & $1.47 \mathrm{E}-07$ & $1.44 \mathrm{E}-06$ & 105 & 1,690 \\
\hline A3 & Coexpression & M1533 & $\begin{array}{l}\text { All significantly down-regulated genes in kidney glomeruli } \\
\text { isolated from TCF21 [Gene ID=6943] knockout mice. }\end{array}$ & $5.64 \mathrm{E}-10$ & $5.87 \mathrm{E}-06$ & $1.51 \mathrm{E}-07$ & $1.48 \mathrm{E}-06$ & 64 & 830 \\
\hline $\mathrm{A} 3$ & Coexpression & 18535662-TableS2c & Mouse Lymphoma_Wu08_1148genes & $1.15 \mathrm{E}-09$ & $1.20 \mathrm{E}-05$ & $3.01 \mathrm{E}-07$ & $2.95 \mathrm{E}-06$ & 48 & 543 \\
\hline $\mathrm{A} 3$ & Coexpression & 17660535-TableS4b & Mouse InnerEar_Sajan08_2230genes & $1.47 \mathrm{E}-09$ & $1.53 \mathrm{E}-05$ & $3.74 \mathrm{E}-07$ & $3.67 \mathrm{E}-06$ & 115 & 1,948 \\
\hline A3 & Coexpression & 18535662-TableS1a & Mouse Lymphoma_Wu08_1813genes & $1.69 \mathrm{E}-09$ & $1.76 \mathrm{E}-05$ & $4.20 \mathrm{E}-07$ & $4.13 \mathrm{E}-06$ & 67 & 913 \\
\hline A3 & Coexpression & M80 & $\begin{array}{l}\text { Genes up-regulated in TC71 and EWS502 cells (Ewing's } \\
\text { sarcoma) by EWSR1-FLI1 [GeneID=2130;2314] as } \\
\text { inferred from RNAi knockdown of this fusion protein. }\end{array}$ & 2.52E-09 & 2.62E-05 & $6.09 \mathrm{E}-07$ & $5.99 \mathrm{E}-06$ & 84 & 1276 \\
\hline A3 & Coexpression & M2324 & $\begin{array}{l}\text { Genes up-regulated in uterus upon knockout of BMP2 } \\
\text { [GeneID=650]. }\end{array}$ & 2.65E-09 & $2.76 \mathrm{E}-05$ & $6.26 \mathrm{E}-07$ & $6.15 \mathrm{E}-06$ & 58 & 745 \\
\hline A3 & Coexpression & 15361855-SuppTable1 & Human Ovarian_Donninger04_1191genes & $3.46 \mathrm{E}-09$ & $3.61 \mathrm{E}-05$ & $7.85 \mathrm{E}-07$ & $7.71 \mathrm{E}-06$ & 69 & 970 \\
\hline A3 & Coexpression & 19139136-TableS3 & Human Prostate_John-Aryankalayil09_2212genes & $3.47 \mathrm{E}-09$ & $3.61 \mathrm{E}-05$ & $7.85 \mathrm{E}-07$ & $7.71 \mathrm{E}-06$ & 96 & 1,547 \\
\hline A3 & Coexpression & 17161497-SuppTable1 & Human Lung_Dracheva07_162genes & 3.74E-09 & $3.90 \mathrm{E}-05$ & $8.30 \mathrm{E}-07$ & $8.15 \mathrm{E}-06$ & 22 & 146 \\
\hline A3 & Coexpression & 17724462-TableS2 & Human StemCell_Nuytten08_2072genes & 4.00E-09 & $4.12 \mathrm{E}-05$ & $8.57 \mathrm{E}-07$ & $8.43 \mathrm{E}-06$ & 105 & 1,753 \\
\hline A3 & Coexpression & 17284527-TableS2 & Human Lypmhoma_Leval07_832genes & 4.11E-09 & $4.28 \mathrm{E}-05$ & $8.74 \mathrm{E}-07$ & $8.59 \mathrm{E}-06$ & 52 & 639 \\
\hline $\mathrm{A} 3$ & Coexpression & 19408105-SuppTable4 & Mouse Breast_Stein09_1780genes & 5.19E-09 & $5.40 \mathrm{E}-05$ & $1.08 \mathrm{E}-06$ & $1.06 \mathrm{E}-05$ & 45 & 514 \\
\hline $\mathrm{A} 3$ & Coexpression & 19061838-TableS7 & Human Viral_Cairo08_982genes & 5.91E-09 & $6.15 \mathrm{E}-05$ & $1.21 \mathrm{E}-06$ & $1.19 \mathrm{E}-05$ & 60 & 801 \\
\hline $\mathrm{A} 3$ & Coexpression & 18381423-SuppTable1a & Human StemCell_Riggi08_262genes & $1.12 \mathrm{E}-08$ & $1.16 \mathrm{E}-04$ & $2.24 \mathrm{E}-06$ & $2.20 \mathrm{E}-05$ & 24 & 182 \\
\hline A3 & Coexpression & M2456 & $\begin{array}{l}\text { Genes requiring MLL [GeneID=4297] for H3K4me3 and } \\
\text { expression in MEF cells (embryonic fibroblast). }\end{array}$ & $1.34 \mathrm{E}-08$ & $1.40 \mathrm{E}-04$ & $2.64 \mathrm{E}-06$ & $2.59 \mathrm{E}-05$ & 31 & 289 \\
\hline A3 & Coexpression & 15972966-TableS2b & Human Colon_Lacroix05_61genes & $1.46 \mathrm{E}-08$ & $1.52 \mathrm{E}-04$ & $2.82 \mathrm{E}-06$ & $2.78 \mathrm{E}-05$ & 12 & 44 \\
\hline A3 & Coexpression & M1941 & $\begin{array}{l}\text { Genes with high-CpG-density promoters (HCP) bearing } \\
\text { histone } \mathrm{H} 3 \text { dimethylation at K4 }(\mathrm{H} 3 \mathrm{~K} 4 \mathrm{me} 2) \text { and } \\
\text { trimethylation at K27 (H3K27me3) in brain. }\end{array}$ & $1.51 \mathrm{E}-08$ & $1.57 \mathrm{E}-04$ & $2.86 \mathrm{E}-06$ & $2.81 \mathrm{E}-05$ & 72 & 1,069 \\
\hline A3 & Coexpression & M17923 & $\begin{array}{l}\text { Genes up-regulated in cultured stromal stem cells from } \\
\text { adipose tissue, compared to the freshly isolated cells. }\end{array}$ & $1.55 \mathrm{E}-08$ & $1.61 \mathrm{E}-04$ & $2.88 \mathrm{E}-06$ & $2.83 \mathrm{E}-05$ & 39 & 425 \\
\hline A3 & Coexpression & M5930 & $\begin{array}{l}\text { Genes defining epithelial-mesenchymal transition, as in } \\
\text { wound healing, fibrosis and metastasis. }\end{array}$ & $1.66 \mathrm{E}-08$ & $1.73 \mathrm{E}-04$ & $3.00 \mathrm{E}-06$ & $2.95 \mathrm{E}-05$ & 25 & 200 \\
\hline A3 & Coexpression & M17788 & $\begin{array}{l}\text { Down-regulated genes whose expression profile is specific } \\
\text { to Custer I of urothelial cell carcinoma (UCC) tumors. }\end{array}$ & $2.15 \mathrm{E}-08$ & $2.24 \mathrm{E}-04$ & 3.79E-06 & $3.73 \mathrm{E}-05$ & 36 & 378 \\
\hline A3 & Coexpression & 19605494-TableS2 & Mouse Skin_Driskell09_1477genes & $2.29 \mathrm{E}-08$ & $2.39 \mathrm{E}-04$ & $3.98 \mathrm{E}-06$ & $3.91 \mathrm{E}-05$ & 62 & 872 \\
\hline A3 & Coexpression & M13256 & $\begin{array}{l}\text { Genes down-regulated in mesenchymal stem cells (MSC) } \\
\text { engineered to express EWS-FLI1 [GeneID=2130;2321] }\end{array}$ & $2.61 \mathrm{E}-08$ & 2.72E-04 & $4.46 \mathrm{E}-06$ & 4.38E-05 & 24 & 190 \\
\hline
\end{tabular}




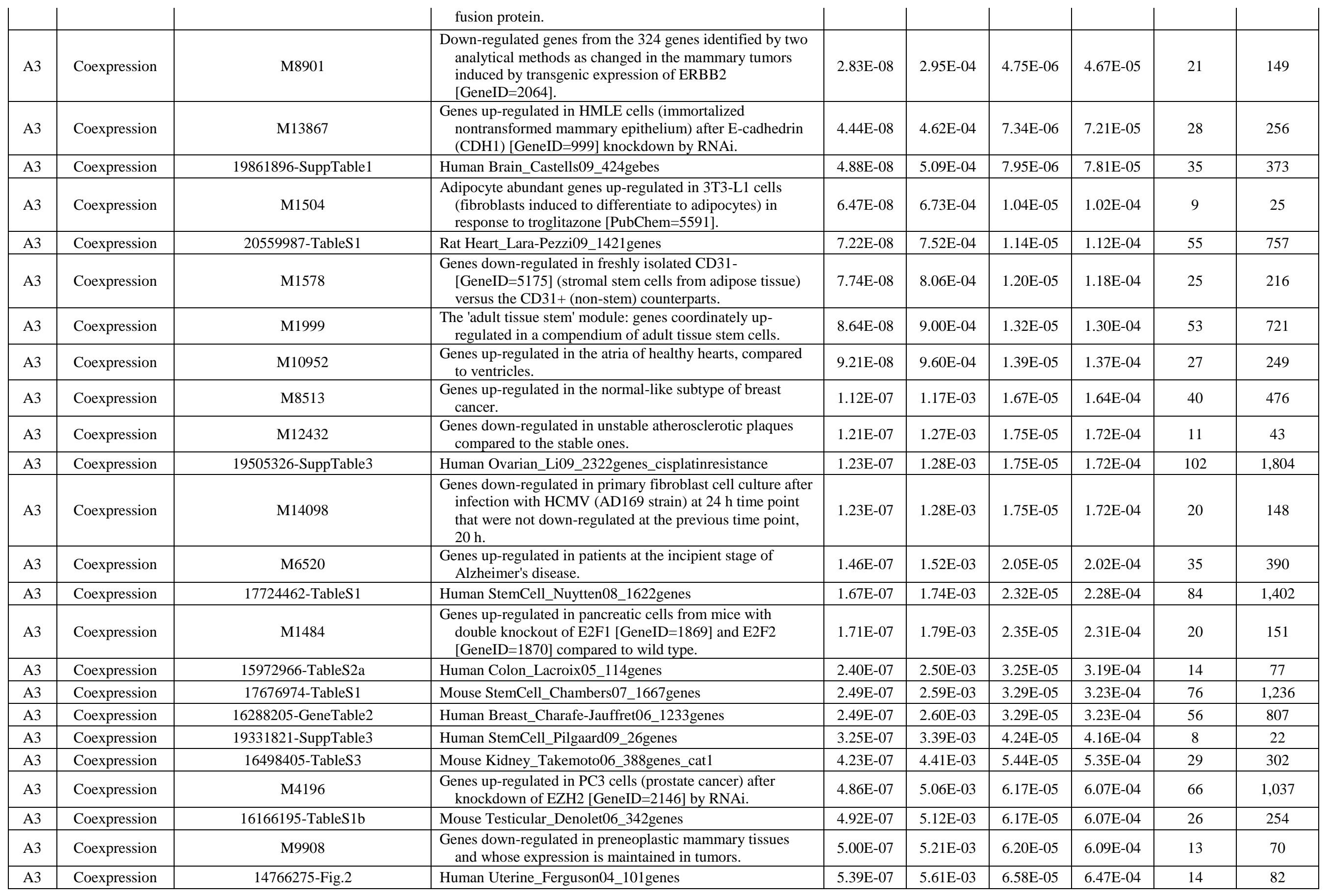




\begin{tabular}{|c|c|c|c|c|c|c|c|c|c|}
\hline A3 & Coexpression & M14027 & $\begin{array}{l}\text { Down-regulated genes in lung tissue of smokers with } \\
\text { chronic obstructive pulmonary disease (COPD) vs } \\
\text { smokers without disease (GOLD-2 vs GOLD-0). }\end{array}$ & $5.43 \mathrm{E}-07$ & $5.66 \mathrm{E}-03$ & $6.58 \mathrm{E}-05$ & $6.47 \mathrm{E}-04$ & 17 & 120 \\
\hline A3 & Coexpression & M4913 & $\begin{array}{l}\text { Genes down-regulated in adipose tissue mesenchymal stem } \\
\text { cells (ASC) vs bone marrow mesenchymal stem cells } \\
\text { (rBMSCs) }\end{array}$ & $6.30 \mathrm{E}-07$ & $6.56 \mathrm{E}-03$ & 7.53E-05 & $7.40 \mathrm{E}-04$ & 16 & 108 \\
\hline A3 & Coexpression & M2571 & $\begin{array}{l}\text { Genes down-regulated during prostate cancer progression in } \\
\text { the JOCK1 model due to inducible activation of FGFR } 1 \\
{[\text { GeneID }=2260] \text { gene in prostate. }}\end{array}$ & $6.37 \mathrm{E}-07$ & $6.63 \mathrm{E}-03$ & 7.53E-05 & $7.40 \mathrm{E}-04$ & 29 & 308 \\
\hline A3 & Coexpression & M13206 & $\begin{array}{l}\text { Genes down-regulated in TC71 and EWS502 cells (Ewing's } \\
\text { sarcoma) by EWSR1-FLI1 [GeneID=2130;2314] as } \\
\text { inferred from RNAi knockdown of this fusion protein. }\end{array}$ & $6.65 \mathrm{E}-07$ & $6.93 \mathrm{E}-03$ & $7.78 \mathrm{E}-05$ & $7.65 \mathrm{E}-04$ & 30 & 326 \\
\hline A3 & Coexpression & M12176 & $\begin{array}{l}\text { Genes down-regulated in robust Cluster } 2(\mathrm{rC} 2) \text { of } \\
\text { hepatoblastoma samples compared to those in the robust } \\
\text { Cluster } 1(\mathrm{rC} 1) \text {. }\end{array}$ & $6.84 \mathrm{E}-07$ & $7.13 \mathrm{E}-03$ & $7.92 \mathrm{E}-05$ & $7.78 \mathrm{E}-04$ & 23 & 210 \\
\hline A3 & Coexpression & 19489030-SuppTable7 & Human Prostate_Chambers09_259genes & $7.18 \mathrm{E}-07$ & $7.48 \mathrm{E}-03$ & $8.22 \mathrm{E}-05$ & $8.08 \mathrm{E}-04$ & 22 & 195 \\
\hline A3 & Coexpression & M1555 & $\begin{array}{l}\text { Adipocyte abundant genes down-regulated in 3T3-L1 cells } \\
\text { (fibroblasts induced to differentiate to adipocytes) in } \\
\text { response to TNF [GeneID=7124]. }\end{array}$ & 7.31E-07 & 7.61E-03 & $8.27 \mathrm{E}-05$ & $8.13 \mathrm{E}-04$ & 14 & 84 \\
\hline A3 & Coexpression & 17273163-Supp1a & Human Skin_Micke07_42genes & $7.42 \mathrm{E}-07$ & $7.73 \mathrm{E}-03$ & $8.31 \mathrm{E}-05$ & $8.17 \mathrm{E}-04$ & 10 & 41 \\
\hline A3 & Coexpression & M2580 & $\begin{array}{l}\text { Genes consistently down-regulated in mature mammary } \\
\text { luminal cells both in mouse and human species. }\end{array}$ & $1.05 \mathrm{E}-06$ & $1.09 \mathrm{E}-02$ & $1.15 \mathrm{E}-04$ & $1.13 \mathrm{E}-03$ & 15 & 99 \\
\hline A3 & Coexpression & M5905 & $\begin{array}{l}\text { Genes up-regulated during adipocyte differentiation } \\
\text { (adipogenesis). }\end{array}$ & $1.11 \mathrm{E}-06$ & $1.15 \mathrm{E}-02$ & $1.17 \mathrm{E}-04$ & $1.15 \mathrm{E}-03$ & 22 & 200 \\
\hline A3 & Coexpression & M8469 & $\begin{array}{l}\text { Genes up-regulated in activated CD4 [GeneID=920] T cells: } \\
\text { wildtype versus MIR17 [GeneID=406952] knockout. }\end{array}$ & $1.11 \mathrm{E}-06$ & $1.15 \mathrm{E}-02$ & $1.17 \mathrm{E}-04$ & $1.15 \mathrm{E}-03$ & 22 & 200 \\
\hline A3 & Coexpression & M8734 & $\begin{array}{l}\text { Genes up-regulated in T cells: control versus IL2 } \\
{[\text { GeneID }=3558] \text { stimulation for } 17 \mathrm{~h} \text {. }}\end{array}$ & $1.11 \mathrm{E}-06$ & $1.15 \mathrm{E}-02$ & $1.17 \mathrm{E}-04$ & $1.15 \mathrm{E}-03$ & 22 & 200 \\
\hline A3 & Coexpression & M13273 & $\begin{array}{l}\text { Genes down-regulated in papillary thyroid carcinoma (PTC) } \\
\text { compared to normal tissue. }\end{array}$ & $1.11 \mathrm{E}-06$ & $1.16 \mathrm{E}-02$ & $1.17 \mathrm{E}-04$ & $1.15 \mathrm{E}-03$ & 24 & 232 \\
\hline A3 & Coexpression & 15489886-TableS1b & Human Sarcoma_Mason04_549genes & $1.19 \mathrm{E}-06$ & $1.24 \mathrm{E}-02$ & $1.24 \mathrm{E}-04$ & $1.22 \mathrm{E}-03$ & 37 & 464 \\
\hline A3 & Coexpression & 17471573-Table2 & Human Viral_Santegoets07_103genes & $1.31 \mathrm{E}-06$ & $1.36 \mathrm{E}-02$ & $1.35 \mathrm{E}-04$ & $1.33 \mathrm{E}-03$ & 14 & 88 \\
\hline A3 & Coexpression & 17206280-TableS1C & Mouse Breast_Park07_173genes & $1.71 \mathrm{E}-06$ & $1.78 \mathrm{E}-02$ & $1.74 \mathrm{E}-04$ & $1.71 \mathrm{E}-03$ & 17 & 130 \\
\hline A3 & Coexpression & M2675 & $\begin{array}{l}\text { Genes down-regulated in HUVEC cells (endothelium) by } \\
\text { treatment with VEGFA [Gene ID=7422]. }\end{array}$ & $2.30 \mathrm{E}-06$ & $2.40 \mathrm{E}-02$ & $2.30 \mathrm{E}-04$ & $2.26 \mathrm{E}-03$ & 21 & 193 \\
\hline A3 & Coexpression & M14142 & $\begin{array}{l}\text { Genes down-regulated in the invasive ductal carcinoma } \\
\text { (IDC) compared to the invasive lobular carcinoma (ILC), } \\
\text { the two major pathological types of breast cancer. }\end{array}$ & $2.32 \mathrm{E}-06$ & $2.42 \mathrm{E}-02$ & $2.30 \mathrm{E}-04$ & $2.26 \mathrm{E}-03$ & 10 & 46 \\
\hline A3 & Coexpression & M5688 & $\begin{array}{l}\text { Genes up-regulated in Paneth cell (part of intestinal } \\
\text { epithelium) of mice with hypomorphic (reduced function) } \\
\text { form of ATG16L1 [GeneID=55054]. }\end{array}$ & $2.59 \mathrm{E}-06$ & $2.69 \mathrm{E}-02$ & $2.52 \mathrm{E}-04$ & $2.47 \mathrm{E}-03$ & 14 & 93 \\
\hline A3 & Coexpression & M13944 & $\begin{array}{l}\text { Genes up-regulated in TMX2-28 cells (breast cancer) which } \\
\text { do not express ESR1 [GeneID=2099]) compared to the }\end{array}$ & $2.72 \mathrm{E}-06$ & $2.84 \mathrm{E}-02$ & $2.63 \mathrm{E}-04$ & $2.58 \mathrm{E}-03$ & 18 & 149 \\
\hline
\end{tabular}




\begin{tabular}{|c|c|c|c|c|c|c|c|c|c|}
\hline & & & MCF7 cells which do. & & & & & & \\
\hline A3 & Coexpression & M14385 & $\begin{array}{l}\text { Genes down-regulated in fibroblasts with defective XPC } \\
{[\text { GeneID=7508] in response to cisplatin [PubChem=2767]. }}\end{array}$ & $2.85 \mathrm{E}-06$ & 2.97E-02 & $2.70 \mathrm{E}-04$ & $2.65 \mathrm{E}-03$ & 23 & 228 \\
\hline A3 & Coexpression & 20937356-TableS1 & Rat Hypothalamic_Mansuy10_1270genes & $2.85 \mathrm{E}-06$ & $2.97 \mathrm{E}-02$ & $2.70 \mathrm{E}-04$ & $2.65 \mathrm{E}-03$ & 57 & 894 \\
\hline A3 & Coexpression & 15845616-SuppTable3 & Mouse Uterine_Jeong05_634genes & $2.92 \mathrm{E}-06$ & $3.04 \mathrm{E}-02$ & 2.74E-04 & $2.69 \mathrm{E}-03$ & 37 & 482 \\
\hline A3 & Coexpression & M2270 & $\begin{array}{l}\text { Genes up-regulated during adipogenesis of 3T3-L1 cells } \\
\text { (fibroblast). }\end{array}$ & $3.58 \mathrm{E}-06$ & $3.73 \mathrm{E}-02$ & $3.33 \mathrm{E}-04$ & $3.27 \mathrm{E}-03$ & 7 & 21 \\
\hline A3 & Coexpression & 20220088-SuppTable1 & Human Immune_Allen10_2900genes & $3.68 \mathrm{E}-06$ & $3.83 \mathrm{E}-02$ & $3.39 \mathrm{E}-04$ & $3.33 \mathrm{E}-03$ & 96 & 1,798 \\
\hline A3 & Coexpression & M7762 & $\begin{array}{l}\text { Genes down-regulated in macrophages: wildtype versus } \\
\text { MYD88 [GeneID=4615] knockout. }\end{array}$ & 4.07E-06 & 4.23E-02 & $3.68 \mathrm{E}-04$ & $3.62 \mathrm{E}-03$ & 21 & 200 \\
\hline A3 & Coexpression & M4545 & $\begin{array}{l}\text { Genes up-regulated in comparison of thymic progenitors } \\
\text { versus DN2 thymocytes. }\end{array}$ & 4.07E-06 & 4.23E-02 & $3.68 \mathrm{E}-04$ & $3.62 \mathrm{E}-03$ & 21 & 200 \\
\hline A3 & Coexpression & 18631401-TableS3 & Human Breast_Yau08_1518genes_OxidativeStress & 4.44E-06 & 4.62E-02 & 3.98E-04 & 3.92E-03 & 67 & 1,129 \\
\hline A3 & Coexpression & 19658189-TableS4 & Human EmbryonicStemCell_Xu09_1801genes & $4.78 \mathrm{E}-06$ & $4.98 \mathrm{E}-02$ & $4.25 \mathrm{E}-04$ & $4.18 \mathrm{E}-03$ & 80 & 1,430 \\
\hline A3 & $\begin{array}{l}\text { Coexpression } \\
\text { Atlas }\end{array}$ & GSM777059_500 & $\begin{array}{l}\text { Stromal Cells, LEC.MLN, gp38+ CD31+, Lymph Node, } \\
\text { avg-4 }\end{array}$ & $2.33 \mathrm{E}-24$ & $1.05 \mathrm{E}-20$ & $6.46 \mathrm{E}-21$ & $5.81 \mathrm{E}-20$ & 68 & 439 \\
\hline A3 & $\begin{array}{l}\text { Coexpression } \\
\text { Atlas }\end{array}$ & GSM777067_500 & $\begin{array}{l}\text { Stromal Cells, St.31-38-44-.SLN, CD45- gp38- CD31- } \\
\text { CD44-, Lymph Node, avg-2 }\end{array}$ & $2.86 \mathrm{E}-24$ & $1.29 \mathrm{E}-20$ & $6.46 \mathrm{E}-21$ & $5.81 \mathrm{E}-20$ & 69 & 453 \\
\hline A3 & $\begin{array}{l}\text { Coexpression } \\
\text { Atlas }\end{array}$ & GSM777063_500 & $\begin{array}{l}\text { Stromal Cells, LEC.SLN, gp38+ CD31+, Lymph Node, avg- } \\
4\end{array}$ & $9.97 \mathrm{E}-24$ & 4.50E-20 & $1.21 \mathrm{E}-20$ & $1.09 \mathrm{E}-19$ & 68 & 450 \\
\hline A3 & $\begin{array}{l}\text { Coexpression } \\
\text { Atlas }\end{array}$ & GSM777046_500 & Stromal Cells, Fi.Sk, gp38+ CD140a+, Skin, avg-4 & $1.07 \mathrm{E}-23$ & $4.83 \mathrm{E}-20$ & $1.21 \mathrm{E}-20$ & $1.09 \mathrm{E}-19$ & 67 & 438 \\
\hline A3 & $\begin{array}{l}\text { Coexpression } \\
\text { Atlas }\end{array}$ & $\begin{array}{c}\text { PCBC_ratio_CardiacMyocyte_vs_SC } \\
\text { _cfr-2X-p05 }\end{array}$ & $\begin{array}{l}\text { Cardiac Myocyte Cells_vs_Pluripotent Stem Cells- } \\
\text { Confounder_removed-fold2.0_adjp } 0.05\end{array}$ & $7.83 \mathrm{E}-23$ & $3.54 \mathrm{E}-19$ & $7.07 \mathrm{E}-20$ & $6.36 \mathrm{E}-19$ & 149 & 1,769 \\
\hline A3 & $\begin{array}{l}\text { Coexpression } \\
\text { Atlas }\end{array}$ & GSM777050_500 & $\begin{array}{l}\text { Stromal Cells, FRC.MLN, gp38+ CD31- CD140a+, Lymph } \\
\text { Node, avg-5 }\end{array}$ & $4.16 \mathrm{E}-22$ & $1.88 \mathrm{E}-18$ & $3.13 \mathrm{E}-19$ & $2.82 \mathrm{E}-18$ & 67 & 467 \\
\hline A3 & $\begin{array}{c}\text { Coexpression } \\
\text { Atlas }\end{array}$ & GSM777032_500 & $\begin{array}{l}\text { Stromal Cells, BEC.MLN, gp38- CD31+, Lymph Node, avg- } \\
5\end{array}$ & $1.20 \mathrm{E}-20$ & $5.43 \mathrm{E}-17$ & $7.75 \mathrm{E}-18$ & $6.97 \mathrm{E}-17$ & 64 & 456 \\
\hline A3 & $\begin{array}{l}\text { Coexpression } \\
\text { Atlas }\end{array}$ & GSM777055_500 & $\begin{array}{l}\text { Stromal Cells, FRC.SLN, gp38+ CD31- CD140a+, Lymph } \\
\text { Node, avg-4 }\end{array}$ & $4.96 \mathrm{E}-20$ & $2.24 \mathrm{E}-16$ & $2.80 \mathrm{E}-17$ & $2.52 \mathrm{E}-16$ & 63 & 455 \\
\hline A3 & $\begin{array}{l}\text { Coexpression } \\
\text { Atlas }\end{array}$ & PCBC_ctl_CardiacMyocyte_1000 & $\begin{array}{l}\text { Progenitor-Cell-Biology- } \\
\text { Consortium_reference_CardiacMyocyte_top-relative- } \\
\text { expression-ranked_1000 }\end{array}$ & $2.41 \mathrm{E}-19$ & $1.09 \mathrm{E}-15$ & $1.21 \mathrm{E}-16$ & $1.09 \mathrm{E}-15$ & 97 & 985 \\
\hline A3 & $\begin{array}{l}\text { Coexpression } \\
\text { Atlas }\end{array}$ & $\begin{array}{c}\text { gudmap_kidney_adult_RenCorpuscGl } \\
\text { omer_1000 }\end{array}$ & $\begin{array}{l}\text { kidney_adult_RenCorpuscGlomer_top-relative-expression- } \\
\text { ranked_1000 }\end{array}$ & $4.11 \mathrm{E}-19$ & $1.85 \mathrm{E}-15$ & $1.85 \mathrm{E}-16$ & $1.67 \mathrm{E}-15$ & 90 & 878 \\
\hline $\mathrm{A} 3$ & $\begin{array}{l}\text { Coexpression } \\
\text { Atlas }\end{array}$ & $\begin{array}{c}\text { gudmap_RNAseq_e15.5_Podocytes_2 } \\
500\end{array}$ & gudmap_RNAseq_e15.5_Podocytes_2500 & $5.32 \mathrm{E}-19$ & $2.40 \mathrm{E}-15$ & $2.19 \mathrm{E}-16$ & $1.97 \mathrm{E}-15$ & 145 & 1,863 \\
\hline A3 & $\begin{array}{l}\text { Coexpression } \\
\text { Atlas }\end{array}$ & GSM777043_500 & $\begin{array}{l}\text { Stromal Cells, Fi.MTS15+.Th, CD45- PDGFRa+ MTS15+, } \\
\text { Thymus, avg-3 }\end{array}$ & $1.49 \mathrm{E}-18$ & $6.72 \mathrm{E}-15$ & $5.60 \mathrm{E}-16$ & $5.04 \mathrm{E}-15$ & 60 & 445 \\
\hline A3 & $\begin{array}{l}\text { Coexpression } \\
\text { Atlas }\end{array}$ & $\begin{array}{c}\text { gudmap_RNAseq_p2_CD2APMEIS } \\
\text { WT_2500 }\end{array}$ & gudmap_RNAseq_p2_CD2APMEISWT_2500 & $3.03 \mathrm{E}-18$ & $1.37 \mathrm{E}-14$ & $1.05 \mathrm{E}-15$ & $9.46 \mathrm{E}-15$ & 141 & 1,822 \\
\hline A3 & $\begin{array}{l}\text { Coexpression } \\
\text { Atlas }\end{array}$ & $\begin{array}{l}\text { PCBC_ratio_BronchSmoothMuscl_vs } \\
\text { _SC_cfr-2X-p05 }\end{array}$ & $\begin{array}{l}\text { Bronchial Smooth Muscle Cells_vs_Pluripotent Stem Cells- } \\
\text { Confounder_removed-fold2.0_adjp0.05 }\end{array}$ & $9.00 \mathrm{E}-18$ & $4.07 \mathrm{E}-14$ & $2.71 \mathrm{E}-15$ & $2.44 \mathrm{E}-14$ & 145 & 1,923 \\
\hline A3 & $\begin{array}{l}\text { Coexpression } \\
\text { Atlas }\end{array}$ & JC_hmvEC_2500_K1 & $\begin{array}{l}\text { JC_hmvEC_top-relative-expression-ranked_2500_k-means- } \\
\text { cluster\#1 }\end{array}$ & $9.90 \mathrm{E}-18$ & 4.47E-14 & $2.80 \mathrm{E}-15$ & $2.51 \mathrm{E}-14$ & 91 & 939 \\
\hline
\end{tabular}




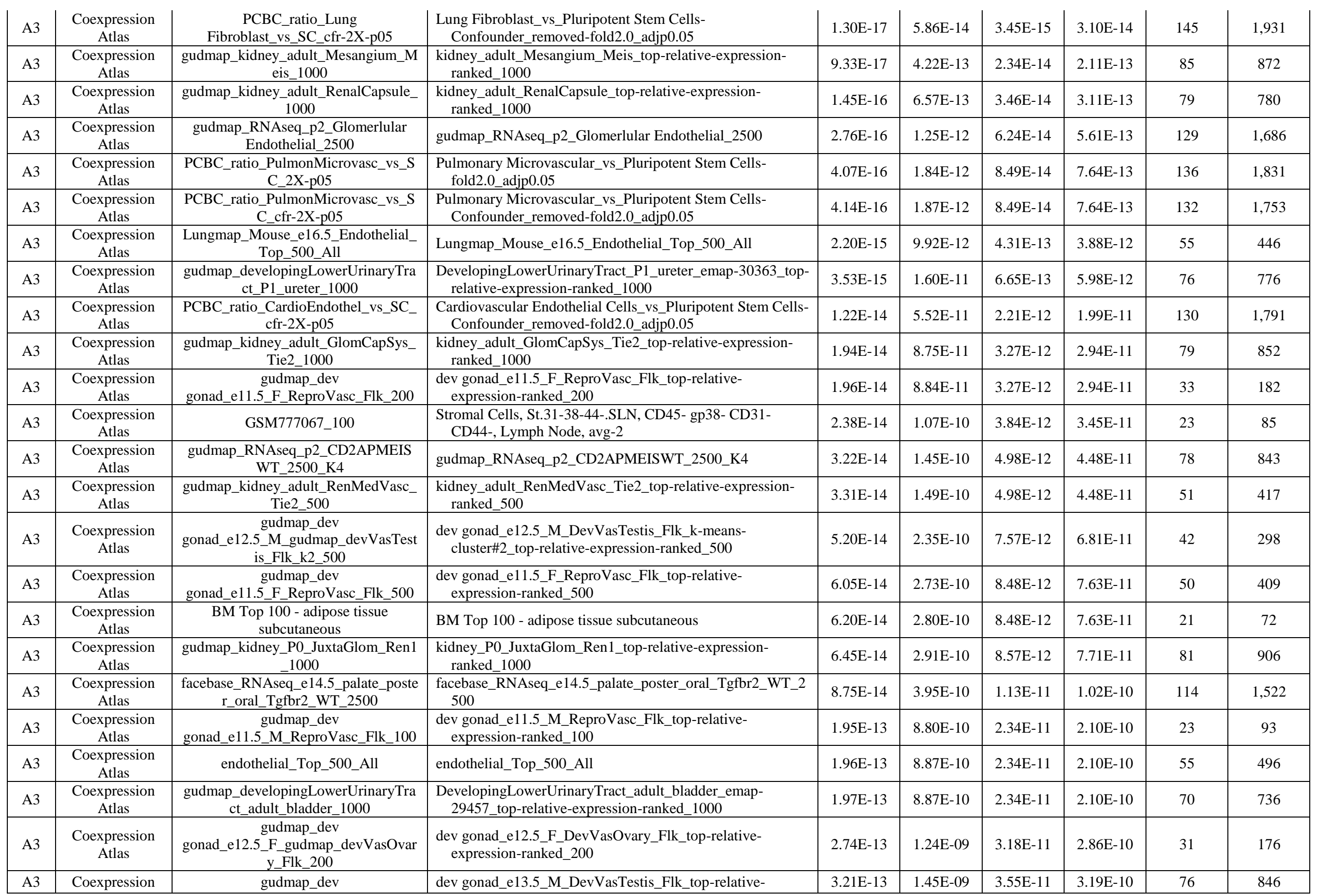




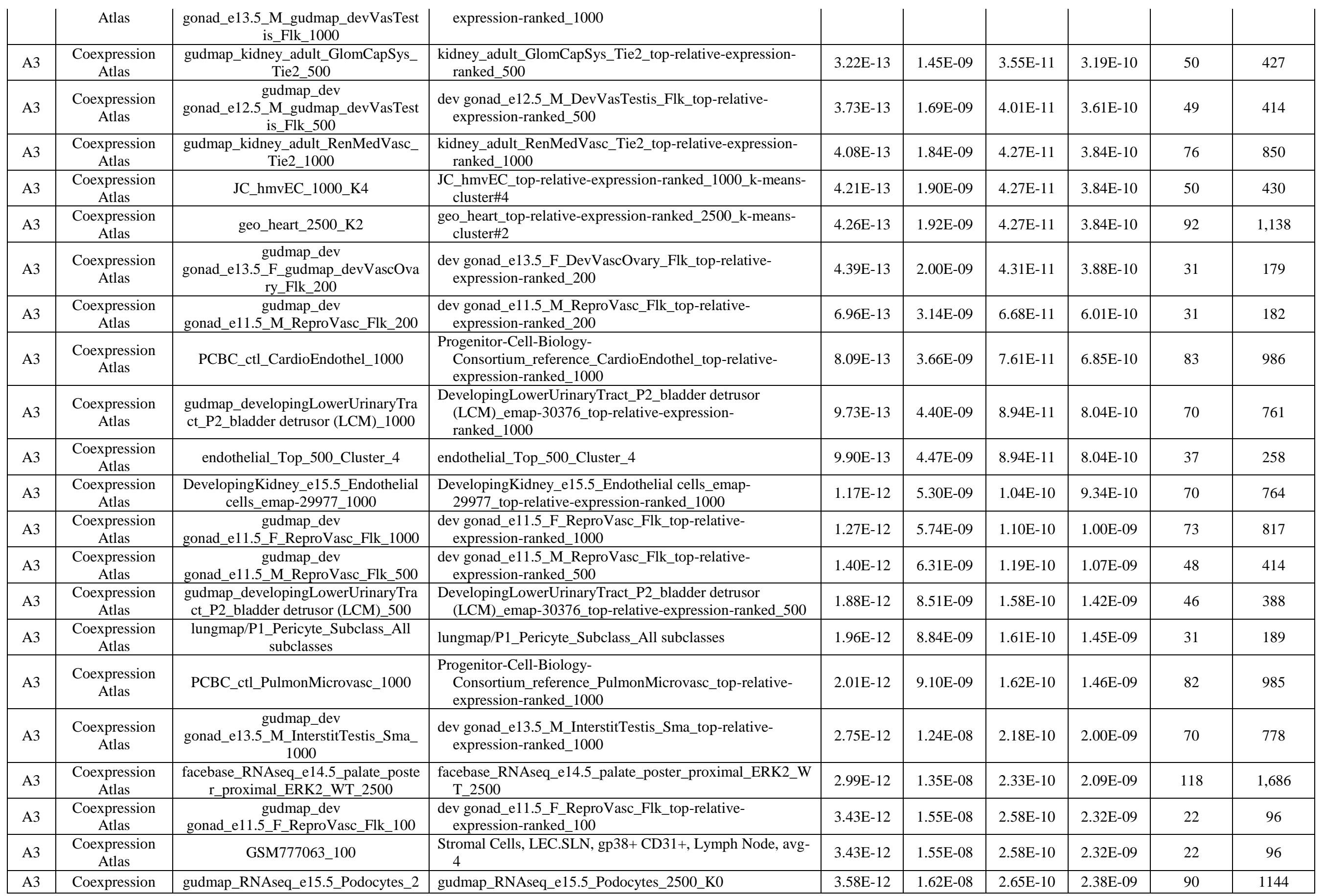




\begin{tabular}{|c|c|c|c|c|c|c|c|c|c|}
\hline & Atlas & 500_K0 & & & & & & & \\
\hline $\mathrm{A} 3$ & $\begin{array}{l}\text { Coexpression } \\
\text { Atlas }\end{array}$ & PCBC_ctl_geo-heart_1000 & geo_heart_top-relative-expression-ranked_1000 & $3.77 \mathrm{E}-12$ & $1.71 \mathrm{E}-08$ & $2.75 \mathrm{E}-10$ & 2.47E-09 & 82 & 997 \\
\hline A3 & $\begin{array}{l}\text { Coexpression } \\
\text { Atlas }\end{array}$ & BM Top 100 - adipose tissue & BM Top 100 - adipose tissue & $4.06 \mathrm{E}-12$ & $1.83 \mathrm{E}-08$ & $2.87 \mathrm{E}-10$ & $2.58 \mathrm{E}-09$ & 19 & 70 \\
\hline $\mathrm{A} 3$ & $\begin{array}{l}\text { Coexpression } \\
\text { Atlas }\end{array}$ & $\begin{array}{c}\text { lungmap/P1_Endothelial_Subclass_En } \\
\text { dothelial-B(2) }\end{array}$ & lungmap/P1_Endothelial_Subclass_Endothelial-B(2) & $4.07 \mathrm{E}-12$ & $1.84 \mathrm{E}-08$ & $2.87 \mathrm{E}-10$ & $2.58 \mathrm{E}-09$ & 35 & 244 \\
\hline A3 & $\begin{array}{l}\text { Coexpression } \\
\text { Atlas }\end{array}$ & $\begin{array}{c}\text { gudmap_kidney_e15.5_SmlBldVes_Ti } \\
\text { e2_k2_200 }\end{array}$ & $\begin{array}{l}\text { kidney_e15.5_SmlBldVes_Tie2_k-means-cluster\#2_top- } \\
\text { relative-expression-ranked_200 }\end{array}$ & $4.29 \mathrm{E}-12$ & $1.94 \mathrm{E}-08$ & $2.94 \mathrm{E}-10$ & 2.64E-09 & 22 & 97 \\
\hline A3 & $\begin{array}{l}\text { Coexpression } \\
\text { Atlas }\end{array}$ & GSM777046_100 & Stromal Cells, Fi.Sk, gp38+ CD140a+, Skin, avg-4 & $4.29 \mathrm{E}-12$ & 1.94E-08 & $2.94 \mathrm{E}-10$ & 2.64E-09 & 22 & 97 \\
\hline A3 & $\begin{array}{l}\text { Coexpression } \\
\text { Atlas } \\
\end{array}$ & $\begin{array}{c}\text { gudmap_kidney_adult_RenMedVasc_ } \\
\text { Tie2_200 }\end{array}$ & $\begin{array}{l}\text { kidney_adult_RenMedVasc_Tie2_top-relative-expression- } \\
\text { ranked_200 }\end{array}$ & $5.01 \mathrm{E}-12$ & $2.26 \mathrm{E}-08$ & $3.38 \mathrm{E}-10$ & $3.00 \mathrm{E}-09$ & 29 & 172 \\
\hline A3 & $\begin{array}{l}\text { Coexpression } \\
\text { Atlas }\end{array}$ & $\begin{array}{c}\text { gudmap_RNAseq_e15.5_Mesangium_ } \\
\text { 2500_K3 }\end{array}$ & gudmap_RNAseq_e15.5_Mesangium_2500_K3 & $5.81 \mathrm{E}-12$ & $2.62 \mathrm{E}-08$ & $3.86 \mathrm{E}-10$ & 3.47E-09 & 35 & 247 \\
\hline A3 & $\begin{array}{l}\text { Coexpression } \\
\text { Atlas } \\
\end{array}$ & $\begin{array}{l}\text { PCBC_ratio_Bronchial Smooth } \\
\text { Muscle_vs_SC_cfr-2X-p05 }\end{array}$ & $\begin{array}{l}\text { Bronchial Smooth Muscle_vs_Pluripotent Stem Cells- } \\
\text { Confounder_removed-fold2.0_adjp0.05 }\end{array}$ & $6.00 \mathrm{E}-12$ & $2.71 \mathrm{E}-08$ & $3.93 \mathrm{E}-10$ & 3.53E-09 & 123 & 1,806 \\
\hline A3 & $\begin{array}{l}\text { Coexpression } \\
\text { Atlas } \\
\end{array}$ & $\begin{array}{l}\text { gudmap_developingLowerUrinaryTra } \\
\text { ct_P1_bladder_1000_B }\end{array}$ & $\begin{array}{l}\text { DevelopingLowerUrinaryTract_P1_bladder_B_emap- } \\
\text { 30374_top-relative-expression-ranked_1000 }\end{array}$ & $6.85 \mathrm{E}-12$ & 3.09E-08 & $4.42 \mathrm{E}-10$ & $4.00 \mathrm{E}-09$ & 69 & 776 \\
\hline A3 & $\begin{array}{l}\text { Coexpression } \\
\text { Atlas }\end{array}$ & $\begin{array}{c}\text { gudmap_dev } \\
\text { gonad_e13.5_F_gudmap_devVascOva } \\
\text { ry_Flk_1000 }\end{array}$ & $\begin{array}{l}\text { dev gonad_e13.5_F_DevVascOvary_Flk_top-relative- } \\
\text { expression-ranked_1000 }\end{array}$ & $7.88 \mathrm{E}-12$ & $3.56 \mathrm{E}-08$ & $5.01 \mathrm{E}-10$ & 4.51E-09 & 72 & 831 \\
\hline $\mathrm{A} 3$ & $\begin{array}{l}\text { Coexpression } \\
\text { Atlas }\end{array}$ & $\begin{array}{c}\text { gudmap_developingKidney_e13.5_po } \\
\text { docyte cells_1000_k5 }\end{array}$ & $\begin{array}{l}\text { DevelopingKidney_e13.5_podocyte cells_emap-27773_k- } \\
\text { means-cluster\#5_top-relative-expression-ranked_1000 }\end{array}$ & $8.55 \mathrm{E}-12$ & $3.86 \mathrm{E}-08$ & $5.36 \mathrm{E}-10$ & $4.82 \mathrm{E}-09$ & 28 & 164 \\
\hline $\mathrm{A} 3$ & $\begin{array}{l}\text { Coexpression } \\
\text { Atlas }\end{array}$ & $\begin{array}{c}\text { gudmap_kidney_adult_JuxtaGlom_Re } \\
\text { n1_Captopr_1000 }\end{array}$ & $\begin{array}{l}\text { kidney_adult_JuxtaGlom_Ren1_Captopr_top-relative- } \\
\text { expression-ranked_1000 }\end{array}$ & $8.66 \mathrm{E}-12$ & 3.91E-08 & $5.36 \mathrm{E}-10$ & 4.82E-09 & 69 & 780 \\
\hline A3 & $\begin{array}{l}\text { Coexpression } \\
\text { Atlas }\end{array}$ & $\begin{array}{c}\text { lungmap/P1_Pericyte_Subclass_Multi } \\
\text { ple }\end{array}$ & lungmap/P1_Pericyte_Subclass_Multiple & $1.05 \mathrm{E}-11$ & $4.76 \mathrm{E}-08$ & $6.43 \mathrm{E}-10$ & $5.78 \mathrm{E}-09$ & 30 & 189 \\
\hline A3 & $\begin{array}{l}\text { Coexpression } \\
\text { Atlas }\end{array}$ & $\begin{array}{c}\text { gudmap_dev } \\
\text { gonad_e12.5_F_gudmap_devVasOvar } \\
\text { y_Flk_100 }\end{array}$ & $\begin{array}{l}\text { dev gonad_e12.5_F_DevVasOvary_Flk_top-relative- } \\
\text { expression-ranked_100 }\end{array}$ & $1.16 \mathrm{E}-11$ & $5.22 \mathrm{E}-08$ & $6.87 \mathrm{E}-10$ & $6.18 \mathrm{E}-09$ & 21 & 92 \\
\hline A3 & $\begin{array}{l}\text { Coexpression } \\
\text { Atlas }\end{array}$ & $\begin{array}{c}\text { gudmap_dev } \\
\text { gonad_e13.5_F_gudmap_devVascOva } \\
\text { ry_Flk_100 }\end{array}$ & $\begin{array}{l}\text { dev gonad_e13.5_F_DevVascOvary_Flk_top-relative- } \\
\text { expression-ranked_100 }\end{array}$ & $1.16 \mathrm{E}-11$ & $5.22 \mathrm{E}-08$ & $6.87 \mathrm{E}-10$ & $6.18 \mathrm{E}-09$ & 21 & 92 \\
\hline A3 & $\begin{array}{l}\text { Coexpression } \\
\text { Atlas } \\
\end{array}$ & BM Top 100 - adipose tissue omental & BM Top 100 - adipose tissue omental & $1.19 \mathrm{E}-11$ & 5.39E-08 & $7.01 \mathrm{E}-10$ & $6.30 \mathrm{E}-09$ & 19 & 74 \\
\hline A3 & $\begin{array}{l}\text { Coexpression } \\
\text { Atlas }\end{array}$ & $\begin{array}{c}\text { lungmap/P3_Endothelial_Subclass_En } \\
\text { dothelial-B(1) }\end{array}$ & lungmap/P3_Endothelial_Subclass_Endothelial-B(1) & $1.26 \mathrm{E}-11$ & $5.68 \mathrm{E}-08$ & $7.28 \mathrm{E}-10$ & $6.55 \mathrm{E}-09$ & 39 & 308 \\
\hline A3 & $\begin{array}{l}\text { Coexpression } \\
\text { Atlas }\end{array}$ & $\begin{array}{c}\text { gudmap_kidney_adult_RenalCapsule_ } \\
500\end{array}$ & $\begin{array}{l}\text { kidney_adult_RenalCapsule_top-relative-expression- } \\
\text { ranked_500 }\end{array}$ & $1.30 \mathrm{E}-11$ & $5.89 \mathrm{E}-08$ & $7.37 \mathrm{E}-10$ & $6.63 \mathrm{E}-09$ & 46 & 410 \\
\hline A3 & $\begin{array}{l}\text { Coexpression } \\
\text { Atlas }\end{array}$ & $\begin{array}{c}\text { lungmap/P1_Endothelial_Subclass_En } \\
\text { dothelial-B(1) }\end{array}$ & lungmap/P1_Endothelial_Subclass_Endothelial-B(1) & $1.31 \mathrm{E}-11$ & $5.90 \mathrm{E}-08$ & 7.37E-10 & $6.63 \mathrm{E}-09$ & 35 & 254 \\
\hline A3 & $\begin{array}{l}\text { Coexpression } \\
\text { Atlas }\end{array}$ & $\begin{array}{c}\text { gudmap_dev } \\
\text { gonad_e11.5_F_ReproVasc_Flk_k4_5 } \\
00\end{array}$ & $\begin{array}{l}\text { dev gonad_e11.5_F_ReproVasc_Flk_k-means- } \\
\text { cluster\#4_top-relative-expression-ranked_500 }\end{array}$ & $1.48 \mathrm{E}-11$ & $6.67 \mathrm{E}-08$ & $8.14 \mathrm{E}-10$ & 7.32E-09 & 33 & 229 \\
\hline
\end{tabular}




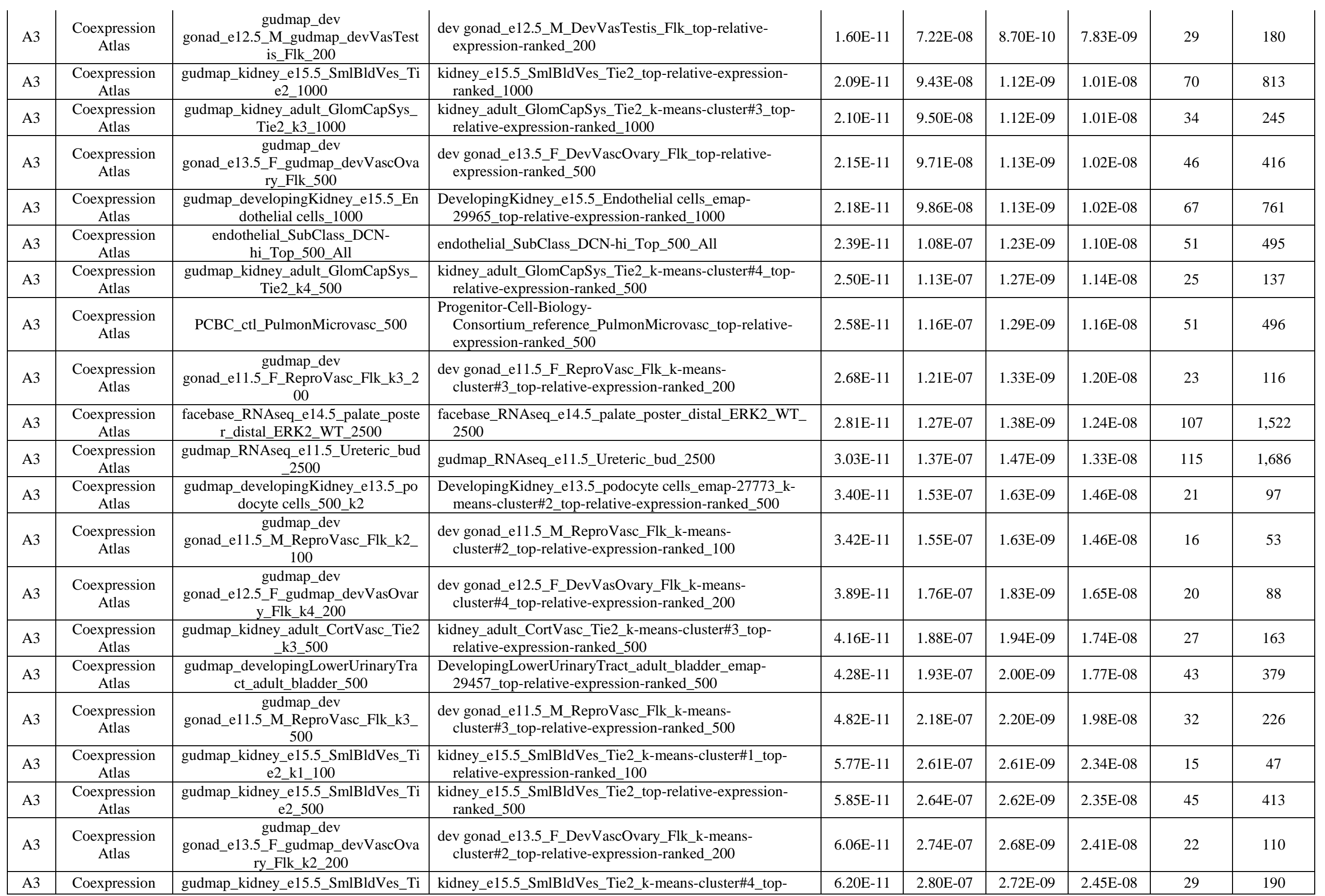




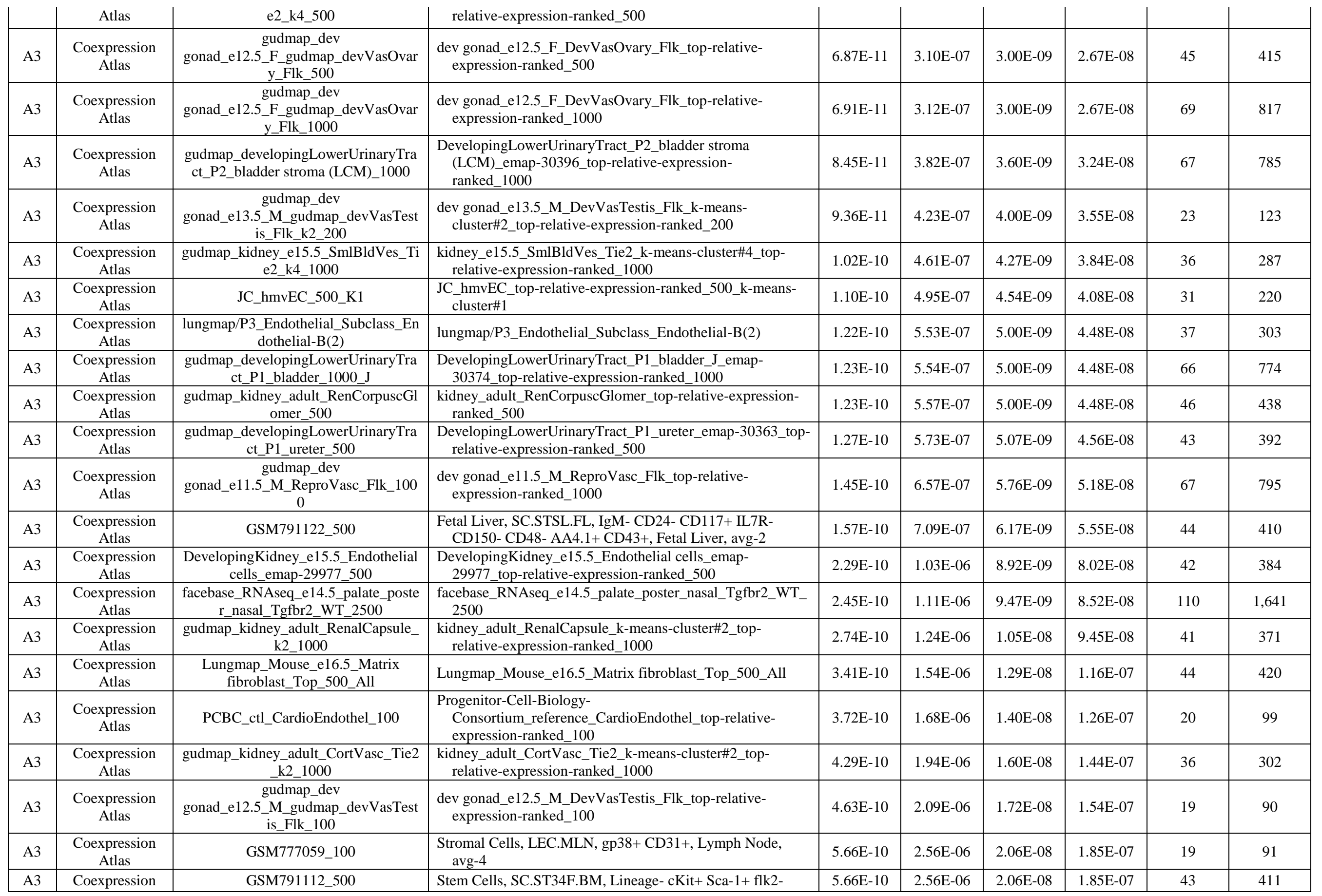




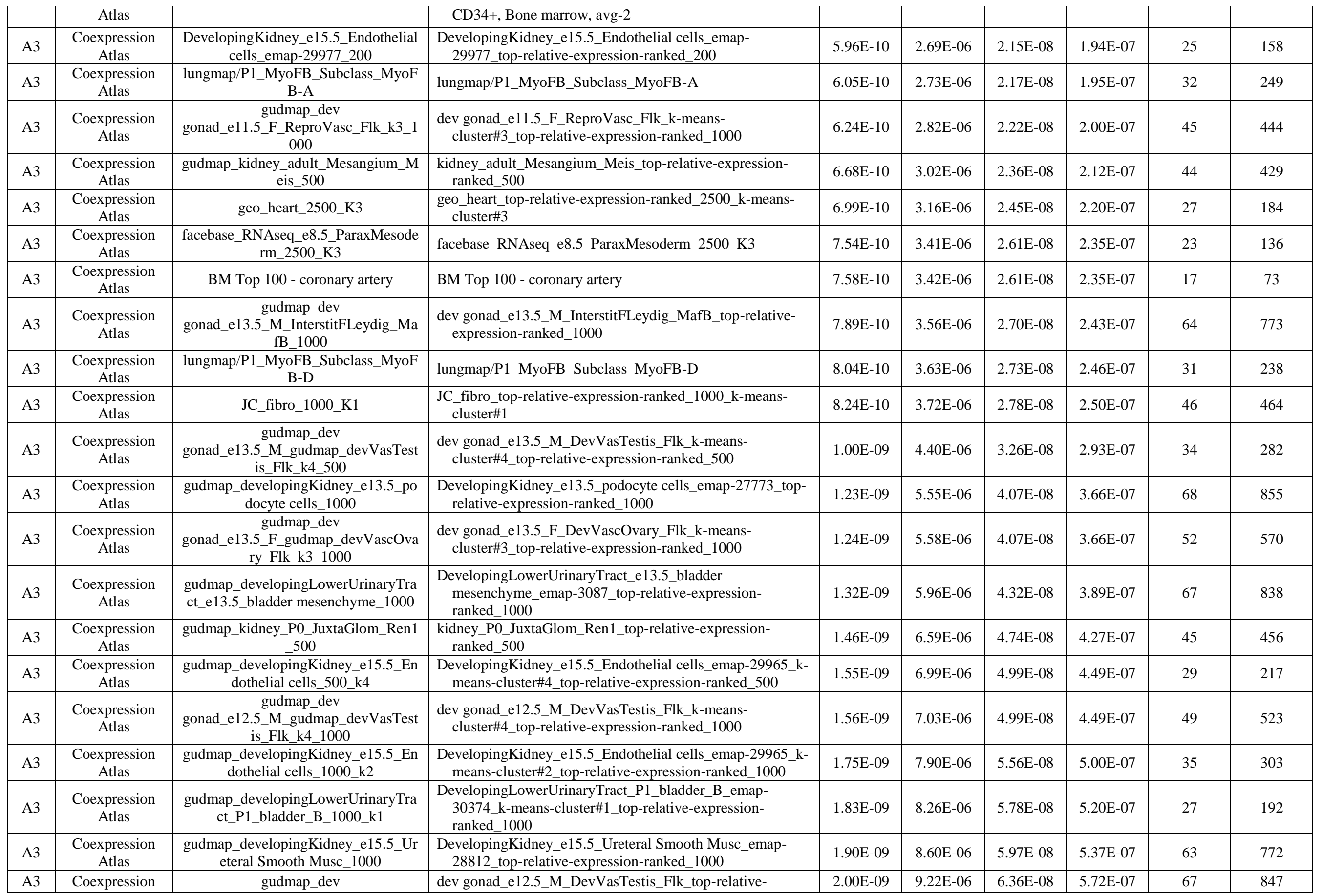




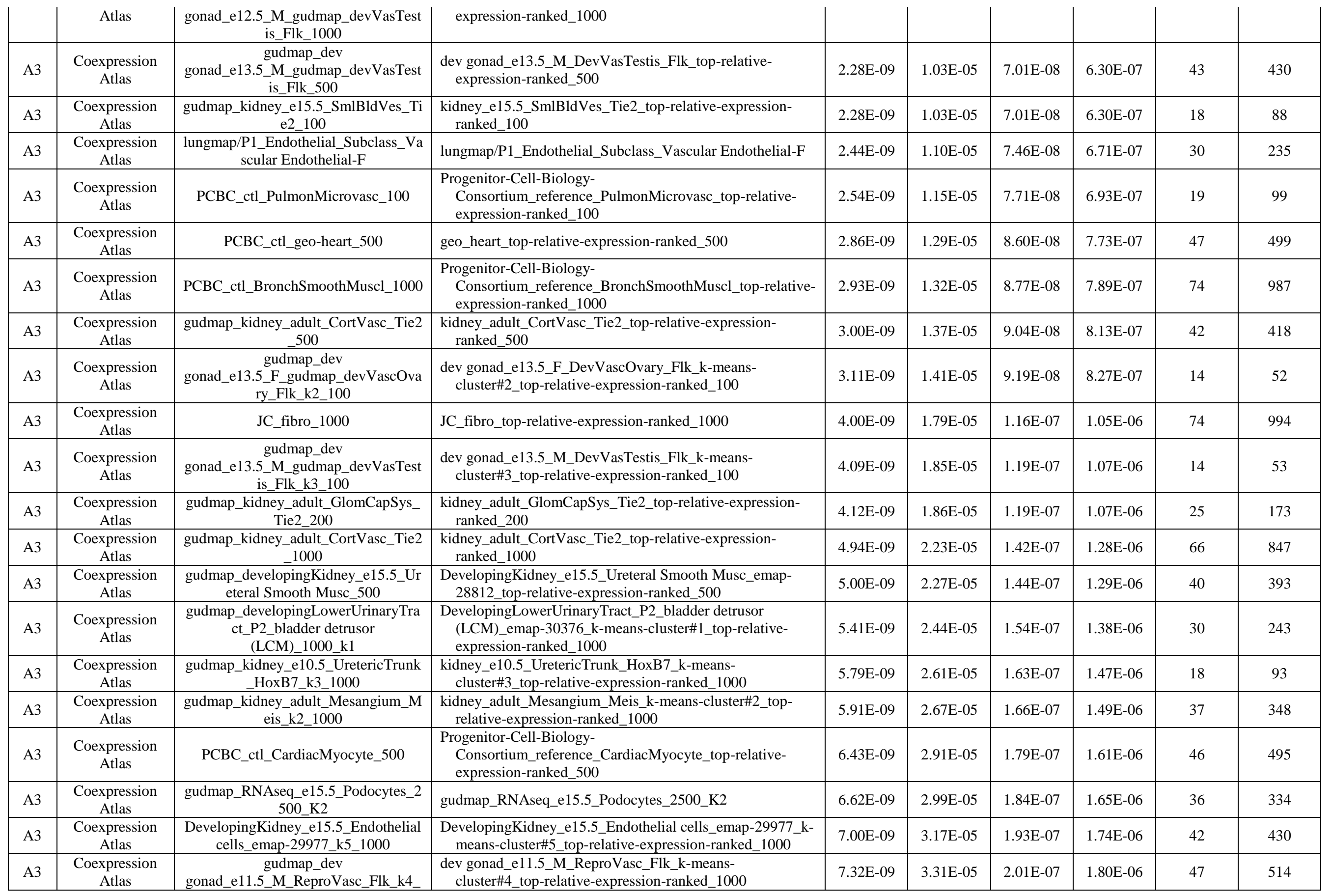




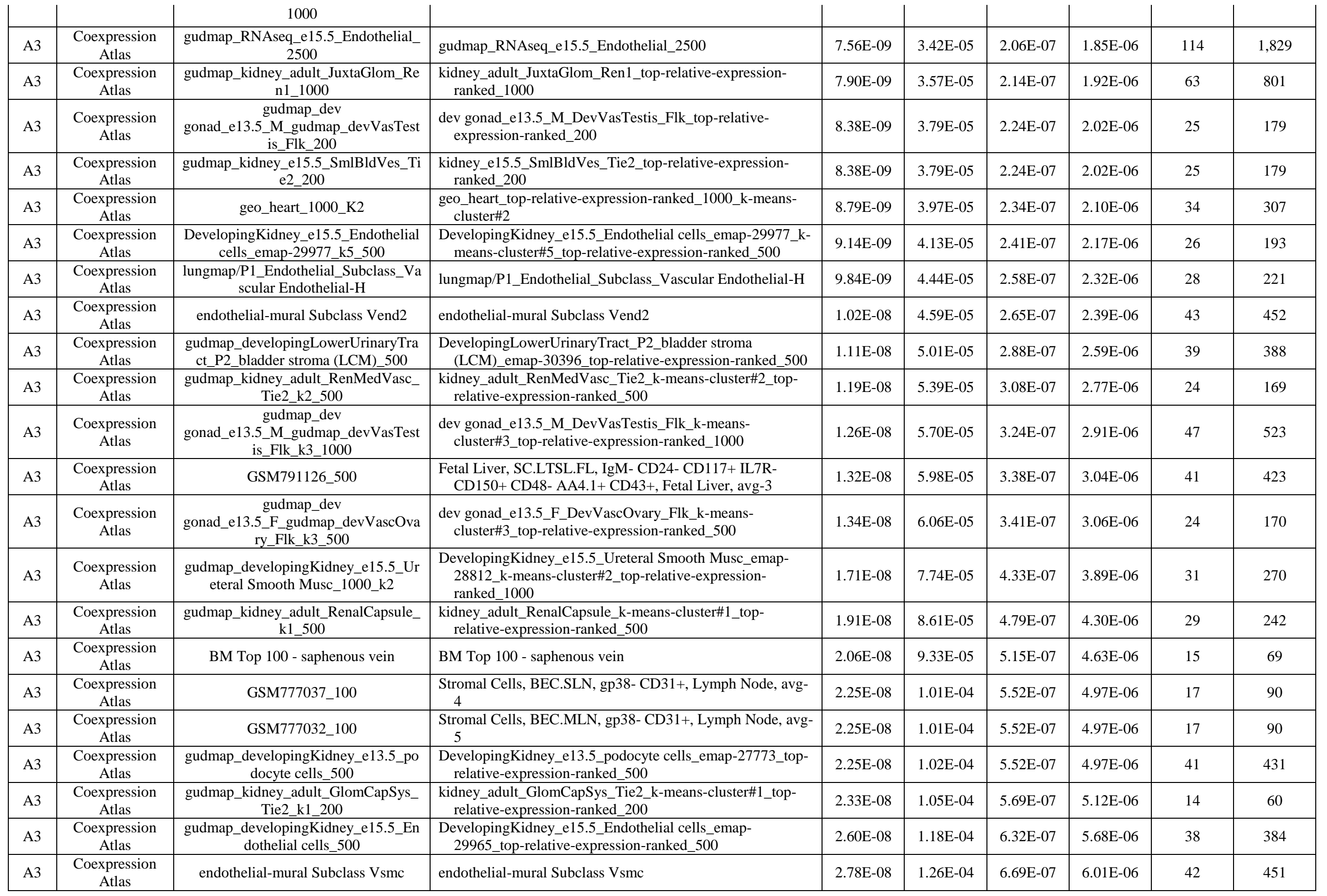




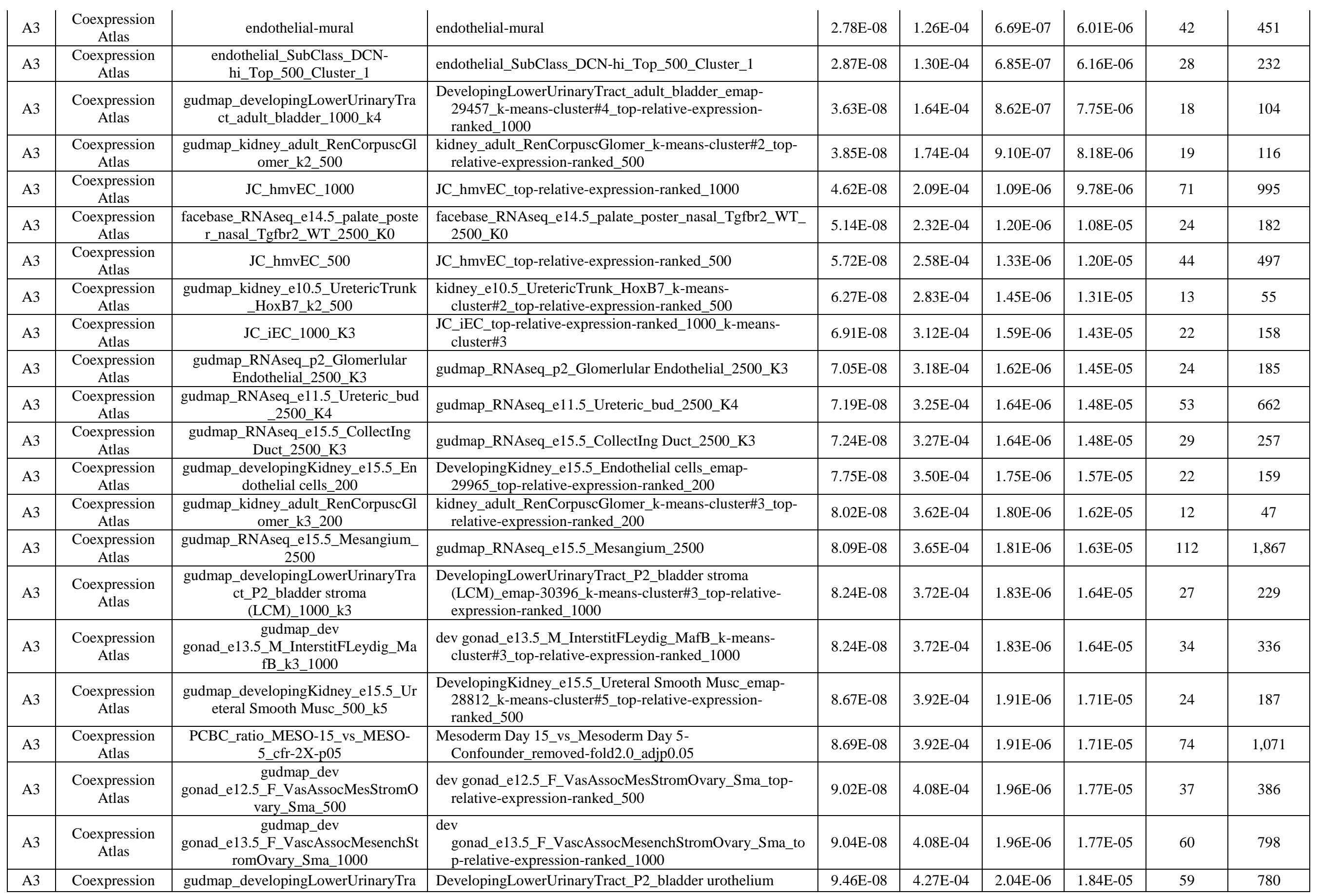




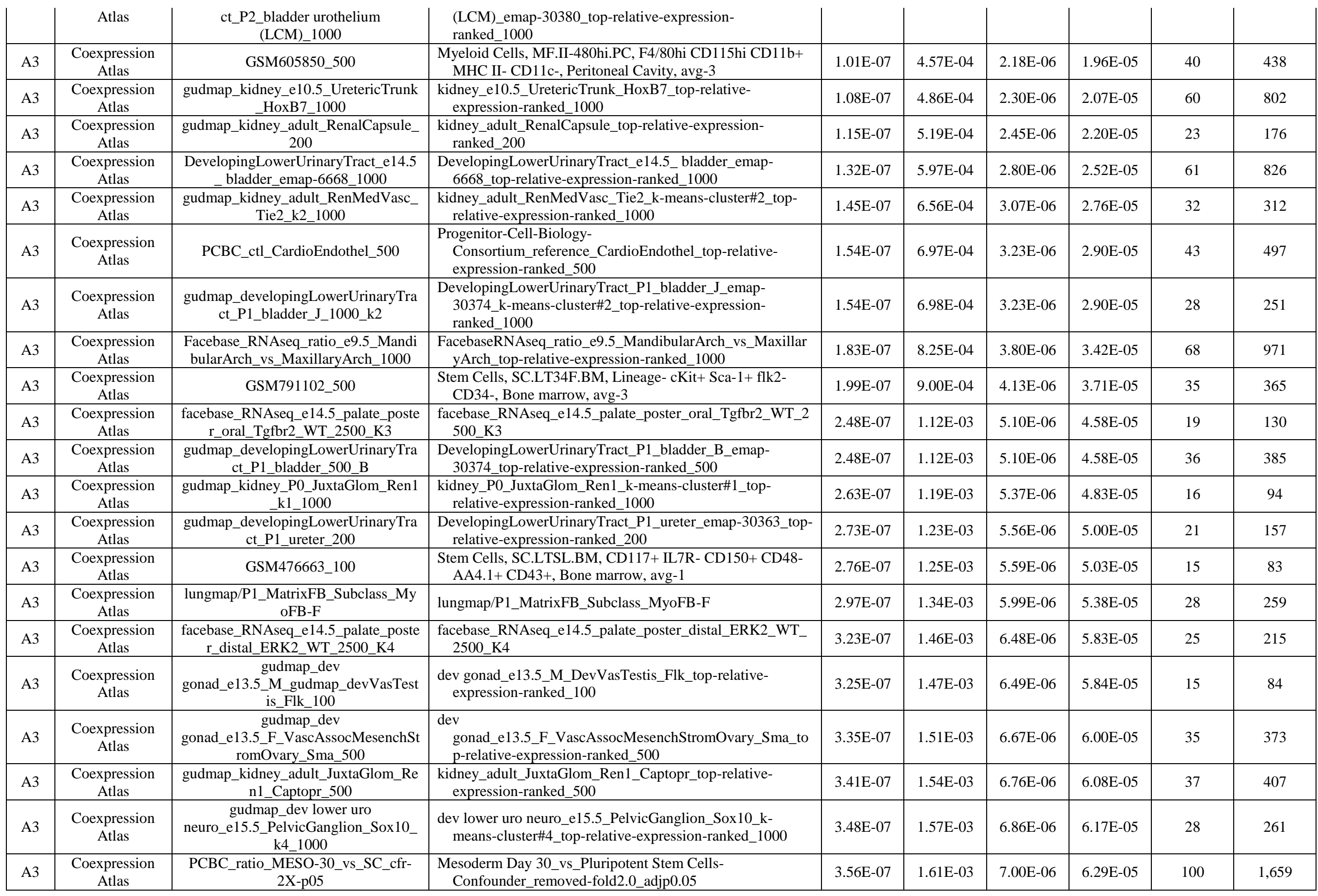




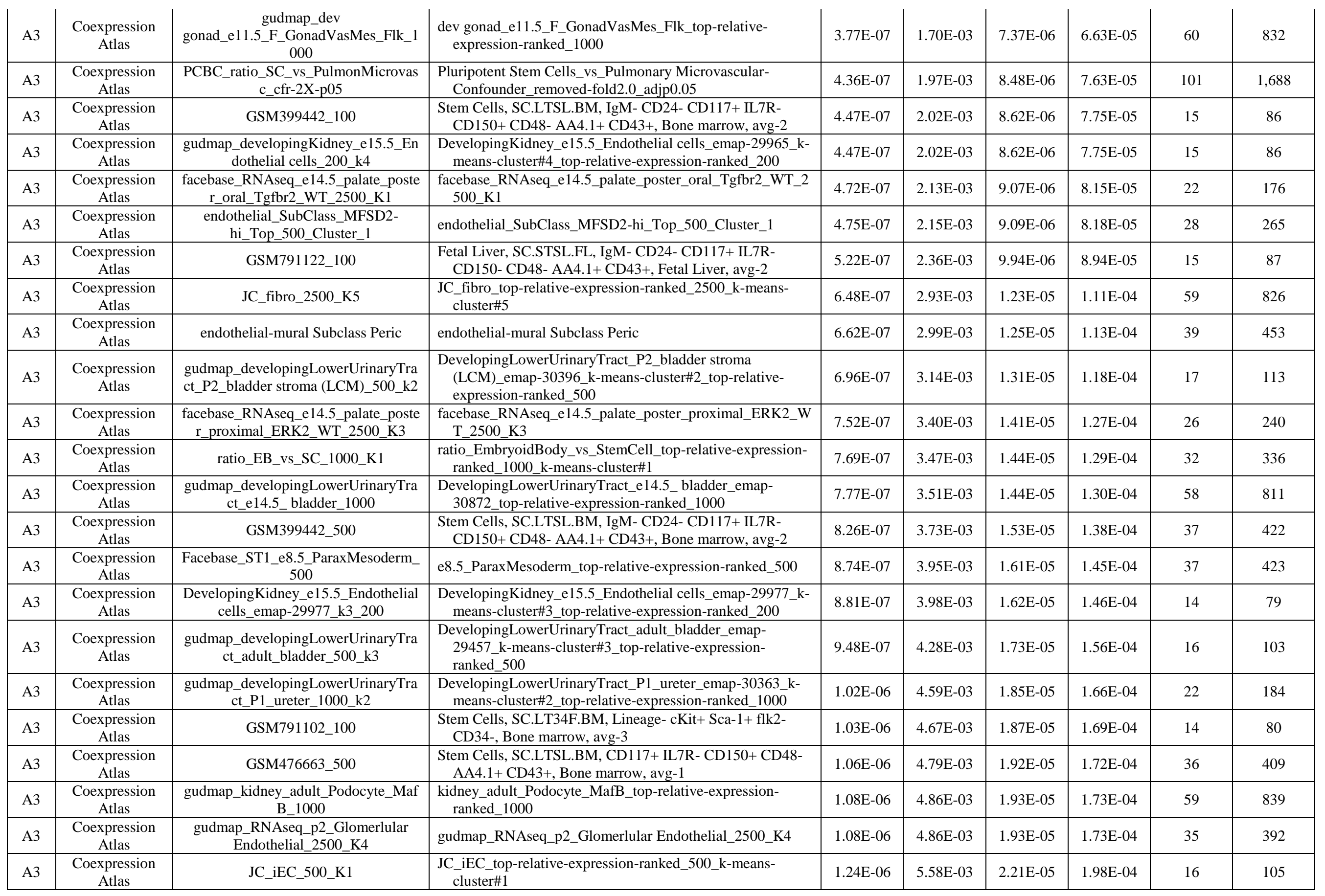




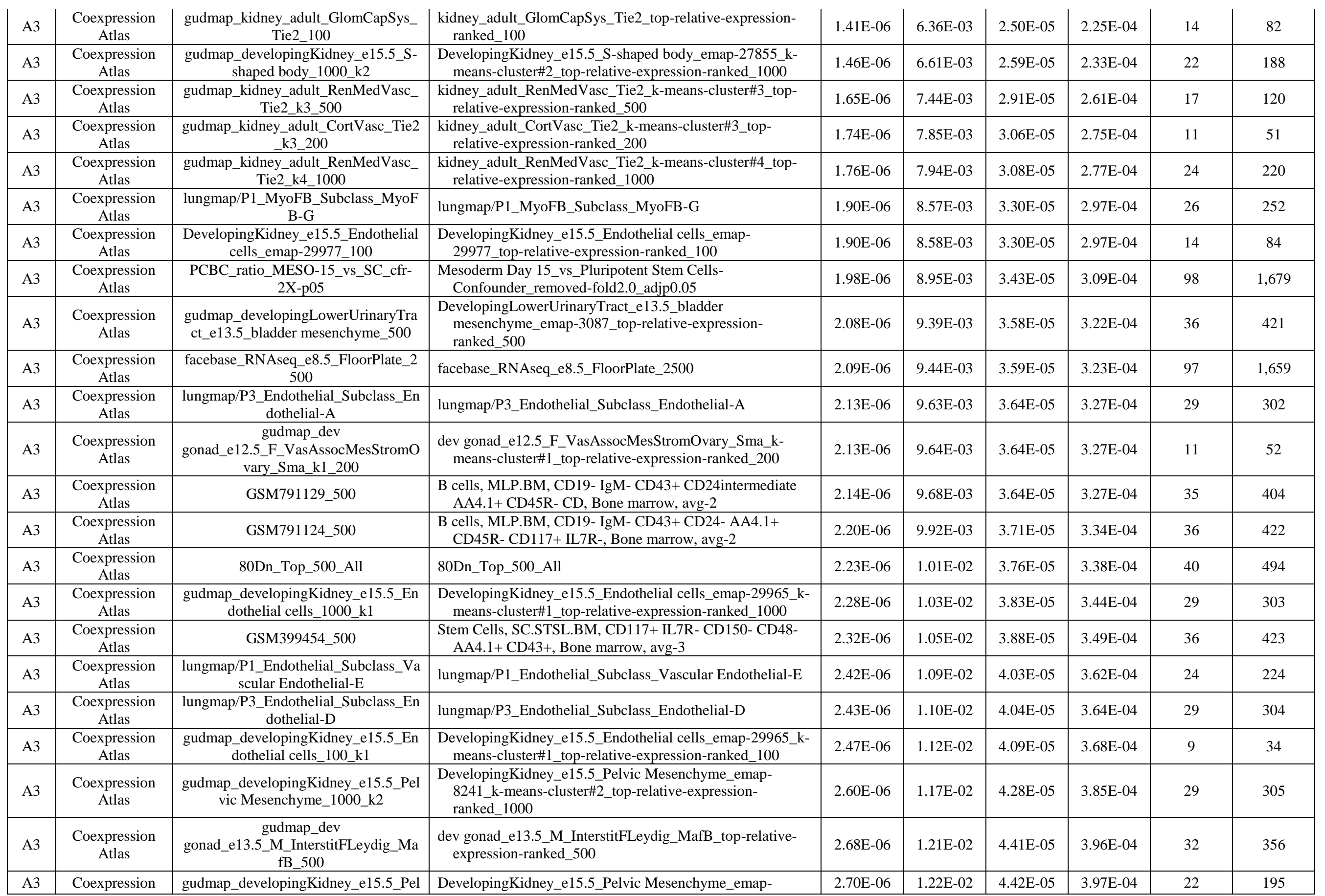




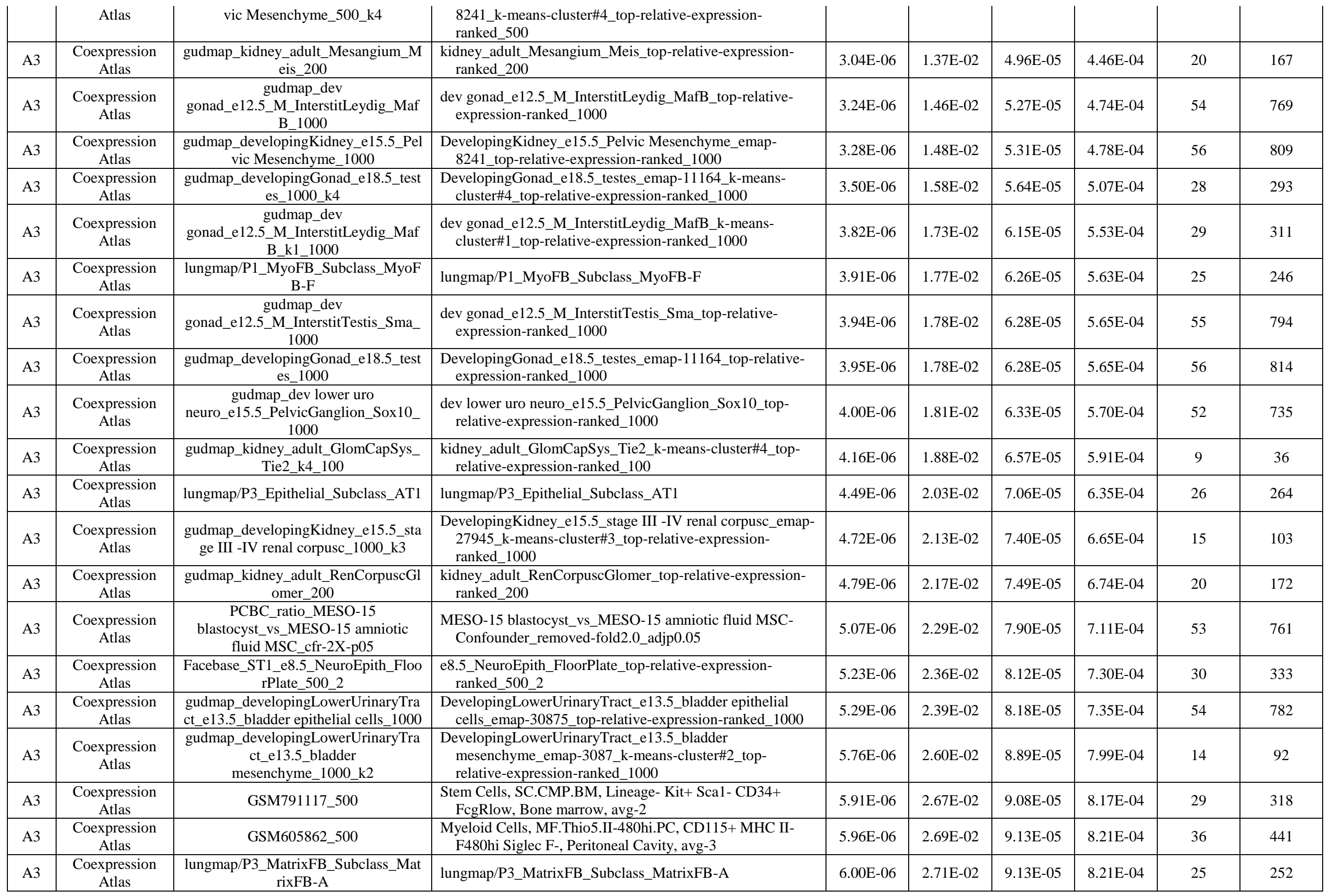




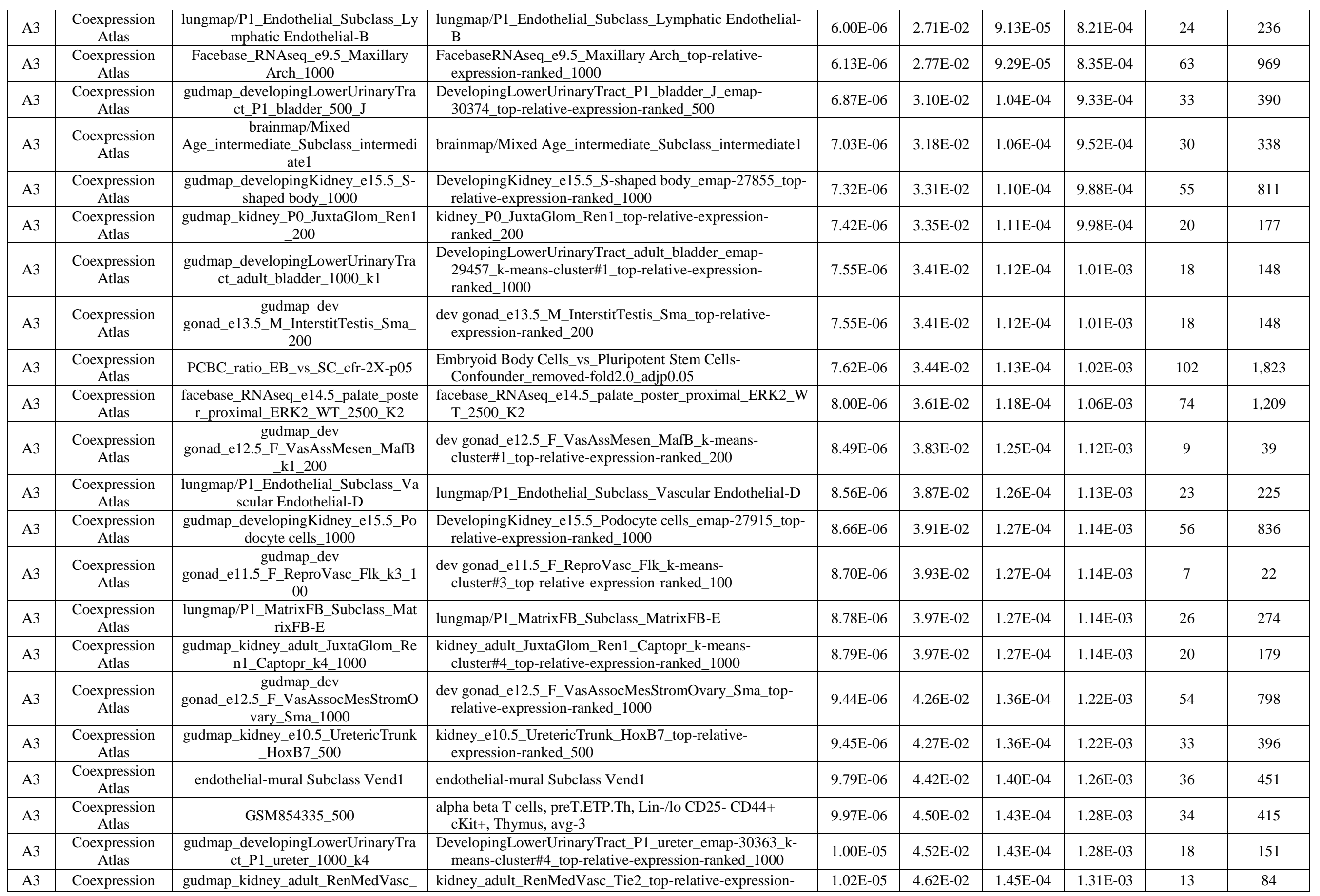




\begin{tabular}{|c|c|c|c|c|c|c|c|c|c|}
\hline & Atlas & Tie2_100 & ranked_100 & & & & & & \\
\hline A3 & Computational & M16395 MODULE_47 & ECM and collagens. & $5.24 \mathrm{E}-09$ & $3.84 \mathrm{E}-06$ & $3.84 \mathrm{E}-06$ & $2.75 \mathrm{E}-05$ & 32 & 225 \\
\hline A3 & Computational & M9982 MODULE_2 & DRG (dorsal root ganglia) genes. & $4.72 \mathrm{E}-07$ & $3.46 \mathrm{E}-04$ & $1.73 \mathrm{E}-04$ & $1.24 \mathrm{E}-03$ & 40 & 384 \\
\hline A3 & Computational & M4051 MODULE_1 & Ovary genes. & $1.90 \mathrm{E}-05$ & $1.39 \mathrm{E}-02$ & $4.64 \mathrm{E}-03$ & $3.33 \mathrm{E}-02$ & 35 & 368 \\
\hline A3 & Drug & D004237 & Diuron & $1.10 \mathrm{E}-27$ & $3.51 \mathrm{E}-23$ & $3.51 \mathrm{E}-23$ & $3.84 \mathrm{E}-22$ & 153 & 1,722 \\
\hline A3 & Drug & $\mathrm{C} 041786$ & palm oil & $8.90 \mathrm{E}-22$ & $2.83 \mathrm{E}-17$ & $1.42 \mathrm{E}-17$ & $1.55 \mathrm{E}-16$ & 138 & 1,675 \\
\hline A3 & Drug & $\mathrm{C} 057693$ & troglitazone & $7.58 \mathrm{E}-12$ & $2.41 \mathrm{E}-07$ & $7.15 \mathrm{E}-08$ & $7.82 \mathrm{E}-07$ & 95 & 1,306 \\
\hline A3 & Drug & D015056 & 1-Methyl-3-isobutylxanthine & $8.98 \mathrm{E}-12$ & $2.86 \mathrm{E}-07$ & $7.15 \mathrm{E}-08$ & $7.82 \mathrm{E}-07$ & 44 & 391 \\
\hline A3 & Drug & $\mathrm{C} 089730$ & rosiglitazone & $1.78 \mathrm{E}-09$ & $5.65 \mathrm{E}-05$ & $9.42 \mathrm{E}-06$ & $1.03 \mathrm{E}-04$ & 100 & 1,552 \\
\hline A3 & Drug & $\mathrm{C} 023035$ & 3,4,5,3',4'-pentachlorobiphenyl & $3.63 \mathrm{E}-09$ & $1.16 \mathrm{E}-04$ & $1.65 \mathrm{E}-05$ & $1.81 \mathrm{E}-04$ & 81 & 1,174 \\
\hline A3 & Drug & C548651 & $\begin{array}{l}\text { 2-(1'H-indolo-3'-carbonyl)thiazole-4-carboxylic acid methyl } \\
\text { ester }\end{array}$ & $7.20 \mathrm{E}-09$ & $2.29 \mathrm{E}-04$ & $2.86 \mathrm{E}-05$ & $3.13 \mathrm{E}-04$ & 61 & 793 \\
\hline A3 & Drug & $\mathrm{C} 015559$ & trimellitic anhydride & $8.20 \mathrm{E}-09$ & $2.61 \mathrm{E}-04$ & $2.90 \mathrm{E}-05$ & $3.17 \mathrm{E}-04$ & 112 & 1,862 \\
\hline A3 & Drug & D004113 & Succimer & $1.63 \mathrm{E}-08$ & $5.18 \mathrm{E}-04$ & $5.18 \mathrm{E}-05$ & $5.67 \mathrm{E}-04$ & 82 & 1,235 \\
\hline A3 & Drug & D007545 & Isoproterenol & $2.10 \mathrm{E}-08$ & $6.68 \mathrm{E}-04$ & $6.07 \mathrm{E}-05$ & $6.65 \mathrm{E}-04$ & 70 & 995 \\
\hline A3 & Drug & C011559 & tributyltin & $7.85 \mathrm{E}-08$ & $2.50 \mathrm{E}-03$ & $2.08 \mathrm{E}-04$ & $2.28 \mathrm{E}-03$ & 32 & 316 \\
\hline A3 & Drug & $\mathrm{C} 023036$ & perfluorooctanoic acid & $1.96 \mathrm{E}-07$ & $6.25 \mathrm{E}-03$ & $4.27 \mathrm{E}-04$ & $4.67 \mathrm{E}-03$ & 97 & 1,638 \\
\hline A3 & Drug & D019324 & beta-Naphthoflavone & $2.01 \mathrm{E}-07$ & $6.40 \mathrm{E}-03$ & $4.27 \mathrm{E}-04$ & $4.67 \mathrm{E}-03$ & 58 & 810 \\
\hline A3 & Drug & D003561 & Cytarabine & $2.16 \mathrm{E}-07$ & $6.88 \mathrm{E}-03$ & $4.30 \mathrm{E}-04$ & $4.71 \mathrm{E}-03$ & 47 & 598 \\
\hline A3 & Drug & C009277 & sodium arsenate & $3.48 \mathrm{E}-07$ & $1.11 \mathrm{E}-02$ & $6.51 \mathrm{E}-04$ & $7.12 \mathrm{E}-03$ & 98 & 1,681 \\
\hline A3 & Drug & CID000082145 & 2-bromopalmitate & $3.86 \mathrm{E}-07$ & $1.23 \mathrm{E}-02$ & $6.83 \mathrm{E}-04$ & $7.47 \mathrm{E}-03$ & 13 & 66 \\
\hline A3 & Drug & 4282_UP & $\begin{array}{l}\text { Hydralazine hydrochloride [304-20-1]; Up 200; 20.4uM; } \\
\text { PC3; HT_HG-U133A }\end{array}$ & $5.28 \mathrm{E}-07$ & $1.68 \mathrm{E}-02$ & $8.84 \mathrm{E}-04$ & $9.67 \mathrm{E}-03$ & 23 & 199 \\
\hline A3 & Drug & C016030 & pantogab & $7.46 \mathrm{E}-07$ & $2.37 \mathrm{E}-02$ & $1.14 \mathrm{E}-03$ & $1.25 \mathrm{E}-02$ & 45 & 586 \\
\hline A3 & Drug & C410127 & PCB 180 & $7.53 \mathrm{E}-07$ & $2.40 \mathrm{E}-02$ & $1.14 \mathrm{E}-03$ & $1.25 \mathrm{E}-02$ & 93 & 1597 \\
\hline A3 & Drug & C510784 & ormosil & $1.27 \mathrm{E}-06$ & $4.03 \mathrm{E}-02$ & $1.83 \mathrm{E}-03$ & $2.00 \mathrm{E}-02$ & 18 & 136 \\
\hline A3 & Drug & C550453 & dioxinodehydroeckol & $1.38 \mathrm{E}-06$ & $4.38 \mathrm{E}-02$ & $1.90 \mathrm{E}-03$ & $2.08 \mathrm{E}-02$ & 6 & 12 \\
\hline A4 & Pubmed & 23020937 & $\begin{array}{l}\text { Range of genetic mutations associated with severe non- } \\
\text { syndromic sporadic intellectual disability: an exome } \\
\text { sequencing study. }\end{array}$ & $1.11 \mathrm{E}-06$ & $2.82 \mathrm{E}-03$ & $2.61 \mathrm{E}-03$ & $2.20 \mathrm{E}-02$ & 2 & 2 \\
\hline A4 & Pubmed & 25162404 & $\begin{array}{l}\text { G protein beta } 5 \text { is targeted to D2-dopamine receptor- } \\
\text { containing biochemical compartments and blocks } \\
\text { dopamine-dependent receptor internalization. }\end{array}$ & $3.32 \mathrm{E}-06$ & $8.45 \mathrm{E}-03$ & $2.61 \mathrm{E}-03$ & $2.20 \mathrm{E}-02$ & 2 & 3 \\
\hline A4 & Pubmed & 16710414 & $\begin{array}{l}\text { The DNA sequence and biological annotation of human } \\
\text { chromosome } 1 .\end{array}$ & $1.31 \mathrm{E}-05$ & 3.33E-02 & $2.61 \mathrm{E}-03$ & $2.20 \mathrm{E}-02$ & 8 & 1,034 \\
\hline A4 & Pubmed & 23020937 & $\begin{array}{l}\text { Range of genetic mutations associated with severe non- } \\
\text { syndromic sporadic intellectual disability: an exome } \\
\text { sequencing study. }\end{array}$ & $1.11 \mathrm{E}-06$ & $2.82 \mathrm{E}-03$ & $2.61 \mathrm{E}-03$ & $2.20 \mathrm{E}-02$ & 2 & 2 \\
\hline
\end{tabular}




\begin{tabular}{|c|c|c|c|c|c|c|c|c|c|}
\hline A4 & Pubmed & 25162404 & $\begin{array}{l}\text { G protein beta } 5 \text { is targeted to } \mathrm{D} 2 \text {-dopamine receptor- } \\
\text { containing biochemical compartments and blocks } \\
\text { dopamine-dependent receptor internalization. }\end{array}$ & 3.32E-06 & $8.45 \mathrm{E}-03$ & 2.61E-03 & $2.20 \mathrm{E}-02$ & 2 & 3 \\
\hline A4 & Pubmed & 16204458 & $\begin{array}{l}\text { Non-EST based prediction of exon skipping and intron } \\
\text { retention events using Pfam information. }\end{array}$ & $3.32 \mathrm{E}-06$ & $8.45 \mathrm{E}-03$ & 2.61E-03 & $2.20 \mathrm{E}-02$ & 2 & 3 \\
\hline A4 & Pubmed & 16710414 & $\begin{array}{l}\text { The DNA sequence and biological annotation of human } \\
\text { chromosome } 1 .\end{array}$ & $1.31 \mathrm{E}-05$ & $3.33 \mathrm{E}-02$ & 2.61E-03 & $2.20 \mathrm{E}-02$ & 8 & 1,034 \\
\hline A5 & $\begin{array}{l}\text { GO: Cellular } \\
\text { Component }\end{array}$ & GO:0044420 & extracellular matrix component & $1.41 \mathrm{E}-05$ & $2.25 \mathrm{E}-03$ & $1.38 \mathrm{E}-03$ & 7.79E-03 & 5 & 141 \\
\hline A5 & $\begin{array}{l}\text { GO: Cellular } \\
\text { Component }\end{array}$ & GO:0005578 & proteinaceous extracellular matrix & $1.72 \mathrm{E}-05$ & $2.75 \mathrm{E}-03$ & $1.38 \mathrm{E}-03$ & 7.79E-03 & 7 & 379 \\
\hline A5 & $\begin{array}{l}\text { GO: Cellular } \\
\text { Component }\end{array}$ & GO:0031012 & extracellular matrix & $4.73 \mathrm{E}-05$ & $7.57 \mathrm{E}-03$ & $2.52 \mathrm{E}-03$ & $1.43 \mathrm{E}-02$ & 7 & 444 \\
\hline A5 & $\begin{array}{l}\text { Human } \\
\text { Phenotype }\end{array}$ & HP:0005622 & Broad long bones & $3.10 \mathrm{E}-06$ & 2.37E-03 & $2.37 \mathrm{E}-03$ & $1.71 \mathrm{E}-02$ & 3 & 10 \\
\hline A5 & Pubmed & 19578796 & $\begin{array}{l}\text { Association of genetic variants with chronic kidney disease } \\
\text { in individuals with different lipid profiles. }\end{array}$ & $1.84 \mathrm{E}-07$ & $1.19 \mathrm{E}-03$ & $8.21 \mathrm{E}-04$ & $7.68 \mathrm{E}-03$ & 5 & 152 \\
\hline A5 & Pubmed & 26915435 & $\begin{array}{l}\text { The anti-oxidative transcription factor Nuclear factor E2 } \\
\text { related factor-2 (Nrf2) counteracts TGF-?1 mediated } \\
\text { growth inhibition of pancreatic ductal epithelial cells - } \\
\text { Nrf2 as determinant of pro-tumorigenic functions of TGF- } \\
\text { ?1. }\end{array}$ & $6.80 \mathrm{E}-07$ & $4.38 \mathrm{E}-03$ & $8.21 \mathrm{E}-04$ & $7.68 \mathrm{E}-03$ & 2 & 2 \\
\hline A5 & Pubmed & 26226105 & $\begin{array}{l}\text { The Crosstalk between Nrf2 and TGF-?1 in the Epithelial- } \\
\text { Mesenchymal Transition of Pancreatic Duct Epithelial } \\
\text { Cells. }\end{array}$ & $6.80 \mathrm{E}-07$ & $4.38 \mathrm{E}-03$ & $8.21 \mathrm{E}-04$ & $7.68 \mathrm{E}-03$ & 2 & 2 \\
\hline A5 & Pubmed & 19389097 & $\begin{array}{l}\text { Intense pulsed light effects on the expression of extracellular } \\
\text { matrix proteins and transforming growth factor beta- } 1 \text { in } \\
\text { skin dermal fibroblasts cultured within contracted } \\
\text { collagen lattices. }\end{array}$ & $6.80 \mathrm{E}-07$ & $4.38 \mathrm{E}-03$ & $8.21 \mathrm{E}-04$ & $7.68 \mathrm{E}-03$ & 2 & 2 \\
\hline A5 & Pubmed & 17146610 & $\begin{array}{l}\text { Expression of decorin and collagens I and III in different } \\
\text { layers of human skin in vivo: a laser capture } \\
\text { microdissection study. }\end{array}$ & $2.04 \mathrm{E}-06$ & $1.31 \mathrm{E}-02$ & $8.21 \mathrm{E}-04$ & $7.68 \mathrm{E}-03$ & 2 & 3 \\
\hline A5 & Pubmed & 27997896 & $\begin{array}{l}\text { Elevated THBS2, COL1A2, and SPP1 Expression Levels as } \\
\text { Predictors of Gastric Cancer Prognosis. }\end{array}$ & $2.04 \mathrm{E}-06$ & $1.31 \mathrm{E}-02$ & $8.21 \mathrm{E}-04$ & 7.68E-03 & 2 & 3 \\
\hline A5 & Pubmed & 21858035 & $\begin{array}{l}\text { The role of serum biomarkers in predicting fibrosis } \\
\text { progression in pediatric and adult hepatitis } C \text { virus chronic } \\
\text { infection. }\end{array}$ & 2.04E-06 & $1.31 \mathrm{E}-02$ & $8.21 \mathrm{E}-04$ & $7.68 \mathrm{E}-03$ & 2 & 3 \\
\hline A5 & Pubmed & 25851604 & $\begin{array}{l}\text { The human } 18 \mathrm{~S} \text { rRNA base methyltransferases DIMT1L and } \\
\text { WBSCR22-TRMT112 but not rRNA modification are } \\
\text { required for ribosome biogenesis. }\end{array}$ & $2.04 \mathrm{E}-06$ & $1.31 \mathrm{E}-02$ & $8.21 \mathrm{E}-04$ & $7.68 \mathrm{E}-03$ & 2 & 3 \\
\hline A5 & Pubmed & 22379029 & $\begin{array}{l}\text { TGF-?-mediated downregulation of microRNA-196a } \\
\text { contributes to the constitutive upregulated type I collagen } \\
\text { expression in scleroderma dermal fibroblasts. }\end{array}$ & $2.04 \mathrm{E}-06$ & $1.31 \mathrm{E}-02$ & $8.21 \mathrm{E}-04$ & $7.68 \mathrm{E}-03$ & 2 & 3 \\
\hline A5 & Pubmed & 24641356 & $\begin{array}{l}\text { In Crohn's disease fibrosis-reduced expression of the miR-29 } \\
\text { family enhances collagen expression in intestinal } \\
\text { fibroblasts. }\end{array}$ & 2.04E-06 & $1.31 \mathrm{E}-02$ & $8.21 \mathrm{E}-04$ & $7.68 \mathrm{E}-03$ & 2 & 3 \\
\hline A5 & Pubmed & 20704113 & $\begin{array}{l}\text { Effects of antisense transforming growth factor-beta1 gene } \\
\text { transfer on the biological activities of tendon sheath } \\
\text { fibroblasts. }\end{array}$ & 2.04E-06 & $1.31 \mathrm{E}-02$ & $8.21 \mathrm{E}-04$ & $7.68 \mathrm{E}-03$ & 2 & 3 \\
\hline
\end{tabular}




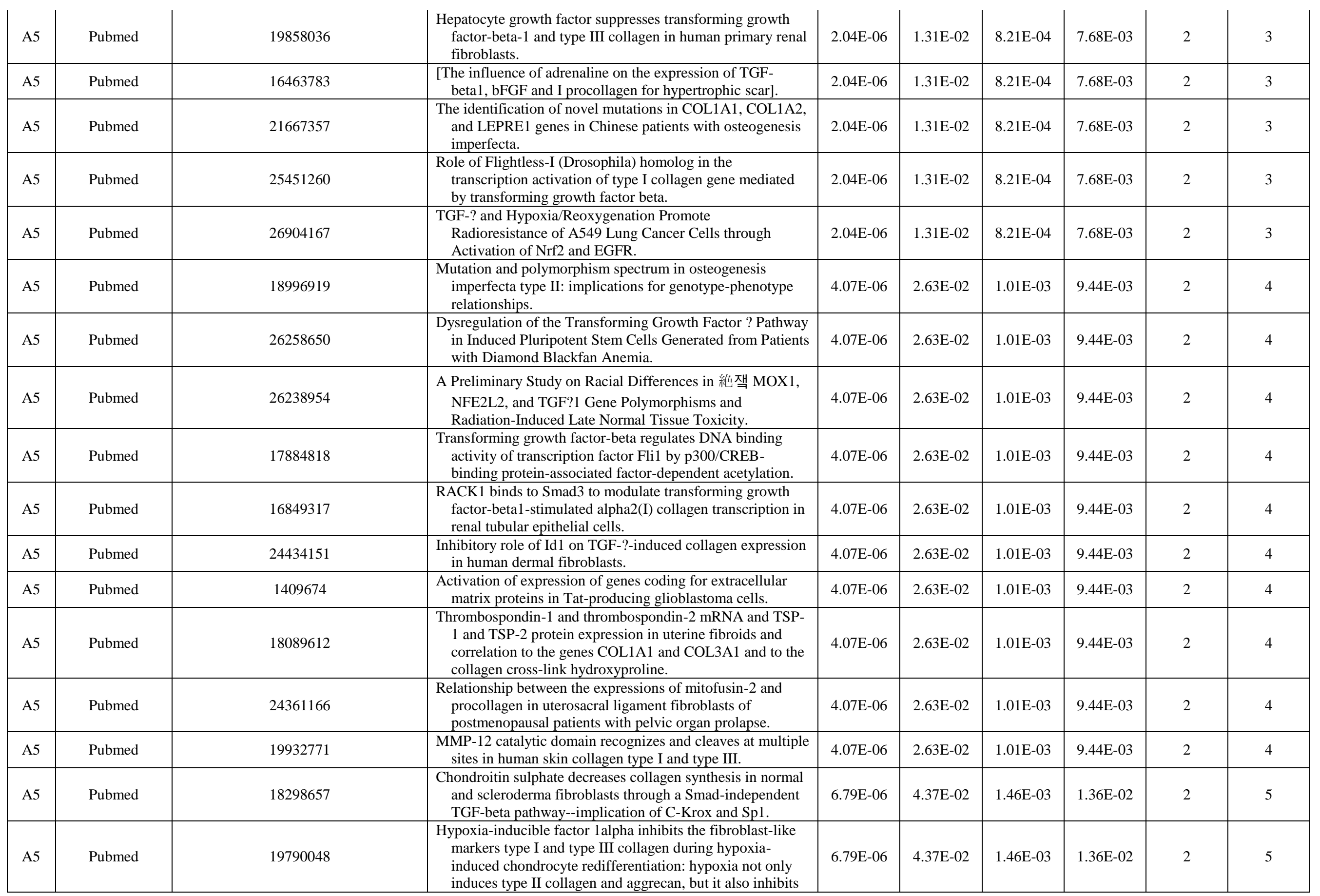




\begin{tabular}{|c|c|c|c|c|c|c|c|c|c|}
\hline & & & $\begin{array}{l}\text { type I and type III collagen in the hypoxia-inducible factor } \\
\text { 1alpha-dependent redifferentiation of chondrocytes. }\end{array}$ & & & & & & \\
\hline A5 & Pubmed & 15365990 & $\begin{array}{l}\text { Stability related bias in residues replacing glycines within } \\
\text { the collagen triple helix (Gly-Xaa-Yaa) in inherited } \\
\text { connective tissue disorders. }\end{array}$ & $6.79 \mathrm{E}-06$ & 4.37E-02 & $1.46 \mathrm{E}-03$ & $1.36 \mathrm{E}-02$ & 2 & 5 \\
\hline A5 & Pubmed & 9675033 & $\begin{array}{l}\text { Decorin core protein fragment Leu } 155-\text { Val260 interacts with } \\
\text { TGF-beta but does not compete for decorin binding to } \\
\text { type I collagen. }\end{array}$ & $6.79 \mathrm{E}-06$ & 4.37E-02 & $1.46 \mathrm{E}-03$ & $1.36 \mathrm{E}-02$ & 2 & 5 \\
\hline A5 & Cytoband & $2 q 31$ & $2 q 31$ & $1.22 \mathrm{E}-04$ & $4.87 \mathrm{E}-03$ & $4.87 \mathrm{E}-03$ & $2.08 \mathrm{E}-02$ & 2 & 13 \\
\hline A5 & Computational & M12987 GNF2_CDH11 & Neighborhood of CDH11 & $2.04 \mathrm{E}-05$ & $3.24 \mathrm{E}-03$ & $3.24 \mathrm{E}-03$ & $1.83 \mathrm{E}-02$ & 3 & 25 \\
\hline A5 & Computational & M11282 GNF2_PTX3 & Neighborhood of PTX3 & $6.23 \mathrm{E}-05$ & $9.90 \mathrm{E}-03$ & $4.95 \mathrm{E}-03$ & $2.80 \mathrm{E}-02$ & 3 & 36 \\
\hline A5 & Drug & C502971 & 2-phenyl-4-(3-pyridin-2-yl-1H-pyrazol-4-yl)pyridine & $1.47 \mathrm{E}-07$ & $1.14 \mathrm{E}-03$ & $1.14 \mathrm{E}-03$ & $1.08 \mathrm{E}-02$ & 3 & 6 \\
\hline A6 & Pubmed & 18303054 & $\begin{array}{l}\text { IKAP localizes to membrane ruffles with filamin } \mathrm{A} \text { and } \\
\text { regulates actin cytoskeleton organization and cell } \\
\text { migration. }\end{array}$ & $3.68 \mathrm{E}-05$ & $4.58 \mathrm{E}-02$ & $1.78 \mathrm{E}-03$ & $1.37 \mathrm{E}-02$ & 2 & 17 \\
\hline A7 & $\begin{array}{l}\text { GO: Cellular } \\
\text { Component }\end{array}$ & GO:0060203 & Clathrin-sculpted glutamate transport vesicle membrane & $6.84 \mathrm{E}-05$ & $1.59 \mathrm{E}-02$ & 7.93E-03 & 4.78E-02 & 2 & 4 \\
\hline A7 & $\begin{array}{l}\text { GO: Cellular } \\
\text { Component }\end{array}$ & GO:0060199 & Clathrin-sculpted glutamate transport vesicle & $6.84 \mathrm{E}-05$ & $1.59 \mathrm{E}-02$ & 7.93E-03 & $4.78 \mathrm{E}-02$ & 2 & 4 \\
\hline A7 & Pubmed & 24728074 & $\begin{array}{l}\text { Enhanced prediction of Src homology } 2 \text { (SH2) domain } \\
\text { binding potentials using a fluorescence polarization- } \\
\text { derived c-Met, c-Kit, ErbB, and androgen receptor } \\
\text { interactome. }\end{array}$ & $1.59 \mathrm{E}-06$ & $7.91 \mathrm{E}-03$ & $4.21 \mathrm{E}-03$ & $3.82 \mathrm{E}-02$ & 4 & 63 \\
\hline A7 & Pubmed & 23799130 & $\begin{array}{l}\text { Expression of ST3GAL4 leads to SLe(x) expression and } \\
\text { induces c-Met activation and an invasive phenotype in } \\
\text { gastric carcinoma cells. }\end{array}$ & $5.20 \mathrm{E}-06$ & $2.58 \mathrm{E}-02$ & $4.21 \mathrm{E}-03$ & $3.82 \mathrm{E}-02$ & 2 & 3 \\
\hline A7 & Pubmed & 25612003 & $\begin{array}{l}\text { Pathologic Regulation of Collagen I by an Aberrant Protein } \\
\text { Phosphatase 2A/Histone Deacetylase C4/MicroRNA-29 } \\
\text { Signal Axis in Idiopathic Pulmonary Fibrosis Fibroblasts. }\end{array}$ & $5.20 \mathrm{E}-06$ & $2.58 \mathrm{E}-02$ & $4.21 \mathrm{E}-03$ & $3.82 \mathrm{E}-02$ & 2 & 3 \\
\hline A7 & Pubmed & 19543515 & $\begin{array}{l}\text { Differential methylation pattern of ID4, SFRP1, and SHP1 } \\
\text { between acute myeloid leukemia and chronic myeloid } \\
\text { leukemia. }\end{array}$ & $5.20 \mathrm{E}-06$ & $2.58 \mathrm{E}-02$ & $4.21 \mathrm{E}-03$ & $3.82 \mathrm{E}-02$ & 2 & 3 \\
\hline A8 & $\begin{array}{l}\text { GO: Cellular } \\
\text { Component }\end{array}$ & GO:0060203 & Clathrin-sculpted glutamate transport vesicle membrane & $6.84 \mathrm{E}-05$ & $1.59 \mathrm{E}-02$ & 7.93E-03 & 4.78E-02 & 2 & 4 \\
\hline A8 & $\begin{array}{l}\text { GO: Cellular } \\
\text { Component }\end{array}$ & GO:0060199 & Clathrin-sculpted glutamate transport vesicle & $6.84 \mathrm{E}-05$ & $1.59 \mathrm{E}-02$ & 7.93E-03 & $4.78 \mathrm{E}-02$ & 2 & 4 \\
\hline A8 & Pubmed & 24728074 & $\begin{array}{l}\text { Enhanced prediction of Src homology } 2 \text { (SH2) domain } \\
\text { binding potentials using a fluorescence polarization- } \\
\text { derived c-Met, c-Kit, ErbB, and androgen receptor } \\
\text { interactome. }\end{array}$ & $1.59 \mathrm{E}-06$ & 7.91E-03 & $4.21 \mathrm{E}-03$ & $3.82 \mathrm{E}-02$ & 4 & 63 \\
\hline A8 & Pubmed & 25612003 & $\begin{array}{l}\text { Pathologic Regulation of Collagen I by an Aberrant Protein } \\
\text { Phosphatase 2A/Histone Deacetylase C4/MicroRNA-29 } \\
\text { Signal Axis in Idiopathic Pulmonary Fibrosis Fibroblasts. }\end{array}$ & $5.20 \mathrm{E}-06$ & $2.58 \mathrm{E}-02$ & $4.21 \mathrm{E}-03$ & $3.82 \mathrm{E}-02$ & 2 & 3 \\
\hline A8 & Pubmed & 19543515 & Differential methylation pattern of ID4, SFRP1, and SHP1 & $5.20 \mathrm{E}-06$ & $2.58 \mathrm{E}-02$ & $4.21 \mathrm{E}-03$ & $3.82 \mathrm{E}-02$ & 2 & 3 \\
\hline
\end{tabular}


between acute myeloid leukemia and chronic myeloid leukemia.

\begin{tabular}{|c|c|c|c|c|c|c|c|c|c|}
\hline & & & $\begin{array}{l}\text { between acute myeloid leukemia and chronic myeloid } \\
\text { leukemia. }\end{array}$ & & & & & & \\
\hline B3 & Pubmed & 20333644 & $\begin{array}{l}\text { Beta1 integrins mediate cell proliferation in three- } \\
\text { dimensional cultures by regulating expression of the sonic } \\
\text { hedgehog effector protein, GLI1. }\end{array}$ & $1.69 \mathrm{E}-06$ & $8.85 \mathrm{E}-03$ & $2.21 \mathrm{E}-03$ & $2.02 \mathrm{E}-02$ & 2 & 2 \\
\hline B3 & Pubmed & 21639857 & $\begin{array}{l}\text { Effect of dexamethasone on human osteoblasts in culture: } \\
\text { involvement of ?1 integrin and integrin-linked kinase. }\end{array}$ & $1.69 \mathrm{E}-06$ & $8.85 \mathrm{E}-03$ & $2.21 \mathrm{E}-03$ & $2.02 \mathrm{E}-02$ & 2 & 2 \\
\hline B3 & Pubmed & 12020426 & $\begin{array}{l}\text { Ionizing radiation induces up-regulation of functional beta1- } \\
\text { integrin in human lung tumor cell lines in vitro. }\end{array}$ & 1.69E-06 & $8.85 \mathrm{E}-03$ & $2.21 \mathrm{E}-03$ & $2.02 \mathrm{E}-02$ & 2 & 2 \\
\hline B3 & Pubmed & 15304053 & $\begin{array}{l}\text { The regulation of integrin-linked kinase in human platelets: } \\
\text { evidence for involvement in the regulation of integrin } \\
\text { alpha } 2 \text { beta } 1 \text {. }\end{array}$ & $5.05 \mathrm{E}-06$ & $2.65 \mathrm{E}-02$ & $2.94 \mathrm{E}-03$ & 2.69E-02 & 2 & 3 \\
\hline B3 & Pubmed & 22345562 & $\begin{array}{l}\text { Insulin-like growth factor-binding protein 2-driven glioma } \\
\text { progression is prevented by blocking a clinically } \\
\text { significant integrin, integrin-linked kinase, and NF-?B } \\
\text { network. }\end{array}$ & $5.05 \mathrm{E}-06$ & $2.65 \mathrm{E}-02$ & $2.94 \mathrm{E}-03$ & $2.69 \mathrm{E}-02$ & 2 & 3 \\
\hline B3 & Pubmed & 26693891 & $\begin{array}{l}\text { Twist induces epithelial-mesenchymal transition and cell } \\
\text { motility in breast cancer via ITGB1-FAK/ILK signaling } \\
\text { axis and its associated downstream network. }\end{array}$ & $5.05 \mathrm{E}-06$ & $2.65 \mathrm{E}-02$ & $2.94 \mathrm{E}-03$ & $2.69 \mathrm{E}-02$ & 2 & 3 \\
\hline B3 & Pubmed & 28188308 & $\begin{array}{l}\text { Tetraspanin } 8 \text { is a novel regulator of ILK-driven ?1 integrin } \\
\text { adhesion and signaling in invasive melanoma cells. }\end{array}$ & $5.05 \mathrm{E}-06$ & $2.65 \mathrm{E}-02$ & $2.94 \mathrm{E}-03$ & 2.69E-02 & 2 & 3 \\
\hline B3 & Computational & M9347 MODULE_176 & Signaling. & $1.82 \mathrm{E}-06$ & $4.17 \mathrm{E}-04$ & 4.17E-04 & $2.51 \mathrm{E}-03$ & 8 & 230 \\
\hline B3 & Computational & M16447 MODULE_112 & Genes in the cancer module 112. & $4.54 \mathrm{E}-06$ & $1.04 \mathrm{E}-03$ & $5.20 \mathrm{E}-04$ & $3.12 \mathrm{E}-03$ & 8 & 260 \\
\hline B3 & Computational & M9982 MODULE_2 & DRG (dorsal root ganglia) genes. & $7.61 \mathrm{E}-05$ & $1.74 \mathrm{E}-02$ & $5.81 \mathrm{E}-03$ & $3.49 \mathrm{E}-02$ & 8 & 384 \\
\hline B4 & Pubmed & 15749123 & $\begin{array}{l}\text { Ethanol potentiates HIV-1 gp120-induced apoptosis in } \\
\text { human neurons via both the death receptor and NMDA } \\
\text { receptor pathways. }\end{array}$ & $2.46 \mathrm{E}-05$ & $1.78 \mathrm{E}-02$ & $2.51 \mathrm{E}-03$ & 1.79E-02 & 2 & 14 \\
\hline B5 & Pubmed & 10343102 & $\begin{array}{l}\text { Assignment of a human cold shock domain protein A } \\
\text { intronless pseudogene (CSDAP1) to human chromosome } \\
16 \text { band p11.2 by in situ hybridization. }\end{array}$ & $1.10 \mathrm{E}-07$ & $1.36 \mathrm{E}-04$ & $1.36 \mathrm{E}-04$ & $1.05 \mathrm{E}-03$ & 2 & 2 \\
\hline B5 & Gene Family & 1010 & Cytochrome P450 family 20 & $6.60 \mathrm{E}-04$ & 7.92E-03 & $3.96 \mathrm{E}-03$ & $1.23 \mathrm{E}-02$ & 1 & 1 \\
\hline B5 & Gene Family & 1015 & Cytochrome P450 family 39 & $6.60 \mathrm{E}-04$ & 7.92E-03 & $3.96 \mathrm{E}-03$ & $1.23 \mathrm{E}-02$ & 1 & 1 \\
\hline B5 & Gene Family & 106 & Y box binding proteins & $1.98 \mathrm{E}-03$ & 2.37E-02 & 7.91E-03 & $2.46 \mathrm{E}-02$ & 1 & 3 \\
\hline B5 & Gene Family & 1127 & Phospholipid phosphatase related & $3.29 \mathrm{E}-03$ & $3.95 \mathrm{E}-02$ & $9.88 \mathrm{E}-03$ & $3.07 \mathrm{E}-02$ & 1 & 5 \\
\hline B6 & $\begin{array}{l}\text { GO: Cellular } \\
\text { Component }\end{array}$ & GO:0030016 & Myofibril & $1.07 \mathrm{E}-08$ & $6.18 \mathrm{E}-07$ & $4.21 \mathrm{E}-07$ & $1.96 \mathrm{E}-06$ & 5 & 220 \\
\hline B6 & $\begin{array}{c}\text { GO: Cellular } \\
\text { Component }\end{array}$ & GO:0043292 & Contractile fiber & $1.45 \mathrm{E}-08$ & $8.42 \mathrm{E}-07$ & $4.21 \mathrm{E}-07$ & $1.96 \mathrm{E}-06$ & 5 & 234 \\
\hline B6 & GO: Cellular & GO:0030017 & Sarcomere & $7.65 \mathrm{E}-07$ & $4.44 \mathrm{E}-05$ & $1.48 \mathrm{E}-05$ & $6.88 \mathrm{E}-05$ & 4 & 198 \\
\hline
\end{tabular}




\begin{tabular}{|c|c|c|c|c|c|c|c|c|c|}
\hline & Component & & & & & & & & \\
\hline B6 & $\begin{array}{l}\text { GO: Cellular } \\
\text { Component }\end{array}$ & GO:0044449 & Contractile fiber part & $1.06 \mathrm{E}-06$ & $6.17 \mathrm{E}-05$ & $1.54 \mathrm{E}-05$ & 7.17E-05 & 4 & 215 \\
\hline B6 & $\begin{array}{l}\text { GO: Cellular } \\
\text { Component }\end{array}$ & GO:0032982 & Myosin filament & $3.88 \mathrm{E}-05$ & $2.25 \mathrm{E}-03$ & $4.50 \mathrm{E}-04$ & 2.09E-03 & 2 & 23 \\
\hline B6 & $\begin{array}{l}\text { GO: Cellular } \\
\text { Component }\end{array}$ & GO:0016459 & Myosin complex & $3.46 \mathrm{E}-04$ & $2.01 \mathrm{E}-02$ & $3.35 \mathrm{E}-03$ & $1.56 \mathrm{E}-02$ & 2 & 68 \\
\hline B6 & $\begin{array}{l}\text { GO: Cellular } \\
\text { Component }\end{array}$ & GO:0015629 & Actin cytoskeleton & $7.89 \mathrm{E}-04$ & $4.58 \mathrm{E}-02$ & $6.54 \mathrm{E}-03$ & $3.04 \mathrm{E}-02$ & 3 & 476 \\
\hline B6 & Domain & PF02736 & Myosin_N & $1.67 \mathrm{E}-05$ & $1.09 \mathrm{E}-03$ & $2.95 \mathrm{E}-04$ & $1.40 \mathrm{E}-03$ & 2 & 15 \\
\hline B6 & Domain & PF01576 & Myosin_tail_1 & $2.43 \mathrm{E}-05$ & $1.58 \mathrm{E}-03$ & $2.95 \mathrm{E}-04$ & $1.40 \mathrm{E}-03$ & 2 & 18 \\
\hline B6 & Domain & IPR002928 & Myosin_tail & $2.43 \mathrm{E}-05$ & $1.58 \mathrm{E}-03$ & $2.95 \mathrm{E}-04$ & $1.40 \mathrm{E}-03$ & 2 & 18 \\
\hline B6 & Domain & IPR027401 & Myosin-like_IQ_dom & $2.72 \mathrm{E}-05$ & $1.77 \mathrm{E}-03$ & $2.95 \mathrm{E}-04$ & $1.40 \mathrm{E}-03$ & 2 & 19 \\
\hline B6 & Domain & 4.10 .270 .10 & - & $2.72 \mathrm{E}-05$ & $1.77 \mathrm{E}-03$ & $2.95 \mathrm{E}-04$ & $1.40 \mathrm{E}-03$ & 2 & 19 \\
\hline B6 & Domain & IPR001609 & Myosin_head_motor_dom & $1.11 \mathrm{E}-04$ & $7.24 \mathrm{E}-03$ & 7.24E-04 & $3.44 \mathrm{E}-03$ & 2 & 38 \\
\hline B6 & Domain & SM00242 & MYSc & $1.11 \mathrm{E}-04$ & $7.24 \mathrm{E}-03$ & $7.24 \mathrm{E}-04$ & $3.44 \mathrm{E}-03$ & 2 & 38 \\
\hline B6 & Domain & PS51456 & MYOSIN_MOTOR & $1.11 \mathrm{E}-04$ & $7.24 \mathrm{E}-03$ & 7.24E-04 & $3.44 \mathrm{E}-03$ & 2 & 38 \\
\hline B6 & Domain & IPR029531 & CAPN3 & $4.27 \mathrm{E}-04$ & $2.78 \mathrm{E}-02$ & $2.52 \mathrm{E}-03$ & $1.20 \mathrm{E}-02$ & 1 & 1 \\
\hline B6 & Domain & SM00015 & IQ & $5.08 \mathrm{E}-04$ & $3.30 \mathrm{E}-02$ & $2.75 \mathrm{E}-03$ & $1.31 \mathrm{E}-02$ & 2 & 81 \\
\hline B6 & Domain & IPR000048 & IQ_motif_EF-hand-BS & $6.27 \mathrm{E}-04$ & $4.08 \mathrm{E}-02$ & $3.11 \mathrm{E}-03$ & $1.48 \mathrm{E}-02$ & 2 & 90 \\
\hline B6 & Domain & PS50096 & IQ & $6.69 \mathrm{E}-04$ & $4.35 \mathrm{E}-02$ & $3.11 \mathrm{E}-03$ & $1.48 \mathrm{E}-02$ & 2 & 93 \\
\hline B6 & Pathway & 1269869 & Striated Muscle Contraction & $1.08 \mathrm{E}-04$ & $5.50 \mathrm{E}-03$ & $5.50 \mathrm{E}-03$ & $2.49 \mathrm{E}-02$ & 2 & 34 \\
\hline B6 & Pathway & 1269896 & Translocation of GLUT4 to the plasma membrane & $3.61 \mathrm{E}-04$ & $1.84 \mathrm{E}-02$ & 7.61E-03 & $3.44 \mathrm{E}-02$ & 2 & 62 \\
\hline B6 & Pathway & P00016 & Cytoskeletal regulation by Rho GTPase & $4.48 \mathrm{E}-04$ & $2.28 \mathrm{E}-02$ & $7.61 \mathrm{E}-03$ & $3.44 \mathrm{E}-02$ & 2 & 69 \\
\hline B6 & Pathway & P00044 & Nicotinic acetylcholine receptor signaling pathway & $7.78 \mathrm{E}-04$ & $3.97 \mathrm{E}-02$ & $9.92 \mathrm{E}-03$ & $4.48 \mathrm{E}-02$ & 2 & 91 \\
\hline B6 & Pubmed & 26871637 & $\begin{array}{l}\text { Widespread Expansion of Protein Interaction Capabilities by } \\
\text { Alternative Splicing. }\end{array}$ & $2.88 \mathrm{E}-05$ & $1.34 \mathrm{E}-02$ & $3.10 \mathrm{E}-04$ & $2.08 \mathrm{E}-03$ & 3 & 374 \\
\hline B6 & Pubmed & 22266860 & $\begin{array}{l}\text { Sp1 phosphorylation by cyclin-dependent kinase } 1 / \text { cyclin B1 } \\
\text { represses its DNA-binding activity during mitosis in } \\
\text { cancer cells. }\end{array}$ & $3.68 \mathrm{E}-05$ & $1.71 \mathrm{E}-02$ & $3.10 \mathrm{E}-04$ & $2.08 \mathrm{E}-03$ & 2 & 54 \\
\hline B6 & Cytoband & $1 \mathrm{p} 31-\mathrm{p} 22$ & $1 \mathrm{p} 31-\mathrm{p} 22$ & $7.79 \mathrm{E}-04$ & $7.01 \mathrm{E}-03$ & $7.01 \mathrm{E}-03$ & $1.98 \mathrm{E}-02$ & 1 & 3 \\
\hline B6 & Cytoband & $4 q 28.1$ & $4 \mathrm{q} 28.1$ & $4.15 \mathrm{E}-03$ & $3.73 \mathrm{E}-02$ & $1.57 \mathrm{E}-02$ & $4.45 \mathrm{E}-02$ & 1 & 16 \\
\hline B6 & Gene Family & 1098 & Myosin heavy chains & $9.50 \mathrm{E}-06$ & $5.70 \mathrm{E}-05$ & $5.70 \mathrm{E}-05$ & $1.40 \mathrm{E}-04$ & 2 & 15 \\
\hline B6 & Gene Family & 777 & Tropomyosins & $1.32 \mathrm{E}-03$ & $7.91 \mathrm{E}-03$ & $1.98 \mathrm{E}-03$ & $4.85 \mathrm{E}-03$ & 1 & 4 \\
\hline B6 & Gene Family & 975 & EF-hand domain-containing|Calpains & $4.94 \mathrm{E}-03$ & $2.96 \mathrm{E}-02$ & 5.93E-03 & $1.45 \mathrm{E}-02$ & 1 & 15 \\
\hline B6 & Drug & CID006419303 & purealin & $1.43 \mathrm{E}-06$ & $2.47 \mathrm{E}-03$ & $2.47 \mathrm{E}-03$ & 1.99E-02 & 3 & 58 \\
\hline B6 & Disease & C1850530 & Flexion contractures of joints & $1.00 \mathrm{E}-05$ & $4.14 \mathrm{E}-03$ & $1.57 \mathrm{E}-03$ & $1.03 \mathrm{E}-02$ & 3 & 93 \\
\hline B6 & Disease & $\mathrm{C} 0333068$ & Flexion contracture & $1.04 \mathrm{E}-05$ & 4.27E-03 & $1.57 \mathrm{E}-03$ & $1.03 \mathrm{E}-02$ & 3 & 94 \\
\hline
\end{tabular}




\begin{tabular}{|c|c|c|c|c|c|c|c|c|c|}
\hline B6 & Disease & $\mathrm{C} 0009918$ & Contracture of joint & $1.14 \mathrm{E}-05$ & 4.69E-03 & $1.57 \mathrm{E}-03$ & $1.03 \mathrm{E}-02$ & 3 & 97 \\
\hline B6 & Disease & C0546264 & Congenital Fiber Type Disproportion & $1.66 \mathrm{E}-05$ & $6.84 \mathrm{E}-03$ & $1.71 \mathrm{E}-03$ & $1.13 \mathrm{E}-02$ & 2 & 13 \\
\hline B6 & Disease & $\mathrm{C} 0009917$ & Contracture & $2.32 \mathrm{E}-05$ & $9.58 \mathrm{E}-03$ & $1.92 \mathrm{E}-03$ & $1.26 \mathrm{E}-02$ & 3 & 123 \\
\hline $\mathrm{B} 7$ & Cytoband & $6 \mathrm{q} 14.2$ & $6 \mathrm{q} 14.2$ & $1.15 \mathrm{E}-03$ & $9.23 \mathrm{E}-03$ & $9.23 \mathrm{E}-03$ & $2.51 \mathrm{E}-02$ & 1 & 5 \\
\hline B7 & Cytoband & $6 \mathrm{p} 25$ & $6 \mathrm{p} 25$ & $3.69 \mathrm{E}-03$ & $2.95 \mathrm{E}-02$ & $1.13 \mathrm{E}-02$ & 3.07E-02 & 1 & 16 \\
\hline B7 & Cytoband & $12 \mathrm{q} 14.3$ & $12 q 14.3$ & $4.61 \mathrm{E}-03$ & $3.69 \mathrm{E}-02$ & $1.13 \mathrm{E}-02$ & $3.07 \mathrm{E}-02$ & 1 & 20 \\
\hline B7 & Cytoband & $1 \mathrm{q} 25$ & $1 \mathrm{q} 25$ & $6.22 \mathrm{E}-03$ & 4.97E-02 & $1.13 \mathrm{E}-02$ & $3.07 \mathrm{E}-02$ & 1 & 27 \\
\hline B7 & Gene Family & 1072 & Alkaline phosphatases & $1.10 \mathrm{E}-03$ & $6.59 \mathrm{E}-03$ & $4.12 \mathrm{E}-03$ & $1.01 \mathrm{E}-02$ & 1 & 4 \\
\hline B7 & Gene Family & 832 & Phospholipases|C2 domain containing phospholipases & $5.21 \mathrm{E}-03$ & $3.13 \mathrm{E}-02$ & $1.04 \mathrm{E}-02$ & $2.55 \mathrm{E}-02$ & 1 & 19 \\
\hline B7 & Gene Family & 778 & Tubulins & $7.13 \mathrm{E}-03$ & $4.28 \mathrm{E}-02$ & $1.07 \mathrm{E}-02$ & $2.62 \mathrm{E}-02$ & 1 & 26 \\
\hline B8 & Domain & IPR024818 & ASXL3 & $1.07 \mathrm{E}-04$ & $1.92 \mathrm{E}-03$ & $7.21 \mathrm{E}-04$ & $2.52 \mathrm{E}-03$ & 1 & 1 \\
\hline B8 & Domain & IPR026905 & ASX-like_PHD & $3.20 \mathrm{E}-04$ & $5.76 \mathrm{E}-03$ & $7.21 \mathrm{E}-04$ & $2.52 \mathrm{E}-03$ & 1 & 3 \\
\hline $\mathrm{B} 8$ & Domain & PF05066 & HARE-HTH & $3.20 \mathrm{E}-04$ & $5.76 \mathrm{E}-03$ & $7.21 \mathrm{E}-04$ & $2.52 \mathrm{E}-03$ & 1 & 3 \\
\hline $\mathrm{B} 8$ & Domain & IPR007759 & HB1/Asxl_HTH & $3.20 \mathrm{E}-04$ & $5.76 \mathrm{E}-03$ & $7.21 \mathrm{E}-04$ & $2.52 \mathrm{E}-03$ & 1 & 3 \\
\hline $\mathrm{B} 8$ & Domain & PF13922 & PHD_3 & $3.20 \mathrm{E}-04$ & $5.76 \mathrm{E}-03$ & $7.21 \mathrm{E}-04$ & $2.52 \mathrm{E}-03$ & 1 & 3 \\
\hline B8 & Domain & PF13919 & ASXH & $3.20 \mathrm{E}-04$ & $5.76 \mathrm{E}-03$ & $7.21 \mathrm{E}-04$ & $2.52 \mathrm{E}-03$ & 1 & 3 \\
\hline B8 & Domain & IPR028020 & ASXH & $3.20 \mathrm{E}-04$ & $5.76 \mathrm{E}-03$ & $7.21 \mathrm{E}-04$ & $2.52 \mathrm{E}-03$ & 1 & 3 \\
\hline B8 & Pubmed & 28100473 & $\begin{array}{l}\text { Delineating the phenotypic spectrum of Bainbridge-Ropers } \\
\text { syndrome: } 12 \text { new patients with de novo, heterozygous, } \\
\text { loss-of-function mutations in ASXL3 and review of } \\
\text { published literature. }\end{array}$ & $5.69 \mathrm{E}-05$ & $1.71 \mathrm{E}-03$ & $1.55 \mathrm{E}-04$ & $6.20 \mathrm{E}-04$ & 1 & 1 \\
\hline B8 & Pubmed & 15138607 & Identification and characterization of ASXL3 gene in silico. & $5.69 \mathrm{E}-05$ & $1.71 \mathrm{E}-03$ & $1.55 \mathrm{E}-04$ & $6.20 \mathrm{E}-04$ & 1 & 1 \\
\hline B8 & Pubmed & 27901041 & $\begin{array}{l}\text { Bainbridge-Ropers syndrome caused by loss-of-function } \\
\text { variants in ASXL3: a recognizable condition. }\end{array}$ & $5.69 \mathrm{E}-05$ & $1.71 \mathrm{E}-03$ & $1.55 \mathrm{E}-04$ & $6.20 \mathrm{E}-04$ & 1 & 1 \\
\hline B8 & Pubmed & 24044690 & $\begin{array}{l}\text { De novo frameshift mutation in ASXL3 in a patient with } \\
\text { global developmental delay, microcephaly, and } \\
\text { craniofacial anomalies. }\end{array}$ & $5.69 \mathrm{E}-05$ & $1.71 \mathrm{E}-03$ & $1.55 \mathrm{E}-04$ & $6.20 \mathrm{E}-04$ & 1 & 1 \\
\hline B8 & Pubmed & 28431838 & $\begin{array}{l}\text { Global developmental delay and postnatal microcephaly: } \\
\text { Bainbridge-Ropers syndrome with a new mutation in } \\
\text { ASXL3. }\end{array}$ & $5.69 \mathrm{E}-05$ & $1.71 \mathrm{E}-03$ & $1.55 \mathrm{E}-04$ & $6.20 \mathrm{E}-04$ & 1 & 1 \\
\hline B8 & Pubmed & 25856206 & $\begin{array}{l}\text { Unlike ASXL1 and ASXL2 mutations, ASXL3 mutations } \\
\text { are rare events in acute myeloid leukemia with } \mathrm{t}(8 ; 21) .\end{array}$ & $5.69 \mathrm{E}-05$ & $1.71 \mathrm{E}-03$ & $1.55 \mathrm{E}-04$ & $6.20 \mathrm{E}-04$ & 1 & 1 \\
\hline B8 & Pubmed & 23736028 & Functional and cancer genomics of ASXL family members. & $5.69 \mathrm{E}-05$ & $1.71 \mathrm{E}-03$ & $1.55 \mathrm{E}-04$ & $6.20 \mathrm{E}-04$ & 1 & 1 \\
\hline B8 & Pubmed & 10723735 & $\begin{array}{l}\text { Evolution of alpha 2-fucosyltransferase genes in primates: } \\
\text { relation between an intronic Alu-Y element and red cell } \\
\text { expression of ABH antigens. }\end{array}$ & $5.69 \mathrm{E}-05$ & $1.71 \mathrm{E}-03$ & $1.55 \mathrm{E}-04$ & $6.20 \mathrm{E}-04$ & 1 & 1 \\
\hline B8 & Pubmed & 27075689 & $\begin{array}{l}\text { Novel splicing mutation in the ASXL3 gene causing } \\
\text { Bainbridge-Ropers syndrome. }\end{array}$ & $5.69 \mathrm{E}-05$ & $1.71 \mathrm{E}-03$ & $1.55 \mathrm{E}-04$ & $6.20 \mathrm{E}-04$ & 1 & 1 \\
\hline B8 & Pubmed & 23383720 & $\begin{array}{l}\text { De novo truncating mutations in ASXL3 are associated with } \\
\text { a novel clinical phenotype with similarities to Bohring- } \\
\text { Opitz syndrome. }\end{array}$ & $5.69 \mathrm{E}-05$ & $1.71 \mathrm{E}-03$ & $1.55 \mathrm{E}-04$ & $6.20 \mathrm{E}-04$ & 1 & 1 \\
\hline B8 & Pubmed & 28097878 & Focusing on frequent ASXL1 mutations in myeloid & $5.69 \mathrm{E}-05$ & $1.71 \mathrm{E}-03$ & $1.55 \mathrm{E}-04$ & $6.20 \mathrm{E}-04$ & 1 & 1 \\
\hline
\end{tabular}




\begin{tabular}{|c|c|c|c|c|c|c|c|c|c|}
\hline & & & $\begin{array}{l}\text { neoplasms, and considering rarer ASXL2 and ASXL3 } \\
\text { mutations. }\end{array}$ & & & & & & \\
\hline B8 & Pubmed & 7876235 & $\begin{array}{l}\text { Sequence and expression of a candidate for the human } \\
\text { Secretor blood group alpha(1,2)fucosyltransferase gene } \\
\text { (FUT2). Homozygosity for an enzyme-inactivating } \\
\text { nonsense mutation commonly correlates with the non- } \\
\text { secretor phenotype. }\end{array}$ & $1.14 \mathrm{E}-04$ & $3.41 \mathrm{E}-03$ & 2.27E-04 & $9.09 \mathrm{E}-04$ & 1 & 2 \\
\hline B8 & Pubmed & 9219535 & $\begin{array}{l}\text { Structure and expression of the gene encoding secretor-type } \\
\text { galactoside 2-alpha-L-fucosyltransferase (FUT2). }\end{array}$ & $1.14 \mathrm{E}-04$ & $3.41 \mathrm{E}-03$ & $2.27 \mathrm{E}-04$ & $9.09 \mathrm{E}-04$ & 1 & 2 \\
\hline B8 & Pubmed & 25450400 & $\begin{array}{l}\text { Repression of LXR? by a novel member of additional sex } \\
\text { comb-like family, ASXL3. }\end{array}$ & $1.14 \mathrm{E}-04$ & $3.41 \mathrm{E}-03$ & $2.27 \mathrm{E}-04$ & $9.09 \mathrm{E}-04$ & 1 & 2 \\
\hline B8 & Pubmed & 18067503 & $\begin{array}{l}\text { Sec1-FUT2-Sec1 hybrid allele generated by interlocus gene } \\
\text { conversion. }\end{array}$ & $1.14 \mathrm{E}-04$ & $3.41 \mathrm{E}-03$ & 2.27E-04 & $9.09 \mathrm{E}-04$ & 1 & 2 \\
\hline B8 & Pubmed & 26167880 & $\begin{array}{l}\text { SR protein kinases promote splicing of nonconsensus } \\
\text { introns. }\end{array}$ & $1.39 \mathrm{E}-04$ & $4.18 \mathrm{E}-03$ & $2.61 \mathrm{E}-04$ & $1.04 \mathrm{E}-03$ & 2 & 361 \\
\hline B8 & Pubmed & 26647312 & $\begin{array}{l}\text { De novo dominant ASXL3 mutations alter H2A } \\
\text { deubiquitination and transcription in Bainbridge-Ropers } \\
\text { syndrome. }\end{array}$ & $1.71 \mathrm{E}-04$ & $5.12 \mathrm{E}-03$ & $2.84 \mathrm{E}-04$ & $1.14 \mathrm{E}-03$ & 1 & 3 \\
\hline B8 & Pubmed & 25835095 & $\begin{array}{l}\text { Functional proteomics of the epigenetic regulators ASXL1, } \\
\text { ASXL2 and ASXL3: a convergence of proteomics and } \\
\text { epigenetics for translational medicine. }\end{array}$ & $1.71 \mathrm{E}-04$ & $5.12 \mathrm{E}-03$ & $2.84 \mathrm{E}-04$ & $1.14 \mathrm{E}-03$ & 1 & 3 \\
\hline B8 & Pubmed & 16412590 & $\begin{array}{l}\text { Characterization of Asxl1, a murine homolog of Additional } \\
\text { sex combs, and analysis of the Asx-like gene family. }\end{array}$ & $2.27 \mathrm{E}-04$ & $6.82 \mathrm{E}-03$ & $3.59 \mathrm{E}-04$ & $1.44 \mathrm{E}-03$ & 1 & 4 \\
\hline B8 & Pubmed & 23932459 & $\begin{array}{l}\text { Genome-wide association study of lung function phenotypes } \\
\text { in a founder population. }\end{array}$ & $6.82 \mathrm{E}-04$ & $2.05 \mathrm{E}-02$ & $1.02 \mathrm{E}-03$ & 4.09E-03 & 1 & 12 \\
\hline B8 & Pubmed & 24941225 & $\begin{array}{l}\text { Genetic variations affecting serum carcinoembryonic antigen } \\
\text { levels and status of regional lymph nodes in patients with } \\
\text { sporadic colorectal cancer from Southern China. }\end{array}$ & 7.39E-04 & $2.22 \mathrm{E}-02$ & $1.06 \mathrm{E}-03$ & $4.22 \mathrm{E}-03$ & 1 & 13 \\
\hline B8 & Interaction & int:ASXL3 & ASXL3 interactions & $3.51 \mathrm{E}-04$ & $2.46 \mathrm{E}-03$ & $1.64 \mathrm{E}-03$ & $4.25 \mathrm{E}-03$ & 1 & 3 \\
\hline B8 & Interaction & int:ANKRD35 & ANKRD35 interactions & $4.68 \mathrm{E}-04$ & $3.28 \mathrm{E}-03$ & $1.64 \mathrm{E}-03$ & $4.25 \mathrm{E}-03$ & 1 & 4 \\
\hline B8 & Cytoband & $18 \mathrm{q} 11$ & $18 \mathrm{q} 11$ & $1.73 \mathrm{E}-04$ & $5.19 \mathrm{E}-04$ & $5.19 \mathrm{E}-04$ & $9.52 \mathrm{E}-04$ & 1 & 2 \\
\hline B8 & Cytoband & $1 q 21.1$ & $1 \mathrm{q} 21.1$ & $7.17 \mathrm{E}-03$ & $2.15 \mathrm{E}-02$ & $9.32 \mathrm{E}-03$ & $1.71 \mathrm{E}-02$ & 1 & 83 \\
\hline $\mathrm{B} 8$ & Cytoband & $19 q 13.33$ & $19 \mathrm{q} 13.33$ & $9.32 \mathrm{E}-03$ & $2.80 \mathrm{E}-02$ & $9.32 \mathrm{E}-03$ & $1.71 \mathrm{E}-02$ & 1 & 108 \\
\hline B8 & Gene Family & 403 & Ankyrin repeat domain containing $\mid$ BRCA1 B complex & $1.33 \mathrm{E}-02$ & $1.33 \mathrm{E}-02$ & $1.33 \mathrm{E}-02$ & $1.33 \mathrm{E}-02$ & 1 & 242 \\
\hline B8 & Disease & C3809650 & BAINBRIDGE-ROPERS SYNDROME & $1.23 \mathrm{E}-04$ & $2.35 \mathrm{E}-03$ & $1.17 \mathrm{E}-03$ & $4.16 \mathrm{E}-03$ & 1 & 1 \\
\hline B8 & Disease & cv:CN180235 & Bainbridge-Ropers syndrome & $1.23 \mathrm{E}-04$ & $2.35 \mathrm{E}-03$ & $1.17 \mathrm{E}-03$ & $4.16 \mathrm{E}-03$ & 1 & 1 \\
\hline
\end{tabular}

